\title{
The PIP-II Conceptual Design Report
}

\author{
V0.00 \\ March 1, 2017
}

\section{Primary authors:}

M. Ball, A. Burov, B. Chase, A. Chakravarty, A. Chen, S. Dixon, J. Edelen, A. Grassellino, D. Johnson, S. Holmes, S. Kazakov, A. Klebaner, I. Kourbanis, A. Leveling, O. Melnychuk, D. Neuffer, T. Nicol, J.-F. Ostiguy, R. Pasquinelli, D. Passarelli, L. Ristori, W. Pellico, J. Patrick, L. Prost, I. Rakhno, A. Saini, W. Schappert, A. Shemyakin, J. Steimel, V. Scarpine, A. Vivoli, A. Warner, V. Yakovlev

\author{
/ Fermi National Accelerator Laboratory \\ P. Ostroumov, Z. Conway \\ / Argonne National Accelerator Laboratory
}

Edited by:

Valeri Lebedev / Fermi National Accelerator Laboratory 


\section{Contents}

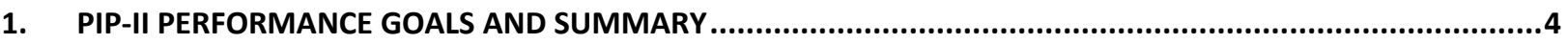

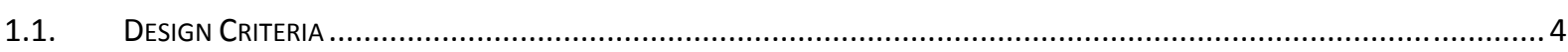

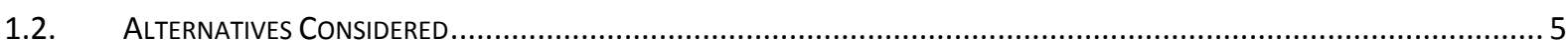

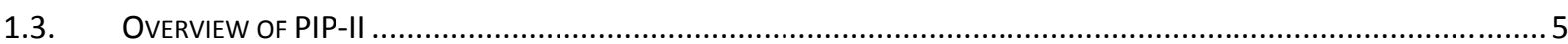

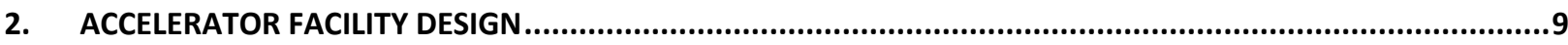

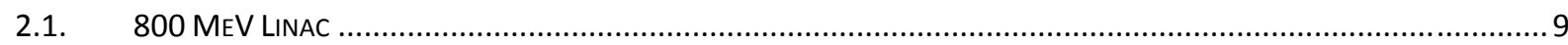

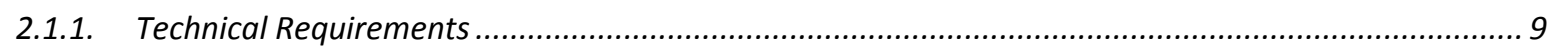

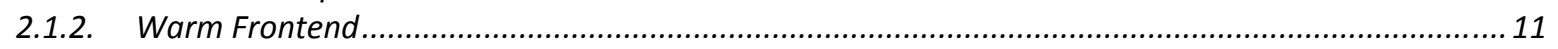

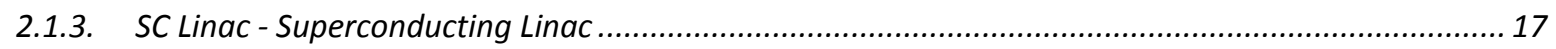

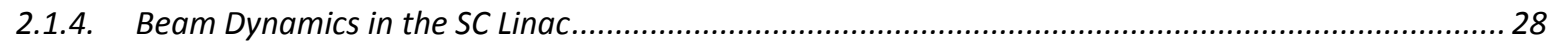

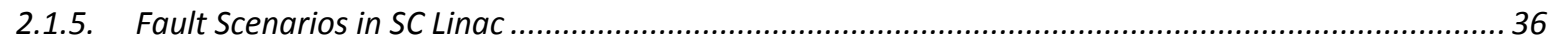

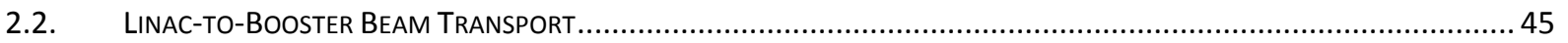

2.2.1. Particle Loss and Limitations on Beam Transport Parameters................................................... 45

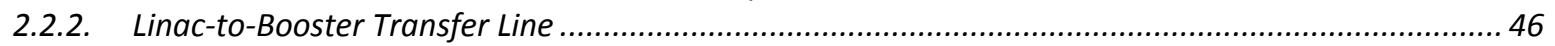

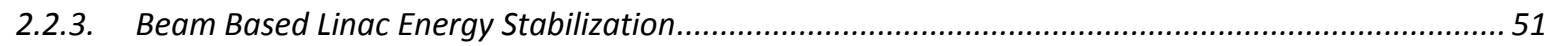

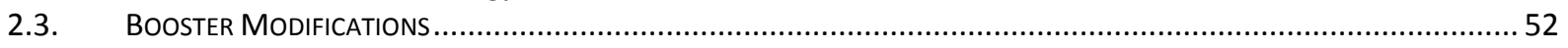

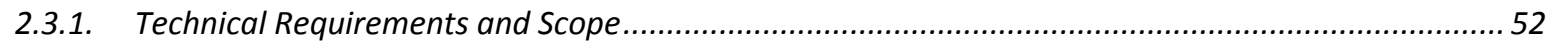

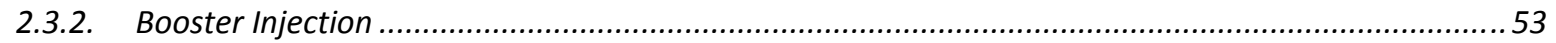

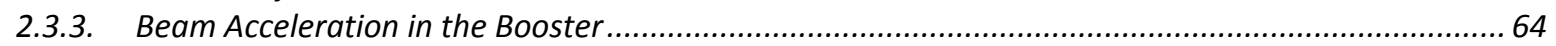

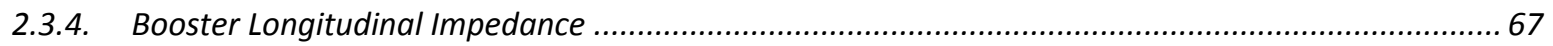

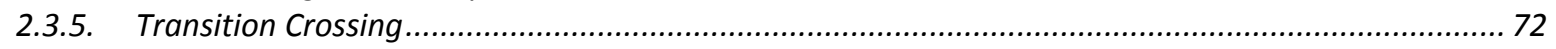

2.3.6. Modifications to the Booster Magnet System Required for $20 \mathrm{~Hz}$ Operation .................................. 76

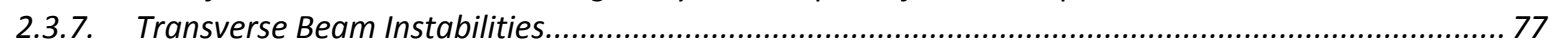

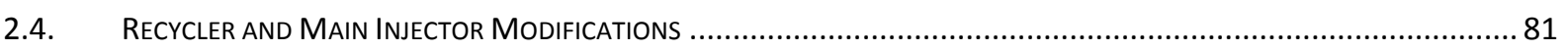

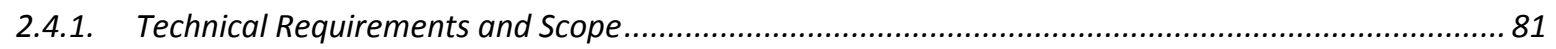

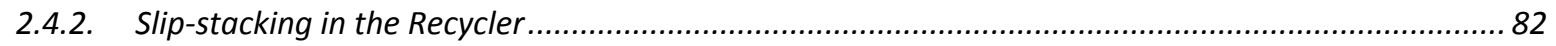

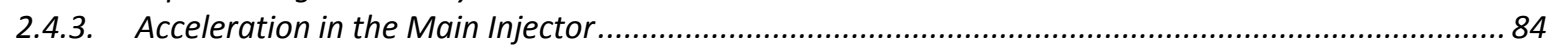

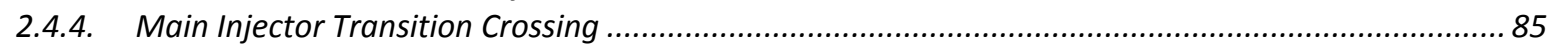

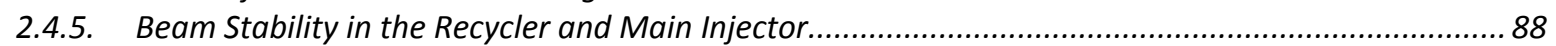

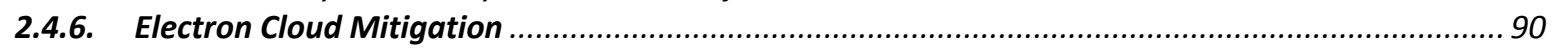

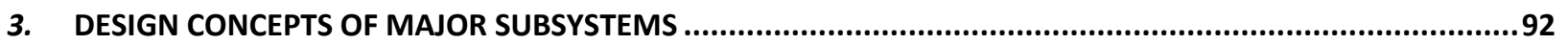

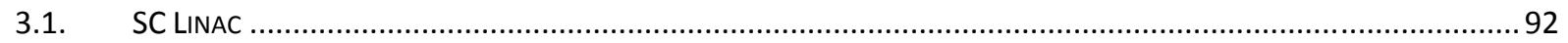

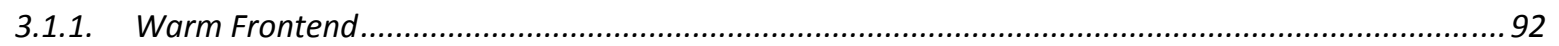

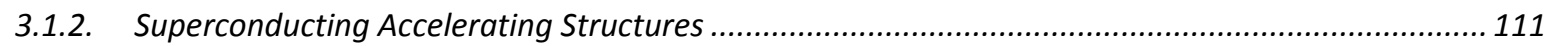

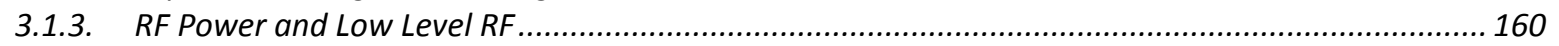

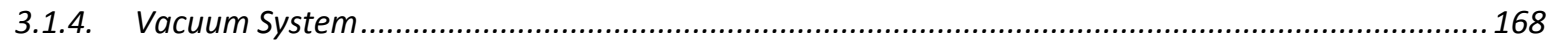

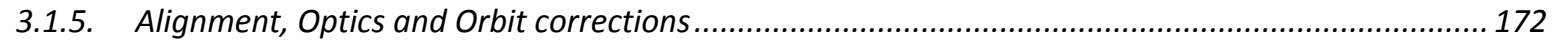

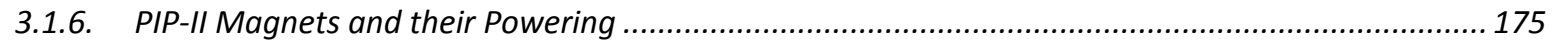

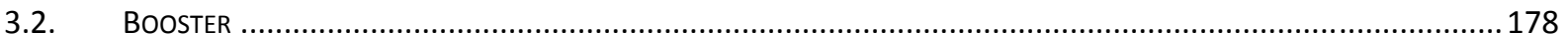

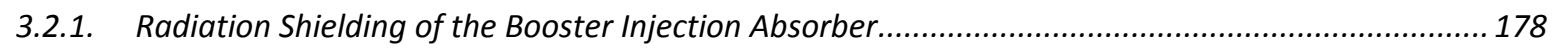

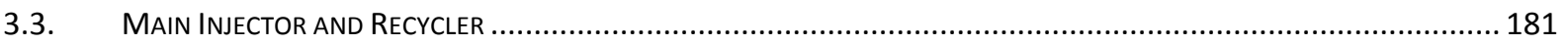

3.3.1. Hardware for Main Injector Transition Crossing .................................................................. 181

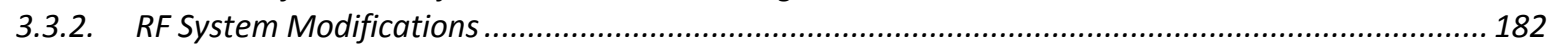

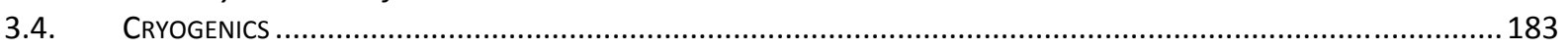

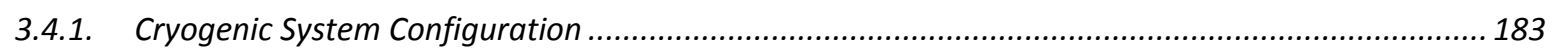

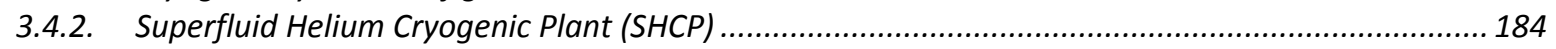

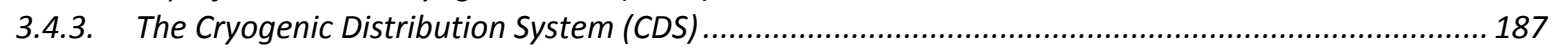

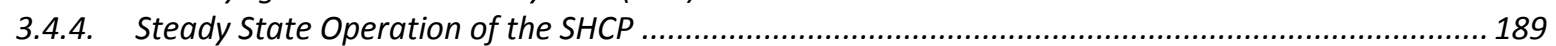




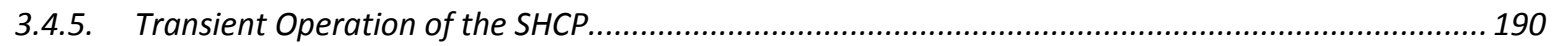

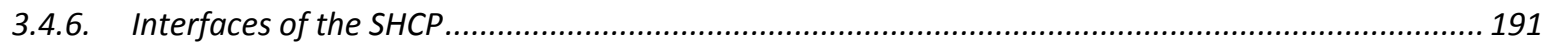

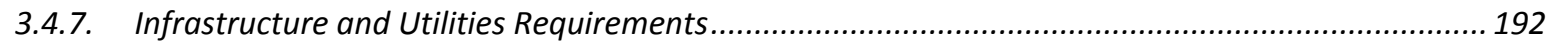

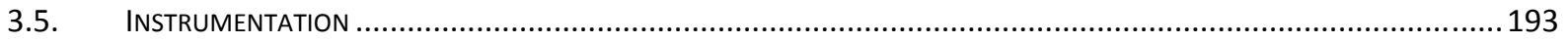

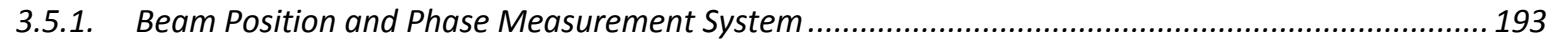

3.5.2. Beam Loss Measurement System........................................................................................ 198

3.5.3. Beam Intensity Measurement System...................................................................................... 199

3.5.4. Beam Transverse Profile Measurement System .................................................................200

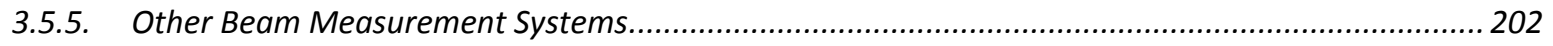

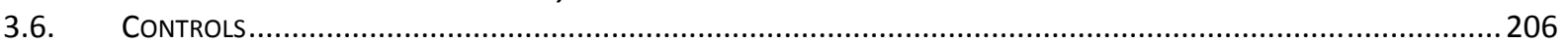

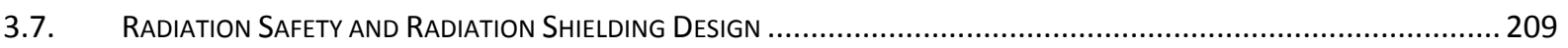

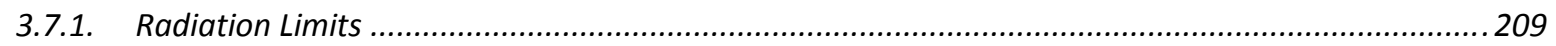

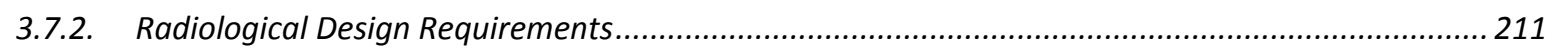

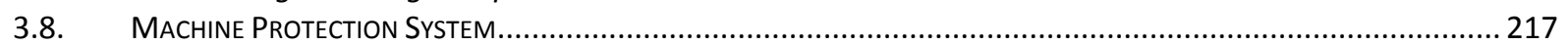

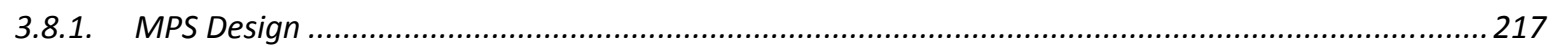

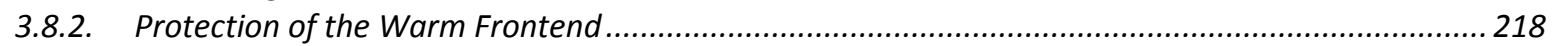

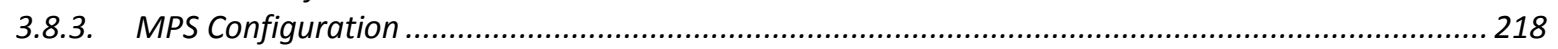

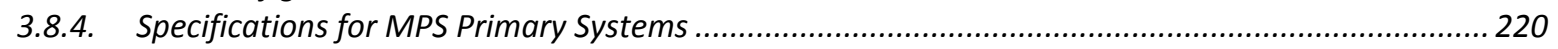

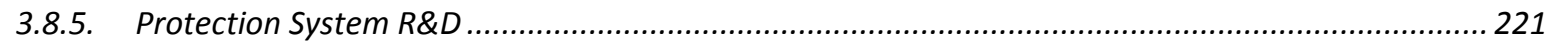

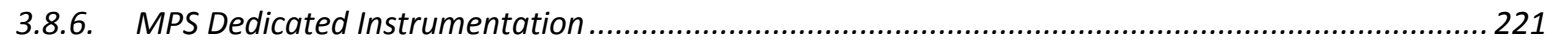

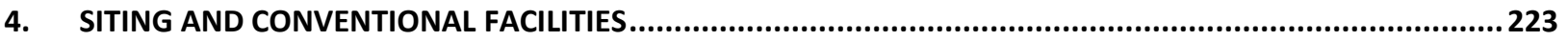

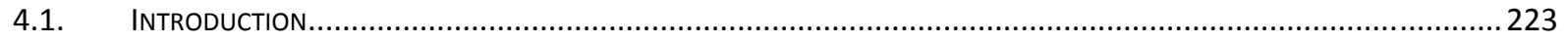

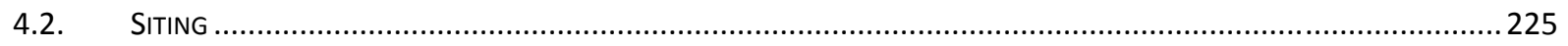

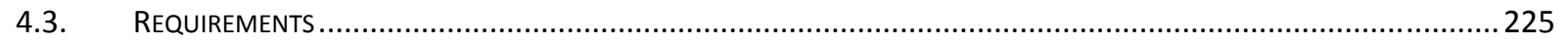

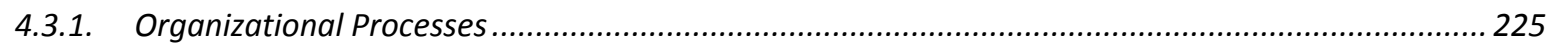

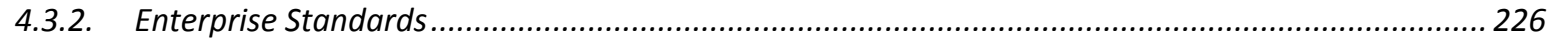

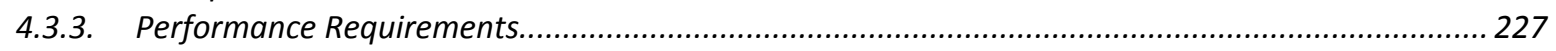

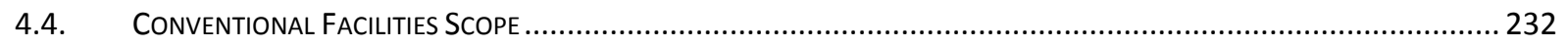

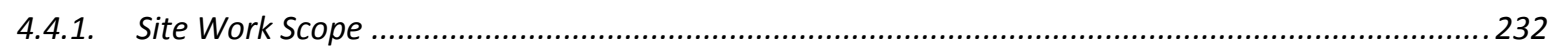

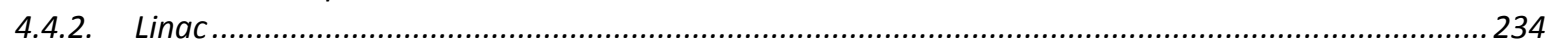

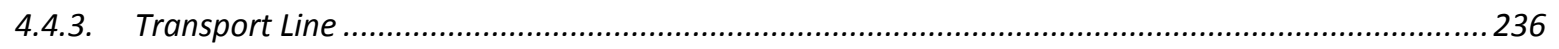

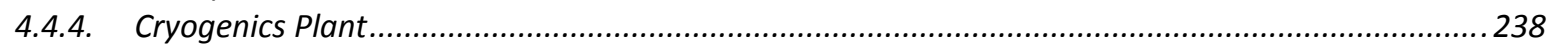

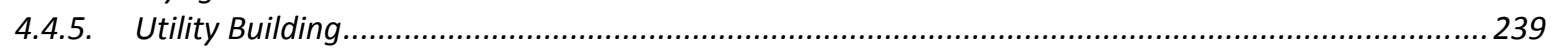

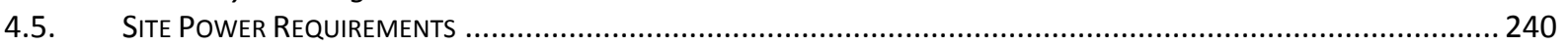

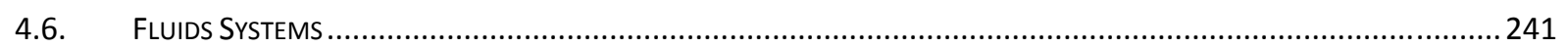

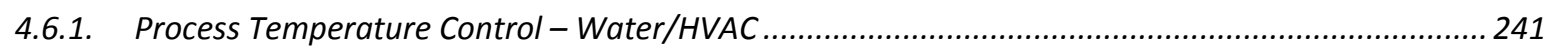

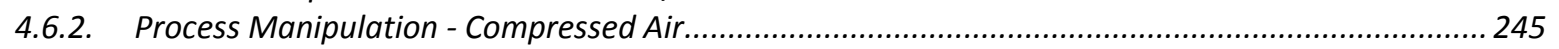

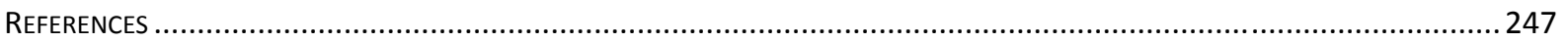

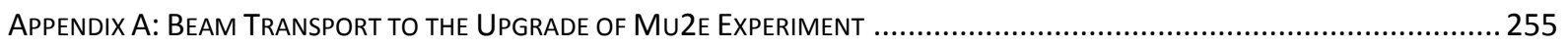

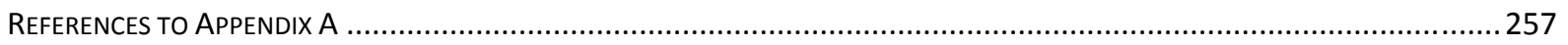




\section{PIP-II Performance Goals and Summary}

The Proton Improvement Plan-II (PIP-II) encompasses a set of upgrades and improvements to the Fermilab accelerator complex aimed at supporting a world-leading neutrino program over the next several decades. PIP-II is an integral part of the strategic plan for U.S. High Energy Physics as described in the Particle Physics Project Prioritization Panel (P5) report of May 2014 [1] and formalized through the Mission Need Statement approved in November 2015. As an immediate goal, PIP-II is focused on upgrades to the Fermilab accelerator complex capable of providing proton beam power in excess of $1 \mathrm{MW}$ on target at the initiation of the Long Baseline Neutrino Facility/Deep Underground Neutrino Experiment (LBNF/DUNE) program [2], currently anticipated for the mid2020s. PIP-II is a part of a longer-term goal of establishing a high-intensity proton facility that is unique within the world, ultimately leading to multi-MW capabilities at Fermilab.

PIP-II builds on three major recent developments at Fermilab: 1) the recently completed upgrades to the Recycler and Main Injector (MI) for the NOvA experiment, 2) the Proton Improvement Plan [3] currently nearing completion, and 3) the development of world-class capabilities in superconducting radio frequency (SRF) acceleration over the last decade. In parallel, the recently launched LBNF/DUNE project has created a compelling need for a revitalization of the Fermilab complex to support a vision of a research program based on very high intensity proton capabilities.

The NOvA project included the conversion of the Recycler Ring from an antiproton storage to a proton accumulation facility, while the Proton Improvement Plan (PIP) consolidates a set of improvements to the existing Linac, Booster, and Main Injector that now supports $15 \mathrm{~Hz}$ Booster beam operation. In combination, the NOvA upgrades and PIP create a capability of delivering 700 $\mathrm{kW}$ beam power from the Main Injector at $120 \mathrm{GeV}$ and set the stage for PIP-II to deliver in excess of $1 \mathrm{MW}$ at the start of the LBNF/DUNE experimental program.

This document describes the Conceptual Design for PIP-II. This concept balances the long-term vision of Fermilab's long baseline neutrino mission with the near- and mid-term goals endorsed by the P5 report and the Mission Need Statement.

\subsection{Design Criteria}

The P5 report and the Mission Need Statement call for a performance upgrade of the Fermilab accelerator complex to support a world-leading neutrino program, while maintaining high-reliability operations through the rejuvenation of aging systems within this complex and providing a platform for future enhancements. Based on these performance requirements pre-conceptual development for PIP-II has proceeded with following design criteria:

- Deliver 1.2 MW of proton beam power from the Fermilab Main Injector, over the energy range $60-120 \mathrm{GeV}$, at the start of operations of the LBNF/DUNE program;

- Sustain high reliability operations of the Fermilab accelerator complex throughout the initial phase of LBNF/DUNE operations;

- Support the currently operating and envisioned 8-GeV program at Fermilab including Mu2e, g-2, and the suite of short-baseline neutrino experiments;

- Provide a platform for eventual extension of beam power to LBNF/DUNE to $>2 \mathrm{MW}$;

- Provide a flexible platform for long-range development of the Fermilab complex; in particular, provide an upgrade path for a factor of $\sim 10$ increase in beam power to the Mu2e experiment, and for extension of accelerator capabilities to include flexible high- 
bandwidth pulse formatting/high beam power operations.

The primary bottleneck limiting beam power to the LBNF target is the existing Linac/Booster. Performance following the completion of the PIP will be limited to about $4.2 \times 10^{12}$ protons per Booster pulse by beam losses - primarily driven by space-charge forces at the $400 \mathrm{MeV}$ Booster injection energy. The secondary bottleneck is slip-stacking of twelve Booster pulses in the Recycler - this performance is determined jointly by characteristics of the Recycler itself and by the characteristics of beam delivered from the Booster. Hence the primary need is to raise the injection energy into the Booster in order to support a roughly 50\% increase in per-pulse beam intensity, while implementing modifications to the Booster, Recycler, and Main Injector to carry this increased beam intensity all the way to the LBNF target. A concept suggested in the PIP-II Reference Design [4] was further developed to satisfy these criteria and is described in this Conceptual Design Report.

\subsection{Alternatives Considered}

A number of different approaches could be used to upgrade the Fermilab accelerator complex to achieve beam power in excess of $1 \mathrm{MW}$ on the LBNF target. The challenge is to identify solutions that provide an appropriate balance between minimizing near-term costs and maintaining the flexibility to support longer-term physics goals. Following the release of the Mission Need Statement, four alternatives, including the Reference Design, were identified for analysis as mandated by DOE 413.3b. All alternatives were based on raising the injection energy of the Booster to provide a $50 \%$ increase in delivered protons per pulse. Paired with a modest decrease in the Main Injector cycle time (from 1.333 to 1.2 seconds) this provides $1.2 \mathrm{MW}$ beam power at $120 \mathrm{GeV}$. Implementation would be via either new or upgraded linacs, based on either superconducting or normal conducting technologies, and with pulsed vs continuous wave (CW) capabilities. The analysis of alternatives report found that all alternatives could meet the near-term needs of the neutrino program as identified within the Mission Need Statement, while the Reference Design (Alternative 1) provided significant long-term opportunities for the development of the Fermilab accelerator complex into a world-leading high intensity hadron facility. Furthermore, Alternative 1 was found to be realizable at a cost comparable to the other alternatives under the assumption that in-kind contributions from international partners are forthcoming as currently envisioned. Based on this analysis the Acquisition Executive has selected the Reference Design, specifically a continuous wave (CW) superconducting linac, as the preferred option and has instructed that this approach be developed to the Conceptual Design level as described in this report. Because the Reference Design represents a natural continuation of the performance improvements being implemented within the PIP, it has been named Proton Improvement Plan-II (PIP-II).

\subsection{Overview of PIP-II}

The goal of Proton Improvement Plan-II is to enhance the capabilities of the existing Fermilab accelerator complex to support the delivery of 1.2 MW beam power to the LBNF production target, while simultaneously providing a platform for subsequent upgrades of the accelerator complex to multi-MW capability. High-level goals, and supporting beam performance parameters, for PIP-II and their comparison to PIP parameters are given in Table 1-1. The central element of PIP-II is a new $800 \mathrm{MeV}$ superconducting linac accelerating $\mathrm{H}^{-}$ions and located in close proximity to the existing Booster as shown in Figure 1-1. This siting offers several advantages in terms of minimizing cost while retaining options for future development; in particular, the site affords direct access to significant electrical, water, and cryogenic infrastructure while providing a straightforward path for eventual replacement of the Booster, which will be required to support beam powers in excess of 2 
MW.

Table 1-1: PIP-II high-level performance goals

\begin{tabular}{llll}
\hline \hline Performance Parameter & PIP & PIP-II & Unit \\
\hline Linac Beam Energy & 400 & 800 & $\mathrm{MeV}$ \\
Linac Beam Current (chopped) & 25 & 2 & $\mathrm{~mA}$ \\
Linac Pulse Length & 0.03 & 0.54 & $\mathrm{~ms}$ \\
Linac Pulse Repetition Rate & 15 & 20 & $\mathrm{~Hz}$ \\
Linac Upgrade Potential & $\mathrm{N} / \mathrm{A}$ & $\mathrm{CW}$ & \\
Booster Protons per Pulse (extracted) & 4.2 & 6.5 & $10^{12}$ \\
Booster Pulse Repetition Rate & 15 & 20 & $\mathrm{~Hz}$ \\
Booster Beam Power @ 8 GeV & 80 & 166 & $\mathrm{~kW}$ \\
8 GeV Beam Power to LBNF & $\mathrm{N} / \mathrm{A}$ & $83-142^{*}$ & $\mathrm{~kW}$ \\
Beam Power to 8 GeV Program & 30 & $83-24^{*}$ & $\mathrm{~kW}$ \\
Main Injector Protons per Pulse (extracted) & 4.9 & 7.5 & $10^{13}$ \\
Main Injector Cycle Time @ 120 GeV & 1.33 & 1.2 & $\mathrm{sec}$ \\
Main Injector Cycle Time @ 60 GeV & N/A & 0.7 & $\mathrm{sec}$ \\
Beam Power @ 60 GeV & N/A & 1 & $\mathrm{MW}$ \\
Beam Power @ 120 GeV & 0.7 & 1.2 & $\mathrm{MW}$ \\
Upgrade Potential @ 80-120 GeV & N/A & 2.4 & $\mathrm{MW}$ \\
\hline \hline
\end{tabular}

* First number refers to Main Injector operations at $120 \mathrm{GeV}$; second number to $60 \mathrm{GeV}$.

The scope encompassed by the PIP-II as described in this document includes:

- An $800-\mathrm{MeV}$ superconducting linac (SC Linac), constructed of CW-capable accelerating structures and cryomodules, operating with an average $\mathrm{H}^{-}$beam current of $2 \mathrm{~mA}$ and a beam duty factor of $1.1 \%$;

- Beam transport from the end of the SC Linac to the new Booster injection point, and to a new $800-\mathrm{MeV}$ beam dump;

- Upgrades to the Booster to accommodate $800-\mathrm{MeV}$ injection, and acceleration of $6.5 \times 10^{12}$ protons per pulse;

- Upgrades to the Recycler to accommodate slip-stacking of $7.7 \times 10^{13}$ protons delivered by twelve Booster batches;

- Upgrades to the Main Injector to accommodate acceleration of $7.5 \times 10^{13}$ protons per pulse to $120 \mathrm{GeV}$ with a 1.2 second cycle time, and to $60 \mathrm{GeV}$ with a 0.7 second cycle time.

- Associated conventional facilities including enclosures, equipment galleries, and utilities. The linac enclosure will be constructed with a length to accommodate a future possible extension of the linac energy to $1.0 \mathrm{GeV}$

The SC Linac energy is selected to support a 50\% increase in Booster beam intensity, accompanied by a $30 \%$ reduction in the space-charge tune shift as compared to the current operations. This choice is conservative and will ensure lower fractional beam loss required at the higher operating intensities and higher injection energy, while preserving the transverse and longitudinal emittance at current levels. The linac is constructed entirely of components that are capable of 
operating in $\mathrm{CW}$ mode. The incremental cost to DOE in constructing the linac from $\mathrm{CW}$ compatible components is minimal ${ }^{1}$.

The SC Linac is followed by a beam transport line to bring the $\mathrm{H}^{-}$beam to the Booster. This line includes an arc bending the beam by about $210^{\circ}$. The bending radius of the arc is maintained above $23 \mathrm{~m}$ to prevent stripping of the beam prior to Booster injection. There is a provision for installation of an RF separator and septum at the SC Linac end. It could be utilized to support the operation of multiple experiments following future linac upgrades.

The Booster repetition rate will be increased from 15 to $20 \mathrm{~Hz}$. This is extremely helpful for reduction of beam losses during slip-stacking in the Recycler due to the larger momentum difference between the two streams of slip-stacked bunches. The repetition rate increase also increases overall particle flux through the Booster and yields higher power for the $8-\mathrm{GeV}$ experimental program. The operation of Booster gradient magnets at $20 \mathrm{~Hz}$ was recently verified [5]. The transition to the higher rate will be achieved by decreasing the value of capacitors in the magnet resonance circuit. Although the acceleration rate increases proportionally to the repetition rate, the peak RF voltage does not change from that being provided by the PIP due to the smaller slip-factor at the higher injection energy. However, doubling the RF power transferred to the beam, to support the increased beam intensity and more rapid acceleration rate, will require additional minor modifications to the present RF power amplifiers.

Protons will be injected into the Booster using multi-turn strip-injection similar to the injection method used in the SNS [6]. The number of injection turns is equal to about 300. Although the number of injection turns is much larger than what is presently used in the Booster, it is still about three times less than that used in the SNS. The large number of injection turns and small emittances of the SC Linac beam allow painting of transverse and longitudinal distributions resulting in a significant reduction of space-charge effects. In contrast to present operations, beam injection will proceed at non-zero RF voltage. This allows one to avoid adiabatic bunching, which is problematic because of the long bunching time ${ }^{2}$. To reduce beam losses during Booster injection the linac bunches arriving at the RF bucket boundaries are removed by a bunch-by-bunch chopper located in the Medium Energy Beam Transport (MEBT) of the linac. The same chopper creates a three-bunch long extraction gap. Slip-stacking in the Recycler and acceleration in the MI will be done in a manner similar to that presently used for NOvA [7].

Upgrades to a number of systems in the Booster, Recycler, and Main Injector will be required in order to support the higher Booster injection energy and higher beam intensities. These include upgrades to the Booster injection system, the RF systems in all rings, implementation of a $\gamma_{t}-j u m p$ in the Main Injector, and various feedback systems. The upgrade to the Booster injection system is the most significant of these.

Modifications to the LBNF target facility to accept 1.2 MW protons are assumed to be undertaken by the LBNF project. However, the requirements for the beam delivery are described in this document. Note that the concept presented here is capable of delivering from 1 to $1.2 \mathrm{MW}$ of beam power to LBNF for the energy range of 60 to $120 \mathrm{GeV}$. Although the LBNF operation is expected to take a major fraction of Booster intensity, considerable power will still be available at $8 \mathrm{GeV}$. In particular, it is expected to be $83 \mathrm{~kW}$ for $120 \mathrm{GeV}$ operations and $24 \mathrm{~kW}$ for $60 \mathrm{GeV}$.

\footnotetext{
${ }^{1}$ The most expensive part of the accelerator making CW operation possible is a new cryo-plant capable to support $\mathrm{CW}$ operation. It is expected to be an in-kind contribution from the India Department of Atomic Energy.

${ }^{2}$ The long bunching time is related to the smaller value of the slip-factor at the higher injection energy. The higher Booster repetition rate additionally magnifies this problem.
} 


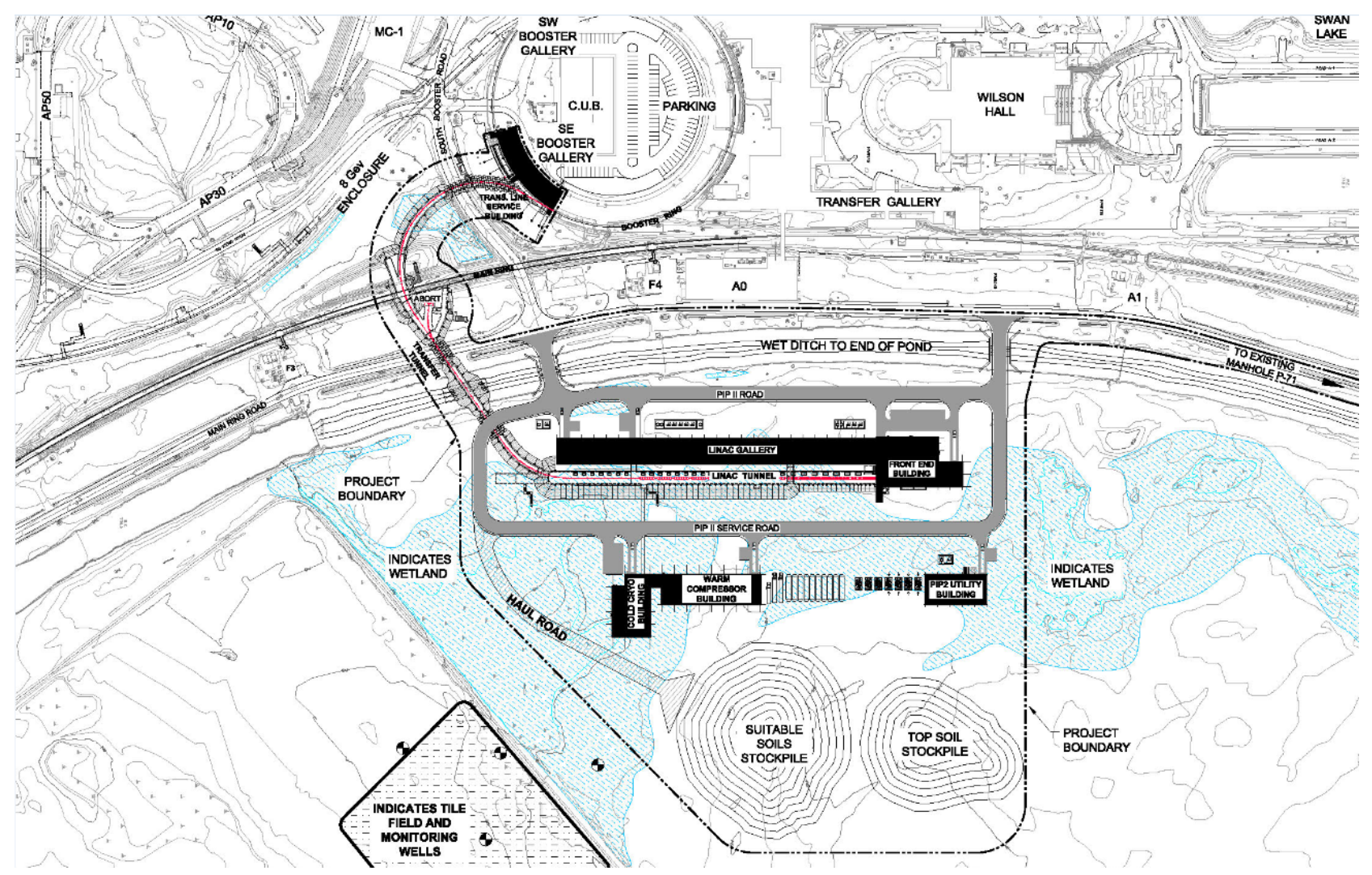

Figure 1-1: Site layout of PIP-II (north is to the right). New construction includes the linac enclosure, transfer line enclosure (including the beam abort area and a stub to facilitate future connection to the Muon Campus), linac gallery, utility building, and cryo/compressor building. The blue areas denote identified wetland areas.

PIP-II provides a variety of straightforward and cost effective upgrade paths. Delivery of more than $2 \mathrm{MW}$ to the LBNF target will require replacement of the existing Booster. The most straightforward strategy would be to extend the $0.8 \mathrm{GeV}$ linac to $1.5-2 \mathrm{GeV}$ and to inject at this energy into a newly constructed 8-GeV rapid cycling synchrotron (RCS). Such a synchrotron could either be of a conventional sort, as currently deployed at the J-PARC facility in Japan, or could incorporate novel design features based on highly non-linear optical elements currently under study at Fermilab. In either case, the siting shown in Figure 1.1 is compatible with siting of such a RCS to the south of the linac, providing capabilities for injecting beam into the Main Injector. 


\section{Accelerator Facility Design}

It is envisioned that the PIP-II construction project will include the superconducting linac (SC Linac), the transfer line connecting the linac and the Booster, and corresponding upgrades to the Booster, Main Injector and Recycler rings. All constituents of the project are closely interconnected, and an achievement of successful operation for each of them represents a considerable challenge. Therefore, they are described below at the same level of detail.

\subsection{MeV Linac}

\subsubsection{Technical Requirements}

The linac includes the following major elements:

- Ion source,

- Low Energy Beam Transport (LEBT),

- RFQ,

- Medium Energy Beam Transport (MEBT), including the bunch-by-bunch chopper and bunching cavities,

- One accelerating section composed of 162.5 MHz Half-Wave Resonators (HWR),

- two accelerating sections composed of $325 \mathrm{MHz}$ Single-Spoke Resonators (SSR1 and SSR2),

- Two accelerating sections of $650 \mathrm{MHz}$ elliptical cavities, one at low beta $\left(\beta_{o p t}=0.64\right)$ and one at high beta $\left(\beta_{\text {opt }}=0.97\right)(\mathrm{LB} 650$ and HB650).

Figure 2.1 shows the structure of the linac. A room temperature (RT) section accelerates the beam to $2.1 \mathrm{MeV}$ and creates the desired bunch structure for injection into the SC Linac. The RFQ and the first SC section (HWR) operate in a $\mathrm{CW}$ mode. In the case when the beam is only delivered to the neutrino program, the rest of the linac is used in a pulsed regime to reduce the required cryogenic power. Otherwise, the entire linac operates in the CW regime. Operation with a peak current of up to $10 \mathrm{~mA}$ is supported by the ion source, LEBT and RFQ. The bunch-by-bunch chopper located in the MEBT removes undesired bunches leaving the beam current at up to $2 \mathrm{~mA}$ (averaged over a few $\mu \mathrm{s})$ for further acceleration. There is also a "slow" chopper in the LEBT with rise and fall times of about $100 \mathrm{~ns}$. It allows forming a macro-structure in the beam timing required for machine commissioning and allows one to avoid unnecessary beam loading in normal operations. Together the LEBT and MEBT choppers form the desired bunch structure.

\section{\begin{tabular}{l|l|l|l|l|l|l|l|} 
LEBT & RFQ & MEBT & $\beta=0.11$ & $\beta=0.22$ & $\beta=0.47$ & $\beta=0.64$ & $\beta=0.97$
\end{tabular}}

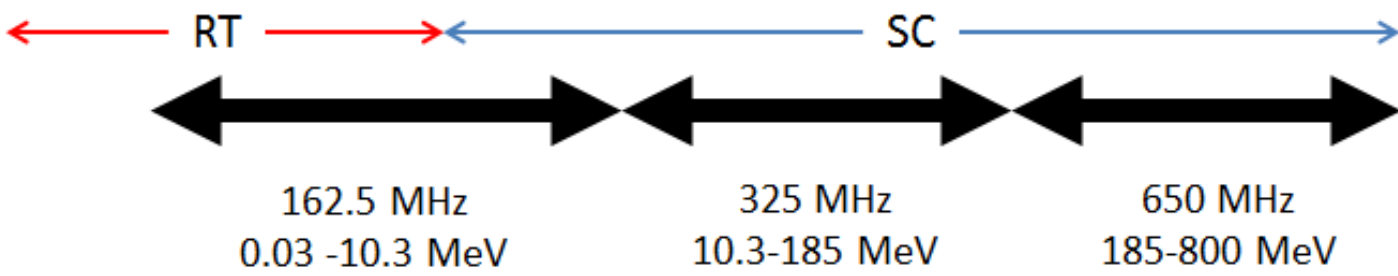

Figure 2.1: The linac technology map. 
The energy stored in the SC cavities is quite large. Consequently, the accelerating voltage variations due to beam loading are below $10^{-3}$ if the bunch structure is repetitive with a period less than about $3 \mu \mathrm{s}$. The SC Linac accelerates up to $2 \mathrm{~mA}$ of beam current to $800 \mathrm{MeV}$ with peak currents of up to $10 \mathrm{~mA}$ for periods of less than a few $\mu \mathrm{s}$. The operational parameters for the $\mathrm{SC}$ Linac are given in Table 2.1 .

Table 2.1: SC Linac Parameters

\begin{tabular}{l|l|l}
\hline \hline Parameter & Requirement & Units \\
\hline Particle species & $\mathrm{H}^{-}$ & \\
\hline Input beam energy (kinetic) & 2.1 & $\mathrm{MeV}$ \\
\hline Output beam energy (kinetic) & 0.8 & $\mathrm{GeV}$ \\
\hline Bunch repetition rate & 162.5 & $\mathrm{MHz}$ \\
\hline RF pulse length & pulsed-to-CW & \\
\hline Sequence of bunches & Programmable & \\
\hline Average beam current in SC Linac & 2 & $\mathrm{~mA}$ \\
\hline Final rms norm. transverse emittance, $\varepsilon_{x}=\varepsilon_{y}$ & $\leq 0.3$ & $\mathrm{~mm}-\mathrm{mrad}$ \\
\hline Final rms norm. longitudinal emittance & $\leq 0.35(1.1)$ & $\mathrm{mm}-\mathrm{mrad}(\mathrm{keV}-\mathrm{ns})$ \\
\hline Rms bunch length at the SC Linac end & 4 & Ps \\
\hline
\end{tabular}

To support beam injection into the Booster, pulsed operation of the linac is sufficient. In this case the linac operates at $20 \mathrm{~Hz}$ with a beam pulse duration of $0.55 \mathrm{~ms}$ resulting in a $1.1 \%$ beam duty factor. The RF cavity filling requires a significantly longer time. The effective cryogenic duty factor is about $6.6 \%$ while the effective duty factor for high power RF is about $15 \%$. To reduce the cryogenic power the phase of the RF amplifiers can be shifted by $180 \mathrm{deg}$. after a beam pulse to accelerate the voltage decay in cavities.

Maintaining sufficiently small emittances through the entire linear accelerator and the beam transport to the Booster is essential for minimizing the beam losses both in the linac and at injection into the Booster. The maximum allowed rms emittances along the accelerator are presented in Table 2.2 for an ion source beam current in the range of $2 \div 5 \mathrm{~mA}$.

Table 2.2: Maximum allowed rms normalized emittances through the accelerator

\begin{tabular}{l|c|c}
\hline \multirow{2}{*}{} & \multicolumn{2}{|c}{$\begin{array}{c}\text { Normalized rms } \\
\mathrm{mrad})\end{array}$} \\
\cline { 2 - 3 } & Transverse & Longitudinal \\
\hline Ion source & 0.14 & - \\
\hline RFQ entrance & 0.18 & - \\
\hline RFQ exit & 0.20 & 0.28 \\
\hline MEBT exit & 0.23 & 0.31 \\
\hline Exit of SC linac & 0.3 & 0.35 \\
\hline
\end{tabular}




\subsubsection{Warm Frontend}

The warm frontend of the PIP-II linac provides an $\mathrm{H}^{-}$beam to the first superconducting module. The frontend beam current specifications go well beyond what is required to support operations of the Booster and Main Injector. While the nominal PIP-II peak current for Booster injection is about $4 \mathrm{~mA}$, the nominal average current of the RFQ is $5 \mathrm{~mA}$ (10 mA maximum). A fast chopper providing bunch-by-bunch chopping is an important part of the frontend. It enables the removal of bunches that are too close to the RF bucket boundaries for in-bucket injection into the Booster. This capability will also be used in future multi-user operations.

The frontend consists of a $30 \mathrm{keV} \mathrm{H}^{-}$ion source, a Low Energy Beam Transport (LEBT) delivering up to $10 \mathrm{~mA}$ DC beam to the entrance of a $2.1 \mathrm{MeV}$ CW Radio Frequency Quadrupole (RFQ) accelerator, and a Medium Energy Beam Transport (MEBT). This is shown schematically by red boxes in Figure 2.1.

The choice for the LEBT energy of $30 \mathrm{keV}$ is a compromise between considerations of beam space charge effects that may increase the transverse emittance at low energy and adiabatic bunching in the RFQ, where the longitudinal emittance is reduced with decreasing the injection energy. This choice balances the final warm frontend emittance among the three degrees of freedom.

The RFQ energy of $2.1 \mathrm{MeV}$ is chosen because it is below the neutron production threshold for most materials, thereby simplifying the RFQ and MEBT maintenance. At the same time, this energy is sufficiently large to mitigate space charge effects in the MEBT with currents as high as $10 \mathrm{~mA}$.

\subsubsection{LEBT - Low Energy Beam Transport}

The conceptual layout of the ion source and LEBT is shown in Figure 2.2. Two ion sources in a "Y"-configuration are installed with a slow switching dipole bending magnet to maximize beam availability. Vacuum valves isolate the ion sources from the rest of the LEBT. Thus, each source can be removed for repairs, reinstalled, and conditioned without interrupting the operation of the other source. The 3-solenoid LEBT (in each leg) transports the beam from the exit of the ion source to the entrance of the RFQ, matching the beam's optical functions to the ones required for low loss acceleration in the RFQ. In addition, a chopping system can form a beam with low duty factor required for commissioning and tuning of the downstream accelerator in a pulsed mode. At the same time, the chopping system interrupts the beam as part of the Machine Protection System (MPS), and prohibits beam to be accelerated in the RFQ accordingly to the safety system status. Ref. [8] presents the Functional Requirement Specifications (FRS) for the LEBT.

Achieving high reliability for the warm frontend and its components is a task of primary importance. With that in mind, a principal goal of the LEBT design is the requirement of maintaining good vacuum in the RFQ, as well as minimizing particle bombardment of the RFQ vanes in order to reduce the frequency of sparking. As a result, a fairly long LEBT $(\sim 2 \mathrm{~m})$ is envisioned in order to isolate the inherently bad vacuum near the ion source exit from good vacuum required at the RFQ entrance. In addition, the bend between the first two solenoids ensures that there is no direct line of sight between the ion sources and the RFQ (as well as the superconducting elements further downstream). This greatly reduces the bombardment of the RFQ vanes by fast neutrals and should help the overall reliability of the RFQ. Good vacuum in the LEBT also reduces the stripping of $\mathrm{H}^{-}$to protons, which can be subsequently accelerated in the RFQ and result in uncontrolled losses in the accelerator downstream.

Similarly, the need for low duty factor operation mainly arises from the desire to limit the potential for failures during tuning of the SRF cryomodules, as well as to limit irradiation of the cavities. 
However, because the neutralization process is not instantaneous, the optimal tune for a short pulse may noticeably differ from long pulse or DC operation. To minimize this effect, the PIP-II LEBT provides an atypical transport scheme in which the beam is not neutralized in the downstream part of the LEBT, independently of its time structure. Figure 2.3 illustrates the geometry of the transport scheme. The vacuum is quite poor near the ion source. It results in a relatively short neutralization time and nearly full beam space charge neutralization in the neutralized section.

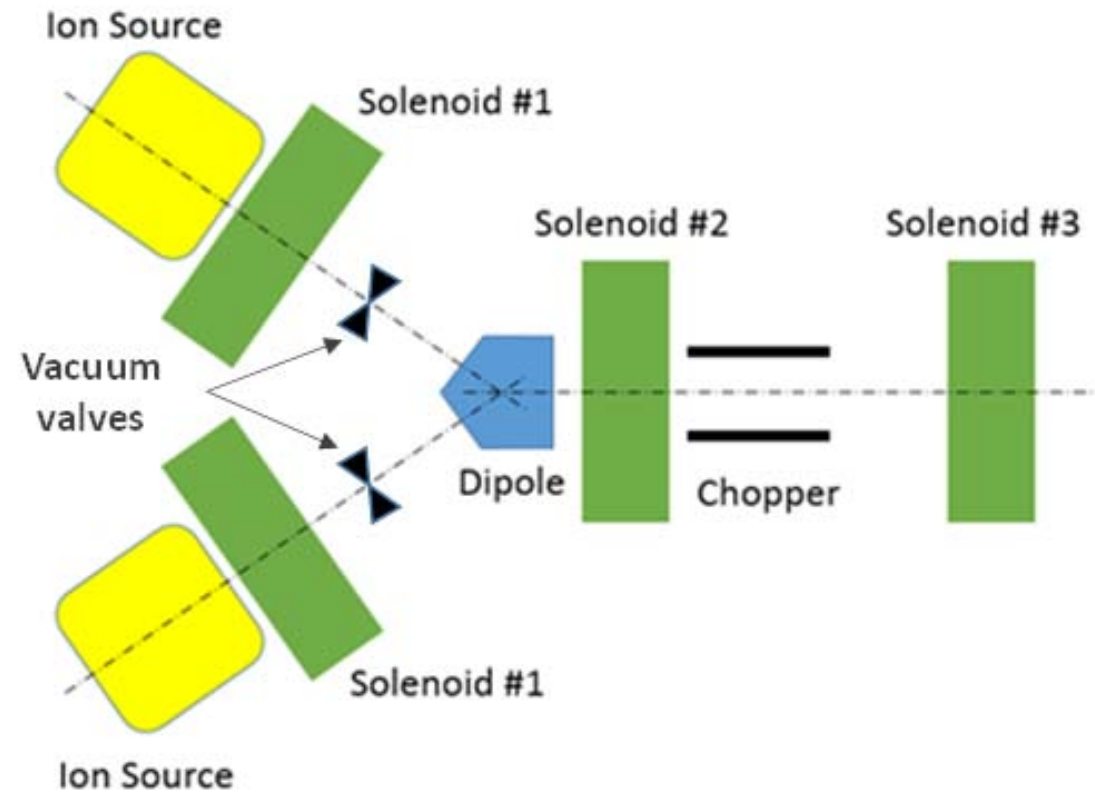

Figure 2.2: Conceptual schematic of the PIP-II LEBT with two ion sources.

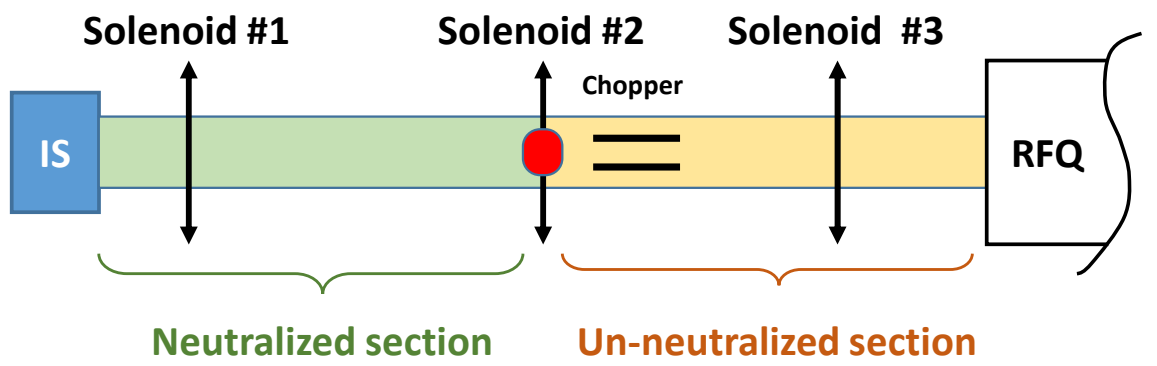

Figure 2.3: Transport scheme concept schematic.

The transition to un-neutralized transport is achieved by combining a potential barrier (red oval on Figure 2.3), which confines neutralized particles upstream, and a clearing electric field removing compensating ions downstream of the potential barrier. The clearing electric field is obtained by applying a DC voltage to one of the chopper electrodes, which sweeps ions out of the beam path. In addition, a low vacuum pressure is maintained between the potential barrier and the RFQ to limit the rate at which neutralized particles are created. The corresponding beam optics is shown in Figure 2.4. This optics has been implemented at the PIP-II Injector Test [9], where a low emittance beam $\left(\varepsilon_{n} \leq 0.18 \mathrm{~mm} \mathrm{mrad}, \mathrm{rms}\right)$ with Twiss parameters adequate for injection into the RFQ was obtained at the end of LEBT.

The location of the chopper, between solenoids \#2 and \#3, hence relatively far from the RFQ entrance, conforms to the principles expressed previously for achieving high reliability (i.e. good vacuum in the RFQ and low particle bombardment of the vanes). In addition, it leaves space for 
diagnostics both before and after solenoid \#3, as well as provides room for a vacuum pump. Note that unlike many other LEBT designs, where a chopping system is located just upstream of the RFQ, the PIP-II LEBT layout provides sufficient room for a simple and robust chopper.

Finally, we would like to stress again that the design of the LEBT described above, beside the beam physics requirements, is driven by requirements of high reliability and efficiency in operation. They call for (1) good vacuum in the RFQ, (2) a simple and reliable chopper, (3) a beam transport scheme, which tuning is independent of the time structure, and (4) sufficient room for diagnostics.

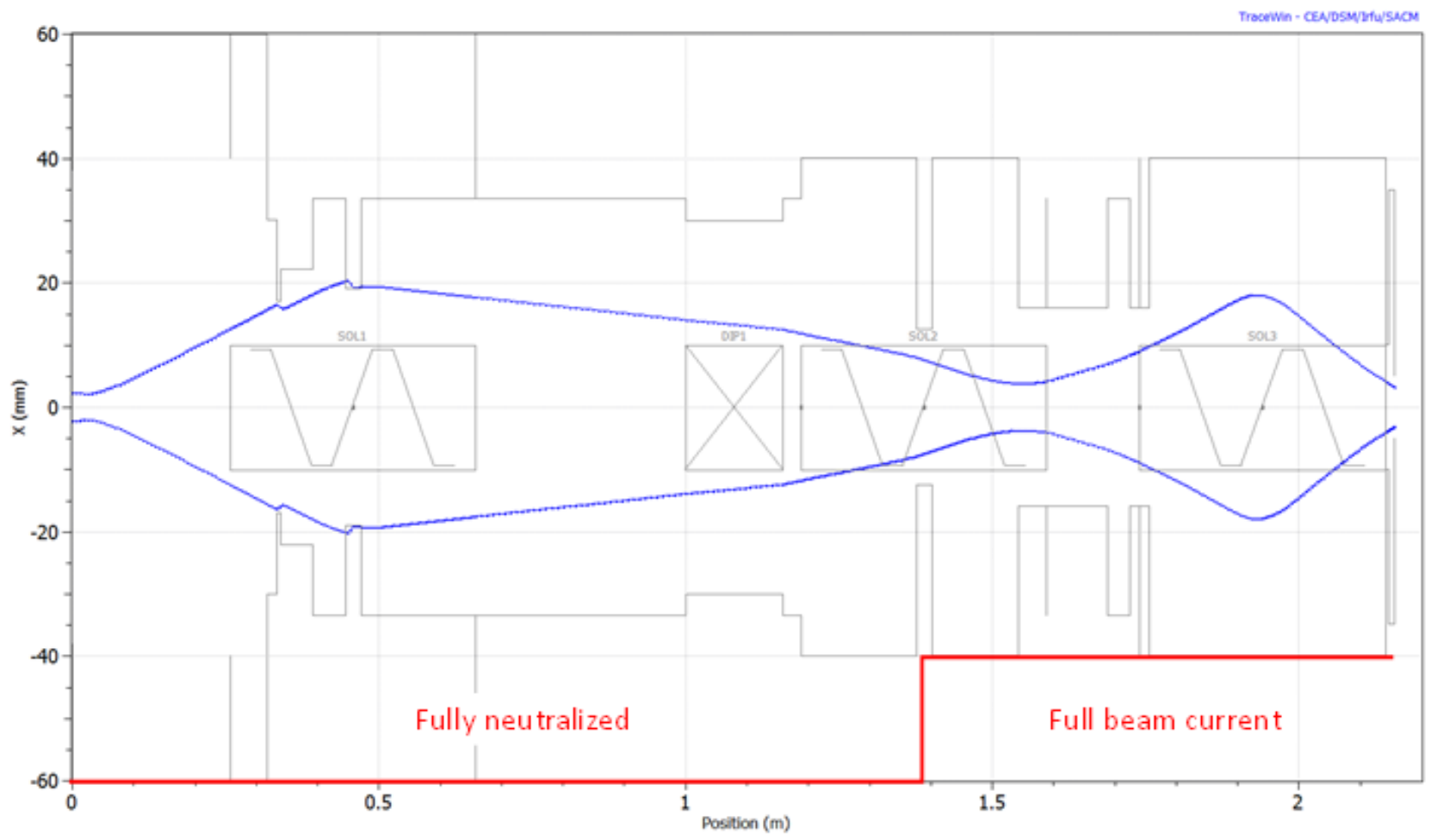

Figure 2.4: Beam horizontal envelope $(2.5 \sigma)$ for the partially un-neutralized LEBT optics solution simulated with TraceWin. The grey lines show aperture limitations. The red line indicates the level of neutralization (from fully neutralized to full beam current of $5 \mathrm{~mA}$ ). Focusing is nearly symmetric which makes the vertical envelope quite close to the horizontal one. The initial distribution was derived from measurements of the ion source phase space carried out initially at LBNL [10] and later at the PIP-II Injector Test (PIP2IT).

\subsubsection{RFQ - Radio Frequency Quadrupole Accelerator}

The $162.5 \mathrm{MHz}$ RFQ accelerates the $30 \mathrm{keV} \mathrm{H}^{-}$ion beam to $2.1 \mathrm{MeV}$ at beam currents of up to $10 \mathrm{~mA} \mathrm{CW}$. The design requirements are presented in Table 2.3.

The requirement of $\mathrm{CW}$ operation forced the design optimization to be aimed at the minimization of RF power loss in the structure since most of RF power is dissipated on the cavity walls creating considerable thermal load. The relatively low nominal vane tip-to-vane tip voltage of $60 \mathrm{kV}$ was adopted to limit the overall RF power requirement, which in turn makes thermal management of the RFQ structure easier. The RF design is based on detailed simulations including 3D electro-magnetic simulations of the entire RFQ. Mode stabilization with pi-mode rods significantly reduces the structure sensitivity to manufacturing errors. The geometry of the vane-ends (vane - to - end-plate transition) was adjusted to achieve good field flatness [11]. It is also supported by 80 slug tuners compensating manufacturing errors and imperfections of simulations. 
The beam dynamics design was simulated using PARMTEQ [12] and it either meets or exceeds all of the requirements in capture efficiency, transmission efficiency and emittance growth. Figure 2.5 shows a PARMTEQ simulation for a $5 \mathrm{~mA}$ beam, ideally matched into the RFQ. The transmission is $99.8 \%$ and output longitudinal emittance is $0.7 \mathrm{keV}-\mathrm{ns}$. For an input emittance of $0.11 \mathrm{~mm} \mathrm{mrad}$, the output emittance is $0.15 \mathrm{~mm} \mathrm{mrad}$, a $35 \%$ increase but still well below the maximum specified. Additionally, error analysis (e.g. mismatch, centroid offsets, field errors...) was carried out and indicate that the design is very robust and error tolerant. Results of simulations of the transverse and longitudinal emittances as functions of the beam current (assuming a $0.11 \mathrm{~mm}-\mathrm{mrad}$ rms normalized emittance at the RFQ entrance) are presented in Figure 2.6.

Table 2.3: Main parameters of the RFQ

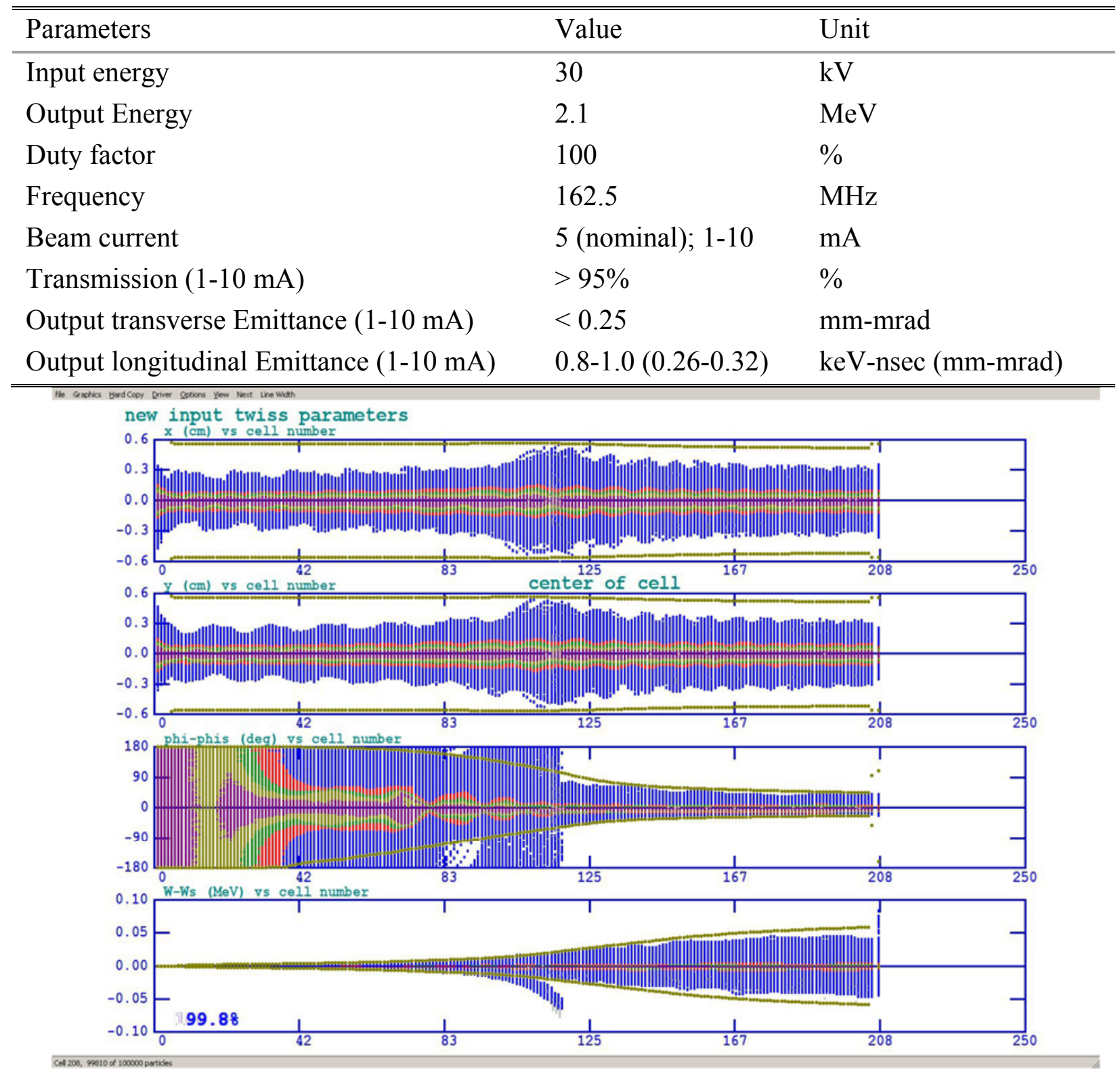

Figure 2.5: PARMTEQ simulation of a $5 \mathrm{~mA}$ beam using 100,000 macro-particles. The initial distribution was derived from the emittance measurements of the ion source performed during acceptance tests. The input Twiss parameters, however, are ideal. 

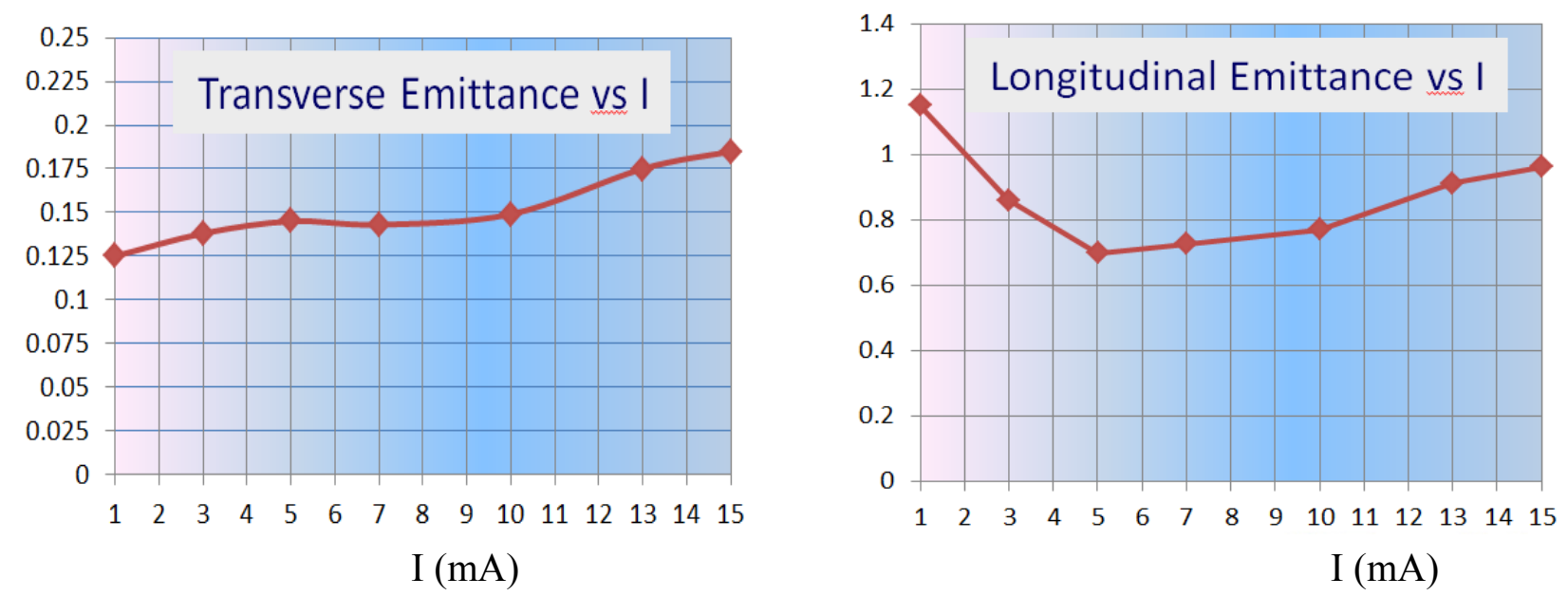

Figure 2.6: Dependence of the calculated transverse (left) and longitudinal (right) rms normalized emittances on the beam current; the transverse emittance is presented in $\mathrm{mm} \cdot \mathrm{mrad}$ and the longitudinal one in $\mathrm{keV} \cdot \mathrm{ns}(1 \mathrm{keV} \cdot \mathrm{ns} \approx 0.32 \mathrm{~mm} \mathrm{mrad})$.
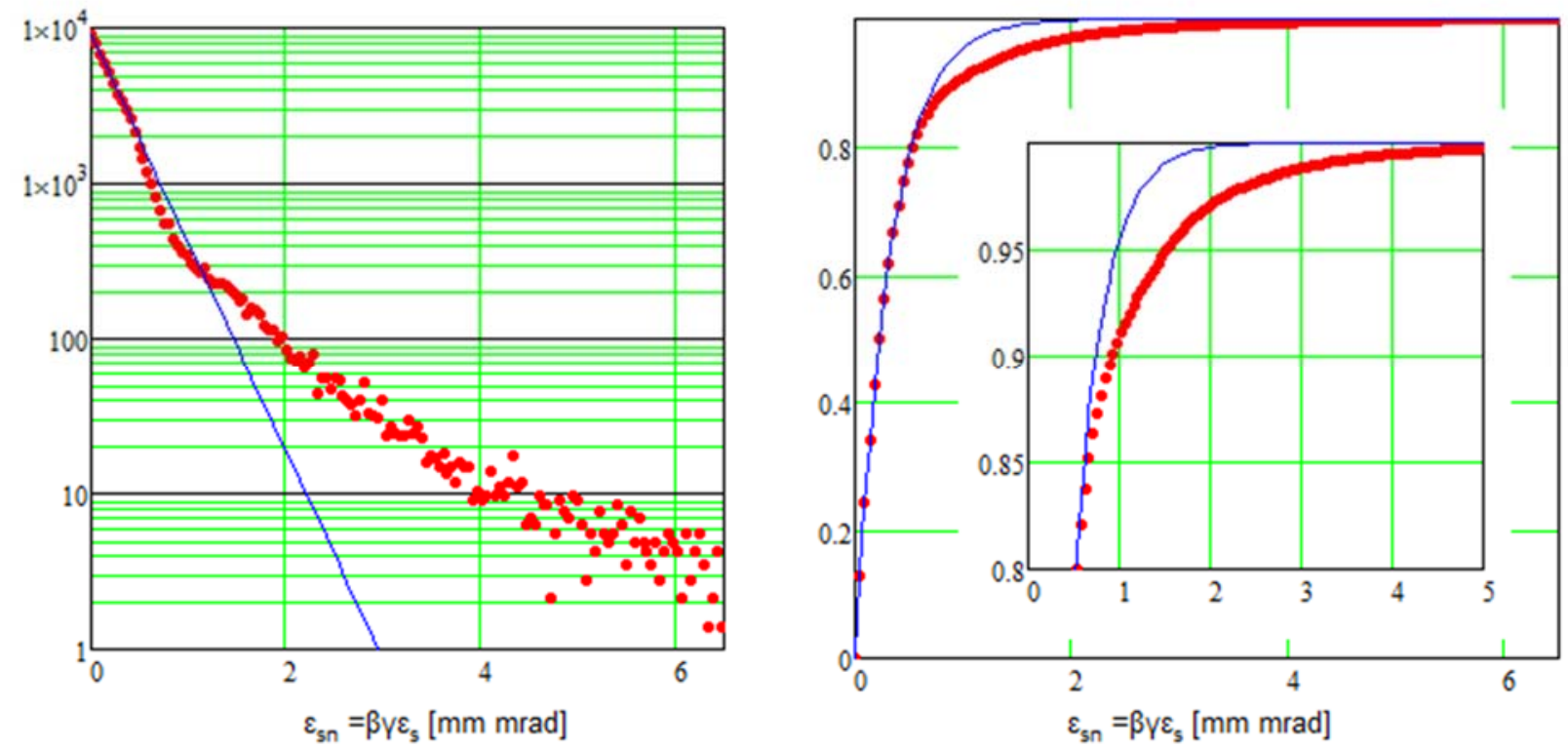

Figure 2.7: The particle longitudinal distribution (left) and its integral (right) at the end of the RFQ simulated for $5 \mathrm{~mA}$ beam current; $\beta_{s}=1.69 \mathrm{~m}, \alpha_{s}=0.087$, rms normalized emittance of $0.162 \mathrm{~mm} \mathrm{mrad}$. Blue lines show the Gaussian distribution and its integral built for longitudinal rms emittance computed from the particle distribution. 100,000 particles were used in the simulations.

Excessive longitudinal tails can result in beam losses in the course of acceleration and can limit the beam extinction of removed bunches ${ }^{3}$. The latter is an important requirement for future high duty factor experiments. Figure 2.7 presents the longitudinal distribution at the exit of RFQ over the

\footnotetext{
${ }^{3}$ A non-zero value for the beam extinction is associated with particles having large momentum deviation. In the course of motion in the MEBT from the bunch-by-bunch kicker to the HWR cryomodule particles with sufficiently large momentum deviations are not captured in the MEBT RF and can drift into a nearby already emptied RF bucket. Then, these particles are captured into HWR RF, which RF bucket height is significantly larger than the bucket height of the MEBT.
} 
longitudinal Courant-Snyder invariant, $\varepsilon_{s}=\left(1+\alpha_{s}{ }^{2}\right) s^{2} / \beta_{s}+2 \alpha_{s} s \Delta p / p+\beta_{s}(\Delta p / p)^{2}$ and the integral of this distribution. Here $\beta_{s}$ and $\alpha_{s}$ are the longitudinal beta- and alpha-functions, respectively, and $s$ and $\Delta p / p$ are the deviations in the longitudinal coordinate and the relative momentum from the reference particle, respectively. Note that $3.1 \%$ of particles located in the halo with the Courant-Snyder invariant above three times of the rms emittance ${ }^{4}$ were not accounted in the computation of $\beta_{s}$ and $\alpha_{s}$ and the rms emittance. One can see from Figure 2.7 that about $2.5 \%$ of particles are in the non-Gaussian tails. This stresses the necessity of accounting for longitudinal tails in the computation of particle losses during further acceleration.

\subsubsection{MEBT - Medium Energy Beam Transport}

The Medium Energy Beam Transport (MEBT) transports the 2.1 MeV, 1-10 $\mathrm{mA} \mathrm{H}^{-}$beam between the RFQ and the HWR cryomodule with low emittance growth $(<10 \%)$ and low beam losses of the passing bunches.

Typically, the main functions of an MEBT are to provide optical matching between the RFQ and the main linac and to include tools for measuring the beam properties. The PIP-II MEBT is envisioned to have, in addition, several other distinctive features.

First, many of the PIP-II MEBT properties are determined by the bunch-by-bunch selection concept. The MEBT wideband chopping system directs unneeded bunches of the initial true-CW 162.5 MHz bunch sequence to an absorber according to a pre-selected pattern and transfers bunches chosen for further acceleration into the SC Linac with minimum distortions. This bunch separation is performed with two kickers separated by $\sim 180^{\circ}$ of betatron phase advance; the absorber is positioned at $\sim 90^{\circ}$ of phase advance downstream of the second kicker. Implementation of the chopping system requires significant elongation of the MEBT. On the one hand, the system itself requires $\sim 5 \mathrm{~m}$ of the beam line. On the other hand, the high power density of the removed bunches on the MEBT beam absorber makes the absorber a critical and inherently risky device. To alleviate possible catastrophic effects on the SRF cavities in a case of an absorber vacuum failure, it is prudent to separate the absorber from the first cryomodule (HWR) by a beam line containing only more conservatively designed devices. The length of this region, determined by the time of closing a fast acting vacuum valve in front of the HWR and the speed of shock wave propagation, needs to be $\geq 5$ m.

The second distinctive feature of this MEBT is a system of scrapers that protects from an errant beam both the SRF cavities and sensitive elements of the MEBT itself.

Finally, the MEBT allocates space for a wall protecting the low-energy part from radiation generated in the high-energy part. It should allow servicing the ion source with the PIP-II linac delivering the beam.

The complete list of the MEBT functional requirements is presented in Ref. [13].

The resulting MEBT design is $14 \mathrm{~m}$ long with transverse focusing provided mainly by equidistantly placed quadrupole triplets with the exception of the two doublets located immediately downstream of the RFQ. In this text, the spaces between neighboring triplets or doublets are referred to as MEBT sections. To keep the beam properly bunched, the MEBT includes four identical bunching cavities. The structure of the MEBT is presented schematically in Figure 2.8.

The period in the regular part of the beam line is $1175 \mathrm{~mm}$, which leaves a $650-\mathrm{mm}$ long (flange-

\footnotetext{
${ }^{4}$ Note that the average of Courant-Snyder invariants of all particles is twice larger than the corresponding rms beam emittance.
} 
to-flange) space for various equipment. The first section labeled \#0 in Figure 2.8 and located between doublets is shorter. It has available space of $480 \mathrm{~mm}$.

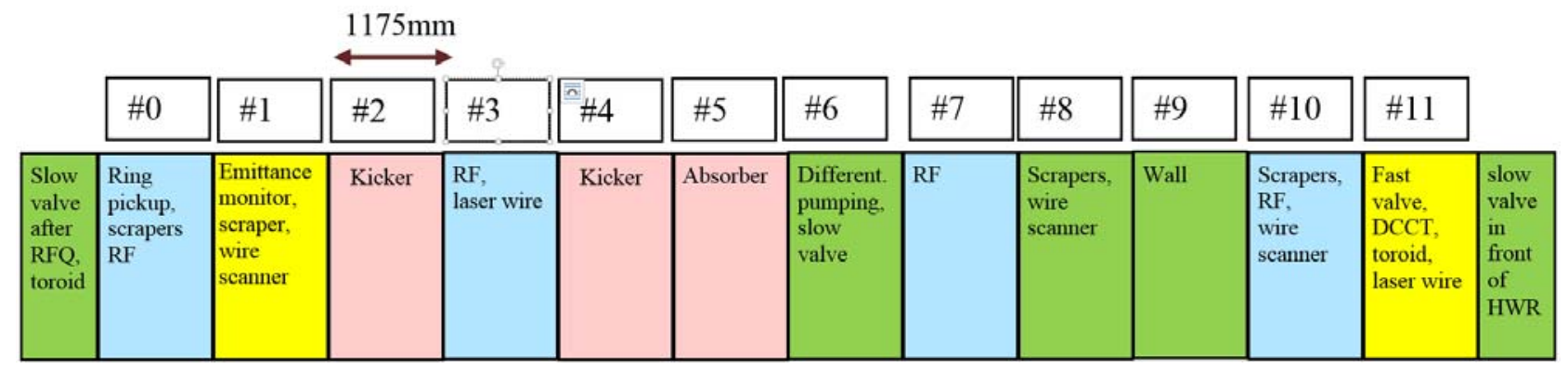

Figure 2.8: The MEBT structure. Sections are color-coded according to their main functions: greenvacuum, blue- RF, yellow- instrumentation, and pink - chopper.

\subsubsection{SC Linac - Superconducting Linac}

The SC Linac starts immediately downstream of the MEBT. It accelerates the beam from $2.1 \mathrm{MeV}$ to $0.8 \mathrm{GeV}$ and includes five types of superconducting (SC) cavities to cover the entire velocity range required for acceleration of $\mathrm{H}^{-}$(or protons).

\subsubsection{Accelerating Cavities}

The cavity frequencies and cell configuration are selected to maximize the acceleration efficiency for each accelerating structure, to minimize the cost of the accelerator and its operation, and to minimize the beam loss.

A primary figure of merit for a RF cavity is the transit-time factor, $T(\beta)$. For a periodic structure with a harmonic distribution of electric field along the axis,

$$
E \propto \sin \left(\omega z / \beta_{G} c\right) \exp (i \omega t),
$$

the transit-time factor for a cavity operating at the $\pi$-mode can be expressed by the following formula:

$$
T(\beta)=\frac{T_{0}(\beta)}{T_{0}\left(\beta_{\text {opt }}\right)}, \quad T_{0}(\beta)=\frac{2 \beta}{\pi n}\left(\frac{\sin \left(\pi n\left(\beta-\beta_{G}\right) /(2 \beta)\right)}{\beta-\beta_{G}}-(-1)^{n} \frac{\sin \left(\pi n\left(\beta+\beta_{G}\right) /(2 \beta)\right)}{\beta+\beta_{G}}\right),
$$

where $n$ is the number of cells in a cavity. Note that $T_{0}\left(\beta_{G}\right)=1$, and $T_{0}(\beta)$ achieves its maximum (exceeding 1) at $\beta=\beta_{\text {opt. }}$. That determines the normalization of $T(\beta)$ so that $T\left(\beta_{o p t}\right)=1$, where $\beta_{\text {opt }}$ is called the optimal beta. Eq. (2.1) approximates well electric field for a typical SC cavity operating in the $\pi$-mode. Consequently, Eq. (2.2) represents a good approximation for the flight factor. Using Eq. (2.2) one can obtain that for sufficiently large number of cells per cavity the geometric and optimal betas are related as follows:

$$
\beta_{o p t} \approx \beta_{G}\left(1+\frac{6}{\pi^{2} n^{2}}\right)
$$

The dependence of the transit-time factors on the beam velocity, $\beta$, is shown in Figure 2.9 for different numbers of cells in a cavity. The figure shows that the range in $\beta$ over which the beam can be efficiently accelerated increases with a decreasing number of cells per cavity. On the other hand, a too small number of cells per cavity reduces the effective gradient and increases costs, due to end effects.

Recent developments in $1300 \mathrm{MHz}$ ILC technology at Fermilab [14] and elsewhere have 
significantly improved SRF technology in general and have made it a preferable choice for the possible future extension of the PIP-II linac to higher energy. This justifies the choice of accelerating frequencies as subharmonics of $1300 \mathrm{MHz}$, and, consequently, 162.5, 325 and $650 \mathrm{MHz}$ are used for the PIP-II. Such choice of frequencies results in a comparatively smooth frequency increase in the course of acceleration, accommodating bunch compression due to adiabatic damping.

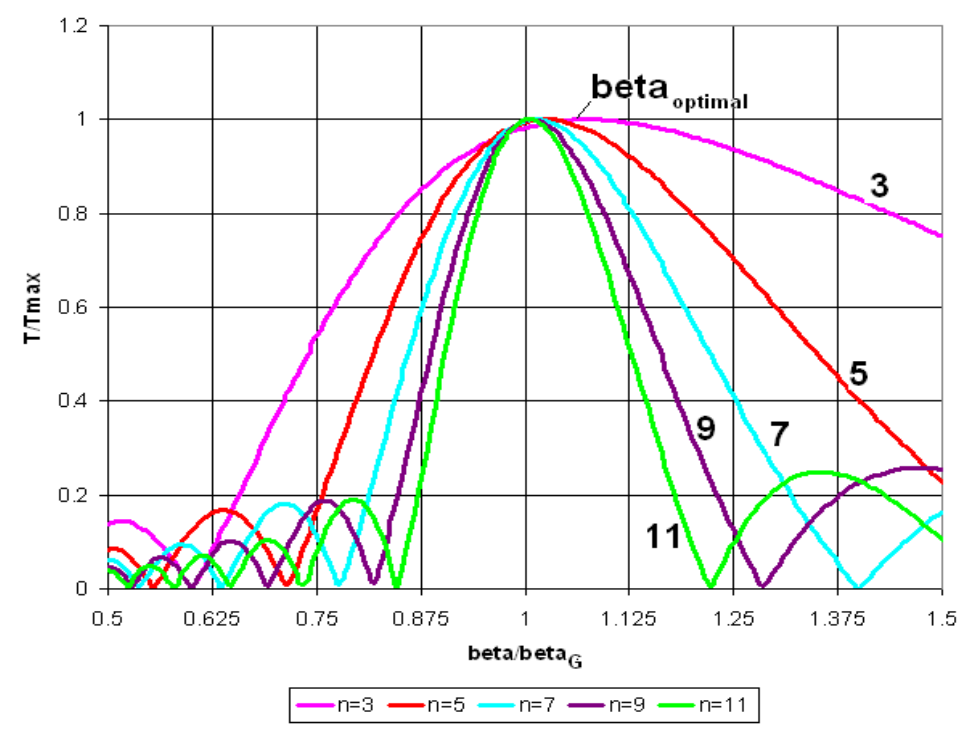

Figure 2.9: Transit-time factor versus the ratio of the beta to the geometric beta, $\beta / \beta_{G}$, for different number of cells in a cavity, $n$.

Table 2.4 and Figure 2.10 present parameters of the cavities for the linac. The acceleration starts with half-wave resonators (HWR) operating at $162.5 \mathrm{MHz}$. They are followed by two types of single spoke resonators operating at $325 \mathrm{MHz}$ (SSR1 and SSR2), and finally by two types of elliptical 5cell cavities at $650 \mathrm{MHz}$ (LB650 and HB650). Figure 2.11 presents the transit-time factors for the SC Linac. The available accelerating voltage increases in each subsequent cavity family. Therefore, the transition occurs before the transit-time factors for two types are equal.

The geometric betas, $\beta_{G}$, presented in Table 2.4 were obtained by fitting Eq. (2.2) to the numerical integration results. This equation approximates well the transit-time factors obtained by numerical integration of the actual time dependent electric field for all PIP-II cavities, including the HWR, SSR1 and SSR2, which have 2 accelerating gaps and therefore $n=2$ is used in the computation of $T(\beta)$. Note that for a typical multicell elliptic cavity the end effects result in that the value of $\beta_{G}$ obtained with described above fitting is slightly higher than the value obtained with another conventional definition, which uses $\beta_{G}$ defined as the ratio of cavity period to the half-wavelength. The corresponding numbers are presented in the note to Table 2.4.

The choice of the RFQ frequency was set by a requirement of bunch-by-bunch chopping. Chopping at $325 \mathrm{MHz}$ is presently beyond the "state-of-the art". It leaves $162.5 \mathrm{MHz}$ as the only viable choice. This frequency is also used for the first superconducting cryomodule (HWR). In comparison with $325 \mathrm{MHz}$ cavities, such choice results in reduced transverse defocusing and reduced longitudinal focusing from cavity fields, which otherwise would limit the accelerating gradient in the first SC cryomodule. The number of cavities and the linac length, required to accelerate the beam to $11 \mathrm{MeV}$ is reduced by more than a factor of 2, compared to cryomodules with $325 \mathrm{MHz}$ cavities. Note that even this frequency choice does not enable a usage of nominal voltage for the first few HWR cavities. In particular, the first cavity uses about half of nominal voltage. 
Table 2.4: Types of accelerating cavities for the SC Linac

\begin{tabular}{l|c|c|c|c|c|c}
\hline \hline $\begin{array}{l}\text { Cavity } \\
\text { name }\end{array}$ & $\beta_{G}$ & $\beta_{\text {opt }}$ & $\begin{array}{c}\text { Freq. } \\
(\mathrm{MHz})\end{array}$ & Cavity type & $\begin{array}{c}\text { Energy gain at } \beta_{\text {opt }} \\
\text { per cavity (MeV) }\end{array}$ & $\begin{array}{c}\text { Energy range } \\
(\mathrm{MeV})\end{array}$ \\
\hline HWR & 0.094 & 0.112 & 162.5 & $\begin{array}{c}\text { Half wave } \\
\text { resonator }\end{array}$ & 2 & $2.1-10.3$ \\
\hline SSR1 & 0.186 & 0.222 & 325 & $\begin{array}{c}\text { Single-spoke } \\
\text { resonator }\end{array}$ & 2.05 & $10.3-35$ \\
\hline SSR2 & 0.398 & 0.475 & 325 & $\begin{array}{c}\text { Single-spoke } \\
\text { resonator }\end{array}$ & $55-185$ \\
\hline LB650 & $0.631^{*}$ & 0.647 & 650 & $\begin{array}{c}\text { Elliptic 5-cell } \\
\text { cavity }\end{array}$ & $11.9\left(11.7^{\star}\right)$ & $185-500$ \\
\hline HB650 & $0.947^{*}$ & 0.971 & 650 & $\begin{array}{c}\text { Elliptic 5-cell } \\
\text { cavity }\end{array}$ & $19.9\left(19.6^{\star}\right)$ & $500-800$ \\
\hline \hline
\end{tabular}

${ }^{*}$ Note that $\beta_{G}$ for the elliptic cavities can be also defined as the ratio of regular cell length to halfwavelength. That yields $\beta_{G}=0.61$ for LB650 and $\beta_{G}=0.92$ for HB650. This definition is used everywhere below, unless it is directly stated.

- It represents a mean value among different field distributions with field flatness of $95 \%$.

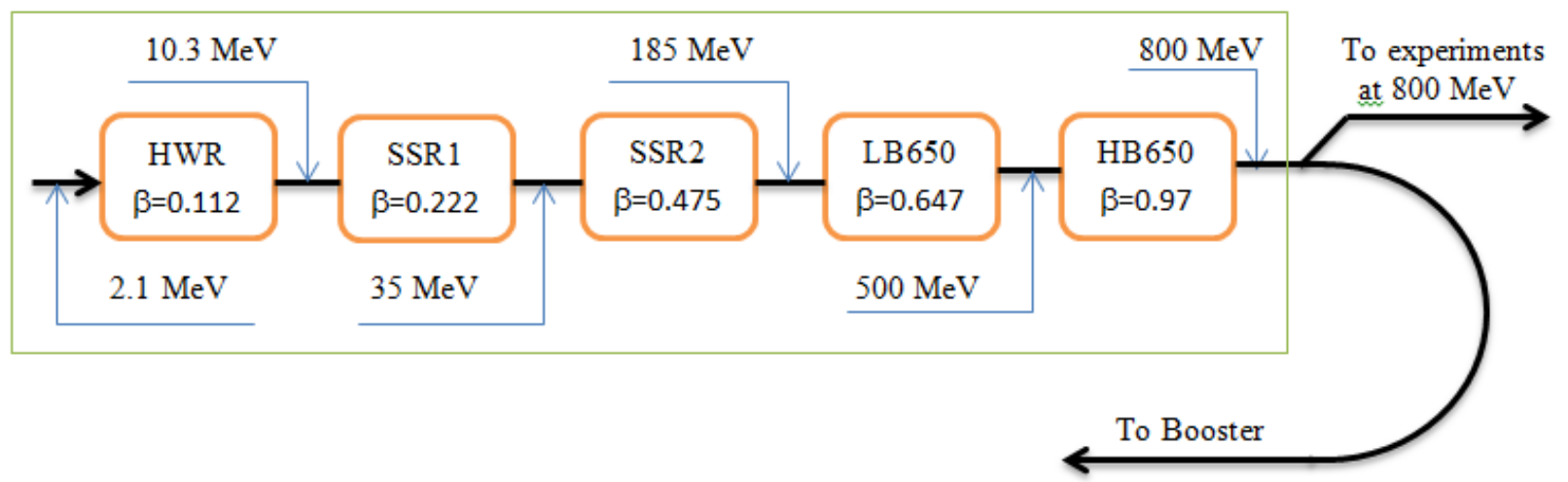

Figure 2.10: Technology map for SC part of PIP-II linac.

The cavity arrangement described above also yields that:

- The frequency jumps do not exceed a factor of 2. That simplifies longitudinal beam dynamics at each transition from one cavity type to another.

- Lower frequency sections used at the linac beginning have larger aperture. That reduces the beam loss.

We emphasize that the choice of cavities with lower frequencies reduces the effects of focusing/defocusing by accelerating cavities, and for a fixed cavity length it decreases the number of cells per cavity (for LB650 and HB650). Consequently, it widens the dependences of transit-time factors on beta resulting in an increase in the effective accelerating gradients and acceleration efficiency.

However, the choice of lower frequencies also presents some disadvantages: 
- Microphonics is a more serious issue at lower frequencies.

- Lower frequency cavities are more expensive (more niobium); though the additional cost is mostly compensated by smaller number of cavities and RF sources.

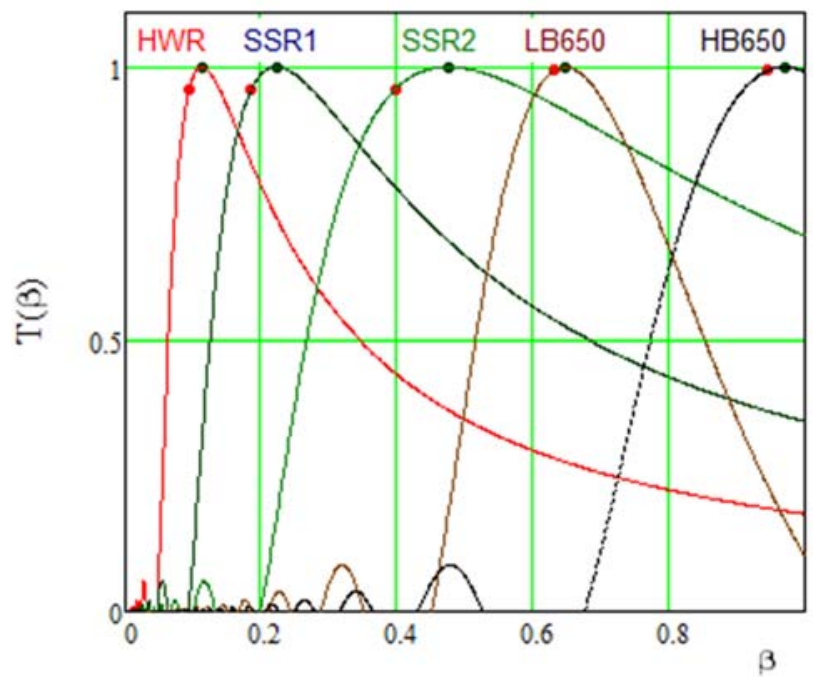

Figure 2.11: Transit-time factors for PIP-II SC cavities; red dots mark the position of $\beta_{G}$, and blue dots position of $\beta_{\text {opt }}$.

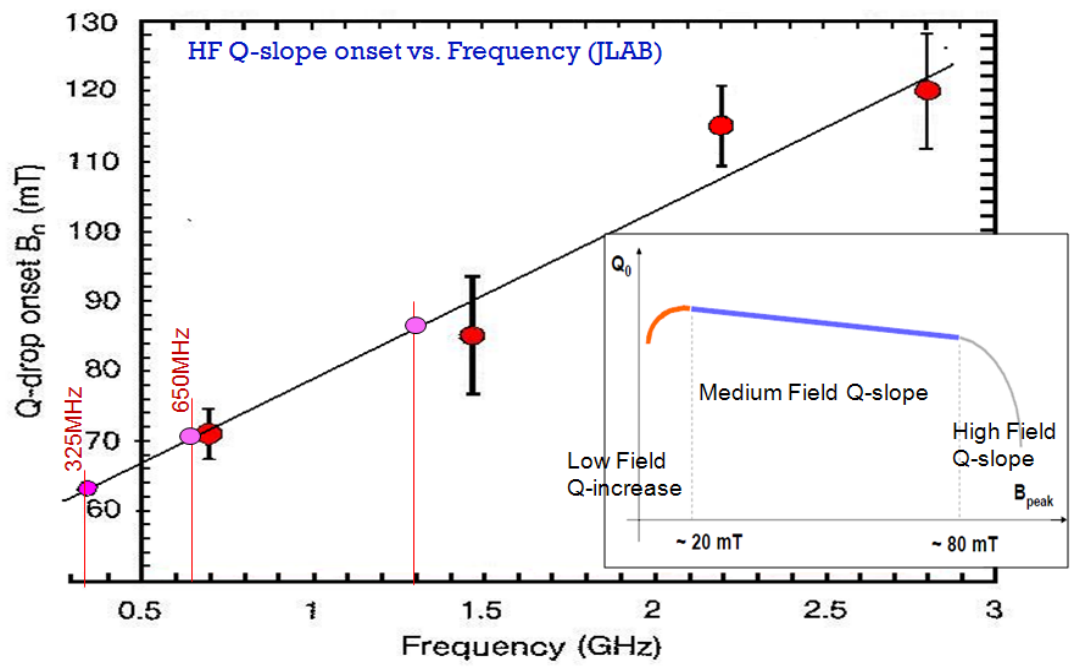

Figure 2.12: High field Q-slope versus frequency.

A few factors limit the accelerating gradient. First, to minimize the cavity power losses the peak surface magnetic field is kept below the high-field Q-slope which is presented in Figure 2.12 (taken from Ref. [15], see also Ref. [16] and Section 3.1.2). That sets the limits: $50 \mathrm{mT}$ at $162.5 \mathrm{MHz}$, and about $75 \mathrm{mT}$ at $650 \mathrm{MHz}$. For all frequencies, the peak surface electric field is kept below $45 \mathrm{MV} / \mathrm{m}$ [17] to reduce the risk of strong field emission (see details in Section 3.1.2). Another important limitation comes from increased difficulties to suppress microphonics and Lorentz Force Detuning (LFD) with an increase of accelerating field. To be compatible with CW operation the PIP-II linac has comparatively small beam current. It results in high loaded Q-values, narrow bandwidths and susceptibility to microphonics and LFD (see details in Section 3.1.3).

The transition from the $325 \mathrm{MHz}$ single-spoke cavities to the $650 \mathrm{MHz}$ elliptical cavities is chosen 
at the energy of about $185 \mathrm{MeV}$, because at lower energies elliptical cavities lose efficiency. It is inefficient to accelerate $\mathrm{H}^{-}$ions from $170 \mathrm{MeV}$ to $0.8 \mathrm{GeV}$ using only one cavity type and, thus, two families of $650 \mathrm{MHz}$ cavities are chosen. Table 2.5 presents the main electro-dynamical parameters of SC cavities. The effective length of a cavity is computed based on $\beta_{o p t}$ so that: $L_{\text {eff }}=n \beta_{\text {opt }} c /(2 f)$, where $n$ is the number of cells in a cavity $(n=2$ for HWR, SSR1 and SSR2; $n=5$ for LB650 and HB650). Consequently, the accelerating gradient is $\Delta \mathrm{E} / L_{\text {eff }}$, where $\Delta \mathrm{E}$ is the net energy gain at the optimal beta.

The transition energies between different types of cavities were optimized to minimize the total number of cavities. As an example of such optimization, Figure 2.13 displays the number of cavities required as a function of geometric betas for LB650 and HB650. Here we additionally assume (1) a linear dependence of the field enhancement factors versus $\beta_{\text {opt }}[18],(2)$ the initial synchronous phase is $-30^{\circ}$, and (3) its modulus decreases inversely proportional to the square root of the energy to keep the desired RF bucket size. As one can see from the left pane of Figure 2.13 the number of cavities has a weak dependence on betas in the vicinity of the minimum. The optimal geometric betas for the two $650 \mathrm{MHz}$ sections are 0.64 and 0.9 respectively (left), and the optimal transition energy is 466 $\mathrm{MeV}$ (right). More accurate simulations, taking into account realistic enhancement factors and other limitations discussed in Section 3.1.2, yield an optimal choice of betas at 0.61 and 0.92 .

Table 2.5: Main electro-dynamical parameters of SC cavities

\begin{tabular}{l|c|c|c|c|c|c|c}
\hline $\begin{array}{l}\text { Cavity } \\
\text { type }\end{array}$ & $\begin{array}{c}\text { Aperture } \\
(\text { diameter }) \\
(\mathrm{mm})\end{array}$ & $\begin{array}{c}\text { Effective } \\
\text { length } \\
(\mathrm{cm})\end{array}$ & $\begin{array}{c}\text { Accelerating } \\
\text { gradient } \\
(\mathrm{MV} / \mathrm{m})\end{array}$ & $\begin{array}{c}\mathrm{E}_{\text {peak }}{ }^{*} \\
(\mathrm{MV} / \mathrm{m})\end{array}$ & $\begin{array}{c}\mathrm{B}_{\text {peak }}{ }^{*} \\
(\mathrm{mT})\end{array}$ & $\begin{array}{c}(\mathrm{R} / \mathrm{Q})^{5} \\
(\Omega)\end{array}$ & $\begin{array}{c}\mathrm{G} \\
(\Omega)\end{array}$ \\
\hline HWR & 33 & 20.7 & 9.7 & 44.9 & 48.3 & 272 & 48 \\
\hline SSR1 & 30 & 20.5 & 10 & 38.4 & 58.1 & 242 & 84 \\
\hline SSR2 & 40 & 43.8 & 11.4 & 40 & 64.5 & 297 & 115 \\
\hline LB650 & 83 & 74.6 & 15.9 & 39.4 & 74.6 & 356 & 187 \\
\hline HB650 & 118 & 112.0 & 17.8 & 38.9 & 73.1 & 610 & 260 \\
\hline \hline
\end{tabular}

$\overline{{ }^{*} \text { For energy gain per cavity presented in Table } 2.4 \text { where the cavity effective length was computed as }}$ $L_{\text {eff }}=c \beta_{\text {opt }} n_{\text {cell }} / 2 f$.

\subsubsection{SC Cryomodules and Cryogenics Requirements}

Cavities and focusing elements, as necessary, are grouped within cryomodules. In the 162.5 and $325 \mathrm{MHz}$ sections transverse focusing is provided by superconducting solenoids, while in the 650 $\mathrm{MHz}$ sections by normal conducting quadrupole doublets located outside of the cryomodules. The main cryomodule parameters and the arrangement of focusing periods by cryomodule type are shown in Table 2.6.

${ }^{5}$ Throughout this document we define $(R / Q)$ so that in the absence of cavity detuning and beam current, the RF power required to create the voltage amplitude $U_{0}$ is equal to: $P_{g}=\left(1+\beta_{c}^{2}\right)^{2} U_{0}^{2} /\left(4 \beta_{c}(R / Q) Q_{0}\right)$, where $\beta_{c}$ is the coupling coefficient, and $Q_{0}$ is the cavity unloaded quality factor. 
The cavity transverse defocusing depends on the particle accelerating phase, i.e. its longitudinal position inside the bunch. To minimize an effect of this defocusing on the beam dynamics the transverse focusing in the accelerator has to be much stronger than this variation of cavity defocusing. That sets the requirement for the length of transverse focusing period for every SC section and, subsequently, determines the number of cavities per period. In the design of the HWR, SSR1 and SSR2 cryomodules all efforts have been made to minimize the distance between cavities inside cryomodule. That minimizes both the longitudinal overfocusing and the transverse focusing variation. The machine optics is built so that at the beginning of each section the betatron phase advance per period is about $90 \mathrm{deg}$. That maximizes the transverse focusing for a given period length.
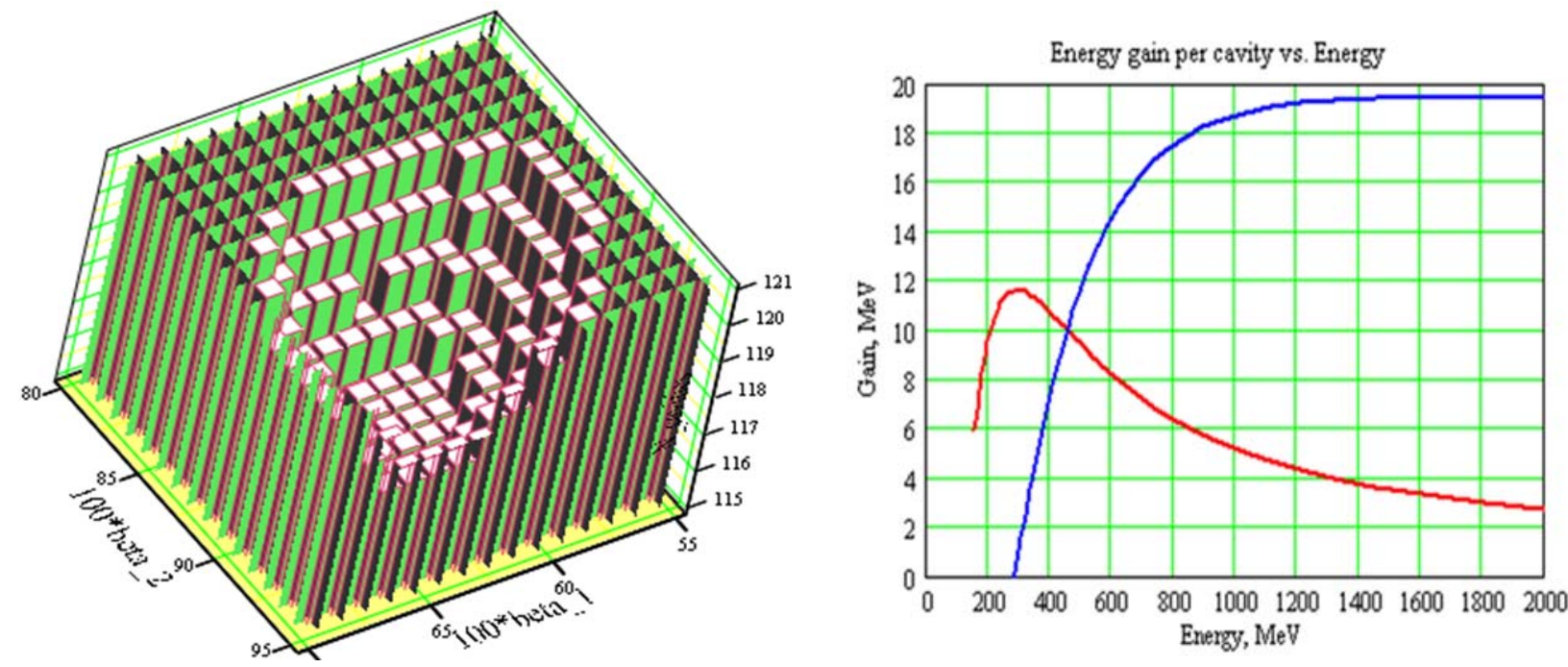

Figure 2.13: Number of cavities required for acceleration from 185 to $800 \mathrm{MeV}$ versus cavity beta in the LB650 and HB650 sections (left) and the energy gain per cavity versus particle energy (right) for LB650 (red curve) and HB650 (blue curve) cavities.

Table 2.6: General parameters of SC cryomodules

\begin{tabular}{l|c|c|c|c|c|c|c}
\hline $\begin{array}{l}\text { CM } \\
\text { type }\end{array}$ & $\begin{array}{c}\text { Cavities } \\
\text { per CM }\end{array}$ & $\begin{array}{c}\text { Number } \\
\text { of CMs }\end{array}$ & $\begin{array}{c}\text { CM configu- } \\
\text { ration }\end{array}$ & $\begin{array}{c}\text { CM length } \\
(\mathrm{m})\end{array}$ & $\begin{array}{c}Q_{0} \text { at } 2 \mathrm{~K} \\
\left(10^{10}\right)\end{array}$ & $\begin{array}{c}\text { Surface resis- } \\
\text { tance, }(\mathrm{n} \Omega)\end{array}$ & $\begin{array}{c}\text { Loaded } Q \\
\left(10^{6}\right)\end{array}$ \\
\hline HWR & 8 & 1 & $8 \times(\mathrm{sc})$ & 5.93 & 0.5 & $9.6\left(2.75^{\star}\right)$ & 2.7 \\
\hline SSR1 & 8 & 2 & $4 \times(\mathrm{csc})$ & 5.2 & 0.6 & $14\left(10^{\#}\right)$ & 3.7 \\
\hline SSR2 & 5 & 7 & sccsccsc & $6.5^{\star}$ & 0.8 & 14.4 & 5.8 \\
\hline LB650 & 3 & 11 & ccc & $3.9^{\star}$ & 2.15 & 8.9 & 11.3 \\
\hline HB650 & 6 & 4 & ccccc & $9.5^{\star}$ & 3 & 8.7 & 11.5 \\
\hline
\end{tabular}

- Within the cryomodule (CM) configuration column "c" refers to an individual accelerating cavity, and "s" to a focusing solenoid.

- This number represents the present estimate of the cryomodule lengths. It will be finalized with advances in the cryomodule design.

* Measured value based on recent measurements of two HWR cavities at $2 \mathrm{MV}$ accelerating voltage.

\# Based on recent measurements of SSR1 cavities made of CABOT niobium. We expect to get better results for the SSR2 cavities to be made of material, which satisfies Fermilab specifications [19]. 
Each magnet package (i.e. solenoid or quadrupole doublet) includes vertical and horizontal correctors and a 3-coordinate beam position monitor ${ }^{6}(\mathrm{BPM})$ required for beam steering and optics measurements. All cryomodules are separated by warm sections. These warm sections are used for additional diagnostics (bunch transverse and longitudinal profile monitors, beam loss monitors, etc.) and for beam collimators required to avoid uncontrolled beam losses inside SC cryomodules. The composition of each of the warm insertions will be determined by requirements of safe and reliable operations, diagnostics, collimation, and cryogenic segmentation constraints. Details of the cryomodule designs are presented in Section 3.1.

The cavity $\mathrm{Q}_{0}$ 's are based on an operating temperature of $2 \mathrm{~K}$ and a conservative approach to the surface resistance based on values already obtained in operating cryomodules. As one can see the measured surface resistance values for the HWR and SSR1 cavities are considerably lower. Table 2.7 presents the cryogenic loads in SC cryomodules for operation in the $\mathrm{CW}$ regime. As one can see, the dynamic cryo-loads in the $\mathrm{CW}$ regime significantly exceed the static loads.

Table 2.7: Cryogenic loads in SC cryomodules for operation in the $\mathrm{CW}$ regime

\begin{tabular}{l|c|c|c|c|c|c}
\hline \hline $\begin{array}{l}\text { CM } \\
\text { type }\end{array}$ & $\begin{array}{c}\text { Number of } \\
\text { CMs }\end{array}$ & \multicolumn{3}{|c|}{ Static loads per CM, (W) } & $\begin{array}{c}\text { Dynamic loads per } \\
\text { CM, (W) }\end{array}$ & $\begin{array}{c}\text { Total load at 2 K } \\
\text { per CM, (W) }\end{array}$ \\
\cline { 2 - 7 } & $70 \mathrm{~K}^{*}$ & $5 \mathrm{~K}^{*}$ & $2 \mathrm{~K}$ & $2 \mathrm{~K}$ & $2 \mathrm{~K}$ \\
\hline HWR & 1 & 250 & 60 & 37 & 23.3 & 60.3 \\
\hline SSR1 & 2 & 255 & 80 & 13 & 23.1 & 36.1 \\
\hline SSR2 & 7 & 145 & 50 & 8.8 & 52.6 & 61.4 \\
\hline LB650 & 11 & 48 & 16 & 2 & 55.5 & 57.5 \\
\hline HB650 & 4 & 86 & 32 & 4 & 130 & 134 \\
\hline Total & & 2647 & 874 & 163 & 1568 & 1731 \\
\hline \hline
\end{tabular}

- Cryo-loads are computed with the nominal accelerating voltage for all cavities. The actual voltages required by the optics are smaller (see Figure 2.20) which yields somewhat smaller heating loads if all the cavities are operating at the design accelerating gradients.

* Static cryo-load includes heat flux from the couplers and current leads of magnets operating at their maximum currents.

The pulsed linac operation (required to support Booster operation) allows a significant reduction of dynamic cryo-loads. In this case the duty factor of dynamic cryogenic load is mainly determined by the time required to pump the energy into the cavity and then to discharge it. For this estimate, we assume that $Q_{0}$ does not depend on the field value. The duty factor for cryogenic operation is then equal to:

$$
\eta_{\text {cryo }}=\frac{f_{\text {rep }}}{E_{\max }^{2}} \int_{\substack{\text { over } \\ \text { pulse }}}(E(t))^{2} d t,
$$

where $E_{\max }$ is the accelerating voltage of a cavity, and $f_{\text {rep }}$ is the repetition rate. Further, we assume

\footnotetext{
${ }^{6}$ The BPM has 4 plates and allows measurements of both transverse beam positions, as well as longitudinal bunch position measured by bunch arrival time.
} 
that the cavity voltage changes as: $E(t)=2 E_{\max }\left(1-e^{-t / 2 \tau}\right), 0<t<2 \tau \ln 2$ during cavity charging, and as $E(t)=E_{\max } e^{-t / 2 \tau}, t>0$ during cavity discharging, where $\tau=Q_{L} / 2 \pi f$ is the time constant for energy decay. The accelerating voltage stays constant during the beam pulse of $0.55 \mathrm{~ms}$. Table 2.8 presents the cryo-duty factors and the dynamic cryo-loads assuming all cavities operate at the nominal voltages presented in Table 2.4. The static losses are the same as for CW operation and are presented in Table 2.7. As one can see for the normal cavity discharge described above the cryo-duty factor is almost an order of magnitude larger than the beam duty factor of $1.1 \%$. The cavity discharge can be accelerated by pumping additional RF power to the cavity after the end of beam pulse. It requires $180 \mathrm{deg}$. phase shift of RF power at the end of beam pulse. The RF pulse ends when the cavity voltage is zero. The corresponding dependence of electric field on time is: $E(t)=E_{\max }\left(e^{-t / 2 \tau}-2\left(1-e^{-t / 2 \tau}\right)\right), 0<t / \tau<0.8109 \ldots$. Such arrangement reduces the dynamic cryoloads by almost factor of two but requires a larger duty factor for RF amplifiers. It also greatly increases the peak power seen by the RF coupler after the phase shift. The couplers have to withstand the resulting four-fold increase in RF power if this mode of operation is used. Note that the HWR cavities are designed so that they cannot be used in a pulsed regime. Therefore they are excluded from Table 2.8 .

Table 2.8: Cavity parameters for operation in the pulsed regime

\begin{tabular}{|c|c|c|c|c|c|}
\hline \multirow{2}{*}{$\begin{array}{l}\mathrm{CM} \\
\text { type }\end{array}$} & \multirow{2}{*}{$\begin{array}{c}\text { Time } \\
\text { constant } \\
\tau(\mathrm{ms})\end{array}$} & \multicolumn{2}{|c|}{ Normal cavity discharge } & \multicolumn{2}{|c|}{ Accelerated cavity discharge } \\
\hline & & $\begin{array}{l}\text { Cryo-duty } \\
\text { factor }(\%)\end{array}$ & $\begin{array}{c}\text { Dynamic cryo-loads } \\
\text { per CM (W) }\end{array}$ & $\begin{array}{l}\text { Cryo-duty } \\
\text { factor }(\%)\end{array}$ & $\begin{array}{l}\text { Dynamic cryo- } \\
\text { loads per CM (W) }\end{array}$ \\
\hline SSR1 & 1.8 & 6.8 & 1.6 & 3.8 & 0.89 \\
\hline SSR2 & 2.9 & 9.9 & 5.2 & 5.3 & 2.8 \\
\hline LB650 & 2.8 & 9.7 & 5.1 & 5.2 & 2.7 \\
\hline HB650 & 2.8 & 9.8 & 12.7 & 5.2 & 6.8 \\
\hline Total $^{*}$ & & & 170 & & 102 \\
\hline
\end{tabular}

This value includes contribution of HWR cryomodule operating in CW mode.

The projected cooling power of a cryo-plant is presented in Table 3.33. It provides sufficient capacity for $\mathrm{CW}$ operation. To minimize the cost of cryogenic system operation, the PIP-II linac can operate in a pulsed mode with a capability to be moved to $\mathrm{CW}$ operations as required. Details of the technical implementation of the cryo-plant are presented in Section 3.4.

Assuming pulsed operation one obtains the total dynamic cryo-loads at $2 \mathrm{~K}$ for all cryomodules to be $170 \mathrm{~W}$ for normal cavity discharge and $102 \mathrm{~W}$ for accelerated cavity discharge. Adding the static cryo-loads yields the total cryo-load at $2 \mathrm{~K}$ to be 333 and $265 \mathrm{~W}$, respectively. As shown in Table 3.33 the cooling power of the cryo-plant at $2 \mathrm{~K}$ is $>1700 \mathrm{~W}$. Therefore, to be efficient for operation in the pulsed regime, the cryo-plant should have corresponding provisions. Note, that the total cryogenic heat load at $2 \mathrm{~K}$ in the pulsed regime is almost equally distributed between the static and dynamic loads and is less than $20 \%$ of the cryogenic load for CW operation. The margin for cryo-plant cooling powers for the $5 \mathrm{~K}$ and $70 \mathrm{~K}$ circuits is large as can be seen from comparison of the total static loads of Table 2.7 with the cryo-plant powers presented in Table 3.33 . Note that the 
$\mathrm{Q}_{0}$ values presented in Table 2.6 are above what is routinely achieved in the previous generation of large superconducting accelerators. This represents a challenge to be addressed by PIP-II R\&D. The present status and expectations for achievable high $\mathrm{Q}_{0}$ value are described in Section 3.1.2.6.

In closing, the Functional Requirements Specifications (FRS) for the cryomodules specify the maximum cryo-loads, which each cryomodule has to withstand. These values determine mechanical designs of cryostats ( sizes of piping etc.). They were chosen so that there would be sufficiently large margins between the expected cryo-loads and actual abilities for cryomodules to remove heat. Table 2.9 summarizes these FRS requirements.

Table 2.9: Maximum allowed heat loads per cryomodule

\begin{tabular}{l|c|c|c}
\hline \hline CM type & $70 \mathrm{~K}$ & $5 \mathrm{~K}$ & $2 \mathrm{~K}$ \\
\hline HWR & $250 \mathrm{~W}$ & $80 \mathrm{~W}$ & $50 \mathrm{~W}$ \\
\hline SSR1 & $250 \mathrm{~W}$ & $80 \mathrm{~W}$ & $50 \mathrm{~W}$ \\
\hline SSR2 & $250 \mathrm{~W}$ & $80 \mathrm{~W}$ & $75 \mathrm{~W}$ \\
\hline LB650 & $100 \mathrm{~W}$ & $15 \mathrm{~W}$ & $100 \mathrm{~W}$ \\
\hline HB650 & $300 \mathrm{~W}$ & $25 \mathrm{~W}$ & $220 \mathrm{~W}$ \\
\hline
\end{tabular}

\subsubsection{RF Power and Suppression of Microphonics}

The RF system has to support $2 \mathrm{~mA}$ beam operation in both $\mathrm{CW}$ and pulsed $(0.55 \mathrm{~ms}, 20 \mathrm{~Hz})$ regimes. The system is based on a single RF source driving each RF cavity, for a total of 113 separate RF sources for SC cavities. It is anticipated that all RF amplifiers will be solid state.

For operation in the pulsed regime, the average RF power delivered to the cavities has two contributions: 1) the energy transferred to the beam, and 2) the energy required to fill and discharge the accelerating cavities. In the first approximation, for a given repetition rate the average power of the RF amplifier does not depend on the pulse duration while the peak power is inversely proportional to it. Note also that the second contribution is about ten times larger than the first one; therefore, only a small fraction of the RF power is transferred to the beam. At fixed average power, the cost of power amplifiers increases with peak power. Therefore, the RF cost minimum is achieved with RF power corresponding to maximum acceptable beam pulse length. For the PIP-II parameters the duty factor for the RF power amplifiers is about 9\% for operation with normal cavity discharge and about $13 \%$ for operation with accelerated cavity discharge. For such long pulses the cost reduction associated with usage of pulsed power amplifiers is modest compared to $\mathrm{CW}$ amplifiers. Therefore $\mathrm{CW}$ amplifiers are planned to be used for both pulsed and CW regimes. The RF power requirements are summarized in Table 2.10. To estimate the peak RF power we assume that the maximum cavity detuning due to microphonics and Lorentz Force Detuning, $\delta f$, is equal to $20 \mathrm{~Hz}$ for all cryomodules [20]. That sets the optimal coupling,

$$
\beta_{c}=\sqrt{\left(1+\frac{I_{b} \cos \phi_{a}(R / Q) Q_{0}}{U_{0}}\right)^{2}+\left(\frac{2 \delta f Q_{0}}{f_{0}}\right)^{2}},
$$

the corresponding cavity bandwidth,

$$
\Delta f=\frac{f_{0}}{Q_{0}}\left(1+\beta_{c}\right)
$$


and the peak RF power,

$$
P_{\max }=\frac{U_{0}^{2}\left(1+\beta_{c}\right)^{2}}{4 \beta_{c}(R / Q) Q_{0}}\left(\left(1+\frac{I_{b} \cos \phi_{a}(R / Q) Q_{0}}{U_{0}\left(1+\beta_{c}\right)}\right)^{2}+\left(\frac{2 \delta f Q_{0}}{f_{0}\left(1+\beta_{c}\right)}\right)^{2}\right) .
$$

Here $I_{b}$ is the beam current, $U_{0}$ is the cavity voltage amplitude, and $\phi_{a}$ is the accelerating phase assumed to be equal to zero in these estimates.

The peak RF power presented in the last column of Table 2.10 determines the peak RF power for each section. It accounts for power loss in transmission (assuming cable for $325 \mathrm{MHz}$ and waveguide for $650 \mathrm{MHz}$ ) and the power margin required for effective operation of voltage control system.

The high accelerating gradient and low beam current result in a narrow cavity bandwidth and, consequently, high sensitivity to microphonics and LFD. The major sources of cavity detuning are:

- Variations in the pressure of the surrounding helium bath,

- Mechanical vibrations driven by external sources,

- Radiation pressure on the walls from the electromagnetic field inside the cavity due to Lorentz force (LFD).

Table 2.10: Requirements for RF power*

\begin{tabular}{l|c|c|c|c|c|c}
\hline $\begin{array}{l}\text { CM } \\
\text { type }\end{array}$ & $\begin{array}{c}\text { Power trans- } \\
\text { ferred to beam } \\
\text { per cav. }(\mathrm{kW})\end{array}$ & $\begin{array}{c}\text { Microphonics } \\
\text { amplitude } \\
(\mathrm{Hz})\end{array}$ & $\begin{array}{c}\text { Cavity half- } \\
\text { bandwidth, } \\
f / 2 Q_{L},(\mathrm{~Hz})\end{array}$ & $\begin{array}{c}\text { Power transfer } \\
\text { efficiency }\end{array}$ & $\begin{array}{c}\text { Power } \\
\text { margin }\end{array}$ & $\begin{array}{c}\text { Peak RF } \\
\text { power per } \\
\text { cavity (kW) }\end{array}$ \\
\hline HWR & 4 & 20 & 33 & $90 \%$ & $80 \%$ & 6.5 \\
\hline SSR1 & 4.1 & 20 & 43 & $90 \%$ & $80 \%$ & 6.1 \\
\hline SSR2 & 10 & 20 & 28 & $90 \%$ & $80 \%$ & 17 \\
\hline LB 650 & 23.8 & 20 & 29 & $94 \%$ & $80 \%$ & 38 \\
\hline \hline
\end{tabular}

${ }^{*}$ Powers are computed for a beam current of $2 \mathrm{~mA}$. Allowances for transmission loss and microphonics suppression are included in the peak RF power.

- Microphonics amplitude represents a target value for maximum cavity detuning due to microphonics.

As can be seen from Eq. (2.7) the power required to maintain a constant accelerating gradient rises rapidly as the cavity detunes. A requirement of sufficient reserve power to drive detuned cavities increases both the capital and the operating costs of an accelerator. If sufficient reserve is not available, the beam may be lost. Thus, all measures minimizing cavity detuning need to be taken to keep RF power at a reasonable level. The measures can be separated into two broad categories: passive compensation and active compensation.

Passive compensation involves designing the machine and its components to minimize cavity detuning. In particular, the following design objectives are aimed at:

- Minimization of the sensitivity of the cavity resonance frequency to variations in the helium bath pressure,

- Minimization of the pressure variations in the $2 \mathrm{~K}$ helium bath; the target value is below 0.1 mbar for rms fluctuations and 1 mbar for maximum pressure deviation,

- Minimization of transmission of external vibrations to the cavities, 
- Minimization of vibrations in the tunnel and the transfer of these vibrations to cryomodules. In particular, large compressors have to be well isolated from ground and be located far enough from the linac tunnel.

Active compensation involves sensing cavity detuning and using feed-forward and feedback to drive a piezo-actuator to compensate detuning in real-time. The detuning of each cavity can be determined from the base-band forward, reflected, and probe RF signals and used to drive a piezo actuator in a combination of adaptive feed-forward and feedback loops.

Table 2.11 presents requirements to cavity detuning due to helium pressure variations [20], estimates for Lorentz force detuning and recently measured values for the HWR and SSR1 dressed cavity prototypes. As one can see the LFD detuning exceeds the cavity bandwidth by about one order of magnitude. That indicates that operation in the pulsed regime is impossible without active frequency control with fast piezo-based tuners. Note that the HWR has no piezo tuner, and thus, cannot be used in a pulsed regime. As was already stated, the cryo-load in the HWR cryo-module is negligible compared to the total cryo-load. That enables its operation with the CW RF.

Table 2.11: Functional requirement specifications on cavity detuning due to helium pressure variations and Lorentz force detuning (LFD)

\begin{tabular}{r|c|c|c|c|c}
\hline \hline & HWR & SSR1 & SSR2 & LB650 & HB650 \\
\hline Sensitivity to He pressure (FRS), $d f / d P, \mathrm{~Hz} /$ Torr & $<25$ & $<25$ & $<25$ & $<25$ & $<25$ \\
\hline$\ldots$ (measurements), $d f / d P, \mathrm{~Hz} / \mathrm{Torr}$ & 13 & 4.0 & - & - & - \\
\hline Estimated LFD sensitivity, $d f / d E^{2}, \mathrm{~Hz} /(\mathrm{MV} / \mathrm{m})^{2}$ & - & -5.0 & - & -1.2 & -1.0 \\
\hline$\ldots .(m e a s u r e m e n t s), d f / d E^{2}, \mathrm{~Hz} /(\mathrm{MV} / \mathrm{m})^{2}$ & $-1.5^{*}$ & -4.4 & - & - & - \\
\hline Estimated LFD at nominal voltage (FRS), Hz & - & -500 & - & -303 & -317 \\
\hline$\ldots$ (measurements ) at nominal voltage, Hz & -122.4 & -440 & - & - & - \\
\hline \hline
\end{tabular}

\footnotetext{
${ }^{*}$ Two cavities were measured in a test stand. The results are: -1.82 and $-1.3 \mathrm{~Hz} /(\mathrm{MV} / \mathrm{m})^{2}$.
} 


\subsubsection{Beam Dynamics in the SC Linac}

High efficiency multi-turn strip injection to the Booster requires small transverse emittances of the injected beam. The rms normalized beam emittance budget for the SC Linac has been established at $0.15 \mathrm{~mm}$-mrad at the ion source and $0.3 \mathrm{~mm}$-mrad at the linac exit. The lattice design and the beam dynamics optimization were performed using the TRACK and TraceWin codes. These codes account for the effects of beam space charge. A considerable effort was made to benchmark the codes and to ensure that they produce reliable calculations.

Figure 2.14 shows the structure of one period inside the HWR, SSR1 and SSR2 cryomodules; and Figure 2.15 shows the structure of one period for the low- and high-beta sections of the SC linac (LB650 and HB650 sections). Other details of the beam optics can be found in Ref. [21]. Transverse focusing in the HWR, SSR1 and SSR2 sections is provided by superconducting solenoids, while normal conducting quadrupole doublets are utilized in the LB and HB sections. To minimize gas penetration from the MEBT into the first SC cavity, the period in the HWR cryomodule starts with a SC solenoid. Its vacuum chamber provides cryo-pumping. The sequence of elements in the SSR1 and SSR2 cryomodules is chosen to minimize optics perturbations at the cryomodule-to-cryomodule transitions.

HWR Section :
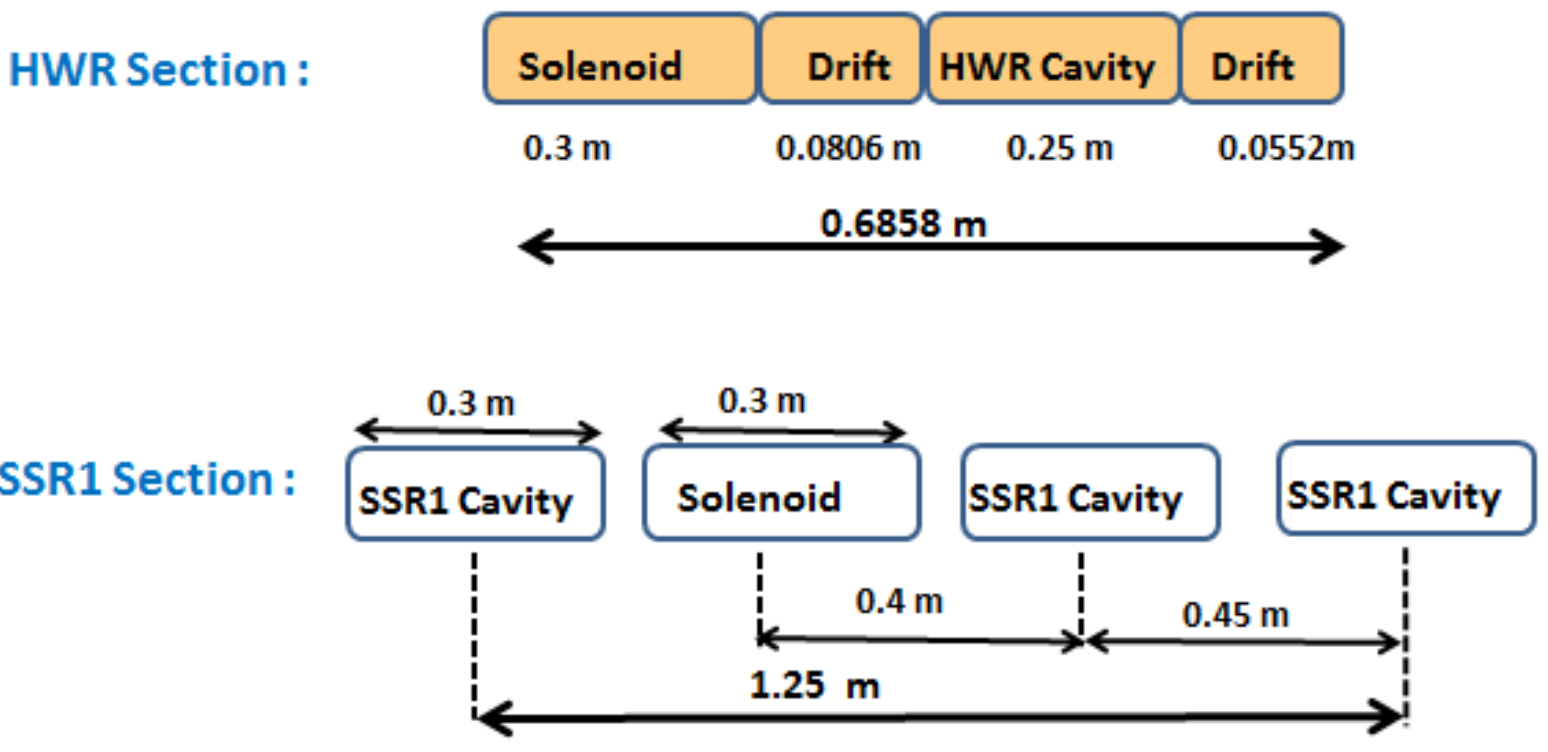

SSR2 Section :

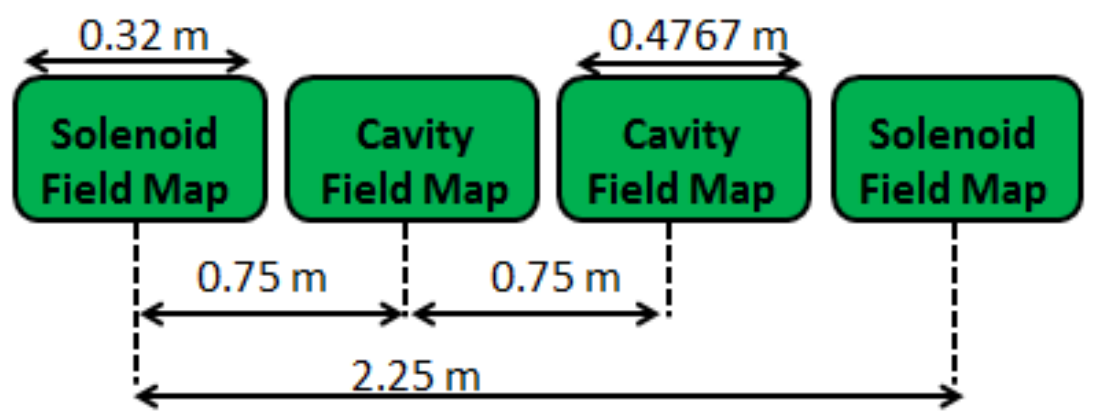

Figure 2.14: Element-to-element distances and element lengths in HWR (top), SSR1 (center) and SSR2 (bottom) sections of the PIP-II SC linac. The structures of cryomodules are: $(\mathrm{sc}) \times 8$ for HWR, (csc) $\times 4$ for SSR1, and (sccsccsc) for SSR2; where $\mathrm{c}$ and s denotes cavities and solenoids, respectively. 

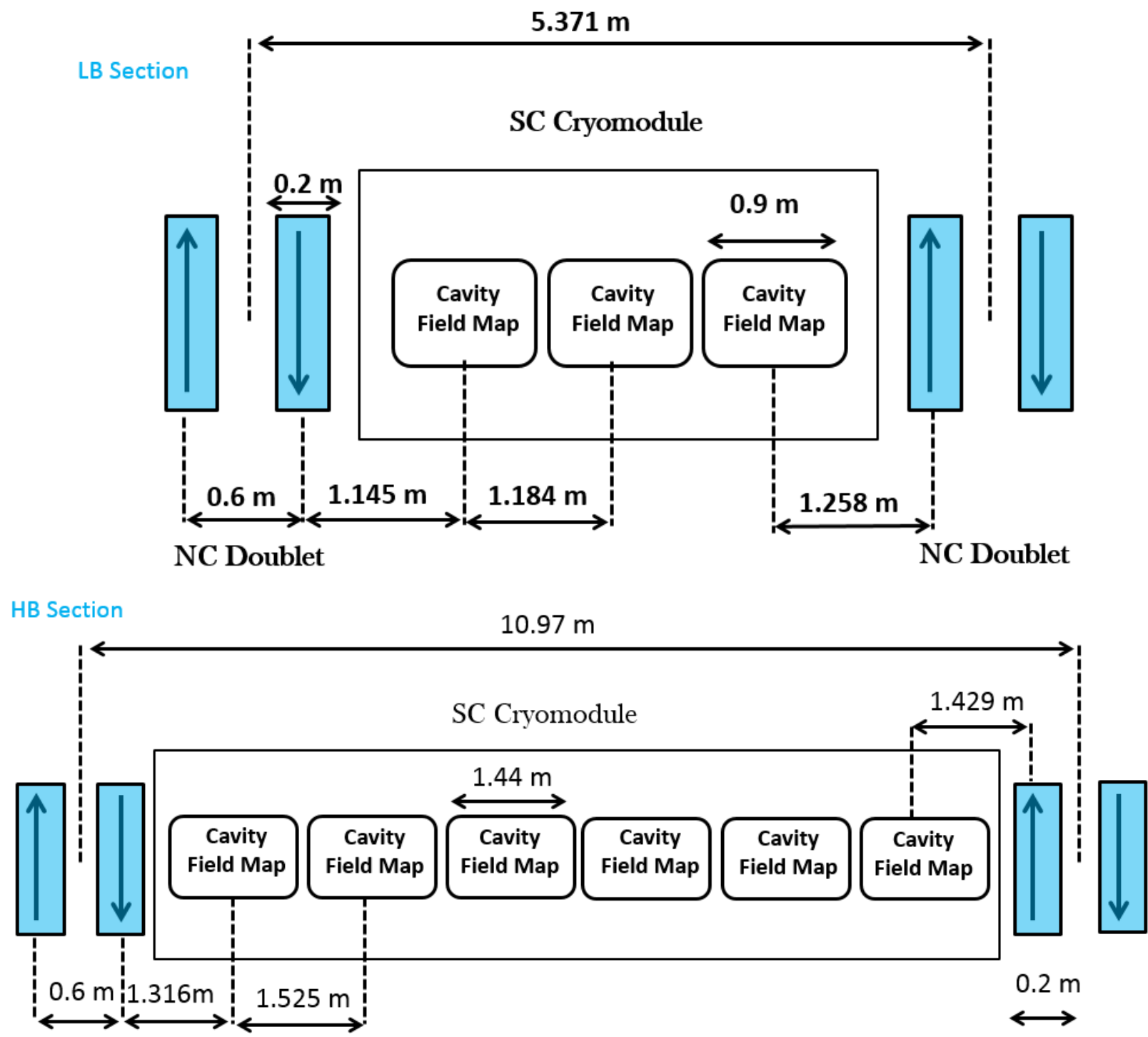

NC Doublet

NC Doublet

Figure 2.15: Focusing period in the LB (top) and HB (bottom) sections of PIP-II SC linac.

Figures 2.16 and 2.17 present the evolution of $1 \sigma$ beam envelopes and the corresponding rms longitudinal and transverse normalized emittances through the entire SC Linac. As one can see there is an emittance growth at the linac beginning where the space charge forces are significant. Simulations show considerable margin for the final value of transverse emittance. Figure 2.18 presents the phase space density projection of a bunch at the linac end. As one can see, there are no significant distortions in the bunch phase space. Simulations show that there are no particles beyond $\sim 6 \sigma$. That result is supported by measurements performed at the SNS, which has a bunch brightness similar to what is expected in the PIP-II. As in the SNS, intrabeam stripping $[22,23]$ is expected to be the main source of particle loss. Figure 2.19 shows the power density of beam losses due to intrabeam stripping. For $\mathrm{CW}$ operation the losses due to this mechanism are below $0.1 \mathrm{~W} / \mathrm{m}$ for the entire linac. Figures $2.20-2.23$ show the strengths of focusing elements, the accelerating voltage and phase. Finally, Figure 2.24 presents the beam energy along the linac, and Figure 2.25 presents the beta-functions computed from the rms beam sizes, angular spreads and emittances, and hence describes the beam transport with the beam space charge forces included. 

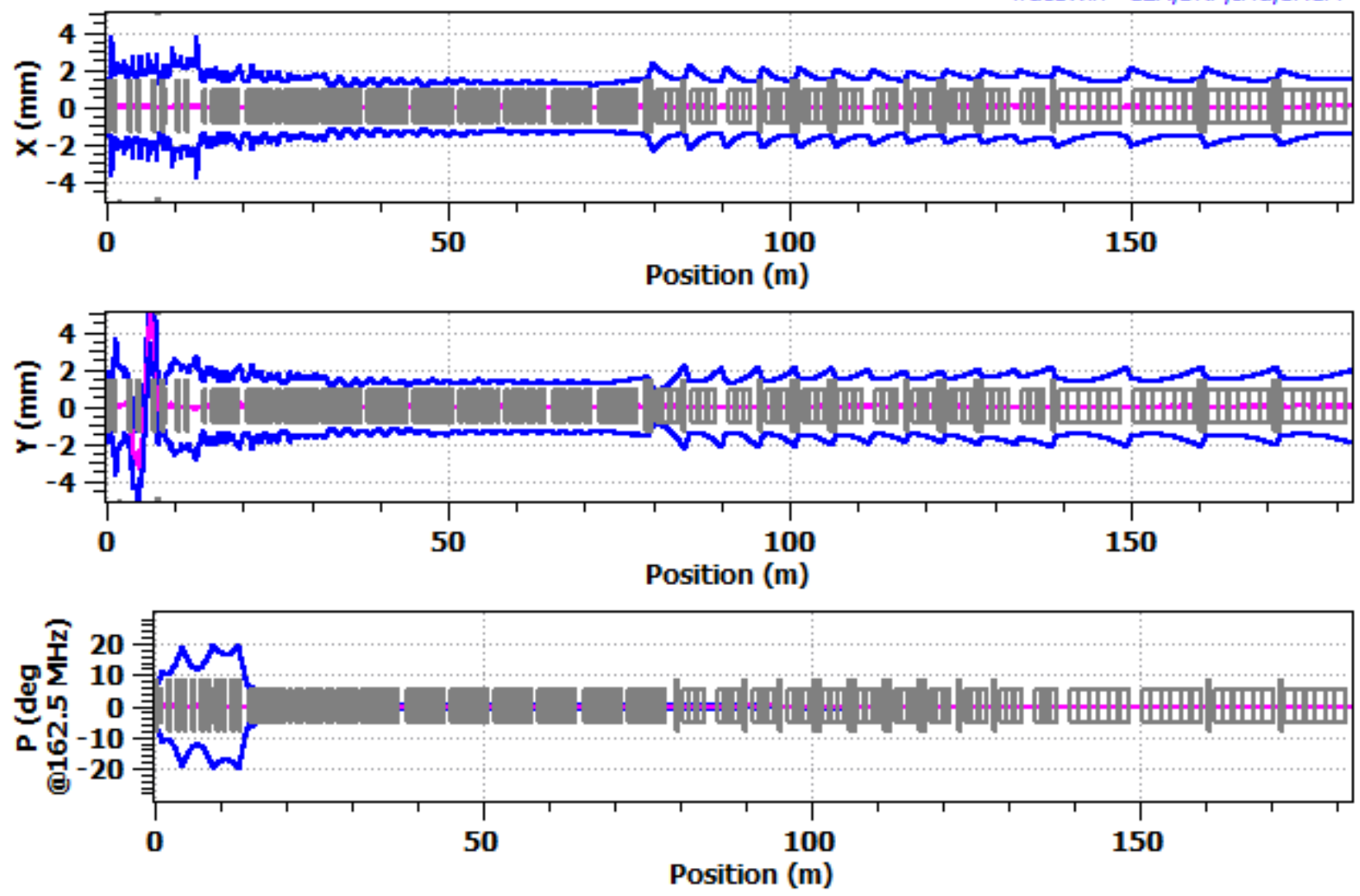

Figure 2.16: Horizontal (top) and vertical (center)) rms bunch envelopes and rms bunch length (bottom) along entire linac (from beginning of MEBT to the end of $0.8 \mathrm{GeV}$ linac); bunch population corresponds to the RFQ beam current of $5 \mathrm{~mA}$. Magenta lines show displacements of the bunch centroid.

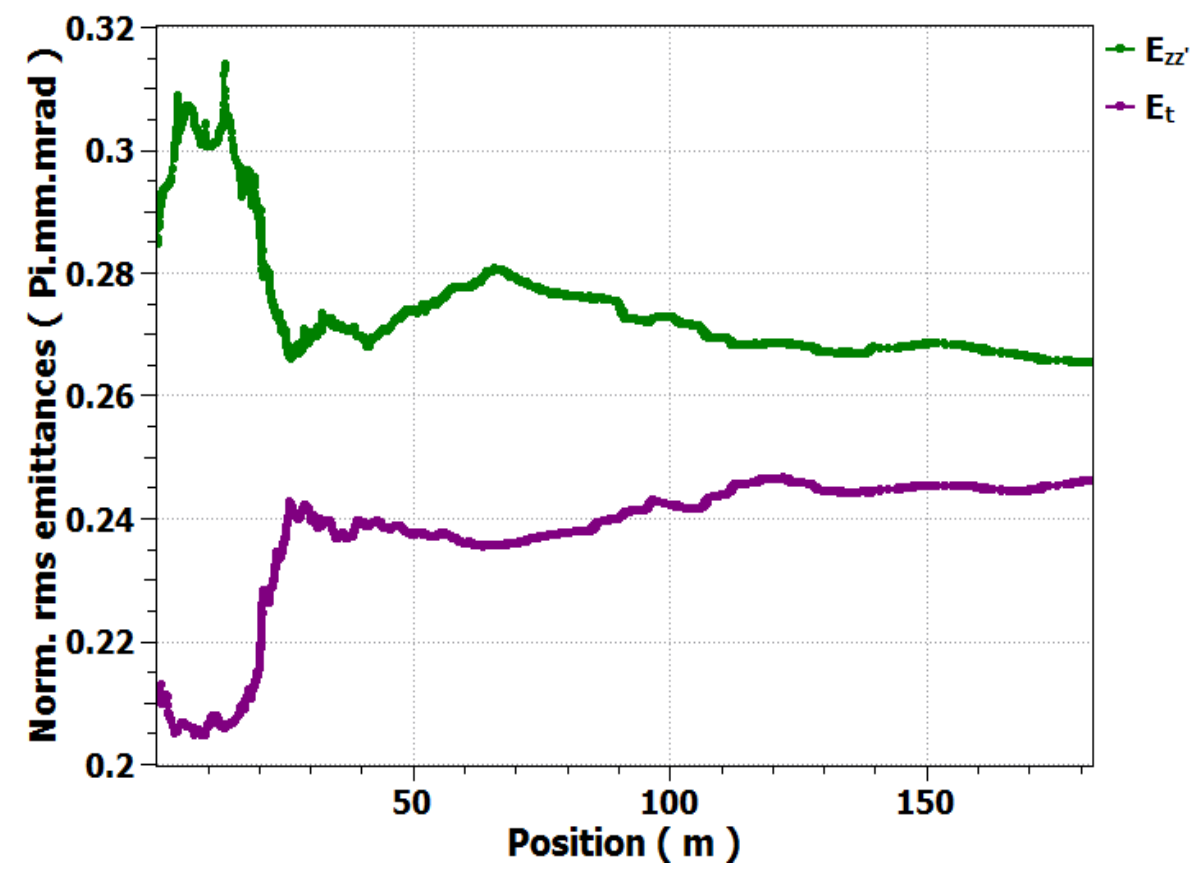

Figure 2.17: Rms normalized transverse (magenta, $\varepsilon_{x}=\varepsilon_{y}$ ) and longitudinal (green) emittances along the linac (from the RFQ exit to the linac end); the beam optics is the same as for Figure 2.16. 
Ele \#1062 [182.04 m] NGOOD : $985741 / 1000000$
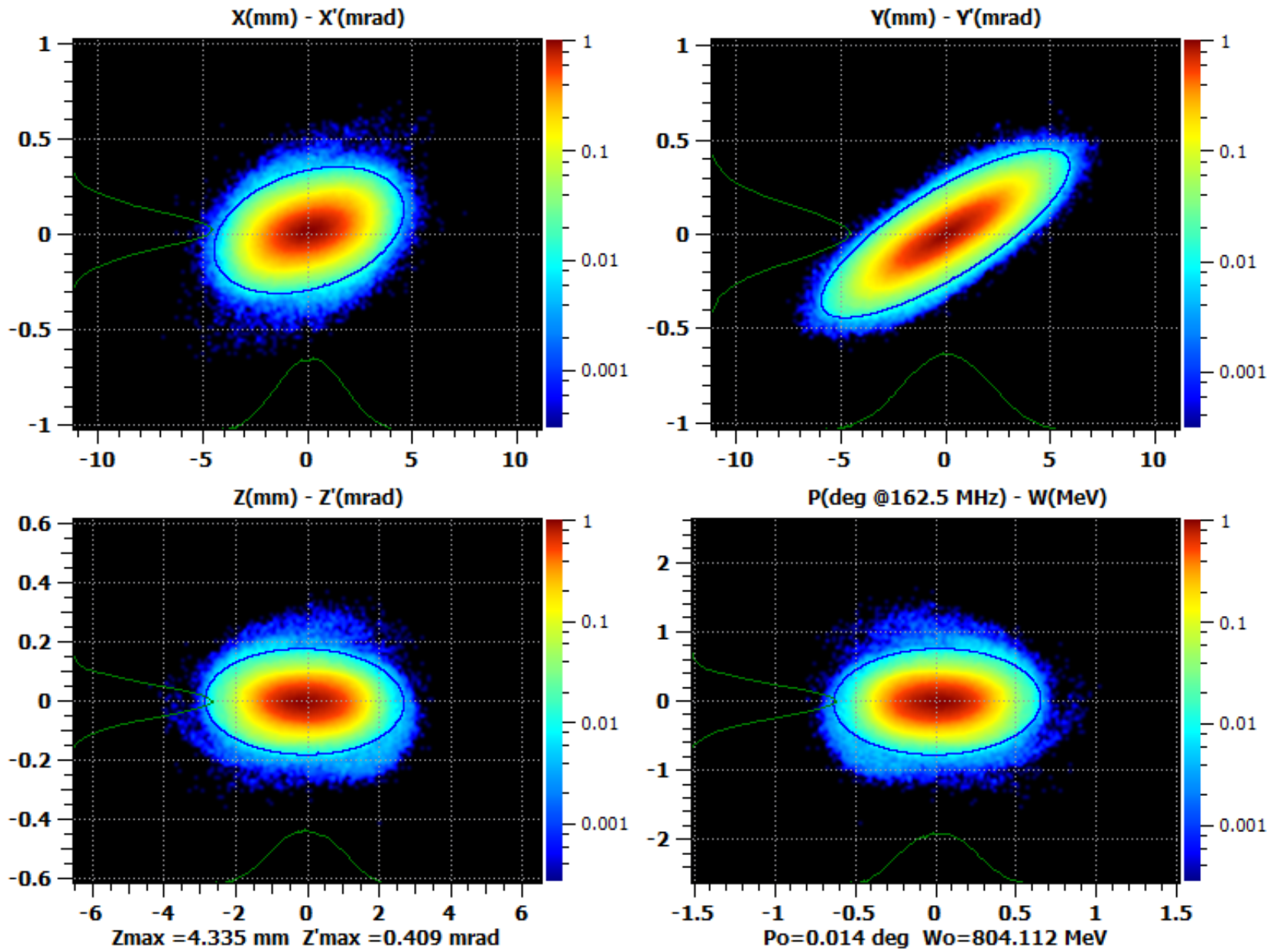

Figure 2.18: Phase space density of a bunch at the linac end; the beam optics is the same as for Figure 2.16.

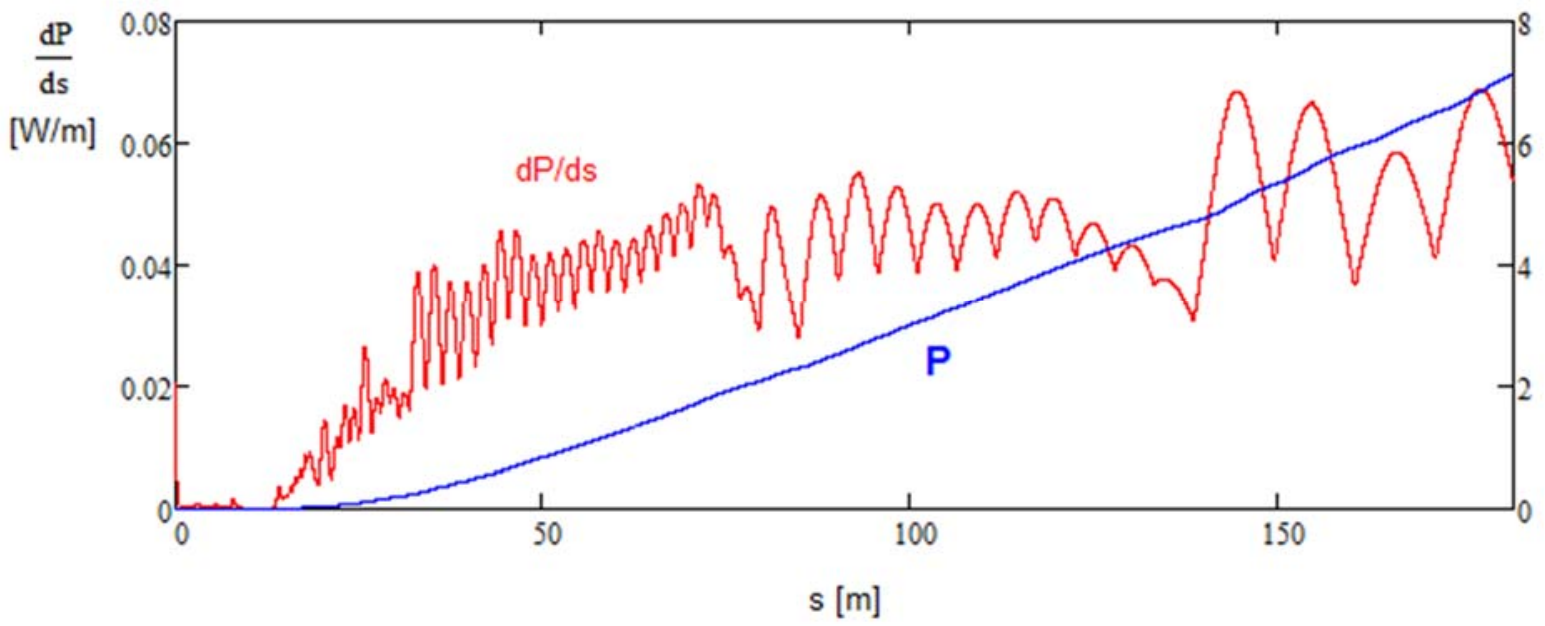

Figure 2.19: The beam power loss per unit length caused by intrabeam stripping (red) and its value integrated along the linac (blue); RFQ beam current $5 \mathrm{~mA}$, CW beam of $2 \mathrm{~mA}$ in the SC Linac ( $60 \%$ of bunches RFQ bunches are chopped off). 

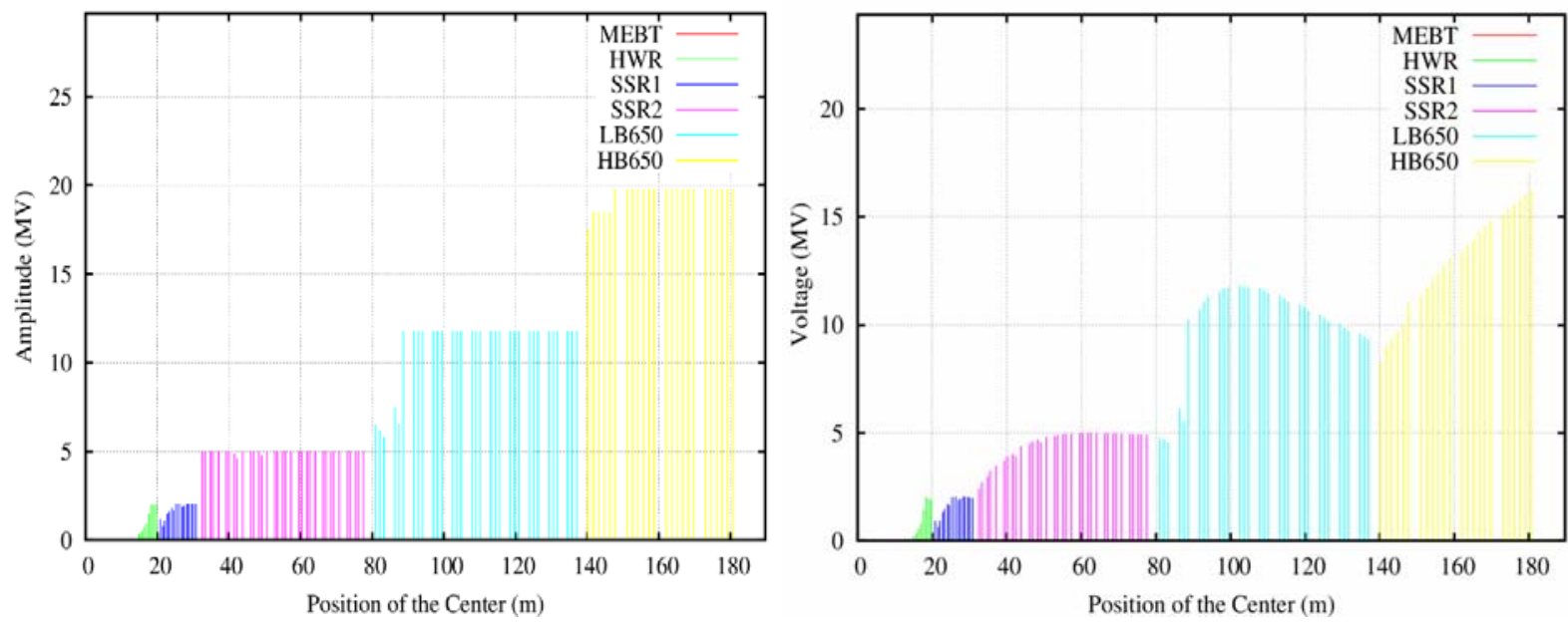

Figure 2.20: Accelerating voltage per cavity along the SC Linac corresponding to the beam optics used for Figure 2.16; left - the voltage amplitude at the optimal beta, right - the voltage amplitude with the transit-time factors accounted.
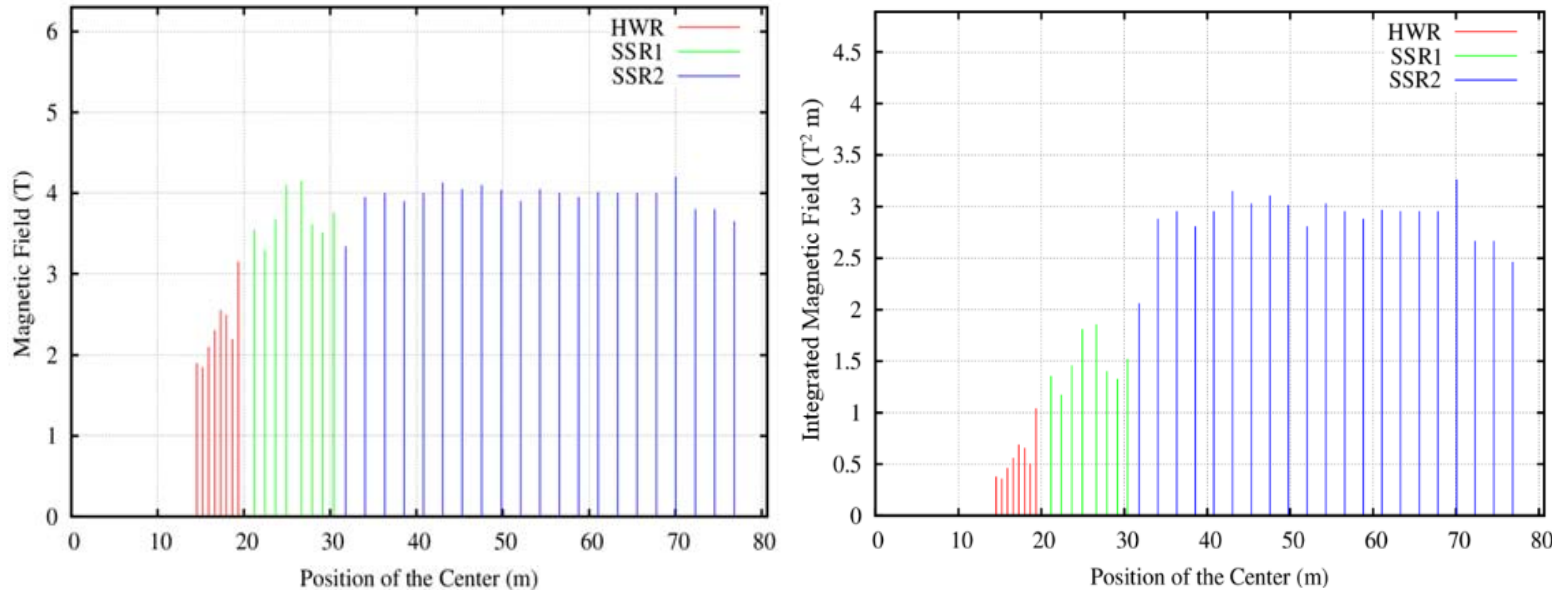

Figure 2.21: Magnetic field of focusing solenoids (left) and their integral strength (right) along the linac corresponding to the beam optics used for Figure 2.16.

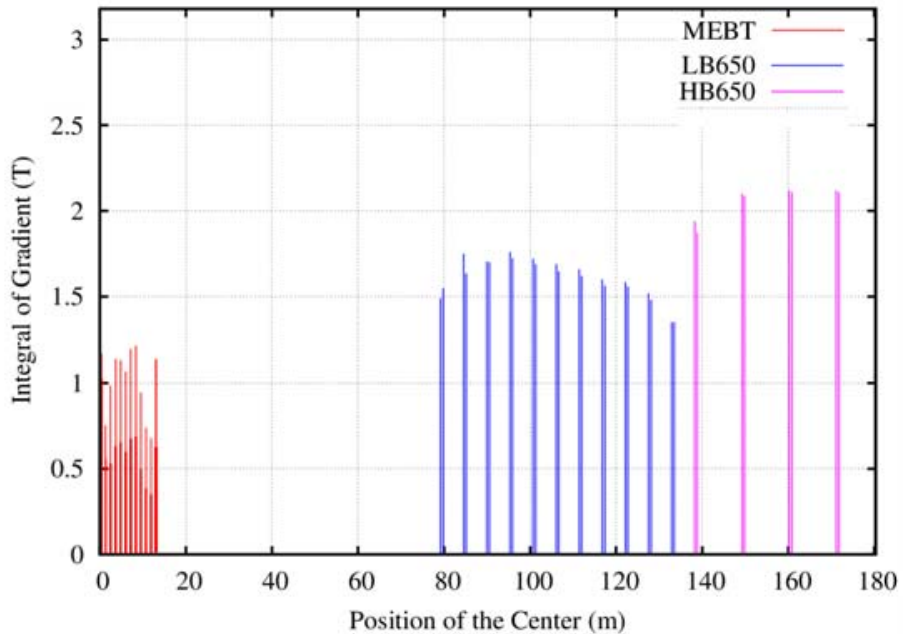

Figure 2.22: Integral strength of quadrupoles along the linac corresponding to the beam optics used for Figure 2.16. 


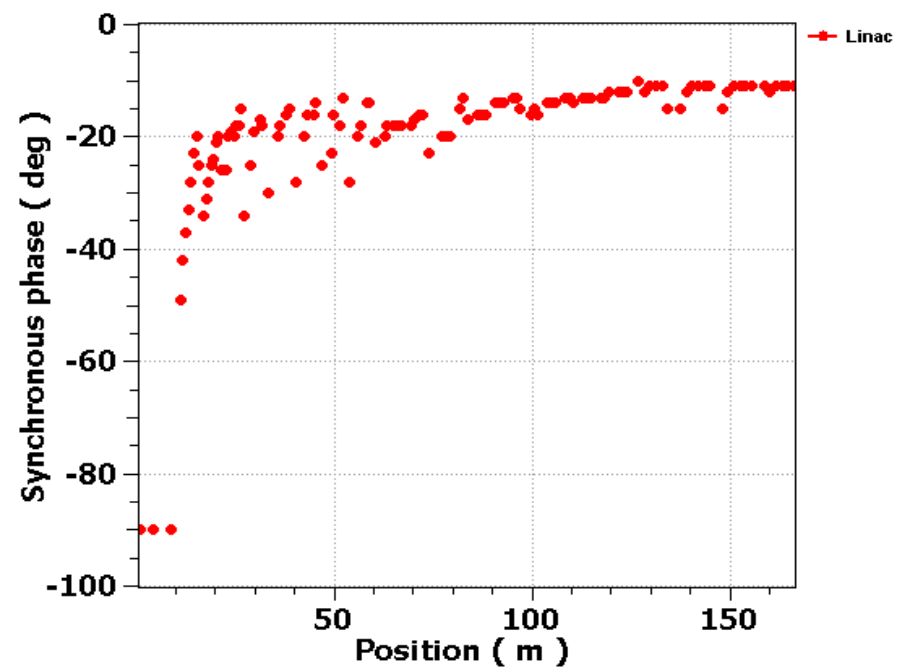

Figure 2.23: Accelerating phase along the linac corresponding to the beam optics used for Figure 2.16. The first three dots belong to the MEBT bunching cavities, which do not produce acceleration.

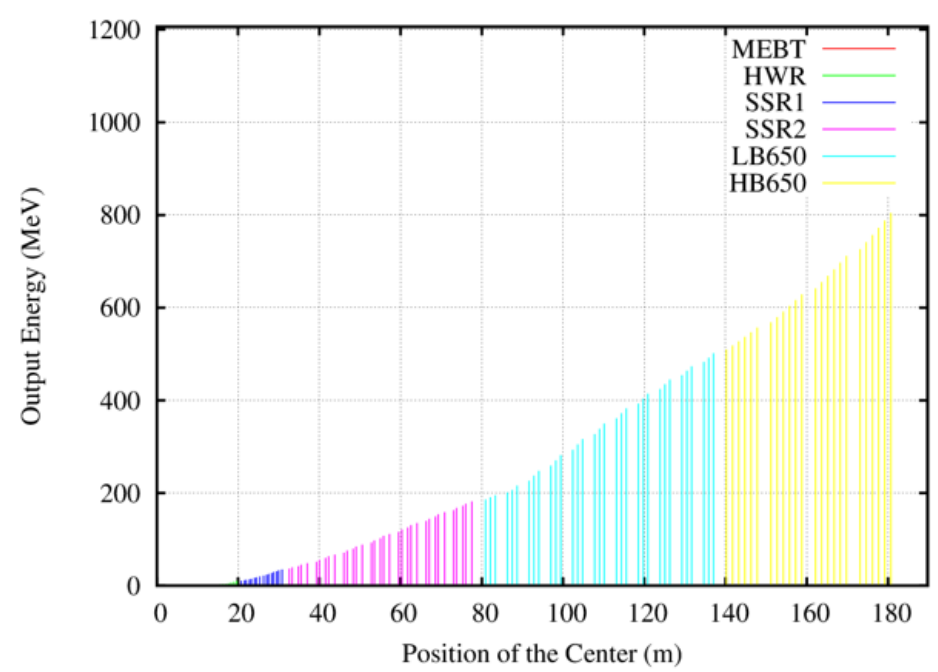

Figure 2.24: The beam energy along the linac.

As shown in Figure 2.16 the transverse rms beam sizes change comparatively little along the linac, and do not exceed $3 \mathrm{~mm}$ in the course of entire acceleration. This weak dependence of beam sizes on the acceleration implies that the adiabatic reduction of beam transverse emittances with beam acceleration is compensated by corresponding increase in the beta-functions. Figure 2.26 presents the beam density projection to the horizontal plane and the aperture limitations along the linac. For the HWR, SSR1, and SSR2 cryomodules, the aperture is limited by apertures in the SC cavities of 33, 30 and $40 \mathrm{~mm}$, respectively. For the LB650 and HB650 cryomodules, the cavity apertures are 83 and $118 \mathrm{~mm}$, respectively. Consequently, the aperture limit for these sections is set by the aperture of the vacuum pipe in the quadrupoles of $46 \mathrm{~mm}$ (standard 2" pipe). As stated above, in the case of a well-tuned machine the intrabeam stripping represents the main source of beam loss. To avoid the beam losses to cryogenic surfaces fixed aperture beam collimators are installed between each cryomodule for HWR, SSR1, and SSR2. Apertures in the collimators are chosen to be $3 \mathrm{~mm}$ smaller than the apertures of the downstream cryomodules. Their thickness increases with energy, reaching $4 \mathrm{~cm}$ of steel at the end of SSR2 section. There are no dedicated collimators in the LB650 and HB650 
sections. As in the SNS, the vacuum chambers in the quadrupoles will perform this role. Taking into account the comparatively small beam losses it appears unnecessary to have additional radiation shielding around the collimators which otherwise would require quite a large amount of shielding material.

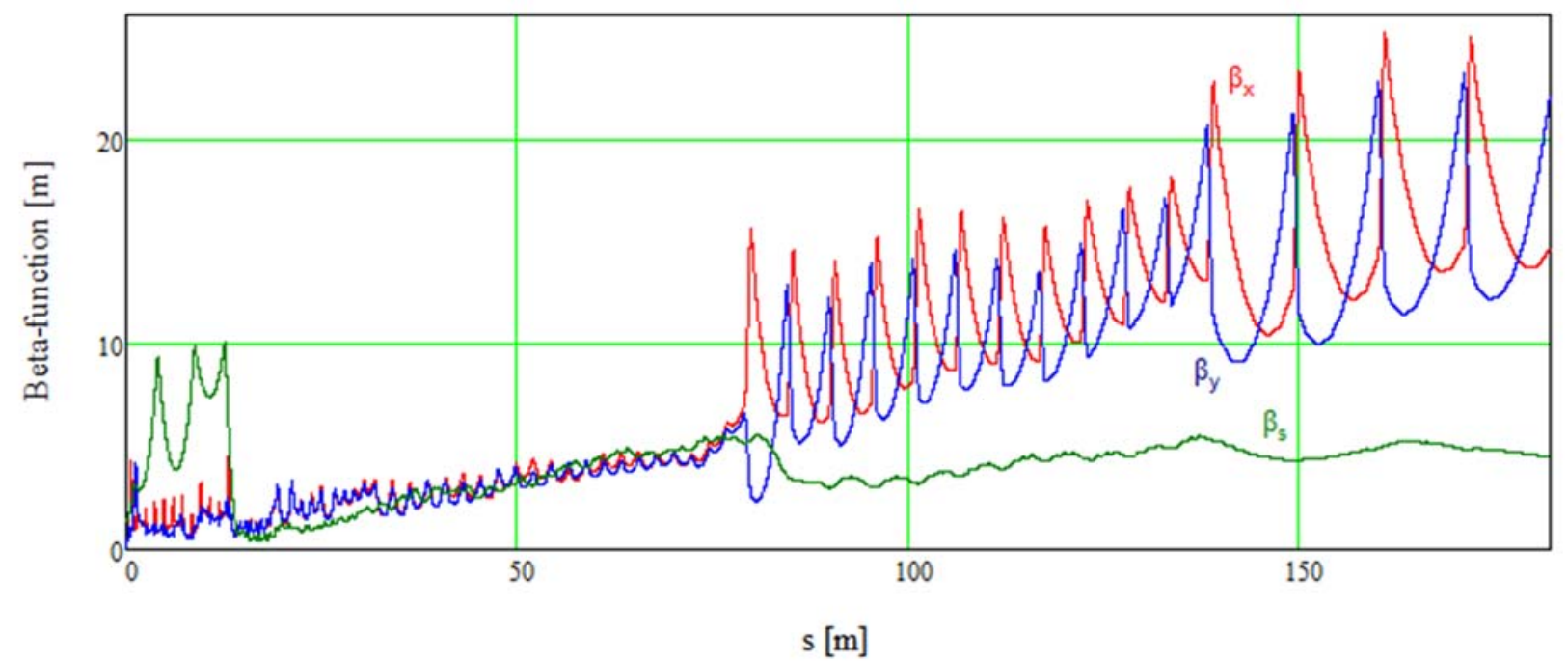

Figure 2.25: The beta-functions for $\mathrm{x}, \mathrm{y}$ and s planes along the linac from the RFQ exit to the linac end. The values were computed from rms beam sizes and emittances obtained by beam tracking with TraceWin for 5 mA RFQ current.

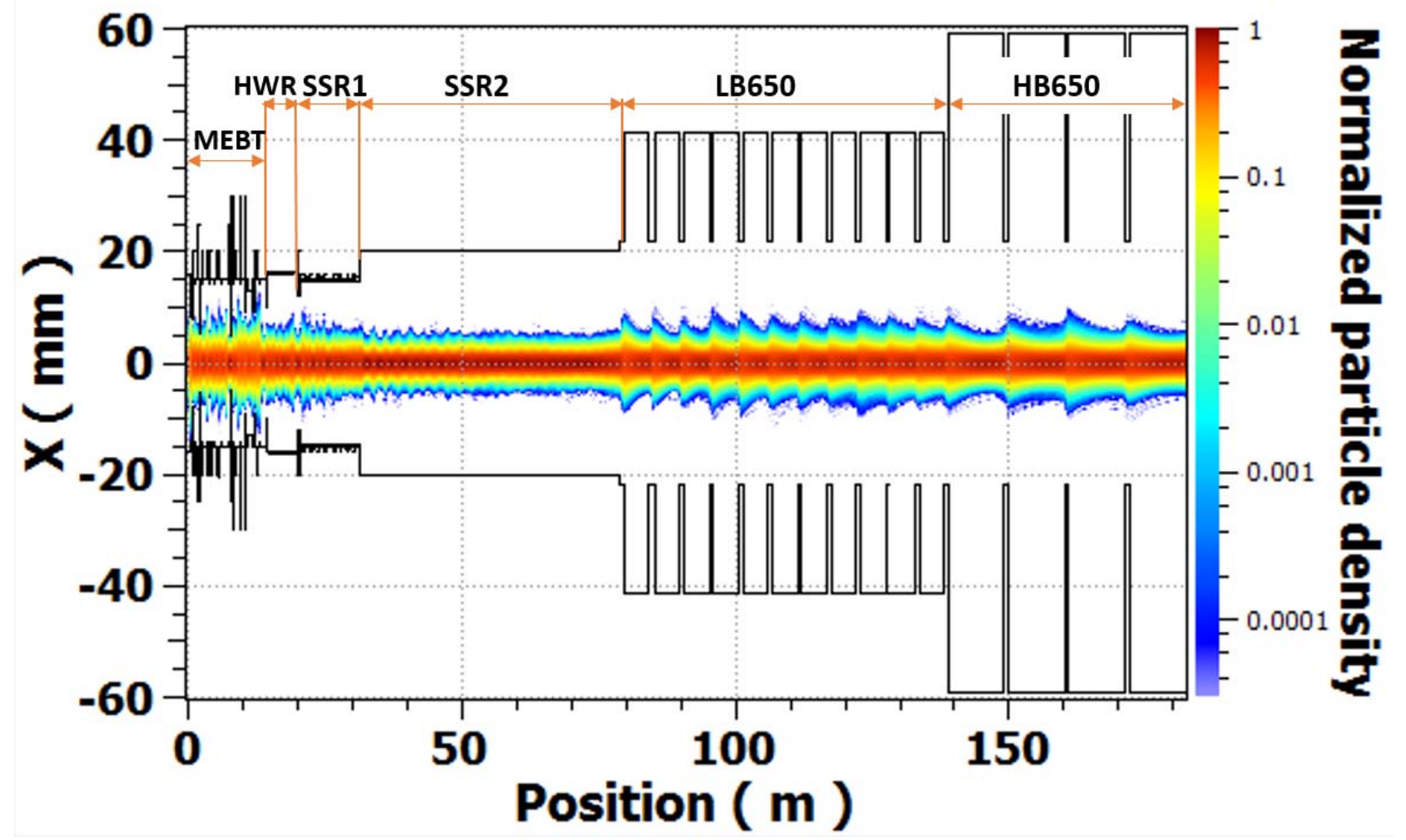

Figure 2.26: Beam density projection in the horizontal plane and aperture limitations along the linac from the RFQ exit to the linac end. 

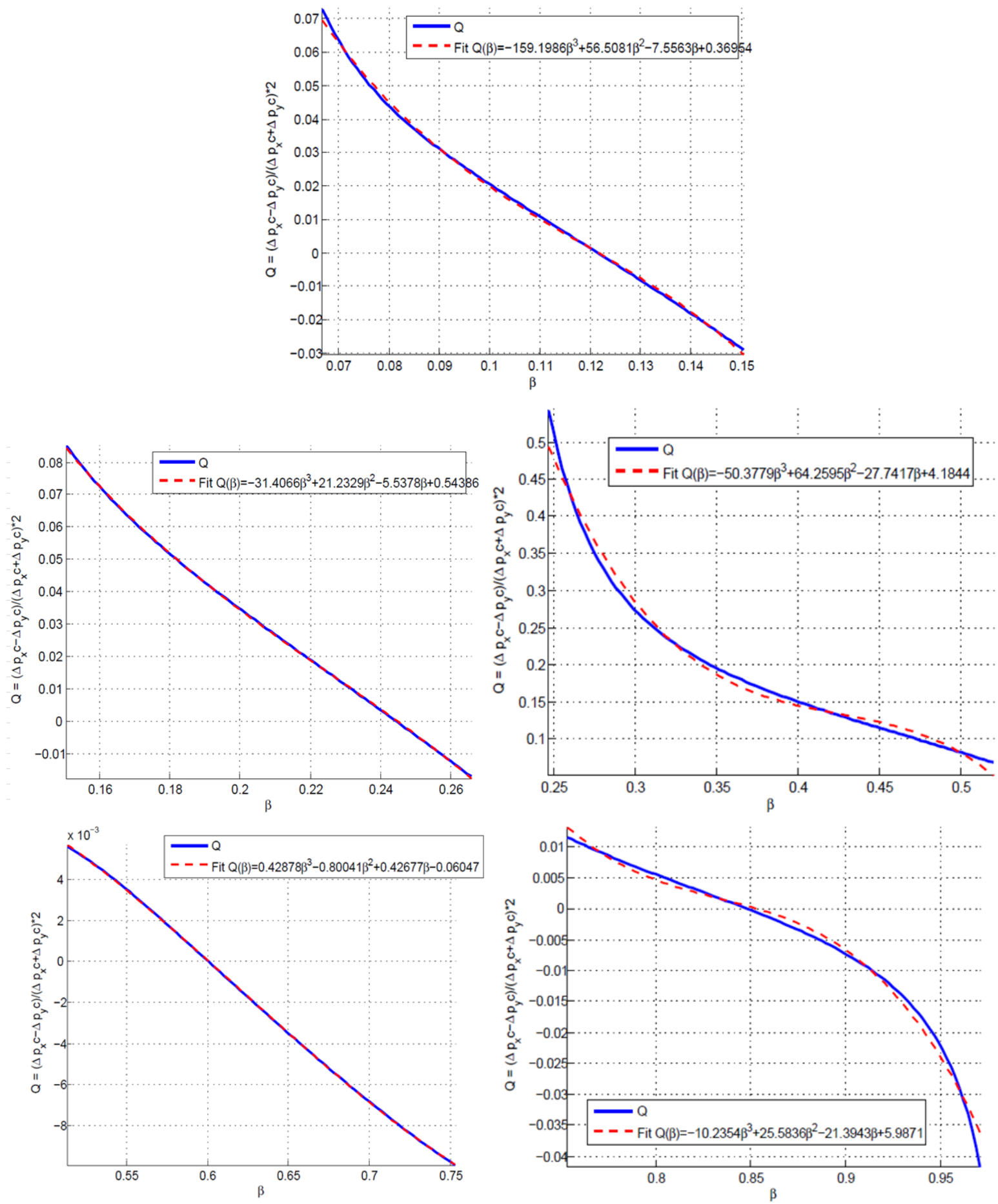

Figure 2.27. The focusing asymmetry parameters (ratio of quadrupolar to radial focusing) for HWR (top), SSR1 (middle-left), SSR2 (middle-right), LB650 (bottom-left) and HB650 (bottom-right) versus the particle velocity $\beta$ in the operating domain; blue and red lines present simulation results and a polynomial fit, respectively.

The HWR, SSR1 and SSR2 cavities are not axially symmetric. Therefore, their quadrupole components cannot be compensated over the entire range of beam velocities. Figure 2.27 presents the dependence of the quadrupole effect on velocity. Numerical simulations verified that in the range of PIP-II parameters the strength of quadrupole field is proportional to the strength of axially 
symmetric cavity defocusing. Both types of focusing are proportional to the sine of the accelerating phase. That motivates the definition of the focusing asymmetry parameter, $Q$, presented in Figure 2.27. The stem in the HWR cavity is located in the horizontal plane and therefore this quadrupole field represents a normal quadrupole. Due to engineering limitations, mainly related to the RF couplers, the SSR1 and SSR2 cavities are rolled by $45^{\circ}$. Consequently, their quadrupole fields are also rolled and are equivalent to a skew-quadrupole field. The cavity quadrupole and skewquadrupole fields have a small but non-negligible effect on beam dynamics. To compensate these fields the SSR1 and SSR2 cryomodules will have skew-quadrupole correction coils located inside the focusing solenoids. The skew-quadrupole field will be created by an imbalance in the currents of independently powered coils of $x$ - and $y$-dipole correctors. Since the solenoids located in the HWR, SSR1 and SSR2 cryomodules rotate the plane of betatron motion, compensation can be improved by appropriate choice of magnetic field signs in the solenoids. The eight cavities of the HWR cryomodule introduce little coupling, and therefore, this cryomodule does not have coupling correction.

The quadrupole fields in the LB650 and HB650 cavities are related to the RF couplers. Compared to the HWR, SSR1 and SSR2 they are significantly smaller as can be seen in the bottom row of Figure 2.27. These quadrupole fields will be corrected by the main focusing quadrupoles located between cryomodules. The RF couplers of LB650 and HB650 cavities also create dipole fields resulting in dipole kicks which are also dependent on the beam velocity. The maximum kicks, $\Delta \mathrm{p}_{\perp} \mathrm{c}$, are about $3.6 \mathrm{keV}$ for LB650 and $2.6 \mathrm{keV}$ for HB650 cavities. The corresponding beam deflections are small and are not expected to produce any visible effect on the beam motion. The coupling accumulated in the linac is corrected by four skew-quadrupoles located at the linac downstream end in two periods immediately following the last cryomodule. Two skew-quadrupoles are placed on both sides of each doublet.

\subsubsection{Fault Scenarios in SC Linac}

An accelerator has to deliver a high quality beam with high availability. The high number of elements in the linac increases the likelihood of failure. In this section we consider ways to mitigate such failures.

Failure of the beam transport elements like cavity, solenoid or quadrupole alters the periodicity of beam focusing, resulting in a mismatch of beam transport with downstream sections. This, in turn, may degrade beam quality and, in the worst case, may cause beam losses. The implication of a failure on machine performance depends on the failure location. In some cases, a failed element results in significant beam losses. If the machine cannot be retuned into an acceptable state, it becomes necessary to replace this element before operation can continue. In some instances, a full cryomodule replacement may be required. It is a time consuming process requiring warmup and subsequent cooldown after repair. To improve reliability optics should be tolerant to at least one major fault in each section. An extensive study has been performed to address this problem for the PIP-II SC linac. Below we discuss failure of beamline elements at critical locations and demonstrate that the optics design of the PIP-II SC linac is sufficiently robust to compensate for failure of a cavity or cryomodule by retuning nearby elements.

\section{Failure of Beamline Elements}

Element failures can be put in two categories: temporary failures and permanent failures. Temporary failures are recoverable after applying appropriate mitigation measures. Quenching of a SC cavity and vacuum breakdown are examples of temporary failures. Such failures do not require 
linac retuning and can be resolved comparatively quickly. A permanent failure occurs when one or more elements become malfunctioning and have to be withdrawn from operations. In this case, retuning of the machine, if possible, can be the most efficient way to maximize machine availability. Such action implies that repairs will be done later when conditions allow. The most probable permanent failures are expected to be:

- Failure of a cavity tuner resulting inability to keep this cavity in the resonance

- Malfunctioning of the power-coupler resulting in reduced accelerating gradient or inability to use this cavity

- Failure of a RF power supply

- Degradation of Q0 resulting in reduced voltage

- Failure of a focusing magnet (short in a coil, quenching, non-functioning power supply, etc.).

In the following sub-sections we discuss permanent failures of accelerating cavities and focusing magnets at critical locations in the PIP-II SC linac.

\section{Local Compensation of Failed Elements}

As will be seen in the following sections, failure of a beamline element causes longitudinal and transverse mismatches leading to emittance growth and beam losses in the downstream part of the linac. In order to restore the beam quality, a local compensation involving adjustments for settings in nearby elements is used. The procedure should minimize excessive oscillations of the bunch sizes. PIP-II has separate powering for each cavity and solenoid. It is greatly helpful for compensation. The constraints and assumptions for local compensation are summarized below:

- Accelerating field in cavity: fields can be varied to recover the beam energy but the surface peak magnetic and electric fields should not exceed their design limits.

- Integral fields in quadrupoles and solenoids should not exceed their design limits.

- Synchronous phases of cavities are varied in such a way that the ratio of synchronous phase (referenced to on-crest acceleration) to longitudinal rms beam size should be greater than 3 . This provides sufficient longitudinal acceptance to accommodate $3 \sigma$ beam.

- A minimum number of retuned elements should be used in order to expedite retuning and to minimize overall effort.

- $100 \%$ beam transmission and minimal emittance growth at the linac end should be achieved.

\section{Failure of the First HWR Accelerating Cavity}

The HWR is the first SC section in the PIP-II linac. It accelerates and focuses the beam coming out of the MEBT. Each focusing period in the HWR section includes a solenoid and a HWR cavity. Failure of the first cavity in the HWR section is considered to be the most serious one due to large transverse and longitudinal beam sizes at this location. At this point, the beam is non-relativistic therefore this failure, if uncompensated, results in both phase and energy oscillations propagating downstream and growing in amplitude along the linac. Furthermore, the space charge forces are quite large resulting in an amplification of beam losses. Figure 2.28 shows the rms longitudinal and transverse beam sizes along the linac after failure of the first HWR cavity. Abrupt changes in the longitudinal beam profile occur due to beam losses happening at transitions between cryomodules. The total beam losses below $0.01 \%$ are observed in the SC linac after failure of the first HWR cavity. The momentum mismatch results in a perturbation of transverse motion. However, as can be seen from Figure 2.28, the transverse beam sizes are comparatively unperturbed due to the small energy gain in the first HWR cavity. This cavity operates at half of nominal voltage and has a large 
accelerating phase offset from on-crest acceleration in order to capture the beam with large longitudinal emittance.

Figure 2.29 shows normalized rms transverse and longitudinal emittances along the linac. The abrupt change in the longitudinal emittance is caused by the beam losses at beginning of the LB650 section. In contrast, there is no significant transverse emittance growth along the linac.
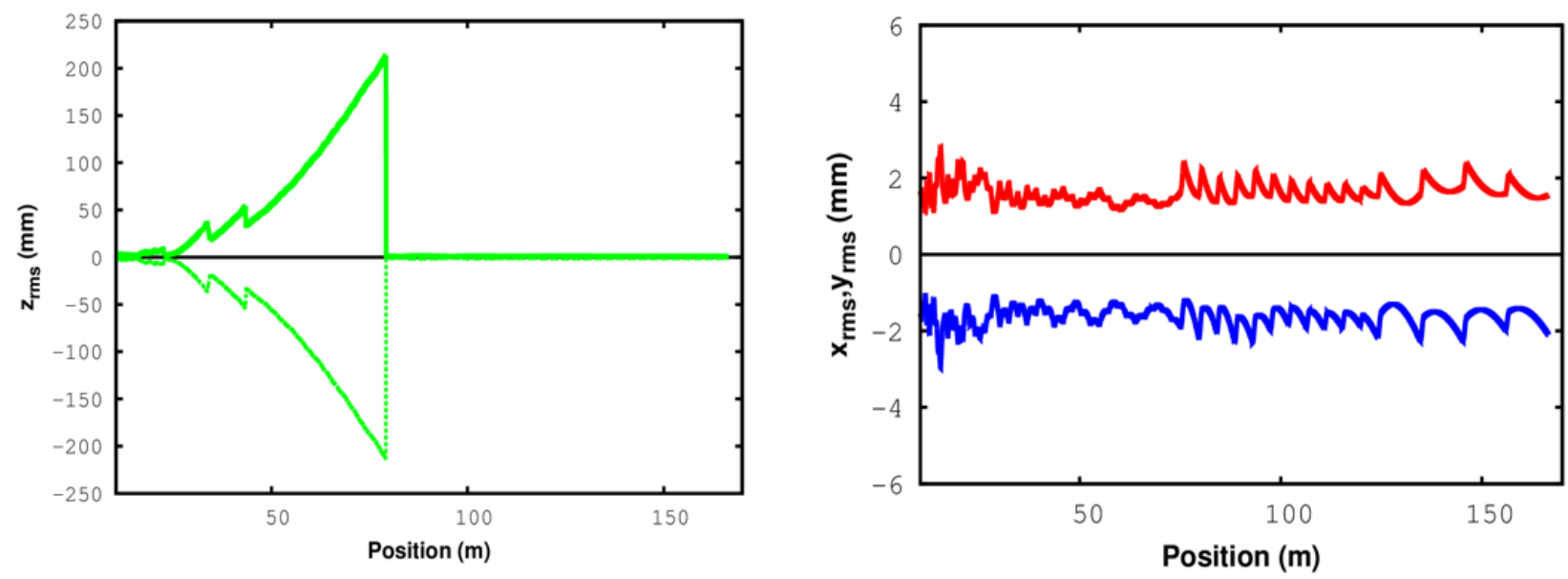

Figure 2.28: Longitudinal (left) and transverse (right) rms beam sizes along the linac (starting at the MEBT end) after failure of the first HWR cavity (horizontal - red, negative vertical - blue).

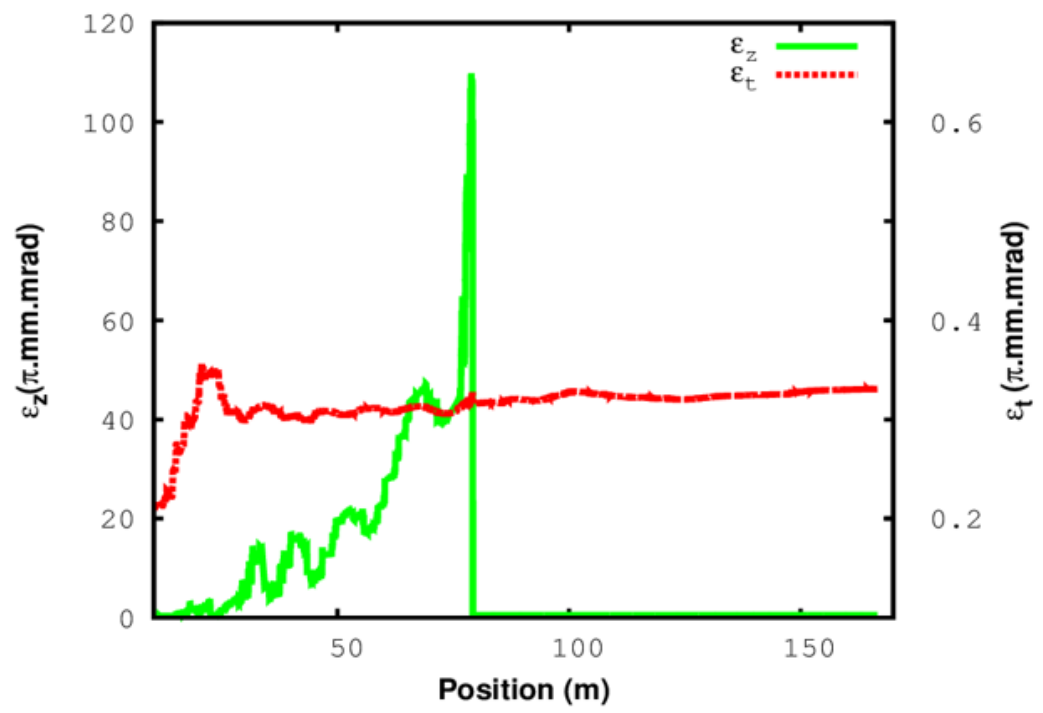

Figure 2.29: Normalized rms longitudinal (green, left scale) and transverse (red, right scale) emittances along the linac after failure of the first HWR cavity.

Figure 2.30 presents results of an application of local compensation used to restore the beam quality. The figure shows the rms bunch sizes and the elements used in the retuning: the last bunching cavity at MEBT and the three HWR cavities downstream of the failed cavity. Additionally, the two subsequent HWR cavities are retuned to recover the beam energy. This prevents excessive bunch size oscillations in the downstream linac without its retuning.

Figure 2.31 presents the bunch envelopes for the entire linac with and without compensation. As can be seen, both the transverse and longitudinal envelopes are completely recovered, and there is no beam losses. Figure 2.32 shows the synchronous phases and the longitudinal beam size. As one 
can see the longitudinal acceptance is large enough to accommodate $6 \sigma$ beam. The left pane in Figure 2.33 shows the normalized rms longitudinal emittances before and after compensation. Note that the emittance after compensation is plotted for the right-hand $y$-axis. As expected, the compensation resulted in smaller emittances; and there is no significant emittance growth in all planes. Table 2.12 presents a comparison of beam emittances at the linac end for different cases.
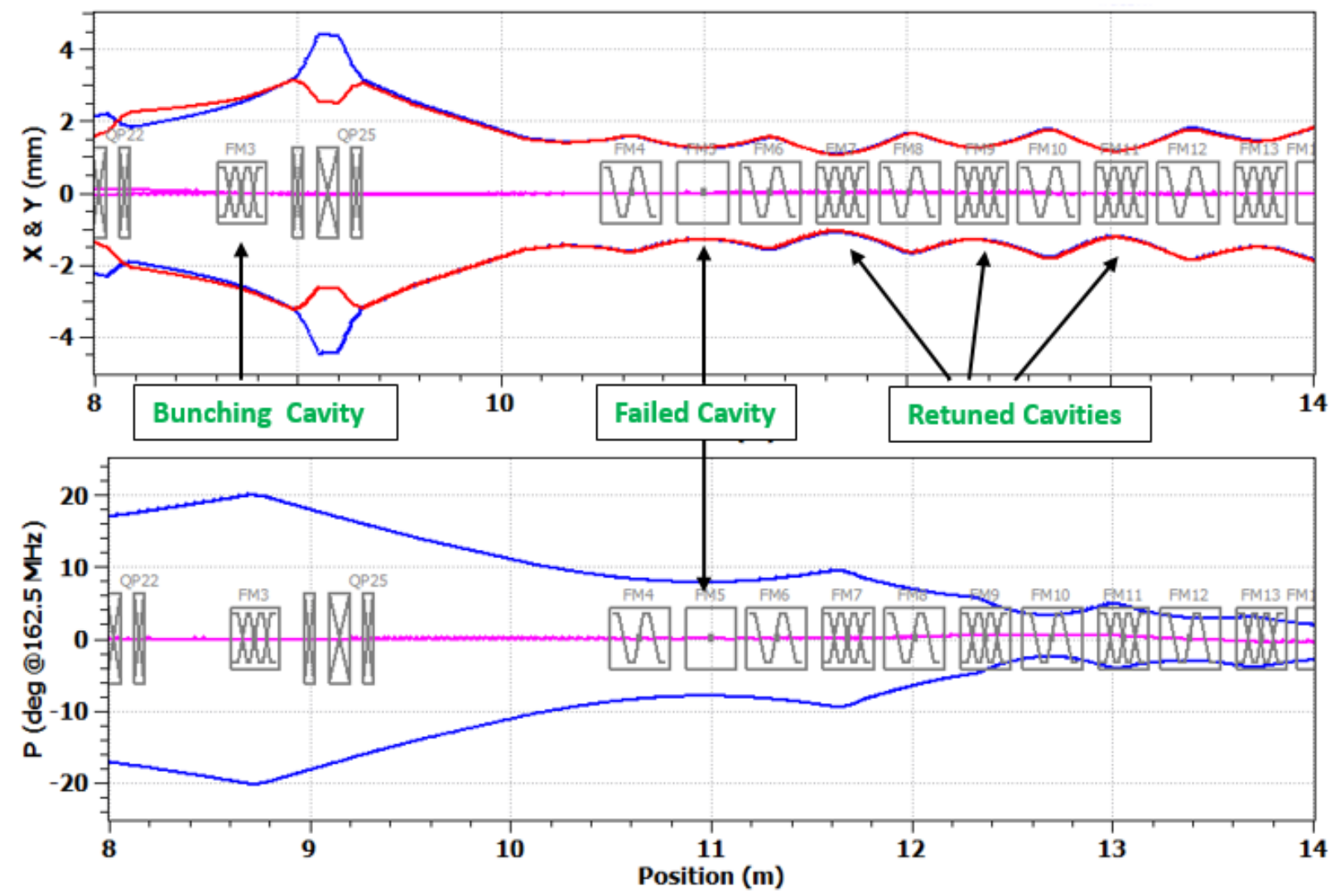

Figure 2.30: Enlarged view of beam profiles in the transverse (top) and longitudinal (bottom) planes after applying local compensation of a first HWR cavity failure. Elements used for retuning are pointed out by arrows.
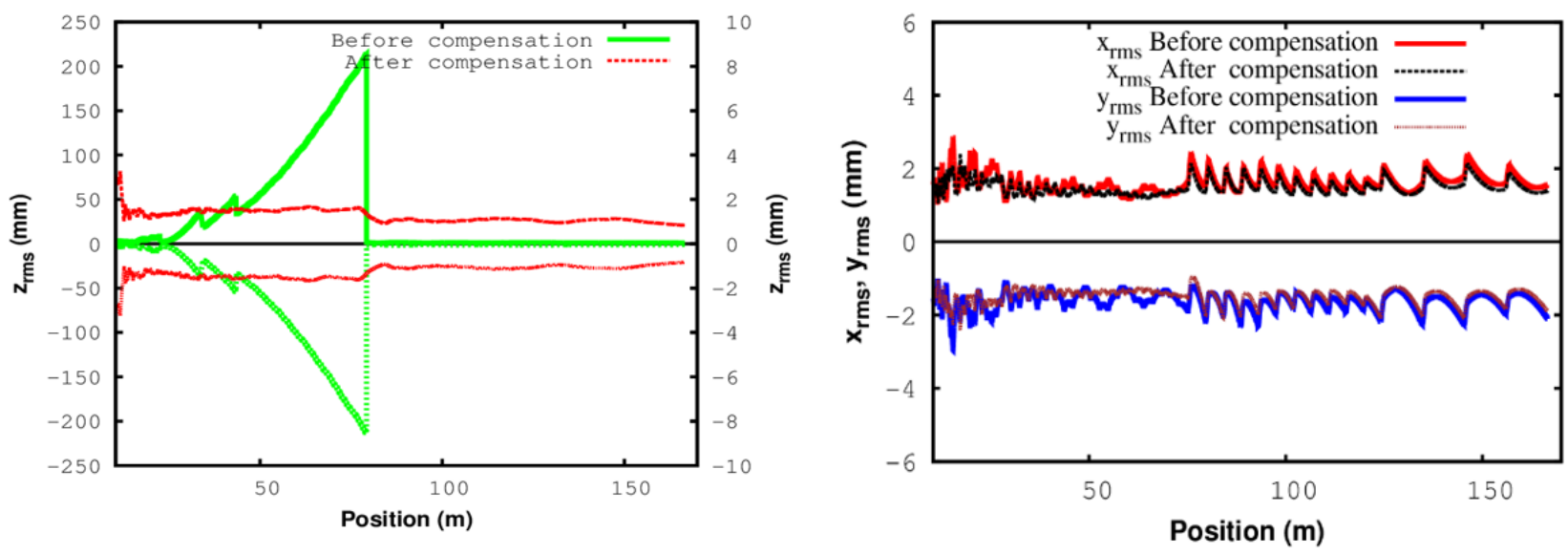

Figure 2.31: Longitudinal (left) and transverse (right) beam sizes along the linac before and after applying local compensation for a failure of the first HWR cavity. 


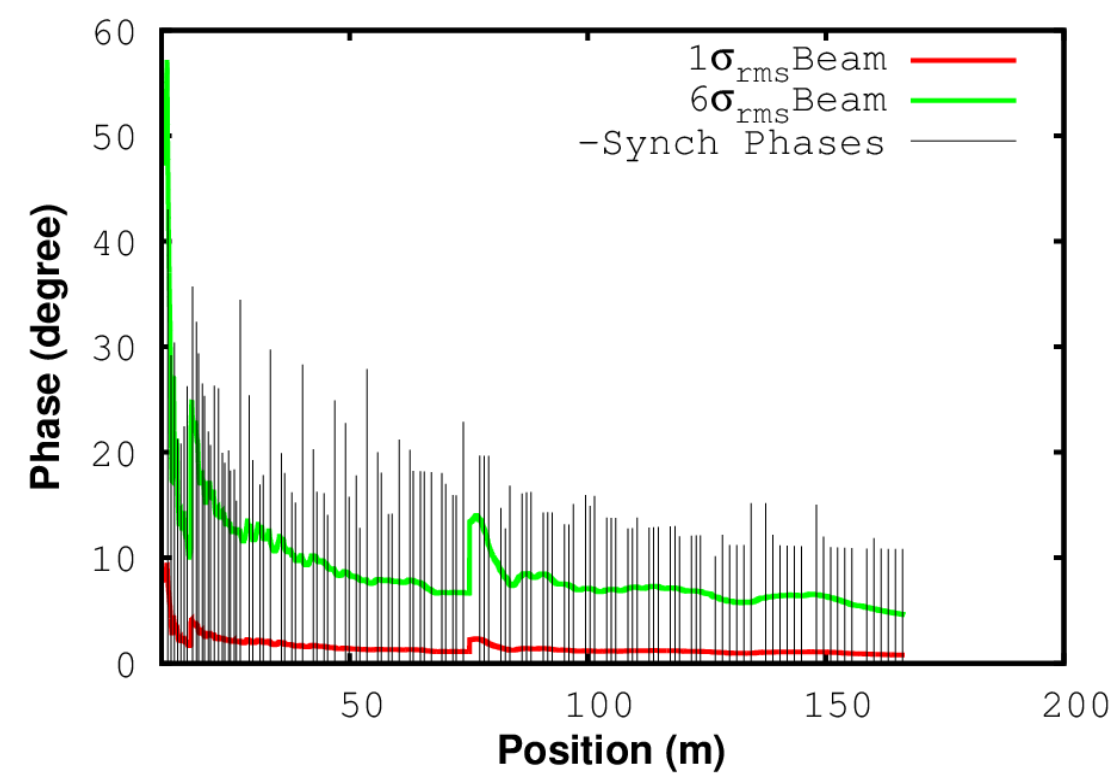

Figure 2.32: Synchronous phases with longitudinal beam size along the PIP-II SC linac.
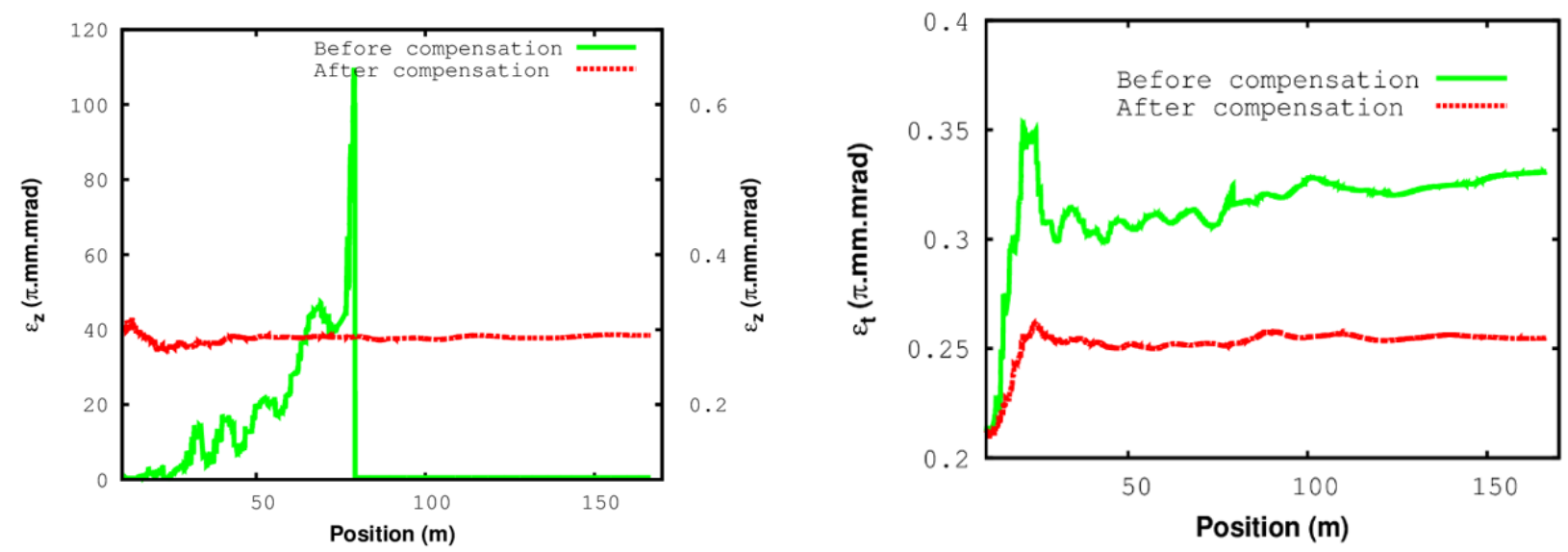

Figure 2.33: Normalized rms longitudinal (left) and transverse (right) emittances along the linac in the presence of first failed HWR cavity. The picture on the left has different scales for the emitances with and whithout compensation.

Table 2.12: Final normalized rms beam emittances

\begin{tabular}{c|l|l|l|l}
\hline \hline Parameters & Unit & Nominal & \multicolumn{2}{|c}{ Failure of first HWR cavity } \\
\hline & & & No Comp. & After Comp. \\
\hline$\varepsilon_{\mathrm{z}}$ & $\mathrm{mm} \mathrm{mrad}$ & 0.28 & 0.52 & 0.29 \\
\hline$\varepsilon_{\mathrm{t}}$ & $\mathrm{mm} \mathrm{mrad}$ & 0.25 & 0.33 & 0.25 \\
\hline \hline
\end{tabular}

\section{Failure of the First Solenoid in the HWR Section}

At the beginning of the linac the transverse beam size is relatively large and the beam barely fits in the physical aperture. A failure of the first solenoid in the HWR section is as critical as a failure of the first HWR cavity. It results in transverse beam size oscillations with large amplitude leading to emittance growth and beam losses. Local compensation is applied by using the same approach as 
discussed above - the neighboring elements are retuned to achieve a smooth beam profile along the linac. Figure 2.34 shows beam envelopes before and after applying the local compensation. The figure demonstrates that the transverse beam oscillations are minimized and the transverse beam profiles are restored. Figure 2.35 shows the corresponding normalized rms emittances before and after applying this compensation.
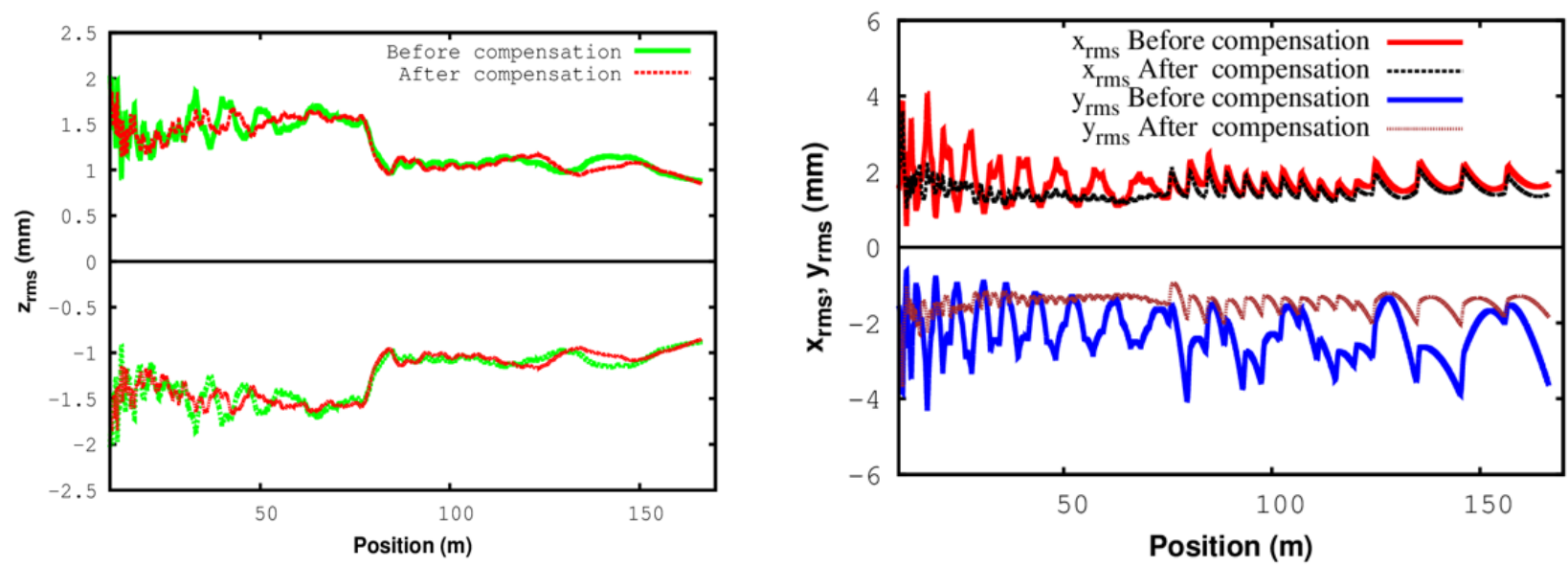

Figure 2.34: Longitudinal (left) and transverse (right) beam sizes along the linac before and after local compensation for a failure of the first solenoid in the HWR section.
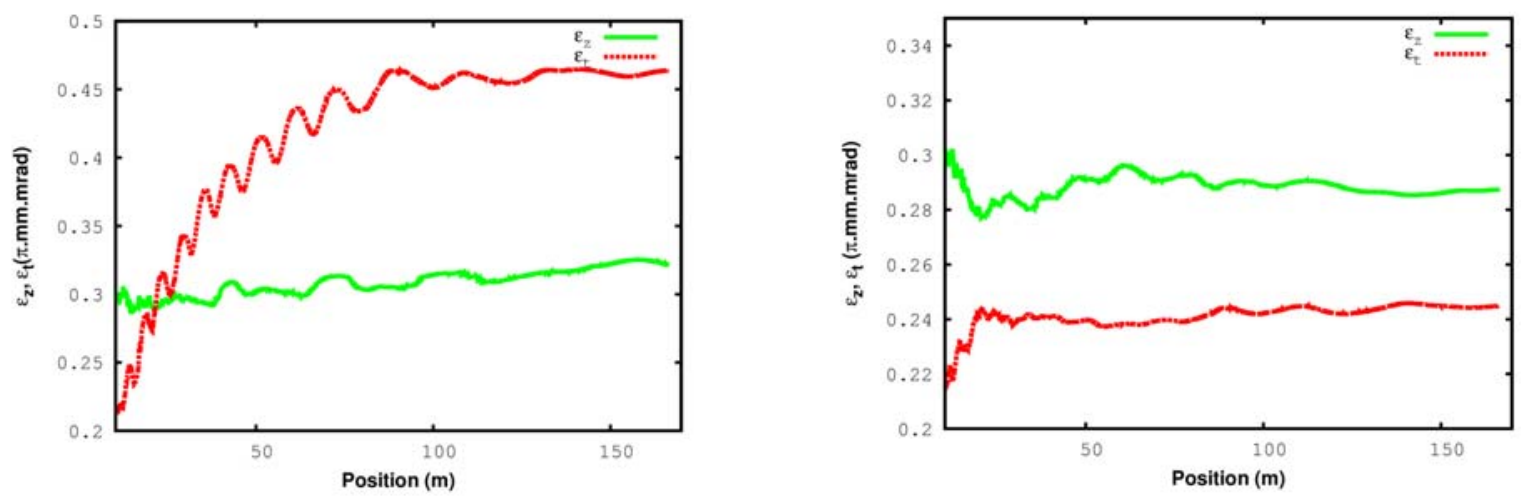

Figure 2.35: Beam emittance growth along the linac before (left) and after (right) the local compensation for a failure of the first solenoid in the HWR section.

\section{Failure of the Last Cavity in the HWR section}

The HWR section is followed by the SSR1 section. There is a short warm section separating the HWR and SSR1 cryomodules. Beam matching is performed using nearby elements of each cryomodule to achieve a smooth beam profile around the transition. Accelerating gradients and synchronous phases in cavities are used to adjust the longitudinal beam profile, while focusing strengths of solenoids are changed to achieve matching conditions in the transverse planes. The left pane in Figure 2.36 shows rms longitudinal beam envelope along the linac after failure of the last HWR cavity. Beam oscillations are observed after the failed cavity. However, a smooth beam profile is obtained after applying the local compensation. It can also be noticed from the right pane in Figure 2.36 that longitudinal emittance is restored. 

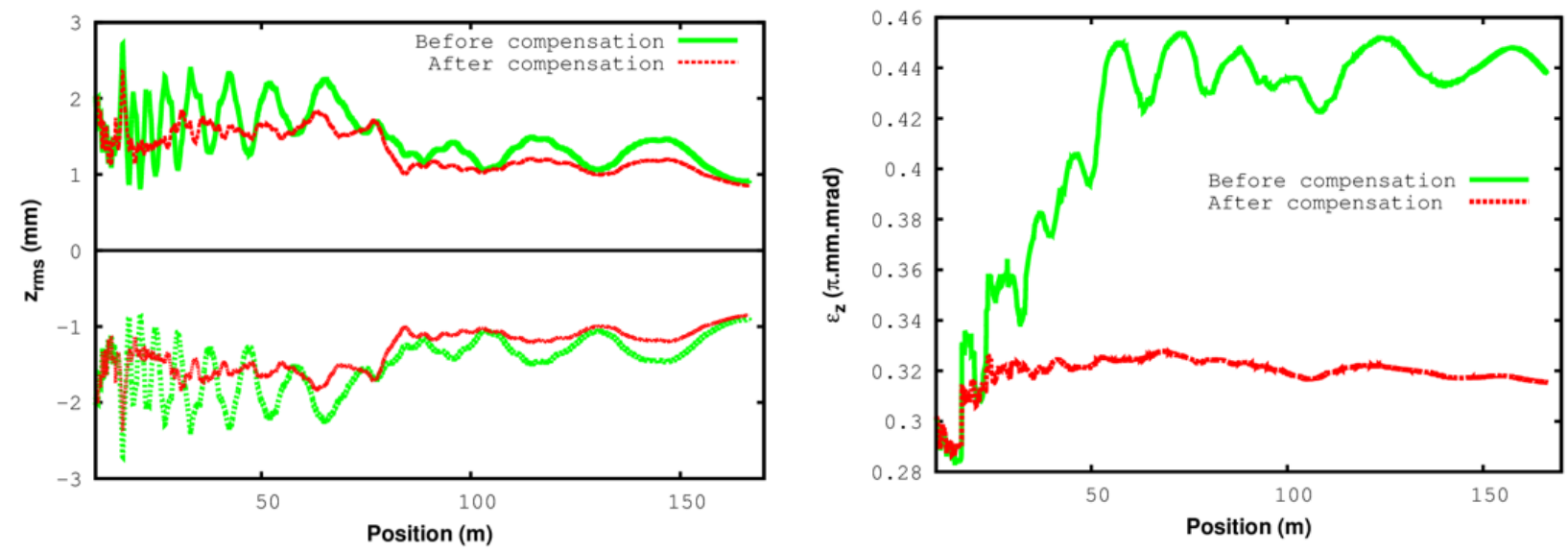

Figure 2.36: Longitudinal rms beam envelopes (left) and normalized rms emiitances (right) along the linac before and after applying the local compensation to the failure of the last HWR cavity.

\section{Failure of the Last Solenoid in the HWR section}

It was found that a failure of the last solenoid in the HWR section represents one of the most dangerous situations with $\sim 13 \%$ beam losses immediately downstream. The center-to-center distance between the last solenoid in the HWR section and the first solenoid in the SSR1 section is about $\sim 1.82 \mathrm{~m}$. A failure of the last HWR-solenoid increases the length of beam transverse focusing cell to $2.51 \mathrm{~m}$. This change in the length and the resulting mismatch due to the absence of focusing lead to a large beam envelope oscillations. Figure 2.37 shows the rms beam envelopes and transverse emittance before and after applying the local compensation.
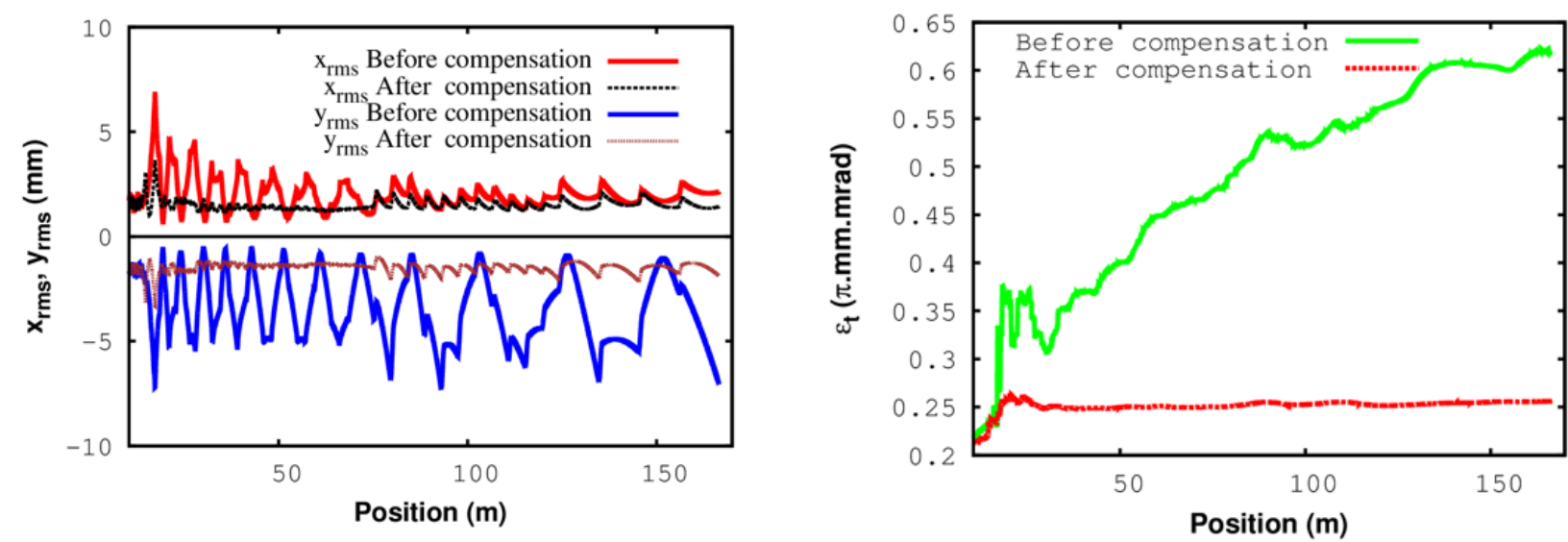

Figure 2.37: Rms transverse beam sizes (left) and normalized rms beam emittances (right) before and after applying the local compensation to failure of the last solenoids in HWR section.

\section{Failures at other Critical Locations}

As the beam energy increases, the machine performance becomes less sensitive to a single element failure. Higher beam energy reduces the impact of non-linear space charge forces as well as a focusing effect of a single element. A study was performed to analyze the performance of the linac in the presence of failed elements at higher energy but in critical locations. The transition from SSR2 to LB650 section is considered to be a vulnerable location in the linac because of two reasons:

- Solenoidal focusing in SSR2 is replaced by focusing with quadrupole doublets in the LB section. 
- A frequency jump from 325 to $650 \mathrm{MHz}$ occurs at this transition.

TraceWin - CEA/DRF/Irfu/SACM

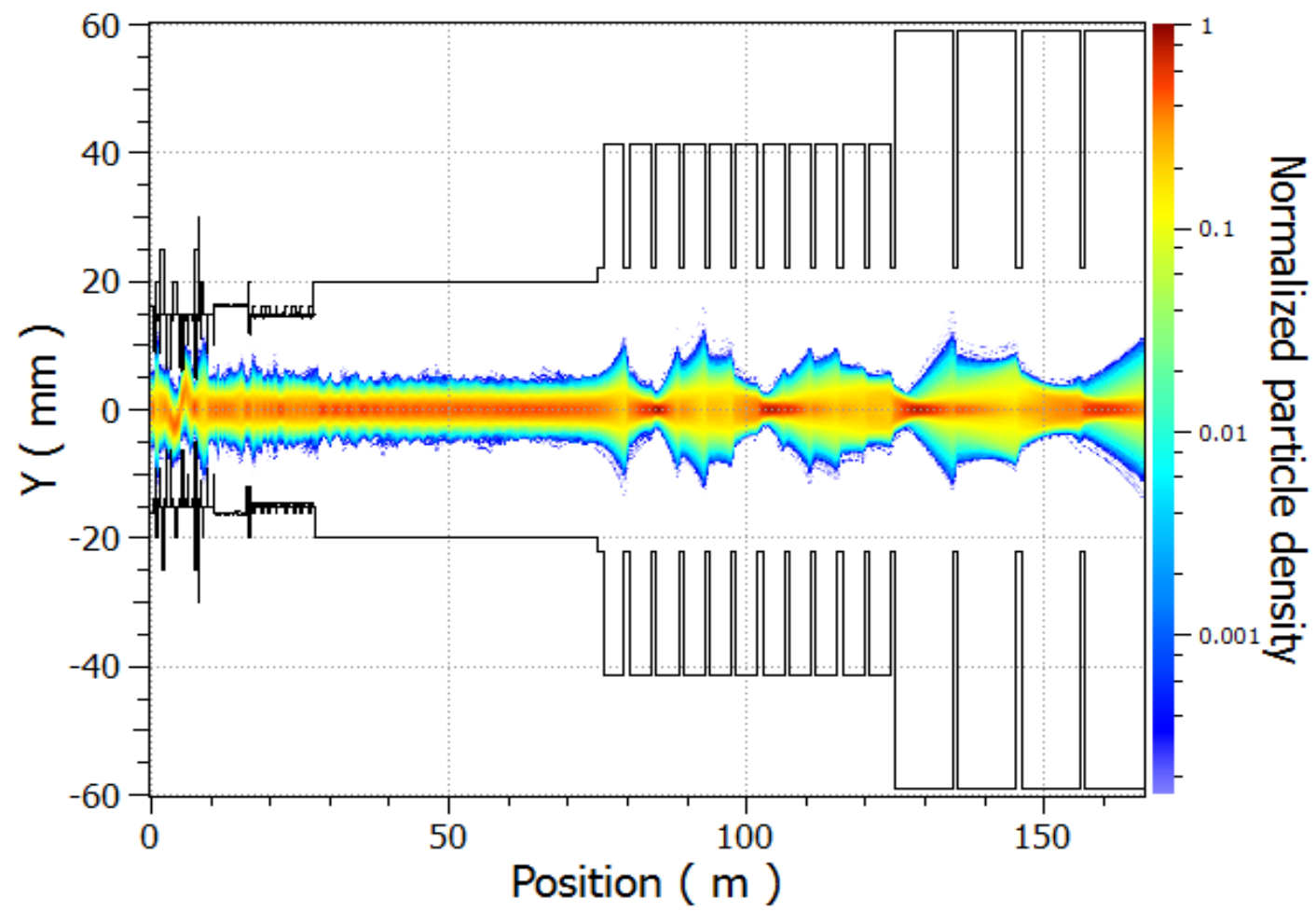

Figure 2.38: Normalized vertical particle density along the linac after a failure of the first quadrupole doublet at the SSR2 to LB650 transition.
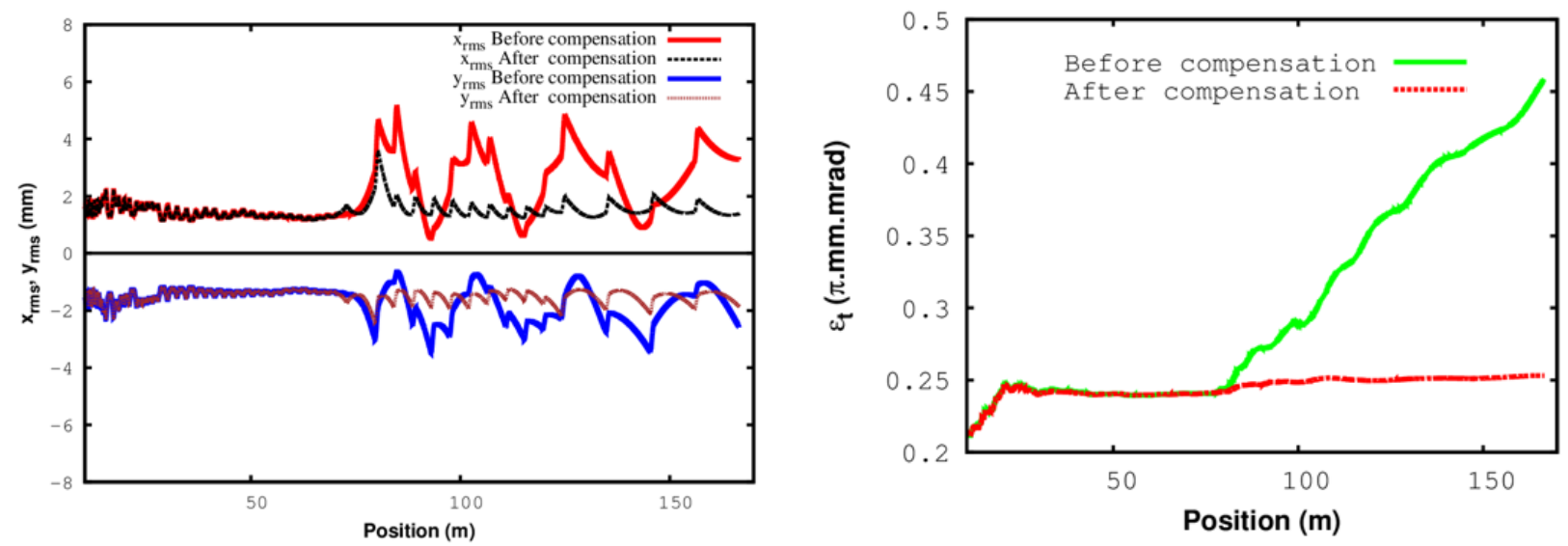

Figure 2.39: Beam transverse sizes (left) and normalized rms transverse emittances (right) before and applying local compensation for a fault of the first quadrupole doublet at the SSR2 to LB650 transition.

Although a replacement of a failed normal conducting quadrupole is much simpler and faster than for a SC solenoid, a study was performed to evaluate performance of the linac in the presence of a failed quadrupole. In this study, we assumed that after failure of one quadrupole in a doublet, the remaining quadrupole is also turned off. Figure 2.38 shows the normalized beam density in the vertical plane along the linac for the case when the first quadrupole doublet in the LB section has failed. In spite of mismatch induced oscillations the physical aperture in the downstream sections is sufficiently large to accommodate the beam. One can see that there is still significant margin (ratio of beam aperture to maximum beam size) even for $6 \sigma$ beam. Figure 2.39 shows the beam transverse 
envelopes and the normalized rms emittances before and after compensation.

A study performed to evaluate implications of cavity failure in the LB section shows no beam losses even after failure of the first complete LB 650 cryomodule. However, the beam energy is reduced to $786.5 \mathrm{MeV}$ and the final normalized rms longitudinal emittance is increased to $0.38 \mathrm{~mm}$ mrad. 


\subsection{Linac-to-Booster Beam Transport}

\subsubsection{Particle Loss and Limitations on Beam Transport Parameters}

A low loss beam transport is critical in the operation of a MW class facility. The $\mathrm{H}^{-}$transport should have sufficiently small losses to minimize residual radiation in the tunnel. It is highly desirable to keep the residual radiation level below $15 \mathrm{mrem} / \mathrm{hr}$ at $30 \mathrm{~cm}$ from component surface. Many facilities use the metric of $1 \mathrm{~W} / \mathrm{m}$ as a limit for "hands on" maintenance; however, at energies about or above $1 \mathrm{GeV}$, a $1 \mathrm{~W} / \mathrm{m}$ loss rate produces a peak contact residual dose rate of $\sim 150 \mathrm{mrem} / \mathrm{hr}$ at $30 \mathrm{~cm}$ on a bare beam pipe [24]. Although magnets shield the radiation and significantly reduce the residual activation on their external surfaces, the radiation of unshielded pieces including magnet interfaces and instrumentation locations has to be sufficiently small. That sets the maximum acceptable loss rate. Setting a desirable activation level to $15 \mathrm{mrem} / \mathrm{hr}$ at $30 \mathrm{~cm}$ results in a loss goal of $\sim 0.1 \mathrm{~W} / \mathrm{m}$ at energies about or above $1 \mathrm{GeV}$. Initial Linac operation will be in a pulsed regime where the total beam power is about $18 \mathrm{~kW}$. Consequently, a fractional loss rate of $5 \times 10^{-6} \mathrm{~m}^{-1}$ is required. Future $\mathrm{CW}$ operation will require a fractional loss rate limit of about $5 \times 10^{-8} \mathrm{~m}^{-1}$.

The primary loss mechanisms for $0.8 \mathrm{GeV}$ transport are the $\mathrm{H}^{-}$intrabeam stripping, Lorentz stripping, inelastic beam-gas scattering, and scraping of beam halo on the apertures. Stripping due to black-body radiation inside the room temperature beam pipe is negligible at this energy.

As shown in Figure 2.19, the intrabeam stripping in the Linac results in an acceptable loss rate even in the case of CW beam. The strength of transverse focusing in the beam line is similar to the focusing strength at the Linac end. Consequently, the beam loss at the transfer line beginning is close to the beam loss at the linac end. The particle momentum spread leads to natural debunching in the course of beam transport from Linac to Booster. It results in a bunch length increase by more than an order of magnitude (from $1.1 \mathrm{~mm}$ to about $14 \mathrm{~mm}$ ), and, consequently, a reduction of intrabeam stripping inversely proportionally to the bunch length resulting in a negligible intrabeam stripping loss of about $100 \mu \mathrm{W} / \mathrm{m}$ at the transport line end for $1 \%$ duty factor. Note that this $1 \%$ duty factor is used for the beam injection to the Booster and the major part of the line sees this beam only; while the initial part of this line is also planned to be used for beam transport to an upgrade of the Mu2e experiment requiring $100 \mathrm{~kW}$ beam (see Appendix A). Consequently, a maximum beam loss of up to $5 \mathrm{~mW} / \mathrm{m}$ is expected.

Beam motion in a magnetic field excites an electric field in the beam frame. If this electric field is sufficiently strong, it can detach a weakly bound outer electron (Lorentz stripping) from the $\mathrm{H}^{-}$ ion. The results of experimental measurements for $\mathrm{H}^{-}$lifetime are presented in Ref. [25] and can be approximated by the following equation:

$$
\tau_{E}(E)=\frac{A_{\tau}}{E} e^{B_{\tau} / E}
$$

where the constants are $A_{\tau}=2.47 \cdot 10^{-8} \mathrm{~s} \mathrm{~V} / \mathrm{cm}, B_{\tau}=44.94 \cdot 10^{6} \mathrm{~V} / \mathrm{cm}$. Figure 2.40 shows the corresponding loss rates per meter for a 0.8 and $1 \mathrm{GeV} \mathrm{H}^{-}$beams as a function of magnetic field. The magnetic field in the dipoles of the transfer line is chosen so that the loss rate would not exceed $10^{-8}$ $\mathrm{m}^{-1}$ for a beam of $1 \mathrm{GeV}$ energy. This corresponds to a limit of $2.77 \mathrm{kG}$ equivalent to a bending radius of $20.431 \mathrm{~m}$. Keeping the same radius of curvature for the $0.8 \mathrm{GeV}$ beam one obtains a limit for the magnetic field in the dipoles of $2.39 \mathrm{kG}$ with a loss rate of $3 \cdot 10^{-13}$.

The loss rate due to $\mathrm{H}^{-}$scattering on residual gas molecules is proportional to their density and their ionization cross sections. The cross sections decrease proportionally to $\beta^{-2}$ and therefore are weakly dependent on the beam energy for energies about or above $1 \mathrm{GeV}$. The cross section of $\mathrm{H}^{-}$ 
stripping for $0.8 \mathrm{GeV}$ beam on residual gas is about $10^{-19} \mathrm{~cm}^{2}$ for atomic hydrogen and $7 \cdot 10^{-19} \mathrm{~cm}^{2}$ for atomic oxygen and nitrogen and grows somewhat slower than proportional to $Z$ for heavier atoms $[26,27]$. The requirement of less than $10^{-8} \mathrm{~m}^{-1}$ for the partial loss rate yields a vacuum requirement of $10^{-8}$ Torr or better for $\mathrm{H}_{2}$ and about an order of magnitude better for heavy molecules (hydrocarbons, water, etc.). Consequently, an application of vacuum practices developed at Fermilab for not-baked vacuum systems, which routinely achieve low $10^{-8}$ Torr, should be sufficient.

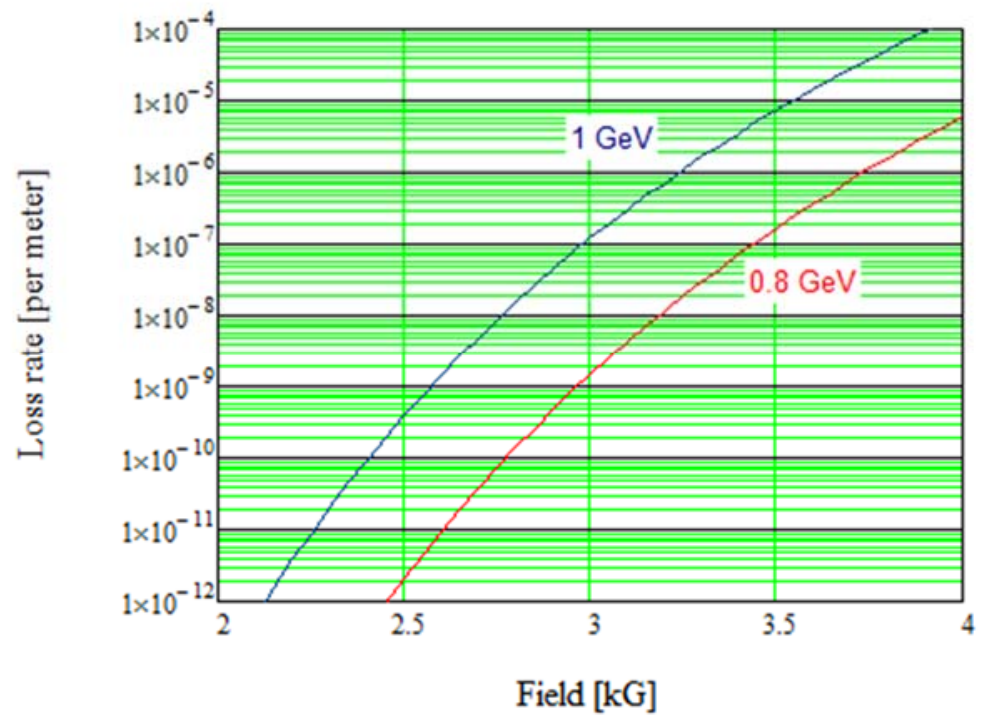

Figure 2.40: Fractional loss due to Lorentz stripping for 0.8 and $1 \mathrm{GeV} \mathrm{H}^{-}$beams traveling in a dipole field.

\subsubsection{Linac-to-Booster Transfer Line}

The Linac-to-Booster Transfer Line transports the beam from the exit of the SC Linac to the injection girder of the Booster. Figure 2.41 presents the transport line layout. The transport line has two arcs and the straight section connecting them. In this straight section the Booster line splits into two additional lines. One goes to the linac beam dump and the other one goes to the Mu2e experiment which future upgrade is expected to receive $800 \mathrm{MeV}$ beam from the SC linac.

As can be seen in Figure 2.41 the second arc crosses the Tevatron tunnel. In the vicinity of this crossing, the line has a local vertical bump bringing the line close to the Tevatron tunnel ceiling. It is done to allow a free passage for people and equipment along the Tevatron tunnel, which contains the operating $120 \mathrm{GeV}$ transfer line. This line brings beams extracted from the Main Injector to the Fermilab Test Beam Facility and is scheduled to be still in use at the time of PIP-II operations.

The SC Linac beam is at the same elevation as the Booster beam; that is $726.48 \mathrm{ft} .(221.431 \mathrm{~m})$ above sea level. The Linac beam is at $1.300 \mathrm{~m}$ above the Linac floor. That yields the floor elevation of $722.215 \mathrm{ft}$. $(220.131 \mathrm{~m})$ above the sea level. The tunnel floors for the SC linac, Transfer Line, and Tevatron are at the same elevation. The injection into the Booster will be vertical with a C-dipole. At its entrance, the beam orbit is located $33.6 \mathrm{~cm}$ (13.23") above the Booster orbit. The angle difference in the horizontal plane between the linac direction and the Booster injection straight is around $-210 \mathrm{deg}$. This bending angle is produced by 32 identical $2.45 \mathrm{~m}$ long dipoles with $2.406 \mathrm{kG}$ field. All these dipoles are connected serially and powered by a single power supply.

The first section of the Transfer Line is located in the extension of the Linac tunnel. This is reserved for a possible future energy upgrade and includes four periods with doublet focusing and 
the same period length as in the HB section of the Linac. Such choice enables the installation of up to four additional HB650 cryomodules in the future, so that the Linac energy could be upgraded to about $1.2 \mathrm{GeV}$.

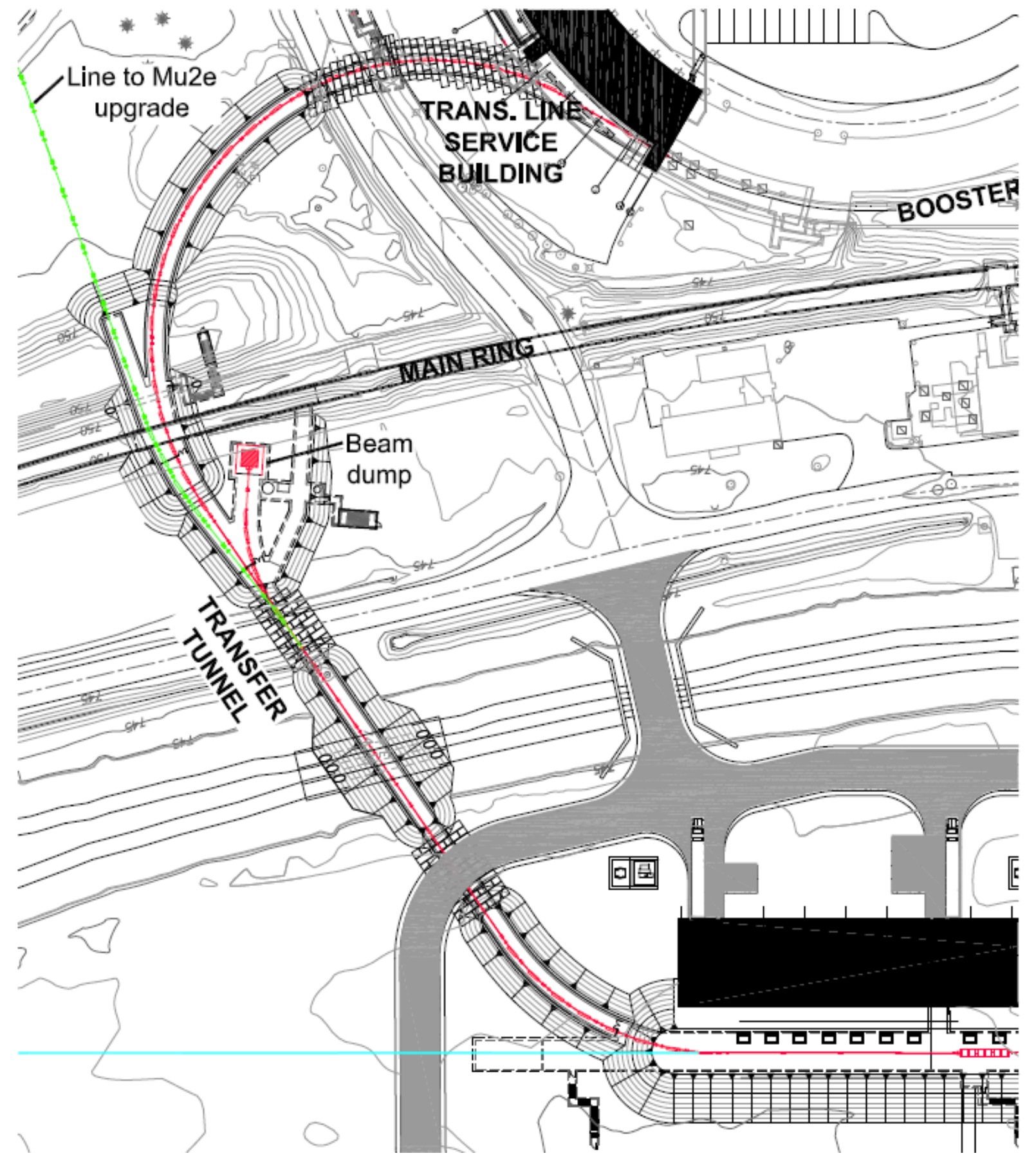

Figure 2.41. Layout of the beam transport line from the SC Linac to Booster. The linac beam dump and future transport line to the Mu2e upgrade are also shown.

The momentum spread of the Linac beam is sufficiently small. Therefore RF debunching is not planned and, consequently, no debunching RF cavities are presently anticipated, although there is sufficient space for debunching cavities if their installation will be required in the future. A FODO lattice is used for the rest of beam transport. Its period is chosen to keep the dispersion and beta- 
functions comparable to their values in the SC Linac and Booster. Geometrical constraints set the cell length to about $11.8 \mathrm{~m}$.

The FODO cells have 90 and $111 \mathrm{deg}$. horizontal and vertical phase advances, respectively, in order to have cancellation of horizontal and vertical dispersions at the end of the arcs. The cells of the straight section and of the arcs are identical except that in the arc cells the dipoles are inserted between the quadrupoles. Such choice automatically ensures that arcs and straight sections are matched to each other, and allows one to connect all focusing (defocusing) quads serially and power them from two power supplies: one for focusing and one for defocusing quads. The two arcs are composed of 4 and 12 cells and are both achromatic. The straight section connecting them consists of 8 cells. Low power adjustable collimators are installed at the beginning and at the center of the first arc. Two collimators at the arc beginning (each collimating on both sides for both the vertical and horizontal planes) separated by one cell remove particles having large betatron oscillations. Another collimator installed in the arc center, where the maximum dispersion is achieved, is used for momentum collimation. The length allocated for each collimator is about $1 \mathrm{~m}$. Since each arc cell includes two dipoles, the packing factor of the arcs is $42 \%$. This choice leaves sufficient space for horizontal and vertical correctors after each focusing and defocusing quadrupole respectively, as well as for ion pumps, BPMs and other instrumentation. It also foresees space for a possible installation of debunching RF cavities and additional collimators if needed in the future. The integrated strengths of the quadrupoles are set by the constraints on the phase advances per cell and are about $0.25 \mathrm{~m}^{-1}$ and $-0.28 \mathrm{~m}^{-1}$ for focusing and defocusing quads, respectively $(L=20 \mathrm{~cm}, G=0.61$ and $-0.69 \mathrm{kG} / \mathrm{cm}$ at $E_{\text {kin }}=800 \mathrm{MeV}$ ).

The second arc of the Transfer Line crosses the Tevatron tunnel. To create the mentioned above vertical bump the first few dipoles of the second arc are rolled around their longitudinal axis in order to increase the beam elevation from $1.3 \mathrm{~m}$ to about $2.4 \mathrm{~m}$ at the location of crossing. A roll of few other dipoles before and after crossing returns the beam to the Linac elevation. This vertical bump creates the vertical dispersion and perturbs the horizontal dispersion. Therefore, in addition to bringing the line to the linac level, rolls of these dipoles are adjusted to cancel both dispersions. Note that the choice of the phase advances was also driven by a necessity of dispersion cancelation. Note also that the dipoles have sufficiently small focusing, and therefore their rolls do not produce measurable $x-y$ coupling.

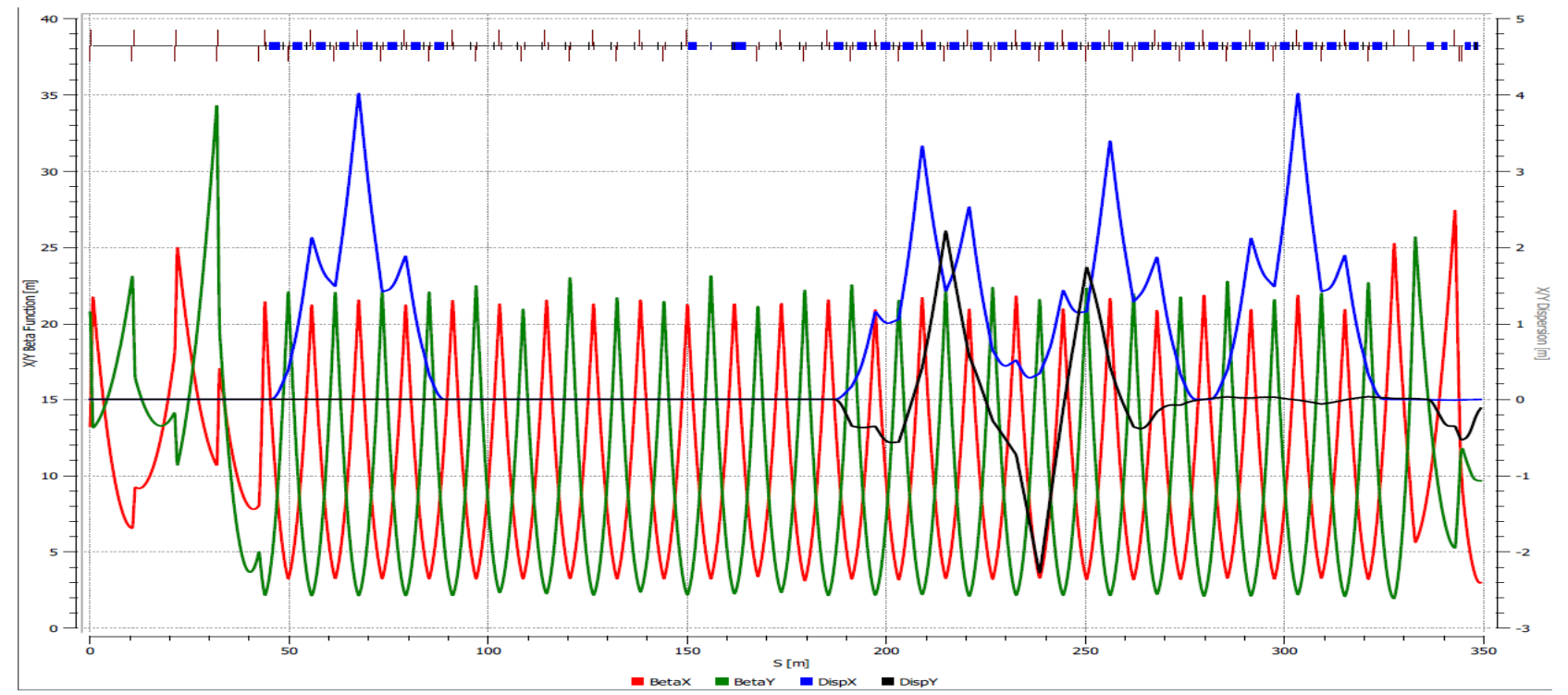

Figure 2.42: Optics of the Transfer Line from the SC linac end to the stripping target. 
The aperture in the first part of the line located in the extension of linac tunnel is the same as in the HB section i.e. $\varnothing 46 \mathrm{~mm}$ (2" stainless steel pipe with $2 \mathrm{~mm}$ wall thickness). Starting from the beginning of the first arc the line aperture is reduced to $34 \mathrm{~mm}(1.5$ " stainless steel pipe with $2 \mathrm{~mm}$ wall thickness). The optical functions of the entire Transfer Line are presented in Figure 2.42 [see optics details in Ref. [21]). The maximum horizontal dispersion in the arcs is about $4 \mathrm{~m}$ and the maximum beta-function is about $23 \mathrm{~m}$. With $5 \mathrm{~mm}$ allowance for orbit distortions, they yield $\pm 13.6 \sigma$ momentum aperture and 5.7 $\sigma$ aperture for betatron motion of each transverse plane. Here we assumed the nominal rms momentum spread at the Linac end of $\sigma_{p}=2.2 \cdot 10^{-4}$ and the transverse normalized rms emittances of $0.3 \mathrm{~mm}$ mrad.

The last straight section of the Transfer Line is downstream of the second arc and is aligned with the Booster injection straight. At its beginning, this line has the same elevation as the Booster and $\mathrm{SC}$ Linac. The Booster injection is in the vertical plane with a C-Dipole bending the beam downward into the Booster as shown in Figure 2.43. To bring the beam to the elevation of the C-Dipole a vertical dogleg is created. It uses two bending dipoles with length of $1.8 \mathrm{~m}$ and magnetic field of $2.5 \mathrm{kG}$. A triplet is placed after the second vertical dipole to match the line Twiss parameters to the values required for efficient injection into the Booster. The $\mathrm{C}$-Dipole is $1.8 \mathrm{~m}$ long with $3.26 \mathrm{kG}$ magnetic field.

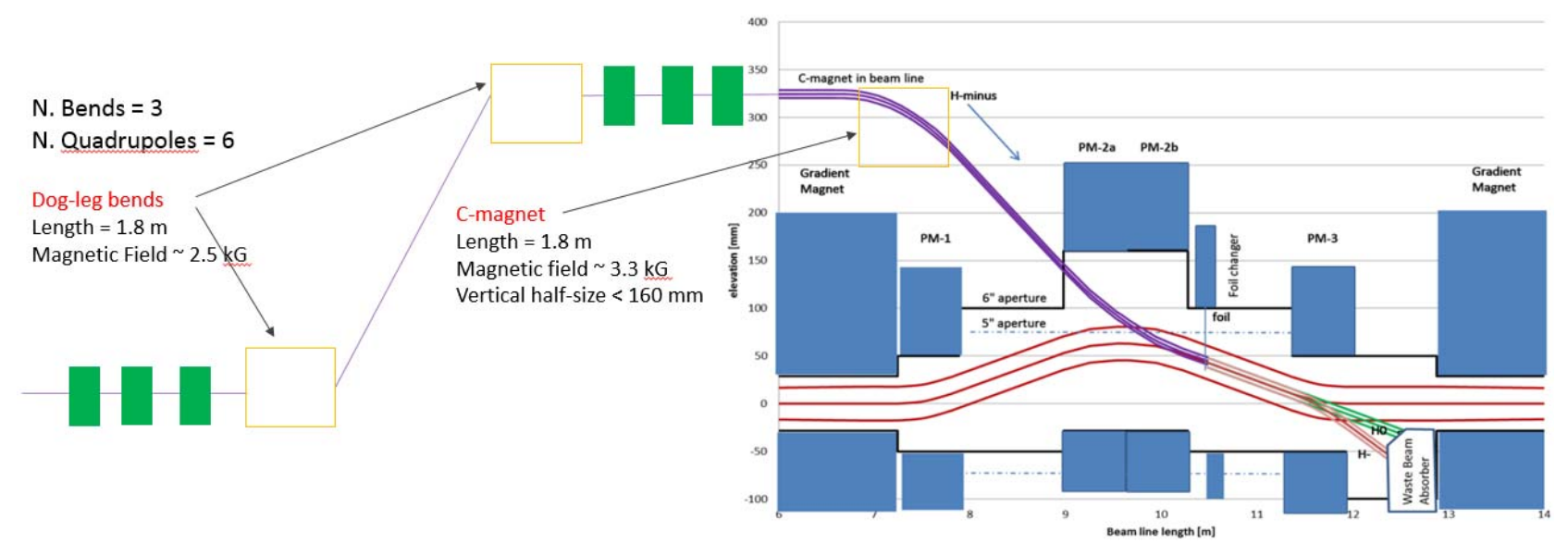

Figure 2.43: Schematic layout of the injection beam line.

A beam switching system is installed in the sixth and seventh cells of the straight section (located between the arcs). The system can switch the beam from the Booster line to the Dump Line or to the line going to the future upgrade of the Mu2e experiment. The Dump Line consists of five dipole magnets of the same design as used in the arcs. It deflects the beam horizontally to the beam dump placed so that there would not be any other beam lines at distances smaller than $10 \mathrm{~m}$. Beam focusing is provided by alternate focusing and defocusing quadrupoles of the same design as used in the arcs, for a total of four. Dipoles and quadrupoles of the Dump Line are powered in series with the corresponding families of the transfer line. A sweeping magnet in the long drift before the dump helps to reduce the power density on the dump entrance. The beam dump is rated for $50 \mathrm{~kW}$ of 800 $\mathrm{MeV}$ beam. Twiss functions of the entire line from the end of the SC Linac to the dump entrance are shown in Figure 2.44. It assumes that the switching system is on and directs the beam to the dump.

Details of the beam transport for the Mu2e upgrade are presented in Appendix A.

The beam switch consists of a fast corrector (located right after the focusing quadrupole of the sixth cell) and a Lambertson septum with 3 apertures (located after the focusing quadrupole of the seventh cell). When the field in the fast corrector is on the beam is deflected from the central orbit 
and passes through two large aperture quadrupoles before it comes to the septum. When the beam reaches the septum, it enters into one of two side apertures which magnetic field deflects the beam to the Dump Line or the Mu2e line. If the fast corrector is off, the beam remains at the vacuum chamber axis and arrives the septum central aperture. There is no magnetic field in this aperture and the beam is directed to the Booster line. The layout of the switching system is shown in Figure 2.45 where $10 \sigma$ envelopes of the deflected and undeflected beams are presented.

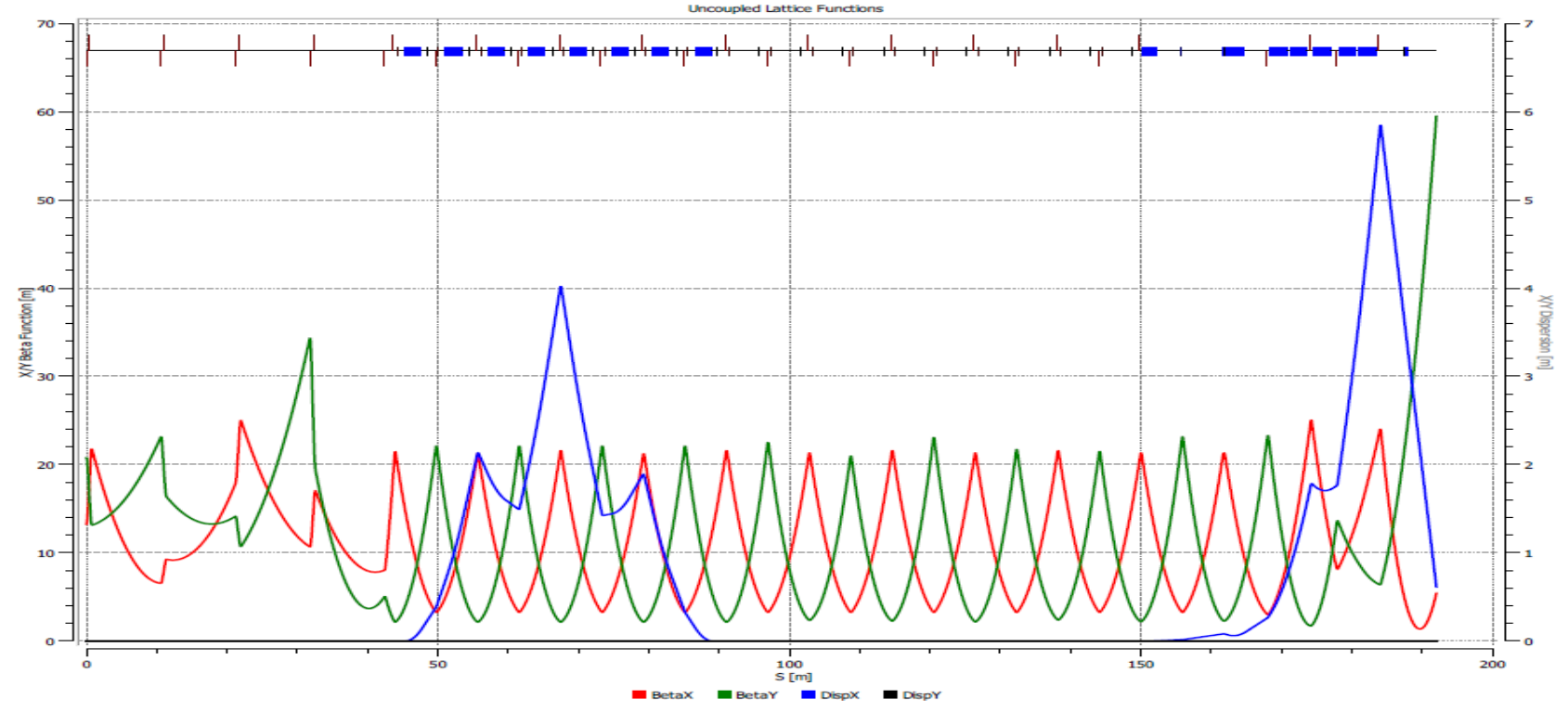

Figure 2.44: Optics of the Transfer Line from the SC Linac end to the dump.

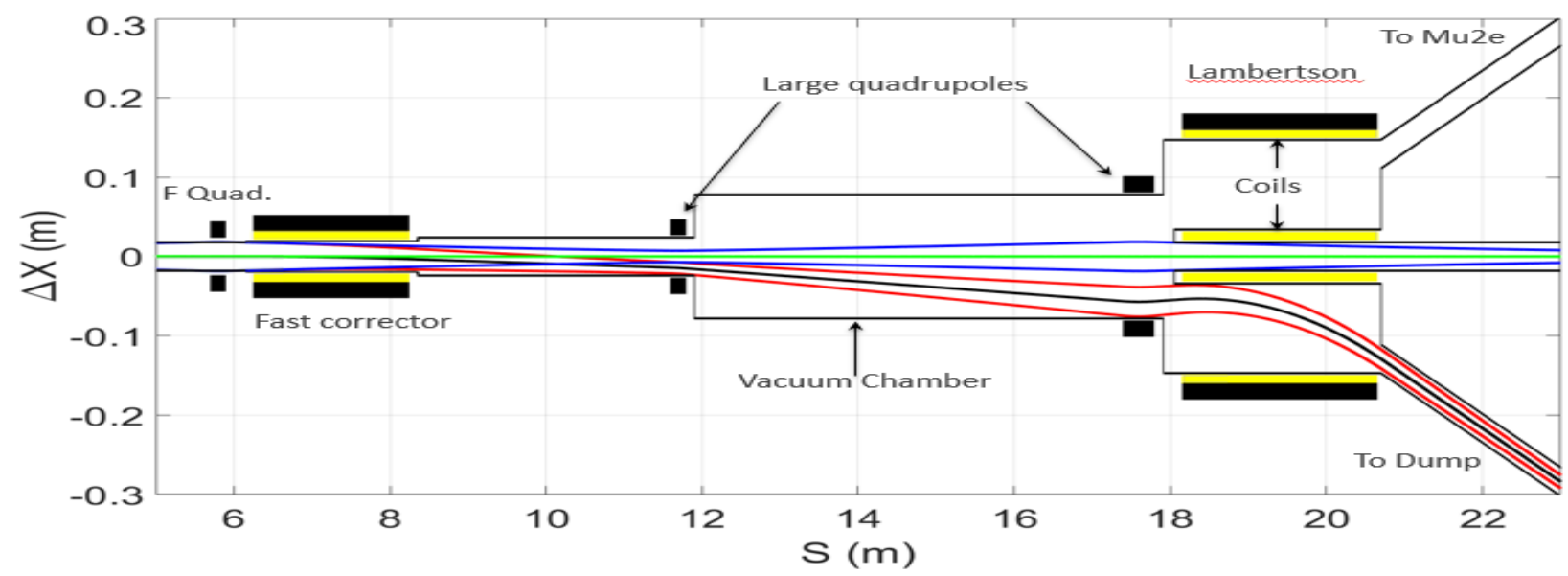

Figure 2.45: Horizontal $10 \sigma$ envelopes of the beam directed to the Booster (blue) and to the dump (red). An inversion of field polarity for the fast corrector directs the beam to a symmetric trajectory leading to the Mu2e upgrade.

The beam based energy stabilization discussed below requires that the fast corrector must be able to switch the beam from one aperture to another nearby aperture in $\sim 20 \mu \mathrm{s}$. The corrector is powered by a special power supply capable to create sufficiently large voltage required for fast switching. The usage of a ceramic vacuum chamber inside the corrector avoids eddy currents, which even for a thin stainless steel chamber would increase the field rise-time to an unacceptably large value. The Lambertson septum, like the other magnets, is powered by DC. The strengths of fast corrector and septum are 0.15 and $3.6 \mathrm{kG} \cdot \mathrm{m}$, respectively. 


\subsubsection{Beam Based Linac Energy Stabilization}

As shown below, high quality injection into the Booster requires relative energy stability better than $10^{-4} \mathrm{rms}$. The present experience does not guarantee that such energy stability can be achieved if the beam energy is stabilized only by internal feedbacks separately stabilizing each cavity voltage. In this case, the beam based energy stabilization is the only possibility to address the problem. A similar approach is used in the present Fermilab linac. The energy correction is applied to the cavities of the last SC cryomodule where the synchrotron motion is greatly slowed down and voltage variation will have little effect on the beam dynamics. It is expected that such a system will improve the energy stability from $\sim 10^{-3}$ level achieved with local feedbacks to the desired value of better than $10^{-4}$.

To avoid unnecessary beam loss in the Booster while the beam energy is still not stabilized to the required level the beam is initially directed to the beam dump located after the first arc of the transport line (see Figure 2.41). After the energy is stabilized to the required level ( $\sim 100 \mu \mathrm{s}$ is required) the beam is switched to the transport line and delivered to the Booster. The switching requires the beam being off and therefore it has to be done sufficiently fast $(\sim 20 \mu \mathrm{s})$ to avoid changes of accelerating voltage during switching time.

The beam energy is measured by BPMs located in the first arc of the transport line. The accuracy of the system is determined by the BPM accuracy and is expected to be $\sim 10^{-5}$ with $\sim 1 \mu$ s sampling time. A time-of-flight system placed in the slots reserved for the energy upgrade of the Linac can also be used. Although it hardly can achieve the accuracy of the BPM based system. This energy measurement will be used to support operation of the beam-based energy stabilization system. A high accuracy absolute energy measurement is not required. 


\subsection{Booster Modifications}

\subsubsection{Technical Requirements and Scope}

The performance requirements of the Booster are summarized in Table 2.13. The $800 \mathrm{MeV}$ injection energy is selected to provide an increase in beam intensity of about $50 \%$ beyond current operations, accompanied by a 30\% decrease in the space-charge (Laslett) tune shift at injection. This choice is made to provide more efficient beam capture and acceleration, in order to minimize losses at the higher beam intensity required in PIP-II. The requirement on the longitudinal beam emittance is set by slip-stacking in the Recycler.

Table 2.13: Performance requirements for the Booster

\begin{tabular}{l|l}
\hline Performance Parameter & Requirement \\
\hline Particle Species & Protons \\
\hline Input (H) Beam Energy (Kinetic) & $800 \mathrm{MeV}$ \\
\hline Output Beam Energy (Kinetic) & $8.0 \mathrm{GeV}$ \\
\hline Protons per Pulse (injected) & $6.7 \times 10^{12}$ \\
\hline Acceleration efficiency & $97 \%$ \\
\hline Protons per Pulse (extracted) & $6.5 \times 10^{12}$ \\
\hline Beam Pulse Repetition Rate & $20 \mathrm{~Hz}$ \\
\hline RF Frequency (injection) & $44.7 \mathrm{MHz}$ \\
\hline RF Frequency (extraction) & $52.8 \mathrm{MHz}$ \\
\hline Total RF voltage & $1.2 \mathrm{MV}$ \\
\hline Injection Efficiency & $98 \%$ \\
\hline Injection Time & $0.55 \mathrm{~ms}$ \\
\hline Injection Turns & 292 \\
\hline Beam Emittance (95\%, normalized; $\left.\varepsilon_{\mathrm{x}}=\varepsilon_{\mathrm{y}}\right)$ & $16 \mu \mathrm{m}$ \\
\hline Maximum Laslett Tune Shift (space charge) & -0.17 \\
\hline Delivered Longitudinal Emittance $(97 \%)$ & $0.1 \mathrm{eV} \cdot \mathrm{s}$ \\
\hline Delivered Momentum Spread $(97 \%$ full height) & $12.2 \mathrm{MeV}$ \\
\hline Delivered Bunch Length (97\% full length) & $8.2 \mathrm{~ns}$ \\
\hline \hline
\end{tabular}

The primary areas that need to be addressed in order to reach the performance goals listed above are given in Table 2.14. Among them the injection and the beam quality are expected to present the primary challenges.

This section will describe concepts and approaches in the areas listed in Table 2.14. This description assumes successful completion of the PIP tasks currently underway. However, this discussion is preliminary and may change after more extensive investigations are completed. It is required that Booster beam losses be maintained at less-than-or-equal-to present levels. The current operating loss limit is $525 \mathrm{~W}$ ring-wide, augmented by independently set beam loss monitor (BLM) trip points in each long and short straight section. 
Table 2.14: Booster areas requiring consideration as part of PIP-II

\begin{tabular}{l|l}
\hline \hline Topic & Associated Items \\
\hline Injection & Injection girder and loss control \\
\hline Capture & RF capture, timing and emittance control \\
\hline Acceleration and Transition & Loss control, RF requirements and transition control \\
\hline Extraction & Loss control, timing and beam manipulations \\
\hline Beam Quality & MI/Recycler requirements \\
\hline Operations & Shielding, Booster Hardware \\
\hline \hline
\end{tabular}

\subsubsection{Booster Injection}

\subsubsection{Present Booster Injection}

The Booster lattice contains 24 periods and can be described as a FDooDFo lattice utilizing combined function dipoles with long straight sections $(\sim 5.7 \mathrm{~m})$ between the defocusing (D) dipoles and a short straight section $(\sim 0.9 \mathrm{~m})$ between the focusing $(\mathrm{F})$ dipoles. The horizontal beta-function varies from about 6 meters in the long straight to $33 \mathrm{~m}$ in the short straights while the vertical beta function varies from $20 \mathrm{~m}$ in the long straights to $\sim 5.3 \mathrm{~m}$ in the short straights. The horizontal dispersion varies between approximately 1.2 (in the long straights) and 2.2 meters (in the short straights). The optical functions for one period are shown in Figure 2.46. It should be noted that the optical functions for all periods are almost equal. Some differences appear due to optics errors and the focusing effect of the extraction dogleg. The latter is quickly reduced with acceleration and has a negligible effect on the beam optics above $1.5 \mathrm{GeV}$ energy.

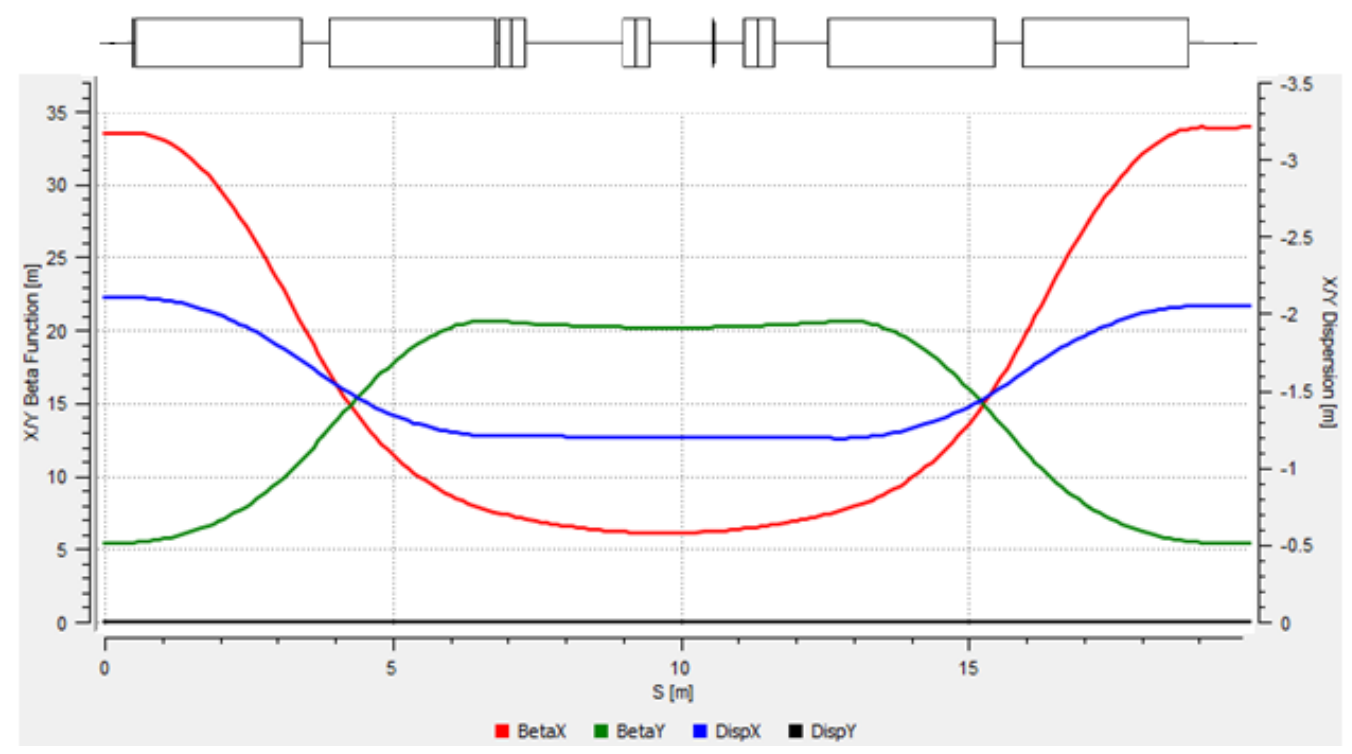

Figure 2.46: Optical functions for one Booster period. The presented period includes the Booster Long 1 straight section where the beam injection is happening. A schematic of the present injection straight is shown at the top of the plot. The locations of pulsed injection dipoles (ORBUMP dipoles) are shown by double rectangles. The vertical line in the center marks position of stripping foil.

The top part of Figure 2.46 also shows the magnet configuration for the present injection straight section which is considered to be the ring beginning and therefore is named Long 1 . The schematic 
of the line is shown at the top of the figure. It includes the combined function dipoles, pulsed injection dipoles (ORBUMP magnets) and correctors. The layout of injection area is shown in Figure 2.47. It is based on the three bump system installed in $2006[28,29]$. The center dipole of this insert is used to merge the incoming $\mathrm{H}^{-}$ions with the proton beam circulating in the ring. The orbit bump is produced by the three pulsed dipoles which displace the closed orbit by $\sim 44 \mathrm{~mm}$ to the stripping carbon foil for the duration of injection $(\sim 35 \mu \mathrm{s})$. The center dipole runs at twice the field of the outer dipoles. The injection foil is located immediately after the middle ORBUMP magnet. The field in the ORBUMP dipoles does not change during the injection, and there is no phase space painting in any degree of freedom. All three dipoles are powered by a single power supply. The angle produced by the center dipole is approximately $\sim 44 \mathrm{mrad}$ which corresponds to an integrated field of $1.4 \mathrm{kG}-$ $\mathrm{m}$. The separation of the ORBUMP magnets is approximately $1.75 \mathrm{~m}$. Lorentz stripping in these magnets is not an issue at $400 \mathrm{MeV}$.

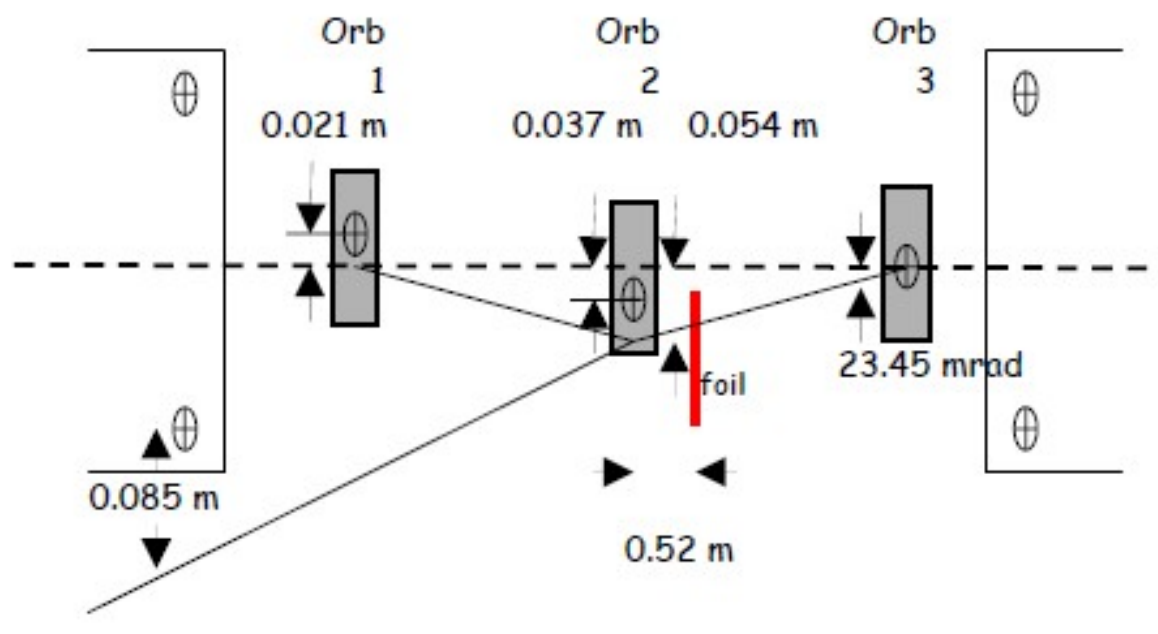

Figure 2.47: The current $400 \mathrm{MeV}$ horizontal injection insert showing the layout of the three chicane dipoles, foil and injection beam line.

The existing ORBUMP magnets cannot be utilized for $800 \mathrm{MeV}$ injection. Their use would require a magnetic field in the center dipole of $\sim 4.5 \mathrm{kG}$, resulting in unacceptably large Lorentz stripping. There is also insufficient space for the beam dump. Addressing these problems requires a complete redesign of the injection straight.

\subsubsection{Conceptual Design of Booster Injection at $800 \mathrm{MeV}$}

The injection into the Booster during the PIP-II era will be moved from "Long 1" to "Long 11" straight section to facilitate injection from the SC Linac located in the Tevatron infield (Figure 2.48). As already noted, all long straight sections have the same geometry and optics. The flange-to-flange length available for the injection insert is $5.6804 \mathrm{~m}$.

The beam current of the SC Linac $(2 \mathrm{~mA})$ is more than an order of magnitude smaller than that of the present $400 \mathrm{MeV}$ linac. It will require much longer injection time ( 300 turns over $\sim 500 \mathrm{us})$ which together with much smaller linac emittance $\left(\varepsilon_{\mathrm{n}_{-} 95 \%} \approx 1.5 \mathrm{~mm}-\mathrm{mrad}\right.$ versus $16 \mathrm{~mm}-\mathrm{mrad}$ specified for the Booster beam) allows us to perform transverse phase space painting. It is expected to be a very efficient cure for suppression of the harmful effects of beam space charge and improvement of the longitudinal beam stability. During the phase space painting process, the Booster closed orbit in each plane will be moved by $\sim 2 \sigma$ of the final Booster accumulated phase space (see 
details below). The vertical displacement will be performed by pulsed magnets of the injection 3bump, the horizontal displacement by regular Booster dipole correctors.

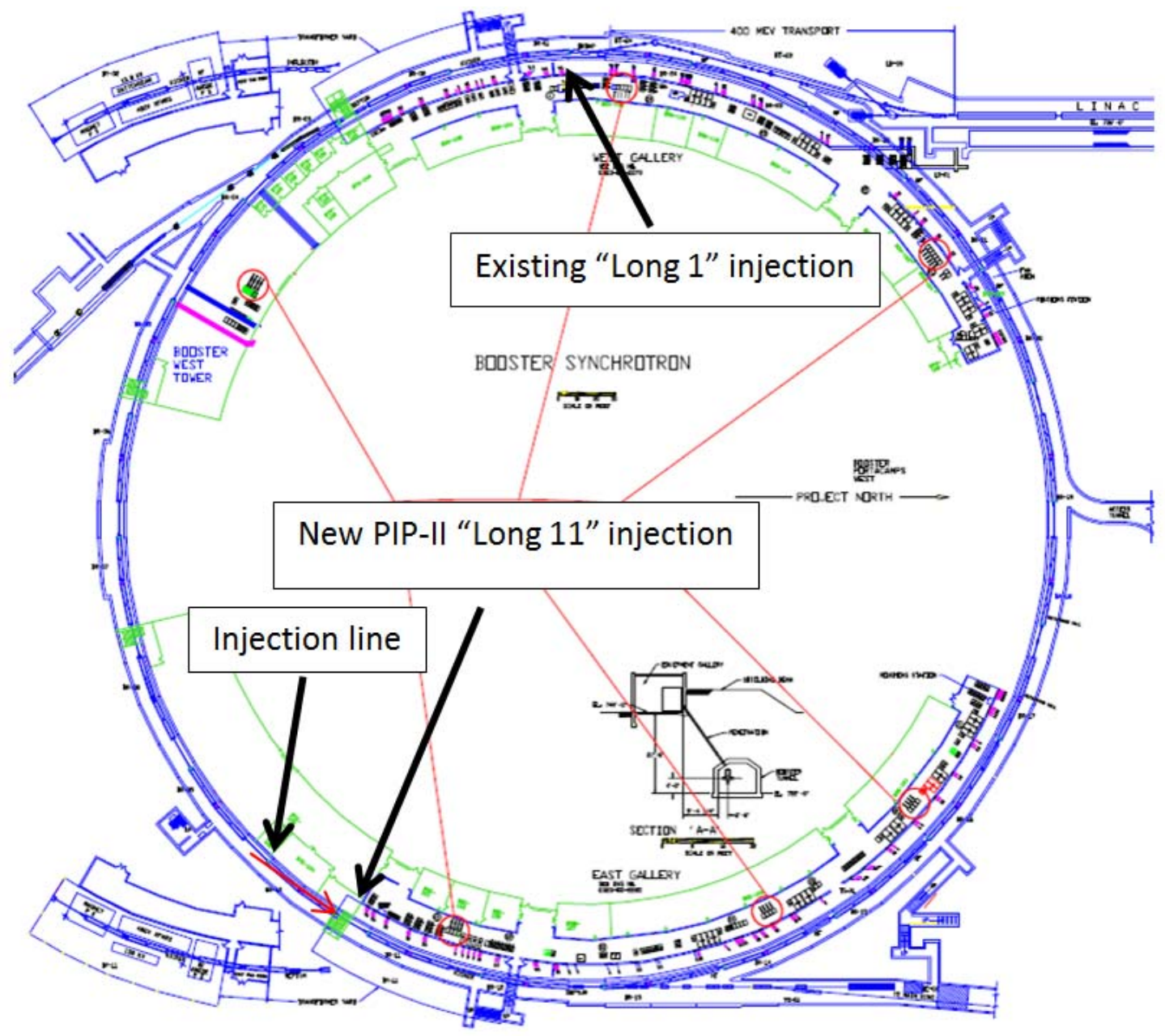

Figure 2.48: Plan view of Booster showing the location of existing and new PIP-II injection insert.

The conceptual design for the straight section is patterned after the present $400 \mathrm{MeV}$ injection three-bump design by adding a second dipole to the middle bend center. The layout of the injection concept, beam envelopes, and apertures are shown in Figure 2.49. The vertical dimensions of the chicane dipoles and foil changer are represented by the blue boxes and are not to scale. The horizontal dimensions of these devices are roughly to scale. The physical aperture is represented by the black line and the absorber is labeled in the open box. The aperture in the D dipoles is set by the pole tip separation on the central orbit of $2.25 "(+/-28.6 \mathrm{~mm})$. The aperture in the central chicane magnets, PM-2a and PM-2b, is determined by the injected beam trajectory.

The chicane dipoles move the Booster closed orbit to $45 \mathrm{~mm}$ at the foil location. During the injection process this closed orbit is collapsed by $\sim 2 \sigma$ of the final vertical rms beam size or $\sim 17 \mathrm{~mm}$ down to $\sim 28 \mathrm{~mm}$. After the end of injection, the chicane dipoles return the closed orbit back to zero. The closed orbit displacement in the center of the two central dipoles is approximately $68 \mathrm{~mm}$. The $\mathrm{H}^{-}$injected beam at the foil position is $45 \mathrm{~mm}$ with a vertical angle of - $40 \mathrm{mrad}$.

Incompletely stripped $\mathrm{H}^{-}$ions (mostly excited states of $\mathrm{H}^{0}$ ) and $\mathrm{H}^{-}$ions which miss the foil will be intercepted by the beam absorber. It is located downstream of the last chicane dipole and upstream of the Booster combined function dipole. For $800 \mathrm{MeV}$ the required space for the dump is $0.5 \mathrm{~m}$ at 
minimum. There are also other equipment (i.e. correction element package, $0.6 \mathrm{~m}$; vacuum bypass and valve, $0.45 \mathrm{~m}$; ion pumps, $0.2 \mathrm{~m}$; horizontal painting dipole, $\sim 0.2 \mathrm{~m}$, and diagnostics/ instrumentation, $\sim 0.25 \mathrm{~m}$ ) which are located in the space between the dipoles.

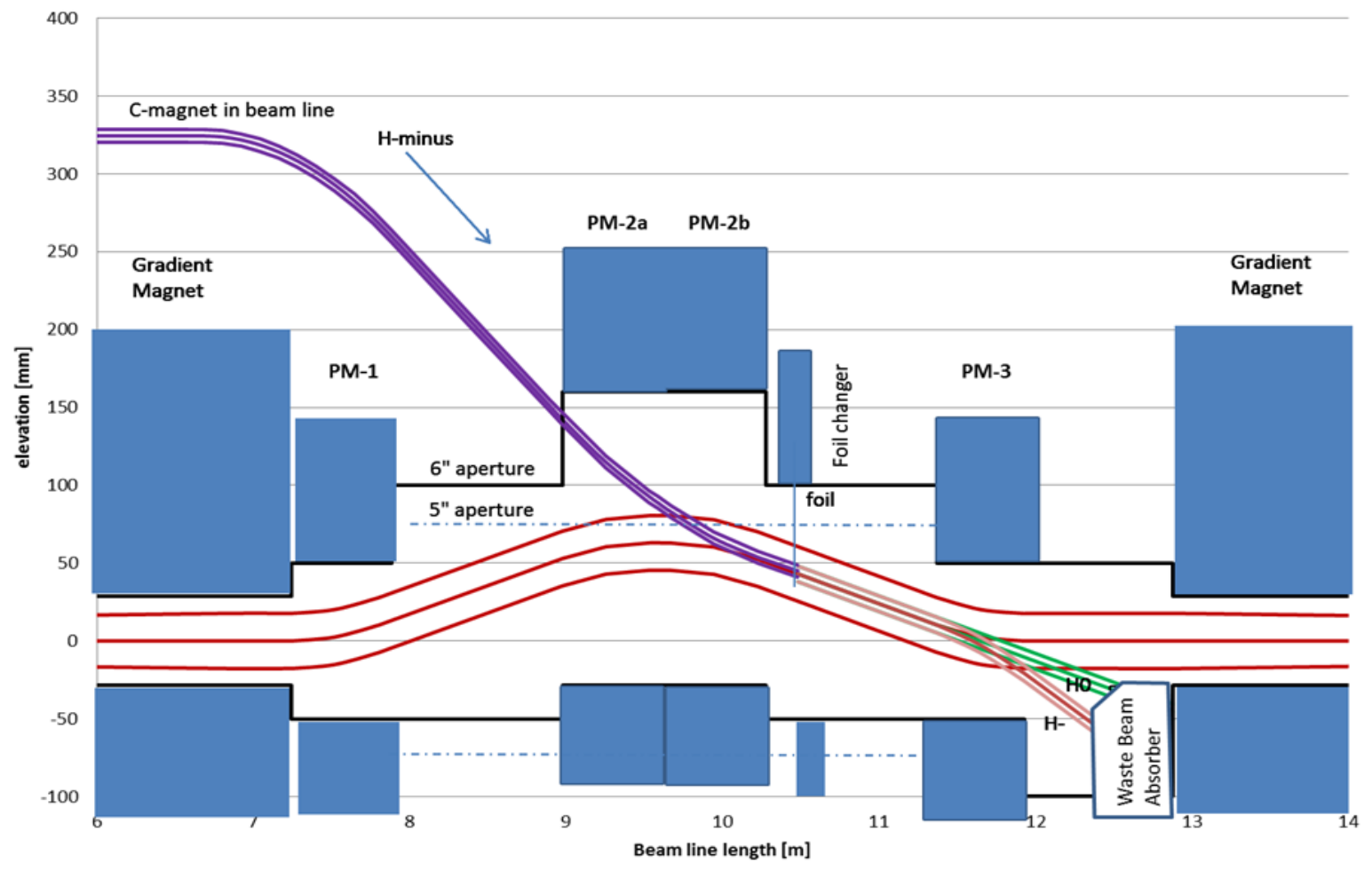

Figure 2.49: Conceptual design for $800 \mathrm{MeV}$ injection using a three-bump chicane within the Booster long straight section.

Table 2.15: Parameters of elements located in the injection straight

\begin{tabular}{c|l|l|l|c|c}
\hline \hline$\#$ & Name & $\begin{array}{c}\text { Accumulated } \\
\text { Length }(\mathrm{m})\end{array}$ & $\begin{array}{c}\text { Flange-Flange or } \\
\text { Drift Length }(\mathrm{m})\end{array}$ & $\begin{array}{c}\text { Magnetic } \\
\text { Length }(\mathrm{m})\end{array}$ & $\begin{array}{c}\text { Magnetic } \\
\text { Field (kG) }\end{array}$ \\
\hline 1 & Drift 1 & .07067 & 0.07067 & & \\
\hline 2 & ORBUMP 1 & 0.7624 & 0.69171 & 0.5585 & 3.496 \\
\hline 3 & Drift 2 & 1.7724 & 1.01 & & \\
\hline 4 & ORBUMP2a & 2.4641 & 0.69171 & 0.5585 & 3.496 \\
\hline 5 & ORBUMP2b & 3.1558 & 0.69171 & 0.5585 & 3.496 \\
\hline 6 & Drift 3 & 3.2570 & 0.1012 & & \\
\hline 7 & Foil changer & 3.5617 & 0.3047 & & \\
\hline 8 & Drift 4 & 4.1667 & 0.6050 & & \\
\hline 9 & ORBUMP 3 & 4.8584 & 0.69171 & & \\
\hline 10 & Drift 5 & 5.1584 & 0.3 & & \\
\hline 11 & Absorber & 5.6584 & .05 & & \\
\hline 12 & Drift & 5.6804 & 0.02202 & & \\
\hline \hline
\end{tabular}


The current placement of the C-dipole in the transport beam line is just over the upstream Booster dipole with a beam elevation of $\sim 0.15 \mathrm{~m}$ above the top of steel of the Booster dipole. The bend angle of the $\mathrm{C}$-dipole is 3 times the angle of the chicane dipoles or approximately $120 \mathrm{mrad}$. To obtain acceptable Lorentz stripping of the incoming $\mathrm{H}^{-}$ions the design of this magnet has to be driven by a requirement of minimization of the peak dipole field. A value less than $4 \mathrm{kG}$ has to be achieved. Table 2.15 present lengths allocated for different elements and their parameters. The accumulated length is between the flanges of the gradient magnets at either end of the straight section and is given at the end of each element. In addition to the elements in the Booster ring, the last magnet in the transfer line (C-dipole) ends 0.505 meters upstream of the ORBUMP2 magnet. It has a magnetic length of 1.8 meters with an angle of $-120 \mathrm{mrad}$ and field of $3.26 \mathrm{kG}$.

\subsubsection{Phase Space Painting}

The small values of the beam emittances of the linac beam allow us to perform phase space painting in all of the degrees of freedom. It reduces the harmful effects of beam space charge on the transverse particle motion due to a reduction of the space charge incoherent tune shifts, and it is expected to be helpful in achieving longitudinal stability (see below).
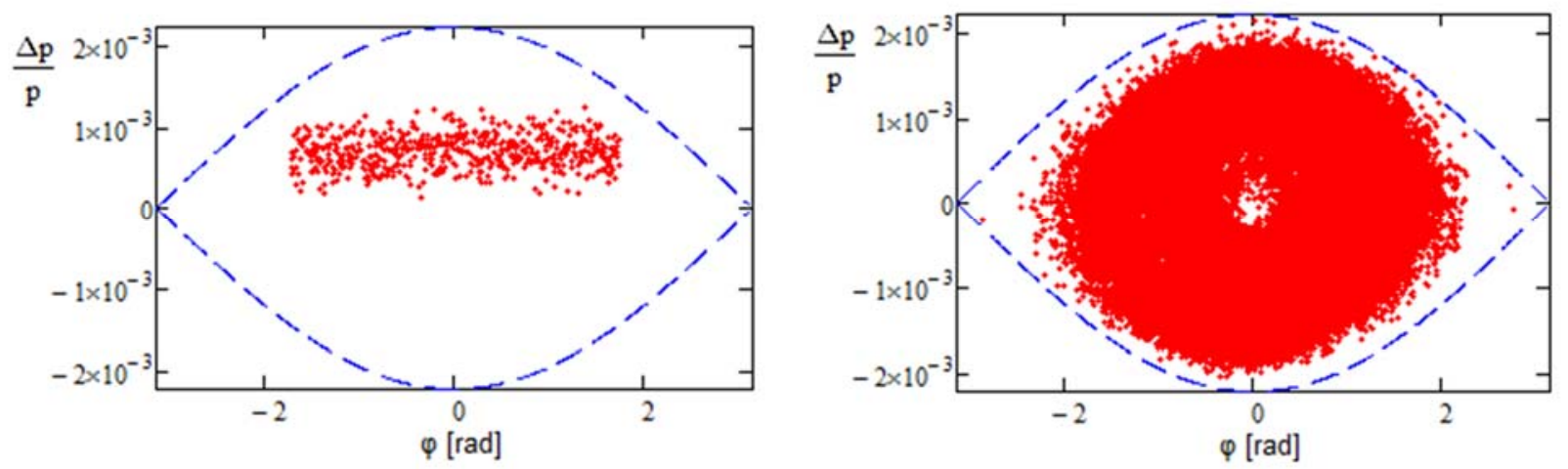

Figure 2.50: The longitudinal phase space of particles incoming to the Booster (left) and the phase space at the end of injection process (right).

The rms momentum spread of the linac beam is $2.2 \cdot 10^{-4}$, an order of magnitude smaller than the RF bucket height in the Booster $\left(2.2 \cdot 10^{-3}\right)$. Together with the long duration of the injection process corresponding to 7 synchrotron periods it enables static longitudinal painting, which greatly simplifies the procedure. In this case the linac energy is offset relative to the Booster reference energy and the synchrotron motion mixes particles in the longitudinal phase space in the course of injection. Linac bunches that would be outside of the RF buckets are removed by the bunch-by-bunch chopper in the MEBT. Figure 2.50 presents the longitudinal phase spaces of the injected beam and the Booster beam at the end of injection. The value of the momentum offset $\left(7 \cdot 10^{-4}\right)$ and the duration of the injection window (55\%) were optimized to minimize the bunching factor. Figure 2.51 presents the longitudinal density along a Booster bunch. The corresponding bunching factor is 2.5 . In average each Booster RF bucket receives two linac bunches per injection turn. The linac bunch frequency of 162.5 MHz and the Booster RF frequency of $44.705 \mathrm{MHz}$ are not related by integers and therefore the injection process is asynchronous. This results in a variation in the number of linac bunches injected in each Booster bunch in the range of $\pm 0.34 \%$ ( \pm 2 bunches out of 588 bunches injected into an RF bucket per injection cycle). The rms bunch lengthening in the course of beam transport from linac to Booster is about $14 \mathrm{~mm}$. This value is much smaller that the RF bucket length of $5.65 \mathrm{~m}$ and can be neglected in most practical considerations. 


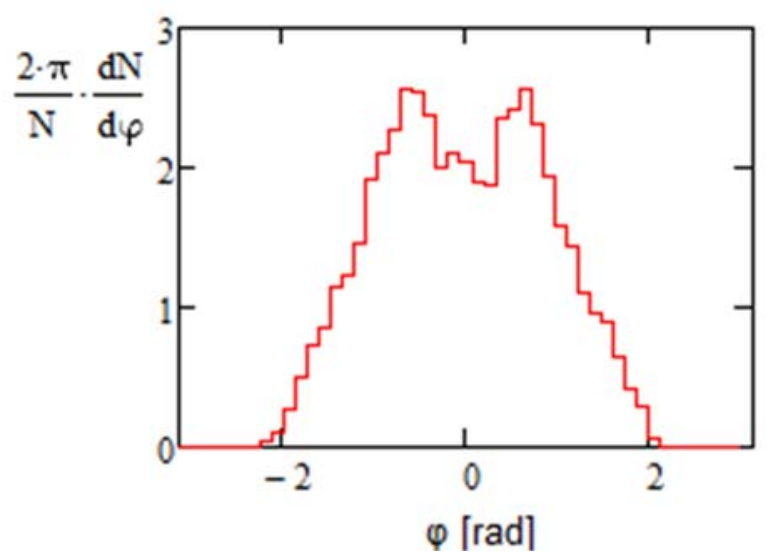

Figure 2.51: The longitudinal density of an injected bunch after injection.

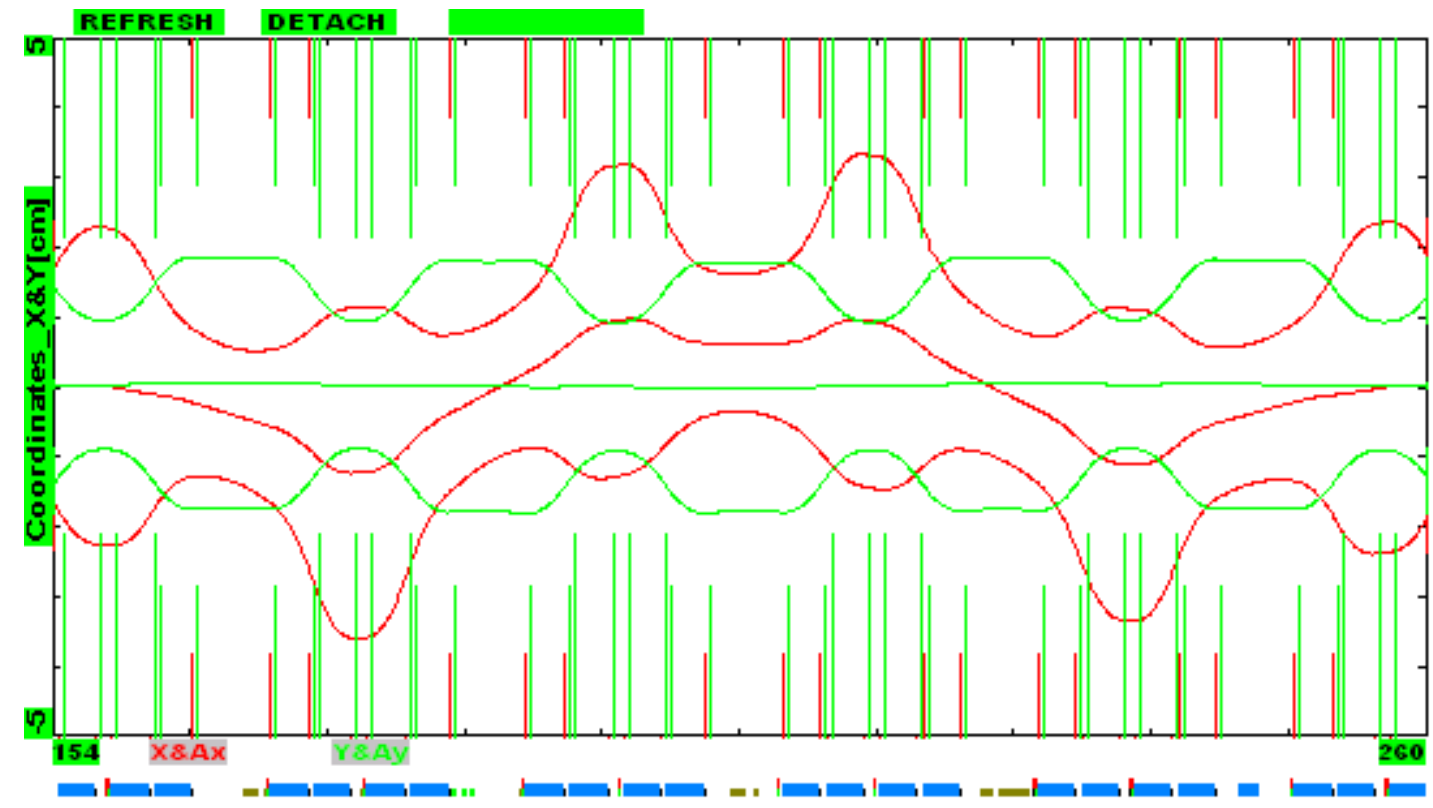

Figure 2.52: The horizontal orbit bump for beam painting at the maximum horizontal (red) and zero vertical (green) displacements. Beam envelopes for the normalized acceptances of $25 \mathrm{~mm}$ mrad are also shown. The injection straight (Long 11) is in the center of the plot ( $s \in[204.3-210.3] \mathrm{m})$. Vertical lines show aperture limitations in the dipoles (horizontal - red, vertical green).

As mentioned above, phase space painting in the vertical plane is performed by the dipoles of the injection chicane. Phase space painting in the horizontal plane is performed by regular Booster correctors located outside of the injection straight. The correctors should create a closed orbit bump with a maximum beam displacement on the foil of $6.1 \mathrm{~mm}$. The shortest orbit bump could be implemented using only the correctors closest to the injection straight (HL10, HS10, HS11, HL12). This choice requires a considerable corrector strength of $6.3 \mathrm{kG} \cdot \mathrm{cm}$. Although the strength of the present horizontal correctors of $9 \mathrm{kG} \cdot \mathrm{cm}$ is sufficient, their slew rate of $3.24 \mathrm{kG} \cdot \mathrm{cm} / \mathrm{ms}$ is about 5 times less than the slew rate required for painting. Therefore, a longer bump using correctors from nearby short straights was chosen. Table 2.16 presents the corrector strengths and Figure 2.52 shows the corresponding beam displacements together with the beam envelopes in the vicinity of the injection straight. The envelopes are plotted for the normalized emittance of $25 \mathrm{~mm} \cdot \mathrm{mrad}$, which includes almost $100 \%$ of the particles (see below). The horizontal beta-function in the short straights 
is about 5 times smaller than in the long ones. This enables a reduction of corrector strengths by $\sim 3.4$ times. The required slew rate of $4.5 \mathrm{kG} \cdot \mathrm{cm} / \mathrm{ms}$ still exceeds the slew rate for the present correctors by about 1.4 times. This problem can be addressed by an upgrade of corrector power supplies or by usage of a longer orbit bump.

Table 2.16: Corrector strengths required to create horizontal injection orbit bump

\begin{tabular}{l|l|l|l|l|l}
\hline Name & HS08 & HS09 & HS011 & HS12 & HS13 \\
\hline BdL $[\mathrm{kG} \cdot \mathrm{cm}]$ & -1.867 & 0.72 & 0.192 & 0.72 & -1.723 \\
\hline \hline
\end{tabular}

The stripping foil and the injected $\mathrm{H}^{-}$beam do not move during injection (see Figure 2.53). Similar to the beam injection in the SNS the rectangular foil is hanged in the vacuum chamber and the linac beam hits it near a corner to minimize number of secondary foil hits by particles of already injected beam. Both betatron and synchrotron oscillations in average push particles out of the foil.
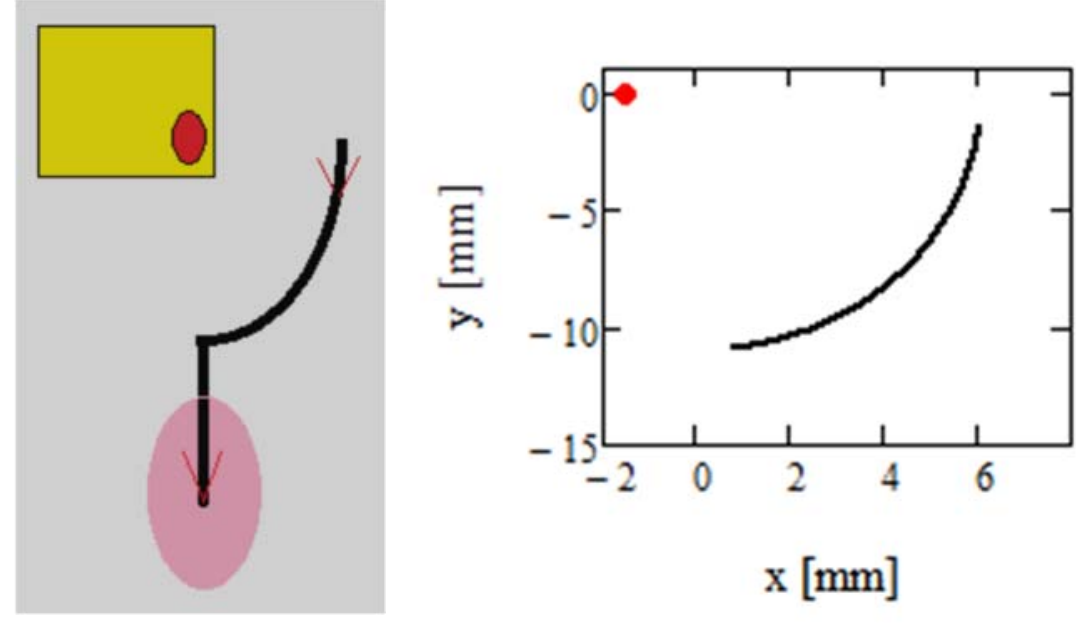

Figure 2.53: Left pane - schematic of injection painting: square (yellow) - stripping foil, small ellipse (red) - linac beam hitting the foil, large ellipse (pink) - circulating beam after the end of injection process, black line - trajectory of closed orbit displacements in the course of beam painting. Right pane - plot of closed orbit displacements in the course of beam painting relative to the linac beam center (shown by the red dot). The reference orbit of Booster beam is located at zero $x$-coordinate. The linac beam is displaced outward by $1.5 \mathrm{~mm}$ to account for the energy offset $\left(\Delta p / p=7 \cdot 10^{-4}\right)$ required for the longitudinal painting. Positive values of beam displacement in the horizontal and vertical planes correspond to inward and upward displacements, respectively (consequence of the right-hand coordinate frame chosen for description of ring orbit and optics).

The horizontal and vertical displacements of the closed orbit are correlated and follow the ellipse as shown in Figure 2.53. The ellipse semi-axes are 6.1 and $11 \mathrm{~mm}$ for horizontal and vertical amplitudes, respectively. To reduce the number of foil secondary hits and improve the uniformity of the distribution the painting curve covers only $84 \%$ of the ellipse quarter as shown in the right pane of Figure 2.53. That results in the actual beam displacements of 5.3 and $9.5 \mathrm{~mm}$ for horizontal and vertical planes, respectively. After painting the closed orbit and, consequently, the beam are moved vertically down to the nominal (central) orbit. As can be seen in Figure 2.53 the center of linac beam is additionally displaced outward by $1.5 \mathrm{~mm}$ to account for the energy offset required for the longitudinal painting. It also reduces the number of foil hits because the synchrotron motion keeps the center of accumulated beam at zero horizontal coordinate. 
The limited space available for beam injection and the small vertical aperture of Booster dipoles force us to obtain vertical painting by changing the magnetic field of the chicane. Unfortunately, it also affects the vertical position of the linac beam on the foil. This displacement is induced by changing the magnetic field of the central chicane dipole (see Figure 2.49) as the linac beam passes through it. This beam position change is corrected by fast dipole correctors located at the end of transport line (just upstream of injection aria).

To minimize the number of passages through the foil the beta- and alpha-functions of the linac beam are scaled from the corresponding values of the Booster by factor of 0.483 so that the linac phase space would be inscribed into the $x$ and $y$ machine acceptances as shown in Figure 2.54. The offsets of linac beam relative to the Booster beam presented in Figure 2.54 are equal to the painting offsets discussed above. Table 2.17 presents Twiss parameters for the linac and Booster beams on the stripping foil. To minimize displacements of linac beam position on the stripping foil we require that the dispersions and their derivatives are equal to zero at the foil. The emittance increase for the injected beam related to the dispersion mismatch to the linac dispersion is negligible. The number of secondary foil hits increases quickly with distance between the linac beam center and the foil edges, i.e. if the foil is moved closer to the circulating beam. To minimize foil hits we assume that one percent of the linac beam can miss the foil. For a Gaussian beam it determines that the distances to the foil edges has to be 2.58 times of the corresponding rms beam sizes or $1.95 \mathrm{~mm}$ and $3.51 \mathrm{~mm}$ for the horizontal and vertical planes, respectively.
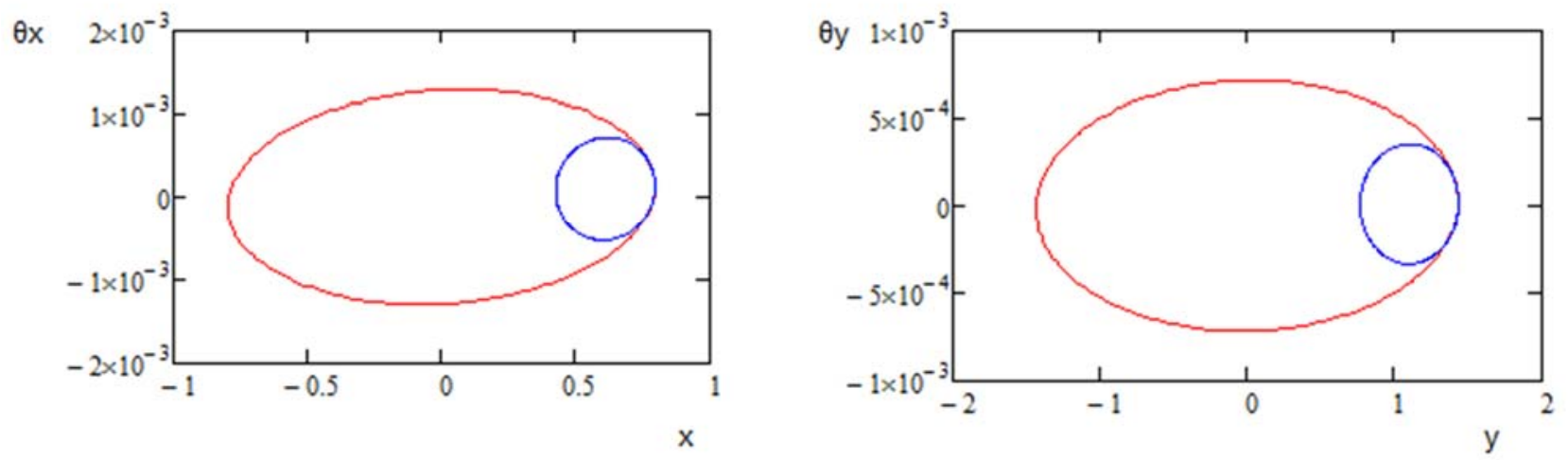

Figure 2.54: Phase space boundaries of the linac (blue) and RCS (red) beams. The linac beam boundaries correspond to the normalized boundary emittance of $1.8 \mathrm{~mm} \cdot \mathrm{mrad}$ ( $95 \%$ normalized linac emittance) the RCS beam boundaries correspond to the normalized emittances of $16 \mathrm{~mm} \mathrm{mrad}$.

Table 2.17: Twiss parameters for the Booster and linac beams at the stripping foil

\begin{tabular}{l|l|l|l|l|l|l}
\hline \hline & $\beta_{x}(\mathrm{~m})$ & $\alpha_{\mathrm{x}}$ & $\beta_{y}(\mathrm{~m})$ & $\alpha_{\mathrm{y}}$ & $D_{x}(\mathrm{~m})$ & $\mathrm{D}^{\prime}{ }_{\mathrm{x}}$ \\
\hline Booster & 6.17 & -0.095 & 20.03 & -0.028 & 2.18 & 0 \\
\hline Linac & 2.98 & -0.046 & 9.67 & -0.014 & 0 & 0 \\
\hline \hline
\end{tabular}

Figure 2.55 presents results of experimental measurements of $\mathrm{H}^{-}$stripping by a carbon foil at 800 $\mathrm{MeV}$, as presented in Ref. [30], with their extrapolation for a thicker foil. The extrapolation assumes the following cross-sections for transitions: $\sigma \mathrm{H}-\rightarrow \mathrm{H} 0=67.6 \cdot 10^{-16} \mathrm{~cm}^{-2}, \sigma_{-} \rightarrow \mathrm{H}^{+}=1.2 \cdot 10^{-16} \mathrm{~cm}^{-2}$ and $\sigma \mathrm{H} 0 \rightarrow \mathrm{H}^{+}=26.4 \cdot 10^{-16} \mathrm{~cm}^{-2}[31]$. It also assumes that there are no transitions with electron capture i.e. $\mathrm{H}^{0} \rightarrow \mathrm{H}^{-}, \mathrm{H}^{+} \rightarrow \mathrm{H}^{-}$and $\mathrm{H}^{+} \rightarrow \mathrm{H}^{0}$. This assumption is well justified for $800 \mathrm{MeV}$ energy. The thickness of stripping foil was chosen to be $600 \mu \mathrm{g} / \mathrm{cm}^{2}$. This is thick enough to strip the major fraction of $\mathrm{H}^{-}$ 
to protons leaving less than $0.1 \%$ particles as $\mathrm{H}^{0}$ and a negligible fraction as $\mathrm{H}^{-}$. As shown below, this thickness does not cause problems with particle scattering in the foil and foil overheating but should support longer foil operation than a thinner foil. Spattering resulting in foil evaporation is expected to be a major mechanism limiting the foil lifetime. As shown in Figure 2.55, operation with foil thickness as thin as $400 \mu \mathrm{g} / \mathrm{cm}^{2}$ is still possible.
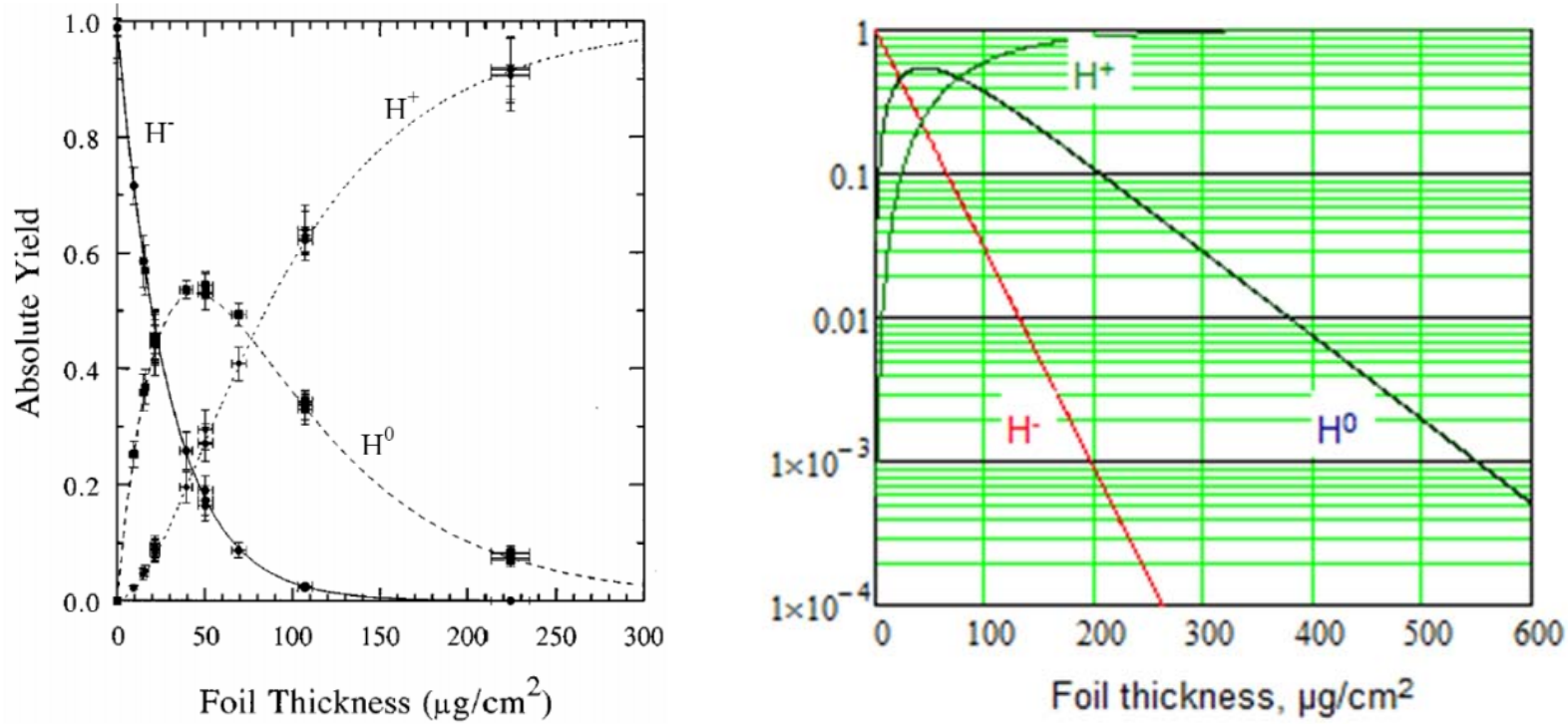

Figure 2.55: Measurement of $800 \mathrm{MeV} \mathrm{H}^{-}$stripping by carbon foil presented in Ref. [30] (left pane) and their extrapolation for a thicker foil (right pane).
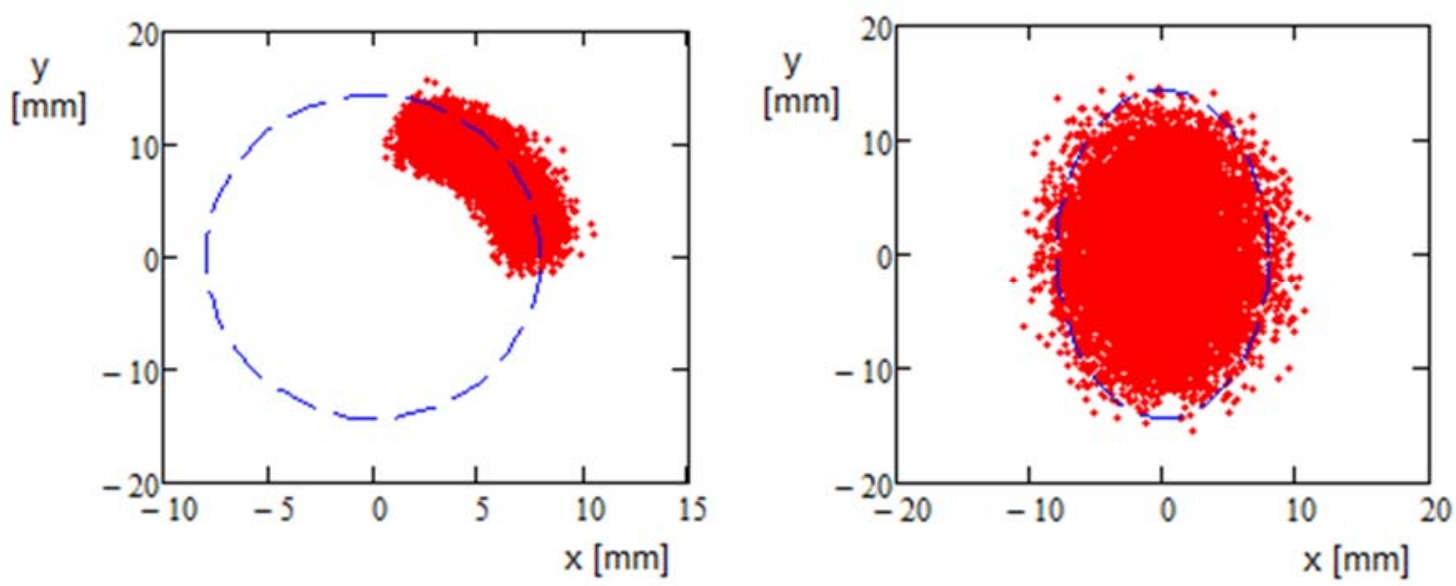

Figure 2.56: $\mathrm{X}$ and $\mathrm{Y}$ coordinates of all injected (in simulation) particles relative to the current orbit position for particles incoming to the Booster (left) and at the end of injection process (right).

Figure 2.56-2.59 present results of numerical simulation of the strip-injection. The simulation includes multiple scattering in the foil, synchrotron and betatron motions and the details of the painting process described above. The beam space charge effects are not taken into account and betatron motion is linear and without $x-y$ coupling. The simulation showed that that the betatron tunes have to be different by more than 0.01 for uniform painting. The results were obtained for the following tunes: $Q_{x}=6.8$ and $Q_{y}=6.81$. Tune values have little effect on the result as long as they are different. The left pane in Figure 2.56 presents $x$ and $y$ coordinates of all particles relative to the orbit position at particle injection time. The right pane presents particle coordinates at the end of the 
injection process. The left pane in Figure 2.57 shows the particle distribution in Courant-Snyder invariants (single particle emittances). One can see that the distribution is somewhere between the Gaussian and K-V distributions. The latter would be represented by the $\delta$-function, $\delta\left(\varepsilon_{4 D n}-\varepsilon_{b n}\right)$, in the 4-dimensional phase space, where $\varepsilon_{4 D n}=\varepsilon_{x n}+\varepsilon_{y n}$, and $\varepsilon_{b n}$ is the normalized boundary emittance of KV-distribution. The right pane in Figure 2.57 shows the integrals of the particle distributions presented in the left pane normalized to 1 . One can see that $95 \%$ of particles are within $17 \mathrm{~mm}$ mrad and almost $100 \%$ within $23 \mathrm{~mm}$ mrad. Similarly, Figure 2.58 presents the longitudinal distribution and its integral. As one can see, $100 \%$ of particles are within $0.06 \mathrm{eV} \cdot \mathrm{s}$.
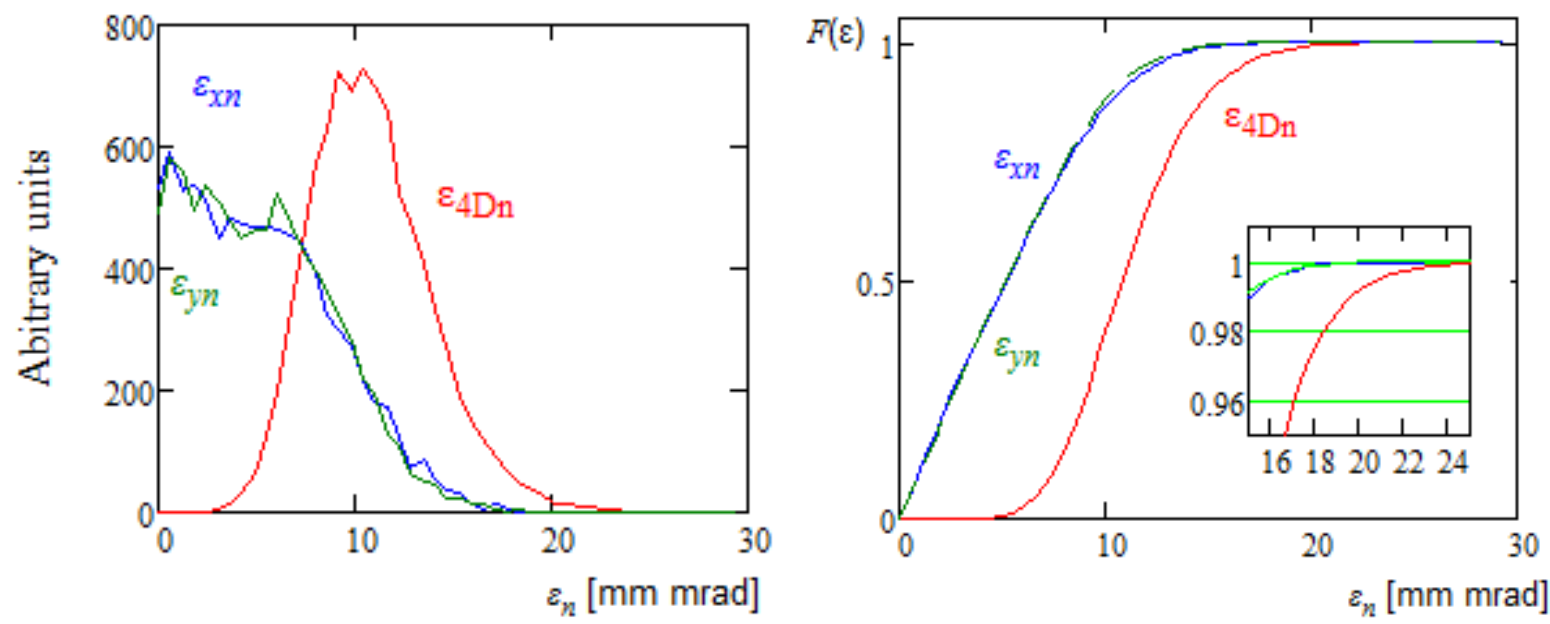

Figure 2.57: Left pane - the particle distribution over particle normalized Courant-Snyder invariants: $\varepsilon_{x n}=\beta \gamma\left(x^{2} / \beta_{x}+2 \alpha_{x} x \theta_{x}+\left(1+\alpha_{x}{ }^{2}\right) \theta_{x}{ }^{2} / \beta_{x}\right)$, (and similarly for $y$ plane); blue - horizontal plane, green - vertical plane, red - the distribution over sum of invariants, $\varepsilon_{4 D}=\varepsilon_{x}+\varepsilon_{y}$. Right pane - the integrals of particle distributions (normalized to unity) presented in the left pane. The insert shows detail near the top of the picture.
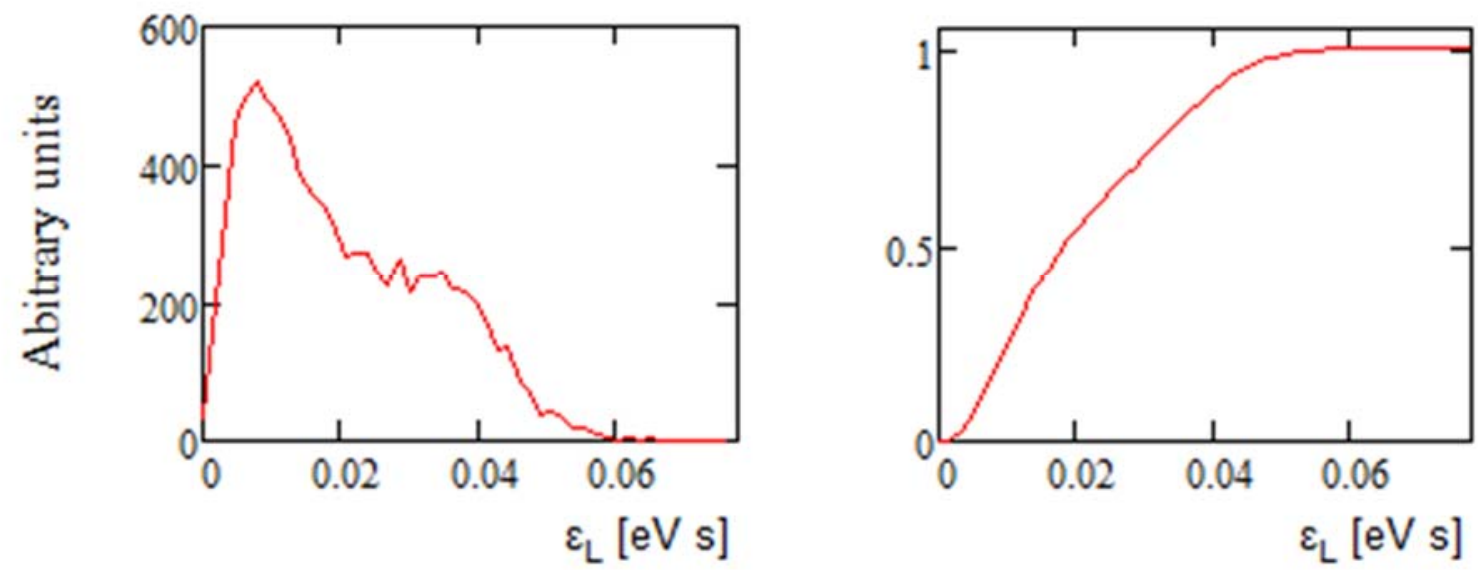

Figure 2.58: Left pane - the particle distribution over particle longitudinal emittance (phase space area subtended by particle trajectory). Right pane - the integral of particle distribution presented in the left pane (normalized to 1). The horizontal axis ends at the bucket boundary.

Figure 2.59 presents the distribution of secondary and primary hits of the surface of stripping foil. The peak of secondary hits is located at the foil corner and is equal to 63 hits per particle per $\mathrm{cm}^{2}$. 
The average number of secondary foil hits is 6.1 per injected particle. The distribution of primary hits is peaked at the center of incoming linac beam and is about 4 times smaller (15.4 hits per particle per $\mathrm{cm}^{2}$ ). As can be seen from the right pane in Figure 2.59 the peak of the total (summed for primary and secondary hits) hit distribution is determined by secondary hits.

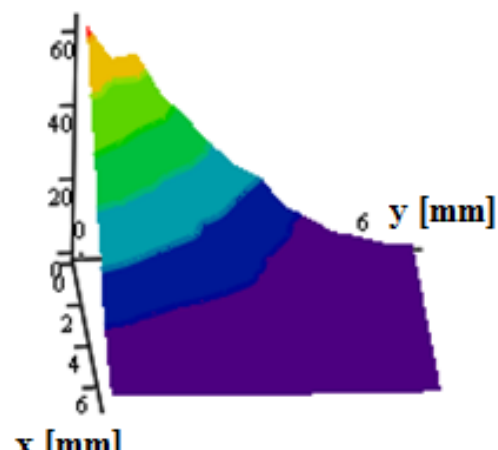

$\mathbf{x}[\mathbf{m m}]$

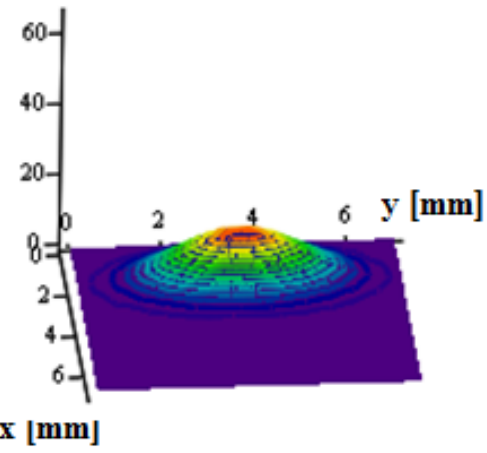

$\mathbf{x}[\mathbf{m m}]$

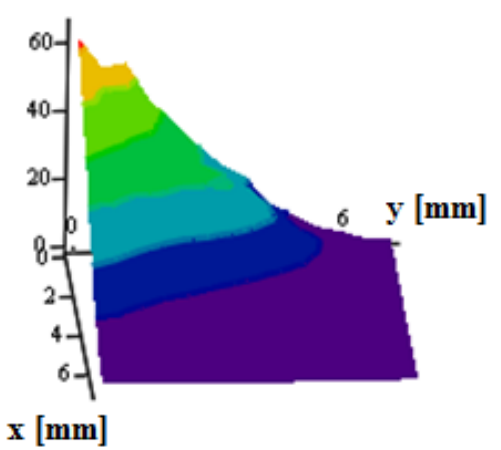

$\mathbf{x}[\mathrm{mm}]$

Figure 2.59: The distribution of particle hits on the stripping foil (hits per injected particle per $\mathrm{cm}^{2}$ ): left - secondary hits, center - primary hits of the incoming linac beam, right - sum of primary and secondary hits.

The beam passing through the foil results in its heating. Although the total deposited power of 33 $\mathrm{mW}$ is small, the power density is still considerable due to small size of the injected beam. At its peak in the foil corner the power density is about $2 \mathrm{~W} / \mathrm{cm}^{2}$. The major cooling mechanism for the foil is the black body radiation. Conservatively assuming the foil emissivity of $50 \%$ one obtains the peak temperature of $640 \mathrm{C}^{\circ}$ at the foil corner. This temperature is sufficiently small to guarantee a long lifetime for the foil. Figure 2.60 presents a dependence of the hottest spot temperature on time after initiation of beam operation. The temperature reaches its peak after the fourth pulse. An estimate shows that accounting for the foil thermal conductivity yields a quite small correction and it was neglected in the above calculation.

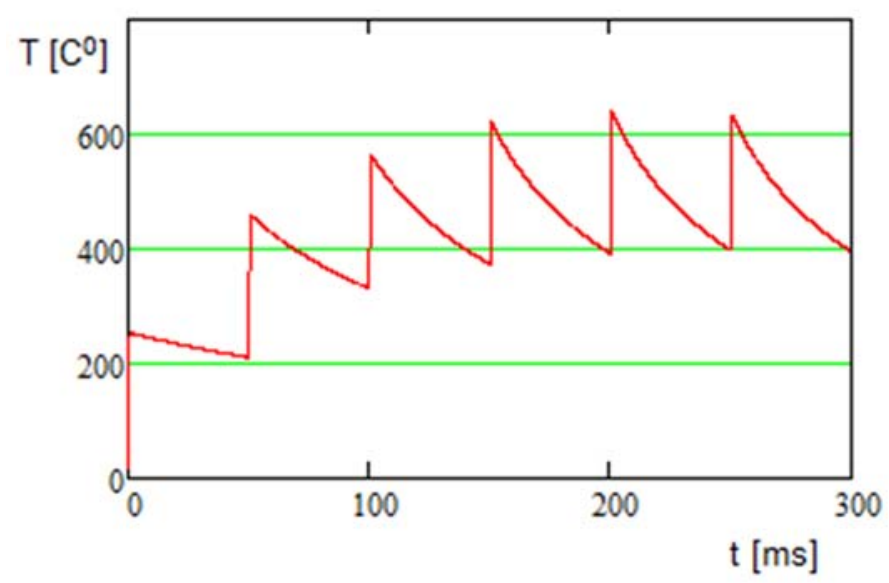

Figure 2.60: The dependence of temperature on time for the hottest place on the foil; $t=0$ corresponds to the first injection pulse.

The total power of the injected beam is about $18 \mathrm{~kW}$. About $1.2 \%$ of these particles are expected to be lost during injection: $\sim 1 \%$ miss the foil, $0.1 \%$ are not completely stripped in the foil, and $0.1 \%$ are lost due to single scattering in the foil (direct and secondary hits are accounted). In normal 
operating conditions, the resulting heat load on the injection beam dump is about $200 \mathrm{~W}$ with $20 \mathrm{~W}$ of uncontrolled beam loss mostly intercepted in the first two dipoles.

Stripping of $\mathrm{H}^{-}$also yields two $400 \mathrm{keV}$ electrons for each stripped $\mathrm{H}^{-}$. These electrons carry a power of about $18 \mathrm{~W}$ that needs to be intercepted by the electron beam dump. After leaving the foil the electrons are reflected from the downstream dipole where they are bent by its magnetic field. It results in their bending by $180 \mathrm{deg}$. and a displacement by a few centimeters in the vertical plane. The design of the electron dump must prevent heating of the foil by these electrons, their accumulation and interaction with the circulating beam.

The injection to the Recycler, and, subsequently, to the Main Injector requires injection gaps of 3 buckets in the bunch structure of the Booster beam. Removal of these bunches will be performed by the bunch-by-bunch chopper located in the linac MEBT.

\subsubsection{Beam Acceleration in the Booster}

The longitudinal emittance of the Booster beam is limited by the RF bucket size in the Recycler. Although the RF bucket size in the Recycler will be larger by $33 \%$ due to an increase of Booster repetition rate from 15 to $20 \mathrm{~Hz}$ (see details below) the longitudinal rms emittance of the Booster beam is required to be about the same. A larger ratio of bucket size to the beam emittance should enable a reduction of beam loss by factor more than 2 , tentatively from $5 \%$ to $2 \%$. Thus, the RF bucket size in the Booster is expected to be close to the PIP value.

An increase of the Booster ramp rate increases the magnetic field ramping rate and the RF voltage required for acceleration. However, for a small intensity beam an increase of the peak RF voltage is not required if the RF bucket area stays the same. Actually, to keep a desirable size of RF bucket during acceleration in the present Booster the maximum $\mathrm{RF}$ voltage is required at the cycle beginning ( $\sim 5 \mathrm{~ms}$ after injection) when the accelerating rate is still comparatively small. The decrease of the slip-factor with higher injection energy used in the PIP-II reduces the RF voltage required for the longitudinal beam focusing, thus resulting in about the same requirements for the peak RF voltage for $20 \mathrm{~Hz}$ ramping rate. Figure 2.61 presents the time dependences of beam and RF system parameters in the course of low intensity beam acceleration. The RF waveform was tuned to keep a fixed value of the bucket size before transition and $\sim 1.5$ times larger value after it. The dependencies presented in the top two rows of the figure were computed assuming adiabatic longitudinal motion in the course of acceleration. The latter is not true in the close vicinity of transition and therefore the divergences for the bucket area and the bucket height do not describe actual beam behavior. The plots in the bottom row were computed by turn-by-turn particle tracking and therefore they describe the transition crossing accurately if the beam intensity is sufficiently small.

As will be seen, the PIP-II intensity requires significantly larger RF voltage. Results of simulations presented below satisfy the PIP-II requirements. They use the peak RF voltage of $1.18 \mathrm{MV}$. Reliable operations require that the Booster should be capable to operate without one cavity yielding the installed RF voltage of $\sim 1.25 \mathrm{MV}$. It will be supported by $22 \mathrm{RF}$ cavities (stations). This goal will be achieved as part of the PIP program. The present RF cavities and power amplifiers are adequate for the beam acceleration although minor modifications to the power amplifiers will be required due to 1.5 times larger beam power. Detailed simulations of transition crossing are presented in the following subsections.

Compared to the present Booster operation, the injection energy increase combined with the beam painting results in a significant decrease of the incoherent tune spread due to beam space charge. This effect is usually characterized by the space charge tune shift for particles with small betatron and synchrotron amplitudes, which, for a Gaussian bunch, is equal to: 


$$
\delta v_{S C_{x, y}}=-\frac{r_{p} q N_{b} B}{2 \pi \beta^{2} \gamma^{3}}\left\langle\frac{\beta_{x, y}}{\sigma_{x, y}\left(\sigma_{x}+\sigma_{x}\right)}\right\rangle_{s} .
$$
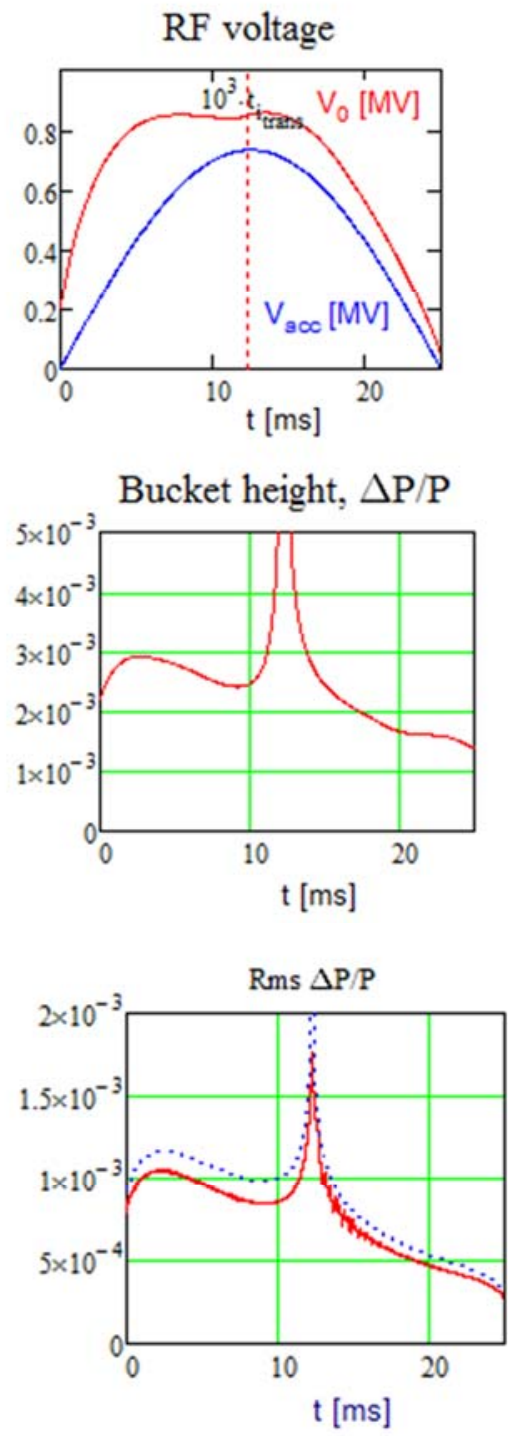

Accelerating phase, deg

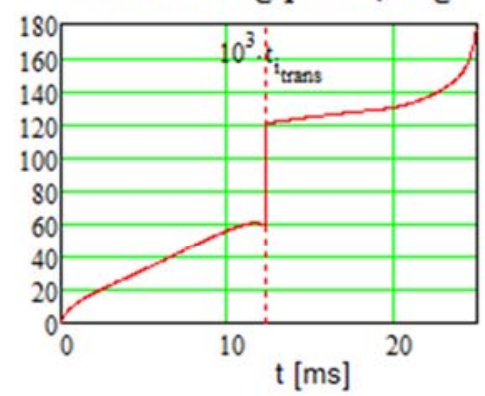

Bucket length, deg

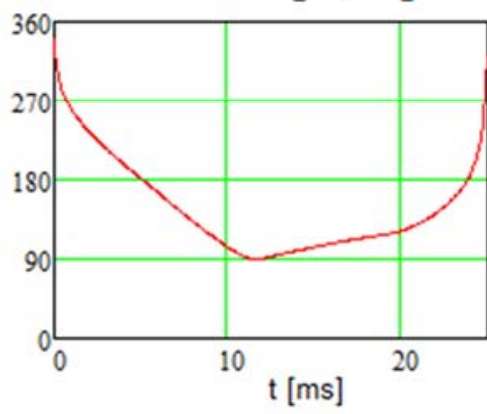

Rms bunch length, deg

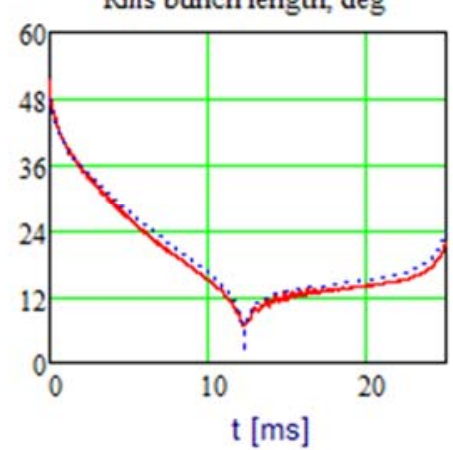

Bucket area, eV s

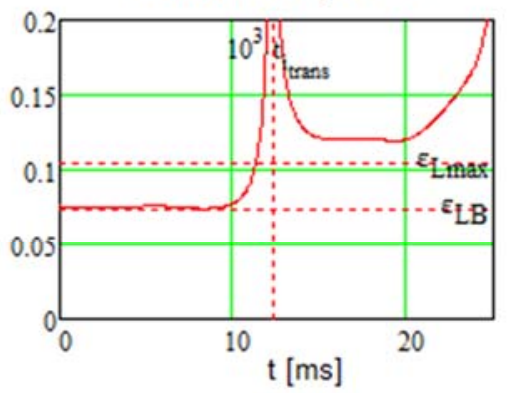

Synchrotron tune

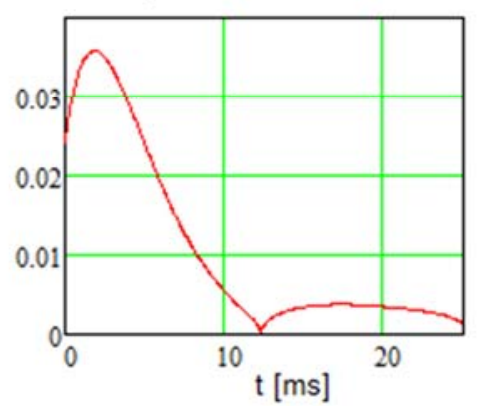

Bunching factor

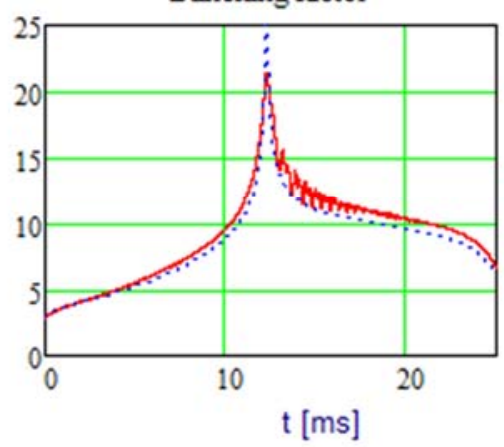

Figure 2.61: Beam and RF system parameters for acceleration of small intensity beam. The initial RF bucket size is $0.075 \mathrm{eV} \cdot \mathrm{s}$, and the $100 \%$ initial longitudinal emittance is equal to $0.06 \mathrm{eV} \cdot \mathrm{s}$. The red lines in the bottom row present the tracking results for a small intensity bunch, and the blue lines the results of adiabatic approximation.

Here $N_{b}$ is the number of particles per bunch, $\beta$ and $\gamma$ are relativistic factors, $\sigma_{x}=\sqrt{\varepsilon_{x} \beta_{x}+D_{x}^{2} \sigma_{p}^{2}}$ and $\sigma_{y}=\sqrt{\varepsilon_{y} \beta_{y}}$ are the rms beam sizes, $\beta_{x}$ and $\beta_{y}$ are the beta-functions, $D_{x}$ is the ring dispersion, $\varepsilon_{x}$ and $\varepsilon_{y}$ are the rms emittances, $\sigma_{p}$ is the rms relative momentum spread, $<>_{\mathrm{s}}$ denotes averaging along the ring circumference, and $B$ is the bunching factor defined as: $B=\left.(d N / d s)\right|_{\max } /\left(N_{b} q / C\right)$ with $C$ being the ring circumference and $q$ the harmonic number. The painting simulations discussed above result in a particle density in the bunch center which coincides with the particle density of Gaussian beam with horizontal and vertical normalized rms emittances equal to $5 \mathrm{~mm}$ mrad. Note 
that the $95 \%$ emittance corresponding to the rms emittance of $5 \mathrm{~mm} \mathrm{mrad}$ is equal to $\sim 30 \mathrm{~mm} \mathrm{mrad}$, while the $95 \%$ emittance of the painted beam is $16 \mathrm{~mm}$ mrad. Thus, the transverse painting decreases the space charge tune shifts by about two times. Figure 2.62 presents the dependence of the space charge tune shifts on time within accelerating cycle. As one can see the space charge tune shifts do not exceed 0.2.

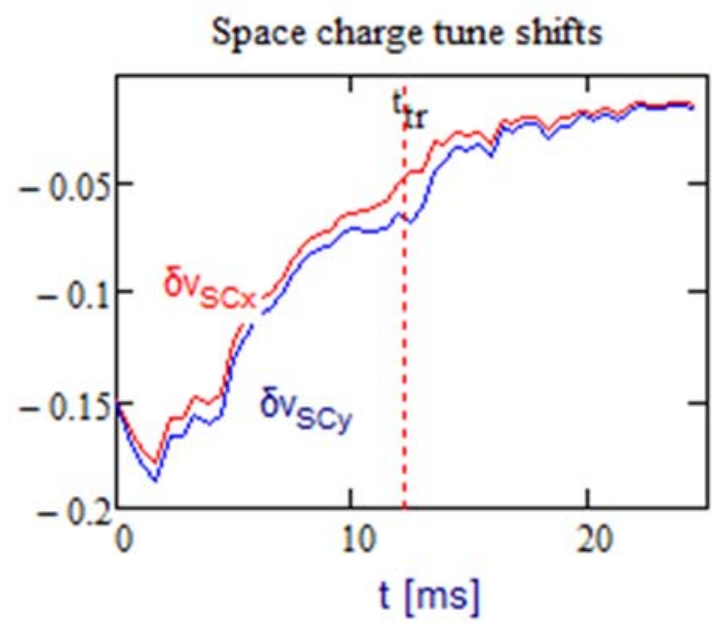

Figure 2.62: The betatron tune shifts due to beam space charge for horizontal and vertical planes within accelerating cycle. The reduction of tune shifts due to non-Gaussian shape of the particle distribution (see details of painting in Section 2.3.2.3) is taken into account. Bunching factor and momentum spread are taken from simulations of full intensity beam acceleration described in Section 2.3.5. Vertical line marks transition crossing.

For the present RF system the beam-induced voltage significantly exceeds the RF system voltage required for beam acceleration and capture. Figure 2.63 and 2.64 present the measured shunt impedance of the present Booster cavities, the beam induced voltage and the beam and cavity powers. As one can see, even at the maximum RF voltage, the beam-induced voltage (at resonance) exceeds the required RF voltage by almost 2 times. This ratio achieves its maximum of about 30 at the end of the accelerating cycle.

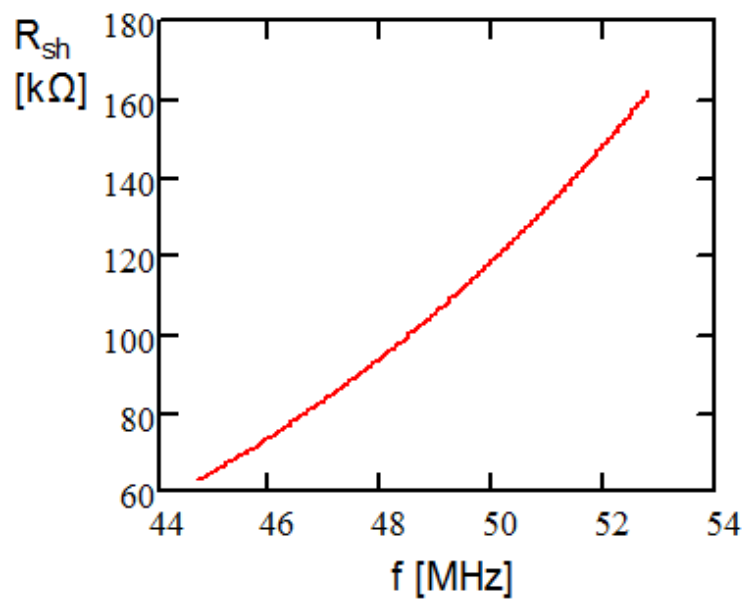

Figure 2.63: The shunt impedance of the Booster RF cavity in the frequency range required for PIP-II operation.

Note that the power loss in the cavity walls presented in Figure 2.64 assumes equal voltage 
distribution in all cavities and their perfect phasing. A practical way of obtaining small RF voltage in the presence of large beam current is paraphasing of two groups of cavities. This technique is presently used in the Booster and will be used in the future for PIP-II. In this case, a small voltage is achieved by operating two groups of cavities with comparatively large and equal voltages with an RF phase difference close to $180 \mathrm{deg}$. That implies that power loss in the cavity walls will be significantly larger at the cycle beginning and the cycle end (where the RF voltage is small) than the value presented in Figure 2.64.
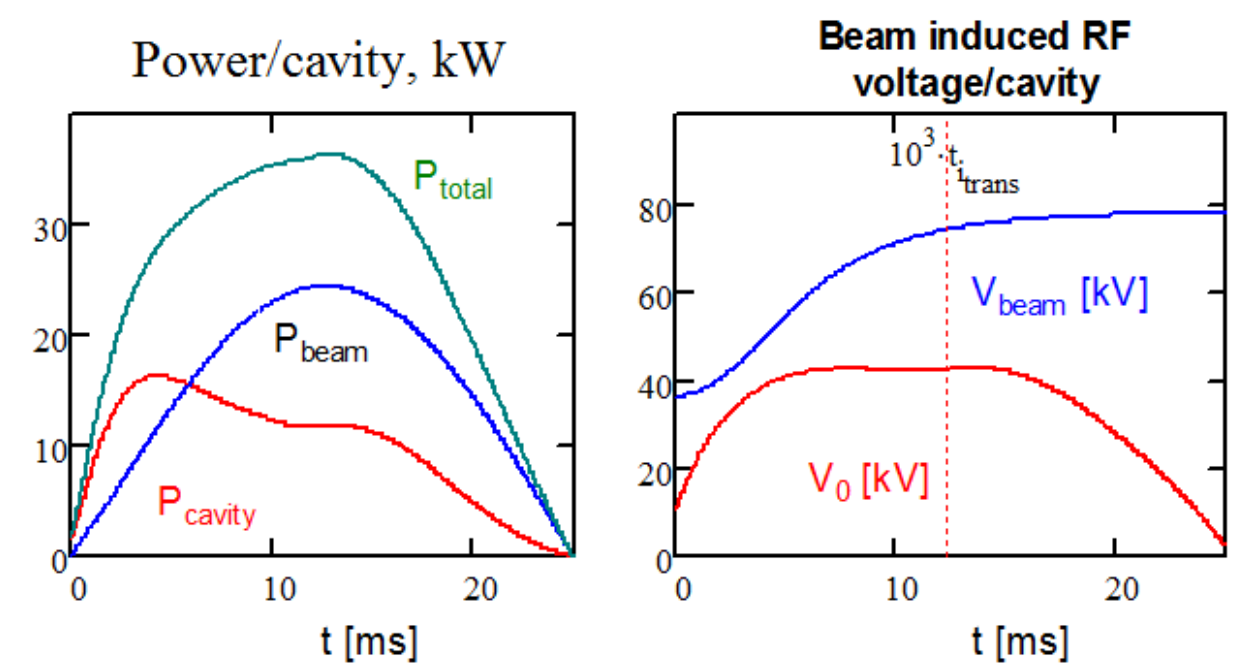

Figure 2.64: Left pane: dependences on time within accelerating cycle for the power loss in the cavity walls (red, $P_{\text {cavity }}=V_{0}{ }^{2} / R_{\text {sh }}$ ), the beam power transferred to the beam (blue) and the total RF power to a cavity (green). Right pane: dependences on time within accelerating cycle for the total RF voltage per cavity (red) and the beam induced voltage. 22 accelerating cavities are implied. The presented cavity voltage is used in the simulations of full intensity beam acceleration described in Section 2.3.5.

An additional reduction of the space charge betatron tune shifts can be achieved with a double harmonic RF system. In this case the voltages of the fundamental (first harmonic) RF system and an additional RF system operating at the second harmonic can create a longitudinal potential well with a flat bottom resulting in a reduction of the bunching factor. The installation of the second harmonic $\mathrm{RF}$ system is planned for the presently proceeding Proton Improvement Plan (PIP). Note that relative misphasing of the first and second harmonic RF systems deteriorates the flat bottom of the potential well. That can result in an increase of longitudinal density and, subsequently, can lead to a particle loss. The required accuracy of relative phasing is about $5 \mathrm{deg}$. of the first harmonic frequency. Addressing the voltage stability and relative phasing will require a sophisticated low level RF.

\subsubsection{Booster Longitudinal Impedance}

To avoid the eddy currents that would be excited in a vacuum chamber by fast changing magnetic field the Booster does not have a vacuum chamber in the usual sense of this word, rather its beam aperture is formed by poles of laminated combined function dipoles. That greatly amplifies its longitudinal and transverse impedances.

To estimate the longitudinal impedance of such a "laminated" beam aperture we use the model presented in Ref. [32], where the longitudinal impedance of a flat laminated dipole with constant gap between poles is derived. The chamber geometry is presented in Figure 2.65. The results of Ref. [32] (see Eqs. (5.12) and (5.19) in there) can be rewritten in the following form, presenting the longitudinal impedance per unit length as a function of frequency: 


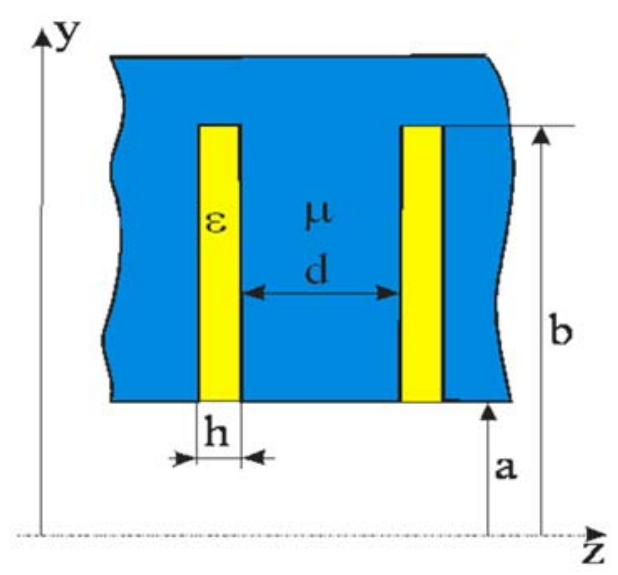

Figure 2.65: Geometry of the laminated beam aperture (or "vacuum chamber"). The beam moves in the z-direction. The chamber is infinite in x-direction (normal to the picture plane). The scale along $z$ axis is magnified for clarity.

$$
Z_{\|_{L M}}(\omega)=i Z_{0} \frac{\omega}{2 \pi c} \int_{0}^{\infty} \frac{F_{L}(\xi)}{1+F_{L}(\xi) \tanh \xi} \frac{d \xi}{\xi \cosh ^{2} \xi}
$$

where

$$
\begin{aligned}
& F_{L}(\xi)=\frac{h}{d+h} \frac{\xi}{k_{y}(\xi)}\left(1+(1-i) \frac{\mu \delta_{S}}{h}\right) \tan \left(k_{y}(\xi)\left(\frac{b}{a}-1\right)\right), \\
& k_{y}(\xi)=\sqrt{\frac{\varepsilon \omega^{2} a^{2}}{c^{2}}\left(1+(1-i) \frac{\mu \delta_{S}}{h}\right)-\xi^{2}},
\end{aligned}
$$

$\mathrm{Z}_{0}=4 \pi / \mathrm{c} \approx 377 \Omega, c$ is the light velocity, $a$ is the half-gap between dipole poles, $(b-a)$ is the depth of laminations, $\varepsilon$ is the effective dielectric constant of the filling (epoxy plus an insulating oxide layer), $h$ is the distance between laminations ${ }^{7}, \delta_{S}=c / \sqrt{2 \pi \sigma_{R} \omega \mu}$ is the skin depth, $\sigma_{R}$ is the steel conductivity, and $\mu$ is the steel permeability. At frequencies of interest the skin depth in soft steel is smaller than, or about the same as, the magnetic domain size, which greatly reduces the magnetic permeability and makes its imaginary part being non-zero. In the below estimate, we use the measured magnetic permeability of soft steel presented in Ref. [33]. The measurements for intermediate values of magnetic field were fitted to the following expression,

$$
\mu(\omega)=\frac{26}{1+i \omega / \omega_{1}}+\frac{9}{\left(1+i \omega /\left(2 \omega_{2}\right)\right)\left(1+2 i \omega / \omega_{2}\right)}, \begin{aligned}
& \omega_{1}=2 \pi \cdot 7 \cdot 10^{7} \mathrm{~s}^{-1}, \\
& \omega_{2}=2 \pi \cdot 3 \cdot 10^{9} \mathrm{~s}^{-1},
\end{aligned}
$$

which plot is presented in Figure 2.66. Other parameters used in Eq. (2.10) are presented in Table 2.18. The steel conductivity was taken from Ref. [33]. Results of the numerical integration of Eq. (2.10) are presented in Figure 2.67. We assume here that the distance between laminations is constant, while in reality it is changing within each gap and from gap to gap in some uncontrolled way. Consequently, Eq. (2.10) has a quite limited accuracy and direct measurements of the dipole impedance are highly desirable. As shown in Ref. [33] the magnetic permeability at high frequency, the same as for DC case, depends on the magnetic field in a dipole. That results in a dependence of

\footnotetext{
${ }^{7}$ The distance between laminations, $h$, was initially estimated from the known packing factor and then was adjusted to match observed and predicted shifts of accelerating phase with beam intensity.
} 
the dipole impedance on its magnetic field. The fitting of Eq. (2.12) was performed for high magnetic field so that it would represent the value of $\mu$ near transition crossing where an accurate estimate of impedance is critical for simulations.

Table 2.18: Parameters of laminations used for the impedance estimates of the Booster laminated dipoles

\begin{tabular}{l|c|l|l}
\hline \hline Dipole type & $\mathrm{F}$ & $\mathrm{D}$ & \\
\hline Dipole length & \multicolumn{2}{|c|}{2.89} & $\mathrm{~m}$ \\
\hline Number of dipoles & 48 & 48 & $\mathrm{~cm}$ \\
\hline Half-gap, $a$ & 2.1 & 2.9 & $\mathrm{~cm}$ \\
\hline Lamina half-height, $b$ & \multicolumn{2}{|c|}{15.2} & $\mathrm{~cm}$ \\
\hline Lamina thickness, $d$ & \multicolumn{2}{|c|}{0.64} & $\mathrm{~mm}$ \\
\hline Dielectric crack width, $h$ & \multicolumn{2}{|c|}{45} & $\mu \mathrm{m}$ \\
\hline Conductivity, $\sigma$ & $2.07 \cdot 10^{16}\left(2.3 \cdot 10^{6} \Omega^{-1} \mathrm{~m}^{-1}\right)$ & $\mathrm{s}^{-1}$ \\
\hline Dielectric permittivity, $\varepsilon$ & \multicolumn{2}{|c|}{2.5} & \\
\hline \hline
\end{tabular}

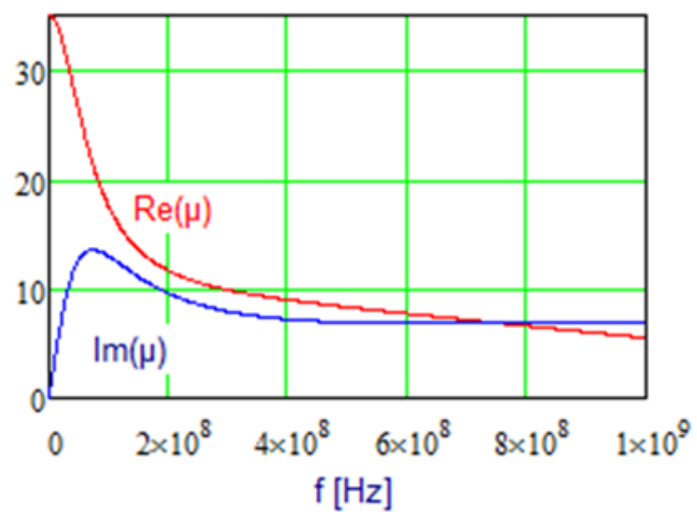

Figure 2.66: The dependence of magnetic permeability on frequency used for computation of the Booster longitudinal impedance.

Two types of impedance measurements were used. The first method is based on stretched wire measurements [34] of a single dipole impedance, and the second one is based on the shift of accelerating phase with beam intensity. The first method yields a dependence of single dipole impedance on the frequency, while the second one allows one to measure only the effective impedance but for the entire Booster.

Figure 2.68 presents results of the longitudinal impedance measurements for two spare Booster dipoles [34] performed with the stretched wire. As on can see from comparison of Figures 2.67 and 2.68 the measurements and the calculations are in a reasonable agreement for D-dipole. However, the agreement is much worse for the F-dipole. It is important to note that the measured impedance of the D-dipole is larger than that of the F dipole while theory predicts the opposite - the dipole with larger aperture should have smaller impedance. Most probably, it is related to a difference in details of lamination packing in these two dipoles. Therefore, we expect that each dipole has a unique impedance. Consequently, a beam-based measurement is the only reliable way to measure the Booster impedance with required accuracy. Unfortunately, the measurements with bunched beam do not measure the dependence of impedance on frequency, and measurements with continuous beam 
at the energy of transition crossing cannot be done. Therefore, taking into account that the frequency dependencies presented in Figures 2.67 and 2.68 are sufficiently close, we use the theoretical prediction of Eq. (2.10) where we adjust the gap between plates to match the measured and observed shifts of accelerating phase with intensity.

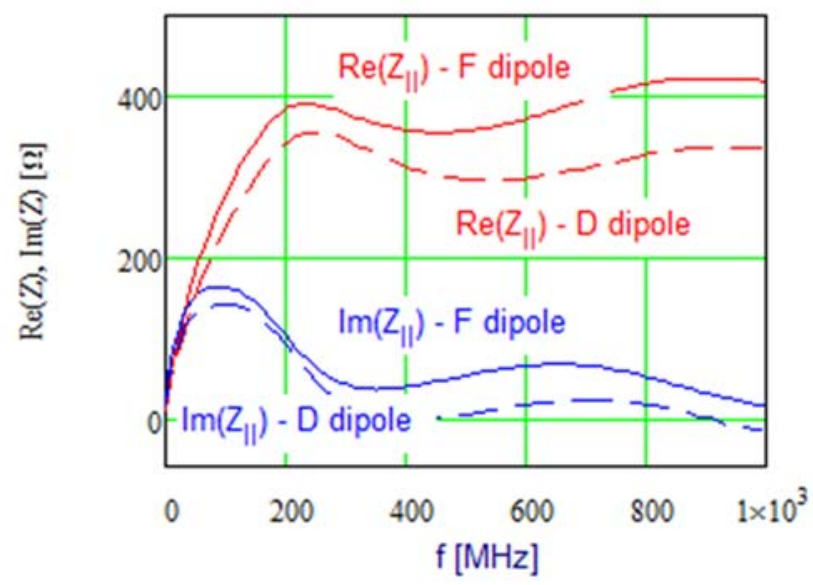

Figure 2.67: The dependences of longitudinal impedance on frequency computed with Eq. (2.10) for the Booster $\mathrm{F}$ and $\mathrm{D}$ dipoles for parameters presented in Table 2.18.

F magnet impedance dotted trace $350 \Omega \| .5 \mathrm{uH}$

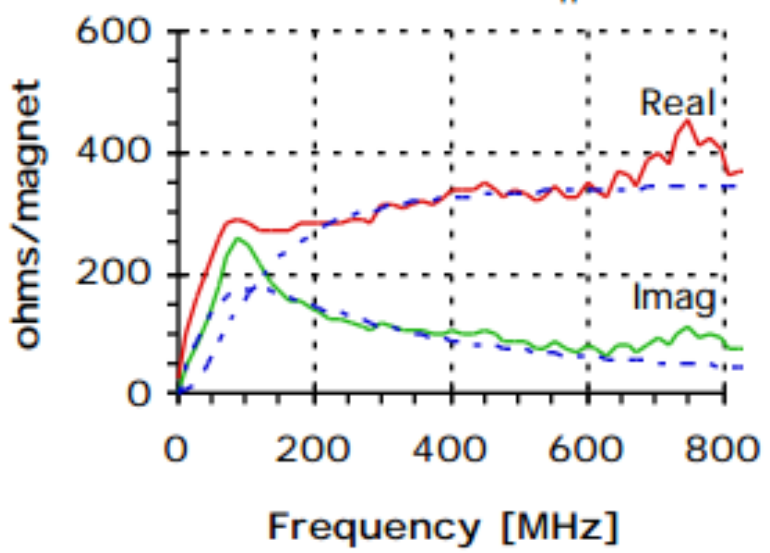

D magnet impedance

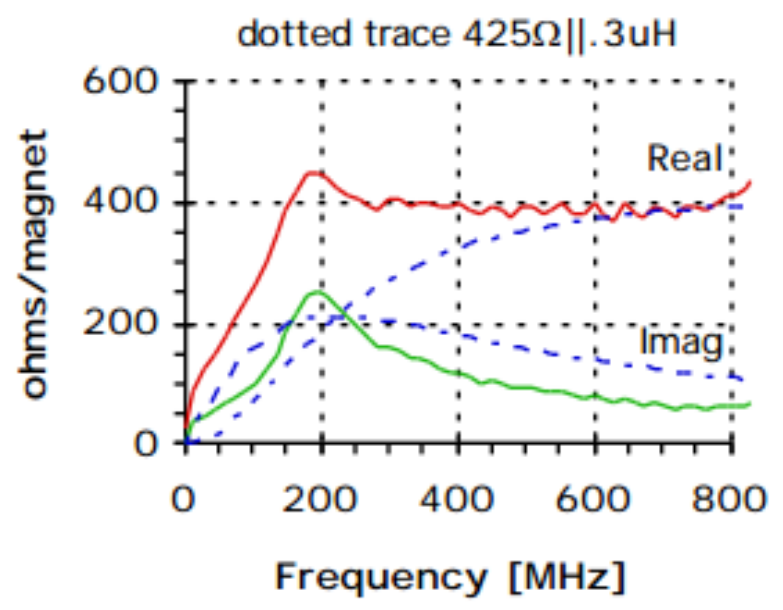

Figure 2.68: Dependence of longitudinal impedance of Booster dipole on the frequency measured with the stretched wire method for $F$ and $D$ dipoles [34].

The beam-based measurements of the effective Booster impedance have been based on the measurements of the accelerating phase shift with beam current [35]. To achieve this we acquired row analog signals coming from the resistive wall monitor (measuring the beam current), the RF sum and the horizontal BPM used in the LLRF for measurements of momentum offset. The sampling rate required to digitize the longitudinal density distribution for each bunch is quite high $\left(\geq 2 \cdot 10^{10} \mathrm{~s}^{-1}\right)$. If the measurements of the entire accelerating cycle would be carried out it would result in $\sim 6.6 \cdot 10^{8}$ samples per channel. This volume of data significantly exceeds memory of the scope used for measurements. Therefore, the data was acquired near injection and around transition crossing. This data was sufficient to reconstruct the beam dynamics for the entire accelerating cycle. The data analysis allowed us to perform calibration for the amplitude of RF voltage near transition to better 
than $1 \%$ and relative timing between RF and beam signals to better than $1 \mathrm{deg}$. [36]. The measured shift of accelerating phase with beam intensity is presented in Figure 2.69. Together with the RF voltage calibration it was employed to finalize the Booster impedance value used in the beam acceleration simulations described in the following section.

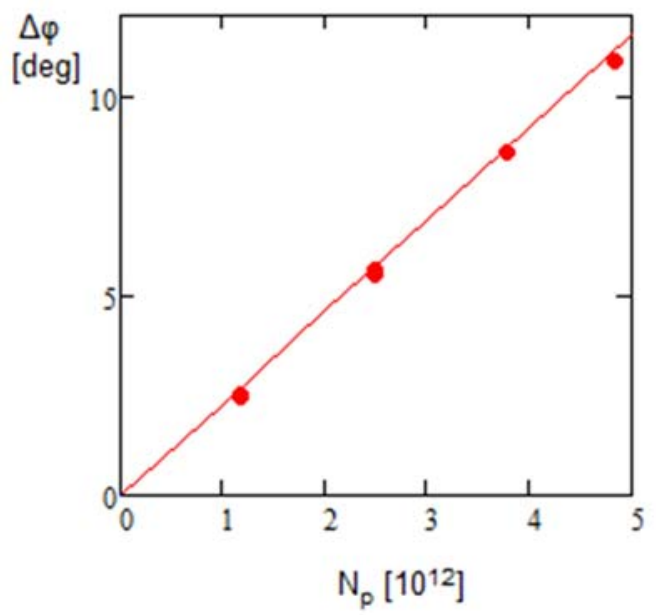

Figure 2.69: The dependence of accelerating phase on the number of particles in the beam shortly before transition; 82 bunches fill 84 RF buckets; the RF voltage is $800 \mathrm{kV}$ and the accelerating phase for zero current is about $50 \mathrm{deg}$.

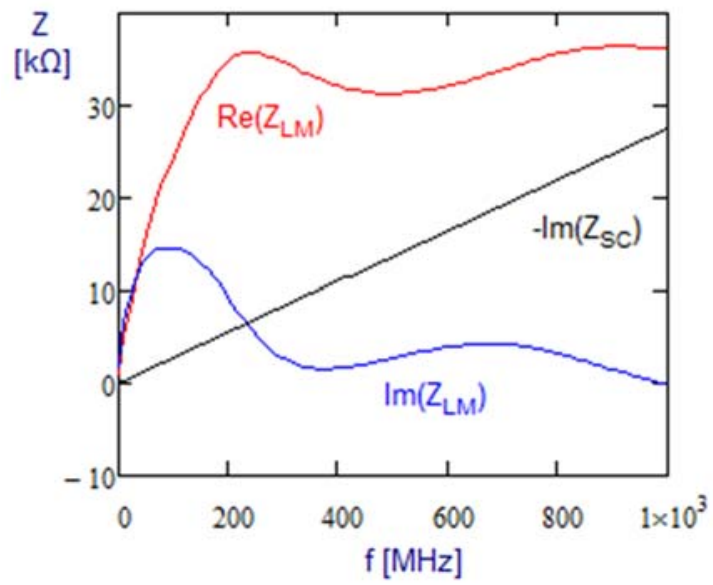

Figure 2.70: The longitudinal impedances of beam space charge, $Z_{S C}$, and all Booster dipoles, $Z_{L M}$, used in simulations of the beam acceleration.

The longitudinal impedance due to bunch space charge is:

$$
Z_{\|_{s c}}(\omega) \approx-i Z_{0} \frac{\omega}{\beta \gamma^{2} \omega_{0}} \ln \left(\frac{r_{c}}{1.06 \sigma_{t r}}\right), \frac{r_{c}}{\sigma_{\perp}} \geq 2,
$$

where $\beta$ and $\gamma$ are the beam relativistic factors, $\omega_{0}$ is the revolution frequency, $r_{c}$ is the vacuum chamber radius and $\sigma_{\perp}$ is the rms transverse beam size. The space charge impedance diminishes fast with beam acceleration. However, its contribution is still essential at the transition crossing. Figure 2.70 compares the space charge impedance and the total resistive wall impedance of all Booster dipoles. Other sources of the impedance are small and can be safely omitted in the described below simulations of beam acceleration. 


\subsubsection{Transition Crossing}

The software used for simulation was benchmarked on the measurements of beam acceleration in the Booster [36, 37]. The measurements resulted in dependences on time for the RF voltage, the accelerating phase, the momentum offset and rms bunch length near transition crossing. The actual transition crossing time and the second order slip-factor ${ }^{8}$ were not known to sufficient accuracy and were used as free parameters. An adjustment of these two parameters yielded good agreement between simulations and measurements. Similar to the actual accelerator the dipole and quadrupole dampers were used in simulations to stabilize the beam.

The simulation for PIP-II were based on the same software, which was developed for analysis of beam acceleration in the present Booster. However, there are a few differences, which have to be taken into account:

- The injection energy is increased from 400 to $800 \mathrm{MeV}$

- The beam injection is much longer and it includes the longitudinal painting as was described in Section 2.3.2.3

- The repetition rate is increased from 15 to $20 \mathrm{~Hz}$ and

- The beam intensity is increased to $6.5 \cdot 10^{12}$.

In addition, a small Q-jump was used in simulations to reduce the emittance growth related to the transition crossing. The jump is supported by existing trim quadrupoles which currents are switched from positive to negative at the maximum rate supported by present power supplies. The peak power supply voltage of $170 \mathrm{~V}$ yields the current rate of $63.7 \mathrm{~A} / \mathrm{ms}$ and the quad strength rate of $1.58 \mathrm{kG} / \mathrm{ms}$. The simultaneous ramping for all 24 quads located in the short straights with $\beta_{x}=34 \mathrm{~m}$ results in the rate of momentum compaction change of $d \alpha / d n=7.56 \cdot 10^{-6}$ per turn. For a ramp duration of 200 turns it results in the total horizontal tune change of $\sim 0.09( \pm 0.045)$.
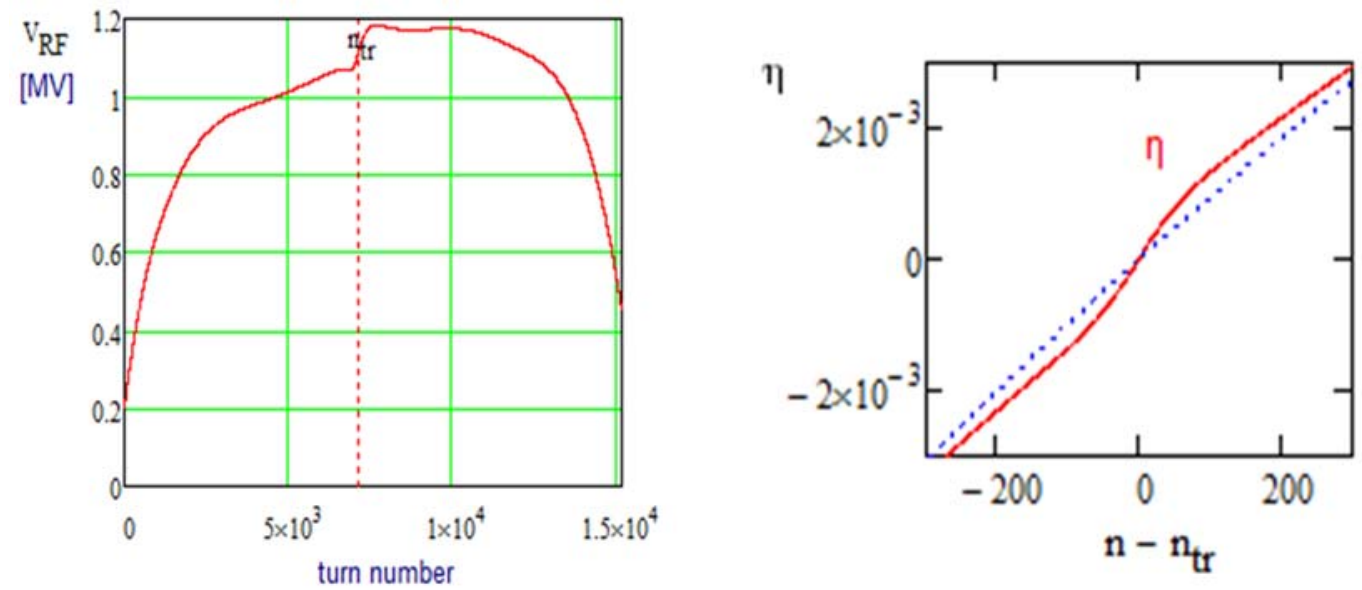

Figure 2.71: Dependence of RF voltage on the turn number (left) and dependance of slip-factor, $\eta$, on the turn number counted from transition crossing (right). The red dashed line shows the location of transition crossing. The blue dashed line presents the slip-factor in the absence of Q-jump.

\footnotetext{
${ }^{8}$ Here we define the $2^{\text {nd }}$ order slip-factor, $\eta^{\prime}$, so that the longitudinal relative particle displacement after one revolution is equal to: $\Delta L / L=\left(\alpha-1 / \gamma^{2}\right) \Delta p / p+\left(\alpha^{\prime}+3 \beta^{2} /\left(2 \gamma^{2}\right)\right)(\Delta p / p)^{2}+\ldots=\eta \Delta p / p+\eta^{\prime}(\Delta p / p)^{2}+\ldots, \quad \eta^{\prime}=\alpha^{\prime}+3 \beta_{t r}{ }^{2} /\left(2 \gamma_{t r}{ }^{2}\right)$, were $\beta_{t r}$ and $\gamma_{t r}$ are relativistic factors at transition, and $\alpha$ and $\alpha^{\prime}$ are the first and second order momentum compaction factors, respectively.
} 
Figure 2.71 shows the RF voltage and the slip-factor used in the described below simulations. As one can see, the peak RF voltage is significantly higher than the maximum accelerating rate of 738 $\mathrm{kV} /$ turn achieved in the middle of accelerating cycle (see Figure 2.61). This large voltage is required for reduction of the longitudinal emittance growth related to the transition crossing. It is more important after transition where it suppresses the growth of tails. Therefore, the RF voltage after transition is close to its maximum of 1.2 MV. Using a Q-jump, as shown in the right pane of Figure 2.71 , increases the rate of slip-factor growth by 1.8 times and results in an additional reduction of the emittance growth related to the transition crossing.
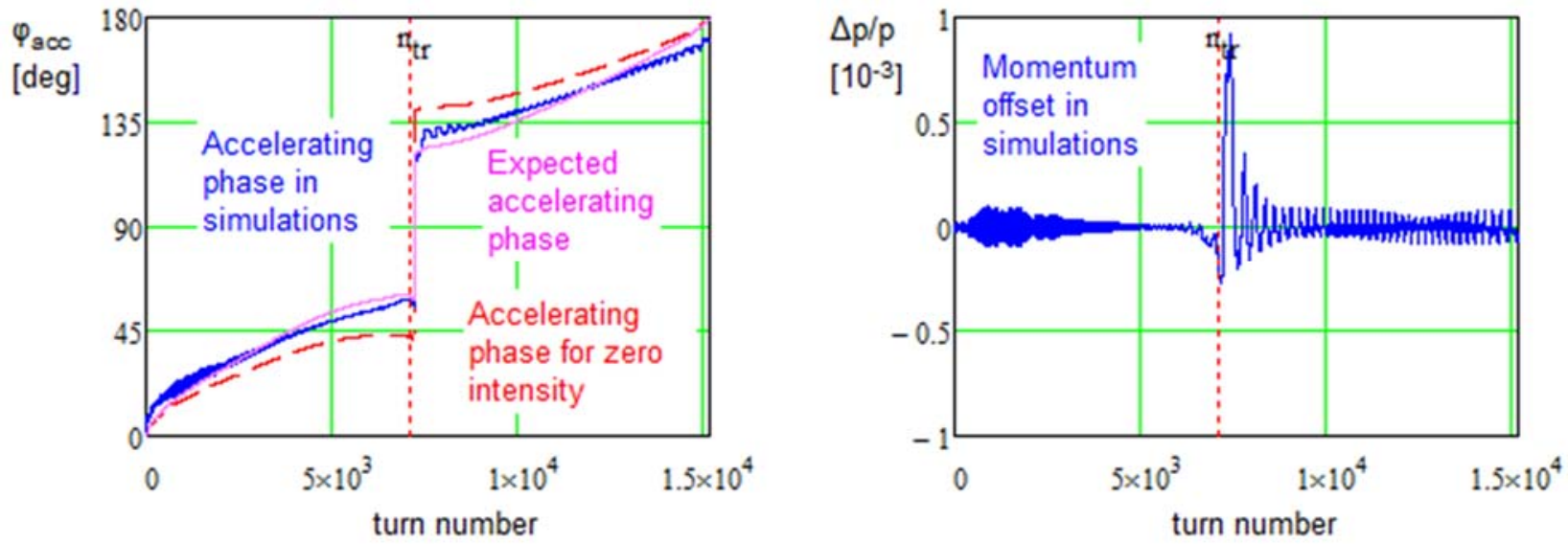

Figure 2.72: Dependence of accelerating phase (left) and the average momentum offset (right) on the turn number.

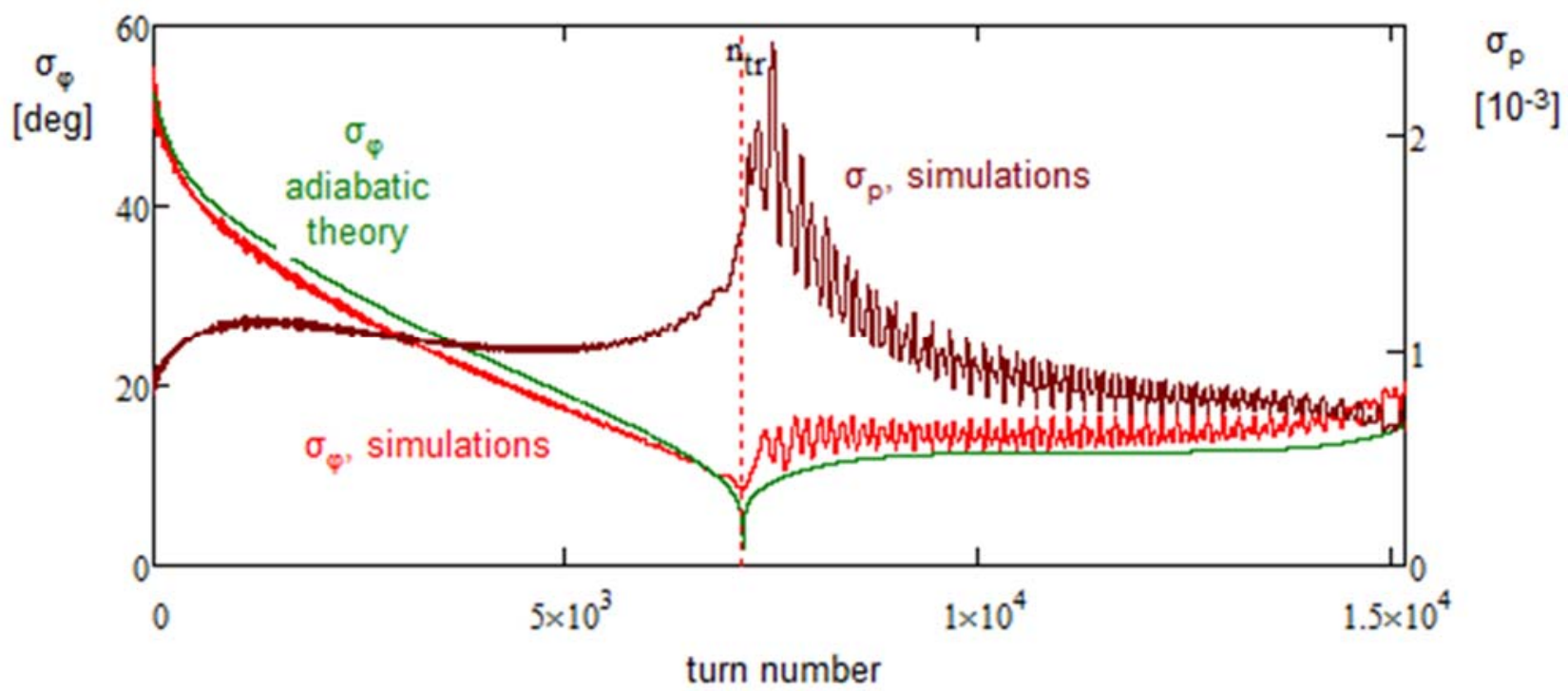

Figure 2.73: Dependence of $r m s$ bunch length, $\sigma_{\phi}$, and $r m s$ momentum spread, $\sigma_{p}$, on the turn number.

Figure 2.72 shows the dependence of accelerating phase on the turn number. The red dashed line presents the accelerating phase required for acceleration of a zero intensity beam. An increase of beam intensity results in an impedance-induced beam deceleration. It shifts the accelerating phase before and after transition (see Figure 2.69) and results in a reduction of transition crossing phase jump as shown by magenta line representing the accelerating phase used in the simulations. The value of the jump was adjusted to minimize oscillations of the average momentum, as presented in 
the right pane of Figure 2.72. Finally, the blue line in the left pane presents the actual accelerating phase obtained in the simulations. As one can see the transition crossing excites oscillations of the accelerating phase and the beam average momentum. It is related to bunch length oscillations excited by transition crossing (see Figure 2.73), which subsequently introduce oscillations of impedanceinduced beam deceleration and the average momentum. These oscillations are suppressed by the feedback system built into the simulations and operating similar to the radial position feedback used in the real Booster. The jump of accelerating phase was delayed by 70 turns. This delay significantly reduces the emittance growth excited by the transition crossing.

Figure 2.73 shows the dependence of rms bunch length on the turn number. A mismatch between the adiabatic theory and simulations in the first half of the cycle (before transition) is mainly related to the focusing force non-linearity due to large bunch length. This non-linearity of focusing strength as well as the impedance-induced voltage are not accounted in adiabatic theory. However, as one can see the bunch length is smoothly changed before transition and there is no longitudinal emittance growth. Transition crossing results in almost instantaneous emittance growth. The bunch becomes quite short near transition and generates enormous voltage, which greatly distorts the bunch phase space in just few tens of turns. This effect is greatly amplified by the absence of synchrotron motion near transition. Figure 2.74 presents the bunch distributions shortly before and after transition and the corresponding impedance-induced voltage. The peak of this voltage is about $250 \mathrm{kV}$. It causes a sag of the beam energy in the bunch center yielding an unrecoverable growth of the effective emittance. The quadrupole damper used in simulations operates similarly to the quadrupole damper in the actual Booster. It prevents uncontrolled emittance growth after transition crossing.
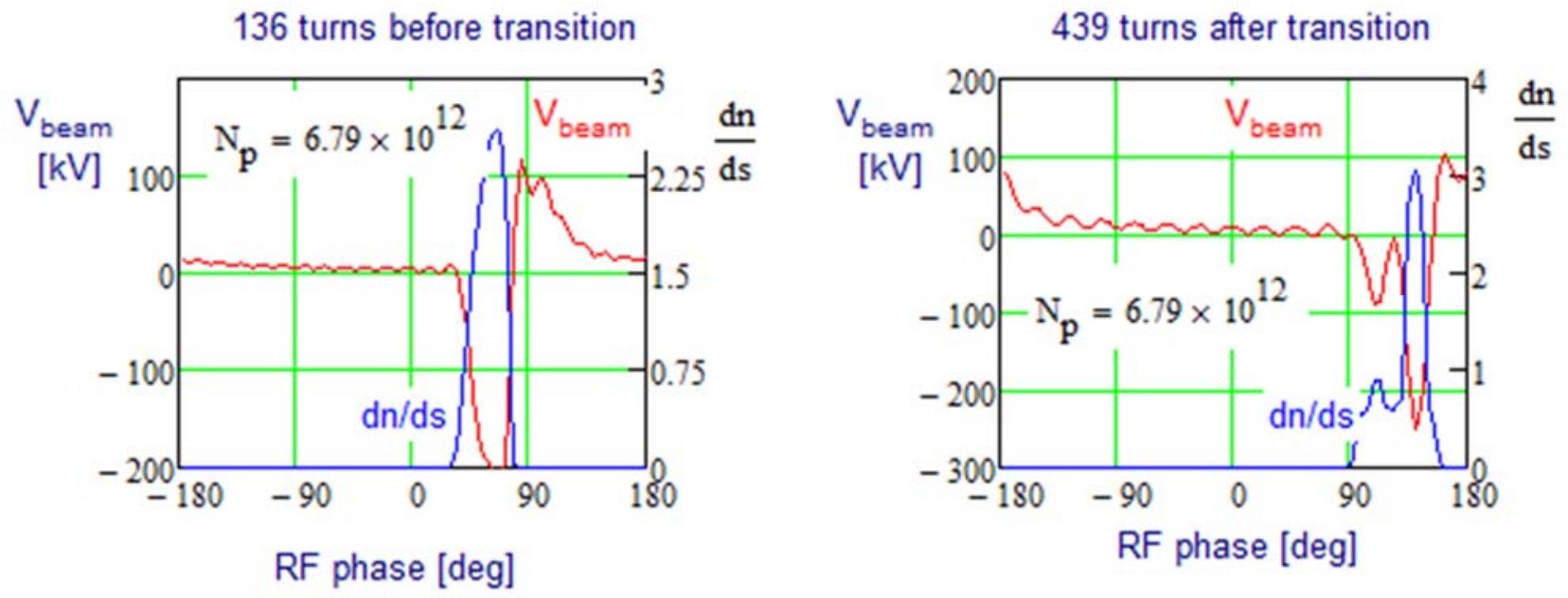

Figure 2.74: Dependences of beam distribution (blue lines) and beam indfuced voltage (red lines) on the RF phase shortly before and after transition. The phase of $90 \mathrm{deg}$. corresponds to on-crest acceleration.

The Booster simulations showed a strong effect of the second order slip-factor on the transition crossing. The second order slip-factor was adjusted to minimize the emittance growth. The optimal value for the second order momentum compaction is equal to $\alpha_{p}=-0.09\left(\eta_{\mathrm{p}}=-0.041\right)$. The value of the second order slip-factor depends on the tune chromaticity; and its value at transition is controlled by the timing of the sextupole polarity flip required to support transverse beam stability before and after transition. Figure 2.75 presents dependence of the second order momentum compaction on the tune chromaticity computed for present Booster optics near transition. One can see that the second order slip-factor can be varied in the required range. It depends linearly on the horizontal chromaticity and is weakly affected by the vertical one. 


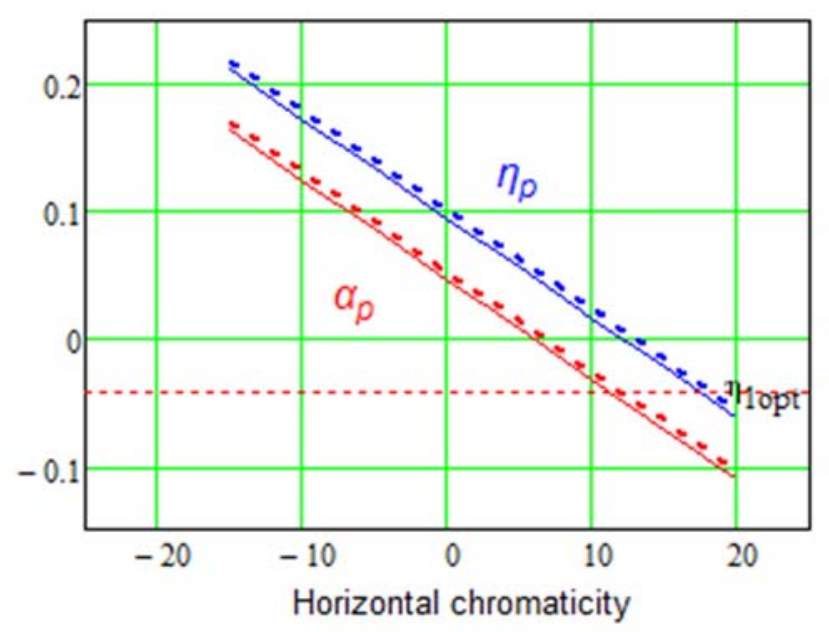

Figure 2.75: Dependance of the second order momentum compaction, $\alpha_{p}$, and slip-factor, $\eta_{p}$, on tune chromaticity computed for present Booster optics near transition; solid lines $-\xi_{v}=0$, dashed lines $\xi_{v}=4$. Dashed horizontal line shows the optimal slip-factor, $\eta_{10 p t}=-0.041$.

An increase of particle intensity greatly amplifies the Booster sensitivity to errors. In particular, the required accuracy for the second order slip-factor should be within \pm 0.02 , and the accuracy for timing of the accelerating phase jump needs to be within \pm 5 turns. The already achieved accuracies of these parameters are close to the above requirements, and their achievement for PIP-II should not present an outstanding problem. Note that presently there is no reliable procedure to determine the transition crossing time and the second order slip-factor at the transition with the required accuracy. Therefore, detailed optics simulations are expected to be helpful in resolving this issue. As presently, the final word will be with empirical adjustments. Note that in the above simulations we did not account for the voltage induced by the beam in the accelerating cavities. Presently, it results in significant variations of accelerating voltage near transition. However, the simulations for the existing Booster show that these details do not play a significant role in the emittance growth and therefore these voltage variations were not accounted. The PIP-II beam intensity increase will result in larger beam induced voltage and, consequently, some improvements in the local cavity feedbacks will be required to suppress these voltage variations. A usage of adaptive feedforward system could greatly simplify requirements to these feedbacks.

Figure 2.76 presents the dependence of effective beam emittance on the turn number. The emittance is defined as $\varepsilon_{L 95 \%}=6 \pi \sqrt{\sigma_{p}{ }^{2} \sigma_{s}{ }^{2}-\langle s \Delta p\rangle^{2}}$, which corresponds to the definition of $95 \%$ emittance for a Gaussian beam. Here $\sigma_{p}$ is the rms momentum spread, $\sigma_{s}$ is the rms bunch length, and $\langle s \Delta p\rangle$ is the corresponding second order moment. One can see almost instant emittance growth after transition crossing. A reduction of the emittance after the jump is related to an imperfection of this definition which does not take into account non-linear distortions of the phase space. However, the emittance growth at the cycle end is real and it is increased if the RF voltage in the second half of the accelerating cycle is reduced. The left pane in Figure 2.77 shows the particle phase space at the end of acceleration. As one can see, the majority of particles are located inside the required phase space ellipse with $0.1 \mathrm{eV} \cdot \mathrm{s}$ area. Note that this ellipse is shifted from the coordinate's origin. It is related to the impedance-induced beam deceleration and residual synchrotron oscillations. The ellipse parameters are shown in the figure. The right pane shows the corresponding particle distribution over single particle emittance (ascribed phase space) and its integral showing the 
percentage of particles located in a given phase space. As one can see about $98 \%$ of particles are located in the target phase space of $0.1 \mathrm{eV} \cdot \mathrm{s}$.

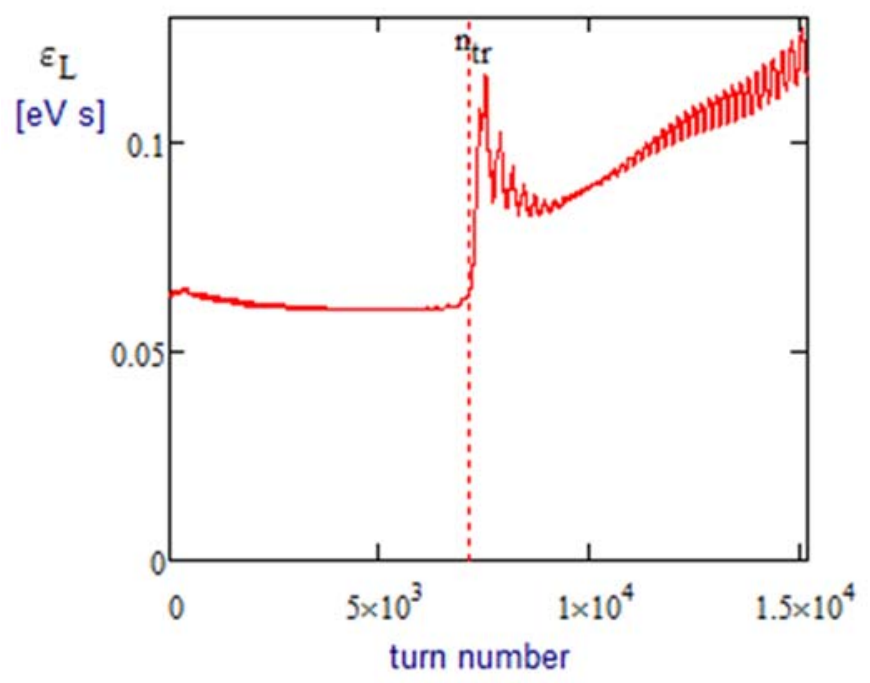

Figure 2.76: Dependence of effective longitudinal emittance on the turn number.
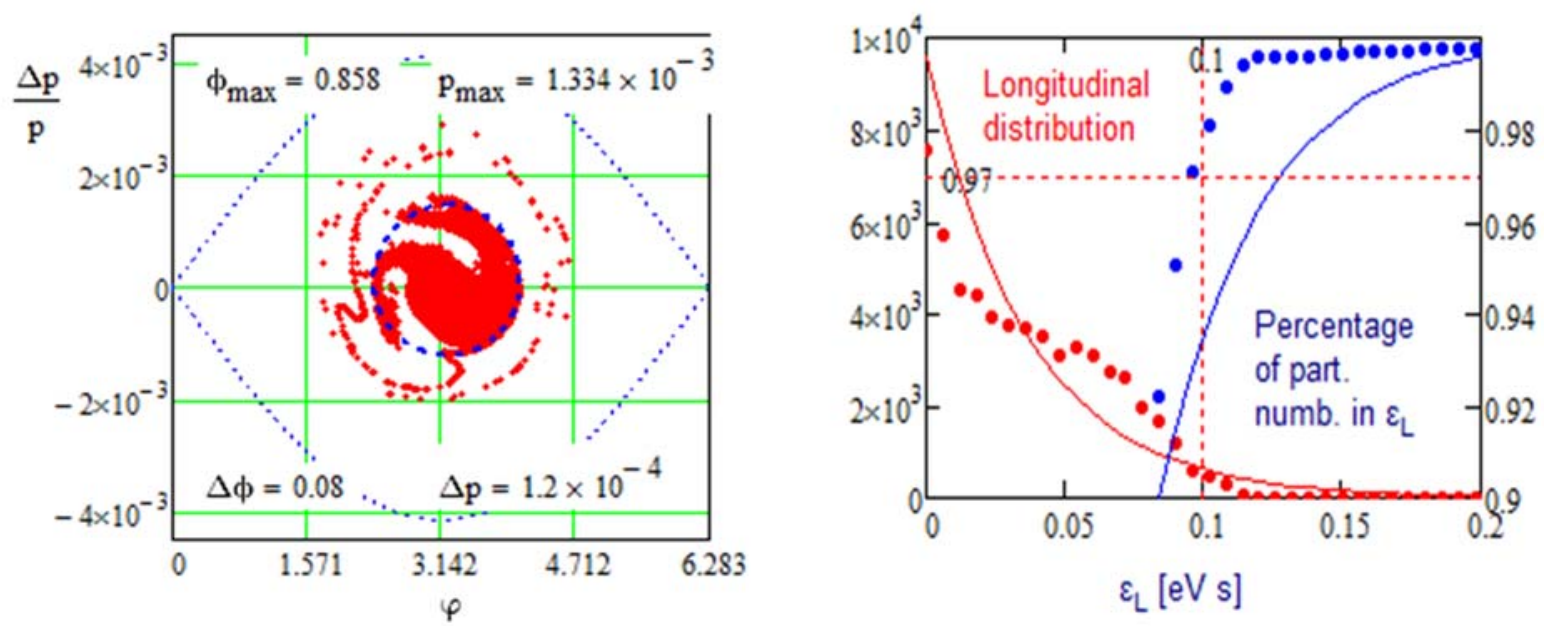

Figure 2.77: The phase space of at the acceleration end (left) and corresponding longitudinal distribution over the single particle emittance and its integral. 50,000 particles were used in this simulation. Dashed lines in the left pane show the boundaries of RF bucket and the target emittance of $0.1 \mathrm{eV} \cdot \mathrm{s}$.

\subsubsection{Modifications to the Booster Magnet System Required for $20 \mathrm{~Hz}$ Operation}

The present system has 96 magnets in a 24 cell arrangement (see Figure 2.78). These are driven by four power supplies that are the MR (Main Ring) style, $720 \mathrm{~Hz}$ update rate SCRs (SiliconControlled Rectifier). Their regulation is based on magnetic field measurements in a reference magnet with B-dot coil and transductor electronics. A sinusoidal drive signal excites the resonant system with a quality factor of about 40 for $15 \mathrm{~Hz}$ operation. Corrections for losses and line voltage variations are done by a card in a VXI crate. Regulation is good to about a part in 4000 .

The conversion of GMPS (Gradient Magnet Power Supply) controls from 15 to $20 \mathrm{~Hz}$ does not 
look difficult. The system was designed to run at $10 \mathrm{GeV}$ and therefore it is capable to operate at higher voltage and power compared to the present $8 \mathrm{GeV}, 15 \mathrm{~Hz}$ operation.

To verify that the Booster dipoles can operate at $20 \mathrm{~Hz}$ an experimental test was carried out [38]. Measurements were performed on both a Booster gradient magnet and a Booster choke with the intent to compare the $15 \mathrm{~Hz}$ losses with the $20 \mathrm{~Hz}$ losses for the proposed Booster upgrade. The analysis carried out after the measurements suggests that running the Booster at $20 \mathrm{~Hz}$ with magnet current ramping in the same range as for the present operation will require about $3.9 \%$ more power. To increase the resonant frequency the resonant capacitor at each "Girder" must decrease from $\sim 8.33$ $\mathrm{mF}$ to $\sim 4.69 \mathrm{mF}$. As result, the capacitor voltage will increase by about $32 \%$ with a subsequent slight increase in the rms current for the choke, magnets and capacitors. This also implies that the rms current per $\mu \mathrm{F}$ will increase as well. The girder drive voltage will increase by about $9.2 \mathrm{~V}(\mathrm{p}-\mathrm{p})$. The present magnet power system runs on 4 power supplies but can operate with only 3 supplies. Booster at $20 \mathrm{~Hz}$ would require all 4 power supplies to operate.

To support effective longitudinal painting the pulse-to-pulse relative stability of injection magnetic field has to be better or about $10^{-4}$. It is almost an order of magnitude better than the present stability. Such stabilization can be achieved by direct stabilization of magnetic field ramp or using field correction with dipole correctors, which are controlled by field measurement in a reference dipole.

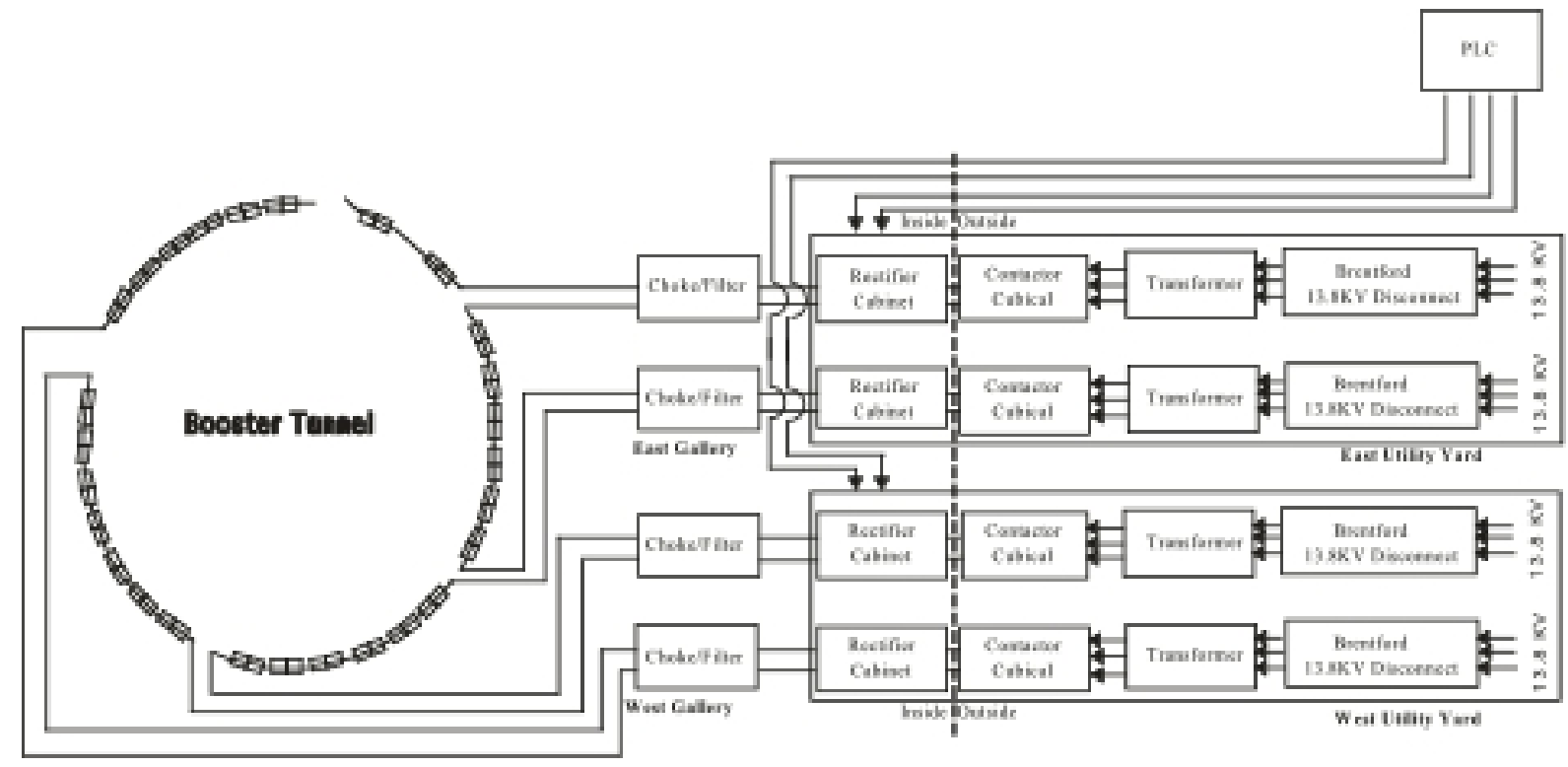

Figure 2.78: Schematic of the Booster magnets power system.

\subsubsection{Transverse Beam Instabilities}

The main challenges for beam stability in the Booster are associated with instabilities at injection and transition. The longitudinal instability at transition is considered elsewhere [39] and is not discussed below. Table 2.19 provides the main beam parameters used in the instability studies presented below.

The Booster impedances are dominated by the impedances of the laminated magnets. The analytical model for these impedances is developed in Ref. [32, 40, 41] and corresponding parameters required to compute them are presented in Table 2.18.

The transverse impedances of the focusing and defocusing magnets are shown in Figures 2.79 and 2.80. The horizontal and vertical impedances of the entire Booster and the corresponding wakes are 
averaged with beta-functions. The corresponding plots for the specified average beta-functions at the magnet locations $\left(\beta_{x F}, \beta_{y F}, \beta_{x D}, \beta_{y D}\right)=(30 \mathrm{~m}, 7 \mathrm{~m}, 11 \mathrm{~m}, 18 \mathrm{~m})$ and the average beta $\bar{\beta}=11 \mathrm{~m}$ are shown in Figures 2.81 and 2.82.

Table 2.19: Beam parameters used in estimates of Booster transverse instabilities

\begin{tabular}{l|l|l}
\hline \hline & Requirement & \\
\hline Bunch population, $N$ & $8.3 \cdot 10^{10}$ & \\
\hline Longitudinal emittance, $\mathrm{rms}, \varepsilon \|=\sigma_{\mathrm{z}} \sigma \mathrm{E}$ & 4.8 & $\mathrm{meV} \cdot \mathrm{s}$ \\
\hline Maximum RF Voltage,$V$ & 0.75 & $\mathrm{MV}$ \\
\hline Maximum acceleration rate, $\dot{\gamma}$ & 0.5 & $\mathrm{~ms}^{-1}$ \\
\hline Transition gamma, $\gamma_{t}$ & 5.47 & \\
\hline \hline
\end{tabular}

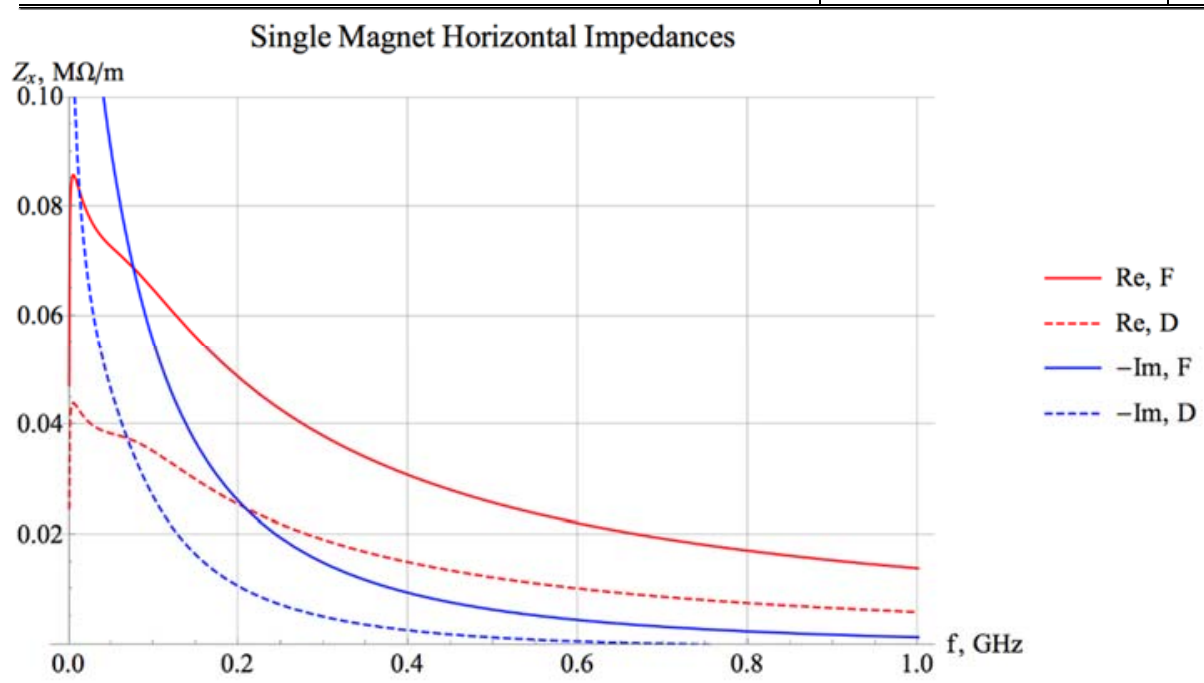

Figure 2.79: Horizontal impedances of the focusing and defocusing magnets.

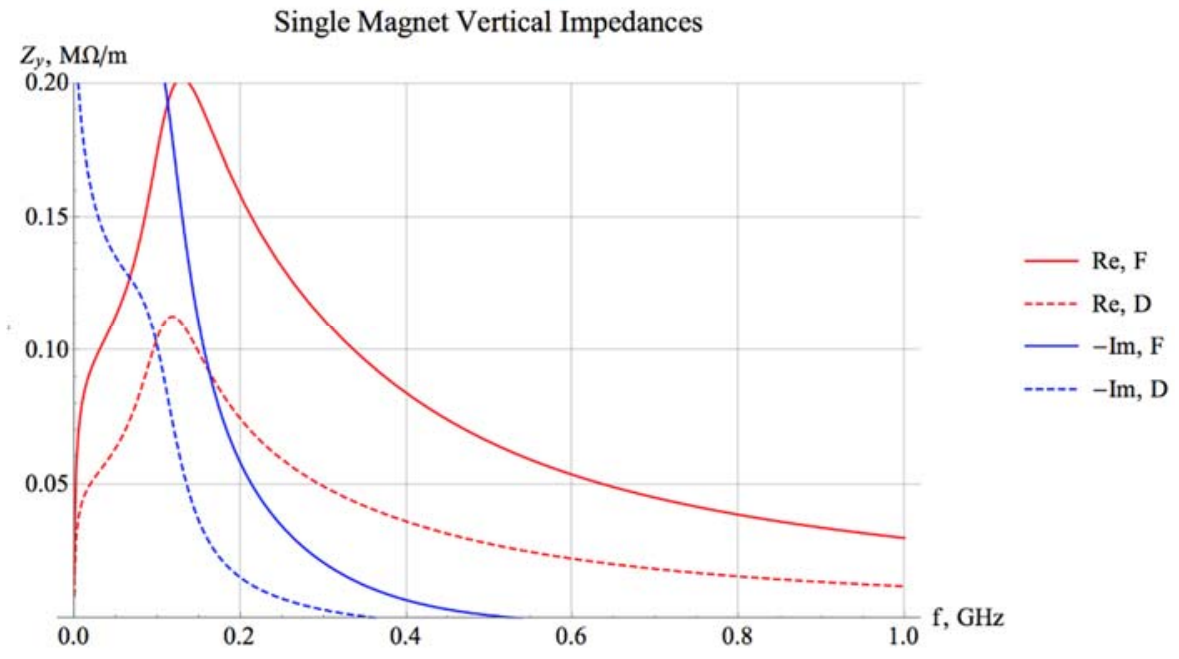

Figure 2.80: Vertical impedances of the focusing and defocusing magnets. 


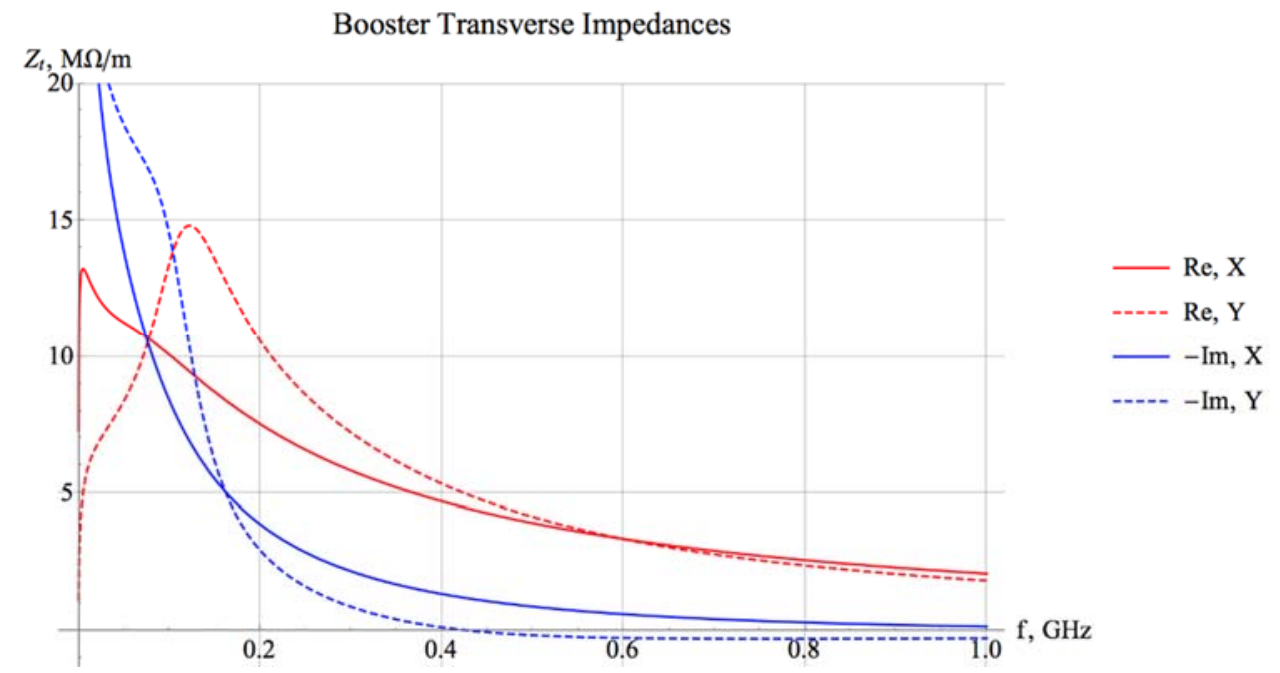

Figure 2.81: Transverse impedances of the Booster.

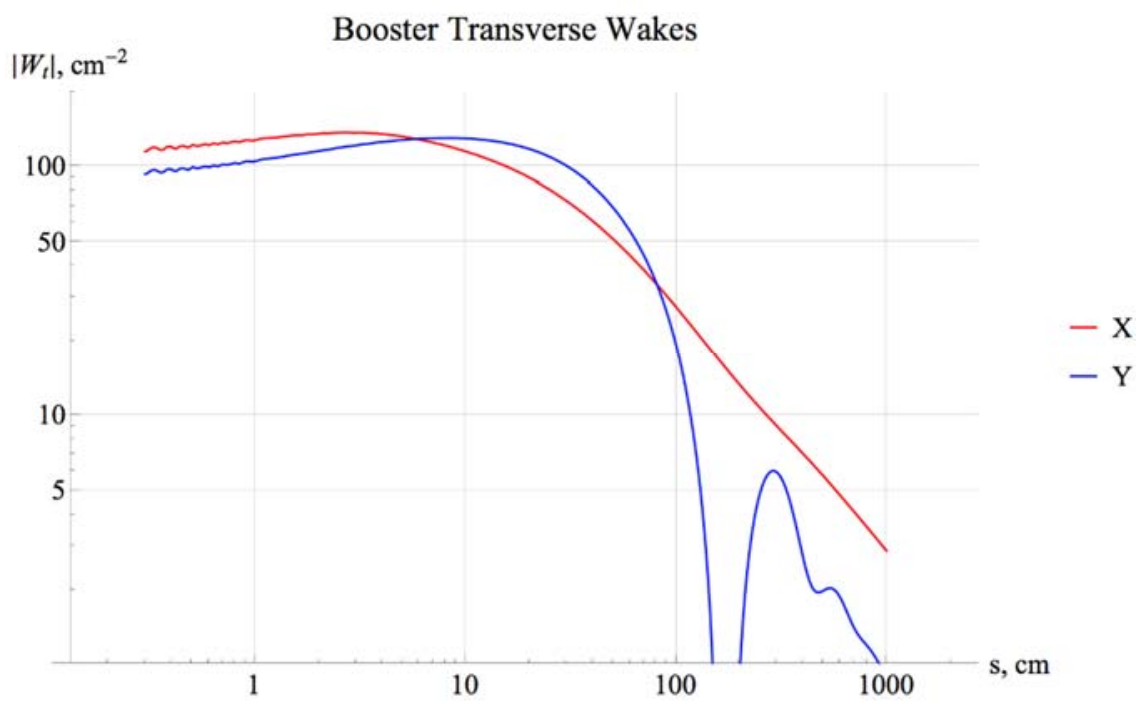

Figure 2.82: Horizontal and vertical wakes of the Booster.

For multi-bunch beams with strong space charge [42], the modes are characterized by two indexes: the single-bunch (head-tail, or intra-bunch) index $n$, and the coupled-bunch (inter-bunch) index $\mu$. At the first order of the perturbation theory over the wake function, the coherent tune shift $\Delta v_{n \mu}$ of the mode $|n, \mu\rangle$ is proportional to the sum of single- and coupled-bunch diagonal matrix elements of the wake function $[42,43]$ :

$$
\begin{aligned}
& \Delta v_{n \mu}=-\frac{N r_{p} \bar{\beta}\left(\hat{W}_{n}^{\mathrm{SB}}+\hat{W}_{n \mu}^{\mathrm{CB}}\right)}{4 \pi \beta^{2} \gamma} \equiv \Delta v_{n \mu}^{\mathrm{SB}}+\Delta v_{n \mu}^{\mathrm{CB}} ; \\
& \hat{W}_{n}^{\mathrm{SB}}=\iint d s d s^{\prime} \bar{W}\left(s-s^{\prime}\right) \exp \left(i \zeta\left(s-s^{\prime}\right)\right) \rho(s) \rho\left(s^{\prime}\right) y_{n}(s) y_{n}\left(s^{\prime}\right) ; \\
& \hat{W}_{n \mu}^{\mathrm{CB}}=W_{\mu}^{\mathrm{CB}}\left|I_{n}(\chi)\right|^{2} ; I_{n}(\chi)=\int d s \exp \left(i \chi s / \sigma_{s}\right) \rho(s) y_{n}(s) ; \\
& W_{\mu}^{\mathrm{CB}}=\sum_{k=1}^{\infty} \bar{W}(k C / M) \exp \left(i k \psi_{\mu}\right) ; \quad \psi_{\mu}=2 \pi\left(\left\{Q_{b}\right\}+\mu\right) / M ; \quad \mu=0,1, \ldots, M-1 .
\end{aligned}
$$


Here $N$ is the number of particles per bunch, $r_{p}$ is the proton classical radius, $\beta$ and $\gamma$ are the relativistic factors, $\chi=\xi \sigma_{s} /(R \eta) \equiv \omega_{\xi} \sigma_{\tau}$ is the head-tail phase (see Eq. (6.187) in Ref. [44]), $\xi$ is the chromaticity, $\eta$ is the slip-factor, $\rho(s)$ is the normalized line density, $y_{n}(s)$ is the $n$-th head-tail eigenfunction, $M$ is the number of bunches, and $\left\{Q_{b}\right\}$ is a fractional part of the betatron tune. At sufficiently small head-tail phase, the single-bunch growth rate can be neglected below the transverse mode coupling instability (TMCI) threshold, while the coupled-bunch growth time is calculated as $\left[\omega_{0} \operatorname{Im}\left(\Delta v_{00}^{C B}\right)\right]^{-1}=80 \mu$ s for the horizontal direction, in agreement with Ref. [45]. A feedback can be taken into account similarly to the coupled-bunch wake [43]; for a bunch-by-bunch damper with a gain $g$ that yields the damping rate:

$$
\operatorname{Im}\left(\Delta v_{n \mu}\right)=-g\left|I_{n}(\chi)\right|^{2}
$$

To make the description complete, the Landau damping has to be taken into account. To do that, the estimations of Landau damping suggested in Ref. [42] have to be compared with dedicated tracking simulations, e.g. with the Synergia [46]. When this is done and the full simulation scheme made complete, predictions and recommendations for various operation scenarios could be derived. An application of the bunch-by-bunch damper is known to be able to reduce the growth rate several times [47].

At transition, the strong head-tail instability is suppressed by the chromaticity, which threshold is proportional to the bunch population. Thus, an increase of the beam intensity by a factor of 1.5 compared with the current value would require similar growth of the chromaticity. 


\subsection{Recycler and Main Injector Modifications}

\subsubsection{Technical Requirements and Scope}

The performance requirements of the Main Injector/Recycler complex are summarized in Table 2.20. The Recycler has recently been reconfigured as a proton accumulation ring in support of the NOvA experiment. For PIP-II an increase in beam intensity of $50 \%$ over current operations is required. It will be accompanied by a modest (10\%) decrease in the Main Injector (MI) cycle time for $120 \mathrm{GeV}$ operation. The primary requirement on the Recycler is to slip-stack twelve Booster batches and to deliver this accumulated beam to the Main Injector in a single turn. In order to maintain losses at current levels the efficiency of this operation has to be at least $97 \%$.

Table 2.20: Main Injector/Recycler requirements for 0.9-1.2MW operations at 60-120GeV

\begin{tabular}{l|c|c}
\hline \hline Performance Parameter & Requirement & \\
\hline Particle Species & Protons & \\
\hline Injection Beam Energy (kinetic) & 8.0 & $\mathrm{GeV}$ \\
\hline Extracted Beam Energy (kinetic) & $60-120^{*}$ & $\mathrm{GeV}$ \\
\hline Protons per Pulse (injected) & $7.7 \times 10^{13}$ & \\
\hline Protons per Pulse (extracted) & $7.45 \times 10^{13}$ & \\
\hline Slip-stacking Efficiency & 97 & $\%$ \\
\hline Controlled 8 GeV losses to Abort & 0.8 & $\%$ \\
\hline Controlled 8 GeV losses to Collimators & 1.7 & $\%$ \\
\hline Uncontrolled 8 GeV losses & 0.5 & $\%$ \\
\hline Transition Losses & 0.2 & $\%$ \\
\hline Cycle Time & $0.7-1.2$ & $\mathrm{~s}$ \\
\hline Beam Power & $1.0-1.2$ & $\mathrm{MW}$ \\
\hline Beam Emittance (95\%, normalized) & 20 & $\mathrm{~mm}-\mathrm{mrad}$ \\
\hline Bunching Factor & 0.5 & \\
\hline Laslett Tune Shift (injection) & -0.06 & \\
\hline \hline
\end{tabular}

* The Main Injector is capable of maintaining beam power of $1.2 \mathrm{MW}$ for energies as low as $80 \mathrm{GeV}$.

In order to provide the RF power required to accelerate $7.5 \times 10^{13}$ protons three options could be considered:

1. Operate the current RF cavities with two power tubes instead of one in a push-pull configuration. This will require doubling of the number of modulators and solid-state drivers.

2. Use a new more powerful power tube, such as the EIMAC 4CW250,000B. This will require a new mounting configuration (to accommodate the much longer tube), new modulators, and upgraded power amplifier cooling.

3. Replace the entire RF system with a new one (new cavities and PAs). The advantage of this solution is that it can accelerate enough intensity to reach $2.3 \mathrm{MW}$ in the next round of Accelerator complex upgrade.

Options 1 and 2 will be considered for PIP-II in more details as they are substantially less expensive 


\section{than Option 3.}

Running the Main Injector at energies as low as $60 \mathrm{GeV}$ will require the $53 \mathrm{MHz}$ cavities in the Recycler operating in CW. The current Recycler slip stacking cavities have a high power dissipation (90 kW, 60\% duty factor) because of their low $R / Q$ and cannot operate in $\mathrm{CW}$. New slip stacking cavities with higher $R / Q$ and active beam loading compensation will be needed. Since the Booster will be running at $20 \mathrm{~Hz}$ instead of the current $15 \mathrm{~Hz}$ the beam separation during slip stacking will be larger $(1680 \mathrm{~Hz}$ instead of $1260 \mathrm{~Hz}$ ). That will require the slip stacking voltage of $140 \mathrm{kV}$ (instead of the current $80 \mathrm{kV})$.

Transition crossing in the Main Injector with the higher beam bunch intensities and larger longitudinal emittances will require a gamma-t jump. That will require up to 8 sets of quad triplets.

\subsubsection{Slip-stacking in the Recycler}

To be ready for the next Booster injection in time, the beam separation for slip-stacking in the Recycler has to be $1680 \mathrm{~Hz}$ or $32 \mathrm{MeV}$ (one Booster batch slippage in one Booster tick). The figure of merit in slip-stacking is the parameter alpha that relates the frequency separation in synchrotron frequency units $\left(f_{s}\right)$ to the energy separation in bucket height units $\left(H_{B}\right)$ :

$$
\alpha \equiv \frac{\Delta f}{f_{s}}=2 \frac{\Delta E}{H_{B}} .
$$

For $\alpha=2$, the hypothetical independent buckets overlap 50\% in energy, and the single particle motion is chaotic everywhere within them. The case of $\alpha=4$ gives tangent boundaries for the hypothetical buckets and in the case of $\alpha=8$ there is space for a complete empty bucket between the upper and lower hypothetical buckets. In practice, we have found that a value of $\alpha$ greater than 5 is adequate. Note that for the given frequency separation further increase of $\alpha$ does not yield larger RF bucket size (area of stable motion). A plot of $\alpha$ as a function of the RF voltage for $1680 \mathrm{~Hz}$ separation is shown in Figure 2.83. From that figure we can see that an RF voltage of $140 \mathrm{kV}$ meets our requirements.

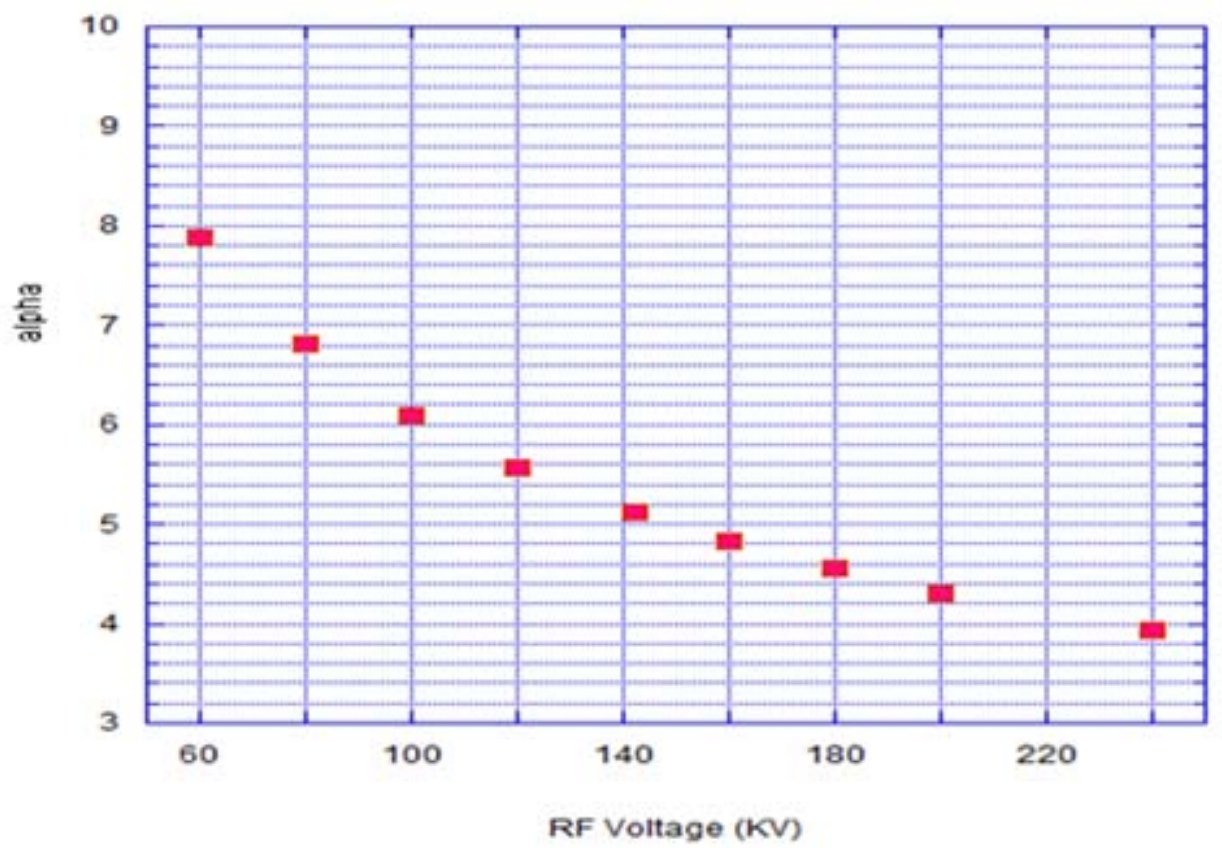

Figure 2.83: $\alpha$ vs. RF voltage for $1680 \mathrm{~Hz}$ separation. 


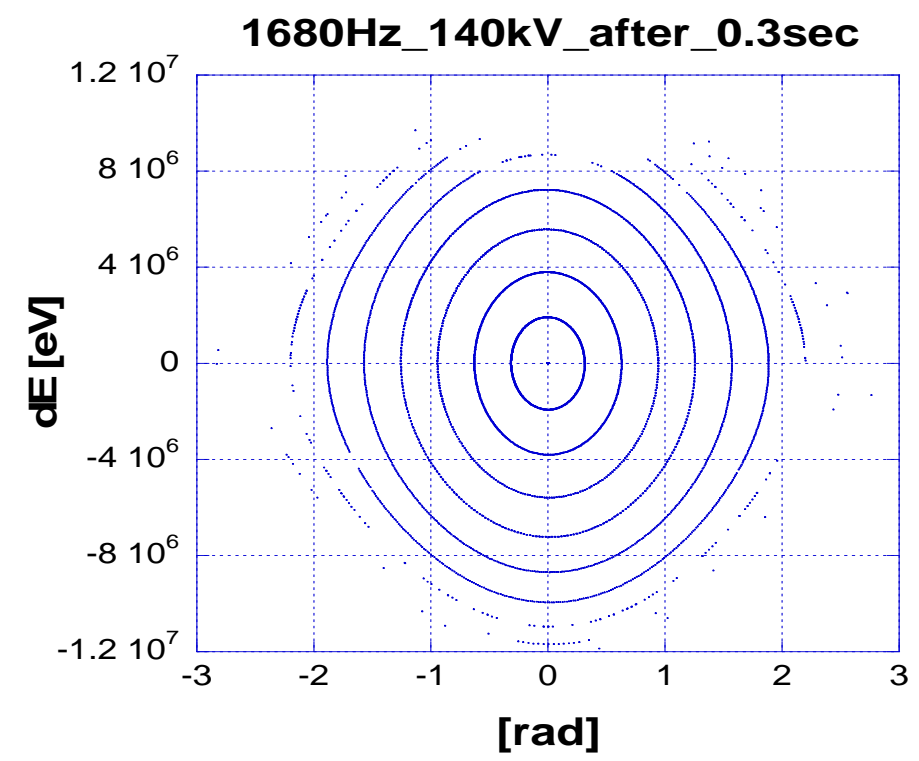

Figure 2.84: Particles on initial matching contours in a $140 \mathrm{KV}$ bucket after $300 \mathrm{~ms}$ of slip-stacking with $1680 \mathrm{~Hz}$ separation.
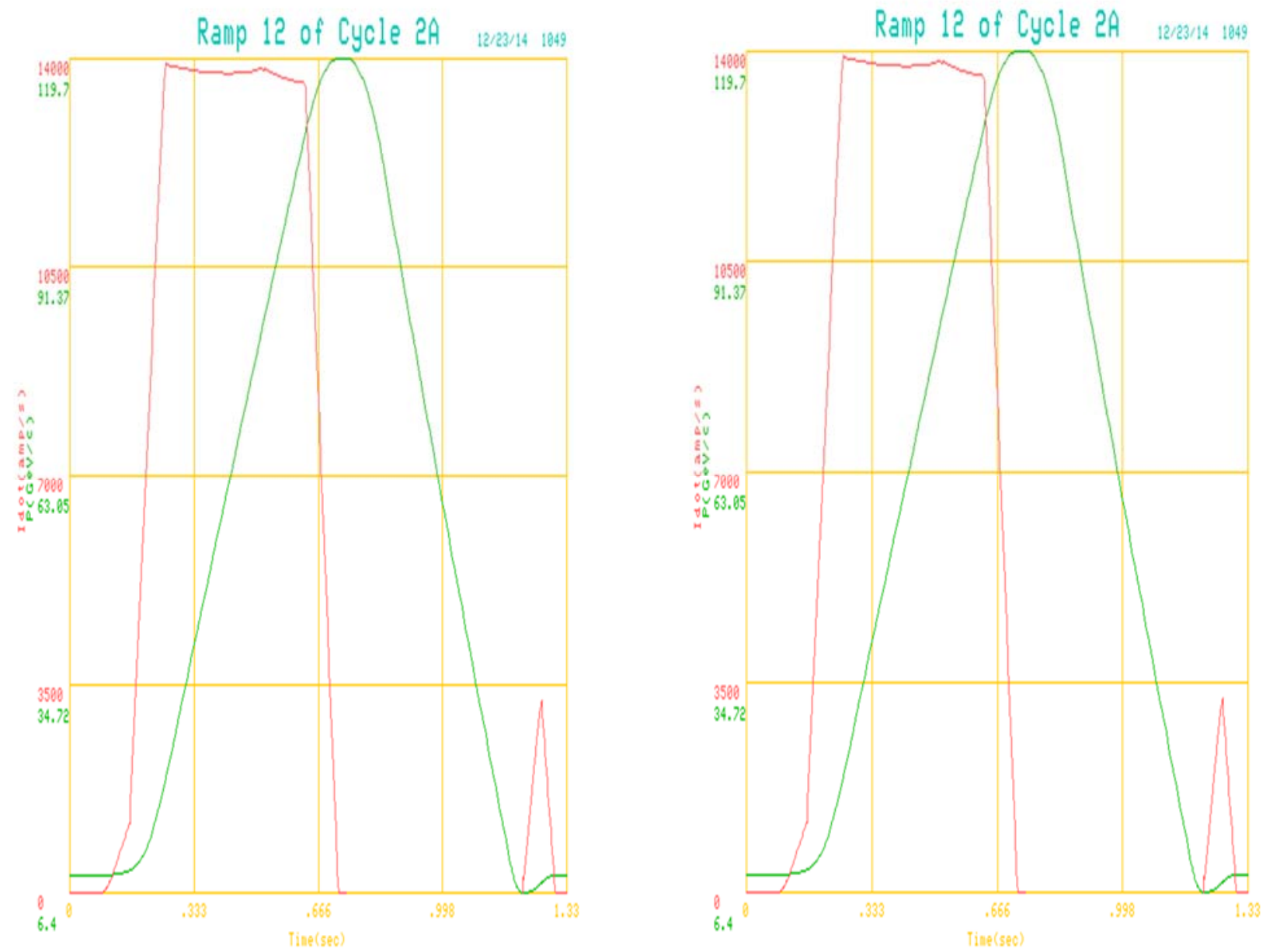

Figure 2.85: The current MI ramp (left) and the faster MI ramp (right). The momentum and the rate of dipole current change (acceleration rate) vs time are shown. 
The choice of RF voltage determines the area of longitudinal phase space where particles will survive for a sufficiently long time. Figure 2.84 shows the particles in the initial matching beam contours that survive after $300 \mathrm{msec}$. The largest beam contour with no particle loss corresponds to an emittance of $0.10 \mathrm{eV}$-sec $( \pm 4.2 \mathrm{nsec}, \pm 8.0 \mathrm{MeV})$. If we want slip-stacking with $97 \%$ efficiency, then $97 \%$ of particles from the Booster should be included in that matching contour.

\subsubsection{Acceleration in the Main Injector}

The current (NOvA) MI Acceleration cycle is $1.33 \mathrm{~s}$ long. The cycle time can be reduced to $1.2 \mathrm{~s}$ by optimizing the 8.9 and $120 \mathrm{GeV}$ dwell times and by speeding up the early parabola. Both the current MI ramp and the faster $1.2 \mathrm{sec}$ ramp are shown in Figure 2.85. The maximum acceleration rate is the same for both ramps. In the same figure the acceleration rate, Idot, vs time is also plotted. From the conversion coefficient of $57.754 \mathrm{~A} / \mathrm{GeV}$ we can see that the maximum acceleration rate is $240 \mathrm{GeV} / \mathrm{s}$. The MI RF has to provide enough voltage to support the above acceleration rate and also to provide the required bucket area. From operational experience with slip-stacked beam a bucket area of at least $2.0 \mathrm{eV} \cdot \mathrm{s}$ is required above transition to contain the beam tails and avoid losses. The total RF voltage of 4.4 MV is needed to accelerate the beam and provide the required bucket area above transition. Since the maximum operational voltage from each RF station is $240 \mathrm{kV}$, all $18 \mathrm{RF}$ stations originally installed in the MI are required. During the NOvA shutdown two spare MI RF stations were installed giving us some margin and enabling operation with up to 2 RF stations down. The RF voltage and bucket area during the current MI ramp are shown in Figure 2.86.

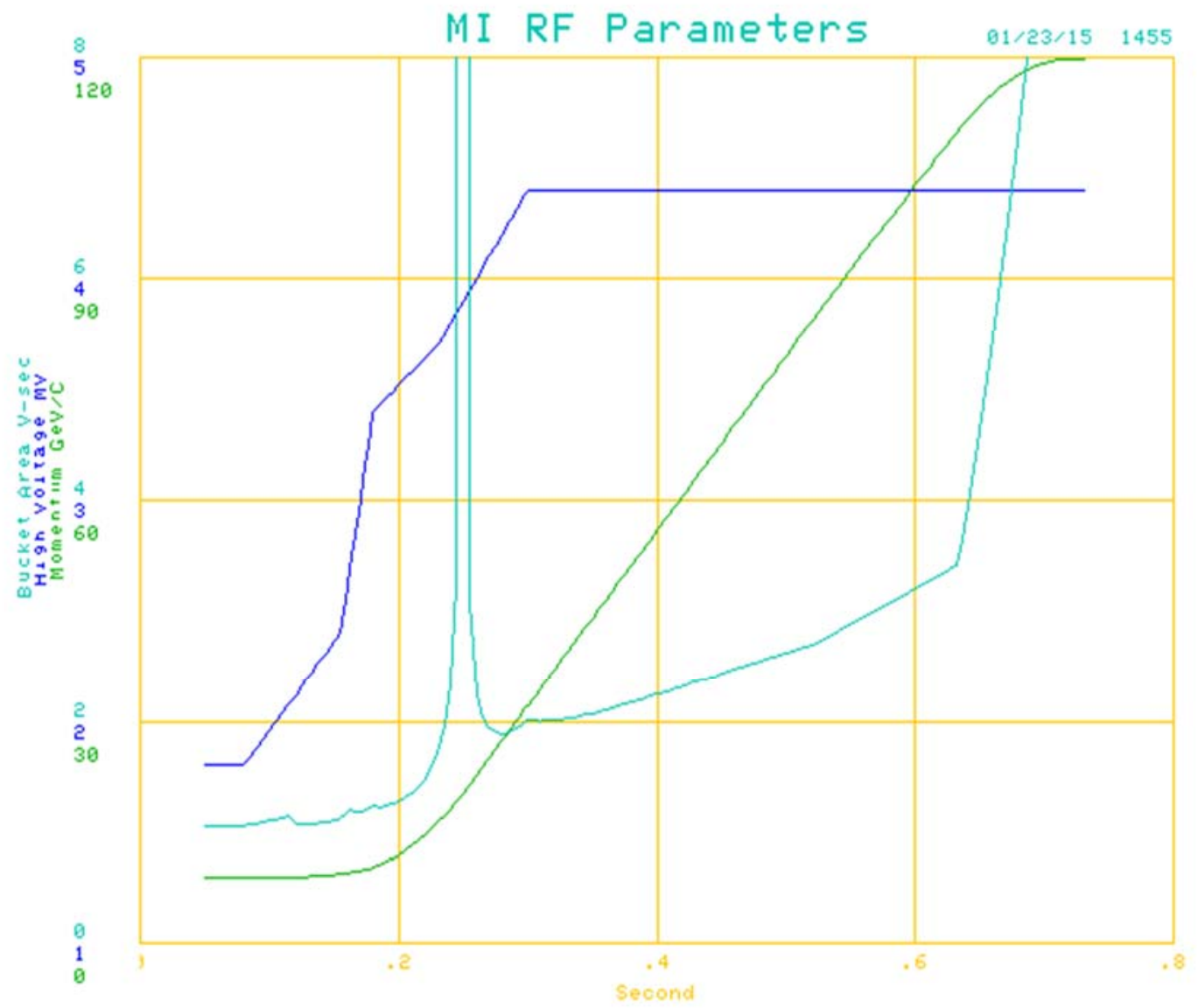

Figure 2.86: RF Voltage, beam momentum and bucket area during the MI acceleration with present cycle. 
The MI ramp can be configured for different momenta. Figure 2.87 presents the MI cycle time vs beam momentum for PIP-II operation. The cycle times have been rounded up to the nearest 1/20 s. Based on the cycle times the calculated MI beam power vs beam momenta is shown in Figure 2.88.

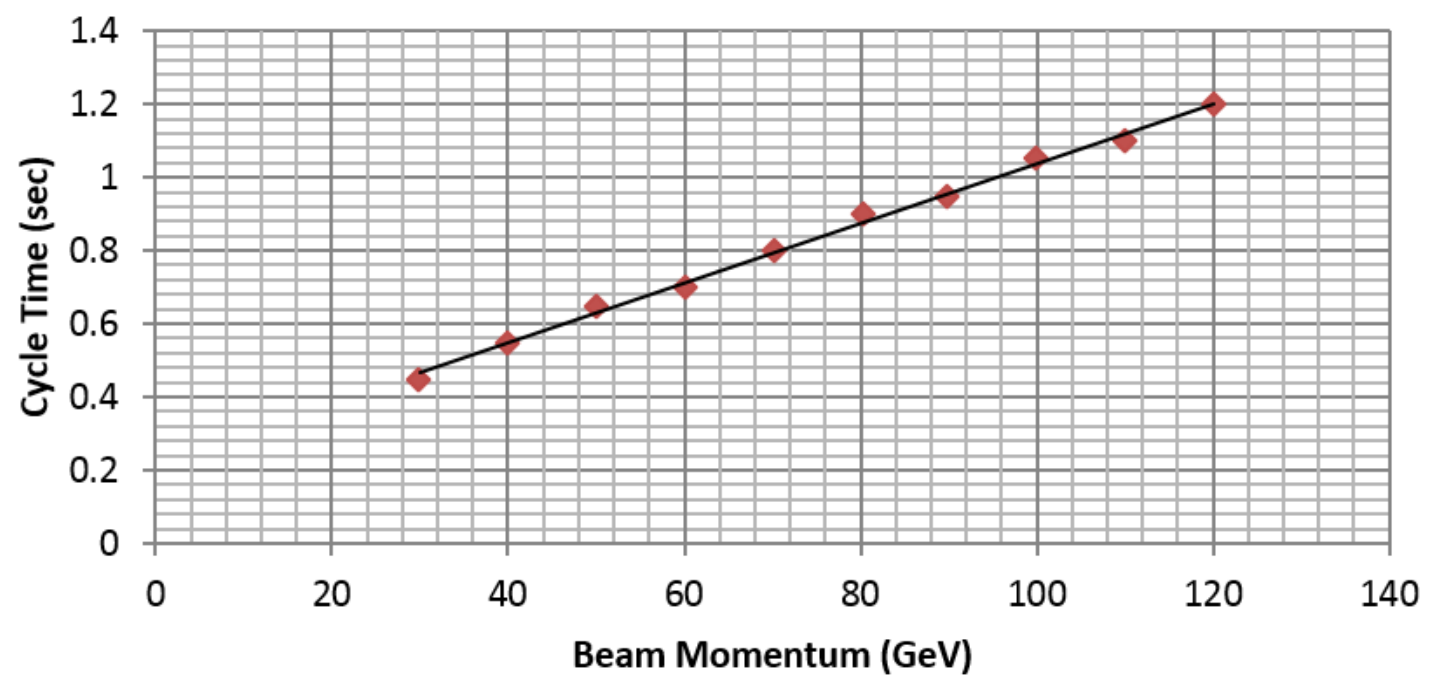

Figure 2.87: MI cycle time vs beam momenta.

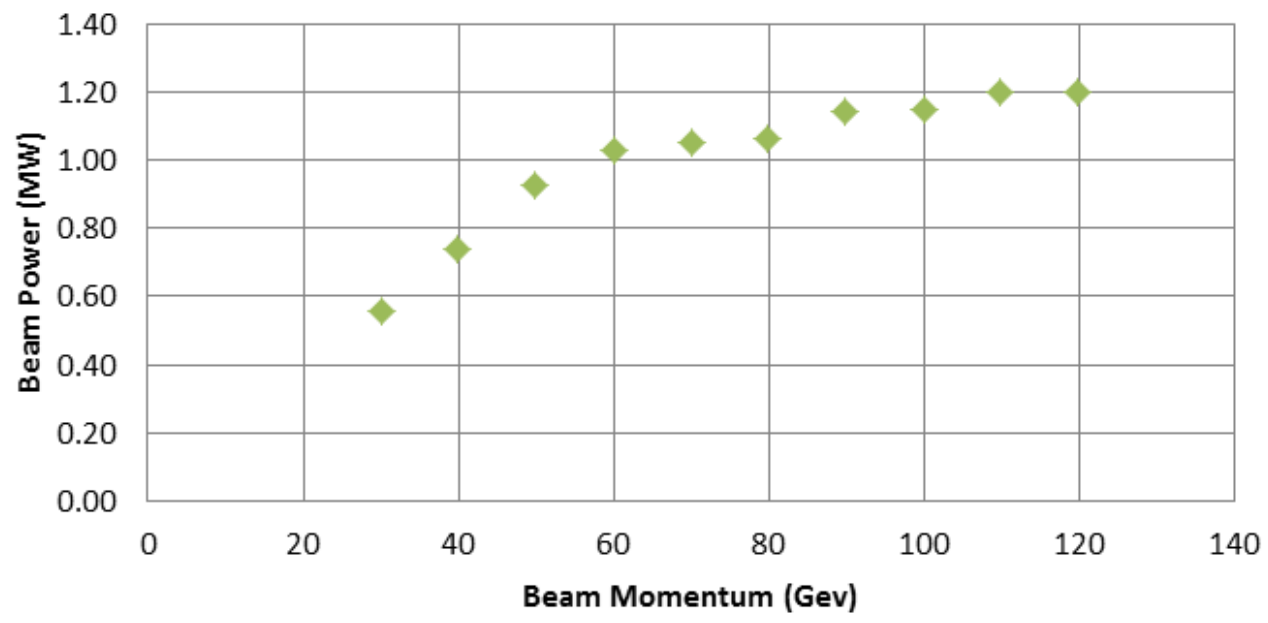

Figure 2.88: MI beam power vs beam momenta.

\subsubsection{Main Injector Transition Crossing}

MI transition crossing is dominated by non-linear effects because of the large longitudinal emittance. Since the Booster will be upgraded to run at $20 \mathrm{~Hz}$ instead of $15 \mathrm{~Hz}$ the frequency separation for slip-stacking will be increased to $1680 \mathrm{~Hz}$ from present $1260 \mathrm{~Hz}$ resulting in larger longitudinal phase space area at injection and, consequently, larger longitudinal emittance after recapture in the MI. To reduce the effects of the transition crossing a first order gamma-t jump has been considered.

For MI the non-adiabatic time $T_{c}$ which represents the time during which the longitudinal motion of the synchronous particle is not well represented by a slowly varying Hamiltonian, is around 1.5 ms. The nonlinear time $T_{n l}$ which parameterizes the Johnsen effect [48, p. 285], in which particles with different momenta cross the transition at different times, is $2.3 \mathrm{~ms}$. The nominal bipolar jump illustrated in Figure 2.89 maintains a clearance of 


$$
\left|\gamma-\gamma_{t}\right| \geq 1 \sim 2 \gamma^{\prime} T_{n l}
$$

except for about $0.5 \mathrm{~ms}$. Transition is crossed at $d\left(\gamma-\gamma_{t}\right) / d t=4000 \mathrm{~s}^{-1}$ almost sixteen times faster than without the jump.

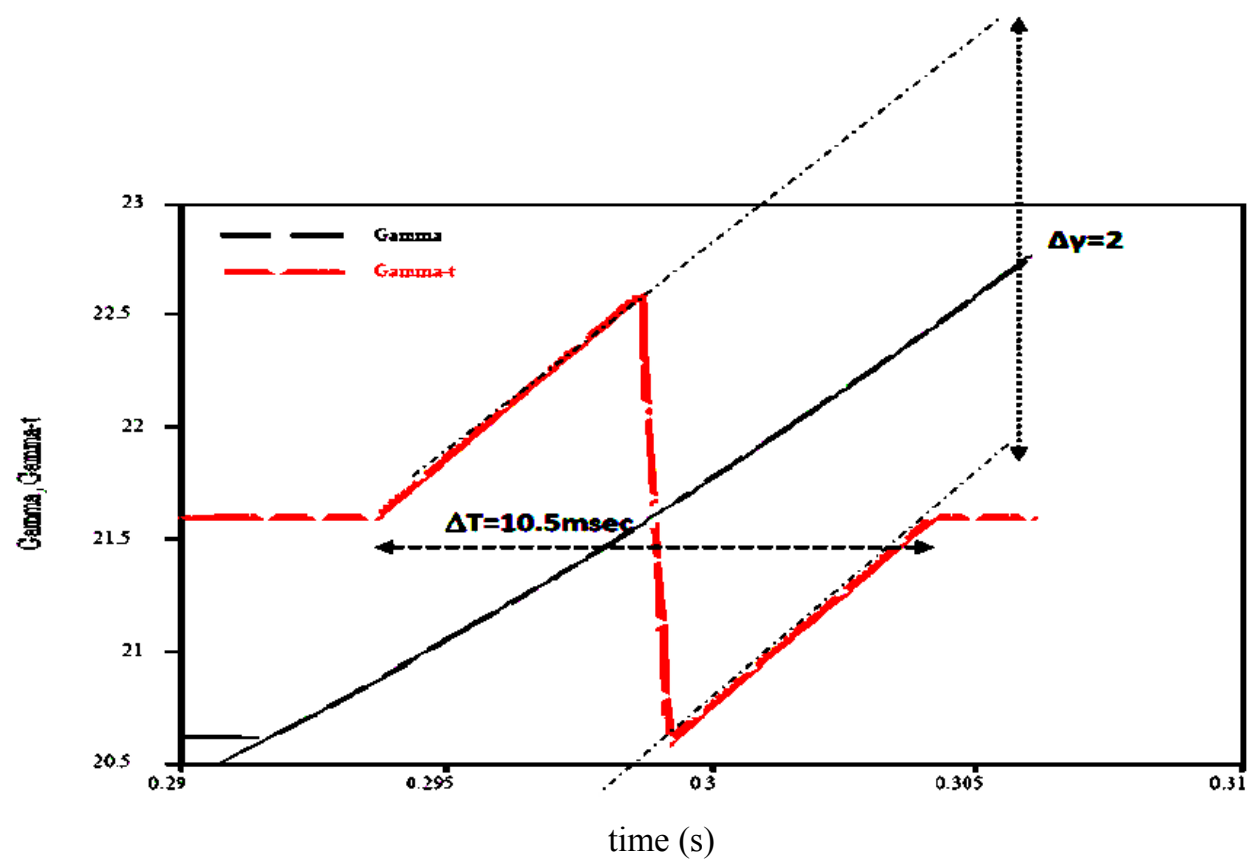

Figure 2.89: MI bipolar gamma-t jump. Transition is crossed 16 times faster with the transition jump than without.
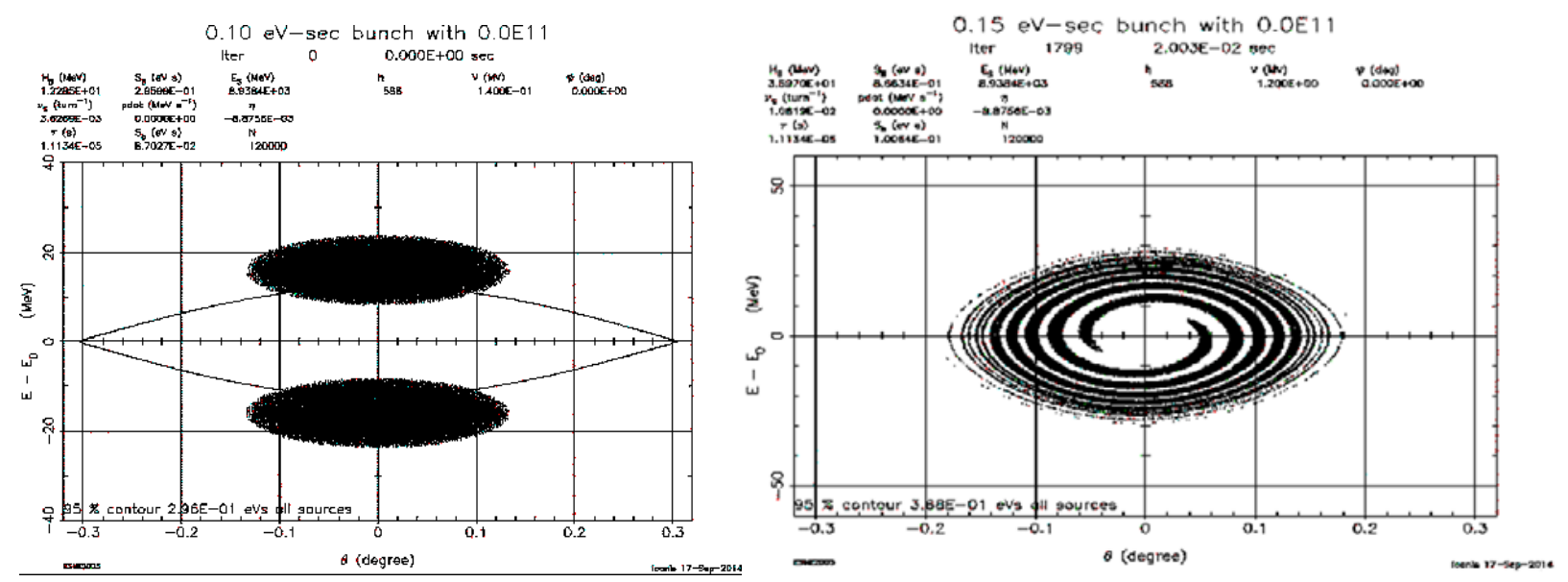

Figure 2.90: Initial condition for the ESME simulation. In the left picture two $0.1 \mathrm{eV}$-sec bunches captured in $145 \mathrm{kV}$ buckets separated by $1680 \mathrm{~Hz}$ are shown. In the right picture the resulting distribution after recapture with 1.2 $\mathrm{MV}$ in $\mathrm{Ml}$ is shown.

A series of ESME simulations were performed. They accounted for the space charge impedance, $Z / n$ and the gamma-t jump. The initial conditions are shown in Figure 2.90. The final bunch distribution after recapture in MI has a bunch area of $0.5 \mathrm{eV}-\mathrm{sec}(100 \%)$. Figure 2.91 presents the pdot and the RF voltage curve used for the MI ramp in the ESME simulations.

The beam distributions after transition with and without gamma-t jump are shown in Figures 2.92 and 2.93. From Figure 2.92 it can be seen that without a gamma-t jump the low energy tail of the 
bunch that develops after transition is similar in the cases with zero charge and full charge indicating that it is non-linear effects and no space charge that is dominating the transition crossing in MI. The low energy tail exceeds the momentum aperture of MI leading in beam scraping and beam loss. With the full gamma-t jump the low energy tail is eliminated while with half the jump the beam is contained within the MI momentum aperture as presented in Figure 2.93.
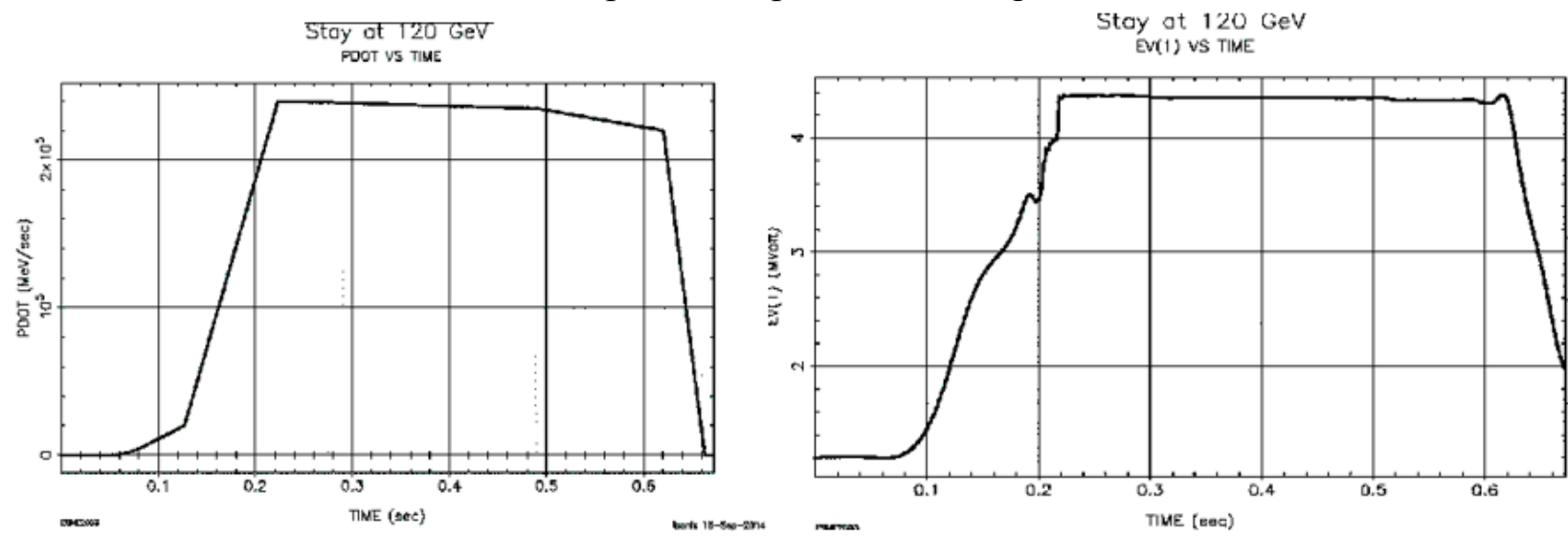

Figure 2.91: Pdot and RF voltage during the MI ramp used in ESME simulations.
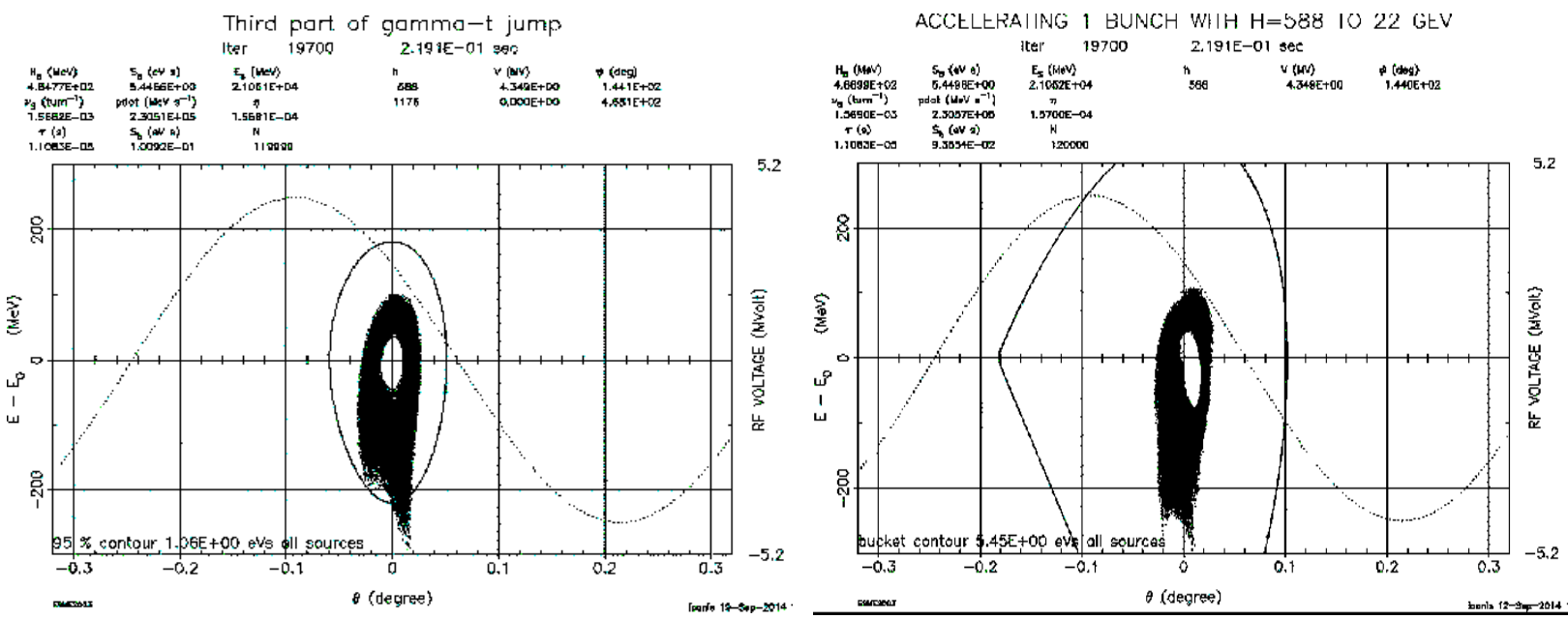

Figure 2.92: Phase space distribution after transition in MI with zero charge (left) and full charge (right).
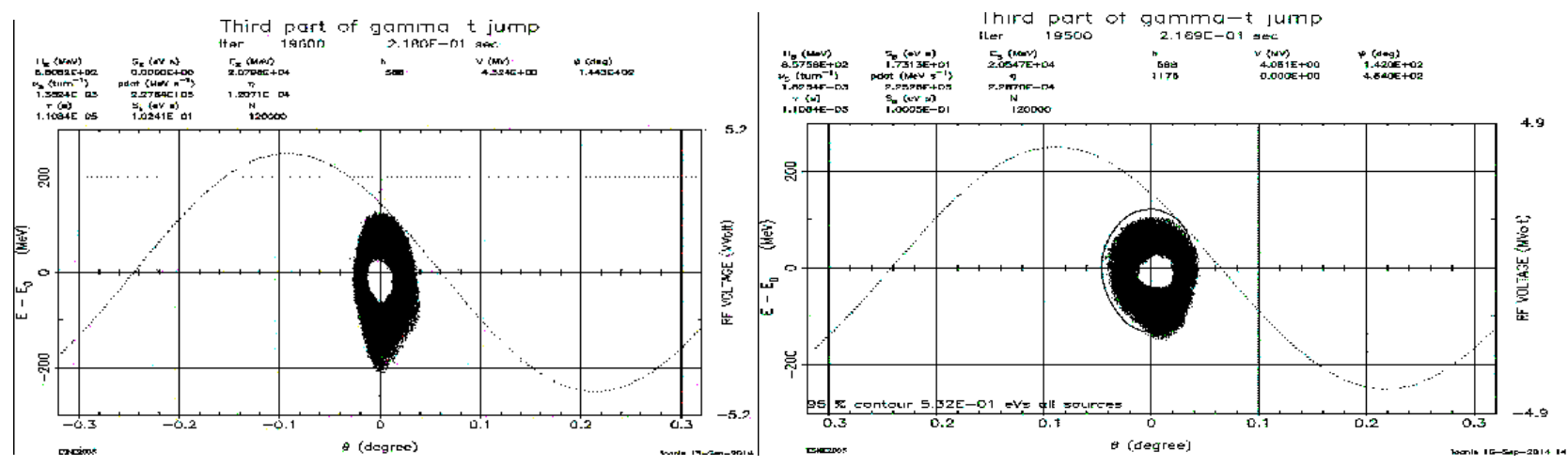

Figure 2.93: Phase space distribution after transition with full charge and two different sizes of the gamma-t jump. Half jump (left) and whole jump (right). 


\subsubsection{Beam Stability in the Recycler and Main Injector}

The main beam parameters used in the Recycler stability estimate are listed in Table 2.21. At injection $(\gamma=9.5)$ the space charge is strong transversely, i.e. the tune shift $\Delta Q_{s c} \approx 0.1$ is much larger than the synchrotron tune $Q_{s} \approx 0.034$; the space charge is also important longitudinally, leading to the synchrotron tune depression of about $20 \%$ [44], and to a possibly of longitudinal instability similar to the "dancing bunches" [49]. The longitudinal instability can be significantly exacerbated by coupled-bunch interaction through high order modes (HOM) in the cavities, leading to the growth rate [50]:

$$
\begin{aligned}
\tau_{\|}^{-1} & =\frac{M N r_{p} \eta R_{s} \omega_{r} \rho_{\omega_{r}}^{2}}{\gamma C Q_{s} Z_{0}} ; \\
\rho_{\omega_{r}}^{2} & =\exp \left(-\omega_{r}^{2} \sigma_{\tau}^{2}\right) ; \quad Z_{0}=377 \Omega .
\end{aligned}
$$

Here $R_{s}$ is the shunt impedance, $\omega_{r}$ is the HOM frequency, and $\sigma_{\tau}$ is the rms bunch length in time units. For $R_{s}=35 \mathrm{k} \Omega, \omega_{r} /(2 \pi)=150 \mathrm{MHz}$, and $\sigma_{\tau}=1.9 \mathrm{~ns}$ this yields a rather high frequency suppression factor $\rho_{\omega_{r}}^{2}=0.03$, leading to $\tau_{\|}=30 \mathrm{~ms}, \tau_{\|}^{-1}=0.02 \omega_{s}$. A narrow-band damper could suppress such slow coupled-bunch motion if it will be required.

Table 2.21: Beam parameters in the Recycler used in beam stability estimates

\begin{tabular}{l|c|c}
\hline \hline Bunch population, $N$ & $8.2 \cdot 10^{10}$ & \\
\hline Number of bunches & $81 \cdot 6 \cdot 2=972$ & \\
\hline Transverse emittance, norm. rms, $\varepsilon_{n \perp}$ & 2.5 & $\mathrm{~mm} \mathrm{mrad}$ \\
\hline Longitudinal emittance, $\mathrm{rms}, \varepsilon \| \sigma_{\tau} \sigma_{E}$ & 3.6 & $\mathrm{meV} \cdot \mathrm{s}$ \\
\hline Maximal RF Voltage,$V$ & 0.125 & $\mathrm{MV}$ \\
\hline Transition gamma, $\gamma_{t}$ & 21.6 & \\
\hline \hline
\end{tabular}

The transverse single-bunch instability is described by a growth rate [42]

$$
\tau_{\perp \mathrm{SB}}^{-1}=F_{\mathrm{SB}}(\chi) \frac{N r_{p} \bar{W}_{\mathrm{SB}} \bar{\beta}}{4 \pi \gamma}
$$

where $F_{\mathrm{SB}}(\chi) \leq 0.1$ is the chromaticity factor determined by the head-tail phase $\chi=\zeta \sigma_{s}, \bar{W}$ is a bunch-averaged wake function, and $\bar{\beta}$ is the average beta-function. For $\chi \geq 1$, the chromaticity factor saturates at its maximum, $F_{\mathrm{S}}(\chi) \simeq 0.1$. For the resistive wall case, with the half-gap $b$ and the conductivity $\sigma$, the average wake is estimated as:

$$
\bar{W}_{\mathrm{SB}}=\frac{\pi^{2}}{12} \frac{2 C}{\pi b^{3} \sqrt{\sigma \sigma_{\tau}}}
$$

Altogether, this leads to single-bunch instability with a growth time $\tau_{\perp \mathrm{SB}}=20 \mathrm{~ms}$ or in other words $\tau_{\perp \text { S B }}^{-1}=0.03 \omega_{s}$.

The coupled-bunch transverse impedance due to wall resistivity is: 


$$
Z_{\mathrm{CB}}=Z_{0} R \frac{\pi^{2}}{12} \frac{\delta}{b^{3}}
$$

where $\delta$ is the skin depth at the corresponding coupled-bunch frequency. The corresponding instability growth rate is:

$$
\tau_{\perp \mathrm{CB}}^{-1}=\left|I_{n}(\chi)\right|^{2} \frac{M N r_{p} \bar{\beta}}{\gamma T} \frac{Z_{\mathrm{CB}}}{Z_{0} R}
$$

where the coupled-bunch chromatic form-factor $I_{n}(\chi)$ is given by Eq. (2.14). For the parameters of Table 2.21, this yields the growth rate close to the synchrotron frequency, $\tau_{\perp \mathrm{CB}}^{-1}=1.2 \omega_{s}=1.8 \cdot 10^{3} \mathrm{~s}^{-1}$. Suppression of that fast instability most likely would require a transverse damper.

The Recycler bunch-by-bunch transverse dampers have been very effective in damping the wall current instability. However, during slip-stacking the previously developed dampers were not effective, and therefore they were turned off. The beam stabilization was achieved with the chromaticity increase by about 11-12 units (to -18 from -6) which resulted in a considerable beam loss.

In the system of slipped bunches the transverse instability mostly develops in the sum mode [51]. This understanding enabled us to build a damper capable to suppress low frequency resistive wall instabilities in Recycler. This so called "frequency" damper was recently commissioned in the Recycler. The "frequency" damper allowed operation at low chromaticity during slip stacking. That greatly reduced the beam losses.

The space charge effects during slip-stacking in the Recycler have been simulated with Synergia for different chromaticities [46]. The space charge footprints at two different chromaticities are shown in Figure 2.94.
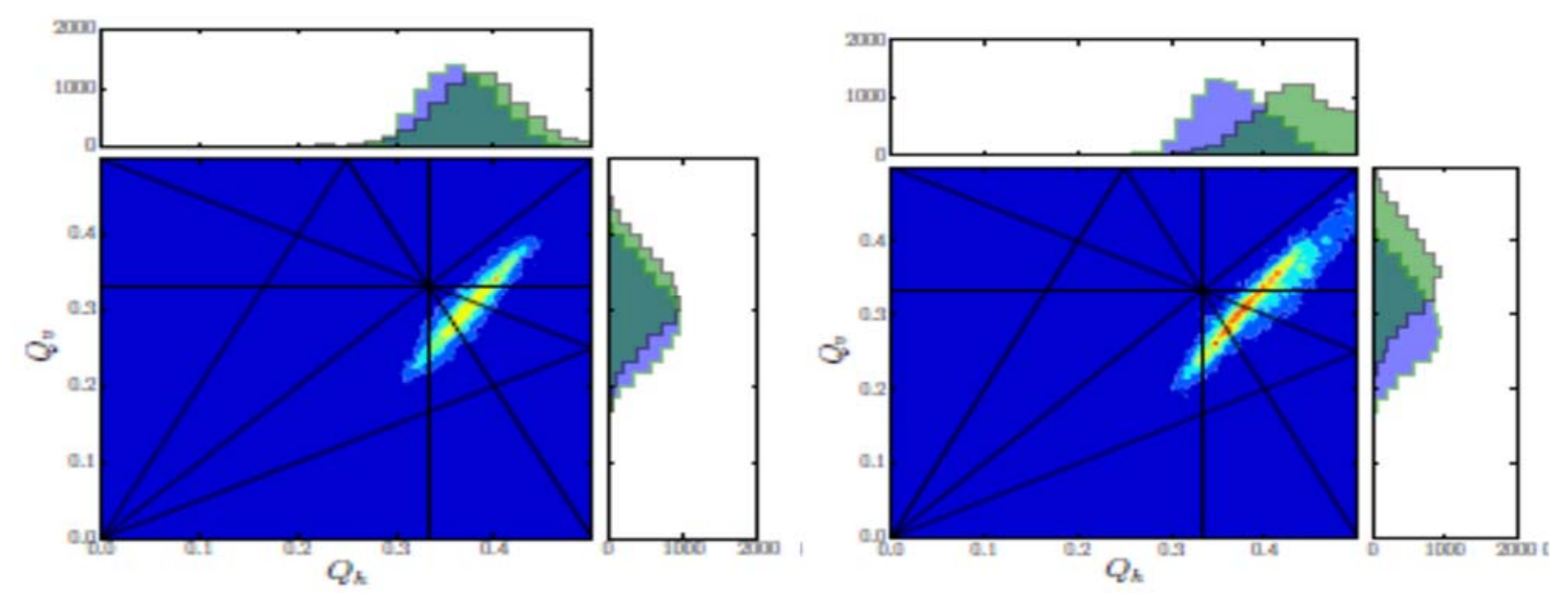

Figure 2.94: Space charge footprints for the PIP II parameters at two different chromaticities. Low chromaticity $(-6)$ on the left and high cromaticity $(-18)$ on the right.

Currently, the Recycler performance is not limited by the electron cloud instability. A series of beam studies and simulations are underway to evaluate if electron cloud will be an issue in the Recycler and Main Injector with the PIP-II beam intensities. So far, beam scrubbing has been effective in reducing the SEY (Secondary Electron Yield) of the stainless steel beam pipe in both rings. A beam pipe coating in both Recycler and Main Injector is also an option. 


\subsubsection{Electron Cloud Mitigation}

Electron cloud generation can be a possible instability source for the PIP-II intensities in the Recycler and Main Injector. The instability growth rates are so high that the instability hardly can be suppressed with a damper. Therefore, the best approach is to mitigate the generation of the cloud itself. There have been a series of measurements in the Main Injector, looking at secondary electron yield and cloud generation. A dedicated measurement setup now exists at MI-52, with newly developed RFA detectors. Both TiN and $\mathrm{C}$ coated beam pipes have been installed and measurements made. Both coatings show significant reductions in secondary electron generation when compared to an uncoated stainless steel pipe. VORPAL simulations are being benchmarked against these measurements. A SEY (Secondary Emission Yield) stand was installed in the Main Injector in order to measure the effect of scrubbing in situ for different kinds of stainless steel. A picture of the SEY measuring stand is shown in Figure 2.95.
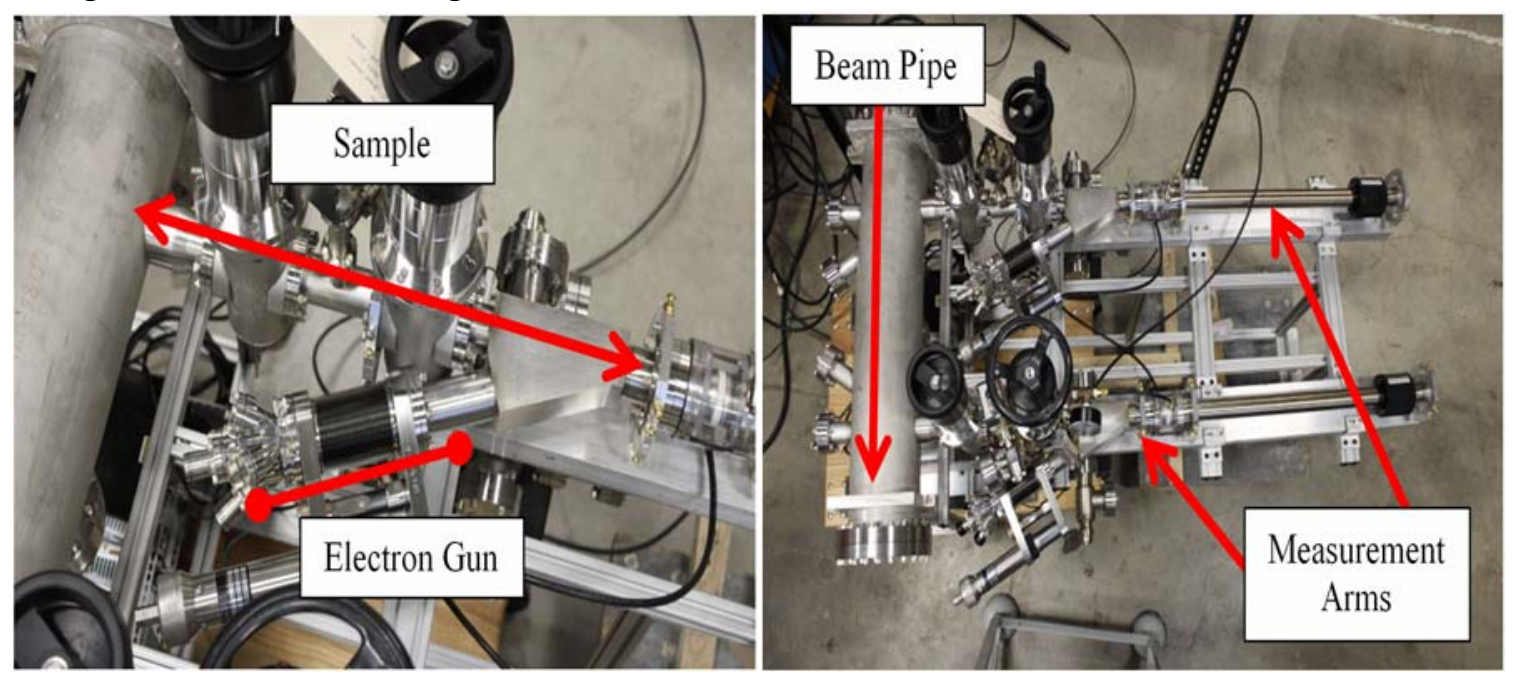

Figure 2.95: SEY Measuring Stand.

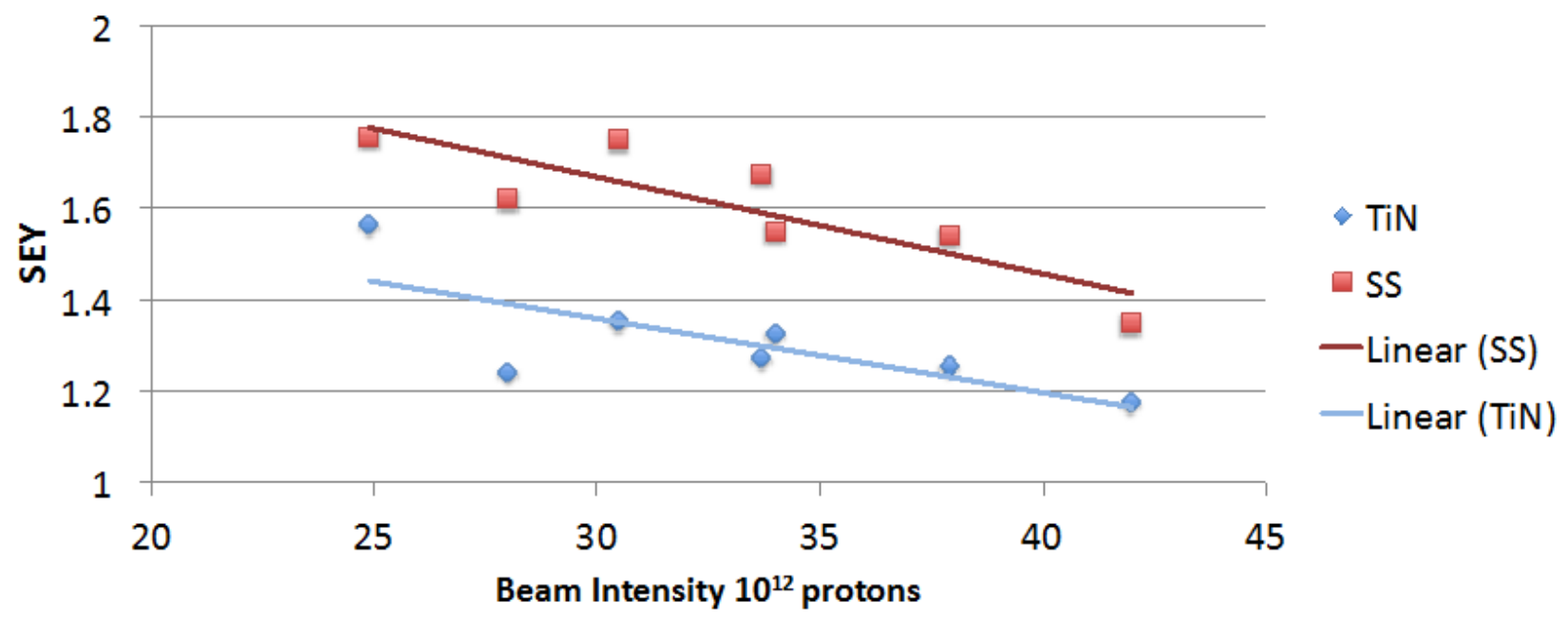

Figure 2.96: The dependence of SEY on the beam intensity measured in the Main Injector for 316L stainless steel with and without TiN coating.

Research continues on the coating process. The MI beam pipe is captured in the dipole magnets, 
so coating needs to take place in situ. A coating (sputtering) facility is set up in E4R, where a $6 \mathrm{~m}$ long piece of round MI pipe was successfully coated with TiN, and the coating thickness along the pipe was measured. Test coupons were also coated by TiN for SEY measurements in the Main Injector. The measurement results for two different test coupons (316L stainless steel with and without TiN) are shown in Figure 2.96. The experience from the coating facility will be used to estimate an effort required to coat the MI beam pipe with TiN in-situ. 


\section{DESIGN CONCEPTS OF MAJOR SUBSYSTEMS}

\subsection{SC Linac}

\subsubsection{Warm Frontend}

The PIP-II warm frontend consists of an ion source, Low Energy Beam Transport (LEBT), Radio Frequency Quadrupole (RFQ), and Medium Energy Beam Transport (MEBT). The $\mathrm{H}^{-}$beam originates from a $5 \mathrm{~mA}$ (nominal, $10 \mathrm{~mA} \max$ ) DC ion source and is transported through the LEBT to a CW normal-conducting RFQ, where it is bunched and accelerated to $2.1 \mathrm{MeV}$. In the MEBT a bunch-by-bunch chopper provides the required bunch patterns, removing $60-80 \%$ of bunches according to a pre-programmed timeline. To foresee possible upgrades, all elements of the frontend are designed for beam currents of up to $10 \mathrm{~mA}$. The beam energy of $2.1 \mathrm{MeV}$ is chosen because it is below the neutron production threshold for most materials. A wall shielding the frontend from radiation generated in the main linac is envisioned in the MEBT to allow servicing the ion sources without interrupting the linac beam.

\subsubsection{Ion Source}

The ion source assembly is a $\mathrm{DC}, \mathrm{H}^{-}$source delivering up to $10 \mathrm{~mA}$ of beam current at $30 \mathrm{keV}$ to the LEBT. The ion source specifications are listed in Ref. [52]. The present scenario uses the volume cusp, filament-driven, ion source presently commercially available from D-Pace Inc. ([53], Figure 3.1). This source is capable of delivering up to $15 \mathrm{~mA}$ with a satisfactory transverse emittance of $<0.2 \mu \mathrm{m}$ (rms, normalized), but its mean time between maintenance is relatively short. The main reason is the source filaments, which need to be replaced every 300-500 hours. To maximize the beam uptime, two ion sources are planned to be installed (see Figure 2.2). Each source can be removed for repairs, installed back, and conditioned without interrupting the operation of the other source. To enable an access to the portion of the beam enclosure, where the ion sources reside, the radiation shielding wall is installed in the MEBT.

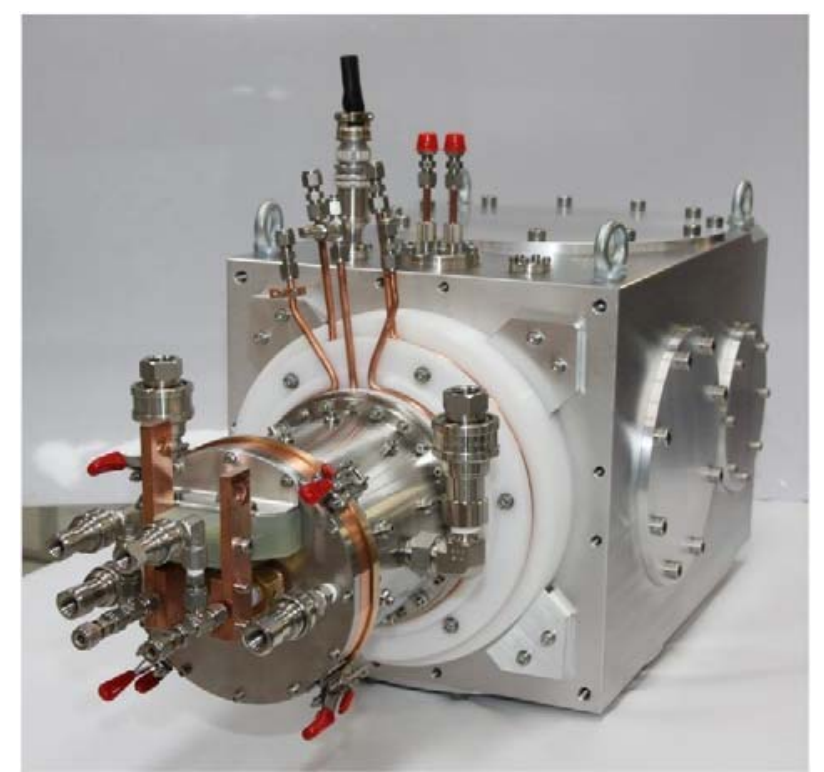

Figure 3.1: Photograph of the D-Pace ion source (foreground) with a vacuum chamber.

The ion source was purchased from D-Pace Inc. to be used in the PIP2IT. It has been in operation 
since 2013. While presently not stated as a requirement for PIP-II, a modulation circuit was built and mounted onto the ion source extraction electrode to provide pulsed operation. This capability brings flexibility to the commissioning of the beam line downstream and provides an additional level of protection in the Machine Protection System.

Alternatives to the D-Pace Inc. ion source may be considered. Several groups around the world have been engaged into R\&D that may lead to an ion source with the proper characteristics for PIPII and a much longer time between maintenance than is currently available.

\subsubsection{LEBT - Low Energy Beam Transport}

The LEBT transports the beam from the exit of the ion source to the RFQ entrance and matches the optical functions into those of the RFQ. In addition, the LEBT forms a low-duty factor beam during commissioning and tuning of the downstream beam line and can interrupt the beam as part of the Machine Protection System (MPS). Pulsed beam operation and fast machine protection are achieved via a chopper assembly, which consists of a kicker combined with an absorber. In addition, in the case of severe failures, the beam is disabled by turning off the ion source's extraction and bias voltages, as well as the switching dipole power supply. In some scenarios, the LEBT chopper assembly can also be used as a pre-chopper to assist the MEBT chopping system.

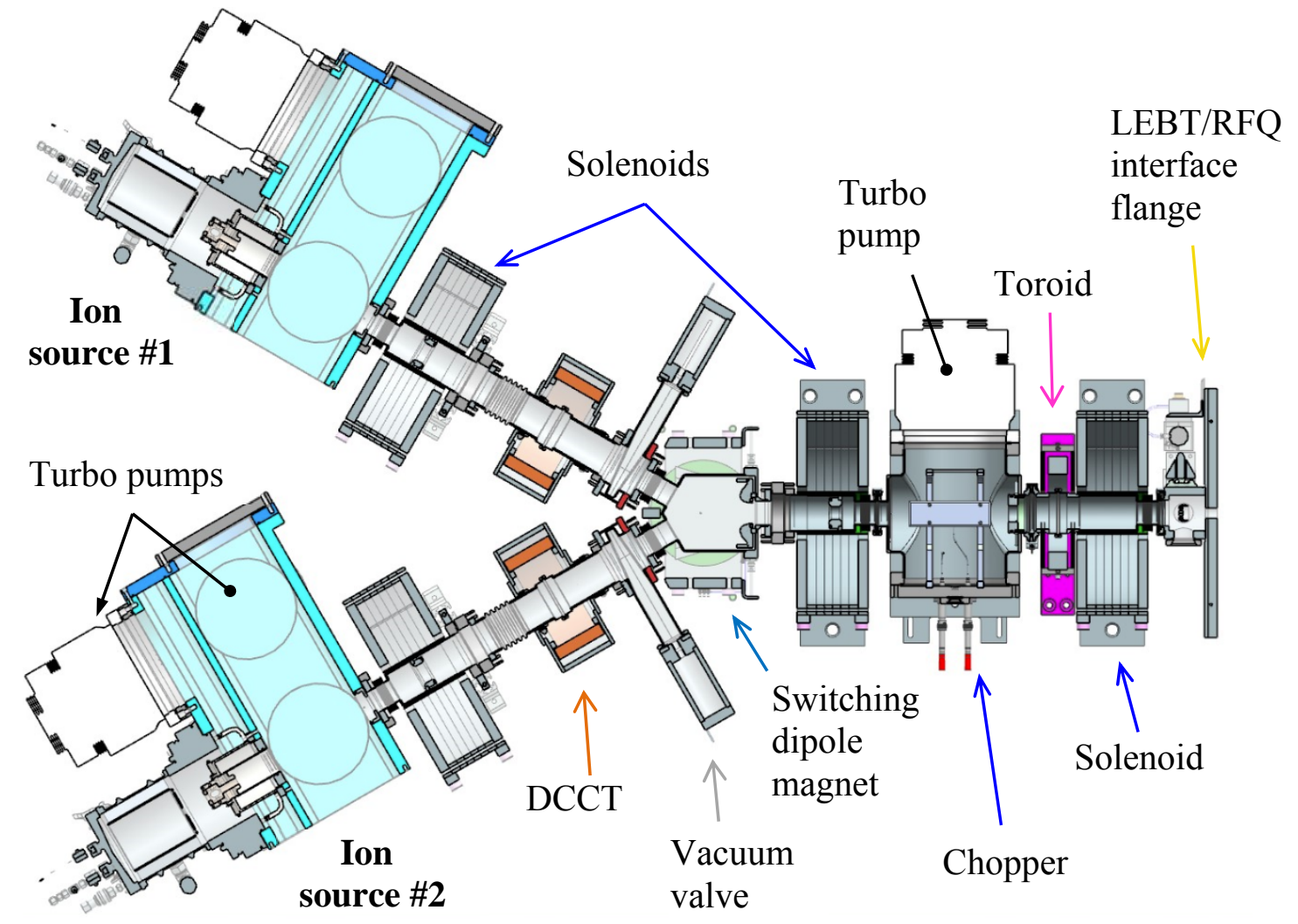

Figure 3 2: Section view of the LEBT with two ion sources.

\section{Layout}

The LEBT consists of 3 solenoids (in each leg), a slow switching dipole magnet, a chopping system, water-cooled Electrically Isolated Diaphragms (EID), an electrically-isolated, water-cooled, movable vertical electrode assembly with 3 apertures, and beam current diagnostics - DCCT after solenoid \#1 and AC current transformer ("toroid") after the chopping system (Figure 3.2). An 
emittance scanner [54] is located at the exit of each ion source. Dipole correctors are mounted inside each solenoid allowing the beam to be steered in any direction. The edge focusing of the switching dipole is adjusted to minimize the asymmetry between horizontal and vertical focusing. As already stated in Section 2.1.2.1, the $\sim 2 \mathrm{~m}$ beam line length ensures that the gas migrating from the ion source to the RFQ is kept at a manageable level.

\section{Chopping system}

For accelerator frontends with beam parameters similar to PIP-II, most LEBT's employ a transport scheme that relies on almost complete neutralization of the beam to counteract the effects of space charge during transport. However, neutralization is inevitably broken in the vicinity of a chopper. In order to decrease the distance that the beam travels with full space charge and low energy, the chopping system is often located just upstream of the RFQ. Such location has several features contradicting the principles described in Section 2.1.2.1. First, absorption of the cut-out beam creates a significant gas load into the RFQ. Second, the short distance required between the last solenoid and RFQ limits the possibilities of fitting in a simple and robust chopper. It also makes it difficult to place diagnostics downstream of the chopper, for instance, to measure the beam current entering the RFQ. Following this logic, the chopping system is placed between solenoids \#2 and \#3.

The chopping system is a simple electrostatic kicker, with the particularity that one of the electrodes is also the absorber. Such a design is inherently robust against un-controlled beam losses, which can quickly become an issue even at the relatively modest maximum beam power of $300 \mathrm{~W}$ (DC). In addition, it removes the need for a dedicated absorber electrode downstream, thus making the overall chopping system more compact. The absorber plate is at the ground potential but electrically isolated in order to measure the primary beam current. When the beam is passing through, the design of the chopper electronics allows applying a DC voltage to the kicking plate to clear secondary ions out of the beam path.

\section{Electrically Isolated Diaphragms (EID)}

The beam line includes 3 water-cooled, Electrically Isolated Diaphragms (EIDs), two of which are located within solenoid \#1 and \#2 and the third is just downstream of the electrostatic kicker.

The primary function of these electrodes is to minimize uncontrolled beam losses. They are sized such that if there is some beam loss (e.g. before the optics is properly tuned, or during the rise and fall time of the kicker (EID \#3)) it would most likely occur at these locations first. The EIDs are electrically isolated to allow the beam loss to be measured.

Second, the EIDs play the role of potential barriers in the transport scheme with an un-neutralized section, confining ions in sub-sections of the beam line.

Finally, they are used to measure the beam size and center the beam. This is achieved by steering the beam with upstream correctors and recording the current drawn by the electrodes.

In addition, there is a scraper assembly just downstream of Solenoid \#3, which is an additional movable electrically-isolated and water-cooled electrode with 3 apertures. ( 2 round apertures and a 'D-shaped' aperture). A small round aperture is used to create a pencil beam. A larger round aperture placed concentrically with the RFQ entrance aperture protects the RFQ vanes during normal operation. The 'D-shaped' aperture is used to measure the beam current density profile by moving its edge across the beam and recording the scraper current as a function of the scraper position. Finally, the scraper can be placed such as to intercept the beam completely. For the larger round aperture, the size of the opening is chosen to scrape the halo particles that otherwise would be lost in the RFQ or MEBT. Also, variation of the beam current intercepted by the scraper while moving the 
beam across the opening with upstream dipole correctors gives information about the beam position and core size for both planes.

\section{Emittance growth mitigation}

To satisfy both the beam physics requirements and the design choices for PIP2IT, a hybrid transport scheme was devised, where the beam propagates through the first 'high pressure' part of the LEBT being neutralized, but neutralization is prevented in the second part, which starts just upstream of the chopper (see Section 2.1.2.1). For a beam with modest perveance, this transport solution nearly preserves the beam emittance if the transition to the un-neutralized section occurs where the beam current density profile is close to the profile corresponding to uniform distribution [55]. While the ion source may not be optimized to deliver a beam with uniform current density, because of the finite extent of the beam emitter (e.g. plasma surface) and the geometry of the extraction system, one would expect the spatial distribution of the beam to have relatively sharp edges. In addition, because of the ion source current overhead, it is possible to implement significant scraping that would result in only keeping the core of the particles' distribution, which is inherently more uniform than the tails. At the same time, the beam formation out of a plasma in a near thermal equilibrium must result in a Gaussian velocity distribution in transverse momentum at the beam emitter location.

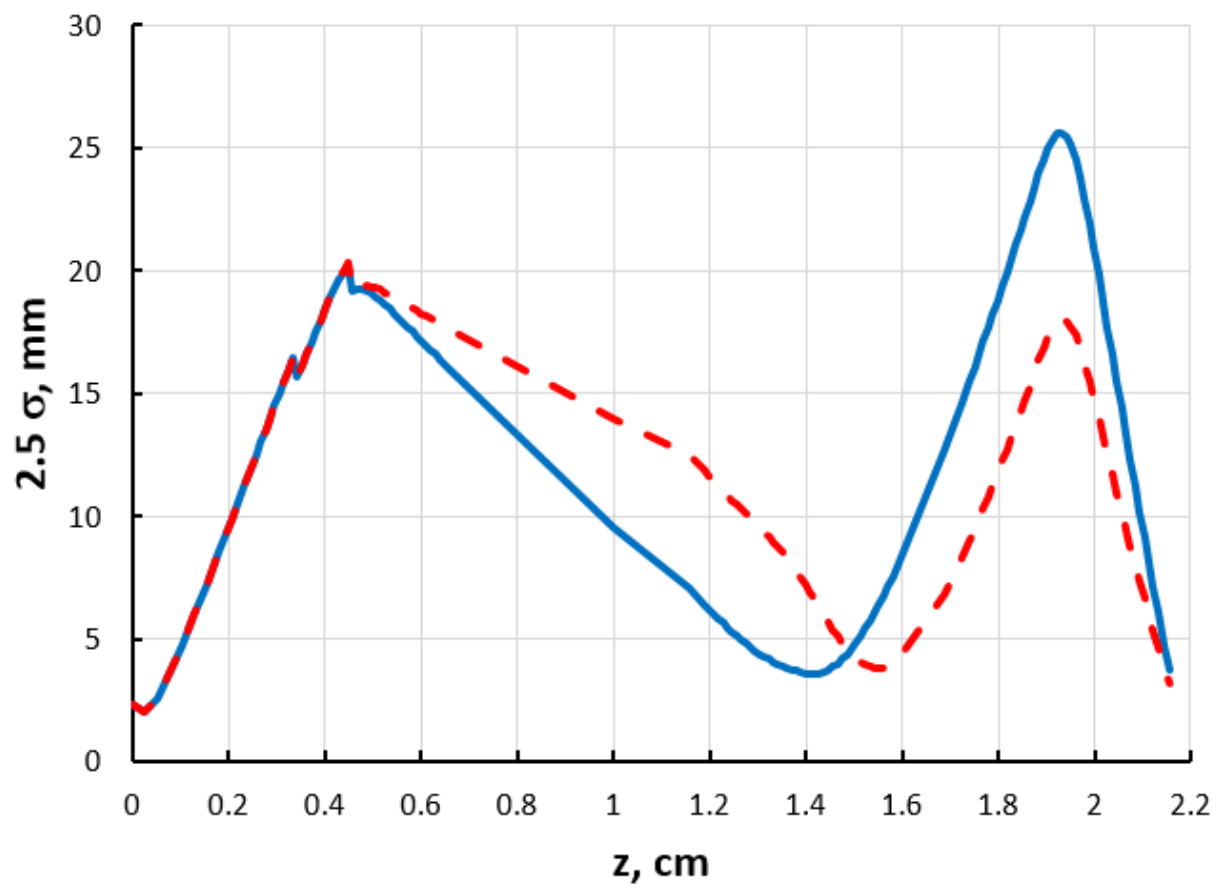

Figure 3.3: Beam envelopes (2.5 $\sigma$ ) obtained with TraceWin. Currents in focusing solenoids are 154, 187 and 223.5 A (blue curve) and 143, 158 and 240 A (red dashed curve) for Solenoids \#1, \#2 and \#3, respectively. Input distributions (uniform current density and Gaussian distribution in velocity subspace) are the same for both simulations; $I_{\text {beam }}=5 \mathrm{~mA}$ (after scraping in the first solenoid).

To illustrate this model, Figures 3.3 to 3.5 show TraceWin simulations, where the initial distribution is uniform in the subspace of transverse coordinates and Gaussian in the velocity subspace. While the final Twiss parameters are nearly equal (Figure 3.3), the solenoids have different current values: in one case (red traces or dots on the plots), the beam is nearly uniform near the chopper, while it is Gaussian for the second case (blue traces or dots on the plots) (Figure 3.4). 
Correspondingly, the emittance growth is lower in the first case than in the second one (Figure 3.5). Note that $\sim 20 \%$ of the beam is scraped off before the first solenoid.

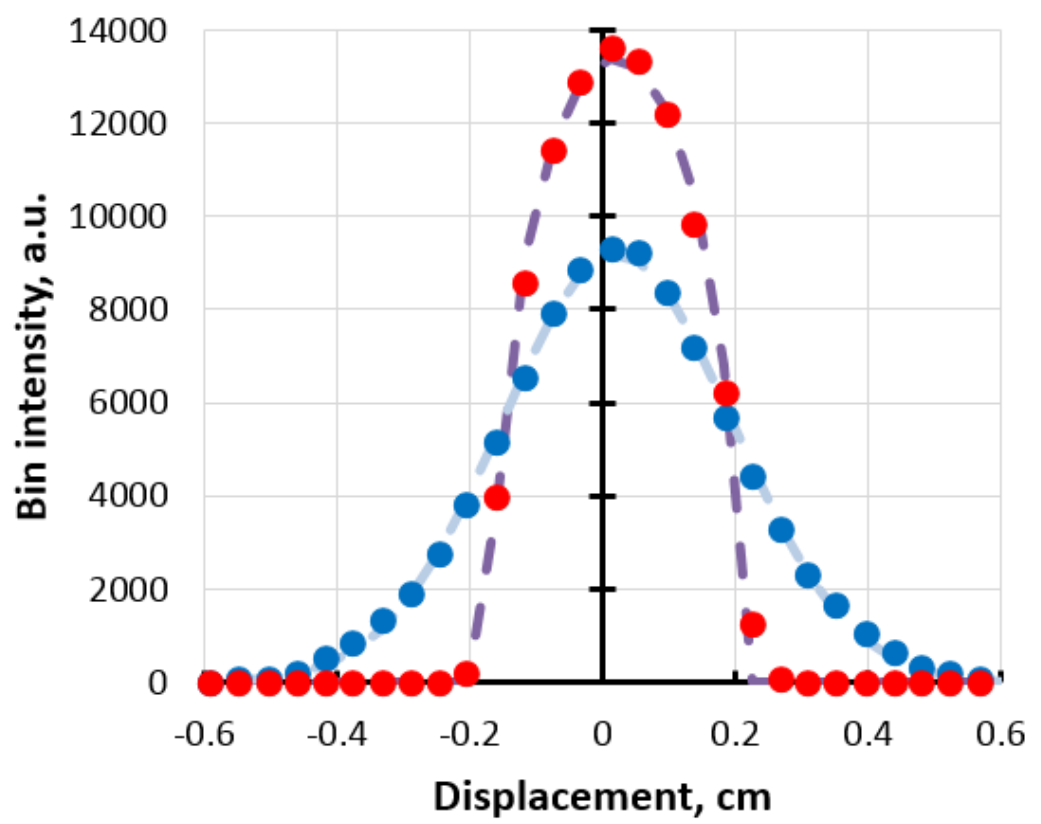

Figure 3.4: 1D beam profiles $20 \mathrm{~cm}$ downstream of EID \#2 corresponding simulations shown on Figure 3.3. Dashed curves are fits, assuming uniform (purple) or Gaussian (light blue) distributions.

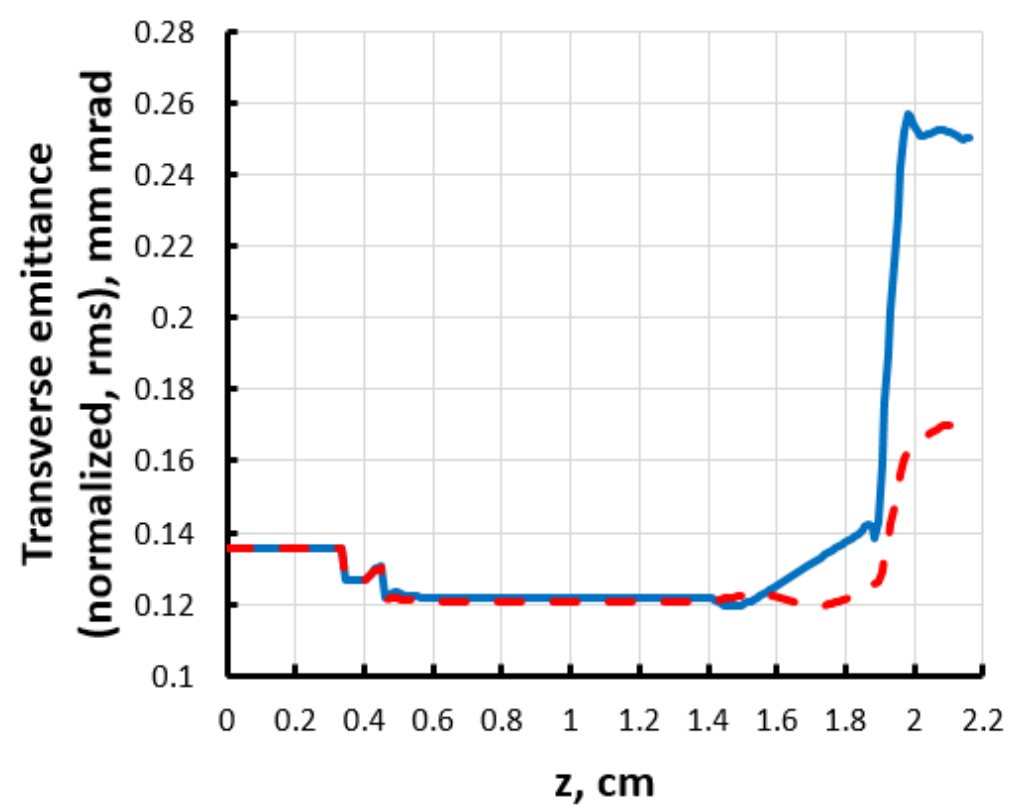

Figure 3.5: Emittance evolution along the beam line corresponding to the simulations shown in Figure 3.3.

Measurements at the PIP2IT were carried out with the transverse emittance station [56] installed close to the location of RFQ input flange. They show a behavior similar to the simulations. Namely, starting with the same ion source tune, different solenoid current settings lead to different emittances for the same measured Twiss parameters at the end of the beam line. In addition, profile measurements carried out between solenoid \#1 and \#2 show that the beam distribution is indeed 
uniform-like or Gaussian-like depending only on the value of the solenoid \#1 current. Thus, both measurements and simulations indicate that the proposed transport scheme allows one to deliver beam with the required properties to the RFQ.

\subsubsection{RFQ - Radio-Frequency Quadrupole Accelerator}

The $162.5 \mathrm{MHz}$ CW RFQ will accelerate an $\mathrm{H}^{-}$ion beam with currents of up to $10 \mathrm{~mA}$ from 30 $\mathrm{keV}$ to $2.1 \mathrm{MeV}$ (see Ref. [57] for specifications). Presently the PIP-II RFQ is assumed to be identical to the one used for PIP2IT, which was constructed and built by LBNL [58], and which design is based on the experience accumulated the LBNL team earlier and, in particular, on the SNS RFQ [59]. Fermilab staff made considerable contributions to the RF design of RFQ and its tuning.

The RFQ is a 4.45-m long, four-vane copper structure composed of four longitudinal modules. The nominal vane-to-vane voltage is $60 \mathrm{kV}$. A series of 32 water-cooled pi-mode rods provides transverse quadrupole mode stabilization, and a set of 80 , evenly spaced, fixed slug tuners is used for the final frequency adjustment and for local field perturbation corrections. It was delivered to FNAL in 2015 and has been commissioned and operated in the PIP-II Injector Test (PIP2IT). The PIP2IT RFQ has already proven that it can operate at full CW power and can accelerate pulsed beam with greater than $95 \%$ efficiency. Preliminary measurements verify its good performance. Capture and transmission efficiency has been verified up to $95 \%$, which is the current limit in instrumentation accuracy. Figures 3.6 and 3.7 present RFQ views as a CAD model and as an actual accelerator component installed in the PIP2IT enclosure.

RF matching into the RFQ cavity is done with two $162.5 \mathrm{MHz}$ input couplers designed and verified at FNAL [60]. A CAD illustration of the coupler is shown in Figure 3.8. These couplers were designed to transport up to $75 \mathrm{~kW}$ of RF power with full reflection, without breakdown. They have two design features that are unique with respect to other RF cavity input couplers: the antenna is forced-air cooled (not water cooled) to avoid potential water leaks to the RFQ vacuum chamber, and the antenna is capacitively coupled to the end-wall, allowing DC biasing to reduce multipacting. The couplers have been tested with up to $120 \mathrm{~kW}$ of CW drive into the RFQ. A bias of about $4 \mathrm{kV}$ on the antennas is required to eliminate multipacting around the couplers at any power.

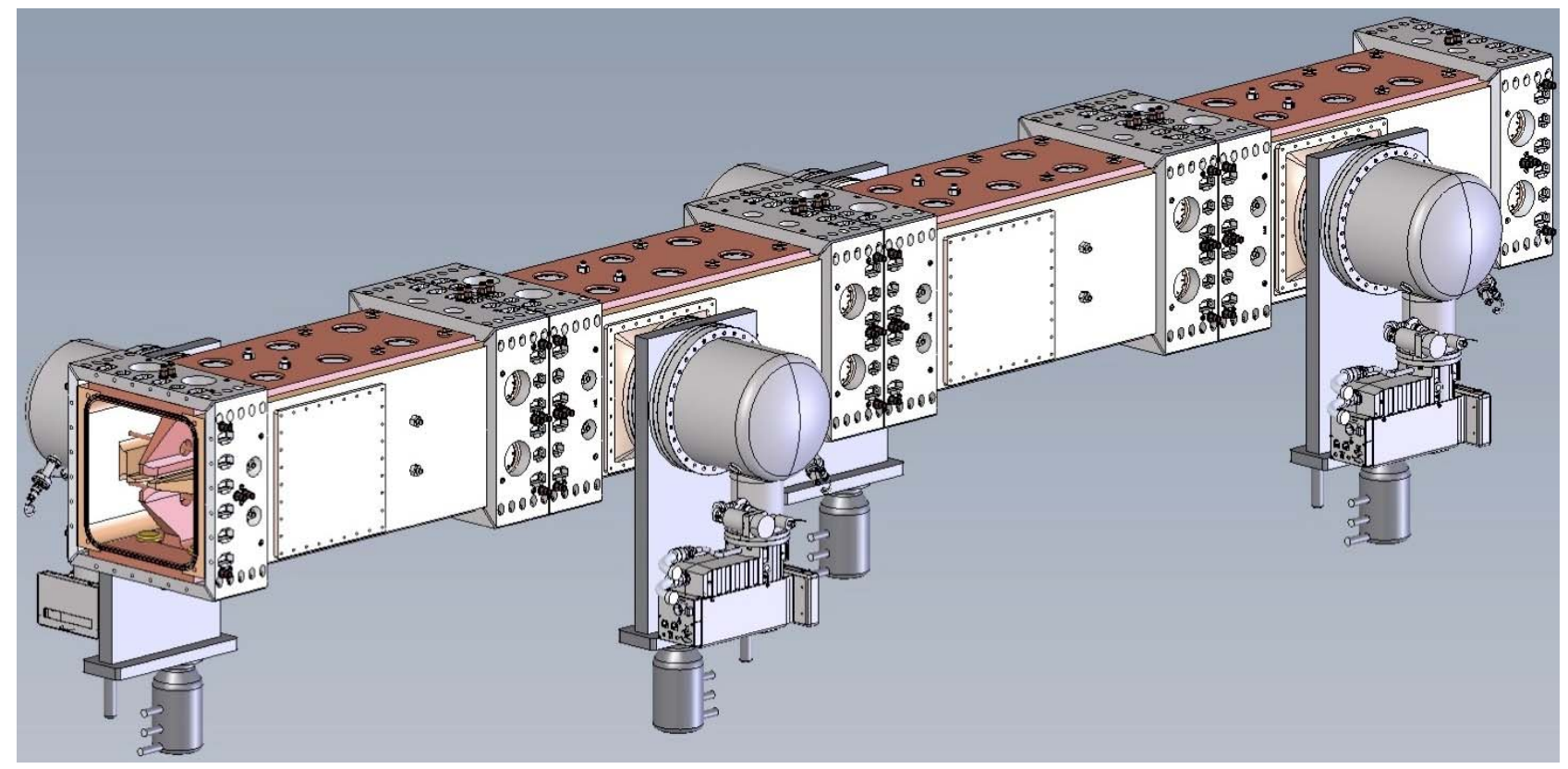

Figure 3.6: CAD model of the full four-module RFQ. 


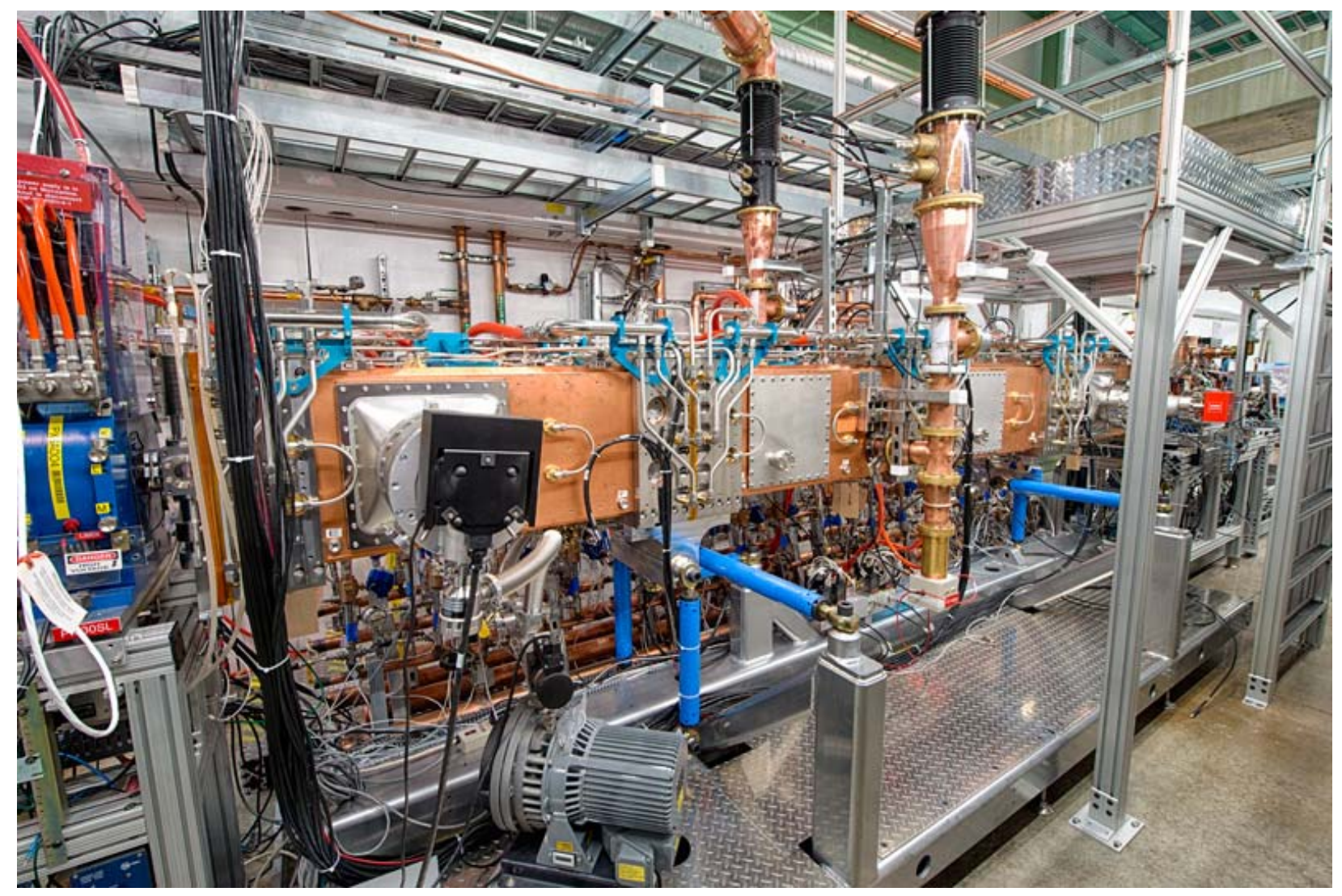

Figure 3.7: RFQ installed in PIP2IT beamline.

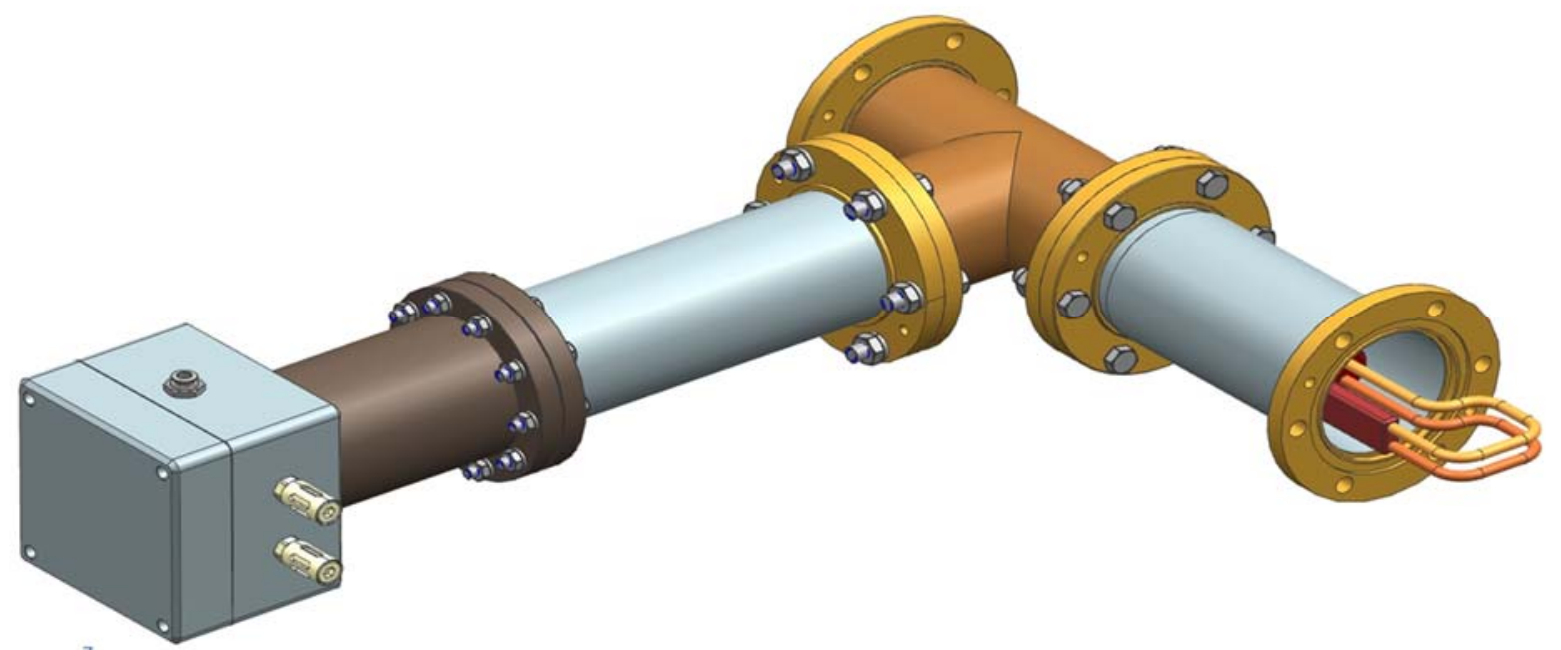

Figure 3.8: Solid model of the RFQ input RF coupler design.

RF power is provided by two, 75kW $162.5 \mathrm{MHz}$ solid-state power amplifiers from Sigma Phi Inc. Each amplifier is protected from power reflected from the RFQ using $75 \mathrm{~kW}$ circulators from Ferrite Inc. The added expense of the RF circulators is justified by the improved stability of RFQ operation. In particular, they allow the RFQ operation over a larger range of resonant frequency offsets and support a pulsed operation with variable duty factors.

The resonant frequency of the RFQ is controlled thermally by adjusting the temperature difference of the water circuits, which cool the vanes and the RFQ walls. The choice of this scheme is supported 
by a much larger sensitivity to the temperature difference than to the average temperature of the circuits. The corresponding frequency response parameters are shown in Table 3.1. The temperature difference is achieved by redistribution of cooling water flow between these two circuits. For pulsed operation, the duration of the RF duty cycle can also be used for temperature stabilization. For $\mathrm{CW}$ operation, the resonant frequency must be maintained by cooling water temperature regulation only. An adaptive control system has been designed that will regulate the steady state temperature of the RFQ cooling system to better than $0.1^{\circ} \mathrm{C}$. A simple block diagram description of the system is shown in Figure 3.9. The system is also designed to respond to sudden changes of thermal load due to RF power trips. The goal is a reduction of the time necessary for bringing the RFQ resonant frequency to the nominal value after a trip. Consequently, it will decrease the beam downtime.

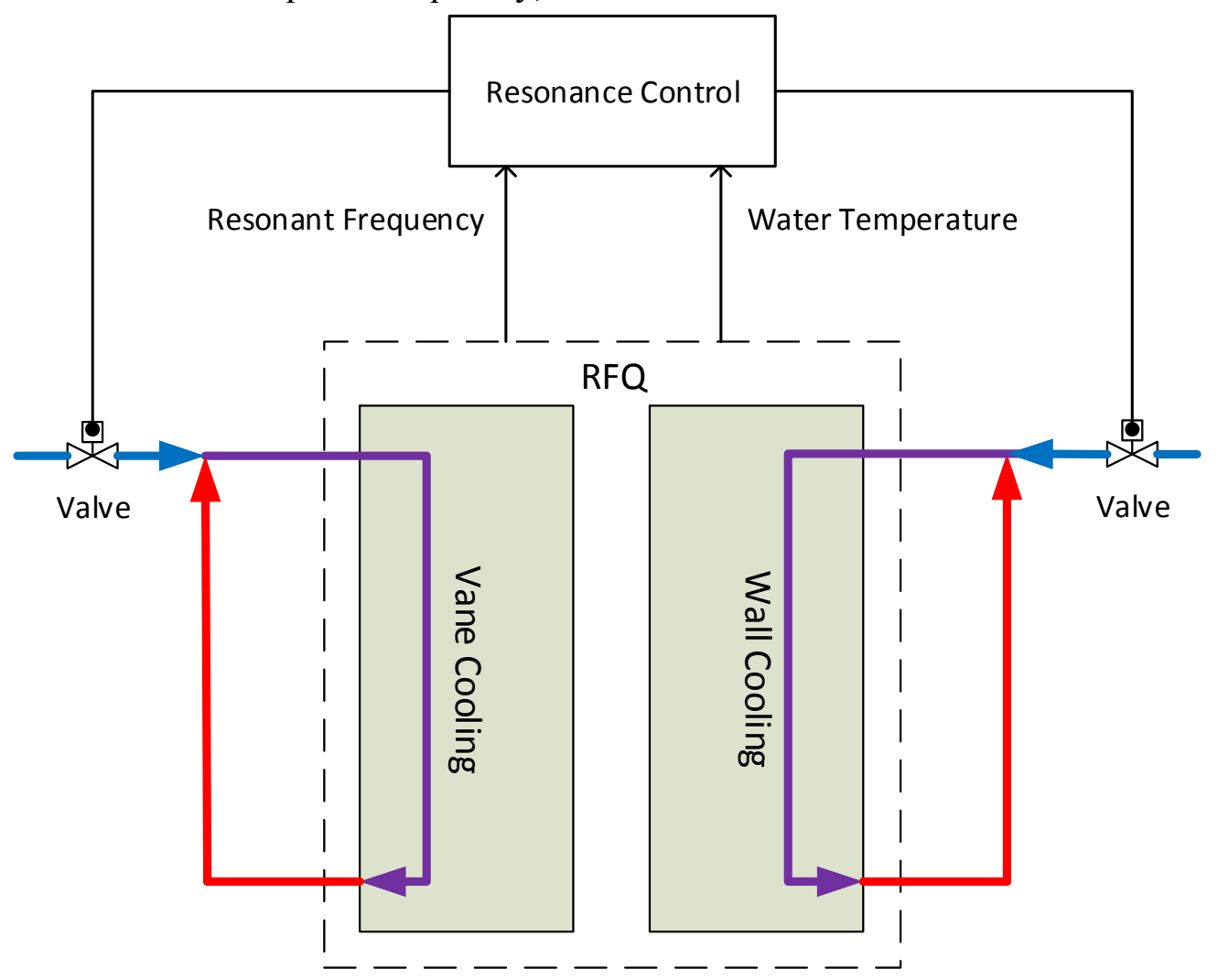

Figure 3.9: A simple block diagram showing the resonant control system of the RFQ. The system monitors the water temperature and the RFQ resonant frequency, and adjusts the flow rate of cooling water into the wall and vane cooling loops.

A series of RF and thermal finite-element models of the RFQ have been developed using ANSYS ${ }^{\circledR}$. An example of the temperature contour plots for the cavity body and vane cutback region is shown in Figure 3.10. From the RF analysis, the average linear power density was determined to be $137 \mathrm{~W} / \mathrm{cm}$ with a peak heat flux on the cavity wall of only $0.7 \mathrm{~W} / \mathrm{cm}^{2}$. With $30^{\circ} \mathrm{C}$ water in the vane and wall cooling passages, the resulting temperature profile in the cavity body ranges between 32 and $37^{\circ} \mathrm{C}$ at full RF gradient.

Additional modeling has been carried out. This includes stress and displacement analyses, thermal analyses of the tuners, pi-mode rods and vane cutbacks, and prediction of the frequency shift of the RFQ cavity due to thermal loading and changes in the cooling water temperature.

The RF design issues [11] include mode stabilization, field flatness, radial matching, and entrance and exit terminations. Table 3.1 summarizes the RF and thermal design results. 

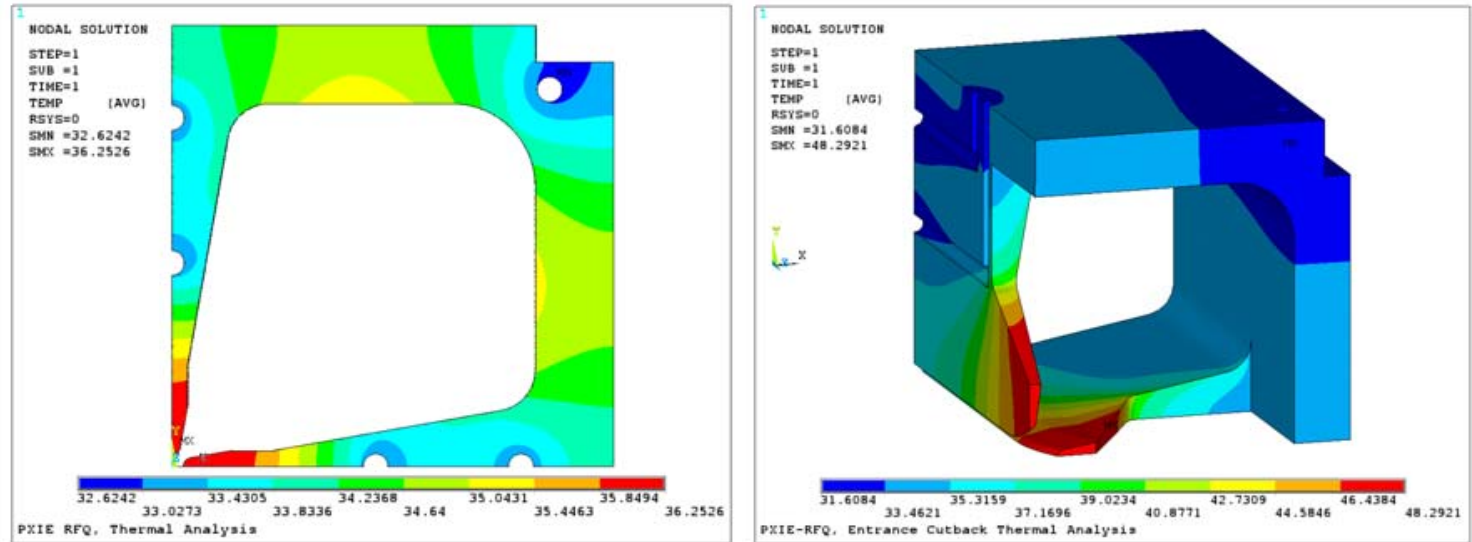

Figure 3.10: Temperature distribution in one RFQ quadrant body (left) and cut-back (right). The color scheme (degrees $\mathrm{C}$ ) is at the bottom of each plot.

Table 3.1: Main parameters of the PIP-II RFQ electromagnetic design

\begin{tabular}{l|l|l}
\hline \hline Parameter & Calculated & Measured \\
\hline Center Frequency, $\mathrm{MHz}$ & 162.493 & 162.445 \\
\hline Frequency of the dipole mode, $\mathrm{MHz}$ & 181.99 & 180.52 \\
\hline Q 0 factor & 14660 & 13000 \\
\hline Total power loss at $60 \mathrm{kV}, \mathrm{kW}$ & 74.6 & 90 \\
\hline Vane cooling resonant frequency parameter, $\mathrm{kHz} /{ }^{\circ} \mathrm{C}$ & -16.7 & -16.4 \\
\hline Wall cooling resonant frequency parameter, $\mathrm{kHz} /{ }^{\circ} \mathrm{C}$ & 13.5 & 13.9 \\
\hline \hline
\end{tabular}

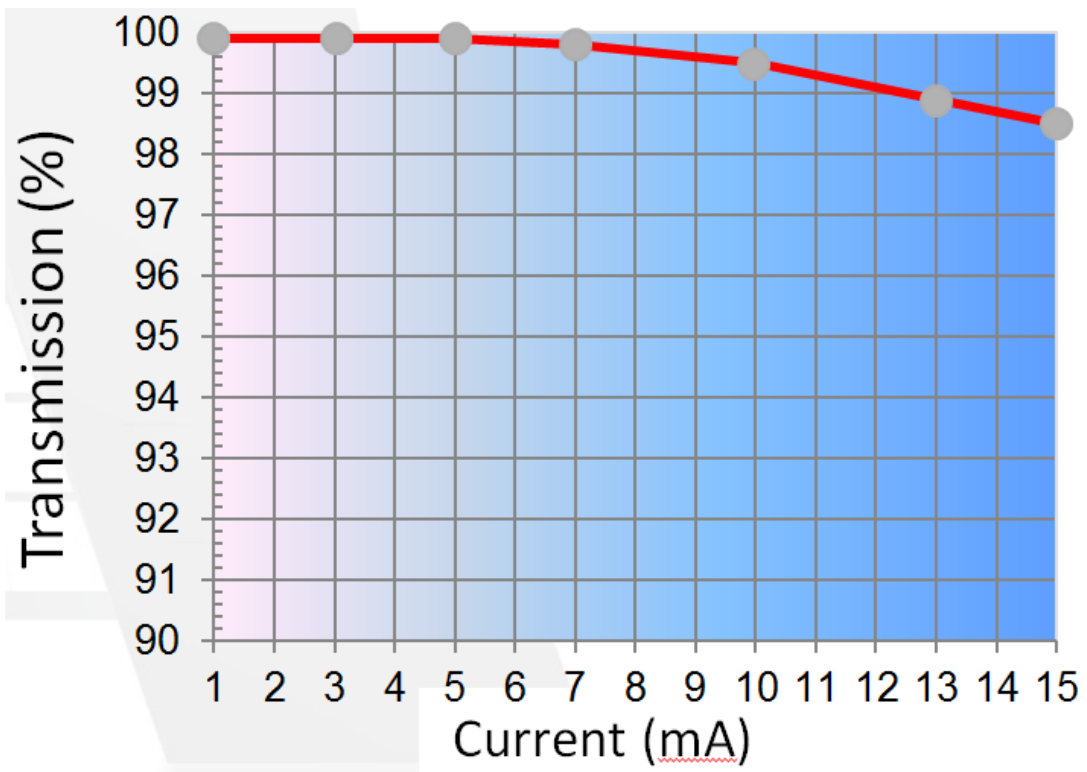

Figure 3.11: Dependence of the calculated RFQ transmission on the beam current.

Beam loss inside the RFQ may result in degradation of the RFQ performance [61]. In the course of its lifetime the PIP-II RFQ is expected to accelerate a very large charge and, consequently, 
minimization of the beam loss has a primary importance. Figure 3.11 presents the dependence of the computed RFQ transmission on the beam current. The design has over $98 \%$ transmission for a beam current from 1 to $15 \mathrm{~mA}$. At the nominal current of $5 \mathrm{~mA}, 99.8 \%$ beam capture is achieved in this simulation.

\subsubsection{MEBT - Medium Energy Beam Transport}

The $\mathrm{H}^{-}$beam accelerated in the RFQ to $2.1 \mathrm{MeV}$ enters the MEBT line, where it is chopped and matched for injection into the HWR cryomodule. The MEBT structure is presented schematically in Figure 2.8, which is replicated below for reading convenience as Figure 3.12.

\begin{tabular}{|c|c|c|c|c|c|c|c|c|c|c|c|c|c|}
\hline & & & $1175 \mathrm{~m}$ & 9 & & & & & & & & & \\
\hline & $\# 0$ & $\# 1$ & $\# 2$ & $\# 3$ & $\# 4$ & $\# 5$ & $\# 6$ & $\# 7$ & $\# 8$ & $\# 9$ & $\# 10$ & \#11 & \\
\hline $\begin{array}{l}\text { Slow } \\
\text { valve } \\
\text { after } \\
\text { RFQ, } \\
\text { toroid }\end{array}$ & 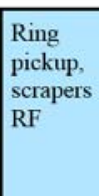 & $\begin{array}{l}\text { Emittance } \\
\text { monitor, } \\
\text { scraper, } \\
\text { wire } \\
\text { scanner }\end{array}$ & Kicker & $\begin{array}{l}\mathrm{RF}, \\
\text { laser wire }\end{array}$ & Kicker & Absorber & $\begin{array}{l}\text { Different. } \\
\text { pumping, } \\
\text { slow } \\
\text { valve }\end{array}$ & $\mathrm{RF}$ & $\begin{array}{l}\text { Scrapers, } \\
\text { wire } \\
\text { scanner }\end{array}$ & Wall & $\begin{array}{l}\text { Scrapers, } \\
\text { RF, } \\
\text { wire } \\
\text { scanner }\end{array}$ & $\begin{array}{l}\text { Fast } \\
\text { valve, } \\
\text { DCCT, } \\
\text { toroid, } \\
\text { laser wire }\end{array}$ & \begin{tabular}{|l|} 
slow \\
valve \\
in \\
front \\
of \\
HWR
\end{tabular} \\
\hline
\end{tabular}

Figure 3.12: The MEBT structure. Sections are color-coded according to their main functions: greenvacuum, blue- RF, yellow-instrumentation, and pink-chopper.

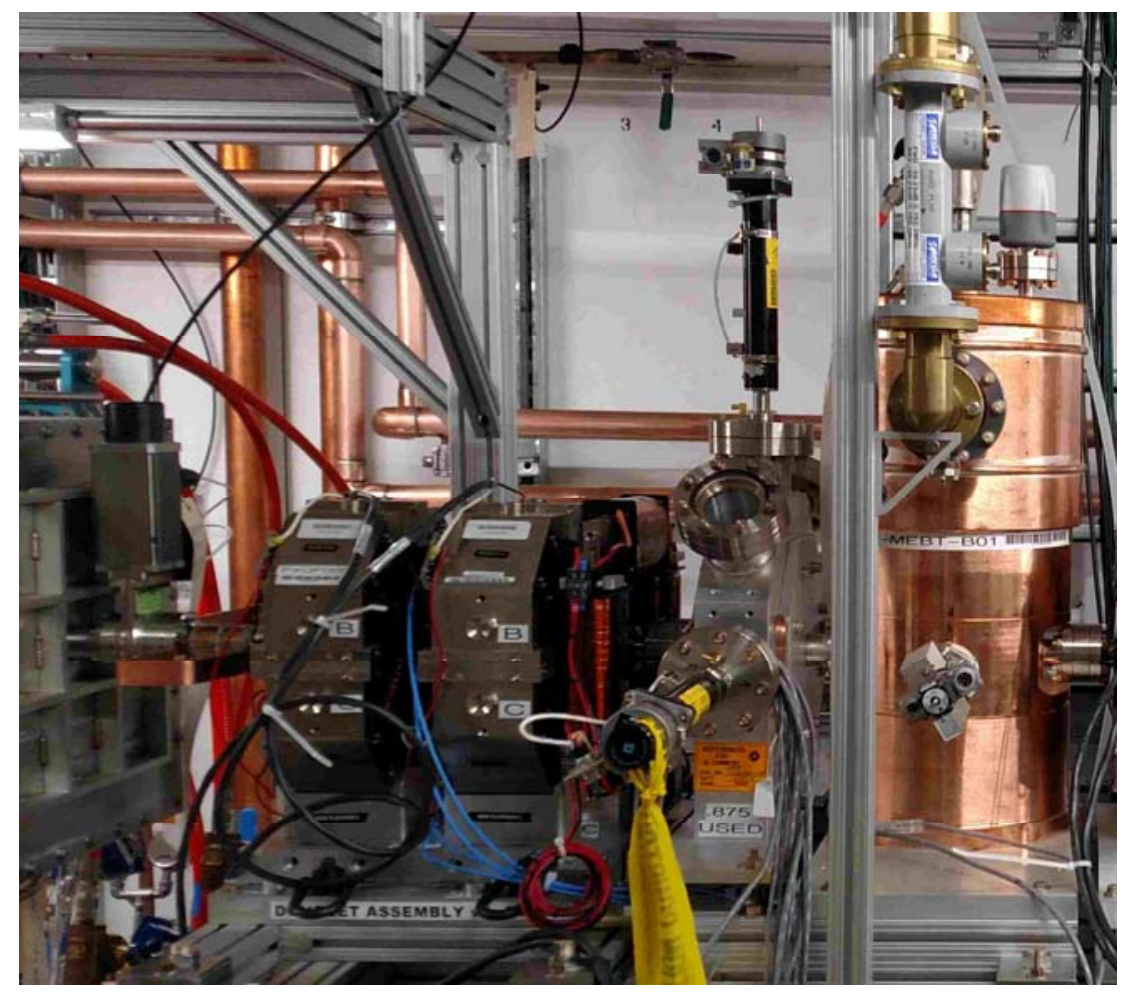

Figure 3.13. The MEBT section \#0 as installed at PIP2IT. A quadrupole doublet is followed by a dipole corrector set, a scraper assembly, and a bunching cavity. A BPM is placed between the quadrupoles and is attached to the pole tips of the upstream quadrupole.

In addition to chopping and matching, the MEBT contains tools to measure the beam properties; a scraping system to protect both the SRF cavities and sensitive elements of the MEBT itself; and a vacuum system. In this chapter, the following major MEBT subsystems are discussed: 
1. Transverse focusing

2. Longitudinal focusing

3. Chopping (kickers and absorber)

4. Scraping

Note that the vacuum system, instrumentation, MPS, HLRF, LLRF, and controls are described in other sections of this document and are mentioned here only as they are relevant to the overall design.

\section{Transverse Focusing}

Transverse focusing is provided primarily by the equidistantly placed quadrupole triplets; the only exception is the two doublets immediately following the RFQ. Each triplet or doublet is followed by a pair of dipole correctors. The design of the central quadrupoles includes the BPMs mounted to the quad pole tips. The specifications for the quadrupoles and the correctors are listed in Ref. [62]. Figure 3.13 shows the air-cooled magnets. They were produced by BARC, India, according to these specifications, and are installed at PIP2IT.

The spaces between neighboring triplets or doublets are referred to as MEBT sections. The section separation in the regular part of the MEBT is $1175 \mathrm{~mm}$, which leaves a 650-mm long (flange-toflange) space for various equipment (only $480 \mathrm{~mm}$ in the section between doublets labeled \#0 on Figure 3.12).

The $3 \sigma$ envelopes of the transmitted bunches are presented in Figure 3.14, and the corresponding simulated emittances along the MEBT are shown in Figure 3.15.

The envelopes vary gently over the MEBT length to avoid emittance growth and are smaller only at the entrance and exit, where matching to the RFQ and the HWR occur. The notable exception is Section \#8, where the vacuum chamber diameter is reduced to $10 \mathrm{~mm}$ over $200 \mathrm{~mm}$. This insert is a part of the Differential Pumping Section, which minimizes a flow of the gas released in the absorber to the SRF.

\section{Longitudinal Focusing}

To keep the beam properly bunched and to match its longitudinal phase space into the first superconducting cryomodule, the MEBT includes four identical room-temperature bunching cavities. The cavities are specified in Ref. [63] and described in [64]. Each cavity is a quarter-wave $162.5 \mathrm{MHz}$ resonator with the nominal accelerating voltage of $70 \mathrm{kV}$ (at $\beta=0.0668$ ). At time of writing this document, one of four cavities is already manufactured and is installed at PIP2IT. Its view is presented in the right-hand side of Figure 3.13.

\section{Chopping System}

The chopping system envisioned for the PIP-II MEBT consists of two identical kickers and a beam absorber. The kickers, which deflect the beam in the vertical (Y) direction, are separated by a phase advance of $\sim 180^{\circ}$ (Y-direction) and synchronized, allowing the summation of their deflections. The absorber is at an additional $\sim 90^{\circ}$ in phase advance with respect to the last kicker so that the angle introduced to a bunch by the kickers is translated at the absorber location into a $6 \sigma$ separation in the vertical plane between the centers of the bunches designated for removal ("chopped-out") and for acceleration ("transmitted"). In the main scenario of the kicker operation, both the chopped-out and transmitted bunches are deflected but into opposite directions, by applying opposite voltage polarities to the kicker plates. The simulated Y beam envelopes for these two cases are compared in Figure 3.16 . 
Both the kickers and the absorber require state-of-the-art designs.

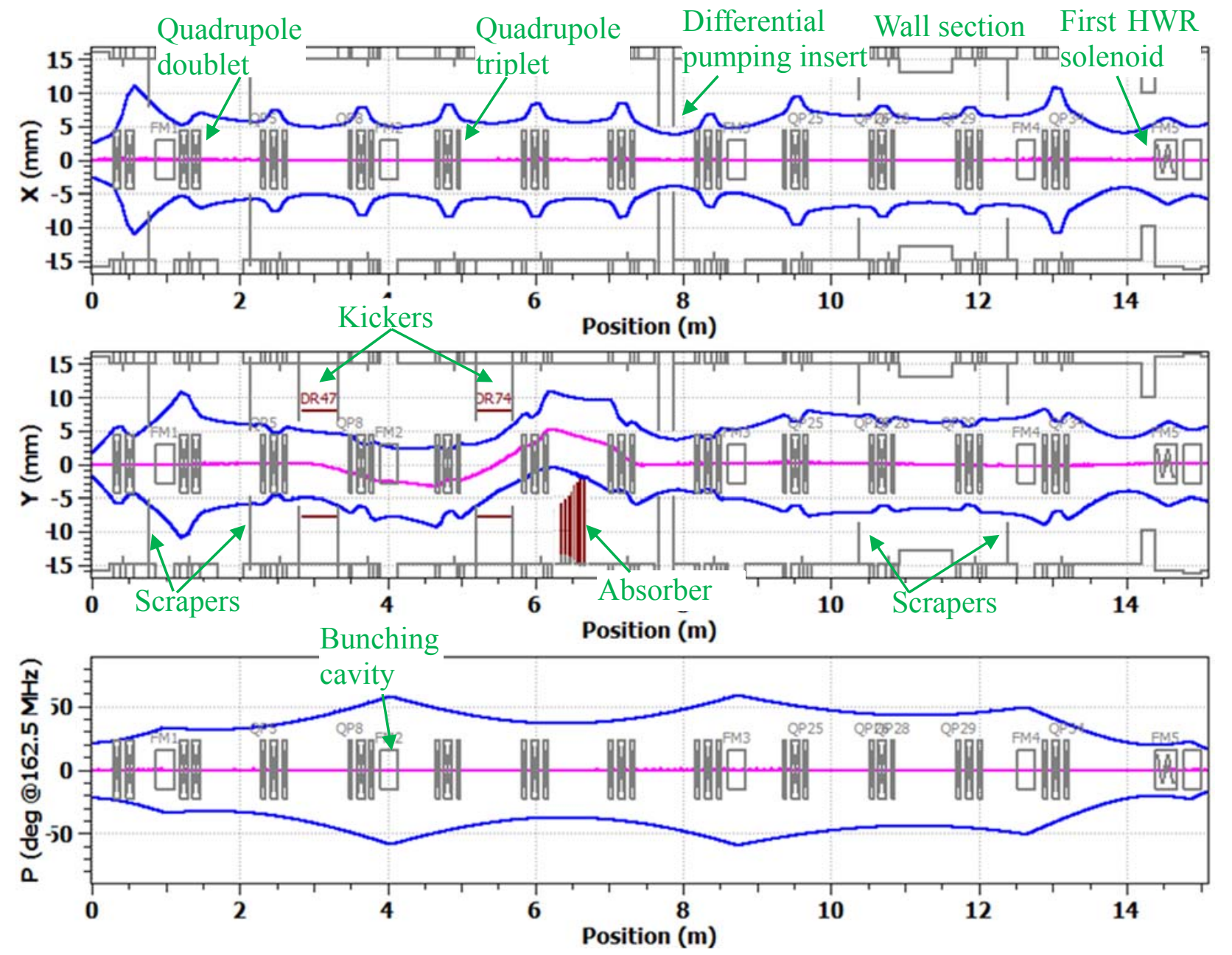

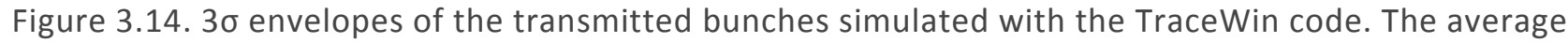
beam current is $5 \mathrm{~mA}$. Zero longitudinal position corresponds to the end of the RFQ vanes. The initial distribution is Gaussian in each of 6 dimensions. The initial transverse emittances are equal, $0.21 \mu \mathrm{m}$, and the longitudinal emittance is also $0.28 \mu \mathrm{m}$ (both rms, normalized).

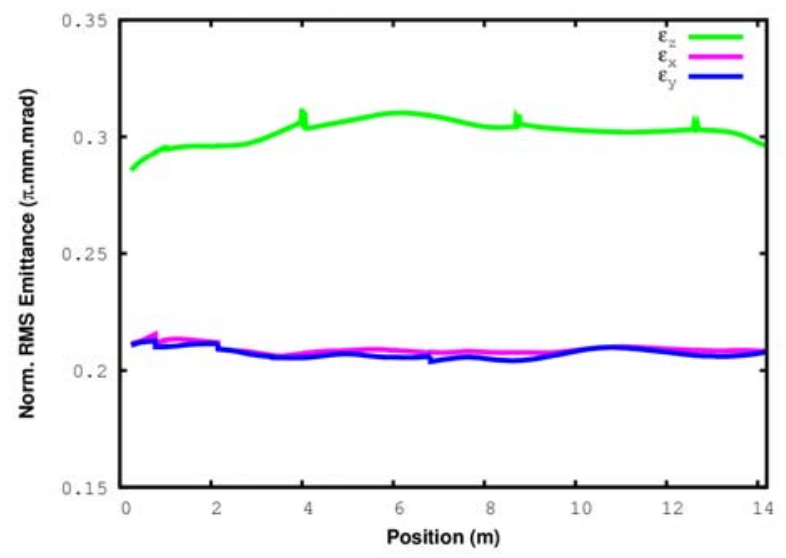

Figure 3.15. Simulated dynamics of the normalized rms beam emittance along the MEBT. 

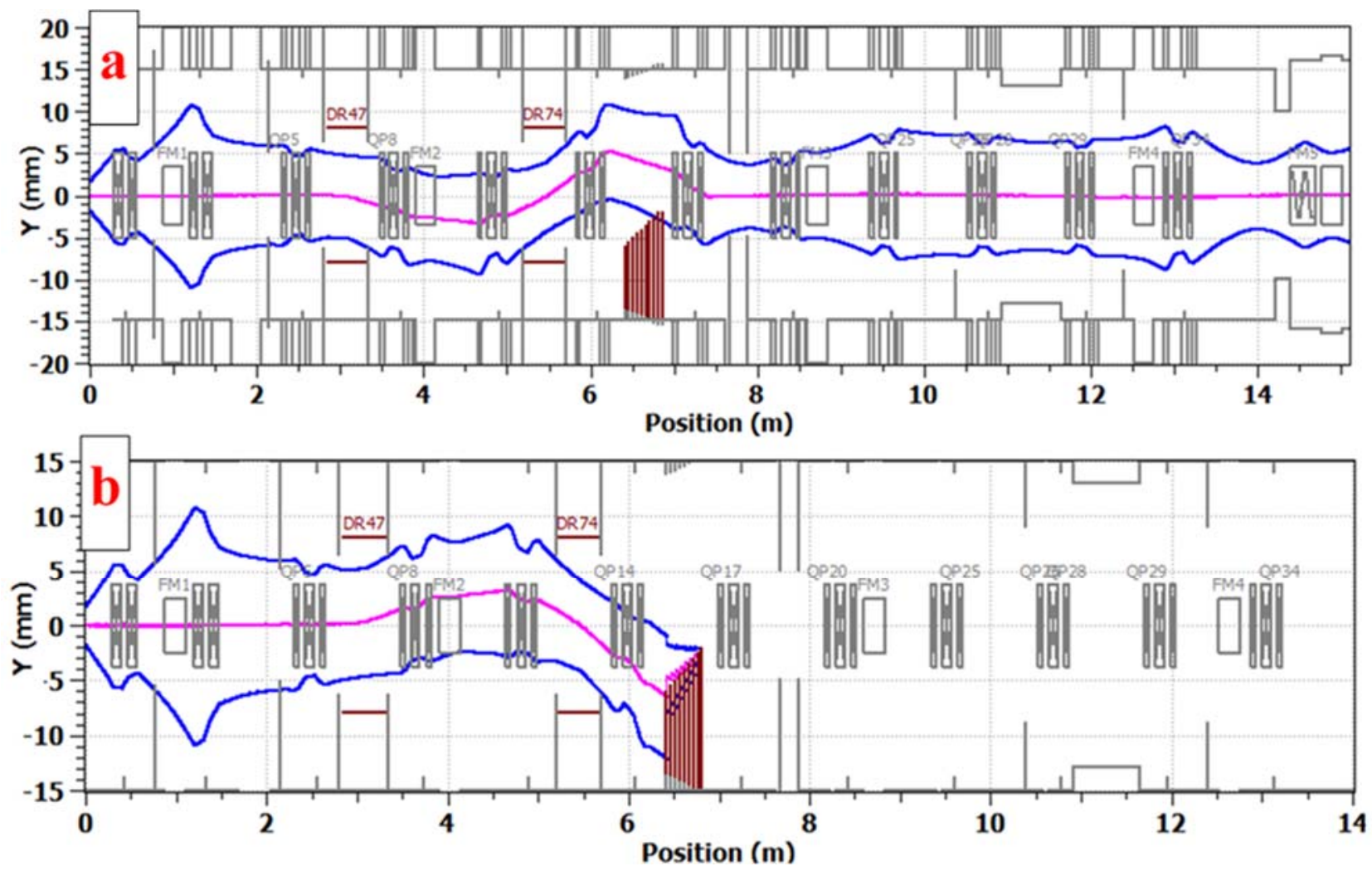

Figure 3.16. $3 \sigma$ envelopes of the transmitted (a) and chopped-out (b) bunches simulated with TraceWin. The top plot is identical to the middle plot of Figure 3.14 and shown for comparison. For the top picture (a), voltages on the top and bottom plates of the first kicker are $-250 \mathrm{~V}$ and $+250 \mathrm{~V}$, correspondingly, and opposite for the second kicker, (+250V, -250V). For bottom picture (b), all voltage values are inverted, i.e. (+250V, $-250 \mathrm{~V})$ for the first kicker and $(-250 \mathrm{~V},+250 \mathrm{~V})$ for the second.

\section{Kickers}

Each kicker has a $50 \mathrm{~cm}$ set of electrostatic plates connected by a broadband, travelling-wave structure. In average the transverse electric field of the kicking pulses propagates through the structure with a phase velocity equal to the speed of the $\mathrm{H}^{-}$ions $(20.0 \mathrm{~mm} / \mathrm{ns}, \beta=0.0668)$. Specifications for the kicker can be found in Ref. [65]. The top and bottom parts of the kicker are powered from pulse generators with voltages of opposite polarity which doubles the kick value. The required voltages are $\pm 250 \mathrm{~V}$. To minimize the emittance growth the electric field, co-propagating with a bunch, has to be uniform within about $10 \%$ along the length corresponding to $6 \mathrm{rms}$ bunch lengths $(1.3 \mathrm{~ns}$ or $26 \mathrm{~mm})$. That requires the voltage for pulses flat-top/bottom to be constant within $25 \mathrm{~V}$. The bunches are separated by a period of the $162.5 \mathrm{MHz}$ RFQ frequency. The corresponding distance, $123 \mathrm{~mm}$, is much larger than the gap between the kicker plates, $16 \mathrm{~mm}$, and, therefore, the electric field deflecting one bunch does not directly affect other bunches.

Based on the experience at other labs (see, for example, [66]), special attention is paid to the survival of the kickers in real operational conditions, where errors are unavoidable. First, the design is specified to withstand a steady-state heating by $20 \mu \mathrm{A}$ beam loss $(0.4 \%$ of the nominal $5 \mathrm{~mA}$ beam $)$ and an accidental loss of $20 \mathrm{~J}(2 \mathrm{~ms}$ at $5 \mathrm{~mA})$. Second, the kicker aperture of $16 \mathrm{~mm}$ is limited to 13 $\mathrm{mm}$ by electrically-isolated protection plates installed on both sides of the kicker so that in the case of mismatched transport or steering error, the beam current intercepted on these plates would trigger the MPS (Machine Protection System) to switch-off the beam. 
Presently two versions of the kicker are being investigated [67]. In this document, they are referenced by the characteristic impedance of their travelling wave structures: $50 \mathrm{Ohm}$ and $200 \mathrm{Ohm}$.

The primary candidate is the $50-\mathrm{Ohm}$ version, where the kicker plates are connected in vacuum by cable delay lines (the purple loops in Figure 3.17a).

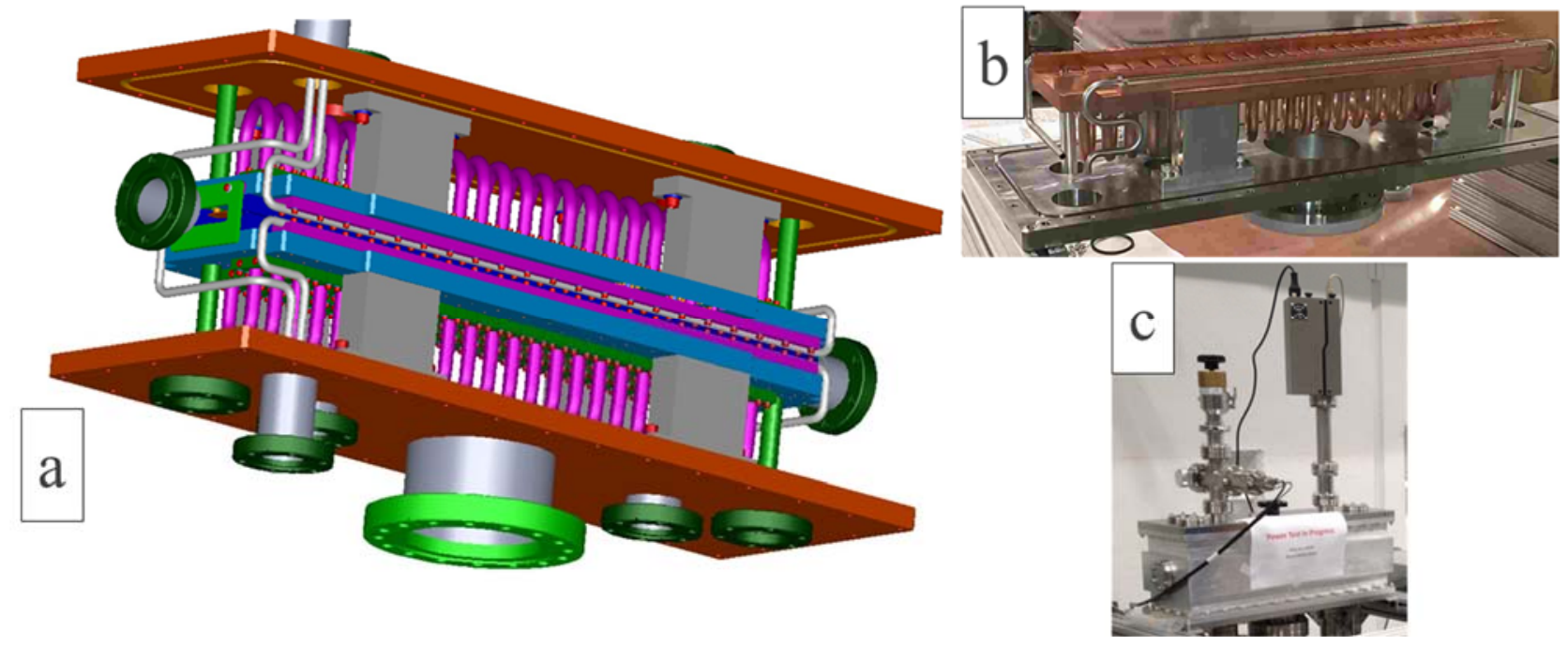

Figure 3.17: (a) 3-D model of the 50-Ohm kicker structure (side walls of the vacuum box are removed for presentation purpose). (b) One plate of the kicker during assembly. (c) Kicker under power testing.

Each kicker is driven by two commercially available linear amplifiers. Because these amplifiers are $\mathrm{AC}-$-coupled, the voltage applied to each plate is bipolar with zero average over a $162.5 \mathrm{MHz}$ period. Signal distortion caused by the imperfections of the amplifier characteristics, cabling, and dispersion in the kicker structure are corrected by a corresponding pre-distortion of the amplifier input signal. The scheme was successfully tested with a similar amplifier of a lower power as shown in Figure 3.18 from Ref. [67].

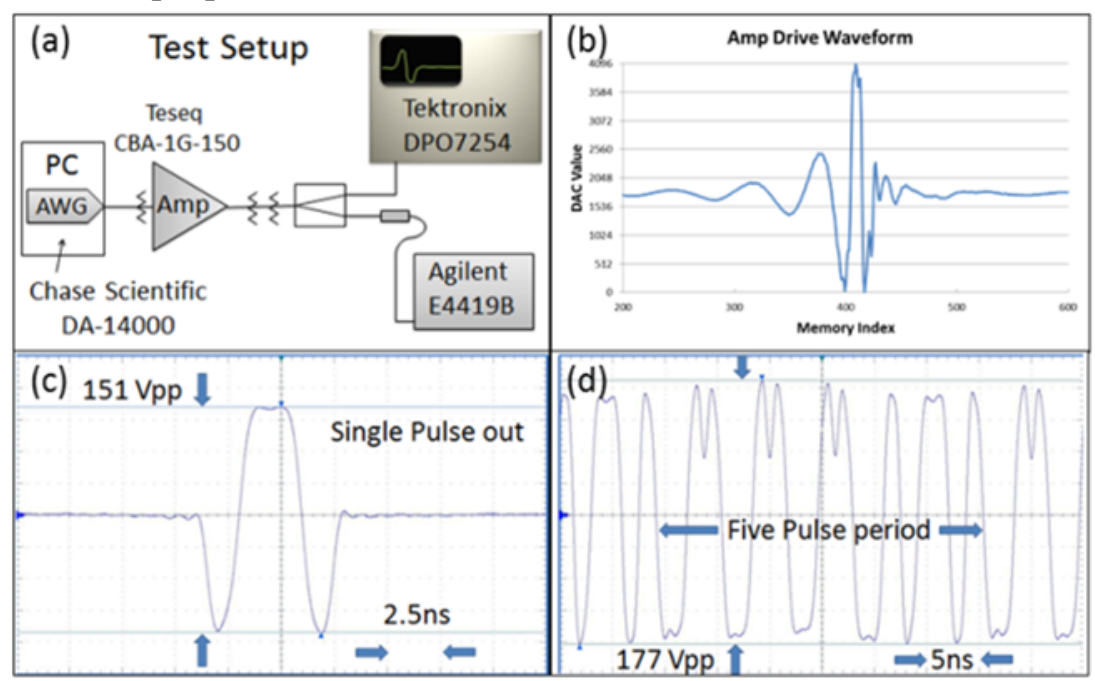

Figure 3.18: Test of the CBA 1G-150 amplifier with pre-distortion. (a) a schematic of the test; (b) the pre-distorted input signal and (c) the corresponding output signal for a single pulse; (d) output for a CW pattern, corresponding to removal of four consecutive bunches followed by a one-bunch passage.

The 200-Ohm travelling-wave structure is a helical winding around a grounded cylinder with plates attached to the windings (Figure 3.19). 
The main idea for this scheme is that the high impedance decreases the power requirement for the driver to a level where a state-of-the-art fast switch can be developed to drive the kicker. Since this driver is DC-coupled, the pulse can remain unipolar during each $162.5 \mathrm{MHz}$ period. Consequently, the 500-V transitions between chopped-out and pass-through states do not require a second part of the pulse with the opposite polarity, thus reducing requirements to the duration of the rise and fall times. It also significantly simplifies the requirements to the dispersion of the travelling-wave structure (e.g. the non-linearity of phase response with frequency). An example of an output pulse of a prototype driver is shown in Figure 3.20. Note that because of the non-standard impedance, custommade feedthroughs, transmission lines, and current loads have to be developed for this scheme.
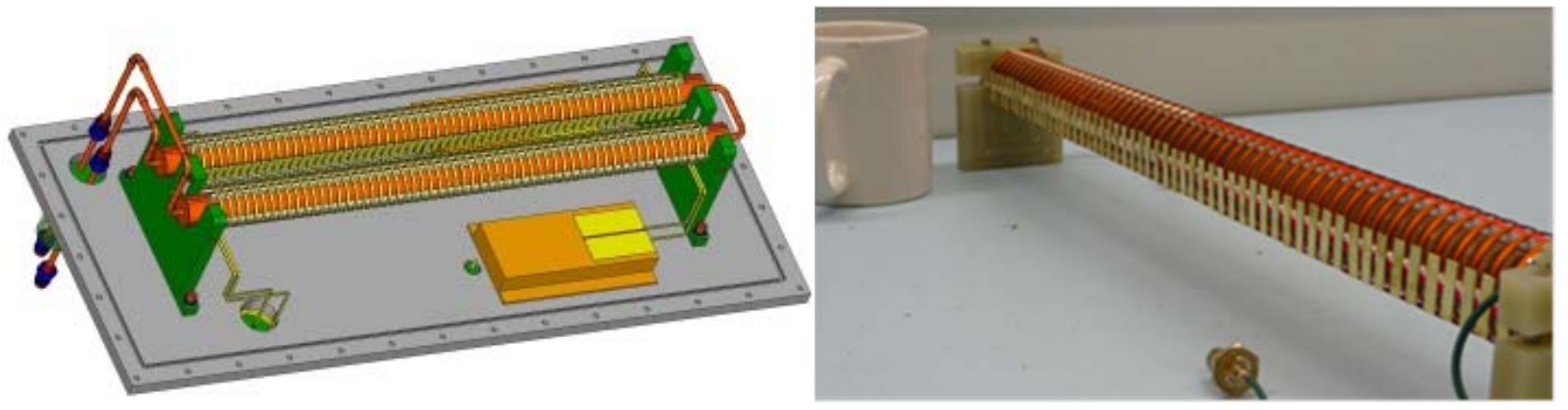

Figure 3.19: Conceptual design (left) and photograph (right) of a single-helix model of the 200-Ohm dual-helix kicker.

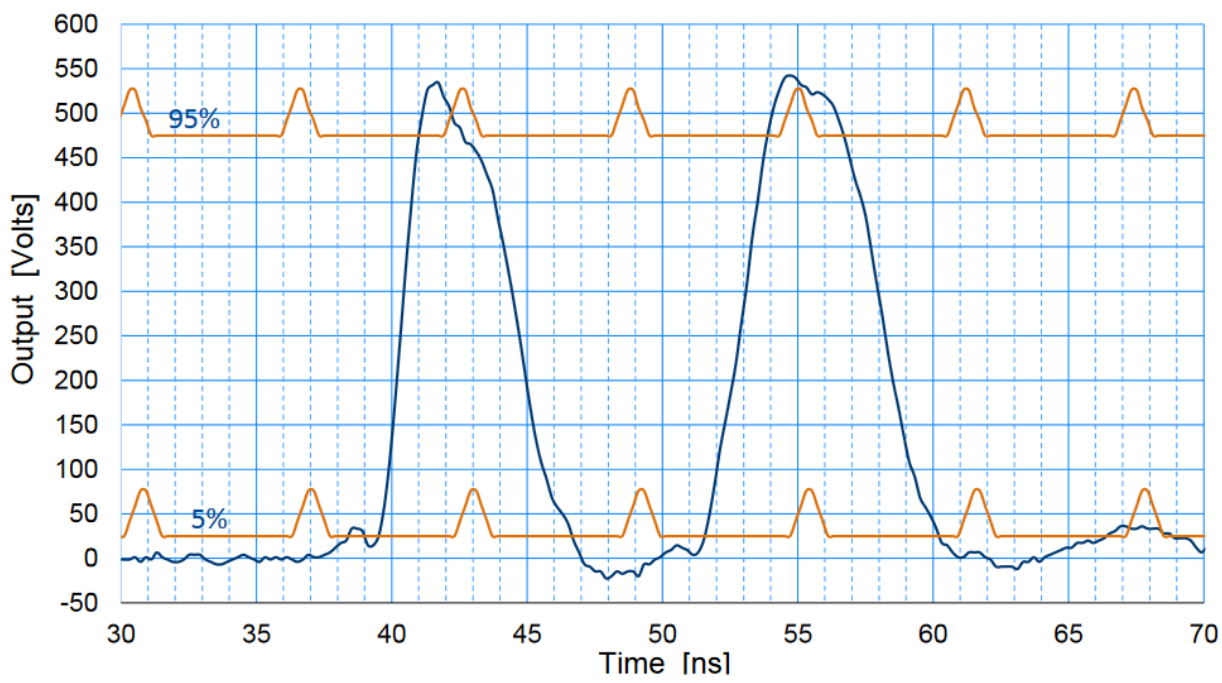

Figure 3.20: An example of an output pulse of a prototype driver being developed at Fermilab. The blue curve is the measured output voltage of the fast switch shaped to remove two bunches out of a $\mathrm{CW}$ sequence. The orange lines indicate the $\pm 5 \%$ tolerance boundary for the voltage stability and mark the assumed position of the bunches and their $6 \sigma$ length.

Development of both versions has not yet found any showstoppers. Prototypes of each kicker went successfully through high-power (thermal) tests and low-power RF measurements. The 50-Ohm kicker development has advanced further, and the amplifiers are available for purchase. The 200Ohm kicker scenario is potentially cheaper, although the driver is still in the R\&D phase, and its CW performance has not been demonstrated. Decision of which version to choose for the final implementation at PIP-II will be based on the results of tests at PIP2IT. 


\section{Absorber}

The undesired bunches are directed onto an absorber that is displaced vertically from the beam line axis. To accommodate the entire beam that the RFQ can deliver, the absorber is specified in Ref. [68] for a maximum beam power of $21 \mathrm{~kW}$ (a 10-mA CW beam completely diverted to the absorber). The power density in the beam with a $\sim 2 \mathrm{~mm}$ rms radius exceeds by an order of magnitude what is technically possible to intercept at a normal angle of incidence without melting the surface. To decrease the surface power density, the absorber is positioned at a small angle $(29 \mathrm{mrad})$ with respect to the beam (Figure 3.21).
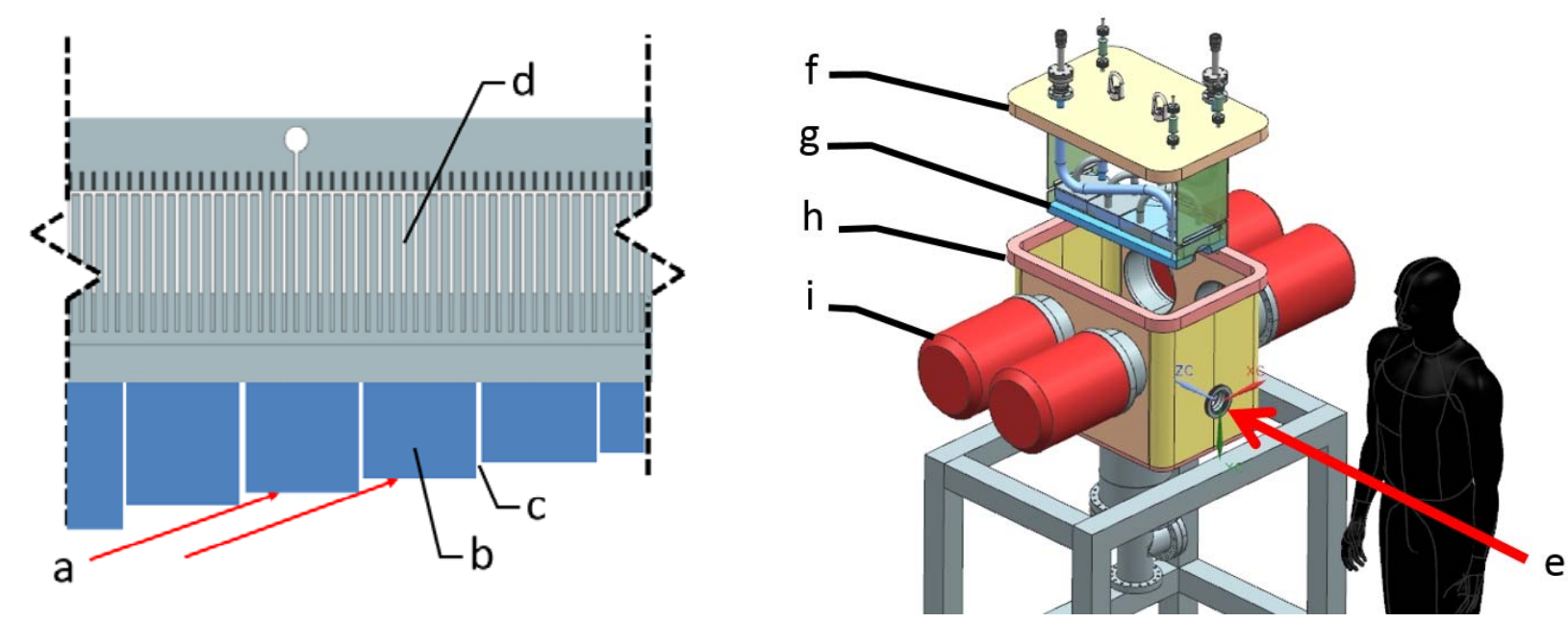

Figure 3.21: A conceptual design of the MEBT absorber. Left: a side-view cross section of the absorber showing (a) the beam incident on the surface, (b) the segmented absorber blocks, (c) the shadowing step increment (magnitude exaggerated), (d) the $300 \mu \mathrm{m}$ wide water cooling channels with $1 \mathrm{~mm}$ pitch. Right: an exploded view of the absorber assembly showing (e) the incoming beam, (f) the flangemounted absorber subassembly, (g) the absorber blocks, (h) the enclosure with provisions for secondary particle absorption, (i) the turbo pumps.

Challenges in the absorber design include spreading the energy deposition, managing surface effects (sputtering and blistering), containing secondary and reflected particles, accommodating radiation effects, maintaining vacuum quality, and survival at high temperatures with temperatureinduced mechanical stresses. Presently the design choice is an absorber with an absorbing surface composed of multiple absorber blocks made of the molybdenum alloy TZM and preloaded against a water-cooled aluminum strongback [69]. In comparison with an initially considered monolithic design, this solution slightly increases the thermal conductance from the absorber surface to water channels but dramatically decreases the chance for catastrophic failure if a crack developed at the absorbing surface propagates all the way to the water channels. Power management in a $1 / 4$-size prototype of such design was successfully tested on an electron-beam test stand [69].

In CW mode, the beam stopped at the absorber delivers a quite large volume of hydrogen as well as additional degassing from the receiving surface of the absorber. If not addressed, this can spoil the vacuum in the MEBT. To suppress $\mathrm{H}^{-}$stripping and to reduce the gas load to the downstream cryomodule, the vacuum in the vicinity of the absorber should be less than or about $2 \cdot 10^{-7}$ Torr. The vacuum is supported by four turbo-pumps installed at the absorber enclosure and the differential pumping pipe installed in the section \#6. 


\section{Scraping System}

Each of the sections \#0, 1, 8, and 10 in Figure 3.12 contains a set of 4 scrapers (Left, Right, Top, and Bottom), totaling 16 plates. A scraper is an electrically-insulated, 75W-rated TZM plate movable across the 30-mm MEBT aperture [70]. The scrapers are used for several purposes: (1) for beam halo measurements and removal, (2) protection of downstream equipment from a beam loss caused by beam envelope and trajectory mismatches, (3) as auxiliary beam density distribution diagnostics, and (4) for formation of a pencil $\mathrm{H}^{-}$beam for measurements downstream. The last two items are associated with the short-pulse mode of operation. The scraper sets in each of the upstream and downstream pairs are separated by a phase advance of $\sim 90^{\circ}$ to ensure effective removal of particles with large transverse actions.

A simulated example of the protection the scrapers provide is shown in Figure 3.22. The simulation considers a mis-phase of the kickers, so that the voltages on all kicker plates are half of the nominal value required for chopping bunches out. In this scenario a significant portion of the beam misses the absorber but is intercepted by a scraper downstream. Consequently, in high power operation this will result in a beam trip by the MPS.

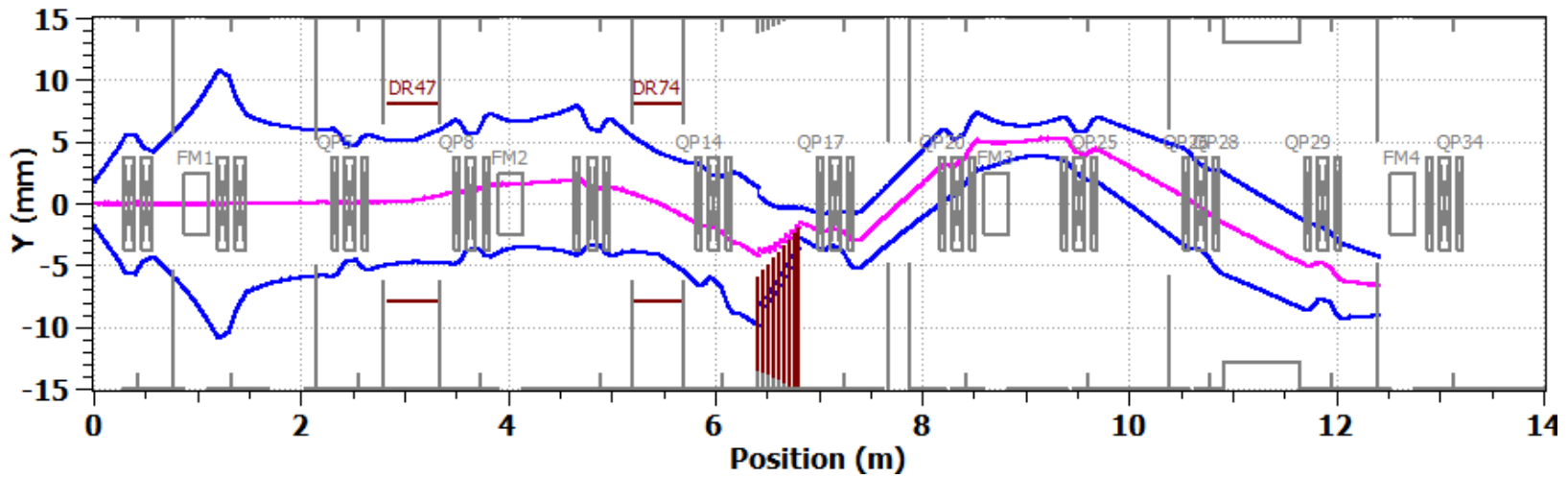

Figure 3.22: Beam Y 3б envelope with voltages on all kicker plates equal to half of the nominal value required for chopping bunches out.

In CW mode, the beam stopped at the scrapers delivers hydrogen and results in degaussing from their surfaces. This scraper irradiation can also generate dust particles due to blistering and sputtering. Considering this, the design assumes that steady-state scraping removes $\sim 1 \%$ of every bunch with the first two scraper sets and $\sim 0.1 \%$ with the third and fourth.

\section{MEBT Bunching Cavities}

To keep the beam bunched and to match its longitudinal phase space to the first superconducting cryomodule, the MEBT includes four identical room-temperature bunching cavities. The functional requirements of the cavities are specified in Ref. [63].

One of the main challenges for the RF design was low operating frequency $(162.5 \mathrm{MHz})$ combined with limited allowed space which flange-to-flange length should not exceed $35 \mathrm{~cm}$. These requirements dictated a choice of quarter-wave principal design for the bunching cavities (see Figure 3.23 .

The power couplers and plunger tuners previously manufactured for the HINS [71] cross-bar CH cavity were used. This power coupler was designed to supply $75 \mathrm{~kW}$ power, that significantly exceeds the maximum expected power of $4 \mathrm{~kW}$. To compensate eventual manufacturing errors a rotatable flange welded into a stainless steel transition allows an independent adjustment of coupling. An alumina ceramic window is utilized to separate cavity vacuum from surrounding air. The custom 
made alumina ceramic window was brazed to two copper sleeves that later were brazed to the inner and outer conductors of the coupler. The motor-driven HINS tuners have the full stroke (the plunger active length) of about $42 \mathrm{~mm}$, which is again more than sufficient to meet tuning range requirement for the bunching cavities.

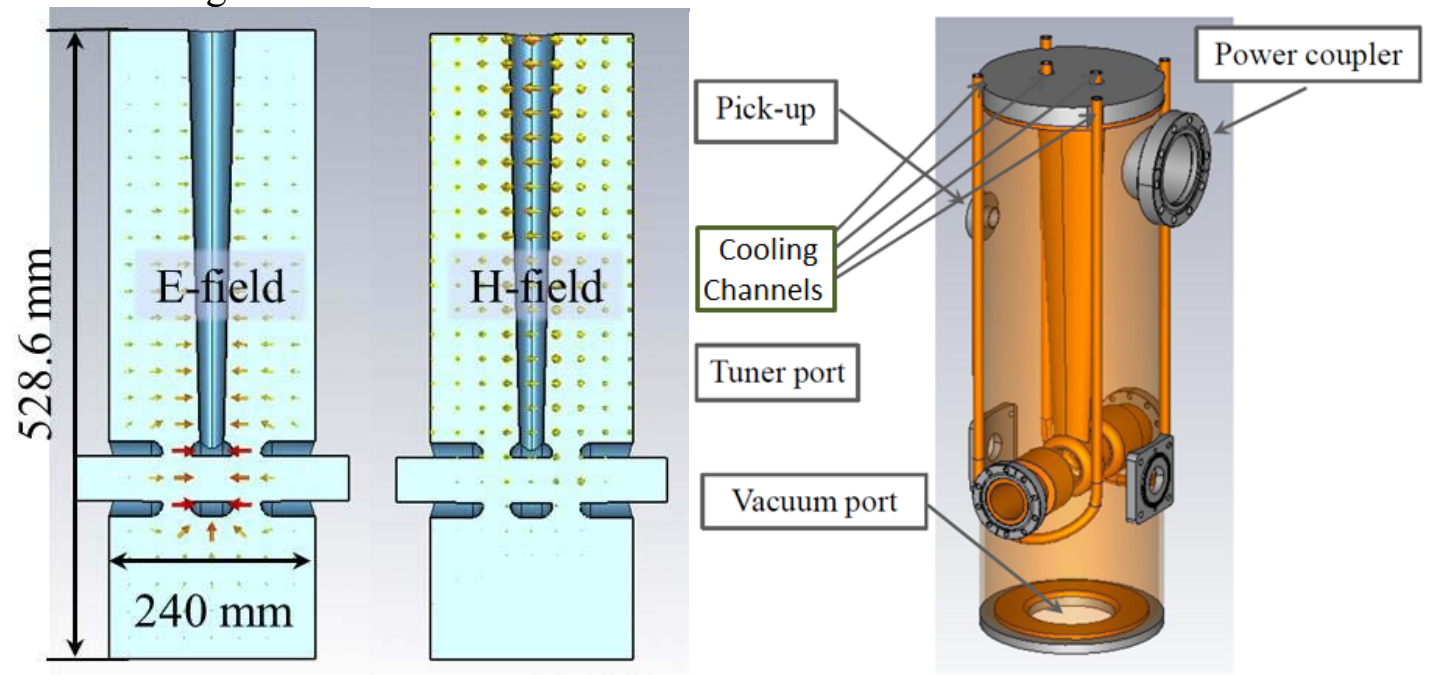

Figure 3.23: Principal RF design and the CST solid model of the bunching cavity.
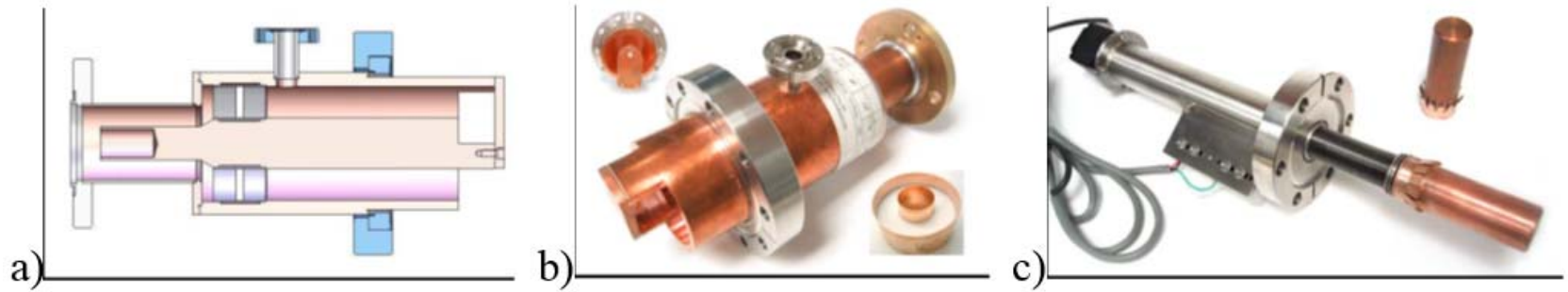

Figure 3.24: a) Principal design of the power coupler; b) Finished input power coupler; c) One of two tuners used on the buncher; Be-Cu contacts are welded to the removable copper fingers, hard stops and limit switches define the stroke, a linear actuator with captive shaft is used to operate the tuner.

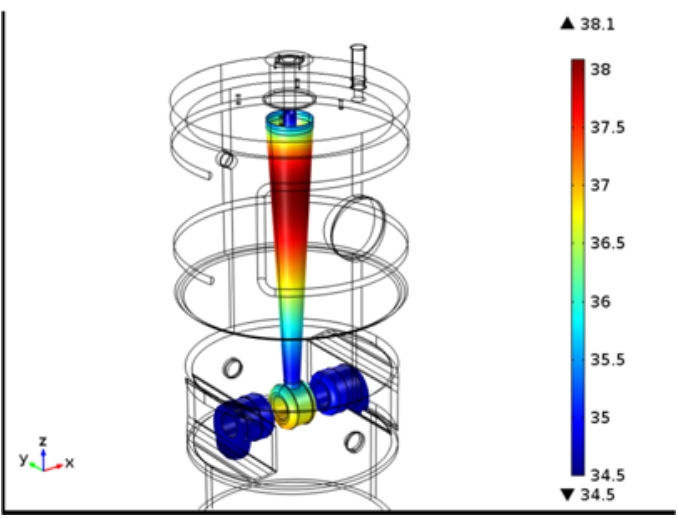

Figure 3.25: Temperature map on the stem from 3-D thermal study.

Matching simulations, preliminary thermal analyses for the power coupler and an estimate of the plunger tuning range have confirmed that the HINS parts can be used [64] (see Figure 3.24). Their usage noticeably reduced the design and manufacturing efforts.

The total power loss in the cavity walls at the effective voltage of $80 \mathrm{kV}$ (nominal $-70 \mathrm{kV}$ ) is $\sim 1200 \mathrm{~W}$ with $\sim 850 \mathrm{~W}$ dissipated in the central stem. A traditional cooling approach was used for 
cooling the cavity body. To remove the power deposited in the central stem, a special coaxial counterflow cooling scheme was developed for the stem [72]. The thermal study and the mock-up experiments confirmed effectiveness of the developed cooling scheme (see Figure 3.25)

At time of writing this document, one of four cavities is already manufactured and is installed at the PIP2IT. Its view is presented in the Figure 3.26. The cavity successfully passed high power tests. Table 3.2 presents a comparison of the functional requirements specifications (FRS) for bunching cavities and the measurement results.
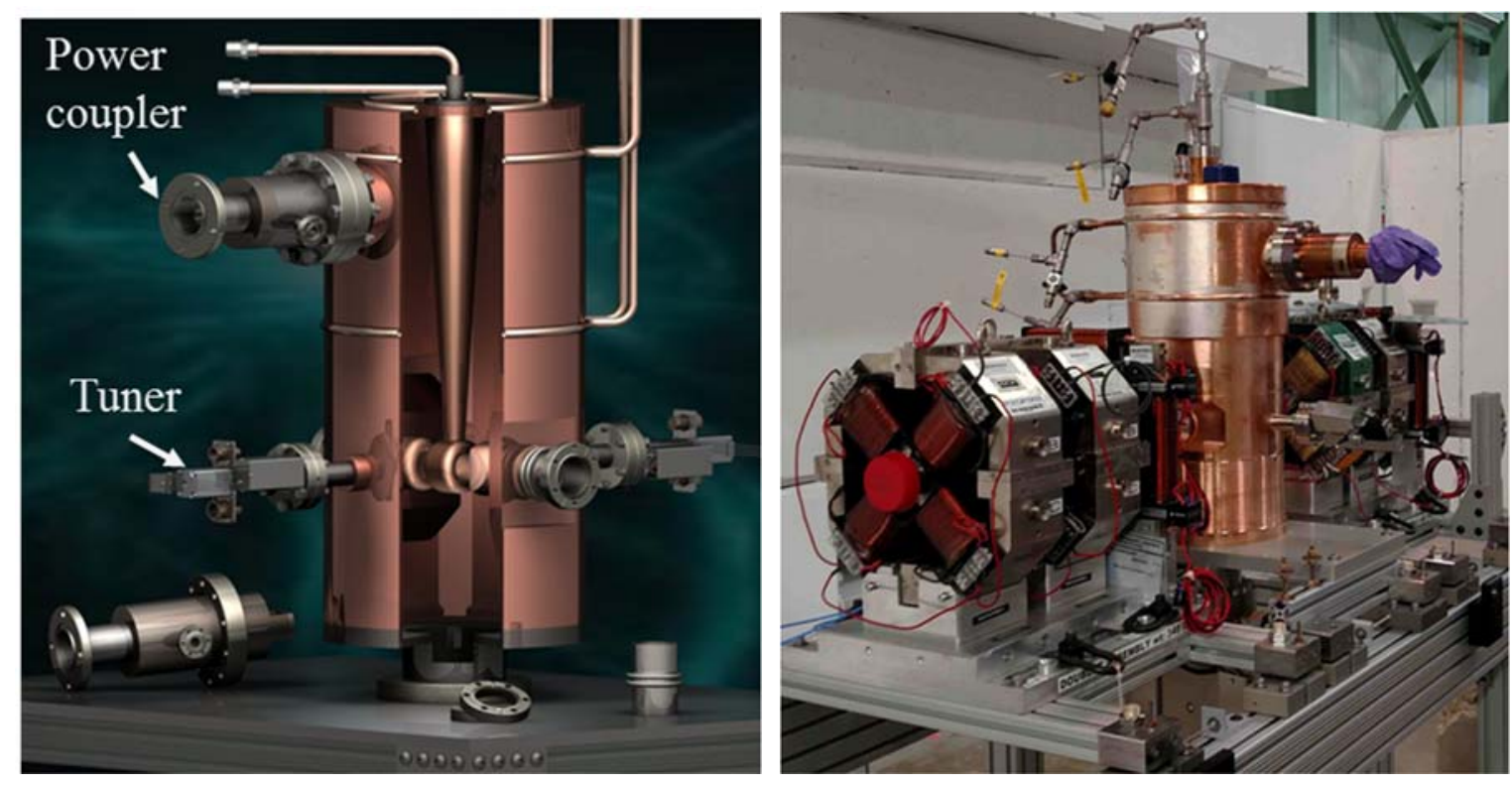

Figure 3.26: Mechanical solid model of the buncher showing the interior of the cavity and the first buncher installed in its place in the beam line of PIP2IT.

Table 3.2: Comparison of the bunching cavities FRS with measurements

\begin{tabular}{l|c|c}
\hline \hline Parameter & Measurements & FRS \\
\hline Frequency, MHz & 162.5 & 162.5 \\
\hline Q-factor & 10030 & 10000 \\
\hline Aperture diameter, $\mathrm{mm}$ & 30 & $>30$ \\
\hline Gap, $\mathrm{mm}$ & $2 \cdot 33$ & - \\
\hline Shunt impedance, $\Omega$ & $5.3 \cdot 10^{6}$ & $5.0 \cdot 10^{6}$ \\
\hline $\mathrm{R} / \mathrm{Q}, \Omega$ & 503 & 500 \\
\hline Effective voltage, $\mathrm{kV}$ & 70 & 70 \\
\hline Power loss in cavity walls, $\mathrm{kW}$ & 1.8 & $\leq 2.2$ \\
\hline Frequency tuning range, $\mathrm{kHz}$ & 440 & 100 \\
\hline Flange-to-flange length, $\mathrm{m}$ & 0.35 & $\leq 0.35$ \\
\hline \hline
\end{tabular}




\subsubsection{Superconducting Accelerating Structures}

The parameters and requirements associated with all of the accelerating structures and cryomodules within the linac are summarized in Tables 2.4, 2.5 and 2.6. This section describes design concepts for the cavity types required in the linac, and the associated cryomodules.

\subsubsection{Half-Wave Resonator (HWR) Cryomodule}

After careful consideration a design based on 162.5-MHz Half-Wave Resonator (HWR) cavities was selected. This design has several substantial advantages with respect to $325 \mathrm{MHz}$ technology used for the downstream portion of the linac:

- Substantially reduces the cavity count. Only 8 HWRs are required to accelerate the beam to $\sim 10 \mathrm{MeV}$.

- Reduces RF transverse defocusing. The lower synchronous phase results in a much faster energy gain without emittance growth.

- Opens the possibility to use $162.5 \mathrm{MHz}$ bunchers in the MEBT enabling longer distances between the latter.

- Cost reduction due to the reduced component count.

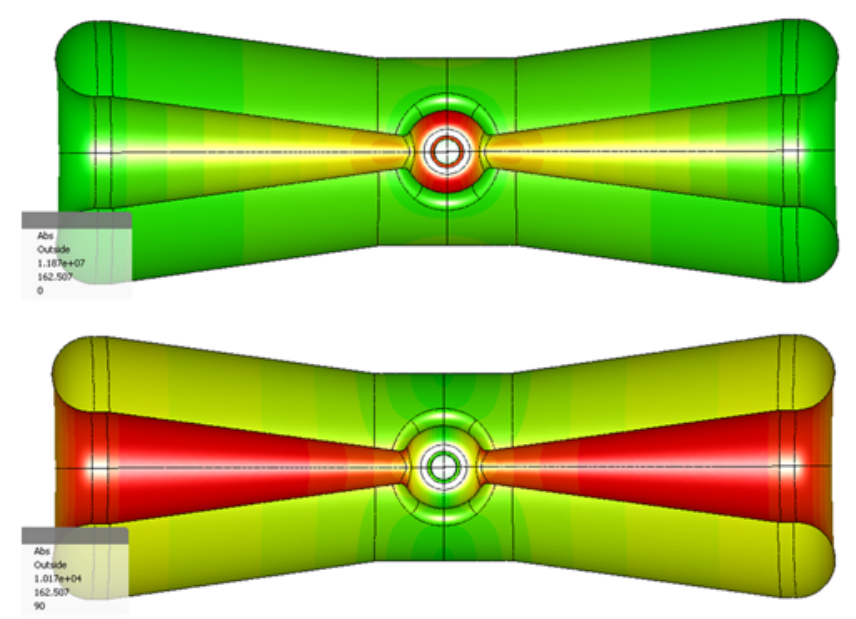

Figure 3.27: Half-wave resonator model in Microwave Studio (MWS). The picture shows electric (top) and magnetic (bottom) field distributions on the surface. Red and green colours correspond to high and low intensities, respectively [73].

Beam dynamics optimization determined the optimal beta of $\beta_{o p t}=0.112$. The cavity design is based on recent advances in SRF technology for TEM-class structures being developed at ANL. Highly optimized EM parameters which maximize the real-estate gradient while maintaining low dynamic cryogenic loads and peak surface fields were achieved using a conical shape for both the inner and outer conductors. A "donut" shaped drift tube in the center conductor (see Figure 3.27) has been developed to minimize the undesirable quadrupole component of the electric field presented in Figure 2.27. Utilization of the HWR requires two major sub-systems: a $10 \mathrm{~kW}$ RF coupler and a slow tuner.

A capacitive adjustable $10 \mathrm{~kW}$ RF coupler prototype has been designed, constructed, and successfully tested. Figure 3.28 shows the $10 \mathrm{~kW} \mathrm{RF}$ coupler attached to a half-wave resonator on a 2" port located in the plane of the beam ports at $90^{\circ}$ to the beam direction. Figure 3.29 shows the coupler performance with full reflection up to $5 \mathrm{~kW}$ - the design goal. However, it is important to note that the coupler bellows and the cold window have previously been tested at up to $9 \mathrm{~kW}$ with 
full reflection. A DC voltage bias will be used to suppress multipacting in the entire range of operating power.

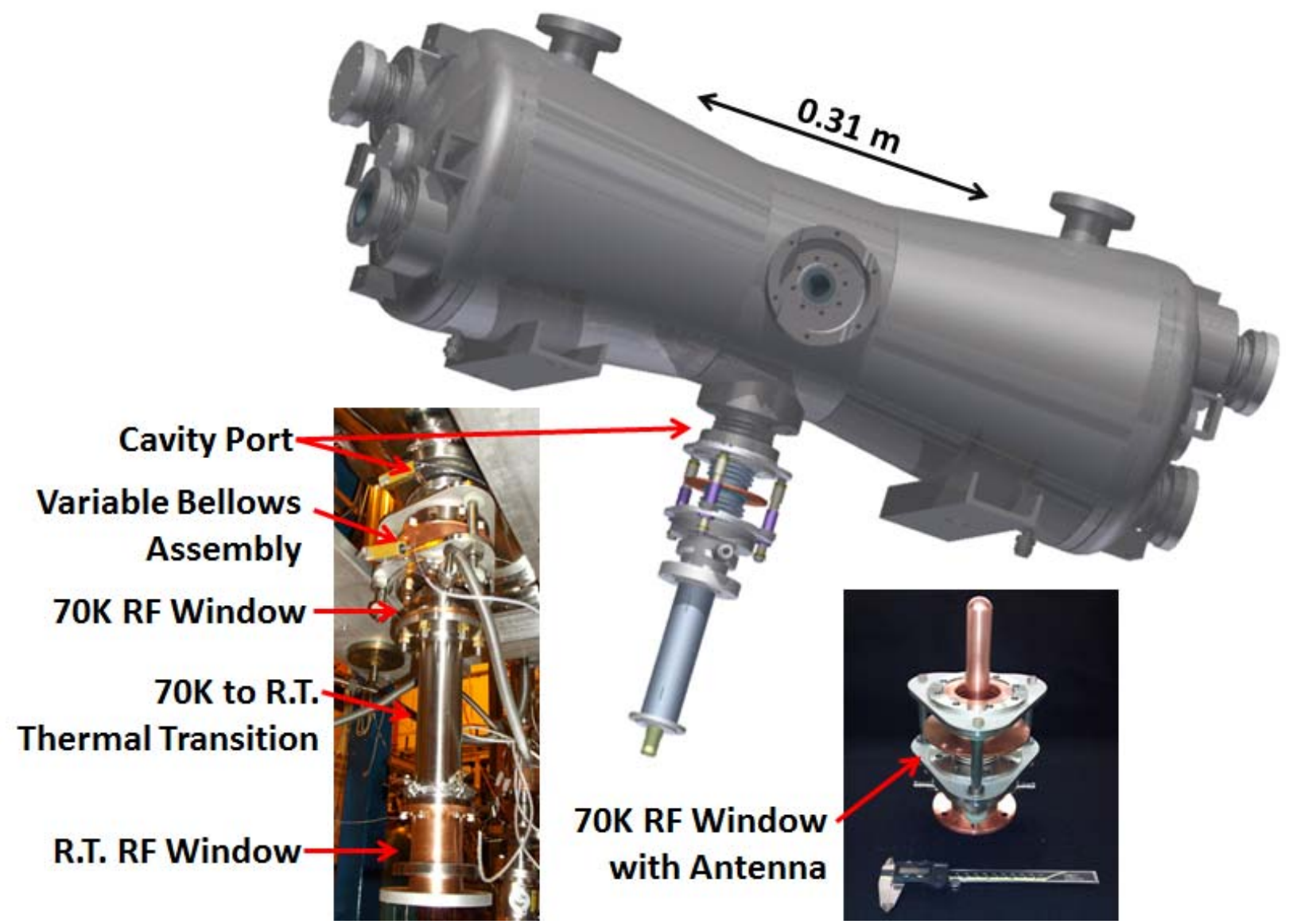

Figure 3.28: HWR cavity 3D model in INVENTOR with coupler hardware, insets. R.T. = room temperature.

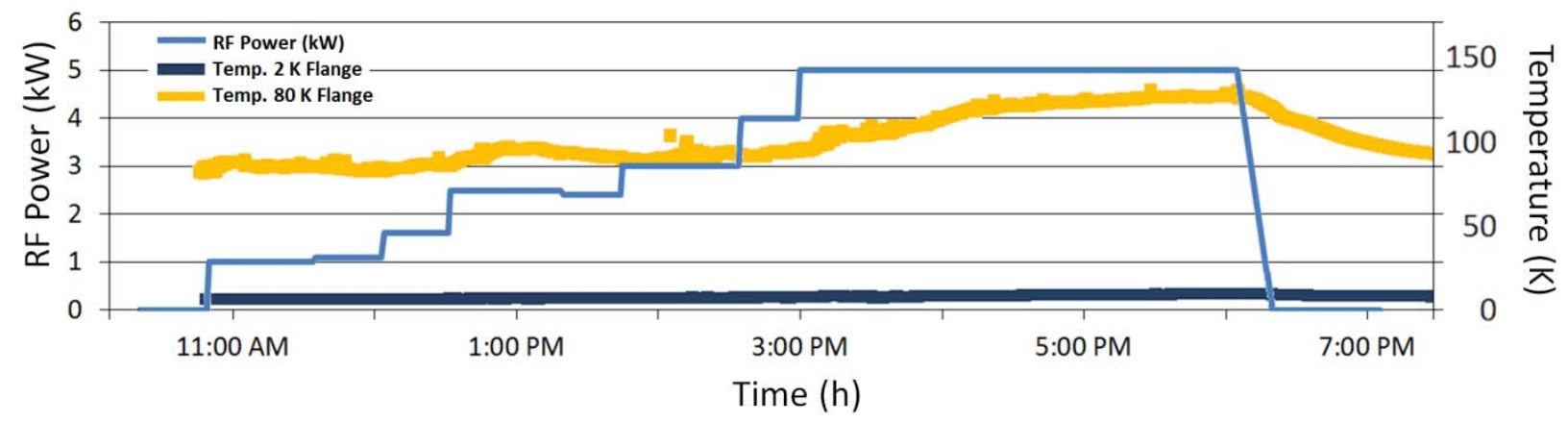

Figure 3.29: Cold testing results from running the RF coupler up to $5 \mathrm{~kW}$, the design operating power level and operating conditions. During testing multipacting between $500 \mathrm{~W}$ and $5 \mathrm{~kW}$ conditioned easily and did not limit the RF power rise.

The first production half-wave resonator with slow tuner installed is shown in Figure 3.30. The slow tuner is a pneumatically actuated device which compresses the cavity at the beam ports. The slow tuner shown in Figure 3.30 is based upon multiple prototype tests conducted at both room temperature and $4 \mathrm{~K}$. Design changes implemented in this unit include the elimination of the bushing/rod assemblies which can bind and gall. The test of the assembly verified the slow tuner 
design and the cavity frequency tuning calculations, and determined its $2 \mathrm{~K}$ performance. Previous testing and evaluation of the microphonic RF noise found the cavity dynamic detuning to be $<5 \mathrm{~Hz}$, thus a fast tuner is not required. The RF power margin (see Table 2.10) was chosen to be sufficient to control microphonic induced RF phase noise (mainly related to helium pressure fluctuations) without a fast tuner at the nominal cavity loaded bandwidth of $66 \mathrm{~Hz}$. The main parameters of the HWR are shown in Tables 2.4 - 2.6.
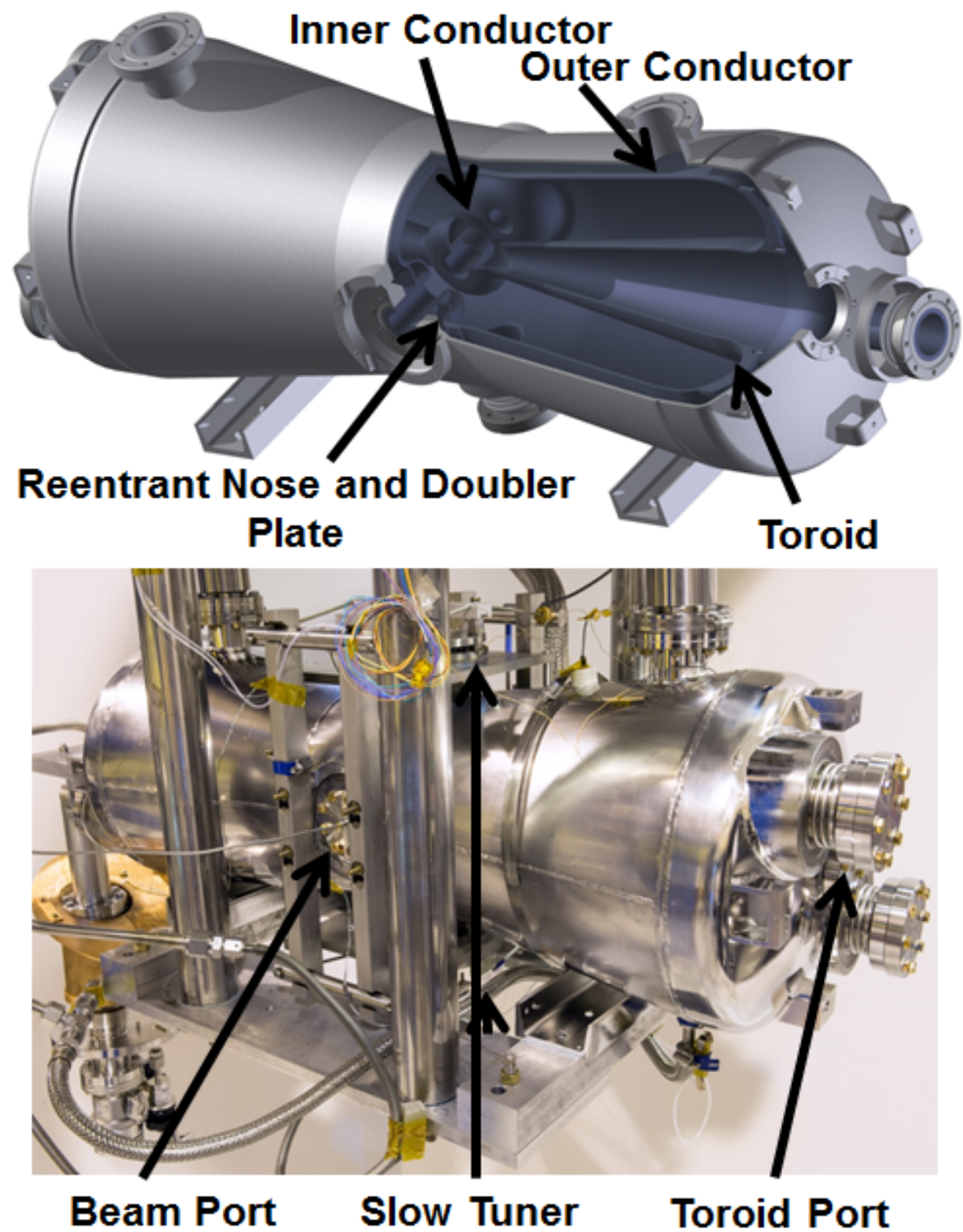

Figure 3.30: Top, 3D cut-away view of the half-wave cavity with stainless steel helium jacket. Bottom, a finished half-wave resonator with tuner ready for testing. The coupler is not visible.

The cavity mechanical design was supported by Autodesk Inventor and ANSYS software. The complete niobium cavity integrated with the stainless steel helium vessel geometry was modelled to analyze the system response to fluctuations in helium system pressure and the appurtenance loading of the slow-tuner, and to verify the safety of the design. Figure 3.30 shows a cut-away view of the cavity together with an image of the finished hardware.

The regions of the cavity with appreciable surface electromagnetic fields are formed from high- 
purity (Residual Resistance Ratio, RRR > 250) 0.125" \pm 0.010 ” thick niobium sheet and are the cavity outer conductor, inner conductor, toroid ends, re-entrant noses, re-entrant nose doubler plates and coupling port extension tubes. Note that the re-entrant nose doubler plates are made from the same size sheet as the re-entrant noses and both parts are made from RRR $>250$ niobium in order to avoid mixing low-/high-purity sheets in fabrication even though the doubler plates are completely shielded from the cavity electromagnetic fields. The toroid gussets were machined from $1 / 2$ " thick RRR $\sim 25$ niobium plate while the beam ports ( RRR $>250)$, coupling ports $(R R R \sim 25)$ and inner conductor drift tube $(R R R>250)$ were machined from bar stock. The sheet niobium forming and subsequent machining was done by Advanced Energy Systems in Medford, New York, and Adron EDM in Menomonee Falls, Wisconsin. The machining of the bar stock was done by Numerical Precision in Wheeling, Illinois, while the toroid gussets were wire-electrostatic discharge machined (EDM) at Adron EDM.

The integral helium vessels surrounding the niobium cavity are fabricated using joint certified 304/304L stainless steel (SST). Niobium-to-stainless steel braze transitions [74] are utilized for joining the SST helium jacket with the niobium cavity at seven locations: 2 for the beam ports, 1 for the power coupler port and 4 for the toroid coupling ports. The cavity mechanical structure is designed to satisfy FNAL's safety requirements. These requirements include the niobium within the pressure boundary, prohibiting the application of any ASME U-stamp. To provide an equivalent level of safety in the design the analysis uses the techniques and rules set forth by the ASME boiler and pressure vessel code Section VIII, Division 2, Part 5, Design by Analysis with niobium material properties given in [75]. Using this the ASME BPVC was applied to the complete niobium/SST structure to demonstrate protection against failure for a 2 bar Maximum Allowable Working Pressure (MAWP) at $293 \mathrm{~K}$ and a 4 bar MAWP at $2 \mathrm{~K}$; we found that any analysis which passes with the 293 $\mathrm{K}$ material properties and 2 bar MAWP would pass at $2 \mathrm{~K}$, even with the greater MAWP. This is due to the increased low temperature strength of the niobium material. The cavity region with the smallest factor of safety, but still an acceptable one, is the area around the inner conductor drift tube. This region was reinforced by increasing the wall thickness of the solid rod used to fabricate the inner conductor drift tube. The thicker wall reinforced the area ensuring safe operation above the safety analysis requirements. Figure 3.31 shows the cavity niobium strain from a limit-load analysis. The central portion of the inner conductor is the most susceptible to plastic collapse but still exceeds the FNAL safety requirements.

The results of the safety analysis studies showed that no gusseting is required. With this model $d f / d P$ equal to $8 \mathrm{~Hz} / \mathrm{mbar}$ was achieved. This value was determined sufficient for operation and further design modifications to reduce $d f / d \mathrm{P}$ have not been pursued. Simulations of the slow tuner were performed by applying a force to the SS flanges of the helium jacket. For example, a $10 \mathrm{kN}$ force results in a frequency shift of $-120 \mathrm{kHz}$. In operation the slow tuners will be limited by mechanical stops to this frequency range and pose no threat of plastically deforming the half-wave resonators.

The primary operational parameters for the HWR presented in Tables $2.4-2.6$ are based on experience with the ATLAS energy upgrade cryomodule and its long-term operation [76], as well as recent tests of the first HWR cavities [77]. As shown in Figure 3.32, the prototype tests of the two half-wave resonators show $<3 \mathrm{n} \Omega$ residual surface resistance at $48 \mathrm{mT}$, which readily supports the design parameters of the HWRs. Recent measurements of the two first HWR cavities showed Q0's exceeding $1.7 \cdot 10^{10}$ at the operating gradient which corresponds to a surface resistance of $2.6 \mathrm{n} \Omega$. This value is slightly larger than that achieved with the $72 \mathrm{MHz}$ quarter-wave cavities installed in the ATLAS heavy-ion linac in 2014 and it is a more optimistic value for the surface resistance if one 
takes into account the 2.2 times increase in the operating frequency. Thus, the measured Qo's provide a margin of more than 3 times relative to a conservative value of $\mathrm{Q}_{0}$ presented in Table 2.6. Note that the ATLAS cavities were measured in a real cryomodule while the HWR cavities were characterized in an off-line test stand. However, the experience accumulated in recent years assures us that there is no significant $\mathrm{Q}_{0}$ increase when a cavity is installed in an Argonne cryomodule.

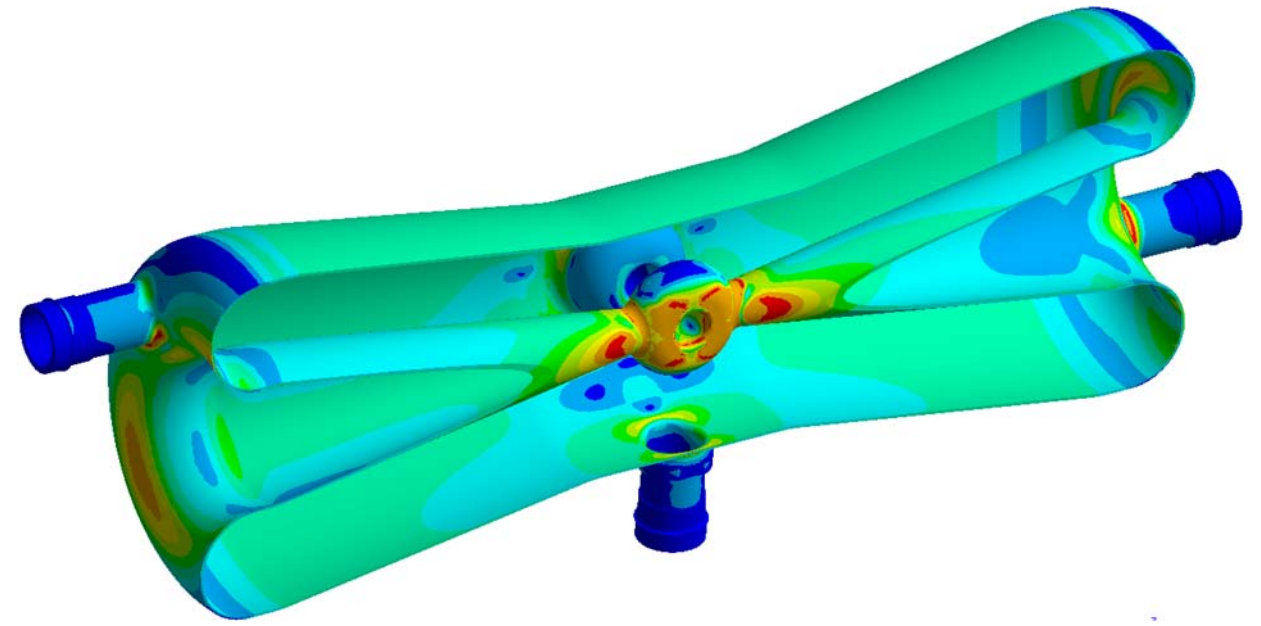

Figure 3.31: Niobium material strain from a limit-load analysis of the half-wave cavity. Areas of high strain are around the ports and in the center of the inner conductor where the beam passes. No strain values are given due to the unrealistic material properties used in a limit-load analysis.
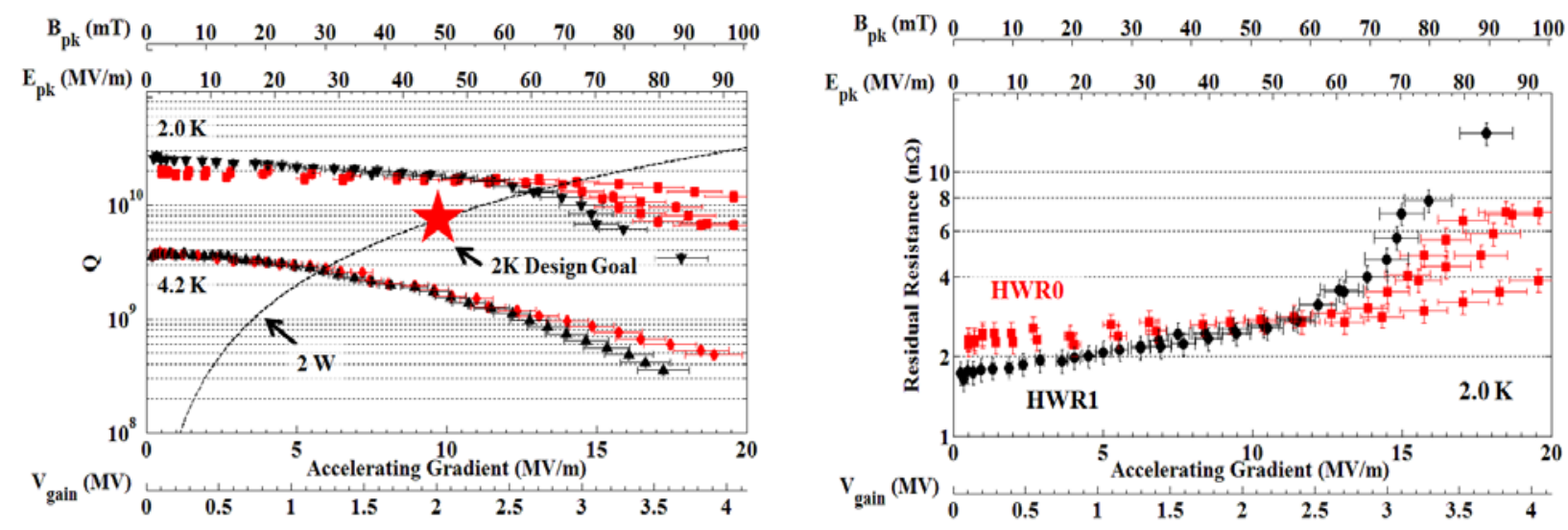

Figure 3.32: Left, the measured $Q_{0}$ vs $E_{\text {acc }}$ for the two half-wave resonator prototypes. Right, the cavity residual resistance corresponding to the $\mathrm{Q}_{0}$ measurements. Note that the operating voltage is $2 \mathrm{MV}$ which corresponds to the peak magnetic and electric surface fields of $48 \mathrm{mT}$ and $45 \mathrm{MV} / \mathrm{m}$, respectively.

The overall design of the half-wave cavity cryomodule is an evolution of the top-loaded box cryomodules used successfully for an energy upgrade of ATLAS in 2009 [78] and for an intensity upgrade of ATLAS in 2014 [79]. The HWR cryomodule is assembled in stages. First, the lowparticulate clean assembly of the cavities, RF power couplers, RF pickup probes, solenoids, beamline gate valves, vacuum manifold and titanium strong-back support structure is carried out in a Class 100 clean room. Then, this assembly is hermetically sealed and removed from the clean room. This separates the "clean" low-particulate beam-line vacuum system assembly from the "dirty" portions of the cryomodule assembly work, preserving, to the best of our ability, the cavity performance. Once out of the clean room the helium distribution system will be installed and the assembly is then hung from the lid of the cryomodule. Once hung, the remainder of the cryomodule subsystems are 
installed such as the slow tuners, instrumentation, alignment targets, solenoid conduction cooled leads, thermal intercepts and RF transmission lines. The complete lid assembly is then lowered into the insulating vacuum vessel completing the cryomodule.

The HWR cryomodule vacuum vessel design balances the need to house a 6 meter long accelerator string with all of its support systems inside the limited space available for assembly while maintaining compliance with FNAL's safety standards. The vacuum vessel has two cryogenic input coolant streams: (1) $5 \mathrm{~K} 3$ bar gaseous helium and (2) $70 \mathrm{~K} 20$ bar gaseous helium. The $70 \mathrm{~K}$ helium stream cools the radiation shielding and the thermally intercepting penetrations running from room temperature. The $5 \mathrm{~K}$ helium coolant stream is split for two separate purposes in the cryomodule. One $5 \mathrm{~K}$ branch is used for thermal intercepting while the second branch is used for the production of $2 \mathrm{~K}$ helium. $2 \mathrm{~K}$ is achieved by heat exchanging the input $5 \mathrm{~K}$ helium gas with the $2.1 \mathrm{~K}$ exhaust gas and then J-T expanding the pre-cooled input to drop the 3 bar supply pressure to 32 mbar for $2 \mathrm{~K}$ liquefaction. The manifolds, heat exchanger and reservoirs that these coolant streams occupy are all designed to comply with ASME B31.3, the process piping standard [80], and the ASME BPVC. All systems have independent safety reliefs sized for the Maximum Allowable Working Pressure (MAWP). The safety reliefs are all located outside of the cryomodule and vent to atmosphere. In this manner, the pressure systems of the cryomodule are separated from the insulating vacuum vessel. This allows us to define the cryomodule box as a vacuum vessel since it is not part of any pressure system boundary, saving considerable design and fabrication costs.

The FNAL safety requirements do not mandate following the ASME BPVC for vacuum vessels but they recommend applying the rules. Because of this the design was developed using the requirements of the 2010 release of the ASME BPVC Section VIII Division 2, Part 5, even though the code explicitly excludes devices with static pressure gradients less than $15 \mathrm{psi}$. This analysis method allowed for relatively rapid evaluation of the complex vacuum vessel design which resulted in significant time-savings relative to traditional hand-based calculations. The ASME BPVC Section VIII Division 2 gives the required procedures for analyzing the 304 SST vessel material properties (yield and ultimate strengths), strain limits, buckling load factors, cyclic loading and collapse criteria. These analysis procedures, when combined with the ASME fabrication and inspection requirements, protect against failure modes of the device: plastic collapse, local failure, buckling and cyclic loading. Our analysis followed these requirements and the results are reviewed below.

The vacuum vessel is evacuated to $<10^{-6}$ Torr and a static pressure gradient of $\sim 14.7$ psi will exist in operation. Rounding up gives a 15 psi MAWP which is used for all analyses. All the simulations presented here were done with ANSYS and used a model with no symmetry planes. The assembly was restrained by placing constraints equivalent to the kinematic mounting system designed for the vessel. The model was also loaded with the weight of all elements and appurtenance loads.

Several analyses are presented here. The first one is a static structural analysis of an elasticperfectly-plastic vessel model which demonstrated the design was protected against plastic collapse and predicted the deflections due to evacuating the vessel. Figure 3.33 shows the cryomodule vessel deflections from the limit load analysis, which the vessel passes. Most areas display stresses well below that allowable for both membrane and bending analyses. Some local high membrane stresses are predicted, and Figure 3.34 shows the primary membrane stress results from a linear elastic analysis of the cryomodule. The contour levels in Figure 3.34 have been adjusted so that all stresses over $20 \mathrm{ksi}$ are red and are indicated with labels. $20 \mathrm{ksi}$ represents the allowable limit for 304 SST in the ASME BPVC at room temperature. The stress concentrations are located on the reinforcing gussets where the largest bending occurs: the weld joint between the cryomodule end-walls and sidewalls and on the 4 mounts on the base of the cryomodule. 


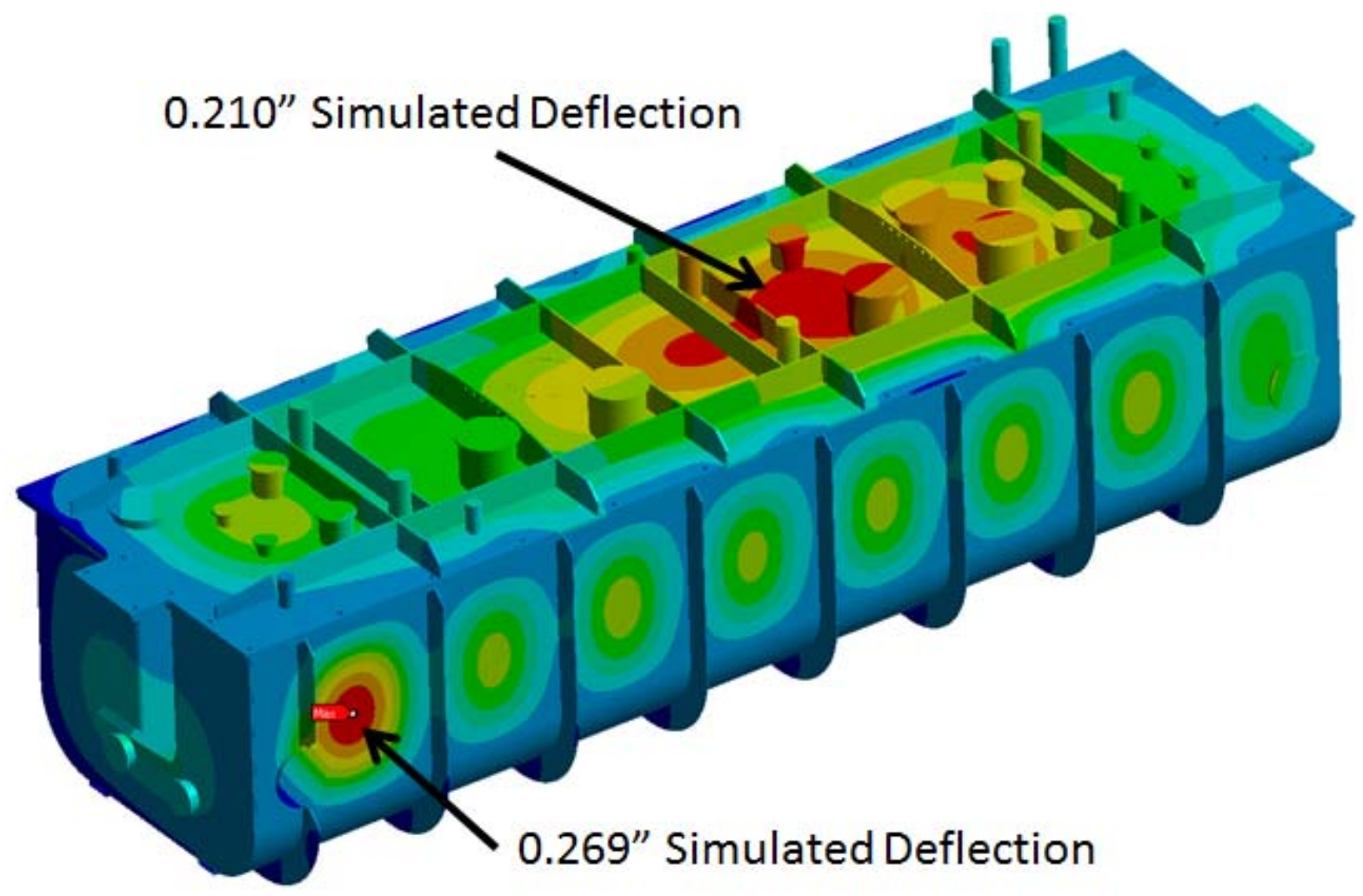

Figure 3.33: Limit-load analysis of the cryomodule vacuum vessel. The labelled area on the side of the cryomodule corresponds to the largest deflection of the vessel with a 15 psig static pressure. The measured deflection during the first pump down was 0.240 " giving good confidence that the vessel exceeds our design expectations.

Beyond demonstrating that the design protects against plastic collapse the ASME BPVC requires several other analyses to demonstrate that the design protects against local failure, buckling and cyclic loading. The local failure analysis requires that at each point in the component the sum of the primary membrane and the principal bending stresses shall not exceed 4 times the allowable stress or $80 \mathrm{ksi}$ for $304 \mathrm{SST}$. This was satisfied with an elastic-material analysis at all locations. Protection against collapse from buckling must also be demonstrated for a vessel with a compressive stress field under the design loads. A bifurcation buckling analysis was performed using an elastic 304 SST material model free of geometric nonlinearities to determine the pre-stress in the vessel. The acceptance criterion is that the buckling load factor be greater than $2 / \beta_{\text {cr }}$ where $\beta_{\text {cr }}$ is the capacity reduction factor given in the ASME BPVC. Since the vessel contains ring stiffened cylinders under external pressure, $\beta_{\mathrm{cr}}=0.80$; and the minimum required buckling load factor is 2.5 . The lowest buckling mode load factor was found to be 3.17 exceeding the minimum requirement of 2.5 predicting that the device will not buckle under the applied loads. Finally, the ASME BPVC requires that the vessel will not fail under cyclic loading. The cyclic loading analysis requires two evaluations: one to protect against high cycle fatigue and another to protect against ratcheting. Evaluation for high cycle fatigue is not required if the total number of cycles is low as defined in the code. We expect to have only 80-100 full and partial loading cycles over the lifetime of the vacuum vessel, which does not come close to the cycle requirement of $>1,000$, the level where a more detailed analysis is required. Therefore, the high-cycle fatigue requirements are satisfied. Finally, a ratcheting analysis to evaluate the performance of the device when the material stresses exceed the yield was done. It demonstrated protection against ratcheting. 

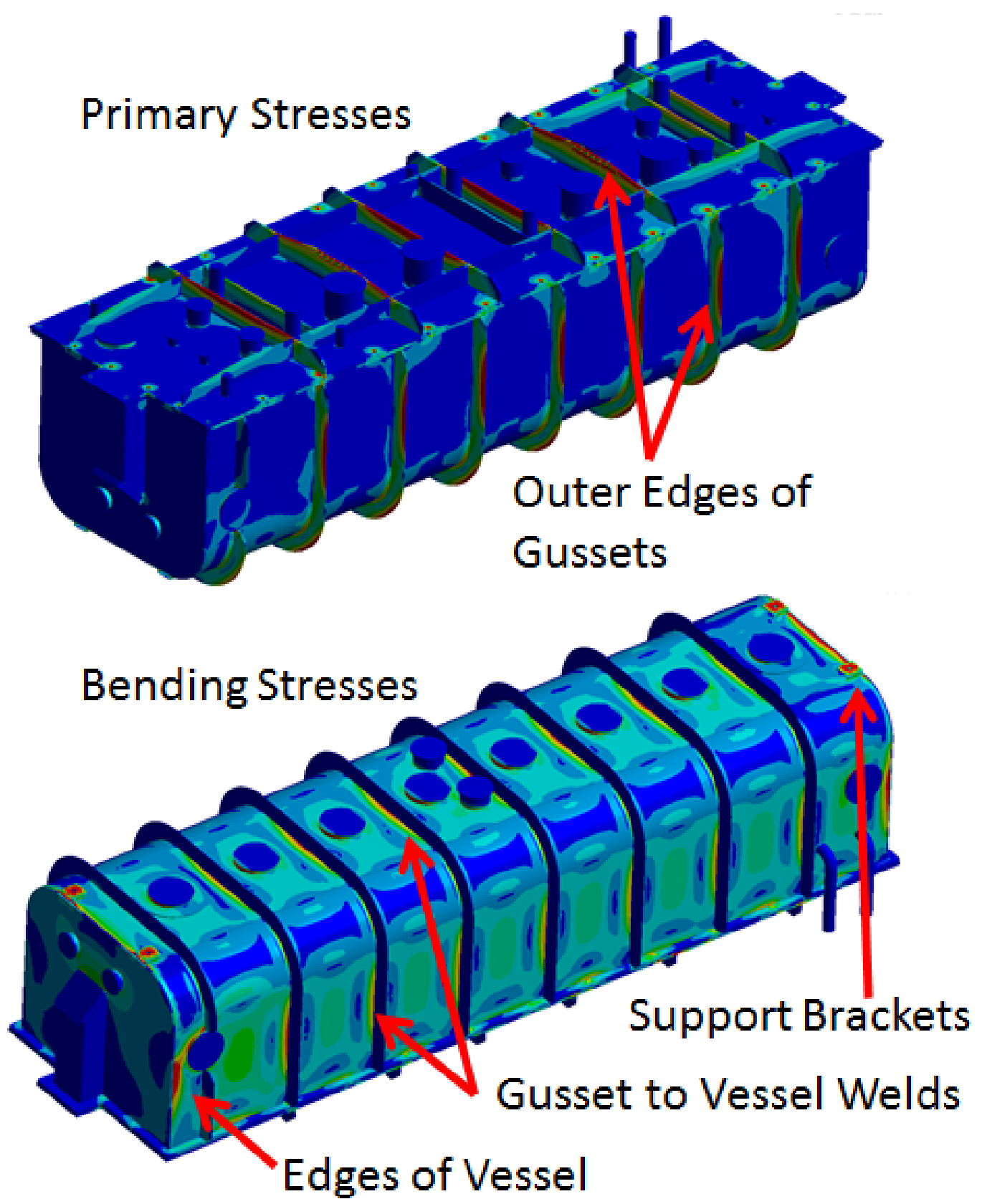

Figure 3.34: The calculated vacuum vessel membrane stresses for an elastic material model for the 304 SST vessel. Top, the primary stress derived from the calculations. Bottom, the secondary bending stress derived from the same calculations. Areas of high stress, 20 ksi for primary and 30 ksi for secondary stresses as set forth in the ASME BPVC, are specified with arrows and labels. This vessel passes the required limit-load analysis for protections against plastic collapse.

The half-wave cryomodule was fabricated by Meyer Tool and Manufacturing in Oak Lawn, Illinois. Meyer Tool assembled the magnetic shielding and the $70 \mathrm{~K}$ radiation shielding with 32 layers of MLI on the exterior and 16 layers on the interior sides as part of the fabrication contract. Figure 3.35 shows the complete vacuum vessel at Argonne. The cryomodule houses 8 sets of identical components. Each set forms a focusing period and includes a resonator, a SC solenoid with 4 dipole coils and a Beam Position Monitor (BPM). 


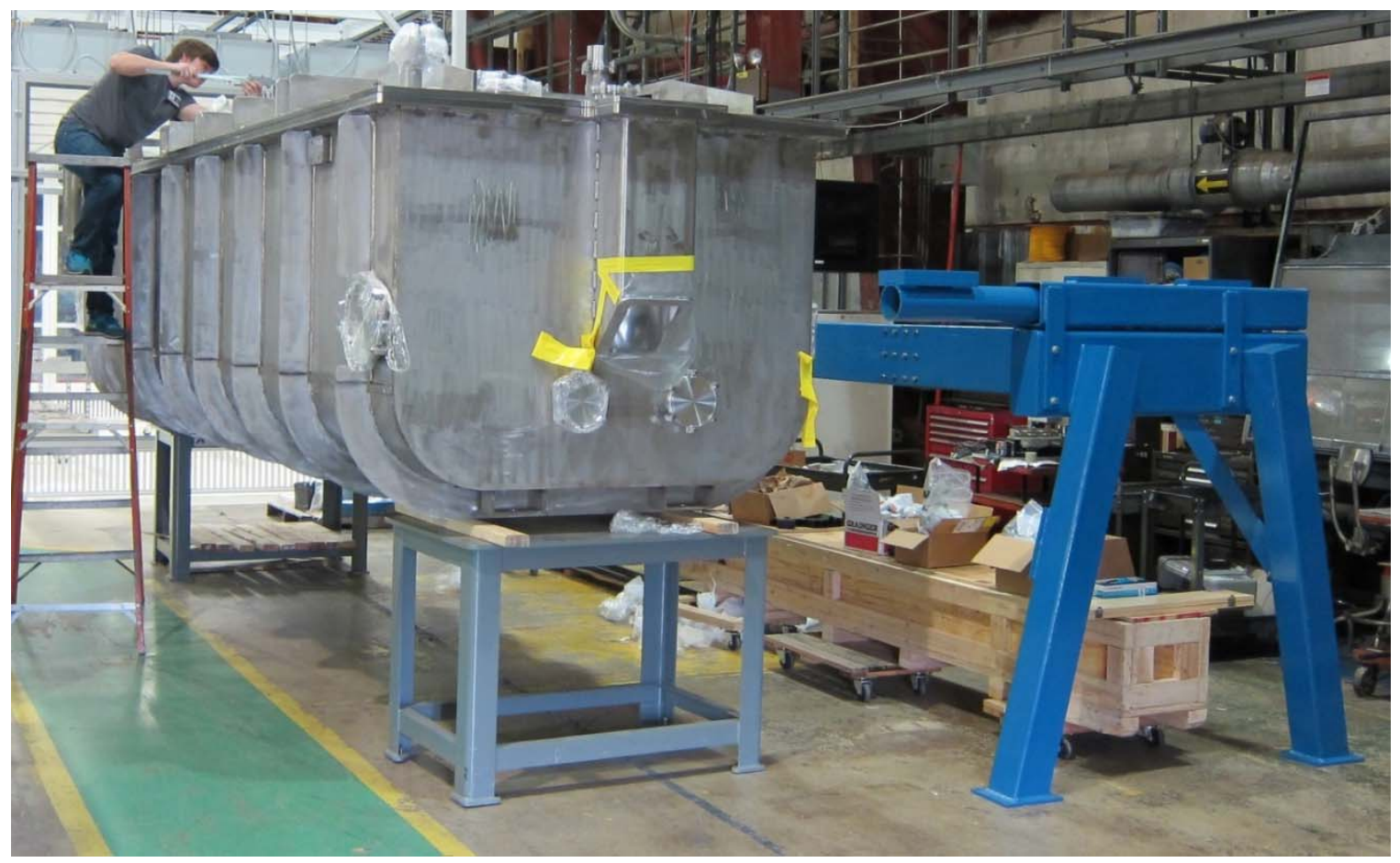

Figure 3.35: The finished vessel assembled at Argonne. The vessel is now being prepared for cryogenic testing.

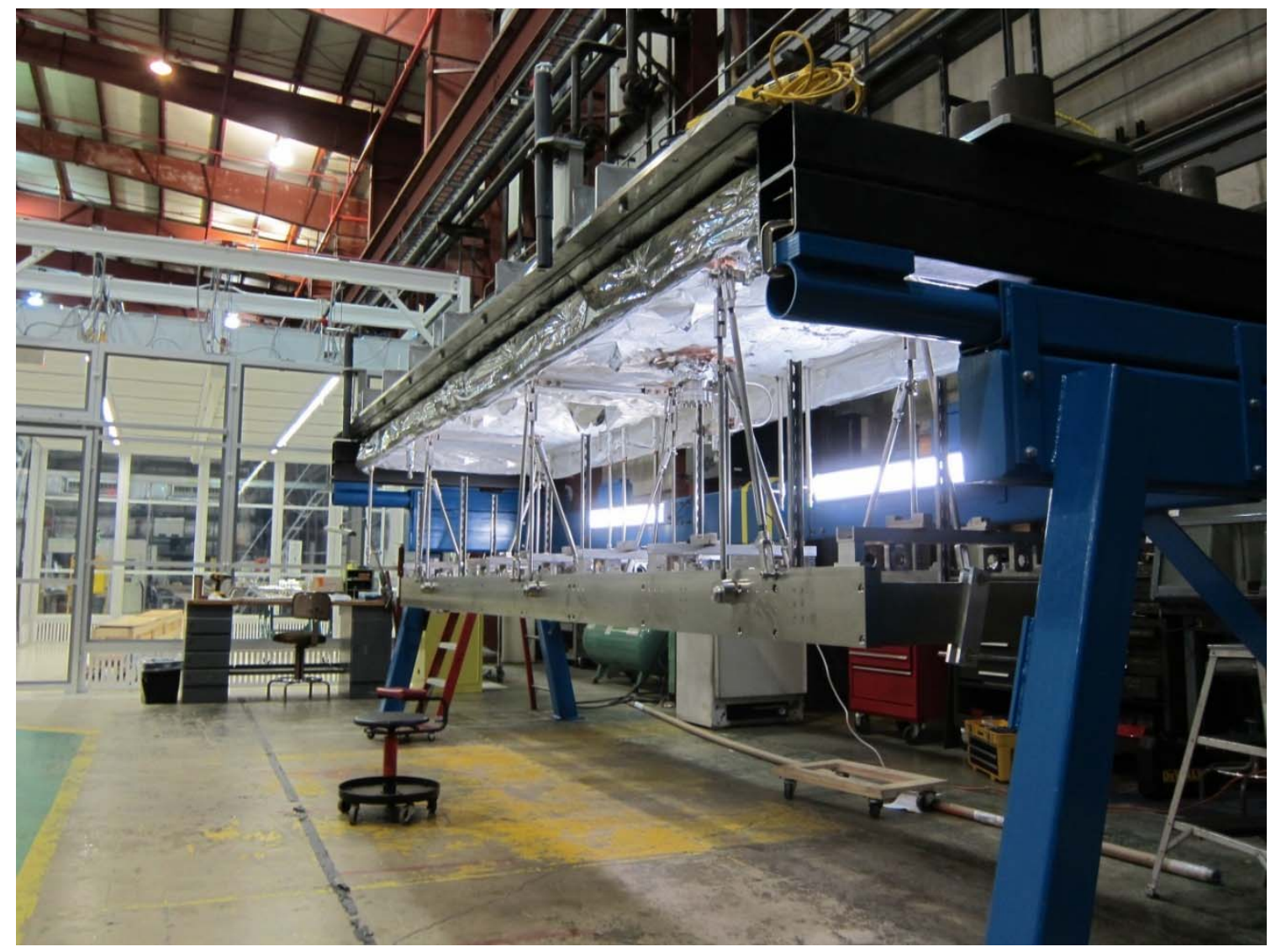

Figure 3.36: The titanium strong-back hung from the lid of the half-wave resonator lid. No cavities or solenoids are mounted on the strong-back. 
Beam dynamics requires the solenoids to be aligned after cooling down to within $\pm 1 \mathrm{~mm}(0.5 \mathrm{~mm}$ $\mathrm{rms}$ ) for both transverse coordinates and within $\pm 2 \mathrm{mrad}(1 \mathrm{mrad} \mathrm{rms})$ for angle between the solenoid magnetic axis and the beam direction. The requirements for cavity coordinate positioning are the same as for the solenoids but the angular alignment can be relieved to $\pm 4 \mathrm{mrad}(2 \mathrm{mrad} \mathrm{rms})$. The beam-line string length is about $6 \mathrm{~m}$ and will be supported and aligned on a cryomodule spanning titanium rail system, called the strong-back as shown in Figure 3.36. The strong-back is composed of $2 " \times 8$ " grade 2 titanium rails with interconnecting plates which hangs from the cryomodule lid. All components are mounted on top of the strong back with independent kinematic-alignment hardware. Initial testing of the alignment systems will begin in late March 2016.

Table 3.3 summarizes the estimated static and dynamic heat loads at each temperature level in the cryomodule assembly from all sources. The following sources were included in the calculation of $2 \mathrm{~K}$ heat load: cavities, RF couplers, helium manifold, radiation from $70 \mathrm{~K}$ to $2 \mathrm{~K}$, instrumentation, high current leads, strong-back hangers, cavity and solenoid cooldown lines, vacuum manifold, slow tuners, and gate valves.

Table 3.3: HWR cryomodule heat load estimate

\begin{tabular}{c|c}
\hline \hline Temperature & Load, $\mathrm{W}$ \\
\hline $2 \mathrm{~K}$, static & 37 \\
\hline $2 \mathrm{~K}$, dynamic & $12^{*}$ \\
\hline $5 \mathrm{~K}$ & 60 \\
\hline $70 \mathrm{~K}$ & 250
\end{tabular}

* This value takes into account the actual voltage distribution on the HWR cavities. Therefore, it is smaller than the value presented in Table 2.7.

\subsubsection{2. $\quad$ Single Spoke Resonator I (SSR1) Cavities and Cryomodules}

Acceleration from 10 to $35 \mathrm{MeV}$ utilizes superconducting cavities with $\beta_{\text {opt }}=0.222$ operating at $325 \mathrm{MHz}$, referred to as Single Spoke Resonator of type 1 or SSR1 (see Figure 3.37) [81].

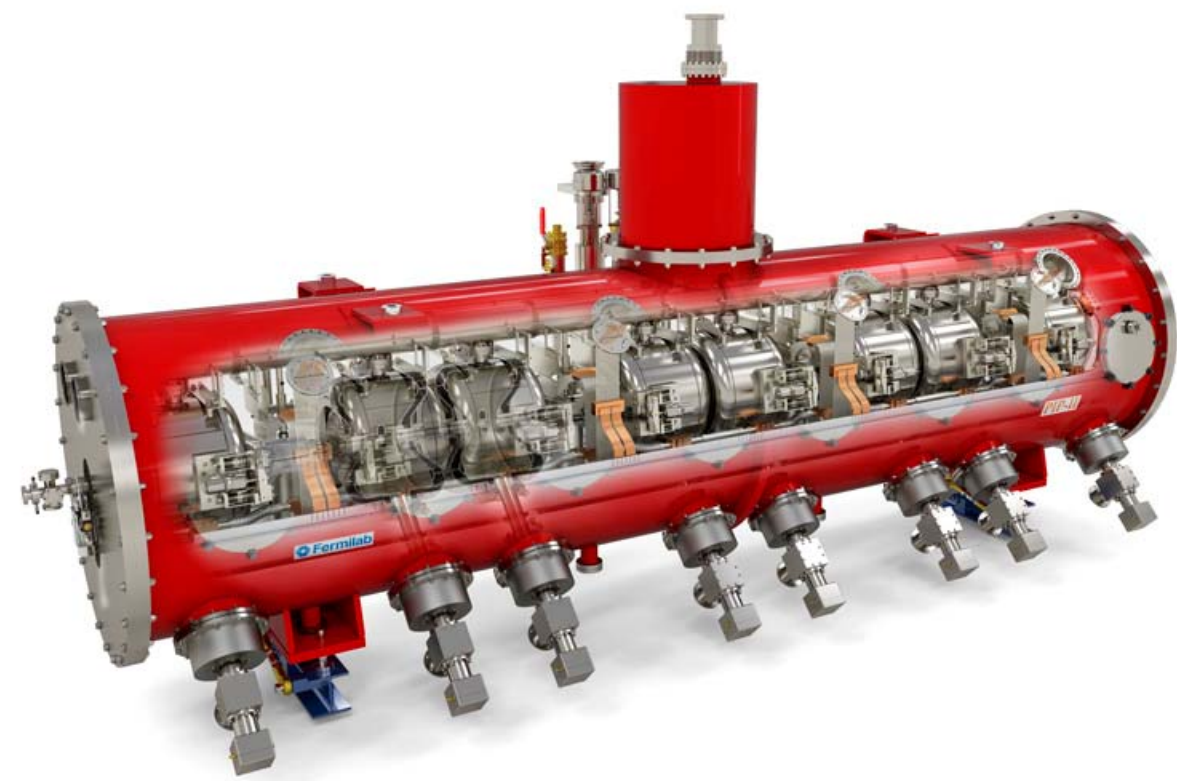

Figure 3.37: Cut-away view of SSR1 cryomodule designed for PIP-II. 


\section{RF Design and Optimization of SSR1 Cavities}

RF design and optimization of the SSR1 cavity was done using Microwave Studio (MWS) software. Figure 3.38 presents the main geometrical parameters used for optimization: $L_{C A V}$ is the cavity length, end-wall to end-wall along beam axis, LIRIS is the iris to iris length, $D$ is the spoke base diameter, $W$ is the spoke width, $T$ is the inner electrode thickness, and $h$ and $\mathrm{v}$ are cylindrical shell and end-wall dimensions. Beam dynamics considerations led to the choice of $\beta=0.21$ and a $30 \mathrm{~mm}$ aperture diameter. Also an iris-to-iris distance, $L_{i r i s}=(2 / 3) \beta \lambda$, was chosen. Table 3.4 lists the general requirements to the electromagnetic parameters.

Table 3.4: SSR1 electromagnetic parameters

\begin{tabular}{l|l}
\hline Parameter & Value \\
\hline Frequency & $325 \mathrm{MHz}$ \\
\hline Shape & Single Spoke Resonator \\
\hline$\beta_{g}, \beta_{\text {opt }}$ & $0.215,0.22$ \\
\hline$L_{\text {eff }}=\beta_{\text {opt }} \lambda$ & $203 \mathrm{~mm}$ \\
\hline Iris aperture & $30 \mathrm{~mm}$ \\
\hline Inside diameter & $492 \mathrm{~mm}$ \\
\hline Bandwidth $\left(f_{0} / Q\right)$ & $90 \mathrm{~Hz}$ \\
\hline$E_{p k} / E_{a c c}$ & 3.84 \\
\hline$B_{p k} / E_{a c c}$ & $5.81 \mathrm{mT} /(\mathrm{MV} / \mathrm{m})$ \\
\hline$G$ & $84 \Omega$ \\
\hline$R / Q$ & $242 \Omega$ \\
\hline \hline
\end{tabular}

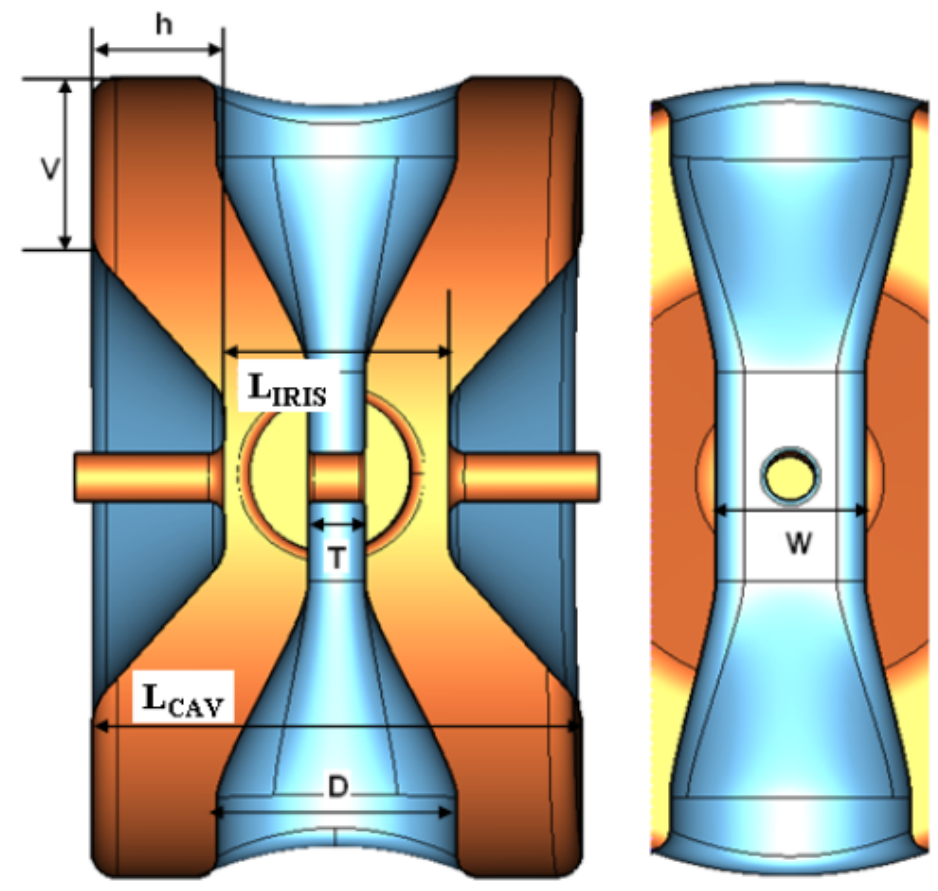

Figure 3.38: Cross section of the SSR1 with the main parameters used in the optimization process. 
Minimization of the peak surface electric field $\left(E_{\text {peak }}\right)$ involves $W, T$ and $L_{i r i s}$ as main parameters, since they define the geometry in the region of high electric field (see Figure 3.39). The ratio $D / L_{c a v}$ and the end cup profile dimensions were optimized to achieve a low peak magnetic field (see Figure 3.40). The corresponding electric and magnetic 3D fields are shown in Figure 3.41. The plots have been generated with Comsol software.
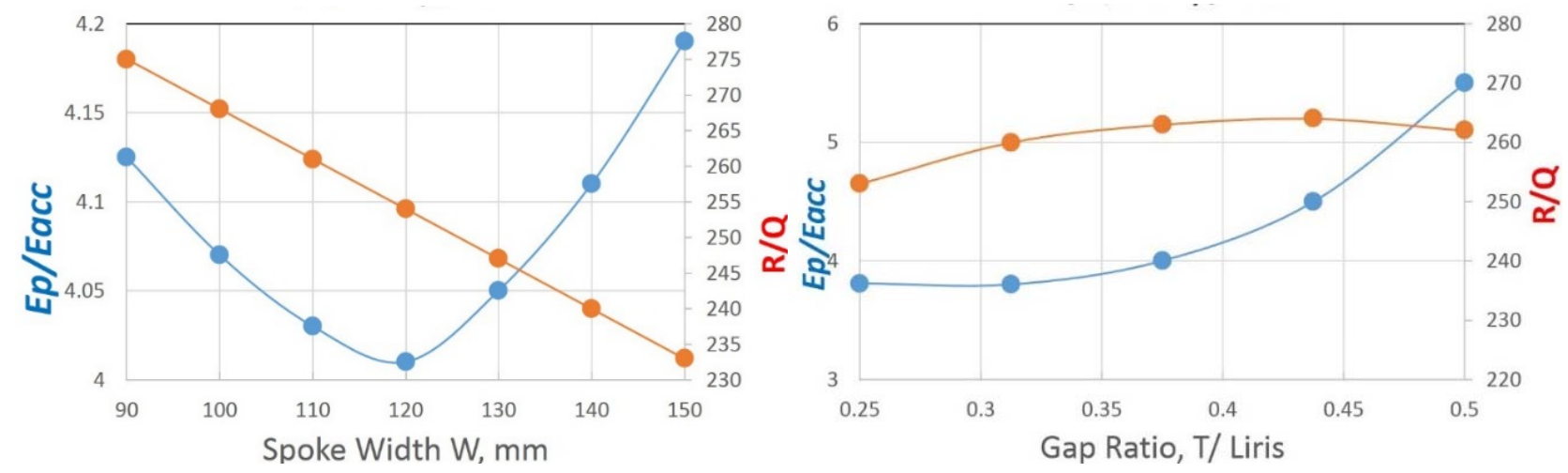

Figure 3.39: Optimization process of $E_{\text {peak }}$.

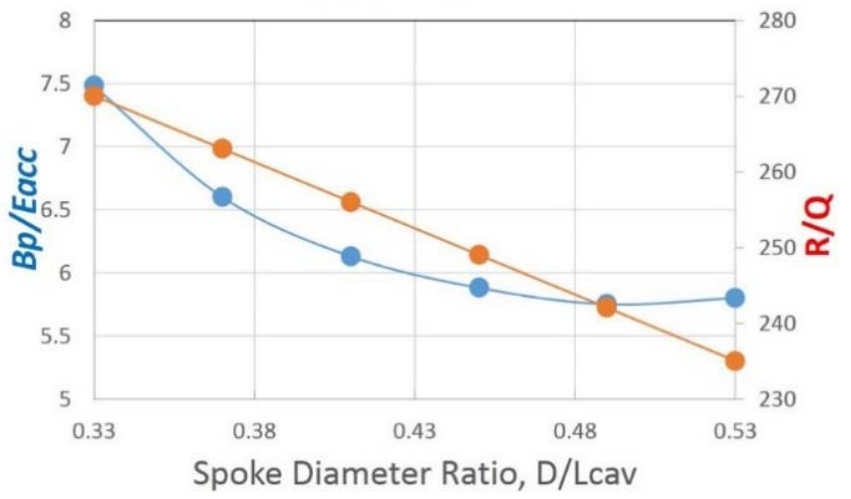

Figure 3.40: Optimization process of $B_{\text {peak }}$.

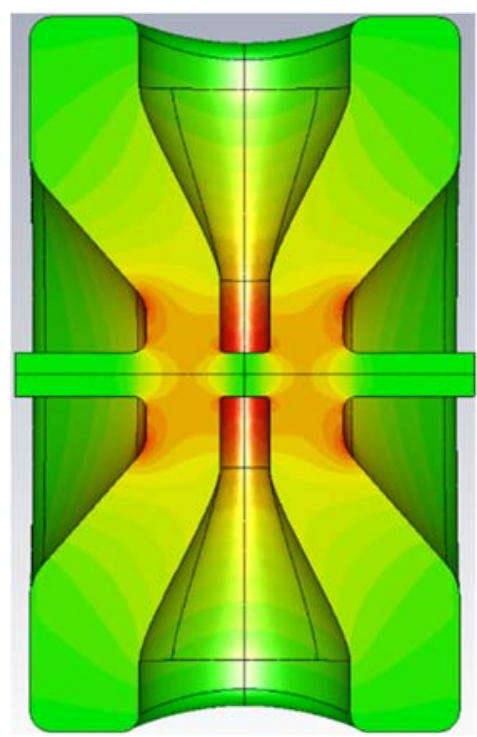

a)

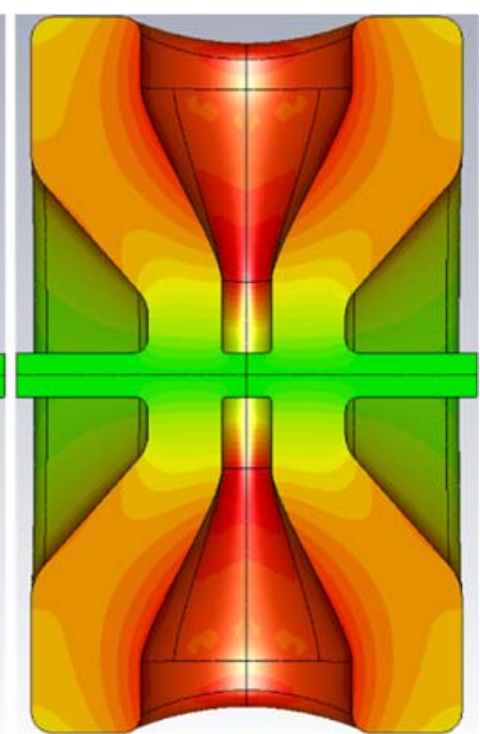

b)

Figure 3.41: Electromagnetic fields of SSR1: (a) Electric field and (b) Magnetic field. The field strength increases as the color changes from green to yellow and to red. 
A spoke cavity has no axial symmetry. Therefore, its quadrupole component cannot be compensated over the entire range of cavity operation. Figure 2.27 presents the dependence of the quadrupole effect on the beam velocity. Due to engineering limitations, mainly related to the RF couplers, the cavities are rolled by $45^{\circ}$; consequently, their quadrupole field is also rolled and is a skew-quadrupole field. The cavity skew-quadrupole fields will be compensated by correction coils located inside nearby focusing solenoids and capable to create dipole and skew-quadrupole fields.

Multipacting, higher order modes and transverse kick are other key concerns in the design of this type of cavity. Multipacting analysis was performed to make sure that no evidence of multipactoring activity appears in the range of the cavity operating gradients. Analysis predicts two multipactoring barriers at $4.5 \mathrm{MV} / \mathrm{m}$ and $7 \mathrm{MV} / \mathrm{m}$. They are sufficiently far from the operating gradient of $12 \mathrm{MV} / \mathrm{m}$. These barriers were later observed in the course of vertical testing of the bare SSR1 cavities, confirming the validity of the analyses.

Higher order modes were investigated in a dedicated simulation study. Monopole, dipole, and quadruple modes were simulated and $R / Q$ for each mode was calculated. Simulated $R / Q$ values are sufficiently small thus enabling operation without any HOM dampers. Bead pull measurements were carried out for all the HOM monopoles up to $2 \mathrm{GHz}$. The measured $R / Q$ of the HOM monopoles is less than $1 \Omega$. Simulated and measured $R / Q$ values are in good agreement. HOMs frequency spread on the tested cavities is within $7 \mathrm{MHz}$.

Geometrical errors in the fabrication process of SSR1 cavity can result in a transverse kick. Fabrication experience has identified three major types of possible variations related to the alignment of both the beam pipe and spoke with respect to the beam axis. Simulation studies have been carried out implementing these variations in the simulation model. Bead-pull measurements were also conducted to measure the transverse kick in the fabricated cavities. The simulations and the measurements are in relatively good agreement. The maximum kick in the fabricated cavities is less than $154 \mathrm{keV}$, which corresponds to a $1.12 \mathrm{mrad}$ beam deflection. It is well within $10 \mathrm{mrad}$ specified for the SSR1 corrector strength.

\section{Structural Design of SSR1 Cavities}

In a machine like PIP-II, operating both in continuous wave $(\mathrm{CW})$ and pulsed regimes, perturbations of cavity fundamental frequency caused by the Lorentz Force Detuning (LFD) and pressure fluctuations in the liquid helium bath need to be minimized.

SSR1 was the first superconducting spoke resonator developed at Fermilab for the High Intensity Neutrino Source (HINS) accelerator [82]. The first prototype of SSR1 cavity with helium vessel (SSR1-01) [83], designed and manufactured for the HINS project, did not meet PIP-II specifications. Sensitivity to helium vessel pressure $d f / d P=140 \mathrm{~Hz} /$ Torr significantly exceeded the PIP-II requirement of $d f l d P \leq 25 \mathrm{~Hz} /$ Torr.

A new generation of jacketed SSR1 cavities was redesigned for the PIP2IT project. The major goal of the helium vessel design was reduction of $d f / d P$. First, the effect of different design modifications was studied. That was helpful to choose the design path and, consequently, avoid costly errors. The minimum value of $d f / d P$ was achieved by coupling the helium vessel with the endwall of the resonator by a bellow of optimal diameter. The cavity tuner stiffness was accounted for in this process. This work resulted in the third generation design for the SSR1 bare cavity. The structural design of the SSR1 cavity is based on the SSR1 cavity Functional Requirement Specification [84]. Its major requirements are presented in Table 3.5. 
Table 3.5: SSR1 cavity requirements

\begin{tabular}{l|l}
\hline Parameter & Requirement \\
\hline Max leak rate (room temp) & $<10^{-10} \mathrm{~atm}-\mathrm{cc} / \mathrm{sec}$ \\
\hline Operating gain per cavity & $2.0 \mathrm{MeV}$ \\
\hline Maximum gain per cavity & $2.4 \mathrm{MeV}$ \\
\hline Max. power dissipation per cavity at $2 \mathrm{~K}$ & $5 \mathrm{~W}$ \\
\hline Sensitivity to He pressure fluctuations, $d f / d P$ & $<25 \mathrm{~Hz} /$ Torr \\
\hline Field flatness & Within $\pm 10 \%$ \\
\hline Multipacting & None within $\pm 10 \%$ of operating grad. \\
\hline Operating temperature & $1.8-2.1 \mathrm{~K}$ \\
\hline Operating pressure & $16-41 \mathrm{mbar}$ differential \\
\hline MAWP & 2 bar (RT), 4 bar (2K) \\
\hline RF power input per cavity & $6 \mathrm{~kW}(\mathrm{CW}$, operating) \\
\hline \hline
\end{tabular}

\section{Bare Cavity}

The first step of SSR1 design was an optimization of RF performance. This was followed by an optimization of resonator structural integrity achieved by adding an adequate system of stiffeners to the outside surfaces, without any modification to the RF shape, to withstand the liquid helium pressure during the vertical test ( 2 bar; 29 psi at $4 \mathrm{~K}$ ).

The cavity parts are formed and machined from high-purity niobium $(\mathrm{Nb})$ joined by electronbeam welding. The cavity has four stainless steel flanges which connect it to the helium vessel. The flanges are connected to the niobium with copper-brazed joints.

The structural design of the bare SSR1 cavity is shown in Figure 3.42.

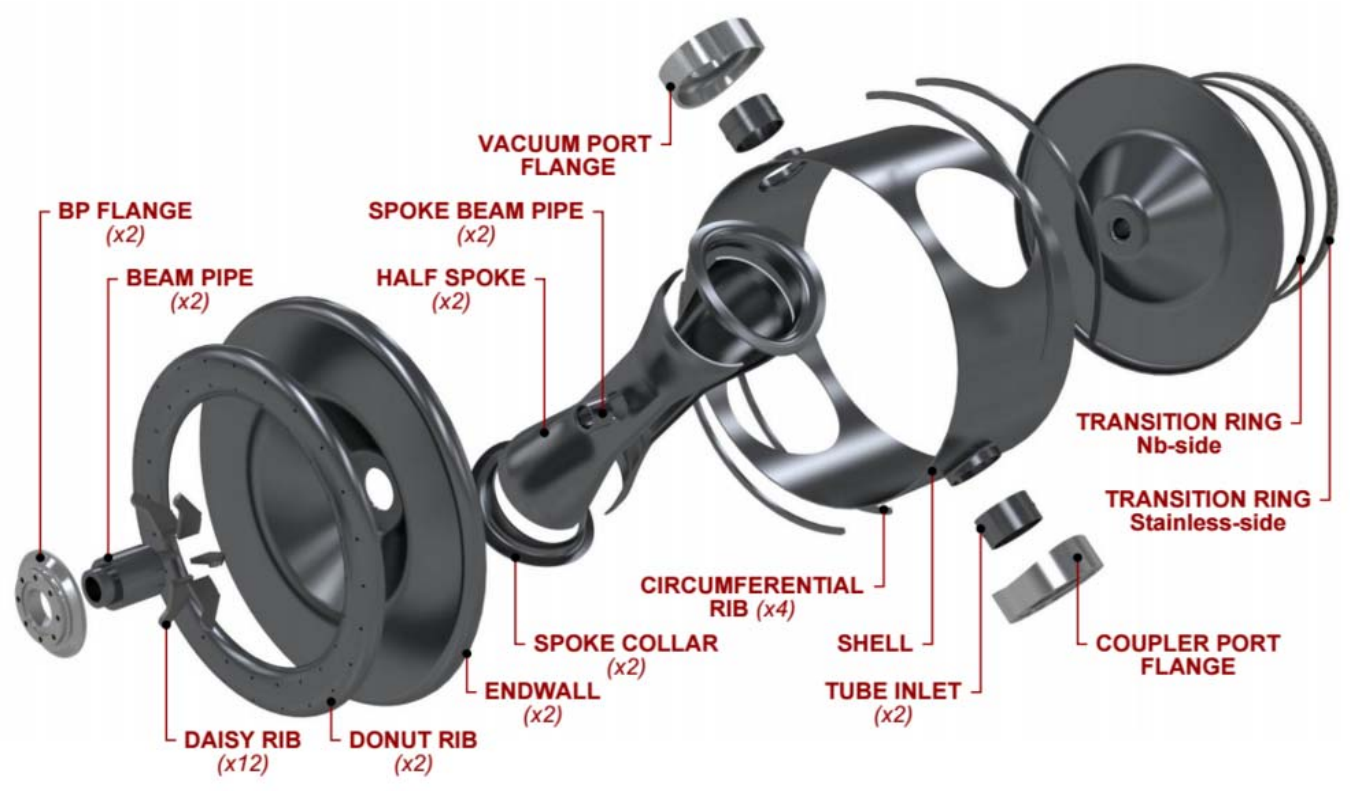

Figure 3.42: Exploded view of the bare niobium SSR1 cavity. 


\section{Jacketed Cavity}

From a mechanical engineering standpoint, a jacketed SRF cavity is comprised of an inner niobium vessel (or SRF cavity) surrounded by a liquid helium containment vessel made of stainless steel or titanium. The helium bath may reach pressures exceeding $15 \mathrm{psi}(0.103 \mathrm{MPa})$ and generally has a volume greater than five cubic feet $\left(0.142 \mathrm{~m}^{3}\right)$. All this leads to consider a jacketed SRF cavity as a system of pressure vessels. Based on the Department of Energy (DOE) directive 10 CFR 851, it is mandatory for safety reasons that all pressure systems designed, fabricated and tested by U.S. National Laboratories conform to ASME Codes. Consequently, jacketed SRF cavities fall within the scope of the following sections of the ASME Boiler and Pressure Vessel Code.

However, a true Code design is not currently possible due to the use of non-Code materials, the unfeasibility of Code required nondestructive examinations of welded joints and the use of unqualified procedures for welding and brazing. A set of rules have been developed by engineers at Fermi National Accelerator Laboratory [85] based on their understanding of best practices in the design, fabrication, examination, testing, and operation of the jacketed SRF cavities. These guidelines comply with Code requirements wherever possible, and for non-Code features, procedures are established to produce a level of safety consistent with that of the Code design [86].

The jacketed SSR1 cavity consists of two nested cryogenic pressure vessels: the inner vessel is the superconducting SSR1 cavity, see Figure 3.42, and the outermost vessel is the helium containment (or helium) vessel, see Figure 3.43. The helium vessel is made entirely of 316L stainless steel and it is assembled around the cavity by full penetration tungsten inert gas (TIG) welds.

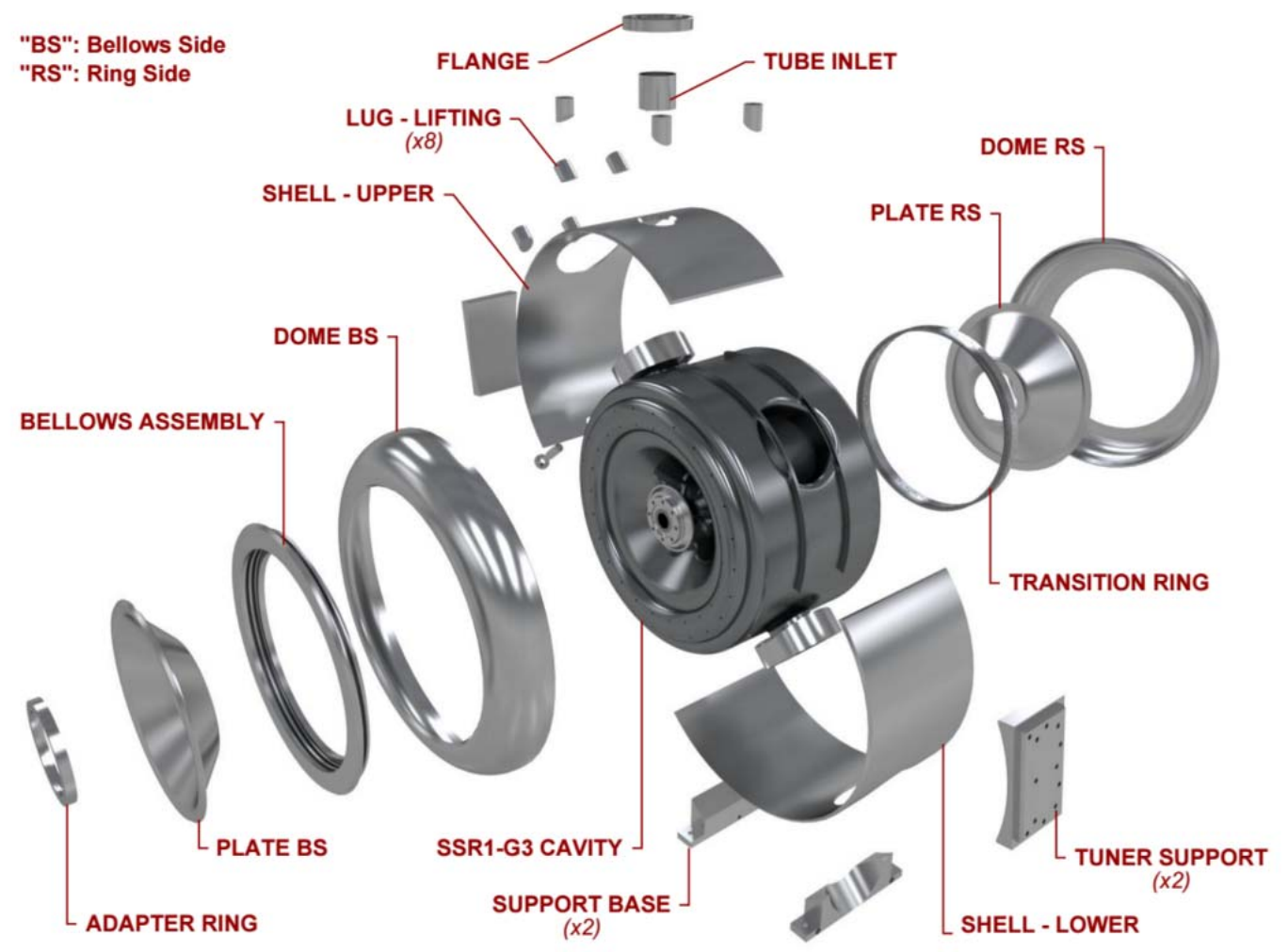

Figure 3.43: Exploded view of the stainless helium vessel surrounding the SSR1 cavity.

The typical operating temperature of an SRF cavity is in the 1.8 to $2.1 \mathrm{~K}$ range. A bath of superfluid helium, confined by the helium vessel, surrounds the cavity exerting a pressure on both 
vessels. The RF volume of the cavity is pumped down to ultra-high vacuum, and the entire jacketed cavity is placed in a cryostat under insulating vacuum, see Figure 3.44. The greatest risk with vessels containing superfluid helium is that an accidental loss of vacuum results in very rapid boiling of the helium, causing a consequent pressurization of the helium space. Moreover, differential pressure appears between the volumes defined by cavity and helium vessel during the first phases of operation before the cooldown (see Figure 3.44). A relief valve setting of $0.2 \mathrm{MPa}$ is needed to ensure the cavity is protected during initial testing and during cooldown. The jacketed SSR1 cavity has two values of maximum allowable working pressure (MAWP), $0.2 \mathrm{MPa}$ at $293 \mathrm{~K}$ when the niobium material strength is low, and $0.4 \mathrm{MPa}$ at $2 \mathrm{~K}$ when the niobium strength is significantly higher. At low temperature higher material strength allows the system piping to be sized for higher short-term pressure increases. This can occur during a loss of cavity vacuum or insulating vacuum when the cavity is cold.

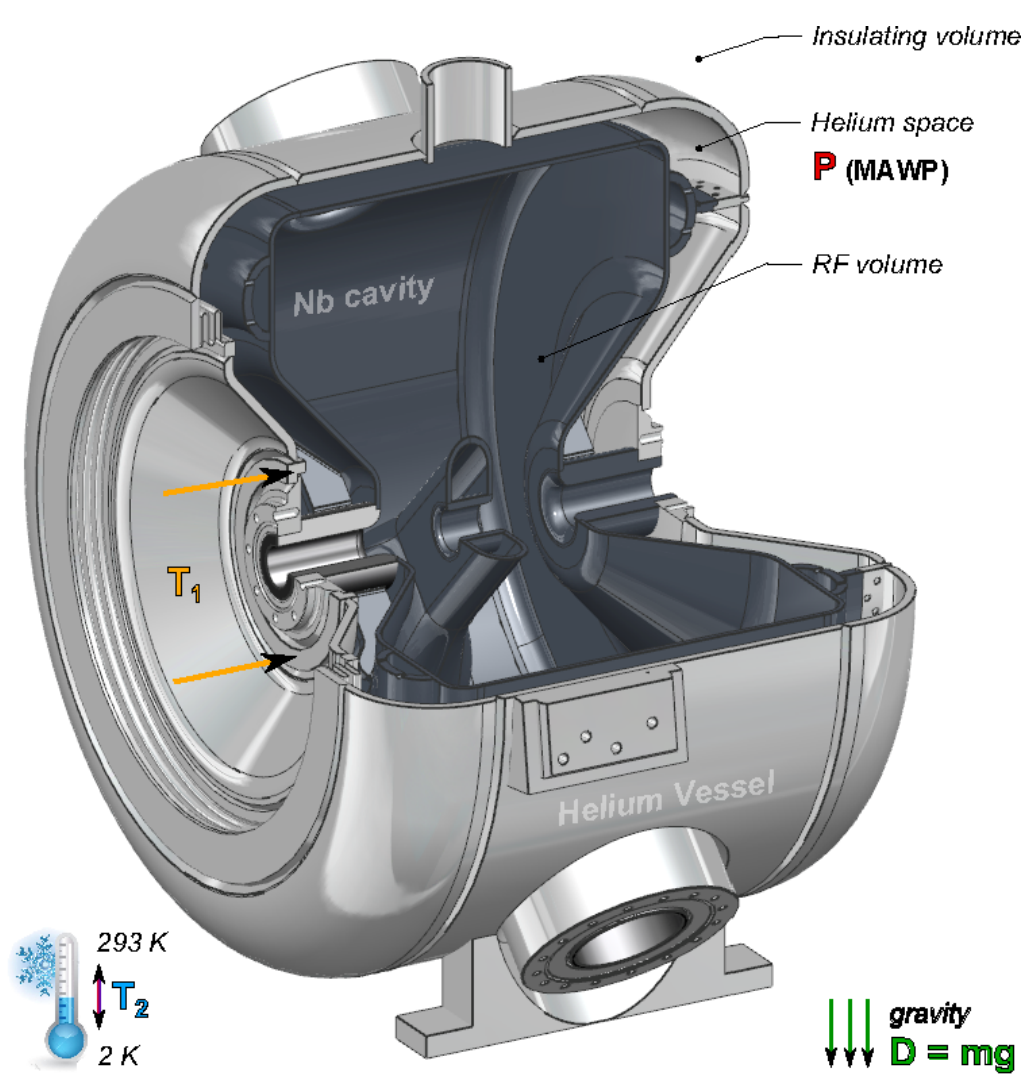

Figure 3.44: Cutaway view of jacketed SSR1 cavity with schematic loads applied. The pressure (P) is applied normal to the surfaces confining the helium space, the frequency tuning displacement $\left(T_{1}\right)$ is applied normal to the beam pipe (along the arrows), the gravitational force (D), and the cooldown ( $\left.T_{2}\right)$ applied to the entire system.

The geometry of the structures and loading conditions of SRF cavities, and therefore the stress distributions, are often complicated and do not lend themselves entirely to design-by-rules method. The design-by-analysis method can be used to optimize those features not amenable to design-byrules method. The design-by-analysis method assumes that a numerical analysis technique will be used, and either elastic or elastic-plastic analysis is carried out. In the case of the SSR1, ANSYS structural analysis software was used to perform the finite element analyses and to ensure protection against four modes of failure: plastic collapse, buckling, cyclic loading and local fracture. 


\section{Testing SSR1 Cavities}

Presently the 12 bare SSR1 cavities have been tested at $2 \mathrm{~K}$ in the vertical test stand. The test results for the 10 qualified bare cavities are presented in Figure 3.45.

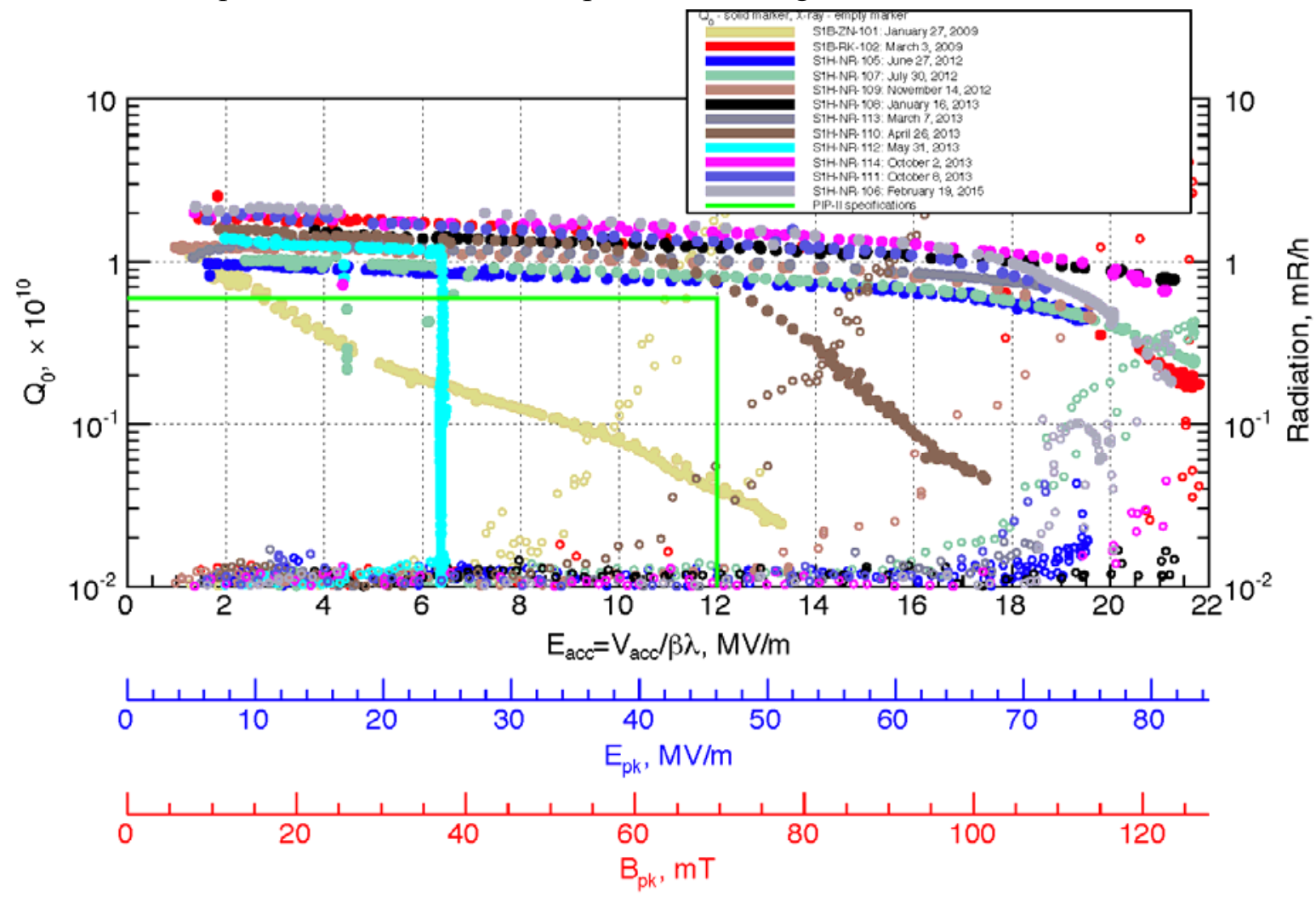

Figure 3.45: Q0 vs. acceleration gradient from the cold test of the twelve bare SSR1 cavities $(\beta=0.222)$.
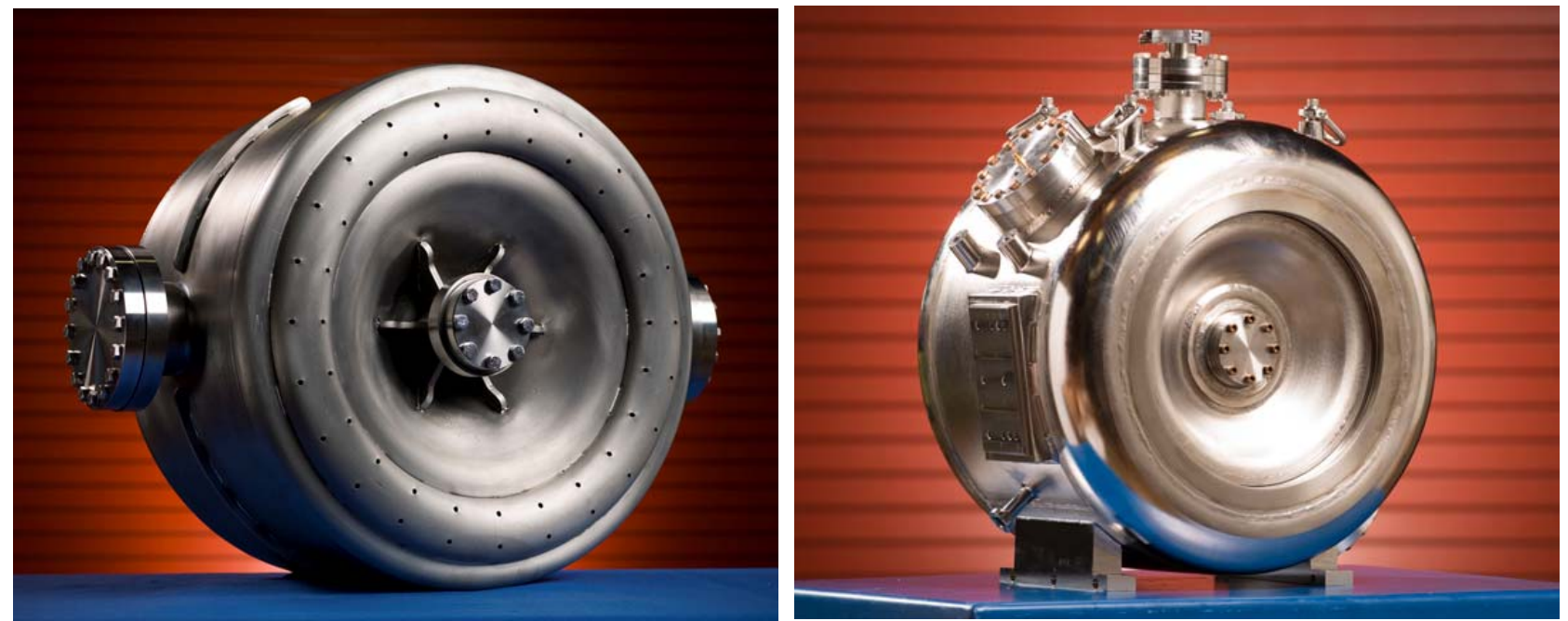

Figure 3.46: SSR1 bare (left) and jacketed (right) cavities.

The qualified cavities were jacketed with helium vessel. Views of bare and jacketed cavities are shown in Figure 3.46. After jacketing mechanical performance of cavities was measured at room temperature. All cavities showed sound structural behavior and very promising performance in terms of sensitivity to He-pressure fluctuations $(d f / d p)$, see Table 3.6. 
Table 3.6: Frequency sensitivity to pressure (df/dp) for the ten jacketed SSR1 cavities manufactured up-to-date

\begin{tabular}{|c|c|c|c|}
\hline Cavity Serial \# & $\begin{array}{c}d f / d p \text { of bare } \\
\text { cavity at } 293 \mathrm{~K} \\
{[\mathrm{~Hz} / \mathrm{Torr}]}\end{array}$ & $\begin{array}{c}d f / d p \text { of jacketd cavity at } \\
\text { 293K (without tuner) } \\
\text { [Hz/Torr] }\end{array}$ & $\begin{array}{c}d f / d p \text { of fully operating } \\
\text { cavity at } 2 \mathrm{~K}^{*} \\
{[\mathrm{~Hz} / \text { Torr }]}\end{array}$ \\
\hline S1H-NR-105 & -560 & 0 & 0* \\
\hline S1H-NR-106 & -564 & 0 & $0 *$ \\
\hline S1H-NR-107 & -561 & 8 & 4 \\
\hline S1H-NR-108 & -553 & -1.2 & 0* \\
\hline S1H-NR-109 & -555 & 5.4 & $2 *$ \\
\hline S1H-NR-110 & -569 & 7.9 & $4^{*}$ \\
\hline S1H-NR-111 & -526 & 2.7 & $2^{*}$ \\
\hline S1H-NR-112 & -525 & 9.0 & $5 *$ \\
\hline S1H-NR-113 & -555 & 6.3 & $3 *$ \\
\hline S1H-NR-114 & -557 & 10 & 5* \\
\hline
\end{tabular}

* Except S1H-NR-107 that was already measured in STC at $2 \mathrm{~K}$, the "df/dp fully operating cavity at 2K" for other cavities is an estimation based on calculations and experience with S1H-NR-107.

The first jacketed SSR1 cavity (S1H-NR-107) was tested on the STC (Spoke Test Cryostat) [87, 88]. Figure 3.47 shows this cavity installed in the cryostat. The measured quality factor and radiation vs accelerating voltage are presented in Figure 3.48. They meet the PIP2IT specifications. Note that the quality factors for the jacketed and bare cavities are quite close. This indicates the jacketing (welding) process has not degraded the niobium cavity performance.

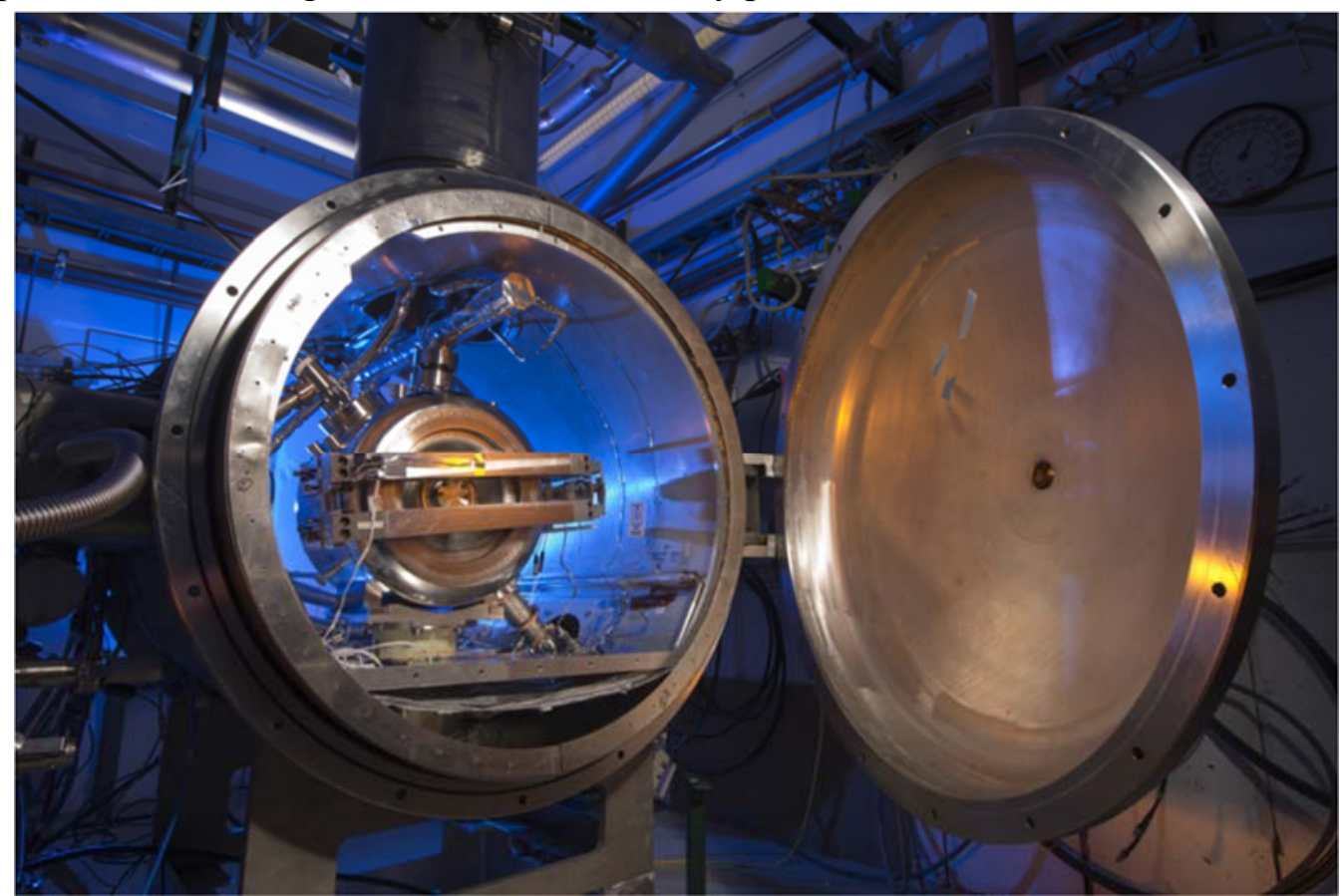

Figure 3.47: Jacketed S1H-NR-107 dressed with tuner mechanism and power coupler, installed in the STC. 


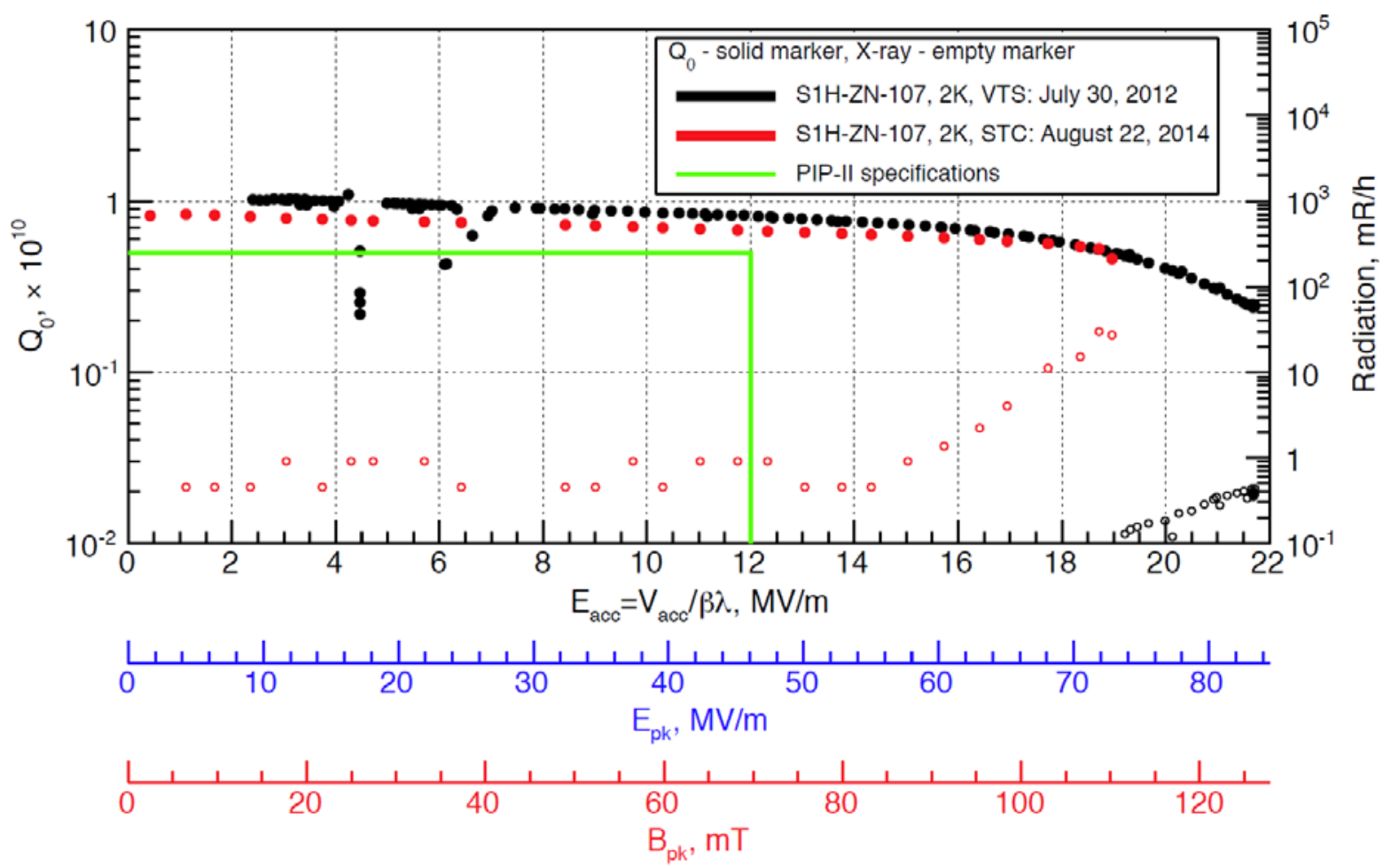

Figure 3.48: Quality factor $\left(\mathrm{Q}_{0}\right)$ and radiation vs accelerating voltage $\left(\mathrm{E}_{\mathrm{acc}}\right)$ measured for the S1H-NR107 at STC before (black) and after (red) jacketing.

After cooling down S1H-NR-107 to $2 \mathrm{~K}, d f / d p$ was measured by varying the helium bath pressure from 18 to 43 Torr and measuring the cavity resonance frequency with a network analyzer. The measurement yielded $d f / d p=4.5 \mathrm{~Hz} /$ Torr. This value is lower than the specification of $25 \mathrm{~Hz} /$ Torr for the PIP-II project, thus validating the design approach for the SSR1 helium vessel.

\section{SSR1 Cryomodule and SSR1 String Assembly}

The cavity string assembly of the SSR1 cryomodule, which constitutes the beamline volume, contains eight superconducting SSR1 cavities with their RF couplers and four solenoids. The sequence of elements in a cryomodule is: $\mathrm{C}-\mathrm{S}-\mathrm{C}-\mathrm{C}-\mathrm{S}-\mathrm{C}-\mathrm{C}-\mathrm{S}-\mathrm{C}-\mathrm{C}-\mathrm{S}-\mathrm{C}$, where $\mathrm{C}$ denotes a cavity and S solenoid. Figures 3.49 and 3.50 show views on the string assembly. Horizontal and vertical dipole correctors are located inside each solenoid. A four-electrode beam position monitor (BPM) is attached to each solenoid.

The connections along the beam-line are made using aluminum diamond seals and stainless steel flanged joints and bellows. At each end, the beam-line is terminated with ultrahigh-vacuum (UHV) gate valves through which the beam-line is pumped down. The planned pumping system for the cryomodule consists of two turbo pumps with a pumping speed of $300 \mathrm{l} / \mathrm{s}$ and an ultimate pressure of $1 \cdot 10^{-8}$ Torr at the pump inlet. The UHV is supported by ion pumps. The cavity string will be assembled in a class 10 cleanroom to minimize particle contamination in the beam-line. The contamination is known to cause field emission and degrade cryomodule performance. Limiting the number of beam-line components and sub-assemblies from the initial stages of design simplifies the installation procedure and promotes a cleaner assembly. A vacuum level of $5 \cdot 10^{-5}$ or lower is achievable by pumping only through the beam-line [89]. The pressure threshold of $5 \cdot 10^{-5}$ Torr was chosen to avoid negative effects such as increased field emission [90] and residual resistance due to condensed gases [91]. This level of vacuum must be reached in less than 12 hours to minimize the 
down-time associated with thermal cycles for the cryomodule.

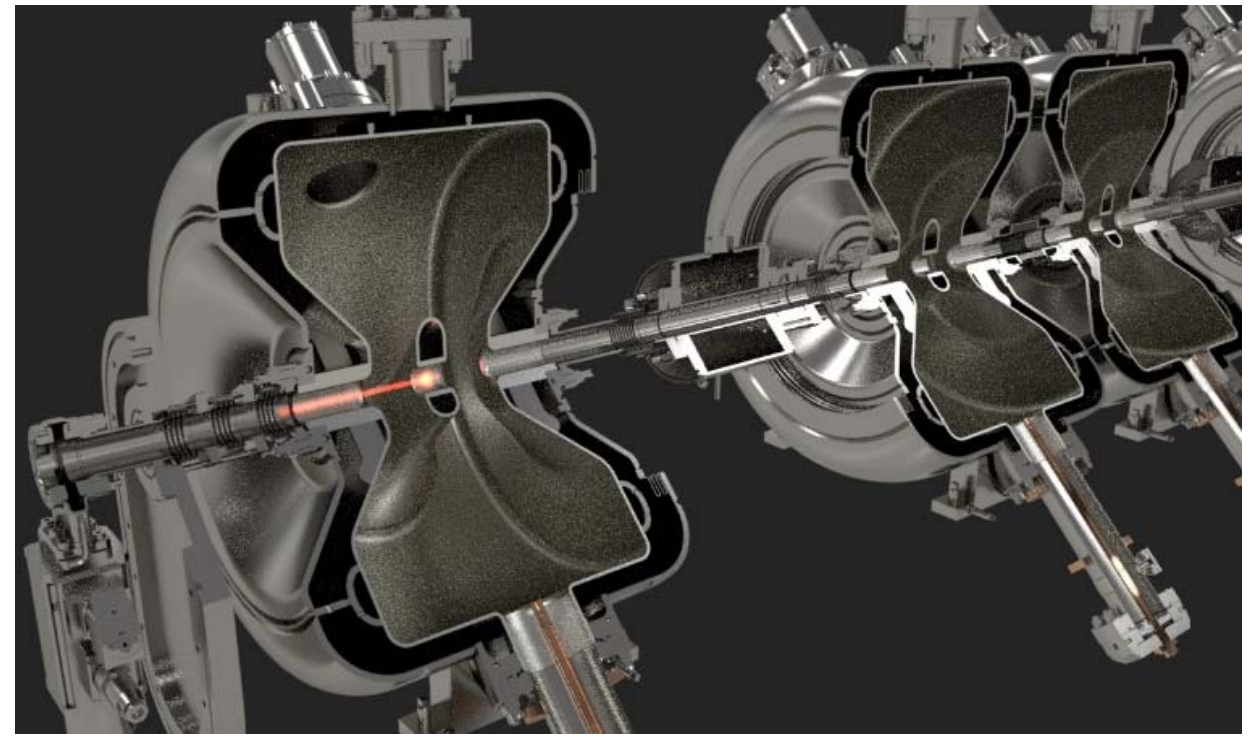

Figure 3.49: View on SSR1 cavity string assembly.

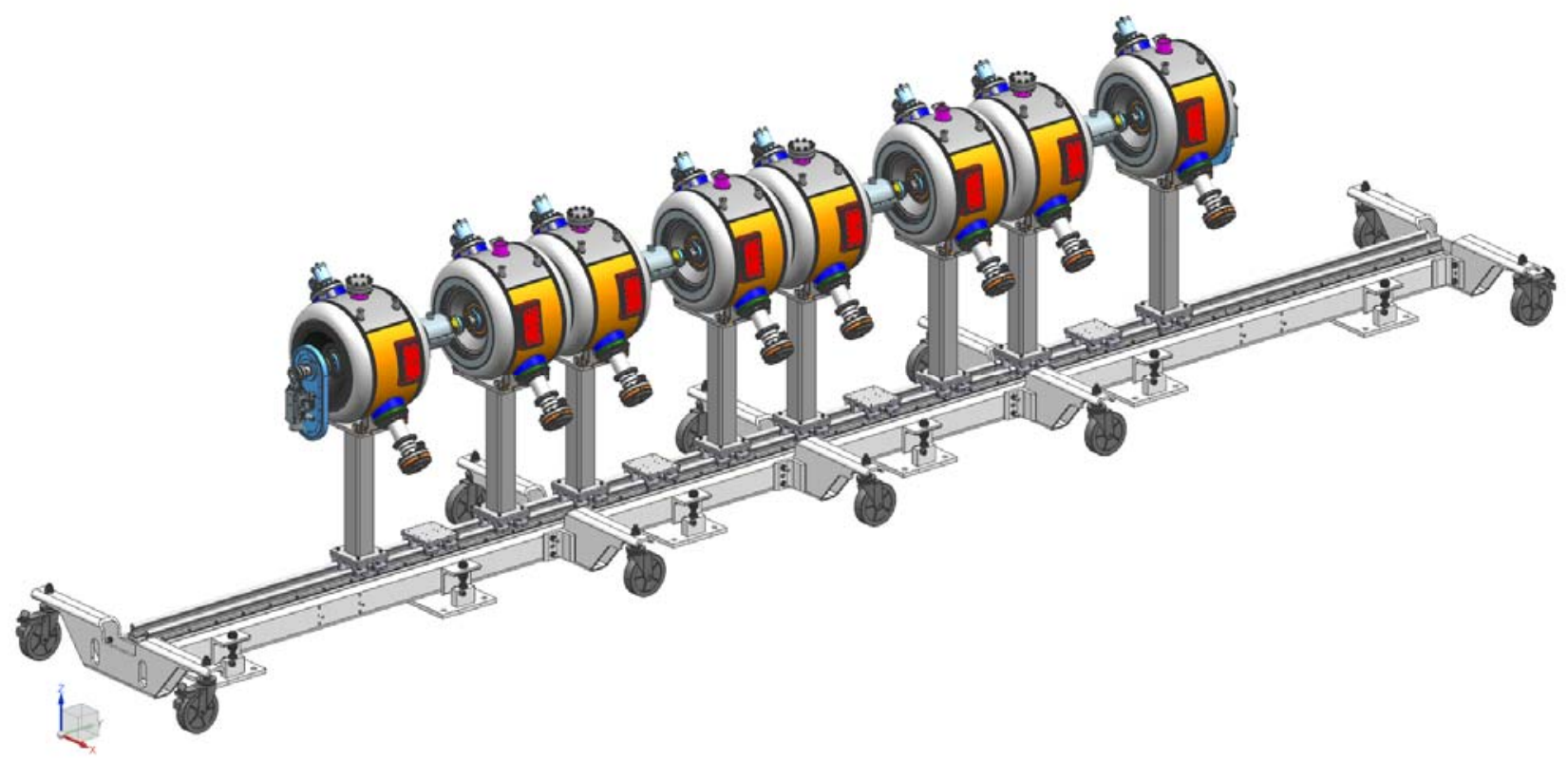

Figure 3.50: SSR1 cavity string assembly.

\section{SSR1 Solenoid, Current Leads and Beam Position Monitor}

There are four magnet packages in the cryomodule. Each contains a focusing solenoid (lens) and four corrector coils, all operating in a helium bath at $2 \mathrm{~K}$. The general design requirements for the lenses in the SSR1 cryomodule are based on Ref. [92] and summarized below:

- Each lens must contain 4 additional coils which fields can be combined into two dipole correctors (x\&y) and a skew-quadrupole;

- The clear aperture in the lens must be not less than $30 \mathrm{~mm}$;

- The uncertainty in the location of the effective magnetic axis in the focusing solenoid of 
the lens relative to reference points on the outer surface of the device must be better than $0.1 \mathrm{~mm}$ rms.

- A liquid helium vessel must be used for cooling the windings down to $2 \mathrm{~K}$;

- The lenses must be quench-protected; the energy deposited in the lenses after quenching must be as low as reasonably achievable;

- The liquid helium vessel must meet the requirements of the Fermilab's ES\&H manual chapters for pressure vessels;

- The design of the LHe vessel must ensure a reliable and reproducible mechanical connection to the alignment fixture of the cryomodule;

- The maximum magnetic field generated by lenses in the cryomodule in the area near the surface of the SSR1 superconducting cavities must not exceed the level that would result in more than two-fold reduction of the intrinsic quality factor after quench event at any point on the surface of the cavity.

The SSR1 magnet assembly was designed and built. The results of magnetic measurements as well as the main FRS requirements to the magnet strength are presented in Table 3.7.

Table 3.7: Main parameters of the SSR1 magnet assembly

\begin{tabular}{l|c}
\hline \hline Solenoid & \\
\hline Maximum current & $65.2 \mathrm{~A}$ \\
\hline Maximum focusing strength, $\int \mathrm{B}^{2} \mathrm{dL}$ & $4 \mathrm{~T}^{2} \mathrm{~m}$ \\
\hline Maximum field integral, $\mathrm{SBdL}$ & $0.763 \mathrm{~T} \cdot \mathrm{m}$ \\
\hline Field in the center at maximum current & $6.82 \mathrm{~T}$ \\
\hline Effective length, $\int \mathrm{BdL} / \mathrm{B}_{0}$ & $11.19 \mathrm{~cm}$ \\
\hline Dipole corrector & \\
\hline Maximum current & $32.5 \mathrm{~A}$ \\
\hline Maximum field integral, $\int \mathrm{BdL}$ & $2.5 \mathrm{mT} \cdot \mathrm{m}$ \\
\hline Beam pipe aperture & $30 \mathrm{~mm}$ \\
\hline \hline
\end{tabular}

As stated above, each focusing element package contains five coils: the main solenoid, and four corrector coils. The conduction cooled current lead design must support $100 \mathrm{~A}$ in the solenoid leads and $50 \mathrm{~A}$ in the corrector leads. Their design is based on the design of similar leads installed in the LHC at CERN. Thermal intercepts at $70 \mathrm{~K}$ and at $5 \mathrm{~K}$ help to reduce the heat load to $2 \mathrm{~K}$, nonetheless, these current leads represent a significant source of heat at the low temperature end. There is one lead assembly for each magnetic element.

The linac lattice, especially the low-beta section, provides limited space along the beamline for beam diagnostics either inside individual cryomodules or between adjacent modules. In order to conserve axial space along the beamline a button-type beam position monitor (BPM) was chosen for installation in the SSR cryomodules [93]. For a non-relativistic beam they also generate larger signal than strip-line BPMs. A total of four BPMs will be installed in the cryomodule, one near each magnetic package. These devices are compact and lend themselves well to incorporation into the solenoid magnet package as shown in Figure 3.51. The bellows at either end of the beam tube allow independent alignment of each magnet package. 


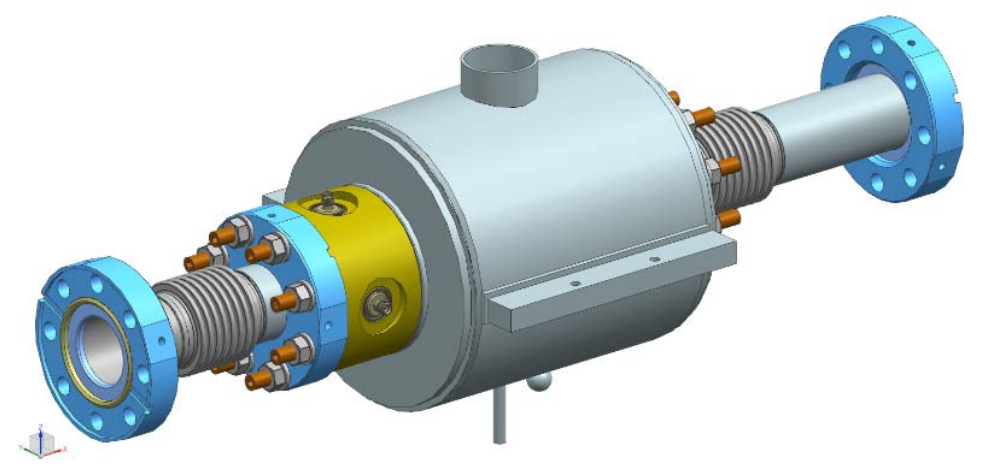

Figure 3.51: Solenoid and BPM assembly.

\section{RF Coupler}

The RF coupler is based on a $105 \Omega$ coaxial design. The power rating of the coupler of $30 \mathrm{~kW}$ is set by the following. The coupler has to supply up to $5 \mathrm{~kW} \mathrm{CW}$ power to each SSR1 cavity. The coupler is expected to have the same design as the SSR2 coupler which is required to deliver up to $14 \mathrm{~kW} \mathrm{CW}$ in the PIP-II era. A possible future PIP-II linac upgrade calls for $30 \mathrm{~kW}$ maximum power [94].

The coupler contains a single warm ceramic window. During cryomodule fabrication, the cold part of the coupler is installed on the cavity in the cleanroom prior to assembly of the string. The warm part of the coupler is installed from outside the vacuum vessel during final assembly. The inner conductor is solid copper with copper plated bellows to accommodate motion due to misalignment and thermal contraction. The cold end of the outer conductor is $316 \mathrm{~L}$ stainless steel. The warm end is copper with copper plated bellows. Heat load estimates do not suggest a significant penalty for not copper plating the outer conductor. A forced-air cooling tube is inserted into the inner conductor. It supplies air to cool the coupler tip. Figure 3.52 shows the current coupler design. The coupler design is described more thoroughly in [95].

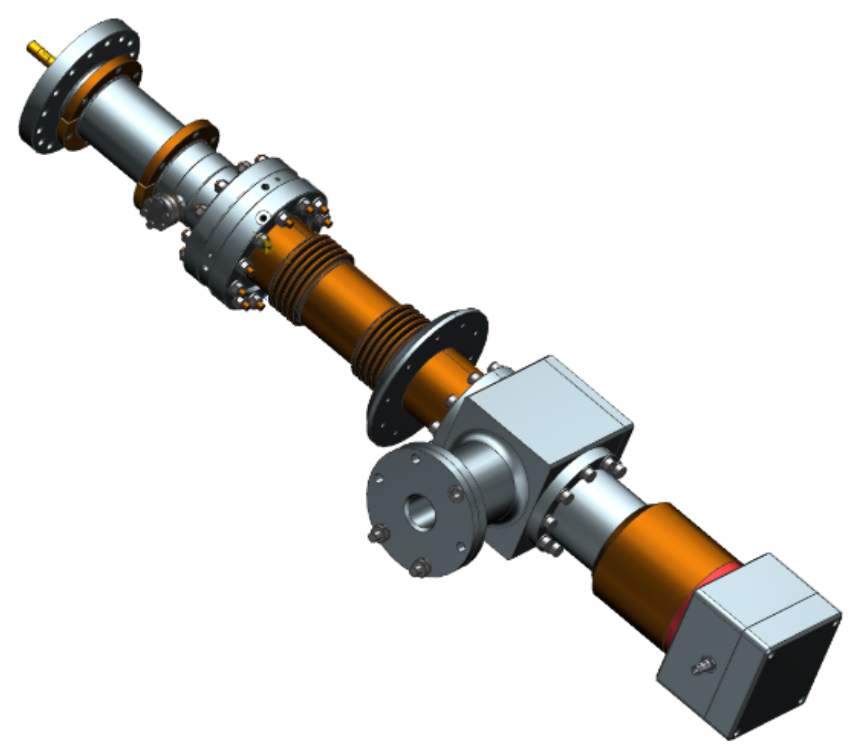

Figure 3.52: RF coupler assembly.

Three prototypes were procured and successfully tested up to $30 \mathrm{~kW}$ [96], in full reflection mode, 
on the RF test stand at room temperature. One of the units was tested to failure at $47 \mathrm{~kW}$, in full reflection mode, on the RF test stand at room temperature. A complete coupler assembly was qualified during tests in the spoke test cryostat (STC) [87].

\section{SSR1 Cold-mass}

Each cryomodule will have a single thermal shield cooled with helium gas, nominally at 45-80 K. It is currently envisioned to be aluminum with cooling channels on both sides. Two 15-layer blankets of multilayer insulation, between the vacuum vessel and thermal shield, will reduce the radiation heat load to the $80 \mathrm{~K}$ shield from the room temperature vacuum vessel to approximately $1.5 \mathrm{~W} / \mathrm{m}^{2}$. A $5 \mathrm{~K}$ circuit will be available to intercept heat on the RF couplers and current leads, but there is no plan to install a full $5 \mathrm{~K}$ thermal shield.

All cavities and solenoids will be mounted on individual support posts, which are in turn mounted to a full-length room temperature strongback located between the vacuum vessel and thermal shield. This enables the entire cavity string to be assembled and aligned as a unit then inserted into the vacuum vessel during final assembly. Presently, the strongback is aluminum, but stainless steel is an option. Maintaining the strongback at room temperature helps to minimize axial movement of the cold elements during cooldown, reducing displacement of couplers, current leads, and other internal piping components. The support posts are similar to supports utilized in the SSC collider dipole magnets, and the ILC and XFEL 1.3 GHz cavity cryomodules. The main structural element is a glass and epoxy composite tube. The tube ends and the intermediate thermal intercepts are all assembled using a shrink-fit technique in which the composite tube is sandwiched between an outer metal ring and an inner metal disk [97]. All of the cavities and solenoids are mounted to the support posts using adjustable positioning mechanisms. Figure 3.53 shows a view of cold-mass assembly.

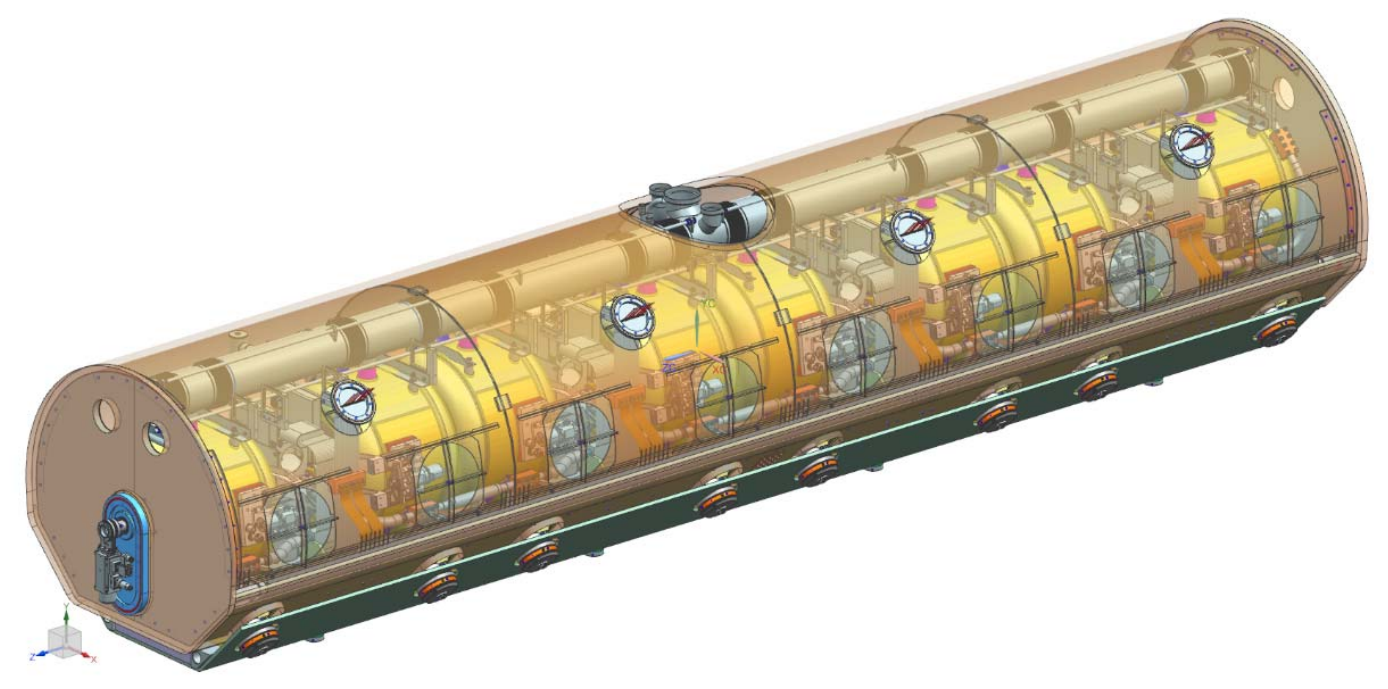

Figure 3.53: SSR1 cold-mass assembly.

Alignment of cavities and solenoids will be accomplished using optical targets installed on the internal assemblies translated to fiducials installed on the vacuum vessel. Changes in alignment due to shipping and handling or during cooldown and operation will be monitored using a series of wire targets on each cavity and solenoid, viewed through optical windows in either end of the cryomodule assembly. The requirements on the alignment of cavities and magnet assemblies are presented in Table 3.8. 
Table 3.8: Requirements on alignment of SSR1 cavities and magnet assemblies

\begin{tabular}{l|c|c}
\hline \hline & Cavities & Magnet assemblies \\
\hline Rms transverse position, $\mathrm{mm}$ & 1 & 0.5 \\
\hline Rms angular alignment, $\mathrm{mrad}$, & 10 & 1 \\
\hline \hline
\end{tabular}

\section{Radio Frequency Tuner}

The cavity tuner has to compensate for its resonant frequency perturbations. In order to meet the requirements for frequency range and resolution, the tuning system integrates coarse and fine mechanisms engaged in series. The former utilizes a stepper motor with large stroke capability and limited resolution, the latter contains piezo-electric actuators with limited stroke but virtually infinite resolution. With the goal of maintaining the cavity at the operational frequency of $325 \mathrm{MHz}$, the tuner has to be designed to compensate uncertainties in the frequency shift due to the cooldown from $293 \mathrm{~K}$ to $2 \mathrm{~K}$, and to minimize detuning caused by microphonics and Lorentz forces. Cooldown uncertainties are estimated to be less than $135 \mathrm{kHz}$, while the amplitude of perturbations is estimated to be less than $1 \mathrm{kHz}$. These two values define respectively the requirements for coarse and fine tuning mechanisms. In order to achieve a low sensitivity to pressure $(d f / d p)$, the stiffness of the tuner seen by the cavity flange (passive stiffness) must be greater than $30 \mathrm{kN} / \mathrm{mm}$. Table 3.9 presents requirements for the tuning system. Each cavity will have its own tuning system.

Table 3.9: SSR1 tuning system requirements

\begin{tabular}{l|c}
\hline \hline & Requirement \\
\hline Coarse frequency range & $135 \mathrm{kHz}$ \\
\hline Coarse frequency resolution & $20 \mathrm{~Hz}$ \\
\hline Fine frequency range & $1 \mathrm{kHz}$ \\
\hline Fine frequency resolution & $\leq 2 \mathrm{~Hz}$ \\
\hline \hline
\end{tabular}

The reliability of a frequency-tuning system is always of great concern. Experience suggests that the active components are prone to failures if not integrated and operated carefully. Failure of the tuning system has an immediate impact on the accelerator complex. Thus, the system shall be designed to allow the replacement of the actuating devices in case of failure or deterioration. Maintenance operations shall be simplified, where possible, considering that the system will be serviced manually through access ports in the vacuum vessel of the cryomodule.

Considering various types of tuner systems [98] and based on experience and lessons learned with the previous SSR1 tuner [99], a lever mechanism was chosen for the control of the resonant frequency in both operating conditions: $\mathrm{CW}$ and pulsed regime [100]. The resonant frequency is changed by adjusting the spacing between the cavity end-wall and the spoke. This will be achieved by acting on the cavity sidewall, which is connected to the helium vessel by the flexible joint (bellows). Controlling this gap by elastically deforming the cavity niobium structure, the resonant frequency has to be kept within the $\pm 20 \mathrm{~Hz}$ from the nominal value of $325 \mathrm{MHz}$.

Figure 3.54 shows the schematic of a double lever mechanism that allows coarse and fine cavity tuning. The large scale tuning ( $135 \mathrm{kHz}$ range) is accomplished via a stepper motor actuating through a threaded rod and traveling nut, translating rotational motion into axial motion of a double-lever 
mechanism, compressing and relaxing the cavity wall at one of the two beam-pipes. The fine-tuning has a tuning range of $1 \mathrm{kHz}$ and is achieved with piezoelectric actuators (or piezos) acting serially with coarse tuning mechanism. This fast tuner has to compensate perturbation related to the helium bath pressure fluctuations, Lorentz force detuning and mechanical vibrations. The two main arms are hinged at one end and connected to the actuation system at the other end. The cavity is tuned by pushing on the beam pipe.

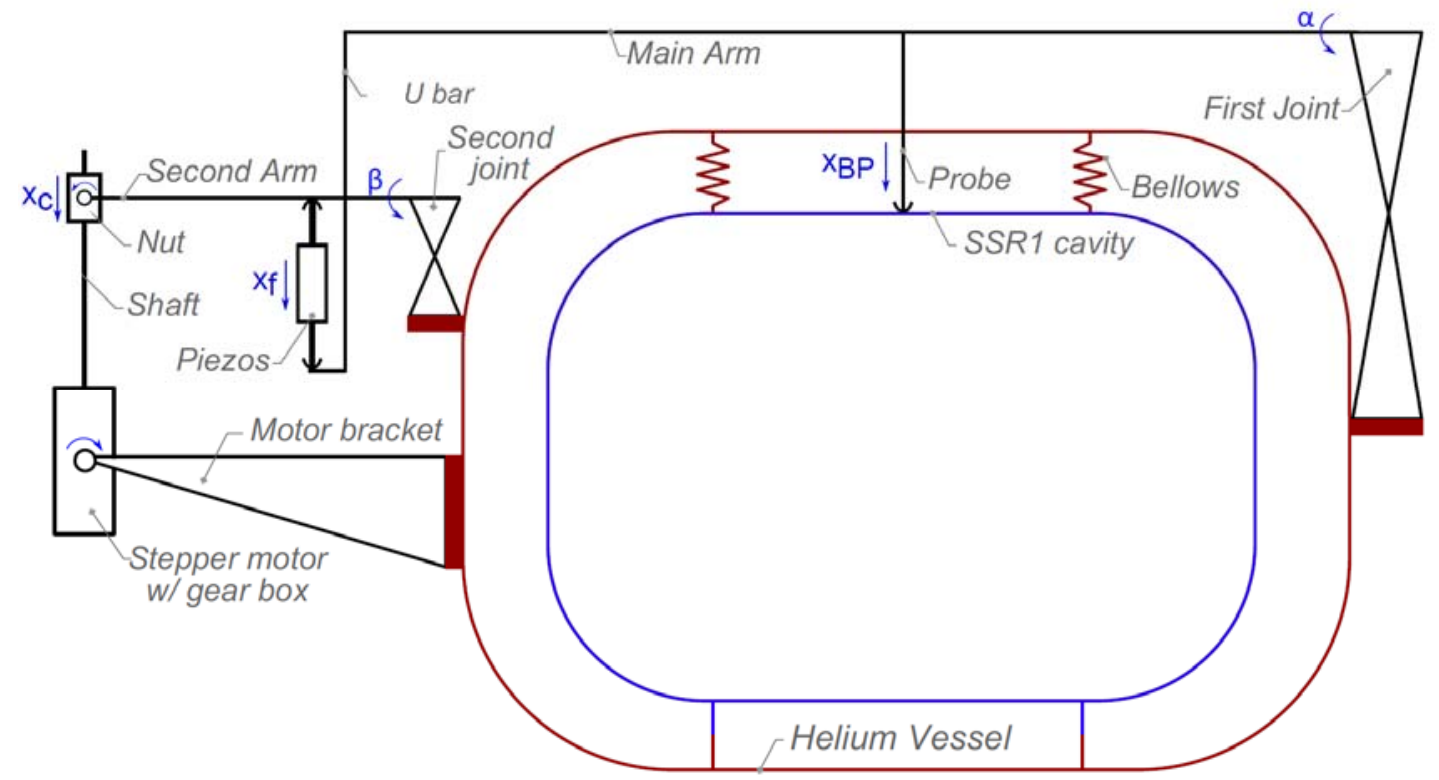

Figure 3.54: Jacketed S1H-NR-107 dressed with tuner mechanism and power coupler, installed in the Spoke Test Cryostat (STC).

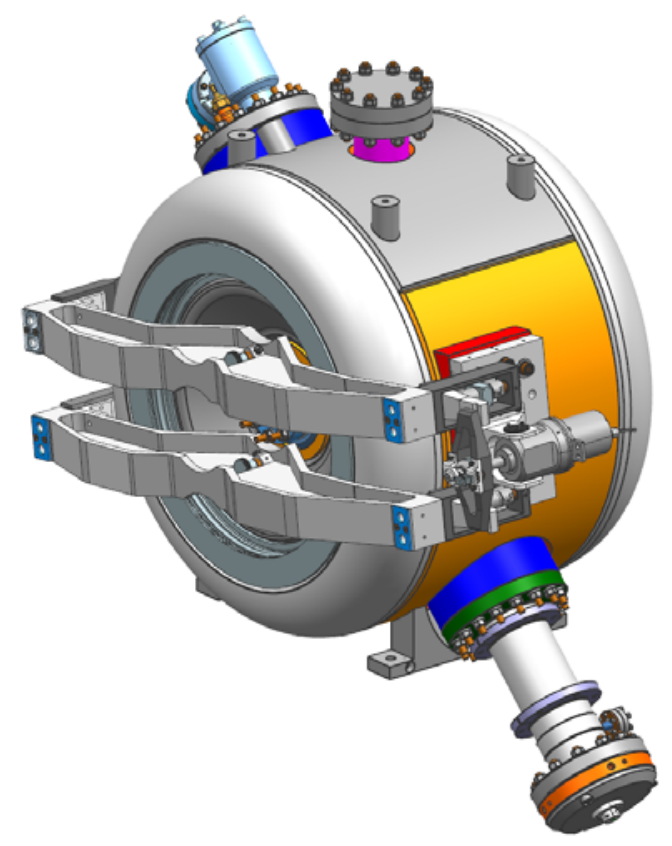

Figure 3.55: SSR1 cavity, helium vessel, tuner and coupler.

The main structural components are made of $316 \mathrm{~L}$ stainless steel. The helium vessel is made of the same material. This has the advantage of having the same thermal contraction ratio during the 
cooldown. Figure 3.55 shows the 3D model of the SSR1 tuner assembled on the jacketed cavity.

Figures of merit for the tuner such as tuning range, components hysteresis and the overall performance were measured during tests in the STC with the prototype tuner at low temperature. The results are presented in Figure 3.56. The tuner mechanism has excellent linearity in the entire tuning range. All specifications for stiffness, tuning ranges, tuning efficiencies, and resolutions of the active components have been met. More details can be found in Ref. [101]. This prototype tuner was also successfully used in studies of resonant control [102] for SSR1 cavities. The fast tuner was used to drive cavity characterization routines, including mechanical-to-electrical transfer functions.

The design of the production tuner is in progress.

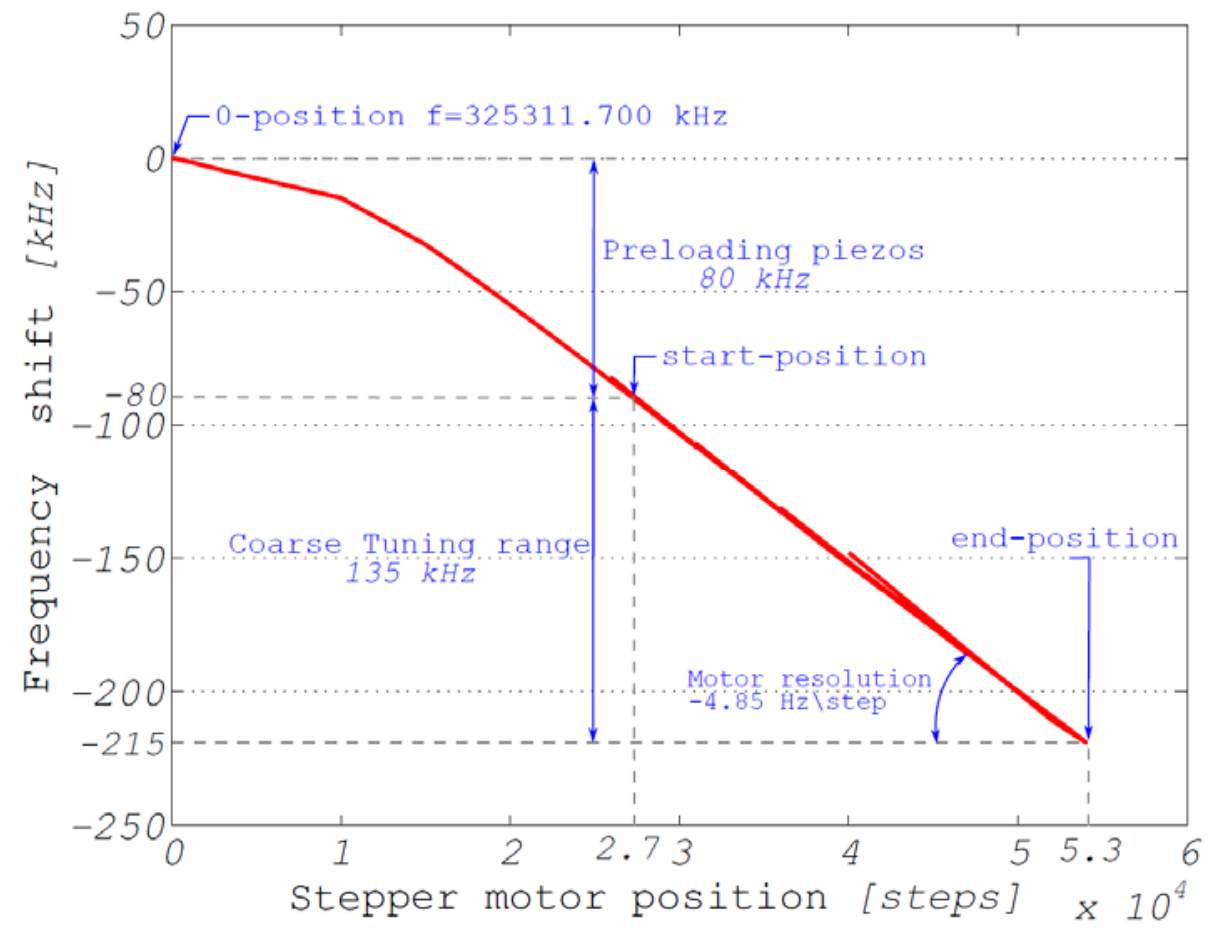

Figure 3.56: The dependence of cavity frequency on the stepper motor position.

\section{SSR1 Cryomodule Final Assembly}

The vacuum vessel contains all cryomodule components in their as-installed positions. It has a secure anchorage to the tunnel floor, thermally insulates all cryogenic components thus minimizing heat loads to $80 \mathrm{~K}, 4.5 \mathrm{~K}$, and $2 \mathrm{~K}$, and maintains the insulating vacuum. The vessel has $1.219 \mathrm{~m}$ (48 inches) diameter. A view is presented in Figure 3.57.

Just inside the vacuum vessel, nearly in contact with the inner wall, is a magnetic shield that shields the cavities from the earth's magnetic field. Tests show that a $1.5 \mathrm{~mm}$-thick mu-metal shield at room temperature reduces the residual field inside the cryostat to less than $10 \mu \mathrm{T}$. It is likely that separate magnetic shields will also be installed around individual magnetic elements to further reduce trapped fields in the superconducting cavities.

The intent is to have all external connections to the cryogenic, RF, and instrumentation systems made at removable junctions at the cryomodule itself. The only connection to the beamline is the beam pipe, which will be terminated by "particle free" valves at both ends. The major goals of the design are minimization of mean time between failures and time required for repairs. The latter is achieved by allowing in-situ repair of some internal systems like cavity tuners. 


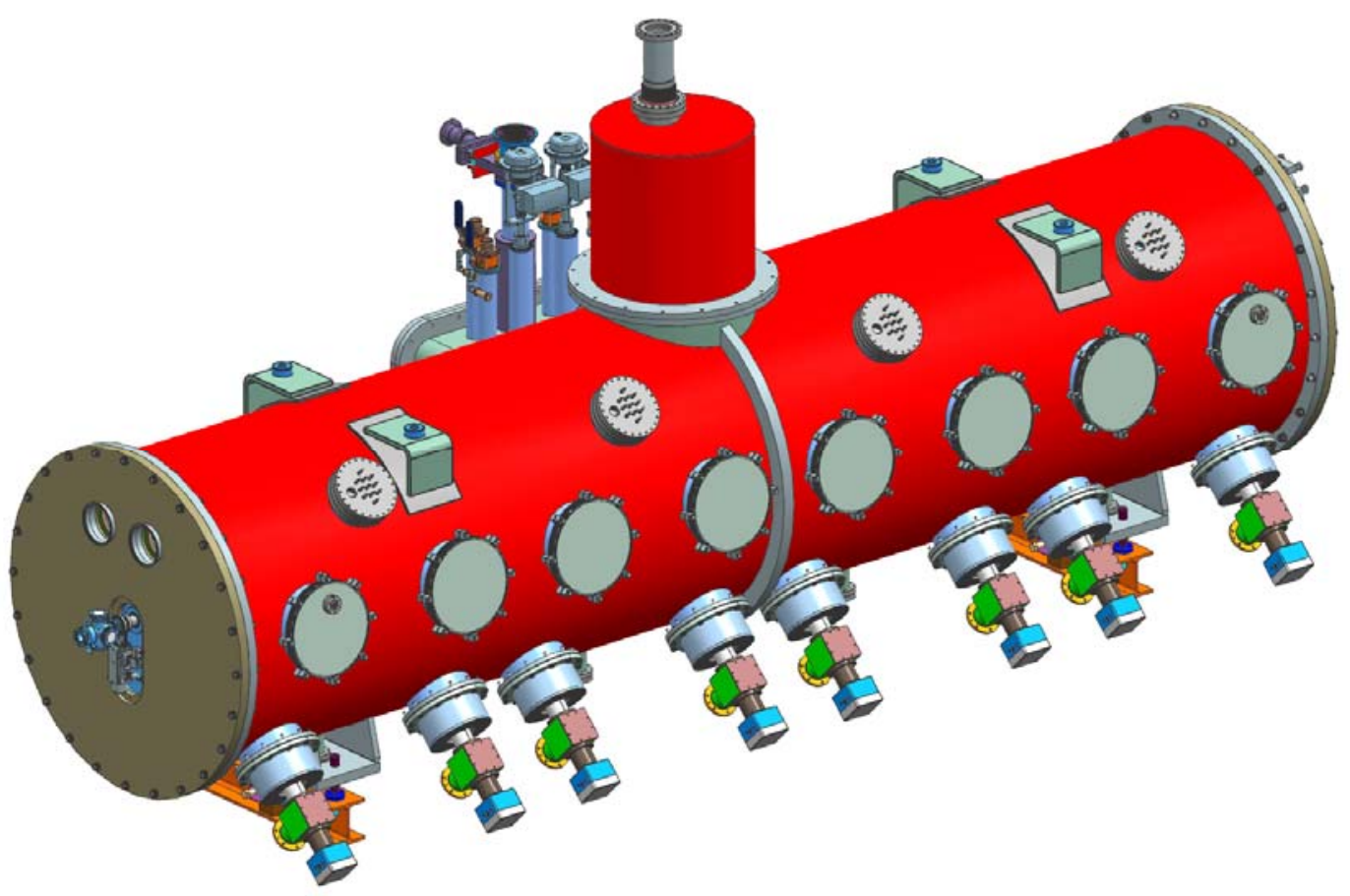

Figure 3.57: SSR1 cryomodule assembly.

\section{SSR1 Heat Load Estimate}

Table 3.10 summarizes the estimated static and dynamic heat loads at each temperature level in the cryomodule assembly from the primary sources. As mentioned earlier, the nominal $70 \mathrm{~K}$ thermal shield and intercepts may operate anywhere between 45 and $80 \mathrm{~K}$.

Table 3.10: SSR1 Cryomodule heat load estimates

\begin{tabular}{l|c|c|c|c|c|c|c}
\hline \multirow{2}{*}{} & \multicolumn{3}{|c|}{ Per Unit (W) } & Units & \multicolumn{3}{c}{ Total (W) } \\
\cline { 2 - 8 } & $70 \mathrm{~K}$ & $5 \mathrm{~K}$ & $2 \mathrm{~K}$ & & $70 \mathrm{~K}$ & $5 \mathrm{~K}$ & $2 \mathrm{~K}$ \\
\hline RF coupler, static & 5.4 & 2.8 & 0.5 & 8 & 43 & 23 & 4 \\
\hline RF coupler, dynamic & 0 & 0 & 0.25 & 8 & 0 & 0 & 2 \\
\hline Cavity, dynamic & 0 & 0 & 1.8 & 8 & 0 & 0 & 14 \\
\hline Support post & 2.8 & 0.4 & 0.05 & 12 & 33 & 4 & 0.6 \\
\hline Conductor Lead Assembly & 36.8 & 13.2 & 1.2 & 4 & 147 & 53 & 5 \\
\hline MLI & 30.5 & 0 & 1.4 & 1 & 31 & 0 & 1 \\
\hline Cold-warm transition & 0.7 & 0.1 & 0.01 & 2 & 1 & 0.2 & 0.02 \\
\hline TOTAL & & & & & 255 & 80 & 27 \\
\hline \hline
\end{tabular}

* MLI stands for multi-layer thermal insulation.

\subsubsection{Single Spoke Resonator II (SSR2) Cavities and Cryomodules}

The design of the SSR2 cryomodule is based on the experience obtained with SSR1 cryomodule. The sequence of cavities, C, and focusing solenoids, S, in a cryomodule is SCCSCCSC. In general, 
the cryomodule structure is similar to the SSR1 cryomodule: 2 cavities per focusing solenoid. However, because the cavities and solenoids are longer the SSR2 cryomodule has fewer cavities and solenoids. The changes in the sequence of elements inside cryomodule are associated with a reduction of longitudinal phase advance per cell due to increased energy. There are many elements in the SSR2 cryomodule, which are identical or nearly identical to the SSR1 cryomodule. In most cases, the elements are scaled due to increased beam aperture and sizes of cavities and solenoids.

\section{RF Design and Optimization of SSR2 Cavity}

SSR2 is a single spoke resonator operating at $325 \mathrm{MHz}$. These cavities will accelerate particles from $35 \mathrm{MeV}$ to $185 \mathrm{MeV}$. Figure 3.58 shows the SSR2 RF geometry in $y$ - $z$ cross-section, where $y$ is directed along the spoke and $z$ is directed along the beam axis. Definitions for the main geometry parameters are shown in the figure, and their values are reported in Table 3.11. Electric and magnetic 3D fields have been simulated with "COMSOL multi-physics" and are plotted in Figure 3.59.

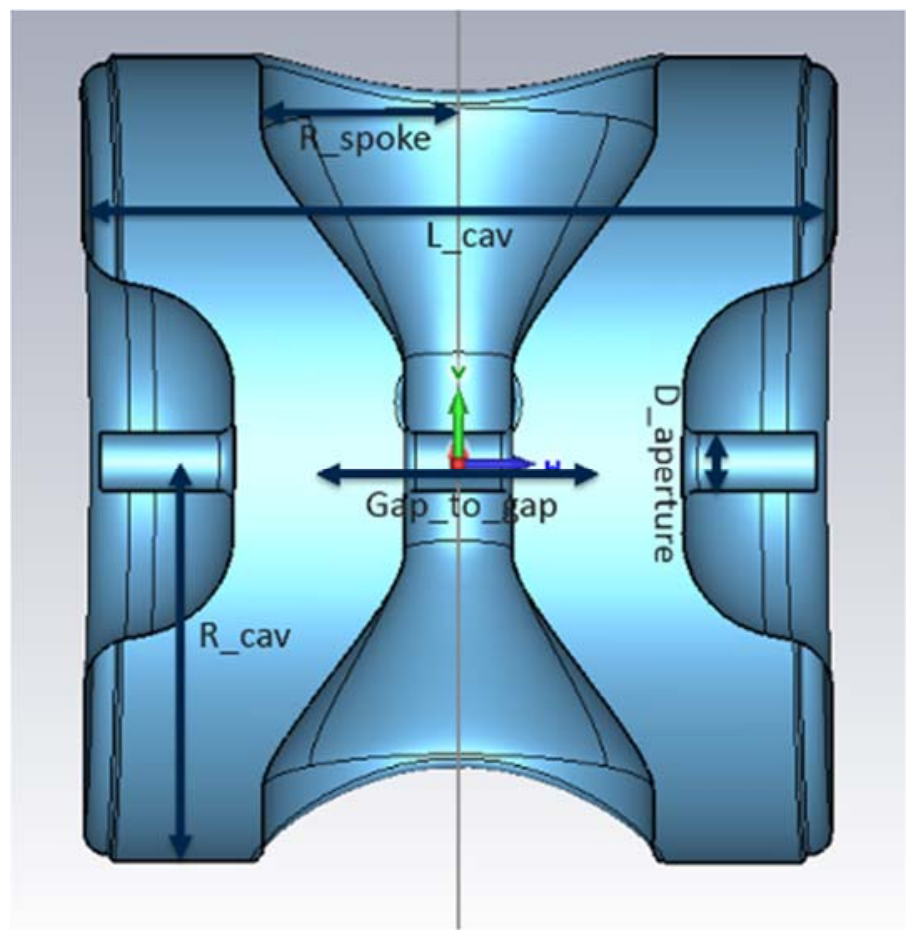

Figure 3.58: SSR2 $y-z$ cross-section and definition of main geometry parameters.

Table 3.11: Main parameters of cavity geometry

\begin{tabular}{l|l}
\hline \hline Parameter & {$[\mathrm{mm}]$} \\
\hline L_cav & 500 \\
\hline R_cav & 271.6 \\
\hline R_spoke & 130.7 \\
\hline D_aperture & 40 \\
\hline Gap_to_gap & 185.9 \\
\hline \hline
\end{tabular}



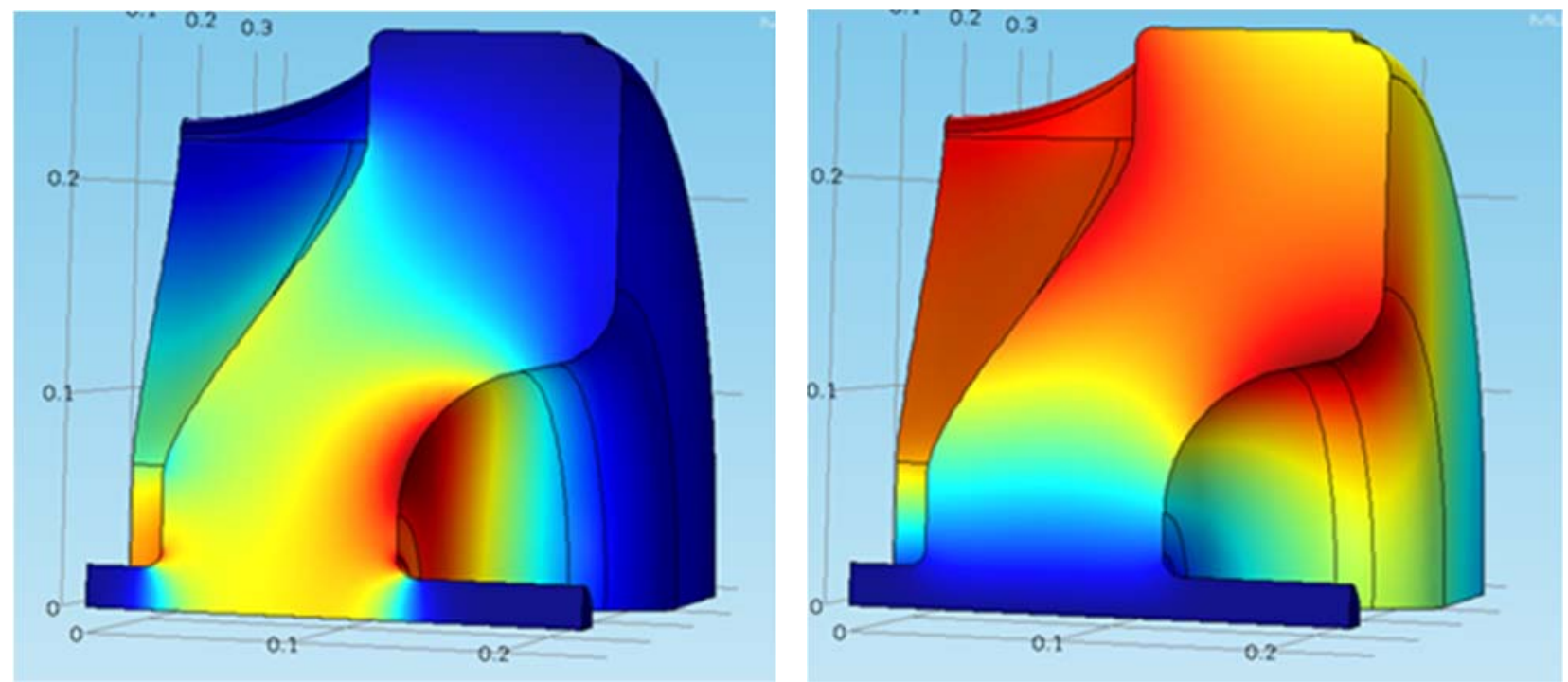

Figure 3.59: SSR2 Electric (left) and magnetic (right) 3D fields computed by COMSOL.

The value of $\beta_{\text {opt }}=0.47$ has been chosen to accommodate the beam dynamics requirements and to minimize the overall number of cavities. The accelerating gradient $E_{a c c}$ is defined over the effective length $L_{e f f}=\beta_{\text {opt }} \lambda$, for acceleration at $\beta_{\text {opt }}$. Here $\lambda$ is the electromagnetic field wavelength at $325 \mathrm{MHz}$. Table 3.12 shows the EM parameters of SSR2 cavity. For given limitations of peak fields $(40 \mathrm{MV} / \mathrm{m}$ and $70 \mathrm{mT}$ ) the SSR2 cavity provides a maximum energy gain of $5.17 \mathrm{MeV}$.

Table 3.12: SSR2 EM parameters

\begin{tabular}{l|c}
\hline \multicolumn{1}{c|}{ Parameter } & Value \\
\hline Frequency $[\mathrm{MHz}]$ & 325 \\
\hline Optimal beta & 0.475 \\
\hline Effective length $[\mathrm{m}]$ & 0.438 \\
\hline Epeak $/$ Eacc & 3.38 \\
\hline Beak $/$ Eacc $[\mathrm{mT} /(\mathrm{MV} / \mathrm{m})]$ & 5.93 \\
\hline $\mathrm{G}[\Omega]$ & 115 \\
\hline $\mathrm{R} / \mathrm{Q}[\Omega]$ & 297 \\
\hline
\end{tabular}

Focusing in the SSR2 cryomodules is provided by solenoids and is axially symmetric. However, spoke resonators have a central electrode which is perpendicular to the particle motion. It breaks the axial symmetry of the system and symmetry of transverse electric and magnetic fields, and results in non-axially symmetric focusing coming from the cavity. Transverse electric and magnetic fields of an SSR2 cavity are shown in Figure 3.60. Together they create a quadrupole component of cavity defocusing (see Figure 2.27). This focusing asymmetry is compensated by skew-quadrupoles located in the magnet assemblies, which are built similarly to the SSR1 magnet assemblies. 

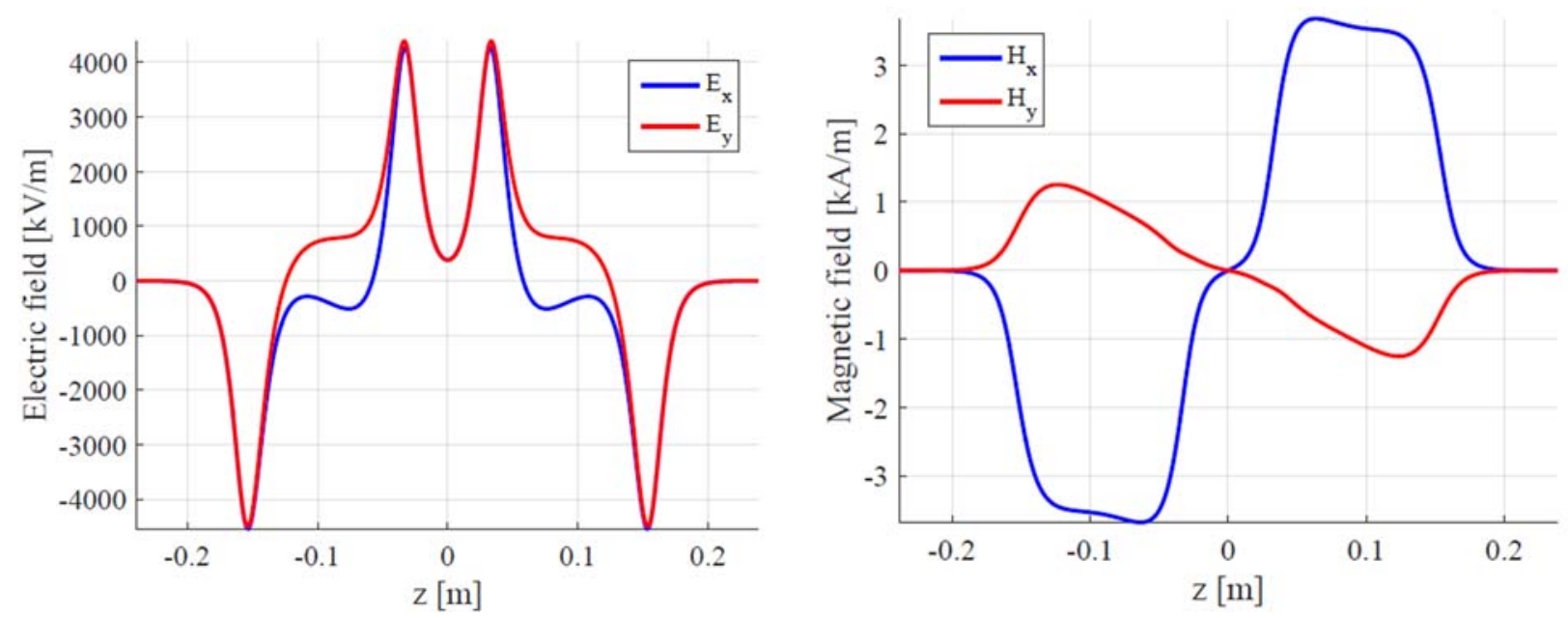

Figure 3.60: SSR2 transverse electric (left) and magnetic (right) fields, calculated at $r=10 \mathrm{~mm}$.

The last iteration on SSR2 electromagnetic design was focused on the reduction of multipacting (MP), since the previous cavity design showed strong and wide MP barriers. The growth rate in the number of electrons is used as a figure of merit in multipacting simulations. Figure 3.61 compares the growth rates for different designs of spoke cavities: SSR1, SSR2 v1.0 (old design) and SSR2 v2.6. One can see significant reduction in multipacting for the latest design. The simulations also demonstrate multipacting levels lower than those for the SSR1 cavity, which is already built and tested, and demonstrated satisfactory multipacting performance.

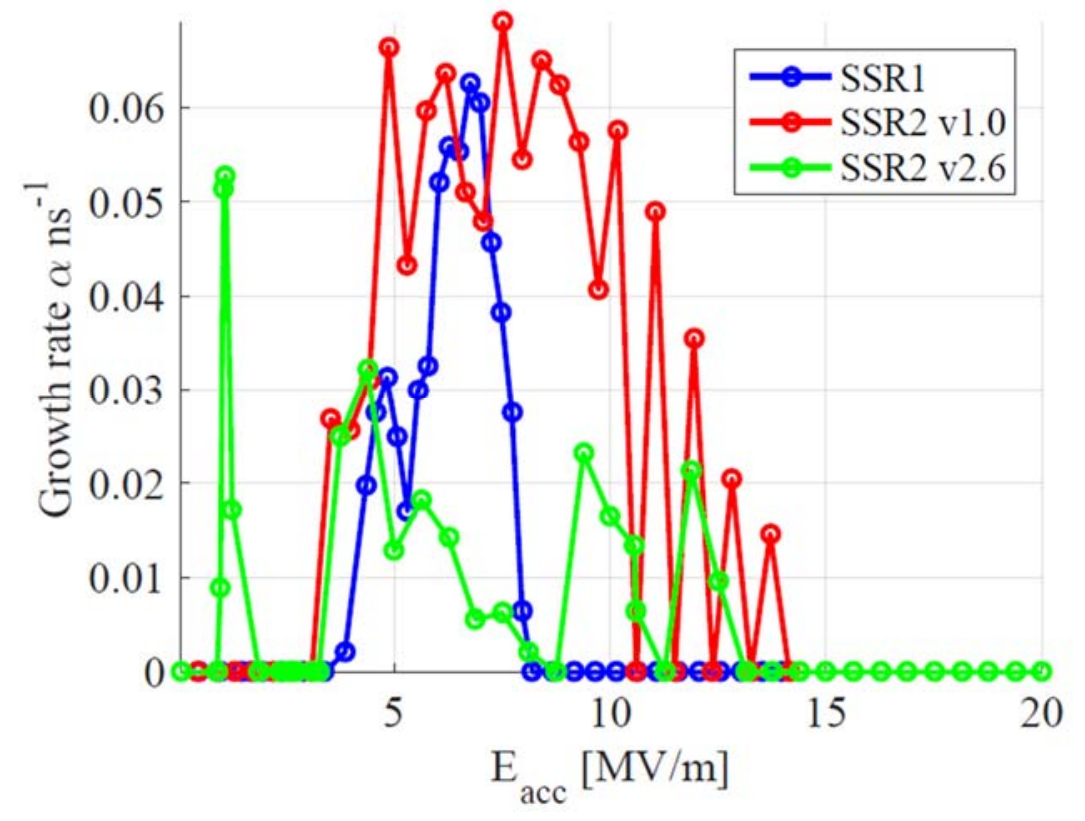

Figure 3.61: Growth rate comparison from CST, SSR1 and both SSR2 designs.

\section{Structural Design of the SSR2 Cavities}

The structural design of the SSR2 cavities will be similar to that of the SSR1 cavities. The IIFC (Indian Institutions Fermilab Collaboration) is working on the cavity design. The design is guided by the Functional Requirement Specifications for the SSR2 cavity [103]. Table 3.13 presents its major requirements. 
Table 3.13: Requirements for the SSR2 cavity structural design

\begin{tabular}{l|l}
\hline \hline Parameter & Requirement \\
\hline Max leak rate (room temp) & $<10^{-10}$ atm-cc/sec \\
\hline Operating gain per cavity & $5.0 \mathrm{MeV}$ \\
\hline Sensitivity to He pressure fluctuations $d f / d P$ & $<19 \mathrm{~Hz} / \mathrm{Torr}$ \\
\hline Lorentz Forse Detuning (when jacketed) & $<4 \mathrm{~Hz} /(\mathrm{MV} / \mathrm{m})^{2}$ \\
\hline Field Flatness & Better than $90 \%$ \\
\hline Operating temperature & $1.8-2.1 \mathrm{~K}$ \\
\hline Operating pressure & $16-41 \mathrm{mbar}$ differential \\
\hline MAWP & 2 bar $(\mathrm{RT}), 4$ bar $(2 \mathrm{~K})$ \\
\hline RF power input per cavity & $17 \mathrm{~kW}(\mathrm{CW}$, operating) \\
\hline \hline
\end{tabular}

\section{SSR2 Solenoid, Beam Position Monitor Current Leads, RF Coupler and Tuner}

There are three magnet packages in the SSR2 cryomodule. As in the SSR1 cryomodule, each package contains a focusing solenoid (lens) and four corrector coils all operating in a helium bath at $2 \mathrm{~K}$. The general design requirements for the lenses in the SSR2 cryomodule are based on Ref. [104]. The main parameters of the magnet assemblies are presented in Table 3.14 [105].

Table 3.14: Main parameters of the SSR2 magnet assembly

\begin{tabular}{l|c}
\hline \hline Solenoid & \\
\hline Maximum focusing strength, $\int \mathrm{B}^{2} \mathrm{dL}$ & $5 \mathrm{~T}^{2} \mathrm{~m}$ \\
\hline Maximum current & $74.97 \mathrm{~A}$ \\
\hline Maximum field integral, $\mathrm{SBdL}$ & $0.978 \mathrm{~T} \cdot \mathrm{m}$ \\
\hline Field in the center at maximum current & $6.024 \mathrm{~T}$ \\
\hline Dipole corrector & \\
\hline Maximum field integral, $\int \mathrm{BdL}$ & $5 \mathrm{mT} \cdot \mathrm{m}$ \\
\hline Maximum current & $37.3 \mathrm{~A}$ \\
\hline Beam aperture & $40 \mathrm{~mm}$ \\
\hline \hline
\end{tabular}

The design of the button-type beam position monitor (BPM) represents a scaled (from 30 to 40 $\mathrm{mm}$ ) version of the SSR1 button BPM design.

The current leads and RF coupler of the SSR2 cryomodules are identical to the corresponding items of the SSR1 cryomodules.

The tuner design is mostly identical to the SSR1 tuner. The requirements for the tuner performance are identical to the SSR1 tuner and are presented in Table 3.9.

\section{SSR2 Cryomodule}

The electromagnetic design of the cavity is the only part of this cryomodule that has been studied and developed so far. 
However, in the design of SSR2 cryomodule we will take into account all the lessons learned during the design, fabrication and testing phase of the SSR1 cryomodule. The general concept of the main components will be as similar as possible. In particular, the tools, which we are developing for assembly of the SSR1 cryomodule, are designed to be also compatible with the SSR2 cryomodule assembly everywhere where it is possible.

\subsubsection{Elliptical $650 \mathrm{MHz}$ Cavities and Cryomodules (LB650 and HB650)}

Acceleration from $185 \mathrm{MeV}$ to $800 \mathrm{MeV}$ is provided by two families of 5-cell elliptical cavities operating at $650 \mathrm{MHz}$ and designed for $\beta_{G}=0.61$ and $\beta_{G}=0.92$. The cavity shape is optimized to decrease the field enhancement factors (magnetic and electric) in order to minimize fields on the cavity walls. A reduction of aperture is greatly helpful in decreasing the field enhancement factors. However, it is limited by the following considerations:

- field flatness,

- beam losses,

- mechanical stability,

- $\quad$ reliable surface processing.

The working gradient is chosen to provide a peak surface magnetic field that allows operation below the high-field Q-slope (see Figure 2.12). For a frequency of $650 \mathrm{MHz}$ the peak magnetic field should be not greater than approximately $70 \mathrm{mT}$. In addition, we require that the peak surface electric field be lower than $40 \mathrm{MV} / \mathrm{m}$ in order to avoid the risk of strong field emission.

The $650 \mathrm{MHz}$ cavities require sufficient wall thickness to minimize sagging caused by the overall weight. Note that stiffening rings are used for both the ILC and $650 \mathrm{MHz}$ cavities to increase the rigidity of cavities. Limiting the maximum cavity sag to $120 \mu \mathrm{m}$ (the same as in the ILC) results in a $4 \mathrm{~mm}$ wall thickness. A small cavity wall slope gives more freedom in decreasing the field enhancement factors. However, the slope is also constrained by surface processing and mechanical stability requirements. The chosen slope of about $2^{\circ}$ results in an acceptable value for the field enhancement.

Optimization of the two $650 \mathrm{MHz}$ cavity shapes was based on the constraints discussed above. The cavity performance parameters are summarized in Section 2. The physical description of the cavity shapes is presented in Figure 3.62 and Table 3.15. All sizes are shown for $2 \mathrm{~K}$ temperature. Requirements for maximum cavity detuning amplitude and cavity sensitivity versus helium pressure fluctuations are discussed in Section 2 (see also Ref. [20]). Note that the $650 \mathrm{MHz}$ cavities have small beam loading, and thus microphonics mitigation is essential. Therefore the cavities are overcoupled; both active and passive means for microphonics compensation are planned to be used [106]. The preliminary mechanical design of the HB650 cavity is shown in Figure 3.63.

Neither of the $650 \mathrm{MHz}$ cavities contain HOM dampers - they are not necessary for the required beam current [77]. This choice is also supported by the experience accumulated in the SNS [6]. An absence of HOM dampers is more problematic for future PIP-II upgrades.

The $650 \mathrm{MHz}$ cavity and cryomodule section of the linac contains a total of 33 low-beta cavities in 11 cryomodules and 24 high-beta cavities in 4 cryomodules. All of the needed focusing magnets are at room temperature and located between cryomodules. Figure 2.1 shows the linac layout and the location of the $650 \mathrm{MHz}$ section.

The cavity string design for each cryomodule is based on the similar designs for SSR1 and SSR2. The cavity string is supported on a room temperature strongback located inside the vacuum vessel. Each cavity is supported by two composite support posts. The attachment to the supports allows axial 
motion during cooldown to prevent excessive loading on the supports with one end fixed. The fixed end is located closer to the input coupler, thus minimizing coupler motion during cooldown.

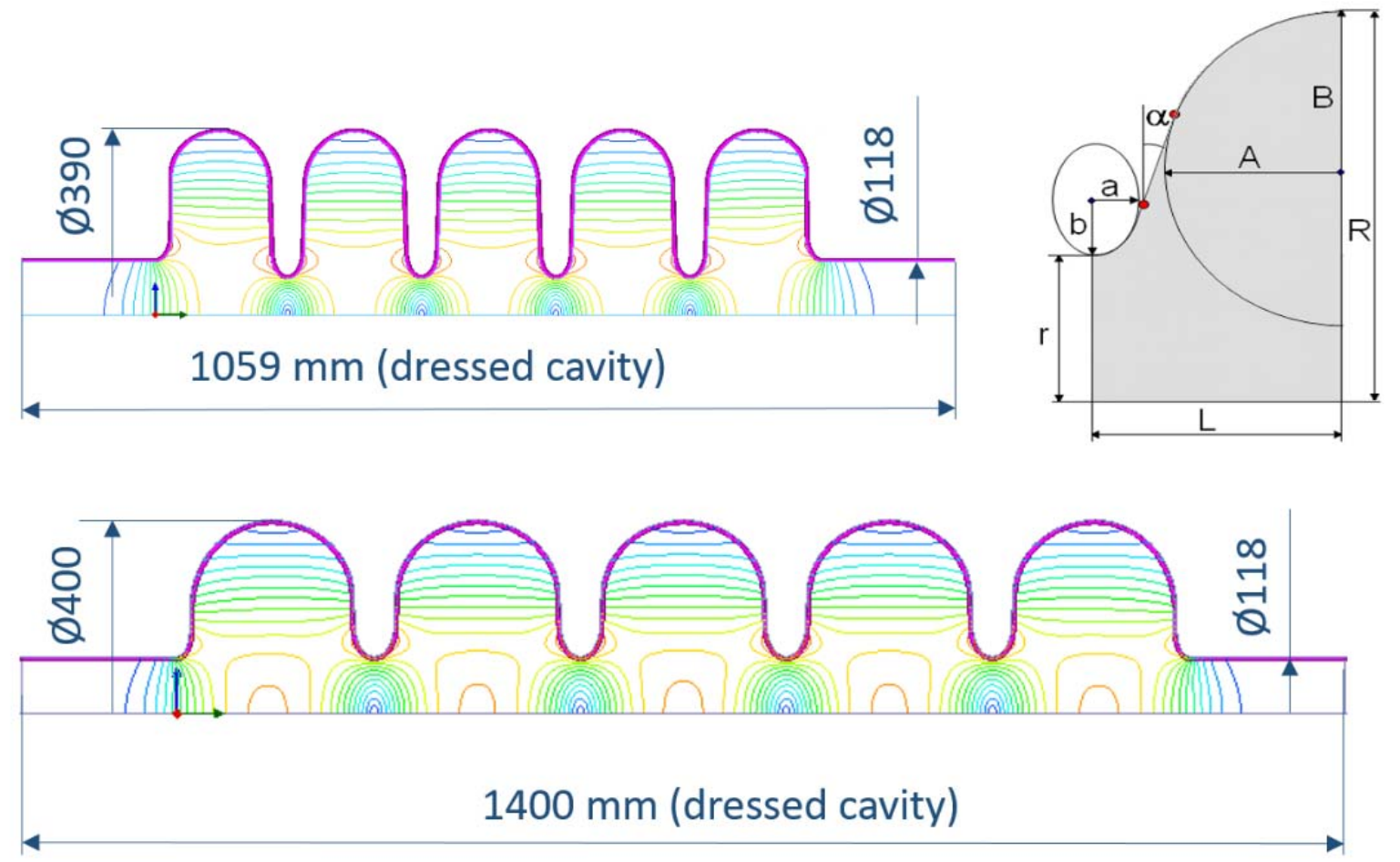

Figure 3.62: Layout of $650 \mathrm{MHz}$ cavities: LB650 - top and HB650 - bottom.

Table 3.15: Dimensions of the $650 \mathrm{MHz}$ cavities

\begin{tabular}{l|c|c|c|c}
\hline \hline \multirow{2}{*}{ Dimension* } & \multicolumn{2}{|c|}{ LB650 } & \multicolumn{2}{c}{ HB650 } \\
\cline { 2 - 5 } & Regular cell & End cell & Regular cell & End cell \\
\hline $\mathrm{r}, \mathrm{mm}$ & 41.5 & 59 & 59 & 59 \\
\hline $\mathrm{R}, \mathrm{mm}$ & 195 & 195 & 200.1 & 200.1 \\
\hline $\mathrm{L}, \mathrm{mm}$ & 70.3 & 68.4 & 106.1 & 97.6 \\
\hline $\mathrm{A}, \mathrm{mm}$ & 54 & 54 & 85 & 84 \\
\hline $\mathrm{B}, \mathrm{mm}$ & 58 & 48 & 78 & 90 \\
\hline $\mathrm{a}, \mathrm{mm}$ & 14 & 14 & 20 & 13 \\
\hline $\mathrm{b}, \mathrm{mm}$ & 25 & 26 & 33 & 28 \\
\hline$\alpha,{ }^{\circ}$ & 2 & 0.7 & 1.9 & 1.3 \\
\hline \hline
\end{tabular}

* See Figure 3.62 for definition of dimensions.

Unlike the cryomodules for XFEL, LCLS-II, and other similar linacs that have their piping continuous across the interconnect region, PIP-II cryomodules are completely self-contained with the only connection between cryomodules being the beamline. Cryogenic connections are made at each cryomodule through bayonet connections at a common connection box that also houses the cryogenic control valves. This eliminates the need for a large $2 \mathrm{~K}$ pumping line within each cryomodule and also makes potential replacement of an individual cryomodule for maintenance more straightforward. Each cavity has its own $2 \mathrm{~K}$ heat exchanger and relief line connection. 


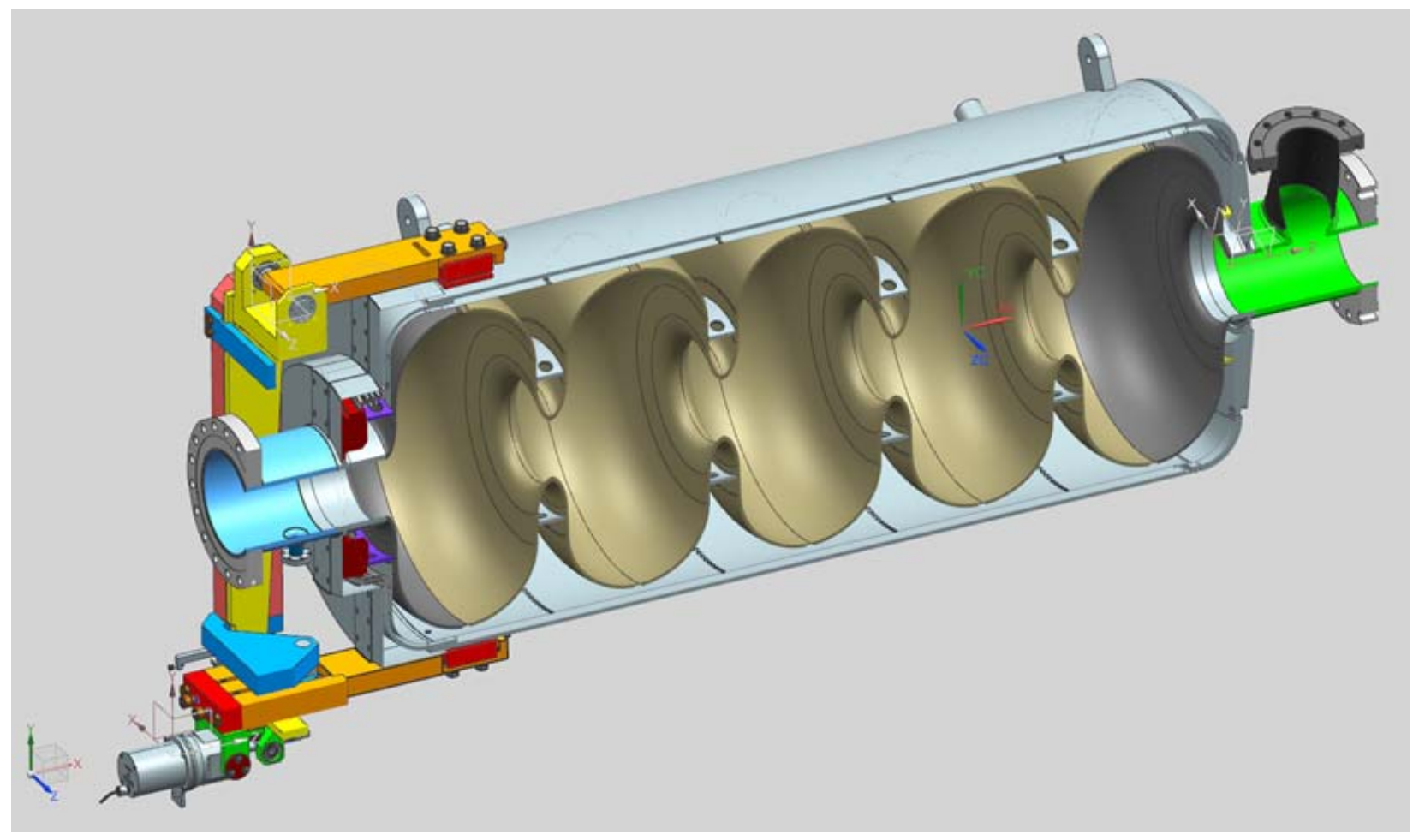

Figure 3.63: High-beta dressed cavity cross section

Figures 3.64 and 3.65 illustrate the cavity string assembly for the high-beta cryomodule with the warm strongback, support posts, 2-phase pipe, and cryogenic piping. Figure 3.66 presents the complete cryomodule assembly.

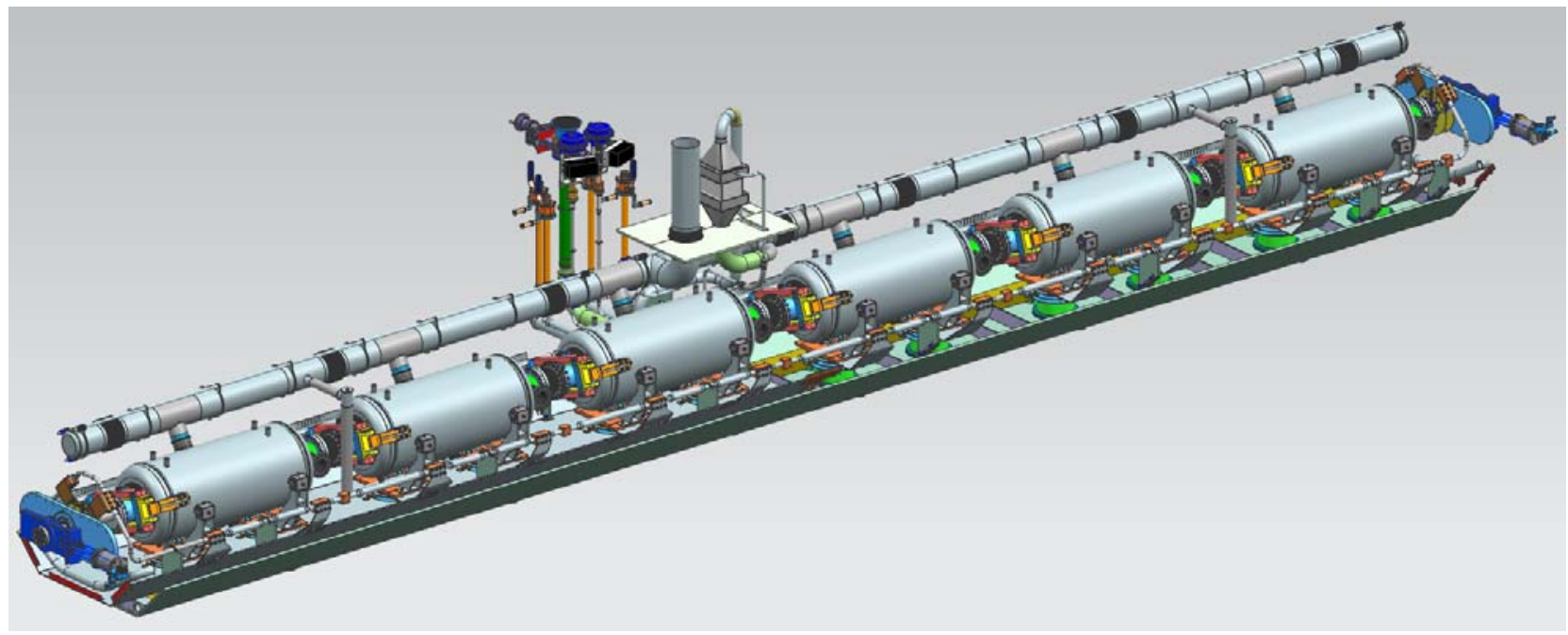

Figure 3.64: $650 \mathrm{MHz}$ high-beta cavity string assembly. 


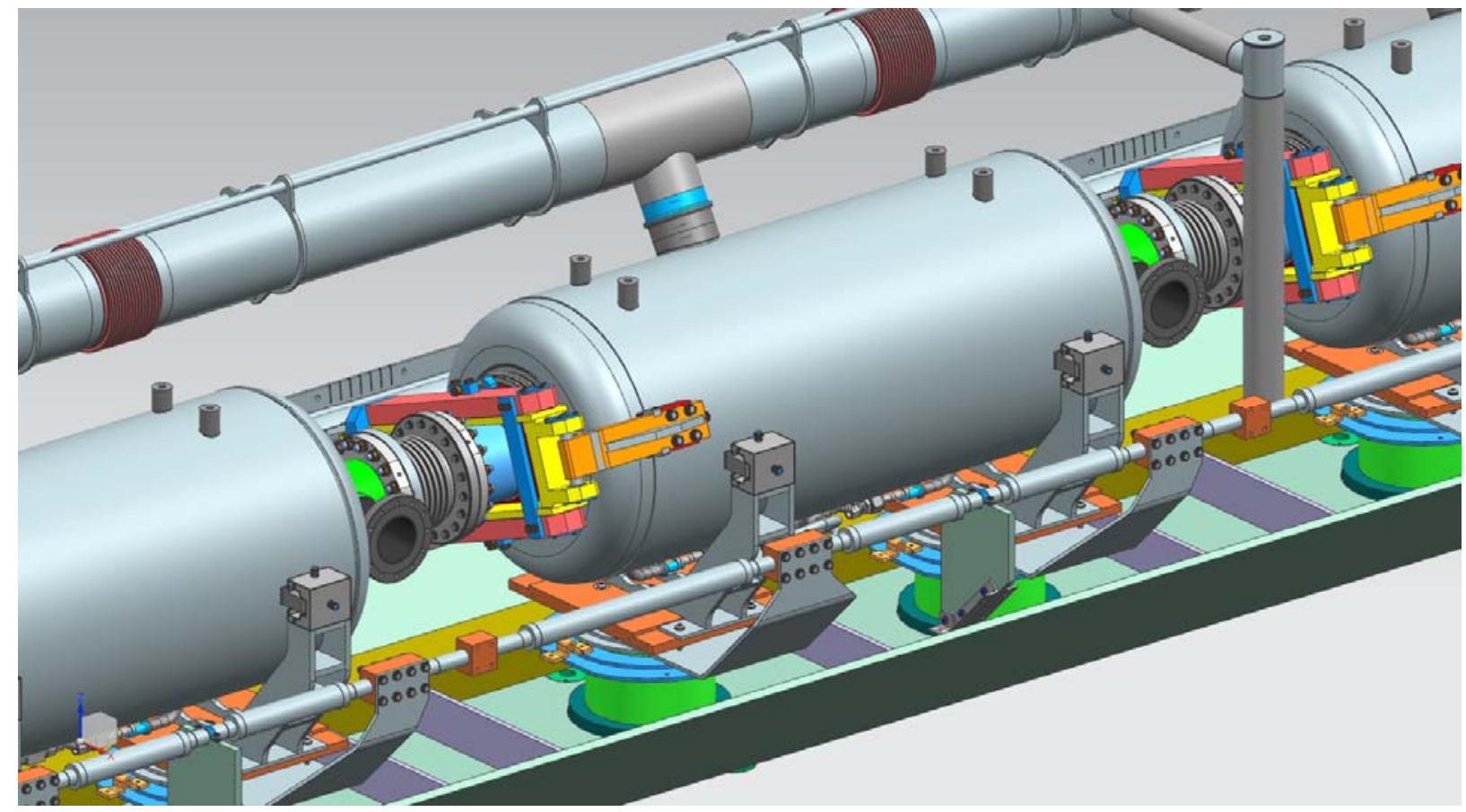

Figure 3.65: $650 \mathrm{MHz}$ high-beta cavity string detail.

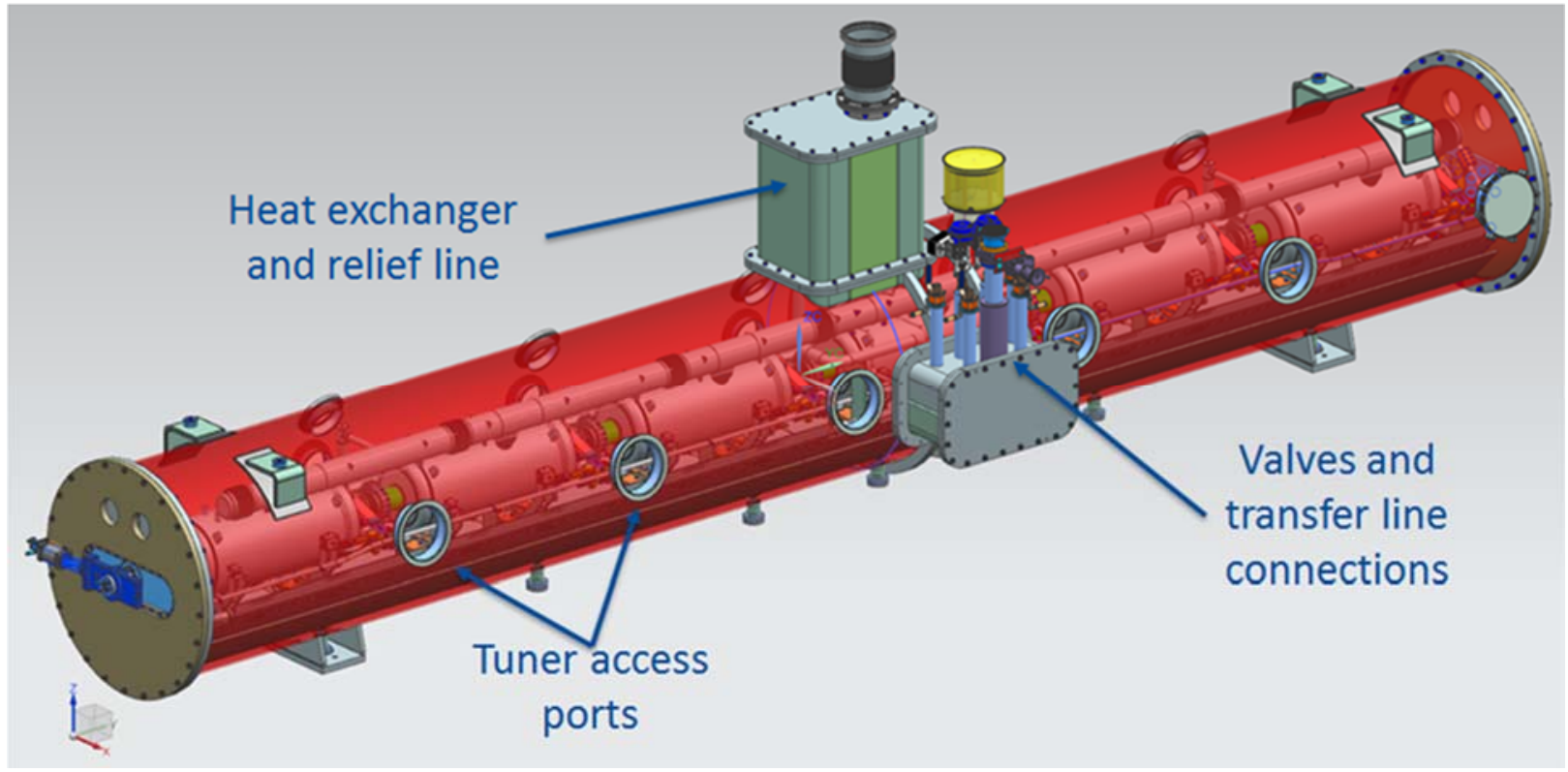

Figure 3.66: $650 \mathrm{MHz}$ high-beta cryomodule assembly

\section{Static Heat Loads}

The static heat load in a cryomodule is the sum of heat flowing from several sources summarized below:

- $\quad$ input coupler,

- mechanical support structure,

- magnet current leads (if any),

- thermal radiation and residual gas conduction, 
- cold-to-warm transition.

Each source represents some form of connection between cryomodule components and the environment. In the case of the input coupler, support structure, current leads, and cold-to-warm transition, they are mechanical connections to the vacuum vessel with intermediate heat intercepts at $70 \mathrm{~K}$ and $5 \mathrm{~K}$. The heat loads to $70 \mathrm{~K}, 5 \mathrm{~K}$, and $2 \mathrm{~K}$ are determined by the temperature dependent thermal conductivities of the materials making up the structures, their lengths and cross-sections. Thermal radiation and residual gas conduction are forms of heat transfer to various temperatures through the multi-layer insulation (MLI) and are estimated to be $1.5 \mathrm{~W} / \mathrm{m}^{2}$ to $70 \mathrm{~K}$ and $0.15 \mathrm{~W} / \mathrm{m}^{2}$ to $2 \mathrm{~K}$ where the effective area is the surface area of the insulating layer.

Separate tables in the sections below summarize the heat loads from each static source for the low- and high-beta 650 cryomodules.

\section{Interfaces}

The cryomodule assembly has interfaces to the following:

- Bayonet connections for helium supply and return,

- Cryogenic valve control systems,

- Cryogenic system interface via a heat exchanger pre-cools helium from approximately $5 \mathrm{~K}$ to $2 \mathrm{~K}$ upstream of the cryomodule liquid level control valve (JT-valve),

- Pumping and pressure relief line connections,

- Cryomodule warm support structures,

- Beam tube connections terminated by a particle free vacuum valve,

- RF and high voltage bias connectors to the input couplers,

- Instrumentation connectors on the vacuum vessel shell,

- Alignment fiducials on the vacuum vessel shell with reference to cavity positions.

\section{Instrumentation}

Cavity and cryomodule instrumentation will include, but not be limited to the following. Internal wiring shall be of a material and size that minimizes heat load to the internal systems:

- Cavity field probes,

- Coupler e-probes,

- Diode x-ray detectors,

- Cavity tuner control and diagnostics,

- Input coupler temperature sensors,

- Thermal shield temperature sensors,

- Cavity helium vessel temperature sensors (externally mounted),

- Helium system pressure taps,

- Helium level probes in the $2 \mathrm{~K}$ phase separator,

- Helium temperature sensors in the $2 \mathrm{~K}$ phase separator,

- Cavity vacuum monitors,

- Insulating vacuum monitors,

- Input coupler vacuum monitors. 


\section{Requirements for the Low-beta and High-beta Cavities and Cryomodules}

The electro-magnetic parameters for the low-beta and high-beta cavities are shown Tables $2.4-$ 2.9. The requirements for the dressed cavities are presented in Table 3.16. Table 3.17 presents requirements to the cavity tuners. Figures 3.67 and 3.68 show the configuration of LB650 and HB650 cryomodules. Table 3.18 summarizes the general and cavity-related requirements for the low-beta cryomodules. Additional details can be found in the corresponding Functional Requirement Specifications [108]-[111].

Table 3.16: Dressed cavity requirements for the low-beta and high-beta cavities

\begin{tabular}{|c|c|c|}
\hline & LB650 & HB650 \\
\hline Operating mode & \multicolumn{2}{|c|}{ Pulsed with CW capability } \\
\hline Maximum leak rate (room temp), atm-cc/s & \multicolumn{2}{|c|}{$<10^{-10}$} \\
\hline Maximum gain per cavity in VTS, MeV & $>14$ & $>24$ \\
\hline Sensitivity to He pressure fluctuations, $\mathrm{Hz} / \mathrm{mbar}$ & $<25$ & $<25$ \\
\hline Lorentz Force Detuning, $\mathrm{Hz} /(\mathrm{MeV} / \mathrm{m})^{2}$ & $<0.8$ & $<1$ \\
\hline Field flatness dressed cavity & \multicolumn{2}{|c|}{$>90 \%$} \\
\hline Operating temperature, $\mathrm{K}$ & \multicolumn{2}{|c|}{2} \\
\hline Operating pressure, mbar & \multicolumn{2}{|c|}{30} \\
\hline MAWP, room temperature $/ 2 \mathrm{~K}$, bar & \multicolumn{2}{|c|}{$2 / 4$} \\
\hline Cavity longitudinal stiffness, $\mathrm{kN} / \mathrm{mm}$ & $<5$ & $<5$ \\
\hline Tuning sensitivity, $\mathrm{kHz} / \mathrm{mm}$ & $>180$ & $>150$ \\
\hline
\end{tabular}

Table 3.17: Tuner requirements for the low-beta and high-beta cavities

\begin{tabular}{l|c|c}
\hline \hline & LB650 & HB650 \\
\hline Coarse tuner frequency range, $\mathrm{kHz}$ & 200 & 200 \\
\hline Coarse tuner frequency resolution, $\mathrm{Hz}$ & 2 & 2 \\
\hline Fine tuner frequency range, $\mathrm{Hz}$ & 500 & 1000 \\
\hline Fine tuner frequency resolution, $\mathrm{Hz}$ & 0.1 & 0.1 \\
\hline \hline
\end{tabular}

$3972.8 \mathrm{~mm}$

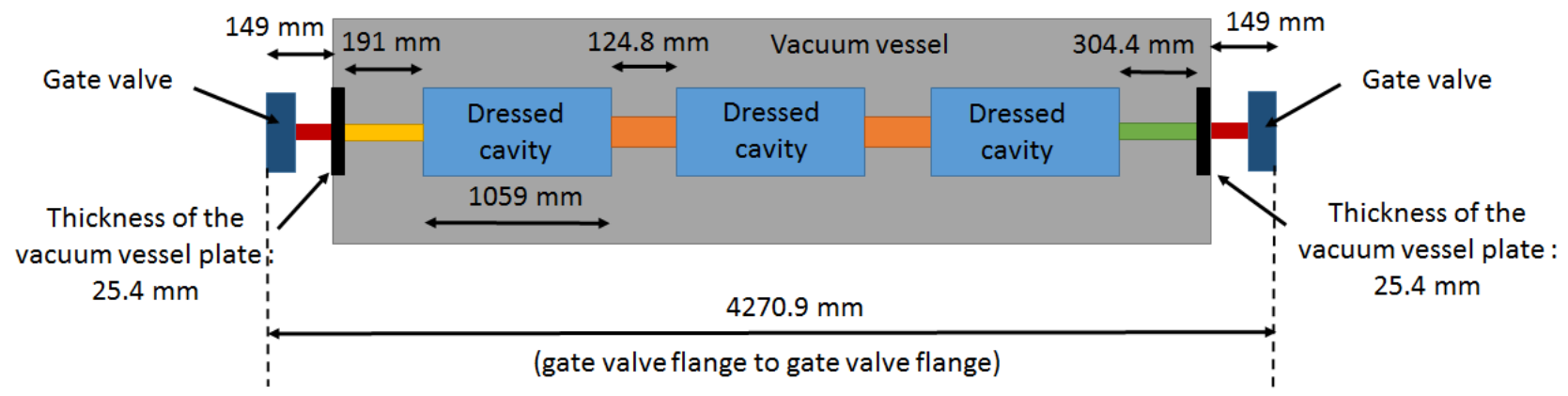

Figure 3.67: Low-beta cryomodule configuration. 


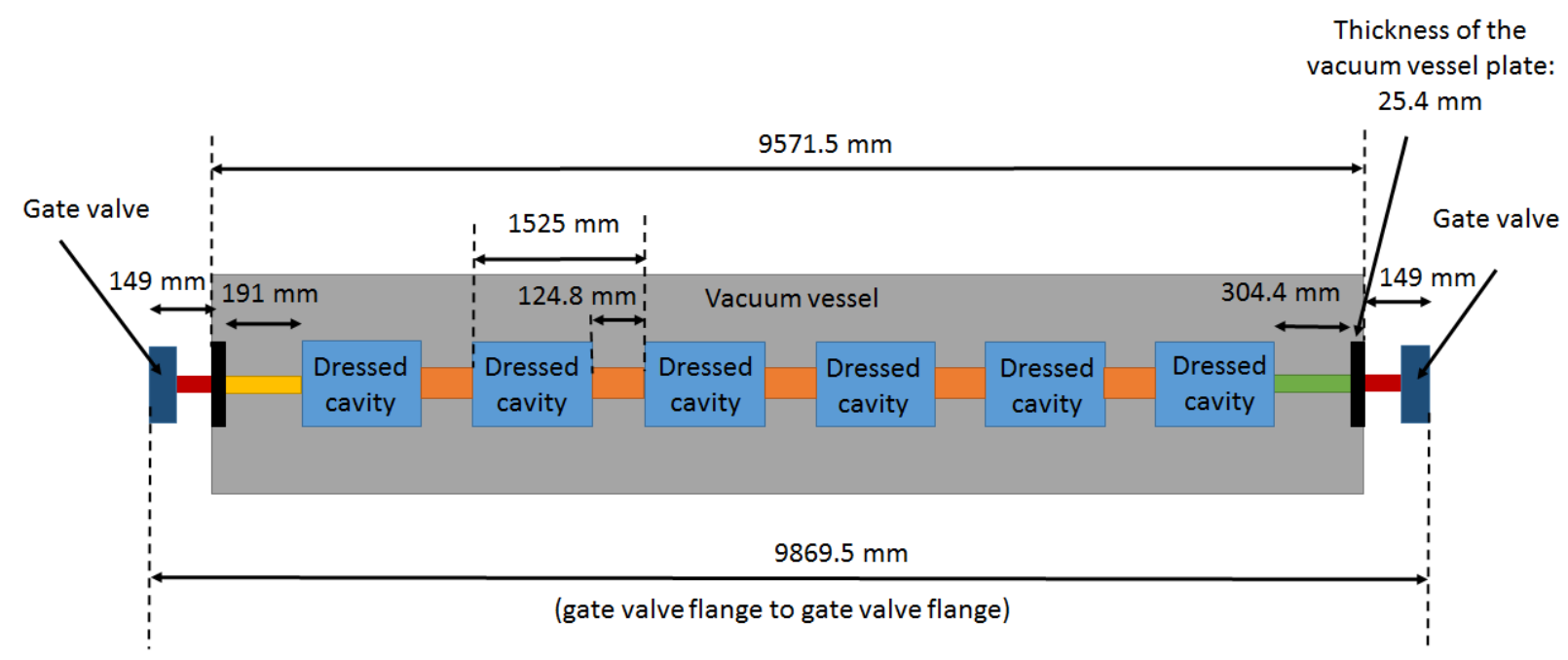

Figure 3.68: High-beta cryomodule configuration.

Table 3.18: Requirements for low- and high-beta cryomodules

\begin{tabular}{|c|c|c|}
\hline & LB650 & HB650 \\
\hline Physical beam aperture, $\mathrm{mm}$ & 83 & 118 \\
\hline Overall length (flange-to-flange), $\mathrm{m}$ & 4.3 & 9.56 \\
\hline Overall width, $\mathrm{m}$ & \multicolumn{2}{|c|}{$\leq 1.6$} \\
\hline Beamline height from the floor, $\mathrm{m}$ & \multicolumn{2}{|c|}{1.3} \\
\hline Cryomodule height (from floor), $\mathrm{m}$ & \multicolumn{2}{|c|}{$\leq 2.00$} \\
\hline Ceiling height in the tunnel, $\mathrm{m}$ & \multicolumn{2}{|c|}{3.20} \\
\hline Max. allowed heat load to $70 \mathrm{~K}, \mathrm{~W}$ & 100 & 300 \\
\hline Max. allowed heat load to $5 \mathrm{~K}, \mathrm{~W}$ & 15 & 25 \\
\hline Max. allowed heat load to $2 \mathrm{~K}, \mathrm{~W}$ & 100 & 220 \\
\hline Maximum number of lifetime thermal cycles & \multicolumn{2}{|c|}{50} \\
\hline Intermediate thermal shield temperature, $\mathrm{K}$ & \multicolumn{2}{|c|}{$45-80$} \\
\hline Thermal intercept temperatures, $\mathrm{K}$ & \multicolumn{2}{|c|}{5 and $45-80$} \\
\hline Cryo-system pressure stability at $2 \mathrm{~K}$ (RMS), mbar & \multicolumn{2}{|c|}{$\sim 0.1$} \\
\hline Environmental contribution to internal field, $\mathrm{mG}$ & $<10$ & $<10$ \\
\hline Transverse cavity alignment error, $\mathrm{mm}$, rms & $<0.5$ & $<0.5$ \\
\hline Angular cavity alignment error, mrad, rms & $\leq 1$ & $<1$ \\
\hline Beam duration for operation in pulsed regime, $\mathrm{ms}$ & \multicolumn{2}{|c|}{$\leq 1$} \\
\hline Repetition rate for operation in pulsed regime, $\mathrm{Hz}$ & \multicolumn{2}{|c|}{$\leq 20$} \\
\hline Number of cavities per cryomodule & 3 & 6 \\
\hline Beta (geometric) & 0.61 & 0.92 \\
\hline Operating temperature, $\mathrm{K}$ & 2 & 2 \\
\hline Coupler power rating (TW and full reflection), $\mathrm{kW}$ & 110 & 110 \\
\hline
\end{tabular}


Tables 3.19 and 3.20 summarize the static heat loads for the low- and high-beta cryomodules. In addition, there is a dynamic heat load to $2 \mathrm{~K}$ somewhat lower than $20 \mathrm{~W}$ per cavity or $60 \mathrm{~W}$ for each LB650 cryomodule and $25 \mathrm{~W}$ per cavity or $160 \mathrm{~W}$ for each LB650 cryomodule (see Table 2.7).

Table 3.19: Low-beta cryomodule static heat loads

\begin{tabular}{l|c|c|c|c|c|c|c}
\hline \hline & \multicolumn{3}{|c|}{ Each cavity (W) } & \multicolumn{3}{c}{ Multiplier } & \multicolumn{3}{c}{ Total per CM (W) } \\
\hline & $70 \mathrm{~K}$ & $5 \mathrm{~K}$ & $2 \mathrm{~K}$ & & $70 \mathrm{~K}$ & $5 \mathrm{~K}$ & $2 \mathrm{~K}$ \\
\hline Input coupler (static) & 4.4 & 1.8 & 0.2 & 3 & 13 & 5 & 1 \\
\hline Support post & 0.4 & 1.8 & 0.0 & 6 & 2 & 11 & 0 \\
\hline MLI & 30.6 & 0.0 & 1.5 & 1 & 31 & 0 & 1 \\
\hline Cold to warm transition & 0.7 & 0.1 & 0.0 & 2 & 1 & 0 & 0 \\
\hline Total static (per CM) & & & & & 48 & 16 & 2 \\
\hline \hline
\end{tabular}

Table 3.20: High-beta cryomodule static heat loads

\begin{tabular}{l|c|c|c|c|c|c|c}
\hline \hline & \multicolumn{3}{|c|}{ Each cavity $(\mathrm{W})$} & Multiplier & \multicolumn{3}{c}{ Total per CM (W) } \\
\hline & $70 \mathrm{~K}$ & $5 \mathrm{~K}$ & $2 \mathrm{~K}$ & & $70 \mathrm{~K}$ & $5 \mathrm{~K}$ & $2 \mathrm{~K}$ \\
Input coupler (static) & 4.4 & 1.8 & 0.2 & 6 & 26 & 11 & 1 \\
Support post & 0.4 & 1.8 & 0.0 & 12 & 5 & 22 & 0 \\
MLI & 53.3 & 0.0 & 2.6 & 1 & 53 & 0 & 3 \\
Cold to warm transition & 0.7 & 0.1 & 0.0 & 2 & 1 & 0 & 0 \\
\hline Total static (per CM) & & & & & 86 & 32 & 4 \\
\hline \hline
\end{tabular}

The pressure rating requirements for each of the systems in the cryomodule are summarized in Table 3.21. The pressure ratings are the same for both low and high-beta cryomodules. All presented pressures are differential pressures.

Table 3.21: System pressure ratings for low- and high-beta cryomodules

\begin{tabular}{l|c|c}
\hline \hline System & $\begin{array}{c}\text { Warm MAWP } \\
\text { (bar) }\end{array}$ & $\begin{array}{c}\text { Cold MAWP } \\
\text { (bar) }\end{array}$ \\
\hline $2 \mathrm{~K}$, low pressure & 2 & 4 \\
\hline $2 \mathrm{~K}$, positive pressure piping & 20 & 20 \\
\hline $5 \mathrm{~K}$ piping & 20 & 20 \\
\hline $45-80 \mathrm{~K}$ piping & 20 & N/A \\
\hline Insulating vacuum & 1 atm external, \\
vacuum inside & 2 bar external, \\
\hline Cavity vacuum & $\begin{array}{l}4 \text { bar external, } \\
\text { vacuum inside }\end{array}$ \\
\hline $\begin{array}{l}\text { Beam pipe outside cavities, includes beam } \\
\text { position monitors and warm to cold transitions }\end{array}$ & $\begin{array}{l}1 \text { atm external, } \\
\text { vacuum inside }\end{array}$ & $\begin{array}{l}1 \text { atm external, } \\
\text { vacuum inside }\end{array}$ \\
\hline \hline
\end{tabular}


Neither HB650 nor LB650 cavities contain HOM dampers - they are not necessary for the required beam current [107]. This choice is also supported by the experience accumulated in the SNS [112]. An absence of HOM dampers is more problematic for future PIP-II upgrades. Here we consider the Project X parameters as an example. Figure 3.69 presents a possible bunch structure considered in Ref. [113] for CW linac operating with $1 \mathrm{~mA}$ average beam current and beam delivery to three experiments running in parallel (muon, kaon, and nuclear experiments) with different beam structure for each experiment. Figure 3.70 shows the corresponding spectrum, assuming very short bunches of equal charge and an absence of timing jitter. The $R / Q$ values for all monopole modes of the HB650 cavity are shown in Figure 3.71.

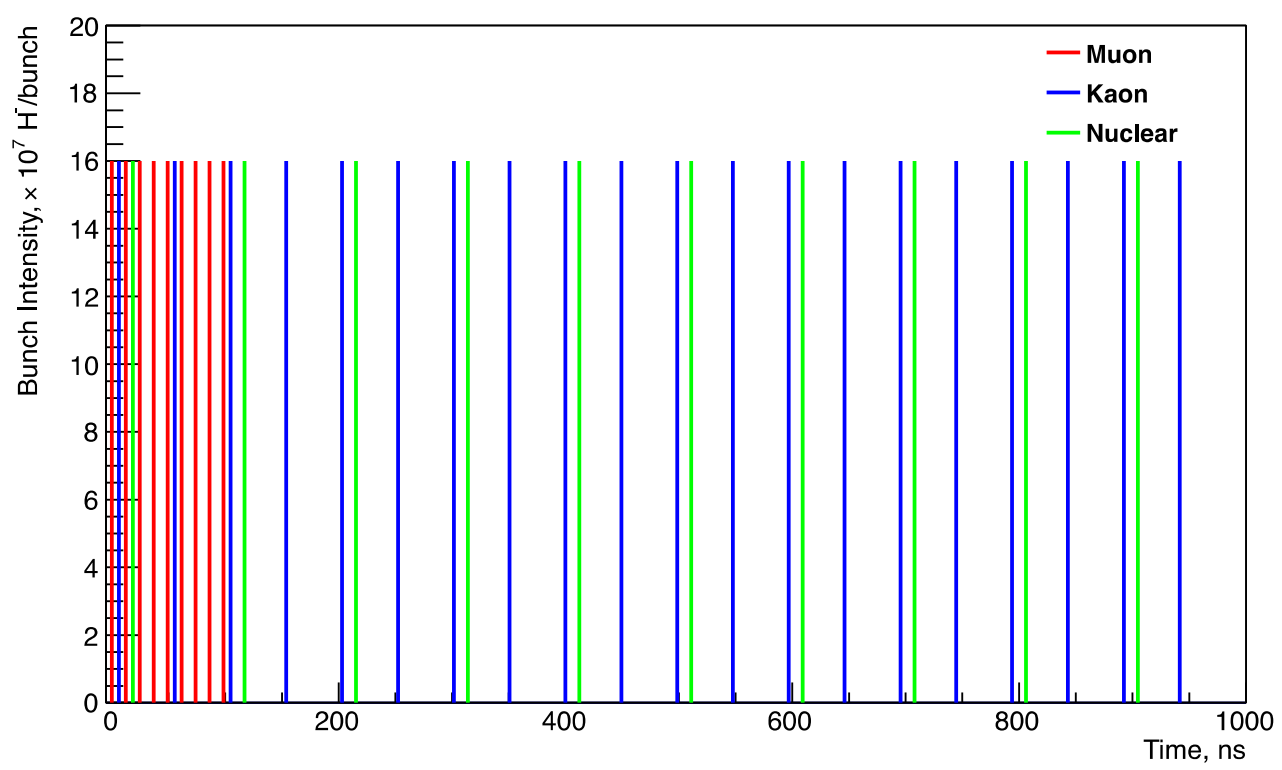

Figure 3.69: Beam structure for a $3 \mathrm{GeV}$ program.

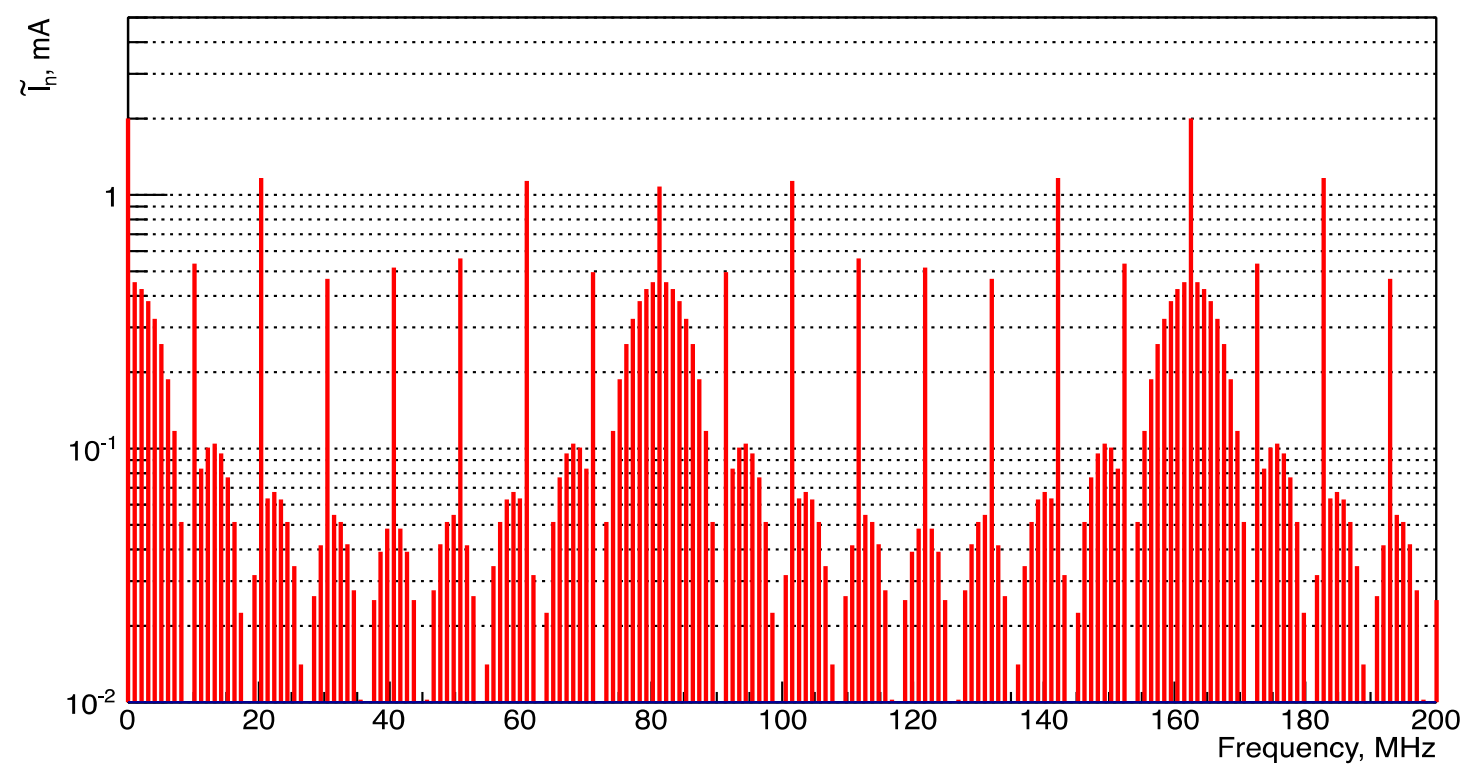

Figure 3.70: Beam spectrum for a $3 \mathrm{GeV}$ program. 


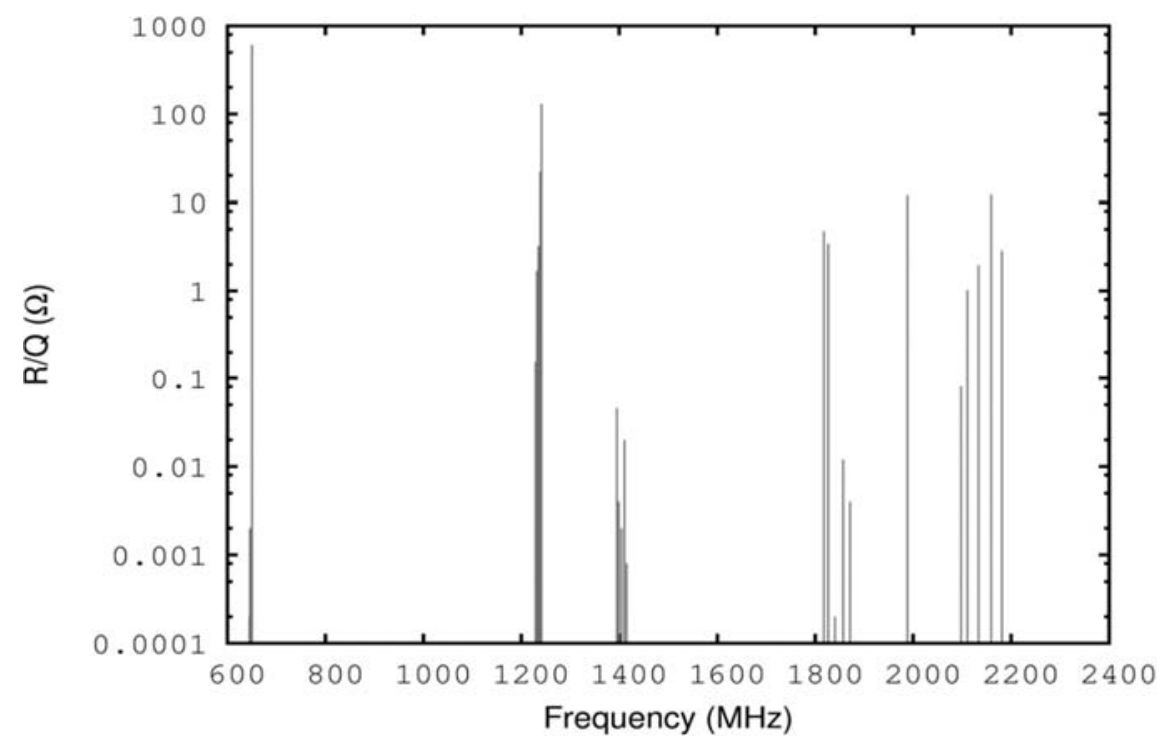

Figure 3.71: (R/Q) of the monopole HOMs in the HB650 cavities.

The amplitude of an excited monopole HOM, $U_{H O M}$, depends on the amplitude of the nearest beam spectrum line, $I$, the detuning $\delta f$, and the distance between the HOM frequency $f$ and the beam spectrum line frequency. It can be estimated for a high $Q$ resonance (assuming $\delta f / f \ll 1 / Q$ ) by the following:

$$
U_{\text {HOM }} \approx \frac{I(R / Q)}{4 \sqrt{2} \delta f / f} .
$$

If the high order mode is exactly at resonance, then

$$
U_{\text {ном }}=\frac{1}{2} I(R / Q) Q_{L},
$$

where $Q_{L}$ is the loaded quality factor of the mode. The cryogenic losses depend on the square of the HOM amplitude:

$$
P_{\text {loss }} \approx \frac{U_{H O M}^{2}}{(R / Q) Q_{0}} .
$$

Requiring $P_{\text {loss }}$ to be much smaller than the sum of the static heat load and the cryogenic losses due to the accelerating mode $(20 \mathrm{~W})$, and assuming the intrinsic quality factor is $Q_{0}=5 \times 10^{9}$, one obtains the maximum allowable value of the monopole HOM loaded quality factor to be: $Q_{L} \ll 6 \times$ $10^{7}$.

Similarly, requiring that excitation of a monopole mode does not increase longitudinal emittance, $\varepsilon_{z} \gg U_{H O M} \sigma_{z} / c$, an estimation of the safe frequency detuning yields:

$$
\delta f \gg f \frac{I(R / Q) \sigma_{z}}{4 \sqrt{2} \varepsilon_{z} c} .
$$

Here $\sigma_{z}$ is the bunch length, and $c$ is the speed of light. The worst case is at the beginning of $\beta_{G}=0.92$ section, where the bunch length is maximum $\left(\sigma_{z} / c=7.7 \cdot 10^{-3} \mathrm{~ns}\right)$. Assuming that the second pass-band monopole $\mathrm{HOM}(1241 \mathrm{MHz}$ and $R / Q=130 \mathrm{Ohm})$ is the nearest beam spectrum line $(I=1 \mathrm{~mA})$, and an emittance of $\varepsilon_{z}=1.5 \mathrm{keV} \mathrm{ns}$, one obtains the following estimate for frequency detuning: $\delta f \gg$ $140 \mathrm{~Hz}$. 
A more accurate estimate of coherent HOM excitation in the Project X linac is performed using statistical analysis based on the expected spread of data for the HOM parameters (frequency, impedance and quality factor). Errors in cavity shape introduced in manufacturing are taken into account by allowing random variations of the cavity profile within $0.2 \mathrm{~mm}$ of ideal shape. In order to estimate the probability of cryogenic losses, and relative change of longitudinal emittance, $10^{5}$ random linacs were generated using predicted deviations of frequency, loaded quality factors and impedances of monopole HOMs. It was found that the probability to have losses above $0.1 \mathrm{~W}$ per cryomodule is extremely small: $10^{-4}$ for an average beam current of $1 \mathrm{~mA}$.

The beam structure, shown in Figure 3.70 consists of three main sub-components $(1 \mathrm{MHz}, 10$ $\mathrm{MHz}$ and $20 \mathrm{MHz}$ ). The phase of the voltage of an HOM excited by a resonance with one of the beam components is random with respect to two other components of the beam. In case of a high- $Q$ resonance such a HOM may introduce a significant energy variation and longitudinal emittance growth along the beam train. Results of statistical analysis show that the probability of the emittance to double is $10^{-3}$ for the beam current of $1 \mathrm{~mA}$. Based on this analysis the conclusion is made that HOM couplers are not needed in $650 \mathrm{MHz}$ cavities. More details can be found in Ref. [107].

\subsubsection{The $325 \mathrm{MHz}$ and $650 \mathrm{MHz}$ Main Couplers.}

PIP-II employs two types of couplers: $325 \mathrm{MHz}$ for SSR1 and SSR2 cryomodules, and $650 \mathrm{MHz}$ couplers for LB650 and HB650 cryomodules. Taking into account that couplers are not adjustable for certain components of the SSR1 and SSR2 couplers, as well as, LB650 and HB650 (most likely the outer conductor) might need to have slightly different dimensions. The power requirements are set by cavities with high power, i.e. SSR2 and SSR1. Since a coupler replacement is very expensive procedure, we additionally require that the couplers have to be able to support an operation with 5 $\mathrm{mA} \mathrm{CW}$ beam, which is presently considered as a probable upgrade for the SC linac. Thus, to assure operational reliability, with suitable overhead at currents between 2-5 mA, all $325 \mathrm{MHz}$ couplers have to be tested at $30 \mathrm{~kW}$ (any reflection) [114]. The only exception is the couplers for the first SSR 1 cryomodule installed in PIP2IT, which will be tested at $20 \mathrm{~kW}$. The $650 \mathrm{MHz}$ couplers have to be tested at $110 \mathrm{~kW}$ (any reflection) [115]. The operating powers for the PIP-II do not exceed 17 $\mathrm{kW}$ at $325 \mathrm{MHz}$ and $64 \mathrm{~kW}$ at $650 \mathrm{MHz}$.

The criteria for coupler design are: (1) reliability and (2) minimization of production and operating costs. The coupler parameters chosen on the base of these requirements are presented in Table 3.22. CAD views of the coupler designs are presented in Figures 3.72 and 3.73. Each coupler uses a high voltage bias to suppress multipacting. To make coupler production more efficient, an approach of maximum unification of parts was chosen during electromechanical design. Couplers for both frequencies should contain a maximum number of common (shared) parts.

The coupler for $325 \mathrm{MHz}$ was designed, built and successfully tested. Its design is based on a 105 $\Omega$ coaxial line. The coupler contains a single warm ceramic window that provides separation of the warm and cold coupler sections. During cryomodule fabrication, the cold section can be installed on the cavity in the cleanroom prior to assembly of the string. The warm section can then be installed from outside the vacuum vessel during final assembly. The inner conductor is solid copper with bellows to accommodate motion due to misalignment and thermal contraction. The cold end of the outer conductor is $316 \mathrm{~L}$-stainless steel. The warm end is copper with bellows. Heat load estimates do not suggest that there is a significant penalty in not copper plating the outer conductor. A forcedair cooling tube is inserted into the inner conductor after assembly that supplies air to cool the coupler tip. 
Table 3.22: Parameters of 325 the $\mathrm{MHz}$ and $650 \mathrm{MHz}$ Main Couplers

\begin{tabular}{|c|c|c|}
\hline Operating Frequency & $325 \mathrm{MHz}$ & $650 \mathrm{MHz}$ \\
\hline Input impedance & $50 \Omega$ & $50 \Omega$ \\
\hline Output diameter & 3", SS, not coated & 4', SS; 3" solid copper \\
\hline Antenna diameter & $0.5^{\prime \prime}$, copper & $0.5^{\prime \prime}$, copper \\
\hline Antenna cooling & Air & Air \\
\hline Window & Single, $\mathrm{Al}_{2} \mathrm{O}_{3}, 3$ "'x $6 \mathrm{~mm}$ & Single, $\mathrm{Al}_{2} \mathrm{O}_{3}, 4$ "' $6 \mathrm{~mm}$ \\
\hline Input & 3-1/8' coaxial & Rectangular waveguide \\
\hline Multipactor suppression & HV bias & HV bias \\
\hline Cryo-load, 2K, 0kW/P $\max *$ & $0.12 \mathrm{~W} / 0.58 \mathrm{~W}$ & $0.15 \mathrm{~W} / 0.55 \mathrm{~W}$ \\
\hline Cryo-load, 5K, 0kW/P $\max *$ & $0.58 \mathrm{~W} / 2.8 \mathrm{~W}$ & $0.6 \mathrm{~W} / 0.93 \mathrm{~W}$ \\
\hline Cryo-load, 70K, 0kW/P $\mathrm{Pax} *$ & $2.0 \mathrm{~W} / 5.4 \mathrm{~W}$ & $3.3 \mathrm{~W} / 6.2 \mathrm{~W}$ \\
\hline
\end{tabular}

$*$ Pmax $=30 \mathrm{~kW}$ traveling wave for $325 \mathrm{MHz}, \mathrm{P}=120 \mathrm{~kW}$ traveling wave for $650 \mathrm{MW}$

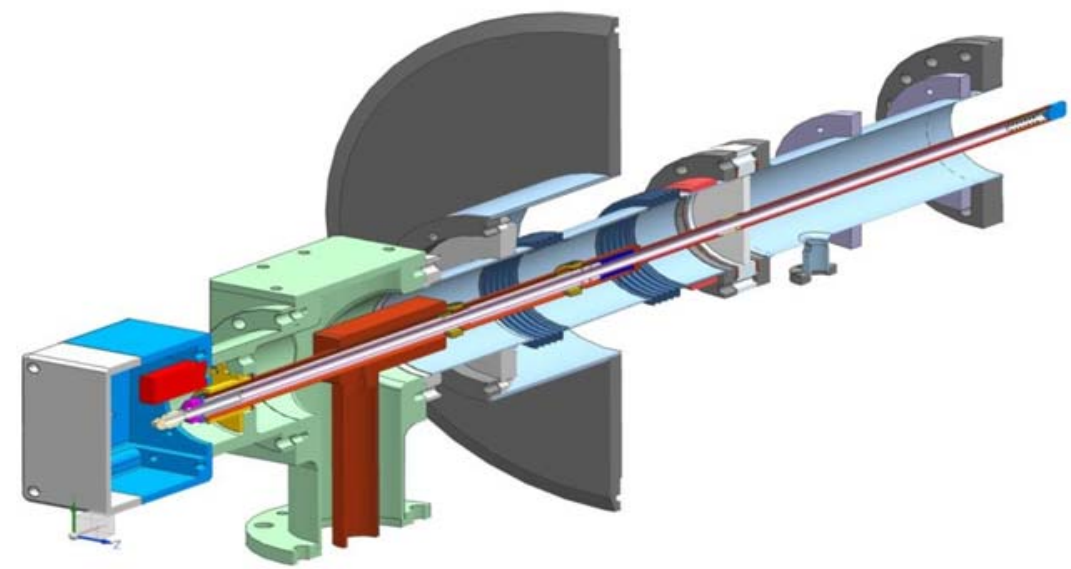

Figure 3.72: View of the $325 \mathrm{MHz}$ input coupler.

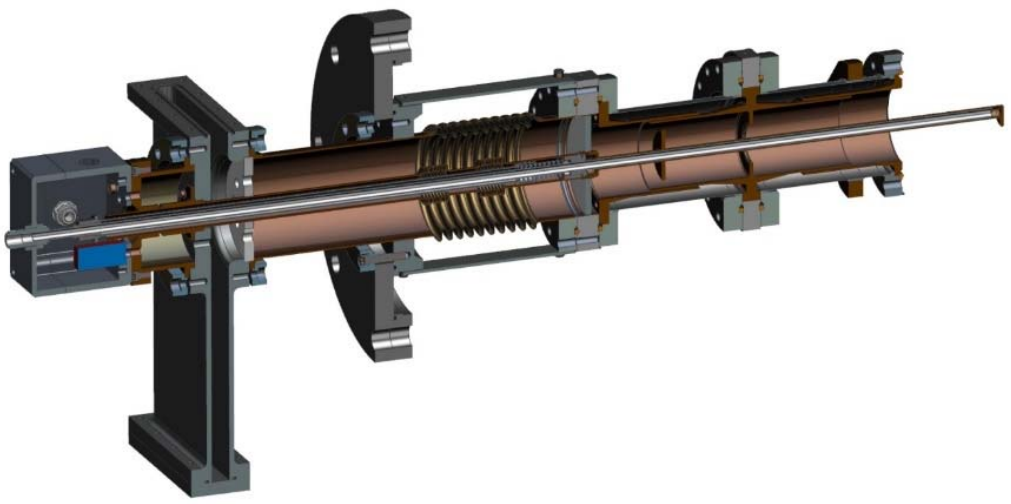

Figure 3.73: View of the $650 \mathrm{MHz}$ input coupler. 
Design of the $650 \mathrm{MHz}$ coupler is based on the same approach. The diameter of the RF window was increased to 4', to handle higher RF power. Electromagnetic shields made of solid copper were installed in stainless tube to decrease resistive losses. Axially non-symmetric antenna tip allows one to adjust coupling by antenna rotation.

\subsubsection{Measures Aimed at Reduction of RF Loss in the Walls of the SC Cavities}

The LB650 and HB650 cavities produce a major fraction of the acceleration and, if not specially treated, they would strongly dominate the heat load of the cryogenic system. Therefore, special attention was paid to reduce $Q_{0}$ for these two types of cavities. Cryogenic loss in a cavity is determined by the $R / Q$ value, $G$-factor and surface resistance. The surface resistance in its turn is a sum of the residual resistance and the BCS resistance.

In this document we use a comparatively conservative approach based on the Q-values recently achieved in large number of experiments with nitrogen doping. The BCS resistance as a function of the frequency and temperature may be estimated using the following formula,

$$
R_{B C S} \approx 2 \cdot 10^{-4} \frac{1}{T}\left(\frac{f}{1.5 \cdot 10^{9}}\right)^{2} e^{-17.67 / T},
$$

where the frequency, $f$, is measured in $\mathrm{Hz}$, and the temperature, $T$, in $\mathrm{K}$. For $650 \mathrm{MHz}$ and $2 \mathrm{~K}$ one obtains RBCS $2.7 \mathrm{n} \Omega$. Modern surface processing technology provides a residual resistance of $\sim 5$ $\mathrm{n} \Omega$ [16] resulting in the total resistance of $\sim 8 \mathrm{n} \Omega$. Assuming a medium field Q-slope at the peak field of $70 \mathrm{mT}$ of about $30 \%$, this yields a target for $\mathrm{Q}_{0}$ value of the $650 \mathrm{MHz}$ cavity of $\sim 3 \times 10^{10}$. This value was used above in Section 2.1.3.2, and it is strongly supported by recent progress in improvement of quality factors of SRF cavities via two breakthroughs: cavity surface doping with nitrogen [116] and manipulation of trapped magnetic flux via cooling [117].

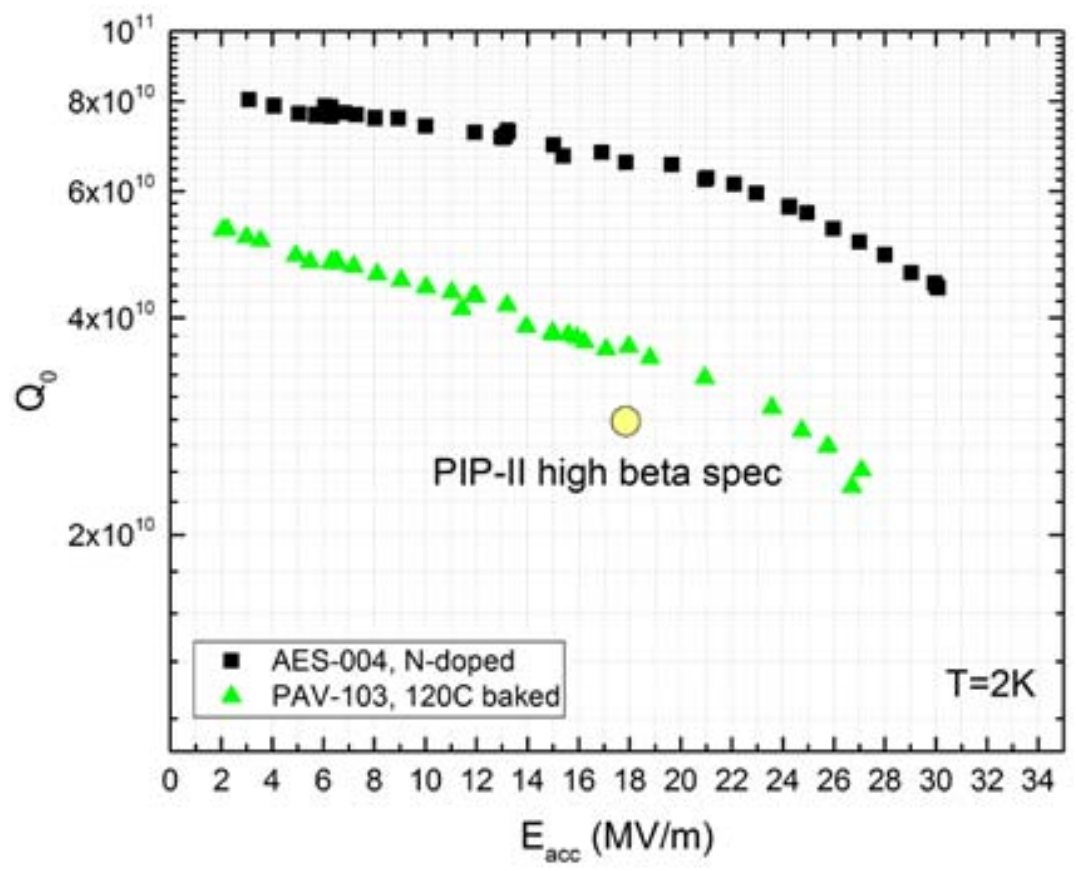

Figure 3.74: The dependence of $\mathrm{Q}_{0}$ on the accelerating voltage for the one-cell $650 \mathrm{MHz}, 120 \mathrm{C}$ baked cavity and the $\mathrm{N}$ doped cavity. The first one was manufactured by AES and another one by PAVAC. The measurements were carried out at $2 \mathrm{~K}$.

The first finding has enabled lowering both $\mathrm{BCS}$ and residual resistance components for $1.3 \mathrm{GHz}$ 
cavities systematically by a factor of more than two. More than twenty $1.3 \mathrm{GHz}$ cell cavities have been treated with $\mathrm{N}$ doping at Fermilab, Jlab and Cornell, achieving an average $\mathrm{Q} \sim 3.5 \cdot 10^{10}$ at 16 $\mathrm{MV} / \mathrm{m}$ and $2 \mathrm{~K}$, which is three times higher than with standard $120 \mathrm{C}$ bake processing (ILC/XFEL recipe). Recently also several $650 \mathrm{MHz}$ cavities have been treated with standard 120C bake processing and $\mathrm{N}$ doping, showing outstanding results exceeding the current specifications for PIP2 of $3 \cdot 10^{10}$ at $2 \mathrm{~K}$ and $17 \mathrm{MV} / \mathrm{m}$, as shown in Figure 3.74. For the $120 \mathrm{C}$ bake cavities the $\mathrm{Q}_{0}$ is $3.5 \cdot 10^{10}$ at $2 \mathrm{~K}$ and $16 \mathrm{MV} / \mathrm{m}$. For the $\mathrm{N}$ doped ones $\mathrm{Q}_{0}$ have reached $7 \cdot 10^{10}$ at $2 \mathrm{~K}$ and $17 \mathrm{MV} / \mathrm{m}$, exceeding the PIP-2 specifications by a factor of more than two.

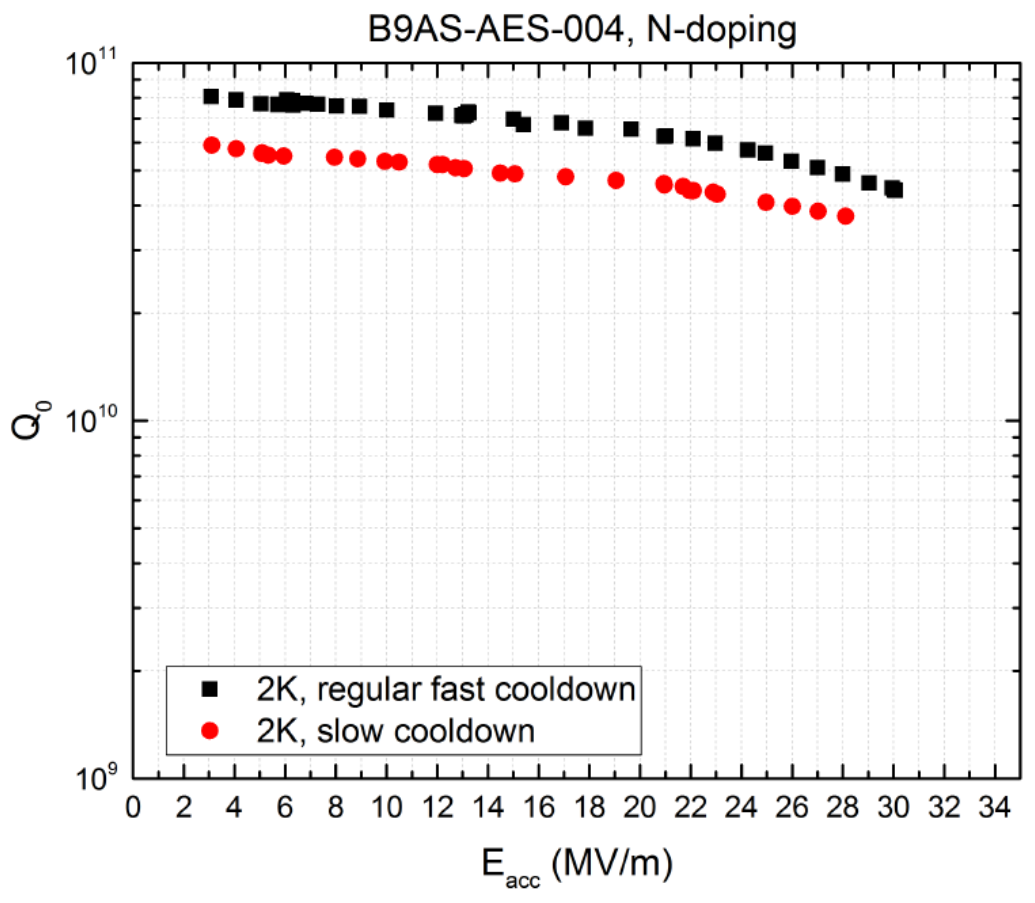

Figure 3.75: The dependence of $\mathrm{Q}_{0}$ on the accelerating voltage for the $\mathrm{N}$ doped $650 \mathrm{MHz}$ cavity for fast and slow cool downs.

Slow versus fast cooling has been demonstrated to significantly deteriorate the performance of 1.3 GHz cavities due to poor flux expulsion efficiency. Recently the same experiment has been performed for $650 \mathrm{MHz}$ cavities and the results showed very little degradation with slow cooling versus fast cooling, as shown in Figure 3.75, hinting to a likely weaker losses dependence of trapped flux due to the lower RF frequency.

The success of the experiments carried out with one-cell cavities supports more detailed studies with 5-cell HB cavities which results are discussed below.

\section{Experimental Studies of Nitrogen Doping in HB650 Cavity}

It has been demonstrated in the vertical tests that the five-cell HB650 cavities, lightly doped with nitrogen exceed the current PIP-II quality factor specification of $3 \cdot 10^{10}$ at $17.8 \mathrm{MV} / \mathrm{m}$ at $2 \mathrm{~K}$. The best $Q_{0}$ for the lightly doped multi-cell cavity at $17.8 \mathrm{MV} / \mathrm{m}$ and $2 \mathrm{~K}$ was measured to be $\sim 4.6 \cdot 10^{10}$. Absence of field emission at $2 \mathrm{~K}$ has also been demonstrated up to fields much higher than the operation field. Detailed description of two HB650 cavities, B9A5-AS-AES008 and B9A5-ASAES009, follows.

Cavity B9A5-AS-AES008 received the following surface processing: bulk electropolishing (EP) at ANL followed by $800 \mathrm{C}$ degassing and "2/6 doping" (the LCLS-II N doping protocol) in the MP9 
furnace followed by generous post doping EP removal ( 20 microns) so cavity is expected to be lightly doped. Lighter doping is beneficial for quench fields (moving them to values higher than 30 $\mathrm{MV} / \mathrm{m}$ ) and for the lower sensitivity to magnetic fields. The optimal doping level is a trade-off among quench field, sensitivity to the trapped magnetic flux and BCS surface resistance, which tends to be reduced with higher doping levels.

The cavity was tested in the FNAL vertical test stand dewar 1 (VTS-1) two times, the second time after an additional HPR cycle. Magnetic fields in the dewar were lower than $5 \mathrm{mG}$. Fluxgate magnetometers were distributed along the cavity equators and showed good flux expulsion property of the cavity as can be seen in Figure 3.76.

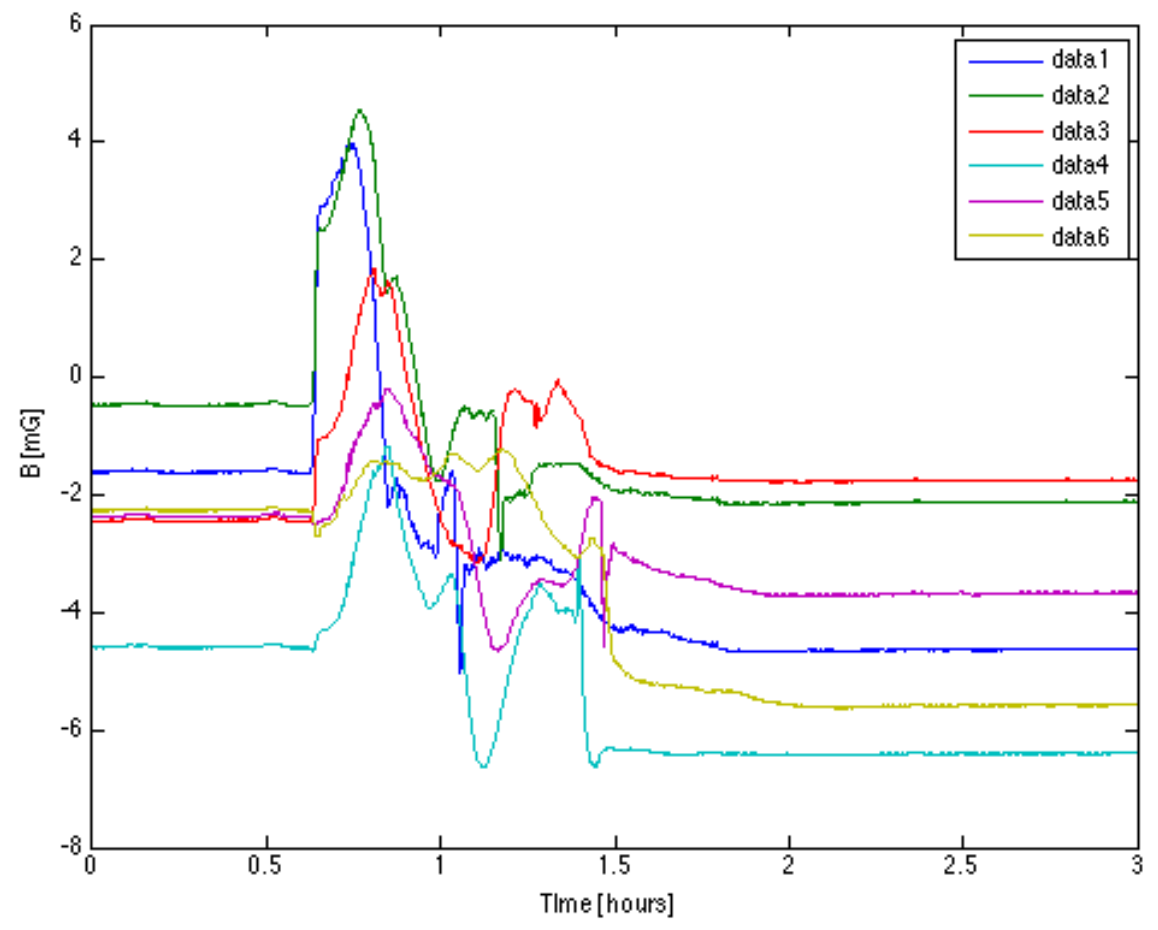

Figure 3.76: Dependence of magnetic field on time during cooldown at different locations near cavity.

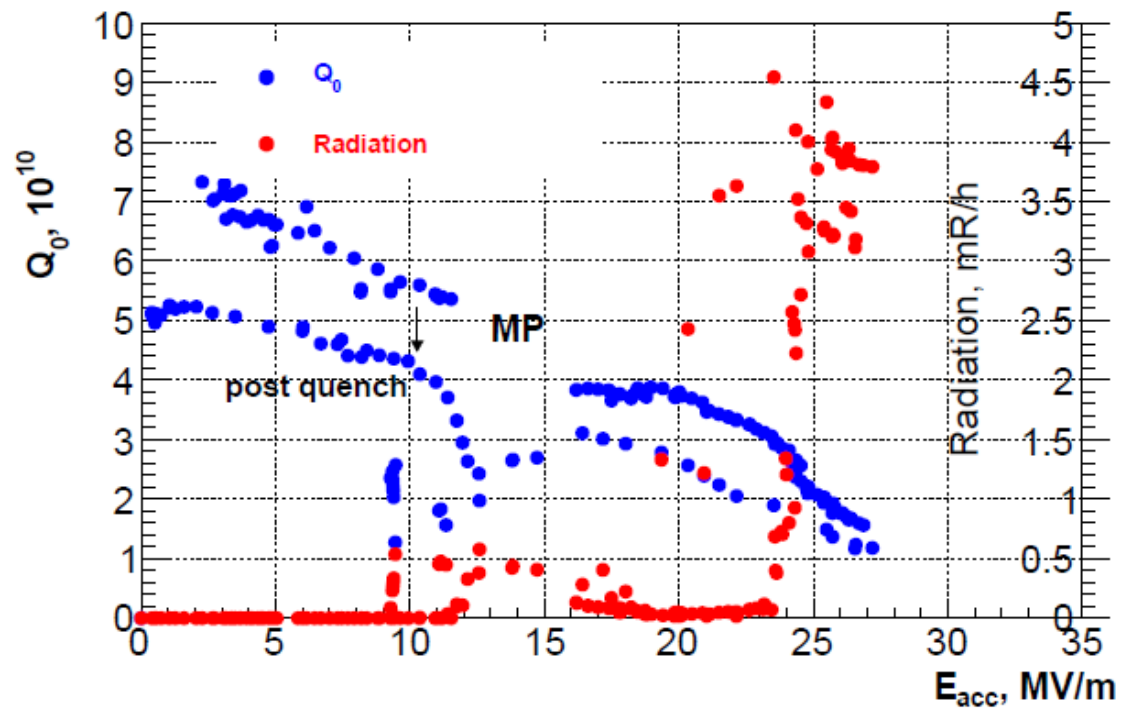

Figure 3.77: First test of HB650 MHz cavity B9A5-AS-AES008. 
The dependences of $Q_{0}$ on the accelerating gradient are shown in Figures 3.77 and 3.78 for the first and second test, respectively.

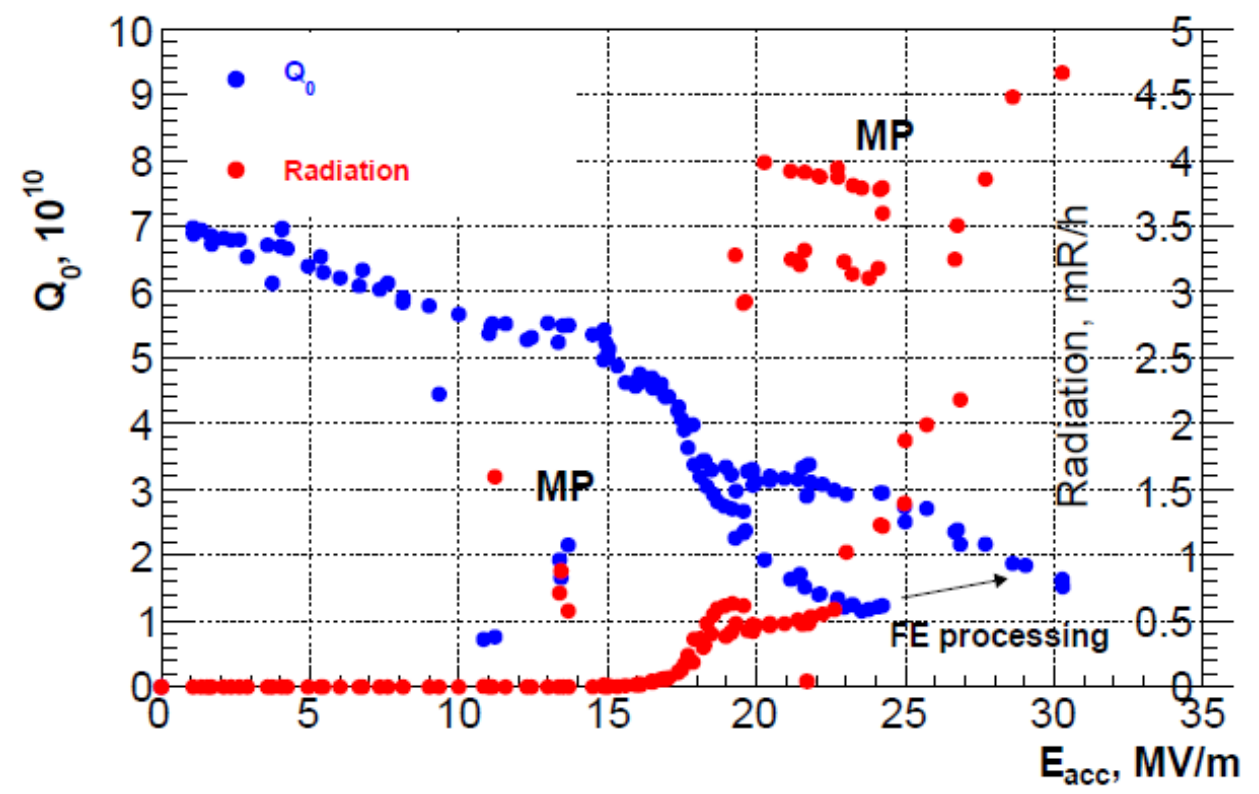

Figure 3.78: Second test of HB650 MHz cavity B9A5-AS-AES008 after addiotional HPR.

The first test of HB650 cavity showed very good $Q_{0}$ at low field, $\sim 7 \cdot 10^{10}$, but a strong multipacting (MP) barrier was encountered at $\sim 11 \mathrm{MV} / \mathrm{m}$, which led to several quenches inducing Q-degradation, and accompanied by very strong X-rays. The MP barrier appeared to extend more widely than for previously studied $650 \mathrm{MHz}$ single cells. In particular, the first MP barrier was encountered in the region of 11-16 MV/m. Then, another softer barrier is encountered at $\sim 17-22 \mathrm{MV} / \mathrm{m}$, followed by a large onset of field emission (FE) at $\sim 24 \mathrm{MV} / \mathrm{m}$. In this test cavity $Q_{0}$ measured near the PIP-II gradient specification of $17.8 \mathrm{MV} / \mathrm{m}$ was $\sim 4 \cdot 10^{10}$, penalized by the presence of some X-rays and by trapped flux caught due to MP-induced quenches. The maximum gradient reached was about 28 $\mathrm{MV} / \mathrm{m}$, the point at which FE was very high and the cavity quenched repeatedly. Significant Qdegradation followed these quenches as demonstrated by a lower Q-curve in Figure 3.77.

It was then decided to re-HPR the cavity with a different nozzle, which had an additional top spray to attempt reaching to top flanges hidden areas. In this test the first MP barrier was much softer than in first test while strong MP was encountered in the region $17-22 \mathrm{MV} / \mathrm{m}$, followed by the onset of FE with large X-rays at $\sim 23 \mathrm{MV} / \mathrm{m}$. Performance in terms of $Q_{0}$ was improved, with $Q_{0}=4.6 \cdot 10^{10}$ at 17 $\mathrm{MV} / \mathrm{m}$ and $2 \mathrm{~K}$ temperature. However, this improvement could be attributed to a lower magnetic field in the dewar in this second test. Ultimate gradient reached was $31.5 \mathrm{MV} / \mathrm{m}$ limited by some issues with electronics in the LLRF or cables, and ultimate quench was not reached. Apart from a softer MP barrier, there was no clear improvement in the radiation level with the additional HPR. Figure 3.79 shows a comparison of the X-rays levels between test 1 and 2 .

For the third test of B9A5-AS-AES008 an additional 3 micron EP was applied and the HPR procedure was modified to reduce field emission. The modifications included decreased translation speed of rail by 50\% and increased number of passes in the first HPR cycle from 1 to 2 . Results of the third test are shown in Figure 3.80. First, the field emission was reduced significantly. The maximum radiation dose rate on the dewar top plate under the radiation shield block dropped to 10 $\mathrm{mR} / \mathrm{hr}$ from $\sim 5000 \mathrm{mR} / \mathrm{hr}$ in the first and second test. Performance in terms of quality factor and quench field was somewhat reduced, although still satisfying PIP-II requirements. Possible 
explanation could be residual MP, as the final dependence of $Q_{0}$ on $E_{a c c}$ still has a dip after processing or/and nitrogen concentration less optimal compared to that obtained with 20 micron EP.

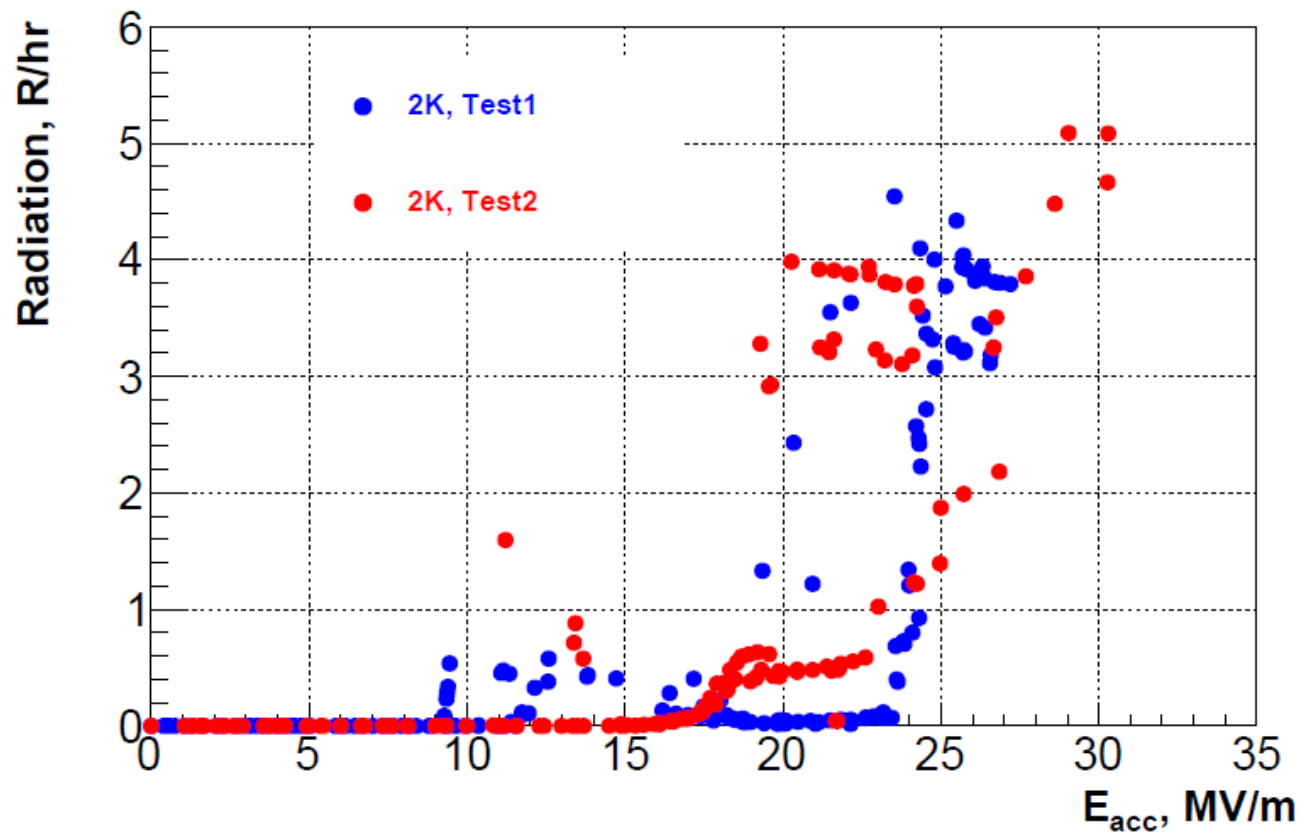

Figure 3.79: Comparison of X-ray radiation between the first and second tests.

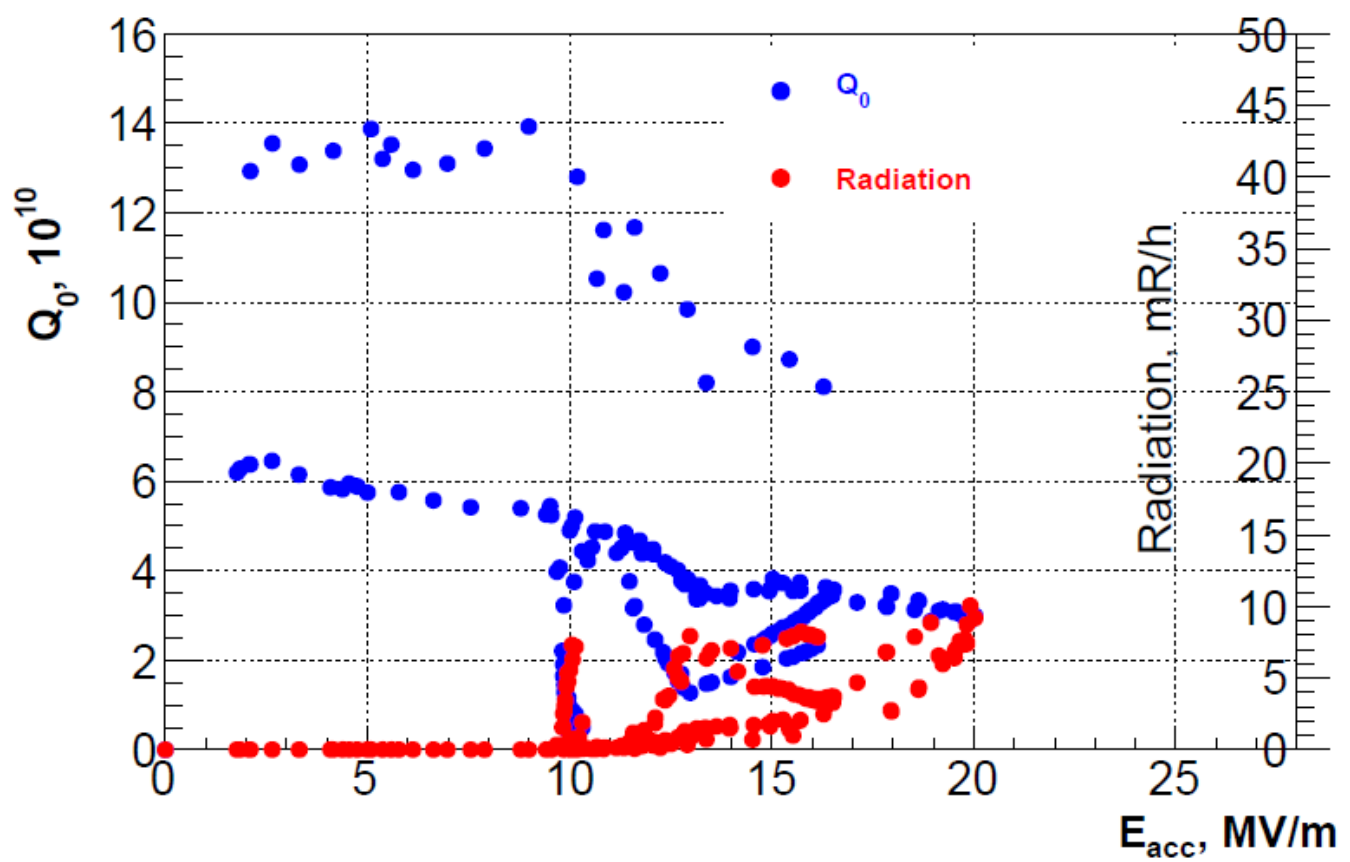

Figure 3.80: Third test of the $650 \mathrm{MHz}$ cavity B9A5-AS-AES008, after additional $3 \mu \mathrm{m}$ EP and modifications in the HPR procedure. Note that the vertical scale on the radiation axis is two orders of magnitude smaller than that on Figures 3.77 - 3.79. The upper and lower $Q_{0}$ curves (blue) present a dependence of $Q_{0}$ on the accelerating field for 1.5 and $2 \mathrm{~K}$, respectevely.

Another HB650 cavity, B9A5-AS-AES009, underwent the same surface preparation as B9A5AS-AES008 but with only 20 micron EP. The improved HPR procedure from the third B9A5-ASAES008 test was used. Results are shown in Figure 3.81. In contrast with B9A5-AS-AES008, the 
B9A5-AS-AES009 cavity did not show any radiation above background level at all fields at $2 \mathrm{~K}$. $Q_{0}$ was $3.8 \cdot 10^{10}$ at $17 \mathrm{MV} / \mathrm{m}$ at $2 \mathrm{~K}$. This test was $\mathrm{RF}$ power limited at $28 \mathrm{MV} / \mathrm{m}$ at $2 \mathrm{~K}$.

In summary, the doping surface treatment produced very good $Q_{0}$ and accelerating gradients. The electropolishing technique for $650 \mathrm{MHz}$ cavities has been developed quickly. The obtained results are already satisfactory. The latest tests, with an improved HPR procedure, showed significantly reduced or zero field emission compared to the initial vertical tests of HB650 cavities. The best measured $Q_{0}$ at $2 \mathrm{~K}$ for the lightly doped multi-cell cavity at $17.8 \mathrm{MV} / \mathrm{m}$ is $4.6 \cdot 10^{10}$. Work is continuing on further optimization of the nitrogen concentration and the best temperature regime for nitrogen doping to achieve even higher performance.

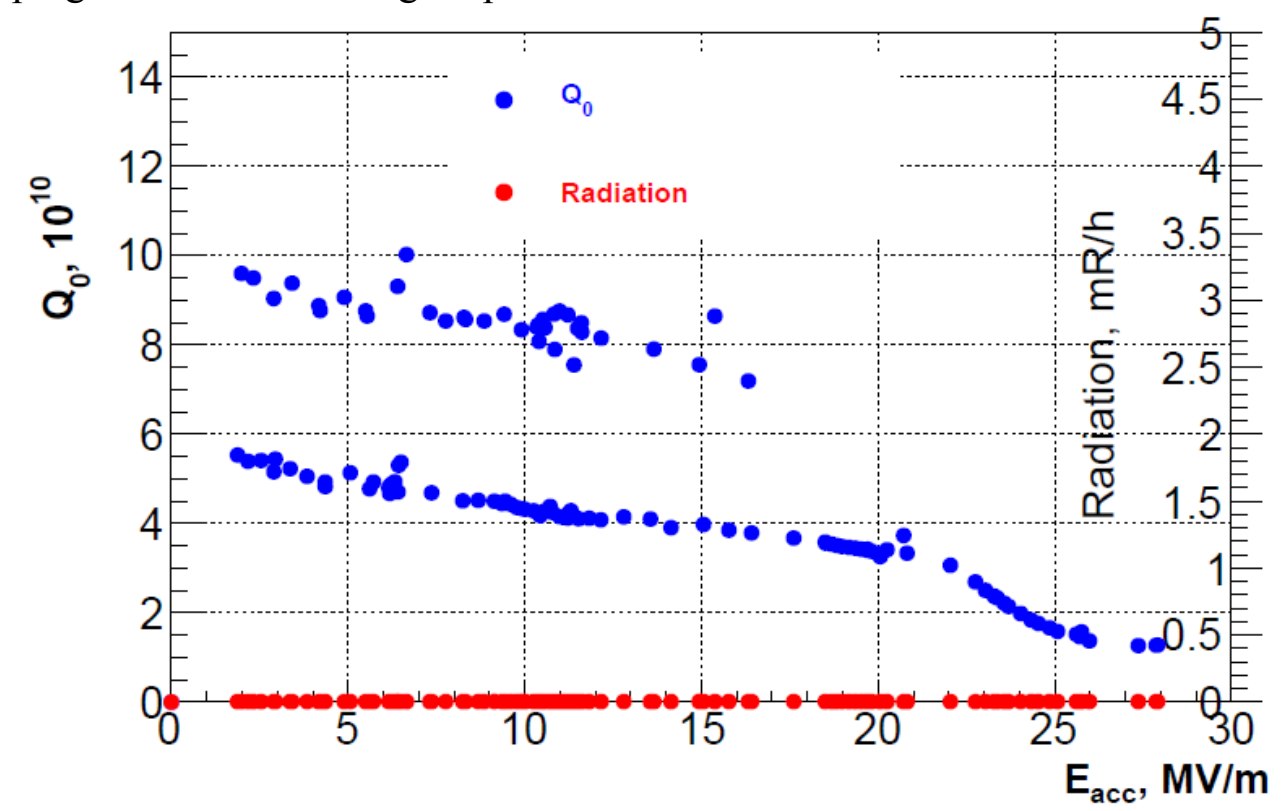

Figure 3.81: Test of $650 \mathrm{MHz}$ cavity B9A5-AS-AES009. The upper and lower $\mathrm{Q}_{0}$ curves present a dependence of $Q_{0}$ on the accelerating field for 1.5 and $2 \mathrm{~K}$, respectevely. 


\subsubsection{RF Power and Low Level RF}

\subsubsection{RF Power}

There are 120 RF systems included in the linac. With the exception of the RFQ they are deployed as one amplifier per cavity. Parameters of the RF amplifiers are presented in Table 3.23. In comparison to the power requirements presented in Table 2.10 the RF amplifier powers were rounded to about $\sim 10 \%$ larger values to get an allowance for operation with some cavities operating at reduced accelerating gradient. All RF systems will utilize continuous wave (CW) amplifiers, although SSR1, SSR2, LB650 and HB650 initially will operate in a pulsed regime to reduce RF cryogenic power. They will be used in CW mode later when the SC Linac will be upgraded to run for muon experiments.

Table 3.23: Parameters of RF amplifiers

\begin{tabular}{l|c|c|c|c|c}
\hline \hline & $\begin{array}{c}\text { Frequency } \\
(\mathrm{MHz})\end{array}$ & $\begin{array}{c}\text { Number } \\
\text { of RF } \\
\text { cavities }\end{array}$ & $\begin{array}{c}\text { Number of RF } \\
\text { amplifiers per } \\
\text { cavity }\end{array}$ & $\begin{array}{c}\text { Regime } \\
\text { of } \\
\text { operation }\end{array}$ & $\begin{array}{c}\text { RF } \\
\text { amplifier } \\
\text { power (kW) }\end{array}$ \\
\hline RFQ & 162.5 & 1 & 2 & $\mathrm{CW}$ & 75 \\
\hline MEBT Bunching cavities & 162.5 & 4 & 1 & $\mathrm{CW}$ & 3 \\
\hline First HWR cavity & 162.5 & 1 & 1 & $\mathrm{CW}$ & 3 \\
\hline Other HWR cavities & 162.5 & 7 & 1 & $\mathrm{CW}$ & 7 \\
\hline SSR1 & 325 & 16 & 1 & Pulsed & 7 \\
\hline SSR2 & 325 & 35 & 1 & Pulsed & 20 \\
\hline LB650 & 650 & 33 & 1 & Pulsed & 40 \\
\hline HB650 & 650 & 24 & 1 & Pulsed & 70 \\
\hline \hline
\end{tabular}

The RFQ has two input ports and is driven by two $75 \mathrm{~kW} \mathrm{CW}$ amplifiers. Three room temperature buncher cavities and one cryomodule containing eight superconducting HWRs operate at 162.5 MHz. They have one solid-state amplifier each operating at power levels of 3 to $7 \mathrm{~kW}$. The first HWR cavity operates at about half of the accelerating voltage and therefore uses smaller power. All amplifiers listed in Table 3.23 are solid state. They will be supplied by the Indian Institutions Fermilab Collaboration (IIFC).

The RF distribution system for the $\mathrm{CW}$ linac will utilize rigid coax commensurate with system power levels, 6-1/8", 3-1/8", or 1-5/8" EIA flanged sections. The final connection to the cryomodules will utilize a section of flexible transmission line to minimize connector location tolerances. Each RF system will have a circulator and a load to isolate the cavity from the power amplifier. This level of protection is essential in SRF systems due to the full power reflection from the cavity in the absence of beam. Cavity and drive sample signals will be provided to the LLRF for vector regulation and frequency control of the cavities. All of the RF amplifiers will be water cooled to minimize the heat load to the building HVAC system.

While each amplifier has built-in protection, which includes water flow, water temperature, pressure differential, and reflected power monitoring; a global interlock and hardware protection system must be designed for all of the RF systems. This will include water flow to loads and circulators, spark detection on cavity couplers, and RF leakage detection. 
The low level RF (LLRF) system will provide a drive signal of on the order of 0 to $+10 \mathrm{dBm}$ for each RF power source. The amplifier(s) will provide sample signals of the pre-driver and final outputs. All amplifiers will be self-contained units complete with integral power supplies, protection circuits, and control interface.

\subsubsection{Active Suppression of Microphonics and Lorentz Force Detuning}

High accelerating gradient and comparatively small beam current result in high values of loaded quality factors, and, consequently, narrow bandwidth and high sensitivity to microphonics (see Section 2.1.3.3). It is difficult to accurately predict uncompensated detuning levels because detuning can be driven by such a variety of different factors. Crude estimates of the range of expected levels can be made by examining the pressure regulation, cavity sensitivity and vibration levels measured in existing machines.

Pressures in large cryogenic systems can be easily regulated to $1 \%$ or better [20]. SNS and JLab are able to maintain steady state pressures to within $100 \mathrm{uBar}$ and $25 \mathrm{uBar}$ respectively but both can experience occasional much larger transients. With careful design the cavity pressure sensitivity $(d f / d P)$ can be reduced to a few Hz/Torr. The sensitivity of the Quarter Wave Resonator and Half Wave Resonators of ISAC-II machine at TRIUMF was $\sim 3 \mathrm{~Hz} /$ Torr and $<1 \mathrm{~Hz} /$ Torr respectively. The sensitivity of the most recent SSR1 prototype developed at Fermilab is $4 \mathrm{~Hz} / \mathrm{Torr}$. This prototype was specifically designed to minimize $d f / d P$. An earlier prototype which was not designed with the explicit goal of minimizing $d f / d P$ exhibited a sensitivity of $150 \mathrm{~Hz} /$ Torr.

Estimates for the expected range of detuning levels due to mechanical vibrations can be extracted from measurements of rms pulse-to-pulse variations in the resonance frequencies of the $1.3 \mathrm{GHz}$ elliptical cavities in the two cryomodules, CM1 and CM2, tested in NML in at Fermilab. Detuning levels ranged between $8 \mathrm{~Hz}$ for cavities at either end of the cryomodules to $2 \mathrm{~Hz}$ for cavities at the center of the cryomodules. The larger values measured for the end cavities were attributed to vibrations caused by nearby vacuum pumps. The levels measured in the NML cryomodules represent only detuning due to mechanical vibrations. The CM1 and CM2 cavities have pressure sensitivities of approximately $50 \mathrm{~Hz}$ /Torr, but the NML detuning control system adjusts the piezo bias voltage on a pulse-by-pulse basis to compensate for variations in the helium bath pressure.

Detuning due to the Lorentz force may vary between cavity types depending on the design. The resonance frequency of the SSR 1 prototype varies by about $0.5 \mathrm{kHz}$ as the cavity is ramped to full gradient. Table 3.24 collects the ranges of detuning expected from each individual source and gives a range of peak uncompensated detuning expected from all sources combined, while Table 2.11 lists the parameters relevant to detuning compensation for each PIP-II cavity type.

A comparison of the narrow matched bandwidths planned for the PIP-II cavities to the expected range of uncompensated detuning emphasizes the importance of exploiting all possible passive compensation measures. Cavities must be designed to minimize sensitivity to pressure; the cryogenic system must be designed to minimize pressure variations; and the cryomodule and other systems of the SC Linac must be designed to minimize vibrations transmitted to the cavities.

Even if all passive measures are fully exploited some form of active detuning compensation will almost certainly be required even for future $\mathrm{CW}$ operation of SC Linac. It is absolutely required for the pulsed operation of PIP-II. 
Table 3.24: Expected ranges of uncompensated detuning for the PIP-II cavities

\begin{tabular}{|c|c|c|c|}
\hline \multicolumn{4}{|c|}{ Pressure Related Detuning } \\
\hline Source & Units & Lower & Upper \\
\hline Peak pressure variation & mbar & 0.02 & 0.3 \\
\hline Mean pressure sensitivity & $\mathrm{Hz} / \mathrm{mbar}$ & 5 & 150 \\
\hline Peak pressure sensitivity variation & $\mathrm{Hz} / \mathrm{mBar}$ & 5 & 45 \\
\hline Peak pressure related detuning & $\mathrm{Hz}$ & 0.2 & 45 \\
\hline \multicolumn{4}{|c|}{ Vibration Related Detuning } \\
\hline RMS detuning due to mechanical vibrations & $\mathrm{Hz}$ & 2 & 8 \\
\hline Peak (6 Sigma) detuning due to mechanical vibrations & $\mathrm{Hz}$ & 12 & 48 \\
\hline \multicolumn{4}{|c|}{ Lorentz Force Related Detuning } \\
\hline Peak detuning due to the Lorentz Force & $\mathrm{Hz}$ & 1 & 50 \\
\hline \multicolumn{4}{|c|}{ Total Uncompensated Detuning } \\
\hline Peak uncompensated detuning $(\mathrm{Hz})$ & $\mathrm{Hz}$ & 13 & 143 \\
\hline
\end{tabular}

\section{Detuning Compensation for PIP-II}

Minimizing detuning in the PIP-II cavities will require a three-pronged approach:

- Fully exploiting all passive compensation measures during cavity and cryomodule design,

- Development and validation of active detuning compensation algorithms, and

- Detuning Control System engineering.

All of the PIP-II cavity types will be designed to minimize $d f / d P$. The pressure sensitivity of the second SSR1 prototype was designed to minimize $d f / d P$ at $5 \mathrm{~Hz} /$ Torr compared to $150 \mathrm{~Hz} /$ Torr for the first prototype. Prototypes of the other four PIP-II cavity types have not yet been constructed or tested. Steps that may be taken to minimize Lorentz force detuning and mechanical vibrations are under investigation but no design goals have been set yet.

The first step towards implementing an active detuning compensation system for the PIP-II cavities is the development, demonstration and validation of an appropriate set of algorithms using single cavities and prototype cryomodules. Once the performance of these algorithms has been satisfactorily demonstrated, they can be integrated into the Low Level RF control system.

While considerable progress has been made in the active stabilization of SRF cavity resonance frequencies using piezo actuators over the last decade, the field is still in its infancy. DESY pioneered the use of piezo actuators to compensate for Lorentz force induced detuning of the SRF cavities. Studies in the HoBiCaT test stand at BESSY, Berlin, showed that the resonance frequency of 1.3 $\mathrm{GHz}$ elliptical $\mathrm{CW}$ cavities could be actively stabilized to better than $1 \mathrm{~Hz}$ rms. An adaptive feedforward algorithm developed at Fermilab has been used to successfully stabilize the resonance for both $\mathrm{CW}$ and pulsed SRF cavities. While these techniques were able to control detuning in individual cavities over the duration of each respective test, it has yet to be demonstrated that any of those techniques can routinely stabilize the resonance frequency for every cavity in a $\mathrm{CW}$ or pulsed machine with narrow-bandwidth SRF cavities to the required level over the entire planned machine lifetime. 


\section{Optimal Control and System Engineering}

In sharp contrast to the ad-hoc techniques employed to date, optimal control offers a well-defined systematic approach to the problem of combined electro-mechanical control of SRF cavities. The Lorentz force couples the electromagnetic and mechanical states of the cavities. Knowledge of one should aid in the compensation of the other but the techniques employed to date treat resonance stabilization independently of the control of the cavity gradient and phase. Optimal control techniques were pioneered by Richard Kalman of Stanford in the 1950s. Since then they have found wide use in a variety of areas of biology, economics, ecology, engineering, finance, management, and medicine but only limited use in accelerator control systems despite the performance improvements they could bring.

The first step in implementing an optimal SRF cavity control system involves characterizing the electro-mechanical response of each individual cavity via a series of stimulus-response measurements including detuning due to piezo response and detuning response to reactive power modulation. Models of the response suitable for use in subsequent steps can be extracted using the minimal state-space realization (MSSR) system-identification algorithm of Kalman and Ho.

The second step involves estimating the cavity electromechanical state at each point in time from real-time measurements of the cavity base-band RF signals. At each time step the Kalman filter determines optimal estimates of the cavity electro-mechanical state by minimizing a quadratic cost function that depends on the measured values of the cavity RF baseband signals, piezo actuator voltage and current, the previous state estimate, the covariance of the signal noise, the covariance of the previous state estimate, and the a-priori cavity response model determined in the first step.

In the final step a Linear Quadratic Gaussian Regulator (LQGR) minimizes at each point in time a cost function similar to that used by the Kalman filter to determine the combination RF and piezo drive signals most likely to keep the cavity gradient, phase and detuning at their target values.

These steps provide a well-grounded chain of deterministic algorithms that can be used to automatically characterize, model and optimally control detuning and field stability of any superconducting cavity or chain of cavities. Steady-state versions of the Kalman filter and LQGR can be implemented in real-time using commercially available FPGA signal processing boards.

The two SSR1 prototype cavities have been used to study microphonics compensation during tests in the STC test stand in the Meson Detector Building (MDB). Tests in 2011 using the first SSR1 prototype showed it was possible to stabilize the resonance frequency to less than $0.5 \mathrm{~Hz}$ over a period of 20 minutes with a quite simple algorithm. Further studies, following quality factor measurements for the second prototype, showed that it was possible to stabilize the resonance frequency of even very narrow band-width cavities for extended periods. Studies in the STC continue with the aim of developing and demonstrating the performance of a set of algorithms appropriate for active compensation of the PIP-II cavities.

Once a well-defined set of detuning compensation algorithms have been developed and their performance has been satisfactorily demonstrated using single cavities and the prototype cryomodules, the algorithms will be integrated into the Low Level RF control system. While the development stage will focus on meeting the required performance goals, the system engineering stage will focus on the large scale deployment of those algorithms. Reliable operation of the PIP-II machine will require an efficient and robust implementation capable of compensating detuning for every cavity for the lifetime of the accelerator. 


\subsubsection{Low Level RF}

The Low Level RF system encompasses the programming and regulation of the cavity field amplitude and phase as required by the longitudinal beam dynamics in the machine. It also controls or interfaces to the ancillary equipment that is involved in the generation of RF. Hardware and software modules include Cavity Field Controller, Resonance Frequency Controller, Master Oscillator, Phase Reference Line, LO distribution, Transfer Synchronization to Booster, Beam Chopper Waveform Generator, and the interface to interlocks, timing systems and the control system (see Figure 3.82). The LLRF system will also be involved in longitudinal phase space painting into the Booster.

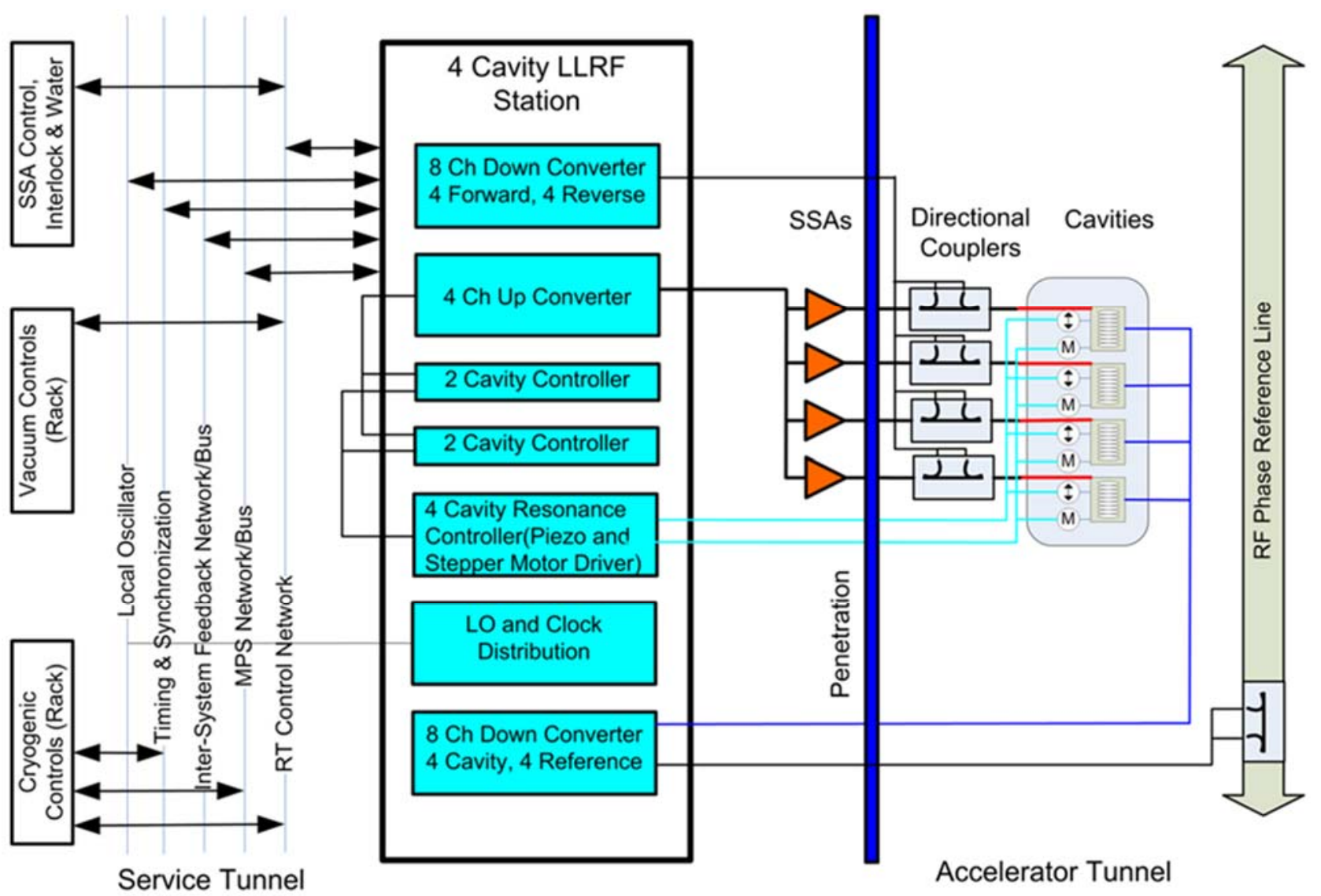

Figure 3.82: Four cavity RF system including LLRF rack.

The Linac is constructed with accelerator sections with the following frequencies; $162.5 \mathrm{MHz}$, $325 \mathrm{MHz}$ and $650 \mathrm{MHz}$. Additionally, $1300 \mathrm{MHz}$ is provided for instrumentation and local clock generation. The basic configuration is that one RF amplifier will drive one cavity with the exception that two amplifiers will drive the RFQ. There is a mixture of warm copper cavities (the RFQ and bunching cavities) and five types of SRF cavities (see Table 2.5). This necessitates three four-cavity stations at $162.5 \mathrm{MHz}, 13$ four-cavity stations at $325 \mathrm{MHz}$, and 15 four-cavity stations at $650 \mathrm{MHz}$. There is also a mixture of CW only systems used the HWR and systems capable to operate both in puled and $\mathrm{CW}$ regimes used for the rest of the linac.

\section{LLRF Regulation Strategy}

The requirements for the linac beam energy stabilization are determined by the Booster RF bucket height and requirements related to the static longitudinal painting in the course of multi-turn beam 
injection (see Section 2.3.2.3). The latter requires the linac rms energy stability better or about $0.01 \%$. The LLRF feedback controller should be capable of regulating to this level over a time period of less than one second. For time periods greater than one second an adaptive feed-forward algorithm will correct for drift in the cavity phase and amplitude calibrations. This will be accomplished by utilizing beam-based diagnostics along the linac and in the Booster as well as RF diagnostics to provide feedforward corrections and slow adjustments to LLRF calibrations.

The same regulation accuracy of about $0.01 \% \mathrm{rms}$ is required for the Booster magnetic field at the injection. It can be achieved with the scope extension of the new Booster cogging system which is presently under commissioning. In particular, the feedback system can be based on the magnetic field measurement in the Booster reference magnet with subsequent average magnetic field correction by Booster dipole correctors - similar to the new cogging system.

The beam current, cavity field gradients, Lorentz Force Detuning (LFD) and worst-case microphonics determine the loaded cavity Qs, bandwidths and RF power requirements (see Section 2.1.3.3). Precision corrections of the LFD and microphonic disturbances through Resonance Frequency Control are required for gradient regulation without exceeding the available RF power overhead. Resonance control to meet these requirements requires a large coordinated research, development, and engineering effort including both mechanical and electrical designs. Resonance control is covered below in more detail.

\section{Phase Reference Generation and Distribution}

The phase reference system starts at the Linac frontend with a $162.5 \mathrm{MHz}$ Master Oscillator (see Figure 3.83). The 162.5 MHz Master Oscillator is inside a temperature controller chassis and generates the $182.5 \mathrm{MHz}$ local oscillator signals for the LLRF up-converters and down-converters for the $162.5 \mathrm{MHz}$ linac section. The $162.5 \mathrm{MHz}$ is driven into a closed loop phase averaging reference line that is located alongside the accelerating structure. The reference line is tapped providing signal to cables run alongside the cavity probe cables providing first order cable temperature compensation. This first reference line provides signal for the main timing system, instrumentation and the beam chopper controller.

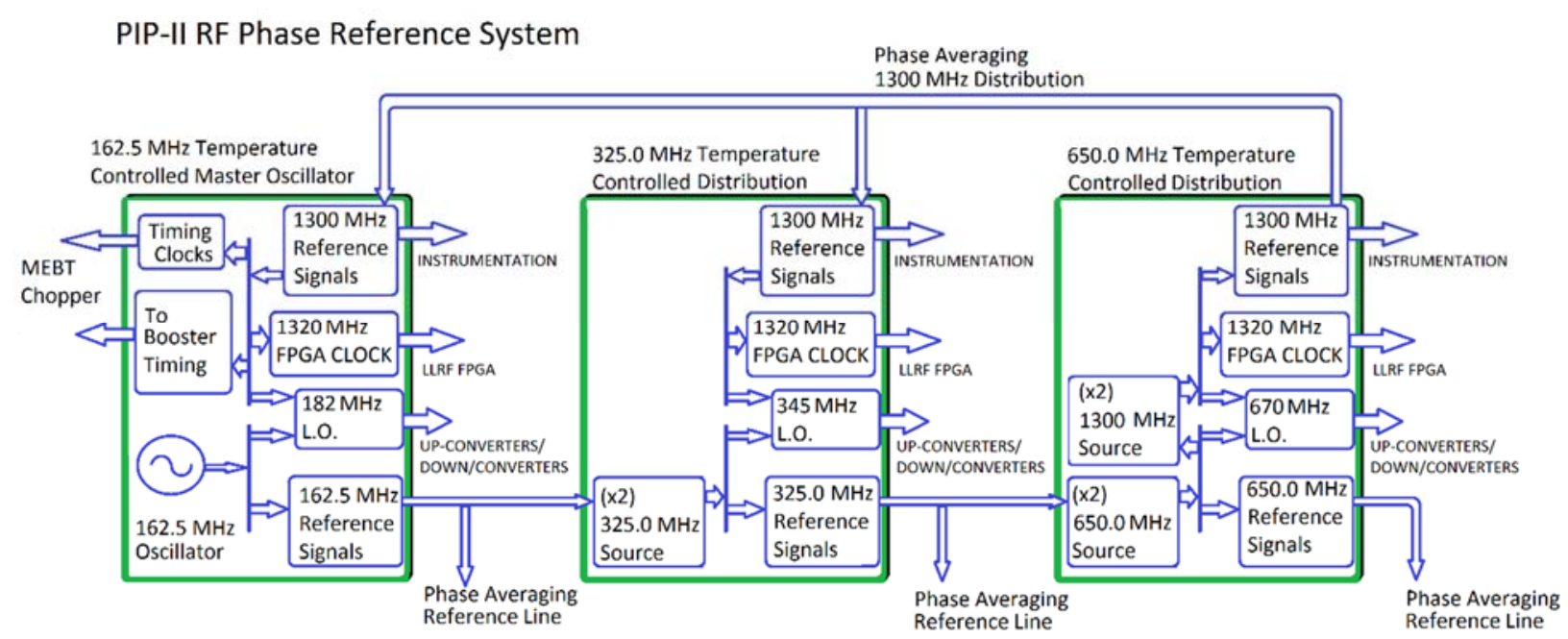

Figure 3.83: Master Oscillator and Multi-frequency Phase Reference Lines

The $162.5 \mathrm{MHz}$ phase reference line also provides signal to the $325 \mathrm{MHz}$ Slave Oscillator that tightly tracks the $162.5 \mathrm{MHz}$ phase. The $325 \mathrm{MHz}$ oscillator generates the $345 \mathrm{MHz}$ local oscillator 
signals in the same fashion as is done in the $162.5 \mathrm{MHz}$ master oscillator. A $325 \mathrm{MHz}$ phase averaging reference line is setup in the same fashion as the $162.5 \mathrm{MHz}$ reference scheme. This chain of frequency multiplication is repeated for the $650 \mathrm{MHz}$ sector. The $1300 \mathrm{MHz}$ serves as the source for a phase averaging reference line that travels back down to the lower frequency sectors, providing $1300 \mathrm{MHz}$ taps for instrumentation, and it will provide for a single $1320 \mathrm{MHz}$ clock for LLRF digital systems distributed through the Linac.

\section{Four Cavity LLRF Control Rack}

The LLRF system is organized in a group of up to four cavities serviced by one rack of electronics as shown in Figure 3.84 with each module described in detail below. The group of four cavities allows for an economy of scale in the hardware design while keeping cable runs as short as possible. The signal path is kept as direct as possible with cables from the accelerator tunnel brought directly to the 8-channel down-converter.

The down-converter is used to translate the RF signals at the various frequencies across the accelerator to a common intermediate frequency (IF) of $20 \mathrm{MHz}$. In order to achieve the regulation requirements the down-converter must be low-noise $(-158 \mathrm{dBc})$, have high channel isolation (greater than $80 \mathrm{~dB}$ ), phase stability to within 0.005 degrees above $1 \mathrm{~Hz}$ and amplitude stability $0.005 \%$ above $1 \mathrm{~Hz}$.

The IF is digitized by a high-speed Analog to Digital Converter (ADC). The ADC must have a sample rate greater than $94 \mathrm{MS} / \mathrm{s}$, and goals of achieving $-155 \mathrm{dBc}$ noise power density and less than $200 \mathrm{~ns}$ of signal latency. There are several ADCs on the market that achieve these specifications; the present choice is the AD9653 from Analog Devices. The digitizer board has 8 channels that are sent to the Field Programmable Gate Arrays (FPGA).

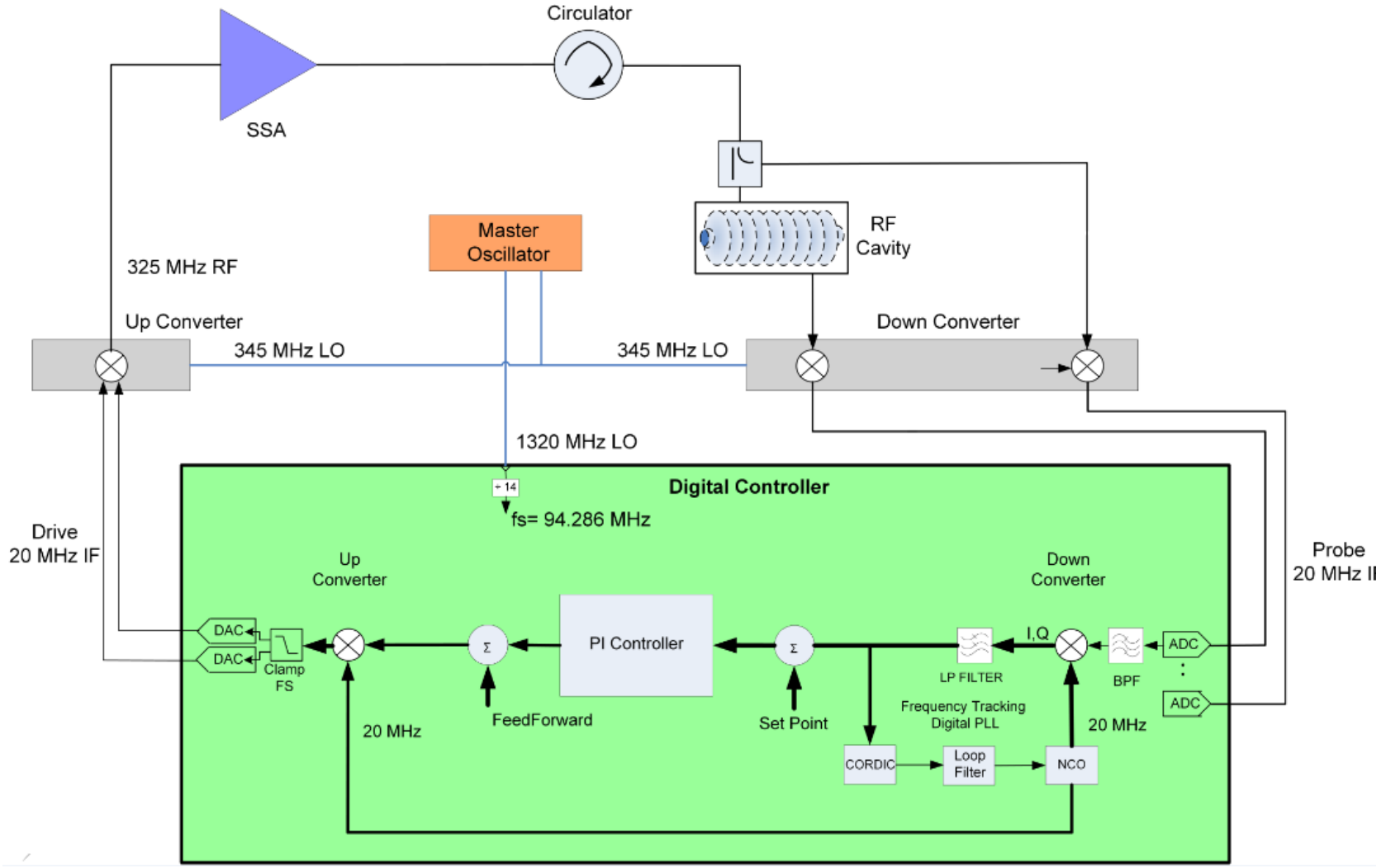

Figure 3.84: Conceptual diagram of the LLRF control system at $325 \mathrm{MHz}$. 
The FPGA processes the digitized signals for both read-back and for input to the controller. The controller will have a closed loop bandwidth of $\sim 30 \mathrm{kHz}$ and is designed to support both $\mathrm{CW}$ and pulsed operation. Each controller will have the ability to run in a self-excited loop where the drive frequency tracks the cavity, and in a generator driven mode where the output is at a fixed frequency. It is expected that each cavity will be operated CW during some part of commissioning and tests. $\mathrm{CW}$ operation typically requires startup in a self-excited loop with a transition to a generator driven loop to align with the beam phase. For cavities with a low bandwidth, pure pulsed operation requires a complex phase trajectory program to fill the cavity with energy. A prototype system is under development for the PIP-II injector test (see Figure 3.84). It includes the LLRF four Station Field Controller Module shown in Figure 3.85. The LLRF system provides both RF waveforms and sampled values to the control system that are calibrated and highly linear. They best represent the cavity field and directional RF signals and will be used for all data analysis.

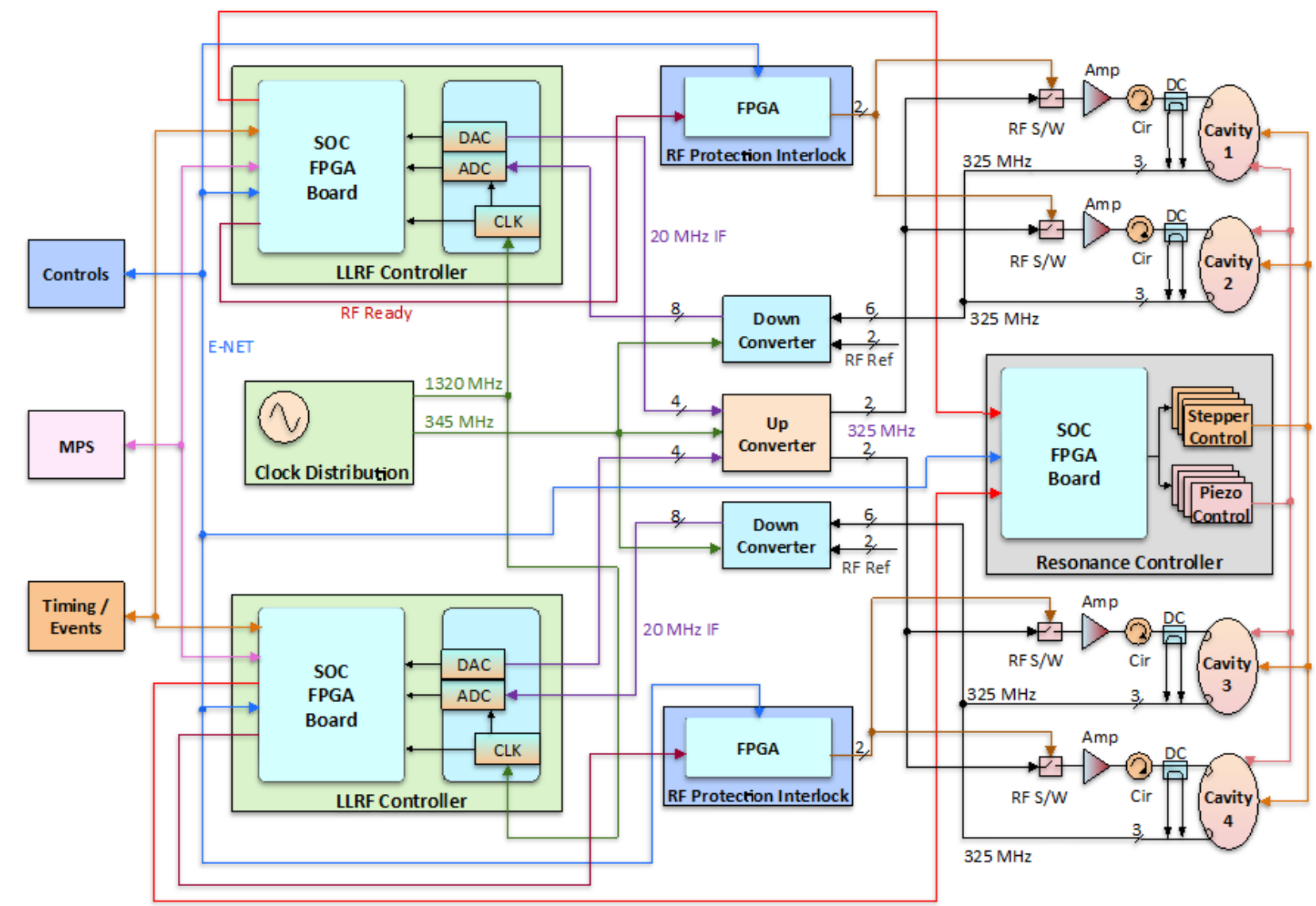

Figure 3.85: LLRF for a four cavity control system architecture. Note that this system is showing an RF frequency of $325 \mathrm{MHz}$, with a corresponding LO of $345 \mathrm{MHz}$. The $162.5 \mathrm{MHz}$ and the $650 \mathrm{MHz}$ systems have an $\mathrm{LO}$ of $182.5 \mathrm{MHz}$ and $670 \mathrm{MHz}$ respectively.

\section{Beam Chopper Waveform Generator and Booster Injection}

LLRF will also generate the waveforms needed for the beam chopper ${ }^{9}$ and the $44.705 \mathrm{MHz}$ RF signal for the Booster to lock to during the 4 millisecond injection period. The waveforms require complex pre-distortion for the chopper amplifiers which is better implemented with the entire

\footnotetext{
${ }^{9}$ The beam chopper removes bunches on the boundary of RF buckets and forms a 3 bunch long extraction gap.
} 
waveforms pre-calculated and played out from tables. There are several advantages to waveform tables: repeatability from pulse to pulse, local storage of beam waveforms in LLRF and instrumentation systems, and the ability to make small corrections utilizing adaptive feed-forward. A multi-channel 4 GSPS arbitrary waveform generator is specified for this purpose.

\subsubsection{Vacuum System}

The PIP-II vacuum system includes all of the vacuum environments which the beam passes through on the way from the ion source to the Booster. The vacuum system includes three regions: (1) the frontend (ion source, LEBT, RFQ, MEBT), (2) Main Linac which includes all cryomodules, and (3) the beamline from the linac to the Booster. There are vacuum gate-valves capable to isolate each section for commissioning or operation. The vacuum system design is focused on the requirement of a low particulate vacuum that is sufficient but practical and cost efficient to ensure reliable performance of the cryomodules. The vacuum system also includes the insulation vacuum of cryomodules, which is absolutely essential for their operation. Requirements for the insulation vacuum systems are covered in the Functional Requirement Specifications and Interface Documents for the cryomodules $[108,110,118,119,120]$.

The PIP-II vacuum system faces significant technical challenges, including a large hydrogen flux from the $\mathrm{H}^{-}$ion source $(500 \mathrm{mTorr} \cdot 1 / \mathrm{s})$ and large outgassing from the MEBT absorber $(\sim 1 \mathrm{mTorr} \cdot 1 / \mathrm{s}$ at $10 \mathrm{~mA}$ ion source current). Therefore, pumps with high effective pumping speed have to be used within available space, which is quite tight in many places. Turbo pumps have been chosen for the ion source, RFQ and the MEBT beam absorber. Ion pumps will be used for distributed pumping among the beamline where applicable.

The greatest challenge is probably the risk minimization for performance degradation of SC cryomodules due to excessive gas load and migration of micro-particles. Therefore, it is important to apply low particulate vacuum practices in vicinity of cryomodules and, in particular, upstream and downstream vicinities of the SC Linac. The roughing/venting ports will be arranged so that to minimize particle migration towards cryomodules. The flow rate of roughing/venting will be regulated to reduce the risk of particle migration in the course of pumping/venting. Differential pumping scheme will be arranged at the vicinity of cryomodules to minimize the hydrogen gas flux towards them.

As usual, the gate valves are included in the interlock system to minimize consequences in the case of vacuum incidents. Data logging will be implemented for operation and analysis.

\section{Functional Requirements}

The requirements for PIP-II vacuum vary depending on the characteristics of the specific regions (see Figure 3.86). A summary of the residual pressure for various regions is shown in Table 3.25. The vacuum requirements can be classified in three levels: 1) UHV and Low Particulate Practice for Main Linac; 2) UHV and Low Particulate Practice for the transition regions located upstream and downstream of the SC Linac; 3) High Vacuum for the region from Ion source to the MEBT absorber and the beam transfer line to Booster. All components shall be vacuum certified accordingly before being installed in the beamline. 


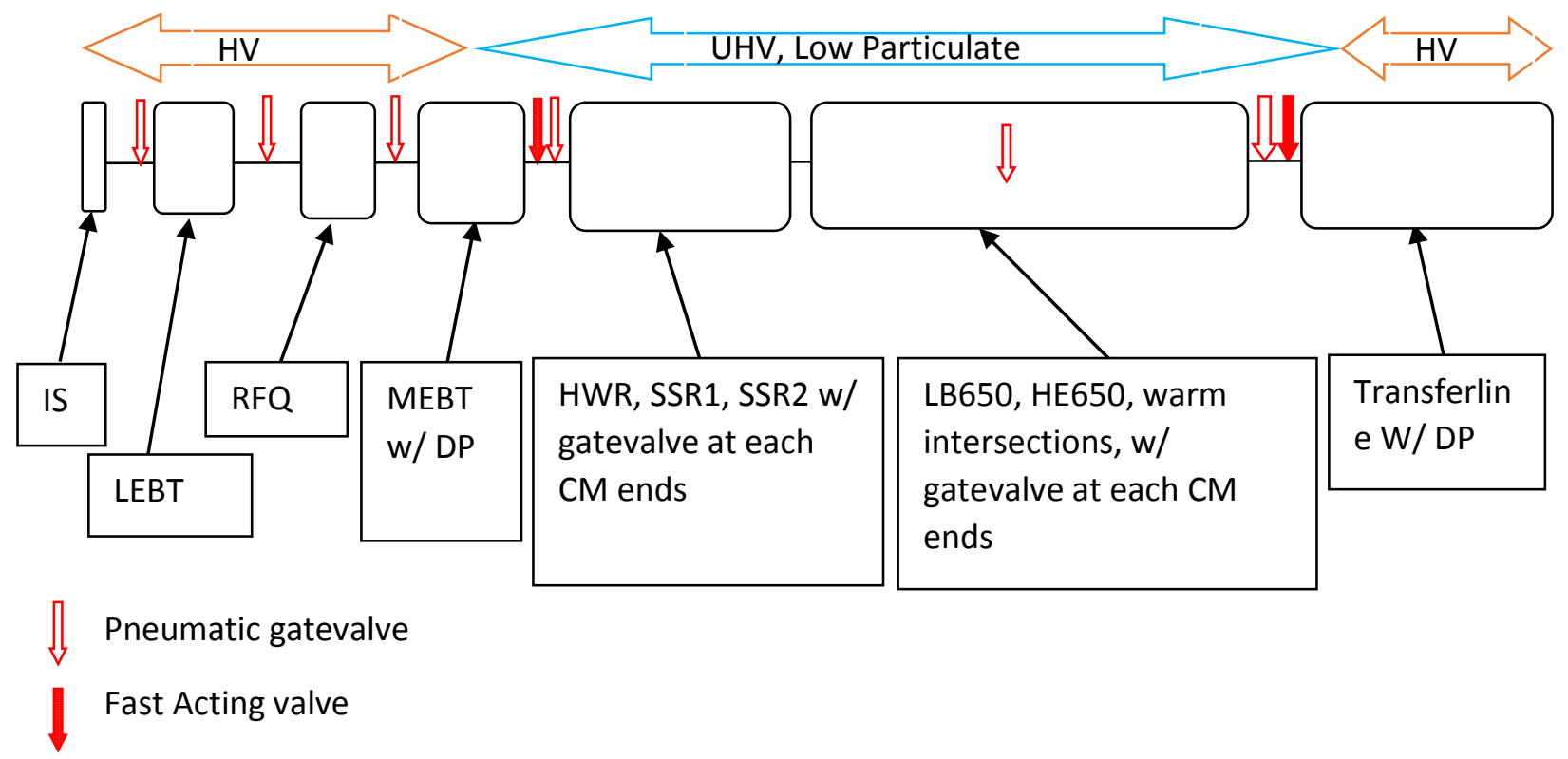

Figure 3.86: PIP-II Vacuum System Layout

Table 3.25: Beam vacuum requirements

\begin{tabular}{|c|c|c|c|c|c|c|c|c|c|}
\hline REQUIREMENT & Ion S. & LEBT & RFQ & MEBT & MEBT_DS & CMs & W-sect & Trans.-US & Transfer \\
\hline Pressure (torr) & $10^{-2-} 10^{-6}$ & $10^{-7}$ & $10^{-7}$ & $10^{-7}$ & $10^{-7}-10^{-10}$ & $10^{-10}$ & $10^{-10}$ & $10^{-10}-10^{-8}$ & $10^{-8}$ \\
\hline $\begin{array}{l}\text { Leak Check } \\
\text { Sensitivity } \\
\text { (mbar.l/s) }\end{array}$ & $2 \times 10^{-9}$ & $2 \times 10^{-9}$ & $2 \times 10^{-9}$ & $2 \times 10^{-9}$ & $2 \times 10^{-10}$ & $2 \times 10^{-10}$ & $2 \times 10^{-10}$ & $2 \times 10^{-10}$ & $2 \times 10^{-9}$ \\
\hline Pumping & \multicolumn{4}{|c|}{ Turbo, Ion Pump } & Ion Pump & Cryo & getter & Ion Pump & Ion Pump \\
\hline $\begin{array}{l}\text { Vacuum Seals of } \\
\text { Joints }\end{array}$ & \multicolumn{4}{|c|}{$\begin{array}{c}\text { metal gasket or elastomer; } \\
\text { CF or KF }\end{array}$} & \multicolumn{4}{|c|}{ CF only; metal gasket only } & $\begin{array}{c}\text { metal gasket } \\
\text { or elastomer; } \\
\text { CF or KF }\end{array}$ \\
\hline Part Cleaning & \multicolumn{9}{|c|}{ UHV procedures } \\
\hline Gauging & \multicolumn{5}{|c|}{ Ion gauge } & \multicolumn{2}{|c|}{ cc gauge } & \multicolumn{2}{|c|}{ Ion Gauge } \\
\hline $\begin{array}{l}\text { Low Particulate } \\
\text { practice }\end{array}$ & no & no & no & no & Yes & Yes & Yes & Yes & no \\
\hline controllers & \multicolumn{9}{|c|}{ equiped with RS485 to communicate with control system ACNET via PLC } \\
\hline
\end{tabular}

\section{Vacuum Sections}

Ion Source --- The ion source is continuously supplied with hydrogen gas in order to produce $\mathrm{H}^{-}$ ions. Inside the plasma chamber a hydrogen pressure is about $3 \cdot 10^{-2}$ Torr, while the pressure downstream of ion source should be better than $10^{-6}$ Torr in order to avoid excessive beam loss due to $\mathrm{H}^{-}$stripping. The pumping system and internal structures of the ion source and the vacuum box are designed to achieve this large pressure gradient within several inches. Classified as HV region.

LEBT --- The low energy beam transfer line provides beam focusing, beam analysis and proper beam parameter prior to entering RFQ. A pressure of $10^{-6}$ Torr is required in this $\sim 2 \mathrm{~m}$ long region. Classified as HV region.

RFQ --- The RFQ accelerates the $\mathrm{H}^{-}$beam to $2.1 \mathrm{Mev}$. It requires $10^{-7}$ Torr vacuum for normal operation. Classified as HV region. 
MEBT --- The medium energy beam transfer line prepares the $\mathrm{H}^{-}$beam time structure prior to entering the HWR (the first cryomodule) by removing (chopping) undesired bunches. Typically, 50 - $80 \%$ of the $\mathrm{H}^{-}$beam is chopped out and directed to the absorber. Sputtering and hydrogen production (due to incoming $\mathrm{H}^{-}$flux) at the absorber are expected in normal operation. From the ion source to the MEBT absorber, only a regular high vacuum is achievable with reasonable cost. This is classified as a HV region. The region from downstream of MEBT absorber to the HWR is the transitional area bridging the regular high vacuum environment to the ultrahigh vacuum and low particulate environment in the cryomodules. This is critical for normal operation of the cryomodules. The UHV and low particulate requirement is applied to the design, cleaning and installation in this region. This is classified as a UHV and Low Particulate region. A fast acting gate valve is installed upstream of cryomodule, in order to protect $\mathrm{CM}$ from contamination due to vacuum failure.

Cryomodule Beam Vacuum --- The cryomodules operate at $2 \mathrm{~K}$. The internal surfaces provide sufficient cryo-pumping effect so that the residual gas pressure will be lower than $10^{-10}$ Torr, mainly driven by the operational temperature. The challenge for this region is to achieve low enough pressure $\left(10^{-7}\right.$ Torr) at room temperature before cooling down. All areas between cryomodules, including the $1100 \mathrm{~mm}$ inserts between $650 \mathrm{MHz}$ cryomodules (see Figure 3.87) will also be classified as UHV and Low particulate as well. Gas flow control, proper filtration and cold trap shall be applied for pumping down and venting up. Cold Cathode (CC) gauges shall be used to minimize the heat load from gauging. This is classified as an UHV and Low Particulate region.

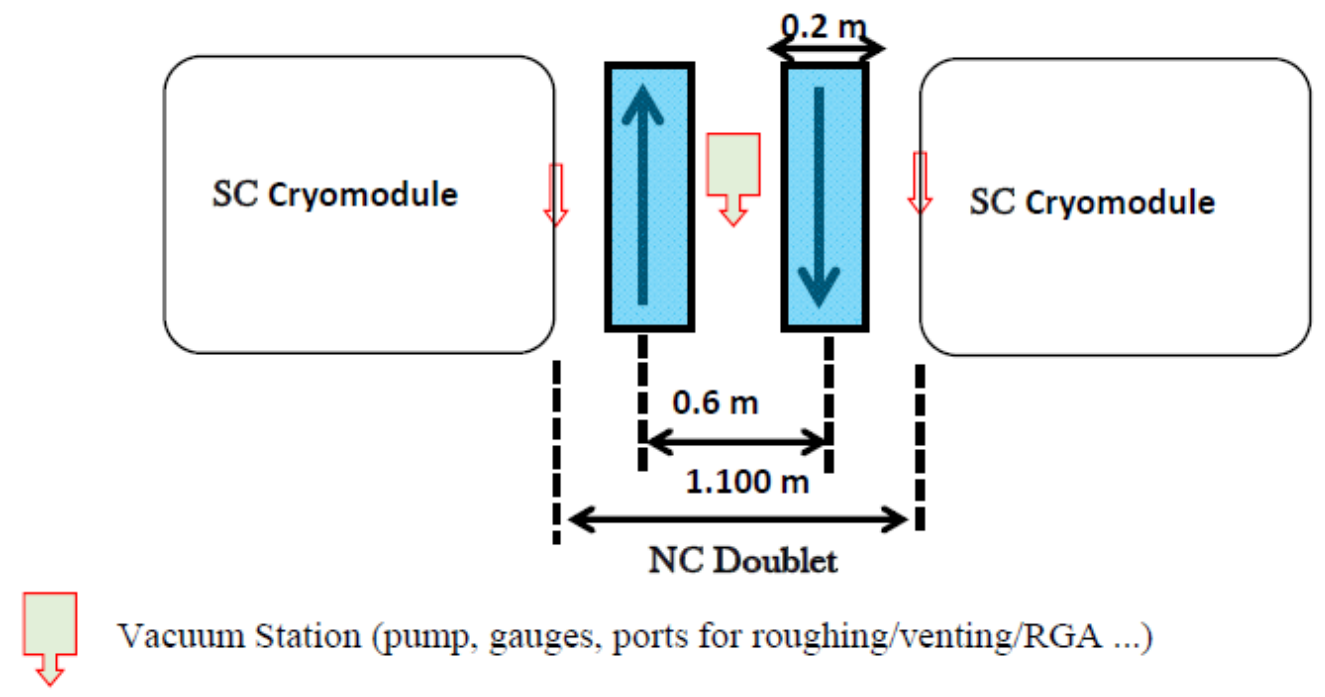

Figure 3.87: Warm Inserts in between $650 \mathrm{MHz}$ cryomodules of the SC Linac.

CM Insulating Vacuum --- The pressure shall be $10^{-5}$ Torr prior to cooldown. This system will be pumped with turbo and roughing pumps. The pressure shall be maintained at $10^{-6}$ Torr or better when cold. CC gauges shall be used. Pumps used in this system should not be used for the UHV system because of contamination. Classified as HV region.

Linac-to-Booster transfer line --- This beamline consists of magnets and beam instrumentations at room temperature. It connects the SC Linac and Booster. In order to maintain a high quality vacuum in the cryomodules at the linac end, the UHV and low particulate requirement shall be applied in its differential pumping section for transition to regular beam transport line. It requires cleaning of beam pipes and clean installation of this region. Classified as UHV and Low Particulate region. A fast action gate valve will be installed at the linac end to protect the cryomodules from contamination due to vacuum failure. 


\section{Components}

Materials --- Materials chosen for vacuum components must not only have a low outgassing rate with no contamination in general, but also must be resistant to radiation and corrosion, and have low magnetic permeability, particulate generation, etc. in the specific environment. Plating or coating is not allowed in areas that require low particulate vacuum. Vacuum personnel shall be informed and/or involved in all material related decisions.

Pumps --- Ion pumps (IP's) will be used in the beamline. The IP's shall have magnetic shields and be positioned so that there is no line of sight from IPs to sensitive areas. Turbo and roughing pumps should be dry. Scroll pumps can introduce TeflonTM and should not be used for beamline or coupler vacuum systems.

Beam pipe --- All beam pipes in an UHV region must be electropolished and hydrogen degassed, an in-situ bakeout may be required. 316L stainless steel must be used in areas sensitive to corrosion, magnetic field and/or high radiation.

Flanges --- $\mathrm{CF}$ flanges $\left(70^{\circ}\right.$ preferred) are required in UHV and preferred in $\mathrm{HV}$.

Gauges --- Inverted magnetron cold cathode gauges (CC's), thermocouple gauges (TC's), ion gauges (IG's), convection gauges (PG's), capacitance manometers, and residual gas analyzers (RGA's) are required.

Hardware --- In the low particulate vacuum regions, standard unplated ConFlat ${ }^{\circledR}$ copper gaskets should be used with the ConFlat ${ }^{\circledR}$ flanges. They should be ordered individually wrapped and already UHV cleaned. Bolts and studs shall be made of domestic 316L unplated stainless steel. Nuts should be 651 silicon bronze. Silicon bronze studs should be used for blind holes. Nuts, bolts, and studs shall be ultrasonically cleaned prior to installation.

Valves --- Manual and pneumatic gate valves will be installed along the beam line for protection and commissioning purposes. All-metal valves are required in UHV regions. Some gate valves, including the fast action valves, will be interlocked as part of machine protection.

Cleaning ---All vacuum parts for PIP-II shall be cleaned as UHV, even the parts for HV regions, and bagged properly (ADDP-ME-000145, procedure for particle free vacuum components) for parts used in regions which require low particulate vacuum.

\section{Certification}

All devices that are part of the beamline vacuum shall be certified by vacuum personnel, prior to being installed in the beam line. The certification must verify that the specific device meets the vacuum requirement. The specific procedures and criteria will be defined accordingly.

\section{Assembly and installation}

The procedures for assembly and installation will be specific to each region.

\section{Roughing and Venting}

Since there is a risk of particle migration towards a CM, the valves that isolate the CM from its vicinity shall remain closed during the period of roughing down and venting up in the adjacent regions. Turbo cart equipped with flow restriction ( $50 \mathrm{mbar} \cdot 1 / \mathrm{s})$ and proper filtration shall be used for such activities, until pressure is below 1 Torr. The locations of pumping/venting ports should be chosen so that the risk of particle migration towards CM minimized.

\section{Basic Low Particulate Requirements}

1. All processes and procedures shall be performed per released written procedures.

2. All processes, procedures and facilities used to install or maintain such hardware shall be 
validated by testing, (as defined in section III. Verification Methods for Measuring Particle Counts) before being approved for use on the PIP-II "low particulate" hardware.

3. All "low particulate" vacuum components shall have the final stages of cleaning performed in ISO Class 4 (FED-STD Class 10) environments per ISO 14644, in the operational state, considered size $0.5 \mu \mathrm{m}\left(352\right.$ particles $\left./ \mathrm{m}^{3}\right)$.

4. All interconnections of "low particulate" vacuum components shall take place in an ISO Class 5 Cleanroom (FED-STD Class 10) or Clean Zone per ISO 14644-1, in the operational state, for 0.5 - $5.0 \mu \mathrm{m}$ particles.

5. Integration, service, or maintenance operations on the Cryomodule beam line vacuum (for example, removing beamline transport spools, connecting the beamline between two Cryomodules, or connecting the beamline between a Cryomodule and a transitional warm beamline section, etc.) shall take place in ISO Class 4 Clean Zones per ISO 14644, rest state, for 0.3 micron particles, 0.5 micron particles and 1.0 micron particles.

6. All test equipment used to validate "low particulate" components, processes, procedures, and facilities shall be properly calibrated, with valid calibration at the time of their use.

7. All personnel entering the Clean Rooms or Zones while "low particulate" hardware is exposed must be trained as cleanroom users.

8. Implementation of low particulate technology in an "as low as reasonably achievable" approach can be justified by the cost of determining the true risk imposed by excessive relaxation of requirements. This approach is adopted for the PIP-II project.

\section{Region Specific Requirements}

For purposes of optimizing the design, fabrication, installation and operation of PIP-II, various regions of the SRF accelerator are categorized into Cleanliness Regions (see Table 3.26.) The most critical regions include the superconducting cavities and their vicinities that share common vacuum space. As one moves further away from the SRF cavity, the probability for particulate migration to the SRF cavity is reduced, so does the requirement.

Table 3.26: Cleanliness Region Description

\begin{tabular}{l|l}
\hline \hline A & SRF cavity, and other surfaces in the same vacuum region (HWR, SSR1, Main Linac) \\
\hline B & $\begin{array}{l}\text { Transitional warm beamline vacuum internal surfaces within "Low particulate Length" of } \\
\text { Cleanliness Region A (MEBT Absorb-HWR, SC Linac - Linac-to-Booster transfer line) }\end{array}$ \\
\hline $\mathrm{C}$ & $\begin{array}{l}\text { External surfaces assembly parts, jigs, fixtures and tools in the cleanliness region A, } \\
\text { transitional warm beamline, within cleanrooms and clean zones during beamline } \\
\text { connections }\end{array}$ \\
\cline { 2 - 2 } & External surfaces of cryo-beamline components \\
\hline \hline
\end{tabular}

\subsubsection{Alignment, Optics and Orbit corrections}

The requirements for the alignment of focusing elements in the PIP-II linac are similar to typical alignment tolerances in other proton accelerators operating in Fermilab. Beam misalignment degrades machine performance due to two major effects: (1) a reduction in geometrical machine acceptance and (2) emittance growth due to spherical aberrations related to the non-linearity of focusing fields, which is increased with beam offsets in focusing lenses. Both effects can result in an increase of beam loss. As can be seen in Figure 2.26, the tightest apertures in the SC part of the linac are at its beginning - in the HWR and SSR1 cryomodules. 


\section{HWR, SSR1 and SSR2 cryomodules}

Focusing in the first three sections of the linac, which include the HWR, SSR1 and SSR2 cryomodules, is achieved with solenoids. Each solenoid has horizontal and vertical dipole correctors located inside and a three-coordinate ${ }^{10}$ BPM mounted on its side. The betatron phase advance per period is about $90 \mathrm{deg}$. at the beginning of each section, and for each section the betatron phase advance decreases gradually with energy increase. The beta-functions through the linac are presented in Figure 2.25. The growth of beta-functions does not increase the transverse beam sizes due to adiabatic damping, as can be seen in Figure 2.26. Similarly, beam betatron oscillations excited by lens displacement or dipole corrector stay with approximately the same amplitude downstream of the linac.

Transverse displacement of a focusing lens excites betatron motion of the beam centroid downstream of this lens. Taking into account that each period of the linac lattice includes one lens and one drift one obtains that the amplitude of betatron motion in close vicinity of the excitation point is:

$$
A \approx 2 \delta x \tan ^{2}(\mu / 2) .
$$

Here $\delta x$ is the value of the lens displacement, and $\mu$ is the betatron phase advance in the period where the excitation occurred. With further beam motion this amplitude is changed proportionally to $\beta / p$. For the present optics it stays approximately constant since the growth of beta-function, $\beta$, is compensated by the increase of beam momentum, $p$. The amplitude of excitation grows with $\mu$ and for the SC linac it achieves the maximum of $A \approx 2 \delta x$ at the beginning of each section, where $\mu=90$ deg.

We require that the maximum deviations of the vacuum chamber alignment from the reference straight line do not exceed $5 \%$ of its aperture or about $1.5 \mathrm{~mm}$. This accuracy is referenced to the fiducials installed on the exterior of cryomodules. Note that this requirement is only applied to possible aperture limitations and is not directly applicable to other features of vacuum chamber. Consequently, the alignment of the magnetic centers of focusing lenses should be at least the same. Another limitation on the alignment of focusing lenses could come from an accumulation of kicks due to misalignments of multiple lenses. However, in the case of the PIP-II linac there is a dipole corrector associated with each lens which enables local compensation of alignment error. This greatly mitigates alignment requirements. In this section we will relate the maximum misalignment mentioned above and its rms value as 3 to 1 . It results in that the mentioned above $1.5 \mathrm{~mm}$ alignment error corresponds to $0.5 \mathrm{~mm}$ rms errors. Note that even in the case of zero corrector strengths in the HWR cryomodule the accumulated orbit excitation after passing 8 solenoids is $2 \cdot 0.5 \mathrm{~mm} \cdot \sqrt{8} \approx$ $2.5 \mathrm{~mm} \mathrm{rms}$, where we assumed the worst case where each solenoid displacement excites twice larger betatron motion. The corresponding maximum of excited amplitude of $\sim 7.5 \mathrm{~mm}$ is small enough to enable sending the beam through an entire cryomodule without internal orbit correction and with small beam loss. This should enable beam observation at all cryomodule BPMs. Consequently, it greatly simplifies further orbit corrections and minimizes the beam hits of the SC cavities in the course of tuning. The alignment of cryomodules relative to other elements of the accelerator is easier than the alignment of cryogenic elements inside cryomodules and therefore the alignment tolerance of cryomodules is twice tighter than the alignment of solenoids inside cryomodules, i.e. it has to be better than $0.25 \mathrm{~mm}$ rms at each end of a cryomodule. This alignment has to be supported on distances not less than on betatron period, i.e. $20 \mathrm{~m}$.

\footnotetext{
${ }^{10}$ Here in addition to two transverse coordinates the bunch arrival time represents the third coordinate.
} 
The beam kick produced by solenoid misalignment includes two contributions. The first one, discussed above, is due to the transverse displacement of the solenoid. That produces a beam deflection equal to $\delta x / F$, where $F$ is the solenoid focusing length, and $\delta x$ is the solenoid transverse displacement. The second contribution is due to the solenoid angular misalignment, $\delta \alpha$, which produces a transverse magnetic field on axis and results in beam deflection by an angle:

$$
\theta=2 \delta \alpha \sqrt{\frac{L_{s}}{F}},
$$

where $L_{s}$ is the solenoid effective length and we assume a solenoid with rectangular field distribution. Equaling this angle with the angle obtained by the beam due to the solenoid offset $\delta x / F$, and expressing $F$ through the length of focusing period, $L$, and the betatron phase advance per period we obtain the value of solenoid angular misalignment which produces the same beam deflection as a solenoid offset by $\delta x$ :

$$
\delta \alpha=\frac{\sin (\mu / 2)}{\sqrt{L L_{s}}} \delta x .
$$

Here the same as above we assume that one period includes one lens and a drift.

Table 3.27 shows the alignment requirements for solenoids presented in the Functional Requirement Specifications (FRS) [118 - 120] for the HWR, SSR1 and SSR2 cryomodules and, for comparison, the solenoid angular misalignments which produce the same beam deflections as solenoid offsets. The solenoid angular misalignments were computed with Eq. (3.6) for $\mu=90 \mathrm{deg}$. where the solenoid length was calculated from computed or, if available, measured field distribution on the axis with following equation: $L_{s}=\int B(s) d s / B_{0}$. Here $B_{0}$ is the field in the solenoid center. As one can see the angular alignment for the solenoids is significantly tighter than the coordinate alignment.

Table 3.27: Requirements on the angular and coordinate alignment of SC solenoids

\begin{tabular}{l|c|c|c}
\hline \hline & HWR & SSR1 & SSR2 \\
\hline Solenoid coordinate alignment, rms, $\mathrm{mm}^{*}$ & 0.5 & 0.5 & 0.5 \\
\hline Solenoid angular alignment, rms, $\mathrm{mrad}^{*}$ & 1 & 0.5 & 0.5 \\
\hline $\begin{array}{l}\text { Angular misalignment computed for the above solenoid } \\
\text { coordinate misalignment with Eq. }(\mathrm{xx}), \mathrm{rms}, \mathrm{mrad}\end{array}$ & 1.2 & 0.94 & 0.58 \\
\hline Effective length of solenoid, $L_{s}, \mathrm{~cm}$ & 12.58 & 11.19 & 16.3 \\
\hline \hline
\end{tabular}

${ }^{*}$ Requirements in the Functional Requirement Specifications.

Alignment of cavities and solenoids will be accomplished using wire targets installed on the internal assemblies which are then translated to the fiducials installed on the vacuum vessel. Changes in alignment due to shipping, handling and during cooldown and operation will be monitored using a series of the wire targets attached to each cavity and solenoid, and viewed through optical windows installed on the upstream and downstream ends of the cryomodule. Two view lines located on the left and right sides enable measurement of both absolute locations and angular misalignments. Note that the alignment accuracy presented in Table 3.27 has to be achieved after cooldown. An additional element alignment after cryomodule cooldown is not anticipated. Therefore, the thermal contraction due to cooldown has to be accounted in the initial alignment of the cryomodule. 


\section{LB650 and HB650 Cryomodules}

The focusing in the LB650 and HB650 sections is obtained by quadrupole doublets located between cryomodules. The two coordinate dipole correctors and three-coordinate BPMs are located in between quads of each doublet. As in the previous sections the betatron phase advance per period is about $90 \mathrm{deg}$. at the beginning of each section, and for each section the betatron phase advance decreases gradually with energy increase. Apertures in LB650 and HB650 cavities are significantly larger than apertures of the beam lines connecting cryomodules, and, formally, they do not require the same alignment accuracy as elements of HWR, SSR1 and SSR2 cryomodules. However, an excitation of axially asymmetric high order modes is increased with beam offset from the cavity axis. Therefore, the alignment of LB650 and HB650 cavities relative to the cryomodule is specified to be the same as for HWR, SSR and SSR2 cryomodules, i.e. $0.5 \mathrm{~mm}$ rms for the center of gravity offset and $1 \mathrm{mrad}$ for the angular misalignment. Note that this requirement also includes possible deformation of SC cavities.

Quadrupoles of the quadrupole doublets which perform focusing between $650 \mathrm{MHz}$ cryomodules are placed on the girders installed between cryomodules. The alignment of quadrupoles relative to their girders has to be better than $0.15 \mathrm{~mm}$ rms [121]. The alignment of girders is the same as for the SC solenoids: $0.5 \mathrm{~mm}$ rms on the distances not smaller than one betatron period or $80 \mathrm{~m}$. Alignment of the cryomodules is the same as for the quadrupole girders, i.e. $0.5 \mathrm{~mm} \mathrm{rms}$.

\section{Beam optics measurements}

The optics measurements will be performed similarly to the optics measurements currently used in the transfer lines of the Fermilab accelerator complex, where transverse beam motion is excited by dipole correctors (one at time), and the dispersion is measured with energy change of the upstream ring. In the case of the PIP-II linac the beam energy change will be achieved by RF phase change in a cavity. At minimum the measurements require an excitation of 2 horizontal and 2 vertical correctors, and 2 RF cavities. In each plane the actuators should be separated by about $90 \mathrm{deg}$. in the betatron/synchrotron phase. Only one actuator is used for one differential orbit. To increase an accuracy of the optics measurements a larger number of dipole correctors and RF cavities will be used.

Strengths of dipole correctors are determined by the requirements of optics measurements. Each corrector must be able to excite at least $3 \mathrm{~mm}$ betatron oscillations in the downstream linac. This requirement results in the corrector strengths presented in Table 3.28.

Table 3.28: Requirements for the corrector strengths of PIP-II linac

\begin{tabular}{l|c}
\hline \hline MEBT & $1.6 \mathrm{mT} \cdot \mathrm{m}$ \\
\hline HWR & $2.5 \mathrm{mT} \cdot \mathrm{m}$ \\
\hline SSR1 & $2.5 \mathrm{mT} \cdot \mathrm{m}$ \\
\hline SSR2 & $5.0 \mathrm{mT} \cdot \mathrm{m}$ \\
\hline Correctors between $650 \mathrm{MHz}$ cryomodules & $10 \mathrm{mT} \cdot \mathrm{m}$ \\
\hline Correctors of the Linac-to-Booster transfer line & $10 \mathrm{mT} \cdot \mathrm{m}$ \\
\hline \hline
\end{tabular}

\subsubsection{PIP-II Magnets and their Powering}

Table 3.29 presents the list of all magnets and kickers of PIP-II project. It includes all elements which affect the beam focusing and deflection. 
Focusing in the LEBT is performed by three normal conducting solenoids. The switching dipole allows one to switch between two ion sources to avoid loss of operating time required for servicing and repairs of the ion sources. The LEBT beam chopper allows to form a pulsed beam for operation in the pulsed regime. Four two-plane $(x \& y)$ correctors control the beam orbit through the LEBT. Three of them are built-in into solenoids, and one is located at the ion source exit.

Quadrupole doublets and triplets are the beam focusing elements in the MEBT. Altogether there are 12 of them. The first two are represented by quadrupole doublets, and the other ten by quadrupole triplets. Each focusing element has a two-plane dipole corrector located in close vicinity. Two fast kickers are used in the MEBT bunch-by-bunch chopping.

The focusing in the HWR, SSR1 and SSR2 cryomodules is performed by SC solenoids. Each solenoid has a two-plane dipole corrector located inside the solenoid and consisting of four SC coils. In the HWR cryomodule each two coils are connected serially to form two dipole correctors - one horizontal and one vertical. In the SSR1 and SSR2 cryomodules each coil has a separate power supply which, in addition to the dipole fields, enable to create a correcting skew-quadrupole field.

Focusing in the part of the linac downstream of SSR2 cryomodules is performed by quadrupole doublets. Each doublet has a two-plane dipole corrector located between quadrupoles. Four skewquadrupoles are located at the linac end (in the slots reserved for 4 additional cryomodules). They correct $x-y$ coupling accumulated in the linac.

Focusing in the SC linac-to-Booster transfer line is FODO. The line includes two arcs and the straight line connecting them. The straight line has the fast dipole corrector which can move beam between 3 apertures of the septum. That enables fast beam switching between the beam dump, Booster and, in the future, the Mu2e experiment. The beam transport to the $\mathrm{Mu} 2 \mathrm{e}$ experiment is not considered being a part of the PIP-II project. Its concise description is presented in Appendix A. From the powering point of view, the line is split into two sections. The first section delivers the beam to the septum; and the second one, which starts after the septum, delivers the beam to the Booster. The first section also includes a short line which transports the beam from the septum to the beam dump. All dipoles of each section are connected serially and powered from one power supply. The quadrupoles for each section are split into two families: F and D-families. Quadrupoles of each family are connected serially and are powered by one power supply. Each focusing quad has a nearby horizontal corrector, and each defocusing quad has a nearby vertical corrector. There are two large aperture quadrupoles installed upstream of the beam-switch septum. Optics correction on the transport line is performed by eight current shunts capable to reduce current in eight quadrupoles. Initially the shunts will be connected to 4 consecutive quadrupoles located in each arc center. The final connection will be determined by the results of optics measurements. The last 5 dipoles of the Booster transfer line are assigned to the beam injection to the Booster. They deflect the beam vertically and expected to be powered from three power supplies. Similarly, the last six quadrupoles of the line are assigned for optics functions matching. Each quadrupole is powered separately. 
Table 3.29: List of PIP-II magnets and kickers

\begin{tabular}{|c|c|c|c|}
\hline Style & Quantity & $\begin{array}{c}\text { Operating } \\
\text { temperature }\end{array}$ & Location (quantity), comments \\
\hline LEBT switching dipole magnet & 1 & warm & LEBT \\
\hline LEBT solenoids & 3 & warm & LEBT \\
\hline LEBT 2-plane dipole corrector & 4 & warm & LEBT \\
\hline LEBT chopper & 1 & warm & LEBT \\
\hline MEBT long quads & 14 & warm & MEBT \\
\hline MEBT short quads & 20 & warm & MEBT \\
\hline MEBT 2-plane dipole correctors & 12 & warm & MEBT \\
\hline MEBT chopper kicker & 2 & warm & MEBT \\
\hline HWR solenoids & 8 & cold & HWR \\
\hline HWR 2-plane dip. correctors & 8 & cold & HWR \\
\hline SSR1 solenoids & 8 & cold & SSR1 \\
\hline SSR1 2-plane dip. correctors & 8 & cold & SSR2 \\
\hline SSR2 solenoids & 21 & cold & SSR2 \\
\hline SSR2 2-plane dip. correctors & 21 & cold & SSR2 \\
\hline SC linac quads & 40 & warm & $\begin{array}{l}\text { LB650 }(2 \cdot 11), \quad H B 650(2 \cdot 4), \text { linac } \\
\text { extension }(2 \cdot 5)\end{array}$ \\
\hline SC linac 2-plane dip. correctors & 20 & warm & $\begin{array}{l}\text { LB650 (11), HB650(4), linac } \\
\text { extension }(5)\end{array}$ \\
\hline SC linac skew-quads & 4 & warm & linac extension \\
\hline Transport line regular quads & 49 & warm & $\begin{array}{l}\text { Arc } 1 \text { and straight (18), beam dump } \\
\text { line (4), Arc } 2(27)\end{array}$ \\
\hline Transport line large bore quads & 2 & warm & Upstream septum \\
\hline End of Booster line quads & 6 & warm & \\
\hline $\begin{array}{l}\text { Transport line one-plane dip. } \\
\text { correctors }\end{array}$ & 56 & warm & $\begin{array}{l}\text { Arc } 1 \text { and straight (18), beam dump } \\
\text { line (4), Arc } 2(33)\end{array}$ \\
\hline Transport line dipoles & 37 & warm & $\operatorname{Arc} 1(8), \operatorname{Arc} 2(24)$, beam dump (5) \\
\hline $\begin{array}{l}\text { End of transport line long } \\
\text { dipoles }\end{array}$ & 3 & warm & bend in vertical plane \\
\hline $\begin{array}{l}\text { End of transport line short } \\
\text { dipoles }\end{array}$ & 2 & warm & bend in vertical plane \\
\hline Fast dipole switch & 1 & warm & \\
\hline Septum magnet & 1 & warm & \\
\hline Beam dump sweep magnet & 1 & warm & \\
\hline
\end{tabular}




\subsection{Booster}

\subsubsection{Radiation Shielding of the Booster Injection Absorber}

\subsubsection{Initial Conceptual Design}

The initial conceptual design for the new Booster injection insert, as described in the PIP-II RDR, utilizes an existing straight section (Long 11) with a flange-to-flange length of $5.68 \mathrm{~m}$ between gradient magnets and a vertical three bump chicane as shown in Figure 2.49. The injection stripping foil is located after the two central chicane dipoles. Here, the vertical chicane dipoles not only move the closed orbit onto the foil, but serve as vertical painting magnets as well. The bending angle of each of the chicane dipoles is approximately $40 \mathrm{mr}$. Horizontal painting magnets are located in the short straight sections on either side of the injection straight. A dipole located over the upstream gradient magnet serves to place the incoming $\mathrm{H}$ - ions onto the proper injection orbit at the foil. As the injected H- pass through the two central bump magnets, this dipole or a corrector in the transport line will needed to ramped to compensate the change in the central dipole field due to painting. As can be seen in Figure 2.49, the injection waste beam absorber can only fit between the last chicane dipole (PM3) and the gradient magnet.
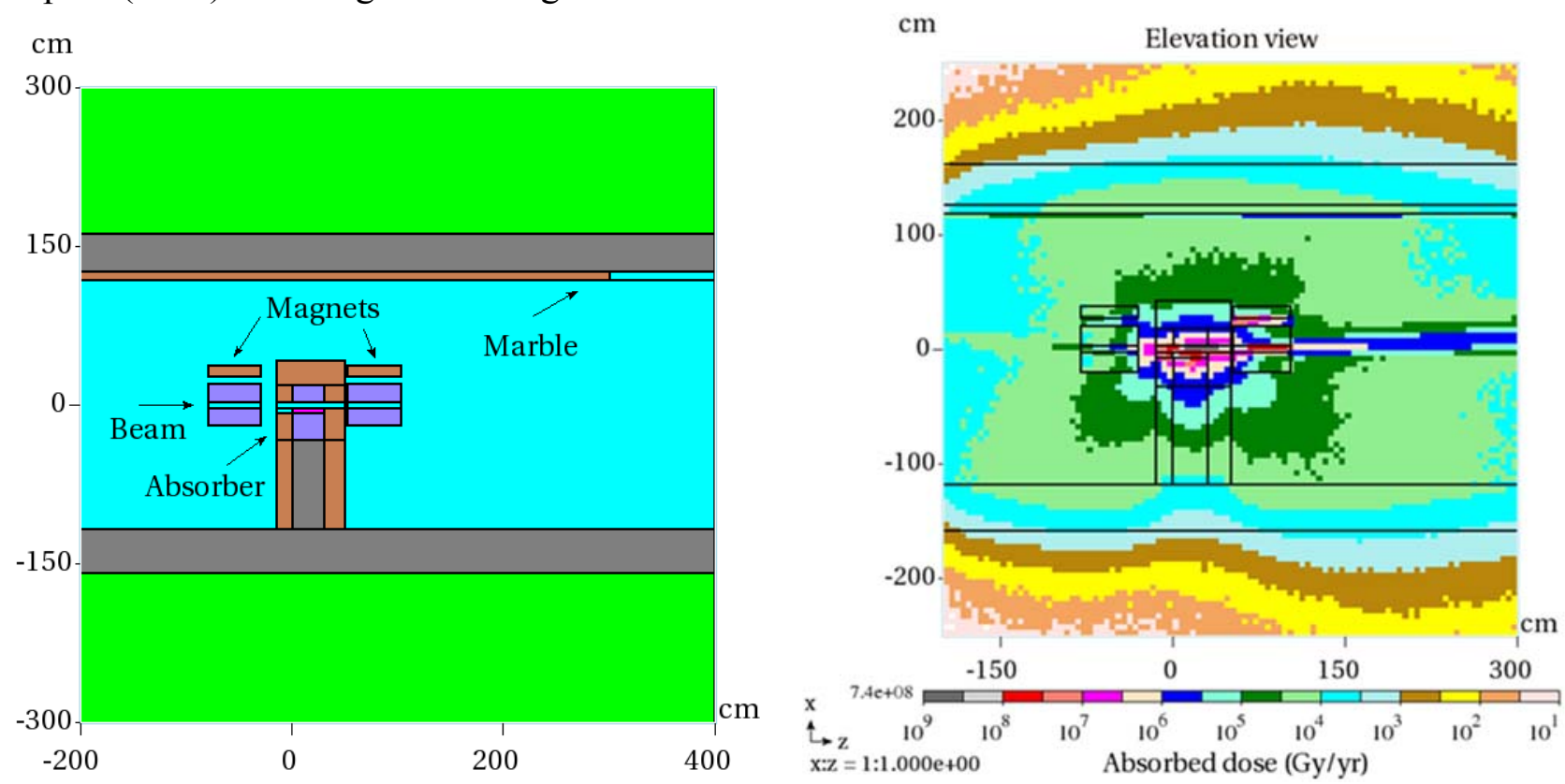

Figure 3.88: Elevation view of a MARS15 model (left) and absorbed dose (right).

A preliminary MARS [122-124] model of this injection layout was developed assuming $400 \mathrm{~W}$ of waste beam power made up of $2 \% \mathrm{H}^{-}$missing the foil and $0.2 \%$ converted to $\mathrm{H}^{0}$. The absorber is made of a small $5 \mathrm{~cm} \times 5 \mathrm{~cm}$ x $30 \mathrm{~cm}$ tungsten core surrounded by $15 \mathrm{~cm}$ steel on a concrete pedestal surrounded by marble shielding. The up and downstream magnets also have a $10 \mathrm{~cm}$ marble shield on the top and aisle side. An estimate for tritium production in the soil around the injection straight indicated that additional shielding is not required. Estimates of the residual activation of components in the area and absorbed dose in the magnets surrounding the absorber were performed. Figure 3.88 shows a side view of the model for the absorber area inside the Booster tunnel and the absorbed dose in the adjacent magnets. The residual activation on the downstream gradient magnet upstream flange is on the order of $100 \mathrm{mSv} / \mathrm{hr}$ and the absorbed dose in the upstream end of the gradient magnet is on the order of $4 \mathrm{MGy} / \mathrm{yr}$. With typical survival dose for kapton and insulation of 20-30 MGy, this 
leads to a requirement for magnet replacement every 5-7 years. This is problematic due to ALARA issues for magnet replacement. It also results in additional costs and downtime.

\subsubsection{Alternative Configuration}

The MARS model indicates that the desire to utilize the existing length of the long straight section is problematic in terms of proximity of the absorber to the gradient magnet, absorbed dose in adjacent magnets, and residual activation of components.

An alternate configuration, which reduces the length of the gradient magnets by $\sim 30 \%$ to increase the straight section length by about 0.86 meter, is considered. This configuration utilizes a vertical four bump chicane with the foil between the two central chicane dipoles allowing for the waste beam to impact the absorber farther from the aperture of the gradient magnet and additional room for shielding of the absorber as shown in Figure 3.89. With the foil located at the peak of the chicane bump, the strength requirements on the chicane dipoles are relaxed. Additionally, there is room for separate painting magnets (for both planes).

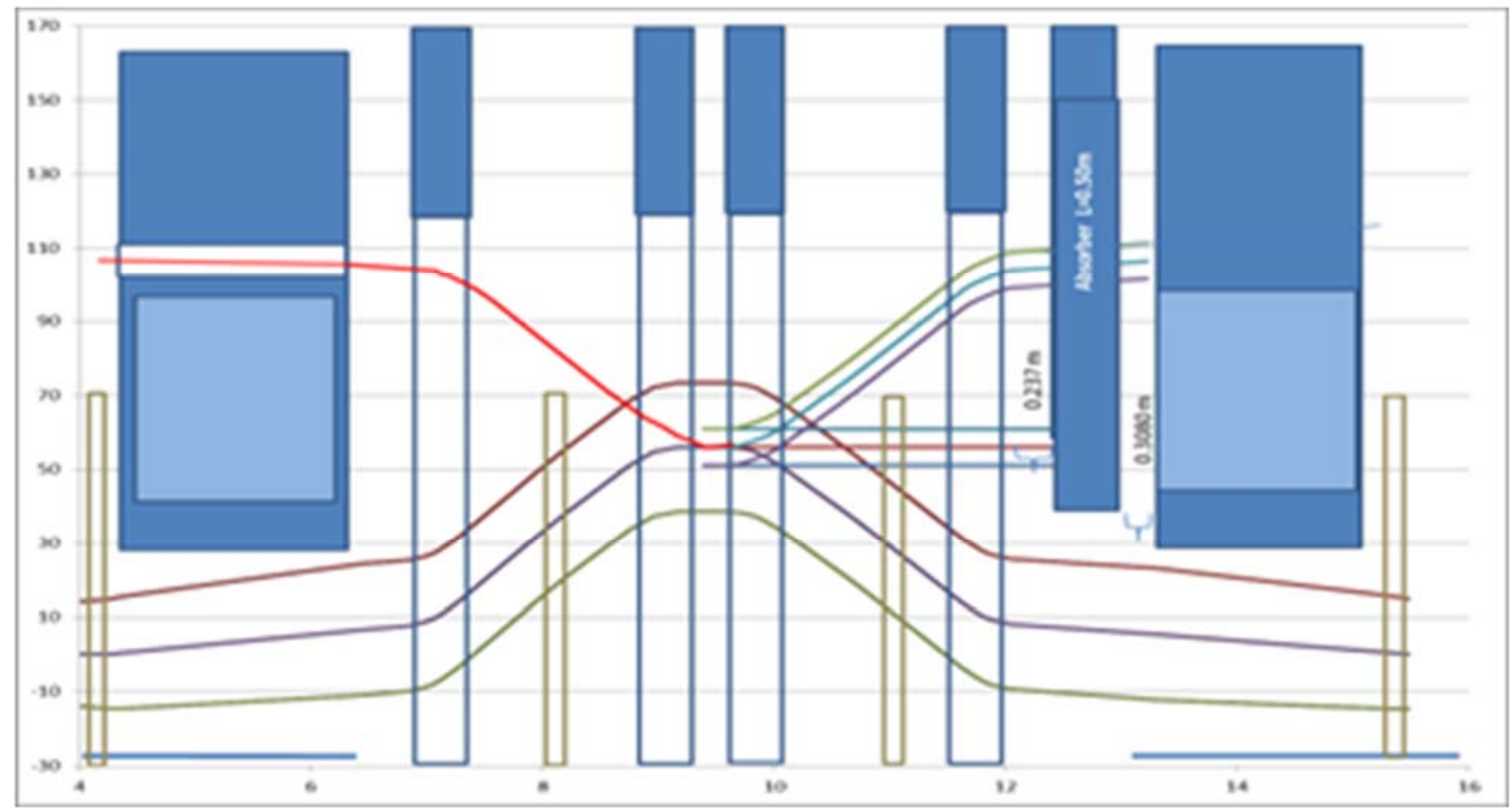

Figure 3.89. Alternative configuration for injection insert using a vertical 4-bump and separate painting magnets; magnet apertures are represented by open boxes.

A preliminary MARS model for this alternative injection layout was developed. It includes the copper coils of the gradient magnet. Figures 3.90 and 3.91 show a fragment of the corresponding MARS15 model and the absorbed dose, respectively. The model includes a $30 \mathrm{~cm}$ tungsten absorber (magenta) surrounded by $10 \mathrm{~cm}$ steel (gray), the upstream chicane dipole ferrite yoke (red), the downstream gradient magnet yoke (tan) and copper coils (dark green). A $10 \mathrm{~cm}$ piece of marble (brown) is upstream of the gradient magnet. The same beam conditions were used as before to look at the absorbed dose and residual activation. The absorbed dose in the coils of the gradient magnet was reduced substantially, to $0.12 \mathrm{MGy} / \mathrm{yr}$. This practically eliminates the issue with magnet replacement due to degradation of kapton and other insulation for a reasonable lifetime of the facility. The residual dose is in the range of $10-100 \mathrm{mSv} / \mathrm{hr}$, still a bit high. Additional optimization is required. 


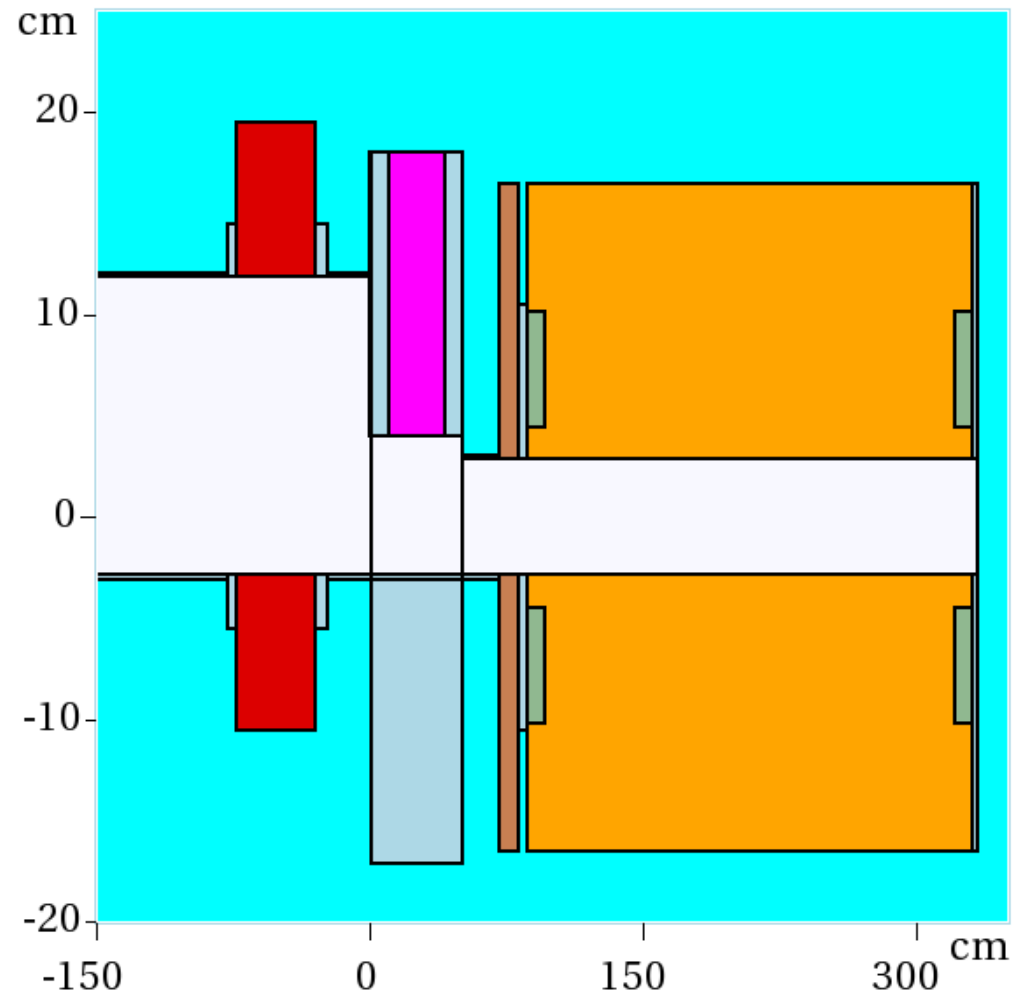

Figure 3.90. A fragment of the MARS15 model for the alternative configuration.

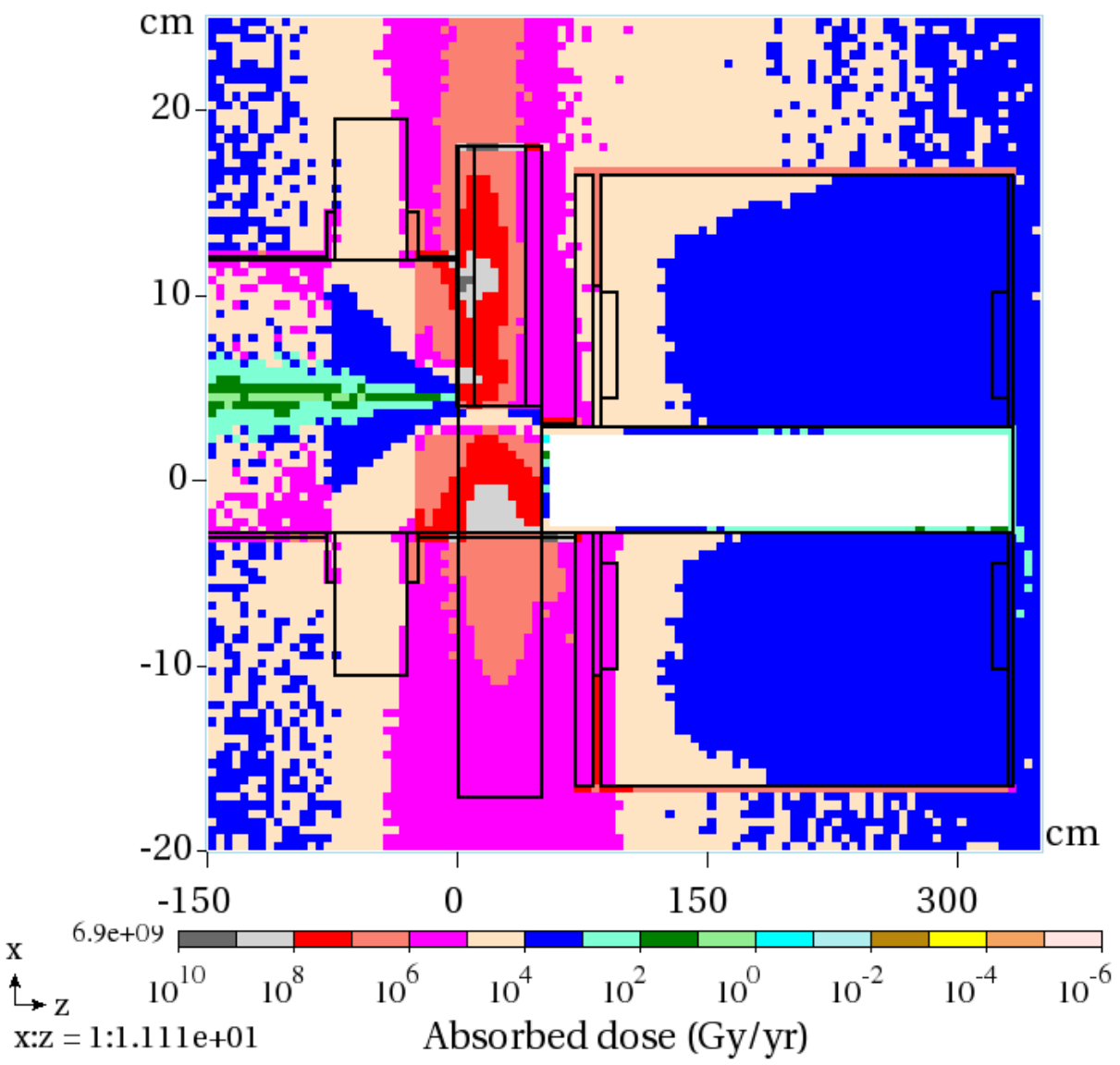

Figure 3.91. Absorbed dose distribution for the region shown in the previous Figure. 


\subsection{Main Injector and Recycler}

\subsubsection{Hardware for Main Injector Transition Crossing}

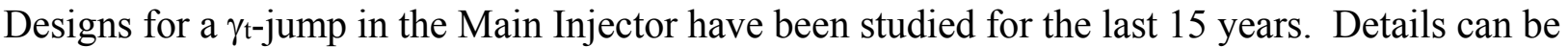
found in the Proton Driver Design Report [125]; a brief summary is included here. The system consists of 8 sets of pulsed quadrupole triplets. Each triplet has two quads in the arc and one of twice the integrated strength in the straight section, with a phase advance of $\pi$ between each quadrupole. The perturbation to the original lattice is localized. In particular, the dispersion increase during the jump is small $\left(\Delta D_{\max } \approx \pm 1 \mathrm{~m}\right)$, which is the main advantage of a first-order jump system. Each triplet is optically independent from the others and provides roughly $1 / 8$ of the total required jump amplitude (i.e., $\Delta \gamma_{t} \approx \pm 0.25$ per triplet). The power supply uses a GTO (gate turn-off thyristor) as the fast switch and a resonant circuit with a $1 \mathrm{kHz}$ resonant frequency. The beam pipe is elliptical and made of Inconel 718. It has low electrical conductivity $\sigma$ and high mechanical strength so eddy current effects are relatively small. The eddy current effects scale as $\sigma d$, where $d$ is the pipe wall thickness. The $\sigma d$ value of Inconel 718 is about four times lower than that of stainless steel.

The 8 pulsed triplet locations are summarized in Table 3.30. Since the original study was performed, there have been changes to the Main Injector and these locations need to be reevaluated. A set of magnet design parameters has been developed and modeled (see Table 3.31).

Table 3.30: Candidate $\gamma_{\mathrm{t}}$ quad triplet locations

\begin{tabular}{l|l}
\hline \hline Pulsed Triplet & Quad Locations \\
\hline 1 & $104,108,112$ \\
\hline 2 & $226,230,302$ \\
\hline 3 & $322,326,330$ \\
\hline 4 & $334,338,400$ \\
\hline 5 & $404,408,412$ \\
\hline 6 & $526,530,602$ \\
\hline 7 & $622,626,630$ \\
\hline \hline
\end{tabular}

Table 3.31: Pulsed quadrupole magnet parameters

\begin{tabular}{l|l}
\hline \hline & Requirement \\
\hline Integrated Gradient & $0.85 \mathrm{~T}$ \\
\hline Vacuum pipe cross section (elliptical) & $2.4 \times 1.125 \%$ \\
\hline Field Quality, 1" radius & $2 \%$ \\
\hline Maximum length & $17 \%$ \\
\hline Maximum Current & $200 \mathrm{~A}$ \\
\hline Maximum Voltage & As low as possible \\
\hline \hline
\end{tabular}




\subsubsection{RF System Modifications}

There are two upgrade options for higher power in the MI RF cavities.

In the first option the MI cavities are operated with two power tubes instead of one in a push-pull configuration. There is already a provision for adding a second power amplifier (PA) in the existing MI RF cavities but this option has never been tested. Running with two PAs per cavity will require to double the number of modulators and the solid state amplifiers.

As a second option we can use a new power amplifier (Eimac 4CW250000B). This is a much longer tube than the current one, consequently, size constraints need to be evaluated. In this option all new modulators will be required.

For the new Recycler $53 \mathrm{MHz}$ cavities we plan to use the cavity design that has been developed in collaboration with SLAC. This design uses perpendicular biased tuners and has $\mathrm{R} / \mathrm{Q} \approx 50 \Omega$. Figure 3.92 shows a view of the present RF cavity and a sketch of the new cavity. The cavity drawing is shown in Figure 3.93.
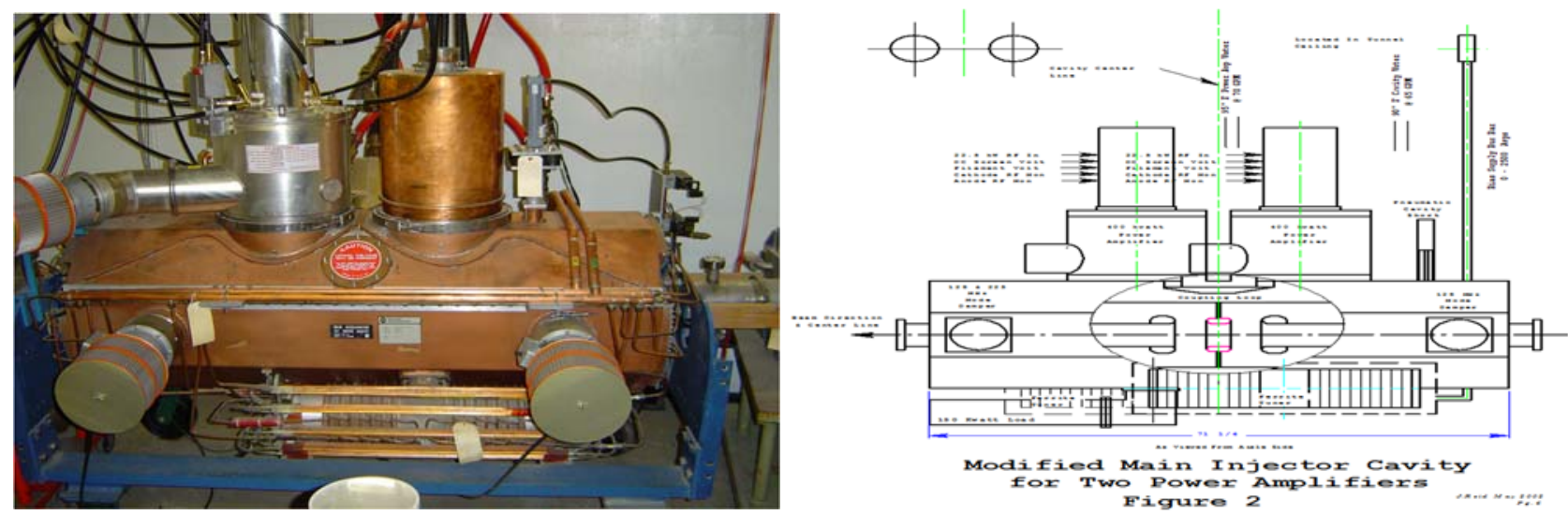

Figure 3.92: Current (left) and Modified(right) MI RF cavity.

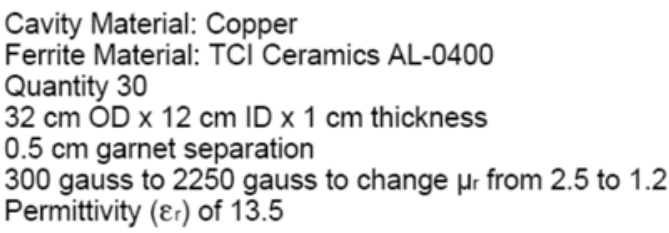

Cavity Material: Copper

Ferrite Material: TCl Ceramics AL-0400 Quantity 30

$32 \mathrm{~cm}$ OD $\times 12 \mathrm{~cm} I D \times 1 \mathrm{~cm}$ thickness

$0.5 \mathrm{~cm}$ garnet separation

300 gauss to 2250 gauss to change $\mu_{\mathrm{r}}$ from 2.5 to 1.2

Permittivity $\left(\varepsilon_{\mathrm{r}}\right)$ of 13.5

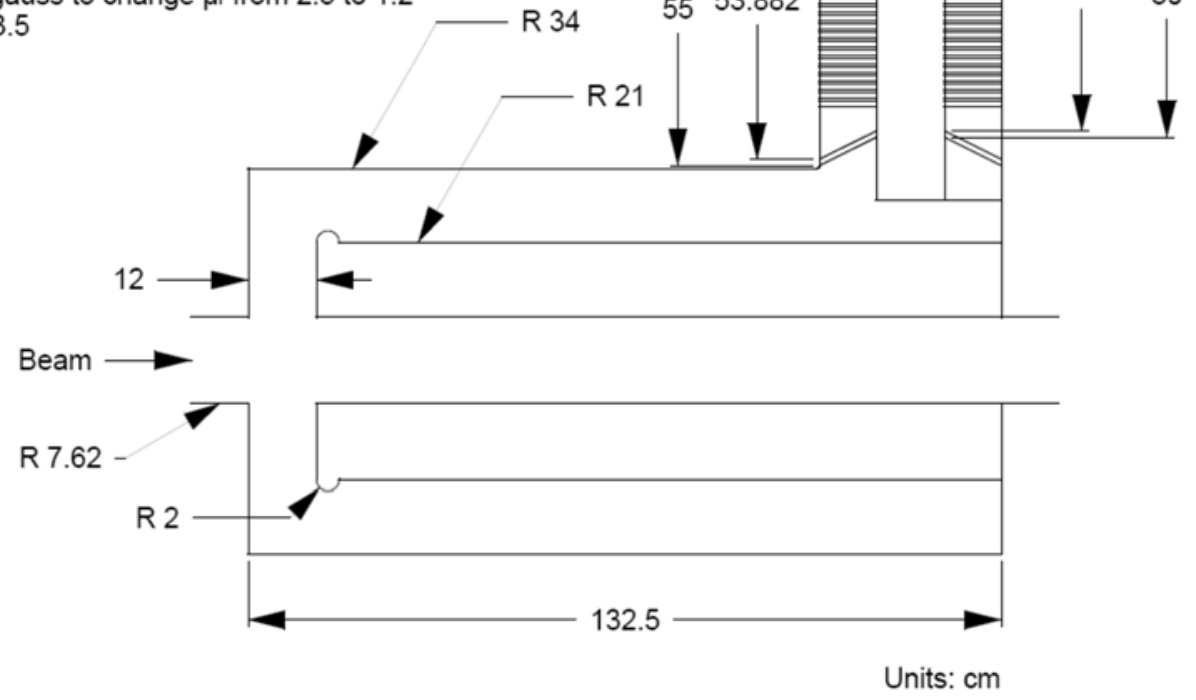

Figure 3.93: Mechanical dimensions of the $53 \mathrm{MHz}$ cavity. 


\subsection{Cryogenics}

\subsubsection{Cryogenic System Configuration}

The Linac cryogenic system (see Figure 3.94) consists of three major subsystems: the Superfluid Helium Cryogenic Plant (SHCP) that produces the refrigeration, the Cryogenic Distribution System (CDS) that delivers the refrigeration from the SHCP to the SRF Linac, and the associated auxiliary systems. The cryogenic system is expected to operate for 20 years, with an estimated continuous operation of two to five years without a scheduled shutdown. The expected availability of the SHCP is $98 \%$, which would define the availability of the entire system. The cryogenic system as a whole is required to perform the following functions:

- Provide sufficient cooling at appropriate temperature levels to enable operation of the SRF cavities and other cryogenic components within their respective operational conditions.

- Ensure that the system shall support controlled cool-down and warm-up of cryomodules.

- Ensure that the system and its components comply with the Fermilab ES\&H manual.

- Provide for proper protection of process fluids from contamination.

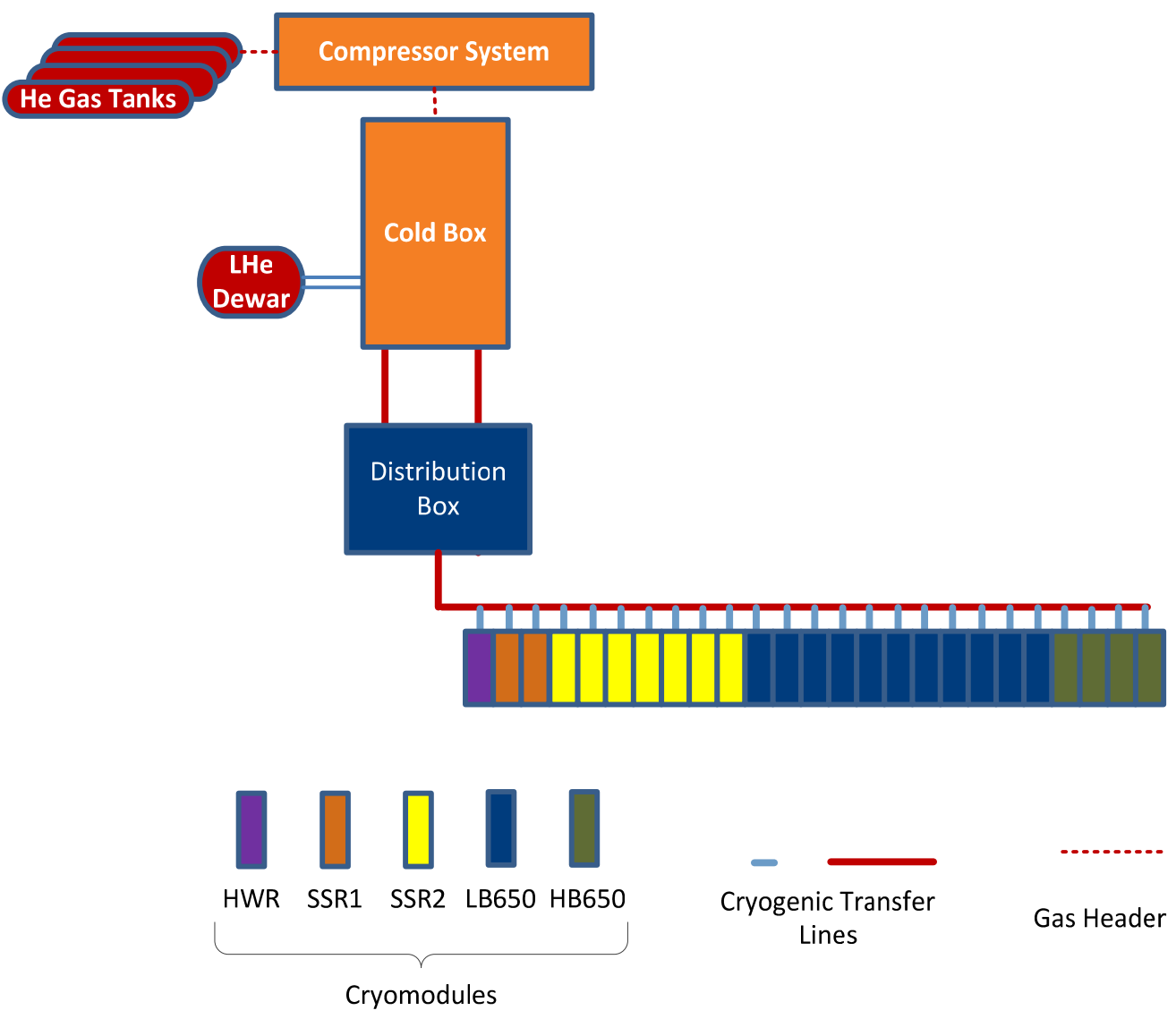

Figure 3.94: Layout of PIP-II cryogenic system.

The cryogenic system will be designed to operate as efficiently as is practical over a wide range of operating requirements [126]. Efficiency is important for the operating modes that are expected to last for extended periods of time, such as normal Linac operation at $2 \mathrm{~K}, 2 \mathrm{~K}$ standby (RF off) and 
4.5 K standby. The refrigeration load requirements for the PIP-II cryogenic system are provided in Table 3.32. Operating procedures for the cryogenic system include:

1. Controlled linac cool down and warm up.

The cryogenic system must be able to reliably cool down and warm up the cryomodule string within the cool down rate and temperature difference constraints imposed by the cryomodule design.

2. Linac liquid helium fill

This represents the $4.5 \mathrm{~K}$ liquefaction capacity of the cryogenic system and determines the time required to fill the cryomodule string with $4.5 \mathrm{~K}$ liquid helium.

3. $4.5 \mathrm{~K}$ standby

During extended shutdown periods, it is desirable to keep the cryomodule string cold and all circuits at positive pressure, thus minimizing the operating cost as well as the risks of contaminating the cold circuits.

4. $2 \mathrm{~K}$ standby

During shorter shutdown periods, it is desirable to keep the cryomodule string at $2 \mathrm{~K}$.

With the RF off, the heat load to the cryogenic system at $2 \mathrm{~K}$ will be about $50 \%$ of the nominal load in the pulsed regime of linac operation.

5. $2 \mathrm{~K}$ operation in the pulsed mode

This represents the normal operation of the Linac in the pulsed mode at the estimated heat loads.

6. $2 \mathrm{~K}$ operation in the $\mathrm{CW}$ mode

This represents the normal CW operation of the Linac at the estimated heat loads.

Table 3.32: Refrigeration load requirement for the PIP-II cryogenic system

\begin{tabular}{l|c|c}
\hline Circuit & $\begin{array}{c}\text { Pulsed operation } \\
{[\mathrm{W}]^{*}}\end{array}$ & $\begin{array}{c}\text { CW operation } \\
{[\mathrm{W}]^{*}}\end{array}$ \\
\hline $2 \mathrm{~K}$ & $\geq 340$ & $\geq 1,700$ \\
\hline Low temperature thermal shield (LTTS), $4.5-9 \mathrm{~K}$ & $\geq 880$ & $\geq 880$ \\
\hline High temperature thermal shield (HTTS), $35-80 \mathrm{~K}$ & $\geq 2,700$ & $\geq 2,700$ \\
\hline \hline
\end{tabular}

${ }^{*}$ The load does not account loss in the cryogenic distribution system and technical margin

\subsubsection{Superfluid Helium Cryogenic Plant (SHCP)}

The SHCP will utilize a mixed compression cycle, as opposed to the all cold compression cycle. The mixed cycle has been successfully used in many superfluid helium cryogenic plants including LHC (CERN) and CMTF (Fermilab). The cycle was also recently chosen by XFEL (DESY) and European Spallation Source (ESS) after independent review of an industrial studies conducted by the helium cryogenic plant manufacturers. A simplified cycle diagram is presented in Figure 3.95. The cycle utilizes a combination of three stages of cold compressors and a sub atmospheric warm compressor operating in series. The major advantage of this cycle is the wide range of efficient capacity modulation.

The PIP-II SHCP design will incorporate the components needed to accommodate all required operating modes of the PIP-II Linac as well as the devices necessary for its function verification. The cryogenic load requirements for the SHCP operation in the CW mode are presented in Table 3.33. 
The "Maximum cooling power" accounts for all the dynamic and static heat loads in cryomodules, loss in the cryogenic distribution system and the required safety factors in the heat loads. The SHCP shall be designed for stable operation for any external load between zero and the maximum values specified. The SHCP shall have a provision to simulate the heat loads for performance tests. The detailed specifications for the SHCP are developed jointly by Fermilab, USA and DAE, India as a part of the ongoing IIFC collaborative efforts. The SHCP shall be procured from industry by DAE and supplied as an in-kind contribution to Fermilab. The major components of the SHCP are the helium warm compression system (WCS) and the cold box (CB), both of which, along with SHCP process control system and instrumentation, are part of the procurement.

Table 3.33: Cryogenic load requirements for the SHCP

\begin{tabular}{l|c|c|c}
\hline \hline & $\begin{array}{c}\text { High temperature thermal } \\
\text { shield (HTTS) } \\
35-80 \mathrm{~K}\end{array}$ & $\begin{array}{c}\text { Low temperature } \\
\text { thermal shield (LTTS) } \\
4.5-9 \mathrm{~K}\end{array}$ & $2 \mathrm{~K}$ \\
\hline Maximum cooling power[W] & $\geq 9,100$ & $\geq 1,500$ & $\geq 2,000$ \\
\hline \hline
\end{tabular}

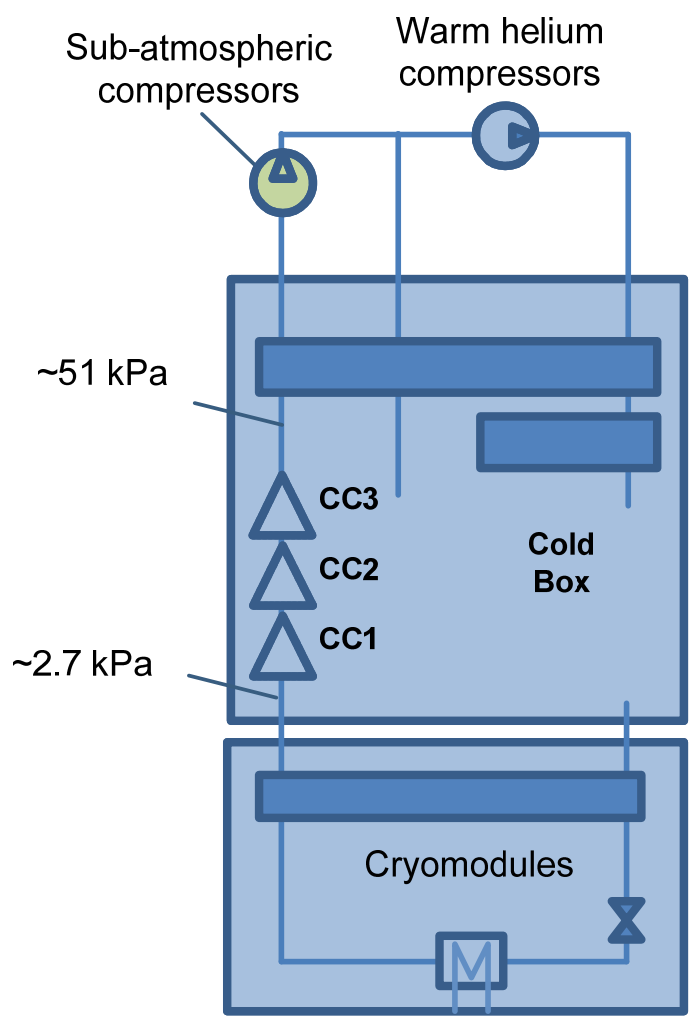

Figure 3.95: Simplified Schematic of a Mixed Compression Cycle.

The WCS consists of the equipment needed to compress the required mass flow of pure helium gas for both atmospheric and sub-atmospheric pressure circuits. The WCS cools gas after compression to ambient temperature and restores its original purity. The performance of the WCS must satisfy the cryogenic load requirements (Table 3.33). The major components of WCS are as follows: 
- Compressor skids;

- Bulk oil removal, cooling and circulation equipment;

- Final oil removal system;

- Charcoal adsorbers;

- Dryer;

- Oil and Gas Filters;

- Gas management panel;

- Helium guard for sub-atmospheric circuits;

- Pump and purge system;

- Gas analysis equipment;

- Safety devices;

- Electrical systems and controls;

- Valves;

- Interfaces for connecting to the $\mathrm{CB}$ and helium buffer vessels.

The CB of the SHCP is a vacuum vessel with clean inner surfaces, covered by multi-layer insulation and housing all the equipment and piping used in the refrigeration process. The CB package also includes instrument panel with transmitters, switches, process and utility instrumentation, instrument air and cooling water connections and distribution, purge connection, electrical cabinet for terminals, transmitters and other hardware. The major components of the CB are as follows:

- Aluminum plate-fin process heat exchangers;

- Cryogenic turbo-expanders (TEX);

- Cryogenic cold compressors (CC);

- Valves at cryogenic temperature with manual or pneumatic actuators;

- Valves at ambient temperature operating at sub-atmospheric pressure;

- Safety valves and other safety devices;

- Dual bed $80 \mathrm{~K}$ adsorbers and single bed full flow 20K adsorber;

- Filters;

- Phase separator and sub-cooler;

- Vacuum insulation system;

- Heaters;

- Temperature sensors and pressure sensing taps;

- Interfaces for connecting to the WCS, liquid helium Dewar and Cryogenic Distribution System (CDS).

SHCP control system will be equipped with all instrumentation necessary for safe and reliable operation. The instrumentation will allow for flow and pressure measurements, temperature, speed controls and impurity monitoring. The control system will consist of a main process control programmable logic controller (PLC), remote input/output modules at equipment positions and Human-Machine-Interface displays. It will include all software required for safe and reliable operation of the system.

Most of the auxiliary equipment, including warm helium storage tanks, liquid helium Dewars, etc., will be reused from the Tevatron. Chillers for turbines and cold compressors, insulation vacuum pumping system (roughing and diffusion pumps), and associated instrumentation will be procured from industry. 


\subsubsection{The Cryogenic Distribution System (CDS)}

The CDS consists of the equipment required to feed and return the cold helium via vacuum insulated pipelines to the SRF Linac components. The equipment includes distribution boxes (DB), cryogenic transfer lines, bayonets and turnaround boxes. The major features of the CDS are:

- A multi-circuit transfer line will run from the SHCP CB (Figure 3.96) into the PIP-II tunnel and along the length of the SRF Linac. The design of this transfer line will be consistent with CW operation.

- In steady state operation, the CB will supply supercritical helium at around $4.5 \mathrm{~K}$ and a maximum supply pressure of 4 bar to the CDS.

- The supercritical helium line is divided into two streams inside the cryomodules, one of which is directed to the sub-atmospheric heat exchanger and subsequent JT valve, while the other is directed to the Low Temperature Thermal Shield (LTTS). This LTTS return line enters the CDS at a temperature of about $9 \mathrm{~K}$ in normal operation.

- The sub-atmospheric return stream from the CDS to the CB has a temperature of about $3.8 \mathrm{~K}$ in normal operation.

- The CB will supply to the CDS the high-pressure helium gas at 35-40 K for the High Temperature Thermal Shield (HTTS). This shield flow is returned from the CDS to the CB at about $80 \mathrm{~K}$.

- The CDS will have a separate cooldown (return) line, which will return helium gas to the CB at different temperatures during the cooldown operation of the CDS and cryomodules.

- The CDS transfer line would make use of 25 inline bayonet cans (one for each cryomodule) and a turnaround box at the end. In parallel, in the vertical plane, there will be a warm helium vent header.

- Connections between the distribution boxes and the cold box are accomplished via removable vacuum insulated cryogenic transfer tubes ("U-tubes"), while the connection to the tunnel transfer line is welded. This architecture provides flexibility for positive isolation of tunnel components and strings of cryomodules from the SHCP during installation, commissioning, operation and maintenance, including repairs.

- Each cryomodule is connected to the transfer line via U-tubes. It provides for maximum segmentation of the Linac.

- The estimated pressure drop for the transfer line elements along the CDS and the cryomodules are presented in Table 3.34.

- The estimated heat load values along the CDS are presented in Table 3.35.

Table 3.34: Estimated pressure drop along the CDS and cryomodules

\begin{tabular}{l|c|c}
\hline \hline Circuit & Operating Pressure[MPa] & Estimated pressure drop[kPa] \\
\hline 2K return & $3.13 \mathrm{e}-3$ & 0.4 \\
\hline $4.5 \mathrm{~K}$ supply & $0.22 \leq \mathrm{P} \leq 0.4$ & 25 \\
\hline LTTS return & $0.22 \leq \mathrm{P} \leq 0.4$ & 3 \\
\hline HTTS supply & $0.3 \leq \mathrm{P} \leq 1.8$ & 5 \\
\hline HTTS return & $0.3 \leq \mathrm{P} \leq 1.8$ & 7 \\
\hline \hline
\end{tabular}


Table 3.35: Estimated heat load along the CDS

\begin{tabular}{l|c}
\hline \hline Circuit & Estimated Heat Load [W] \\
\hline 2K return & 248.7 \\
\hline $4.5 \mathrm{~K}$ supply & 77 \\
\hline LTTS return & 60 \\
\hline HTTS supply & 90 \\
\hline HTTS return & 576 \\
\hline \hline
\end{tabular}

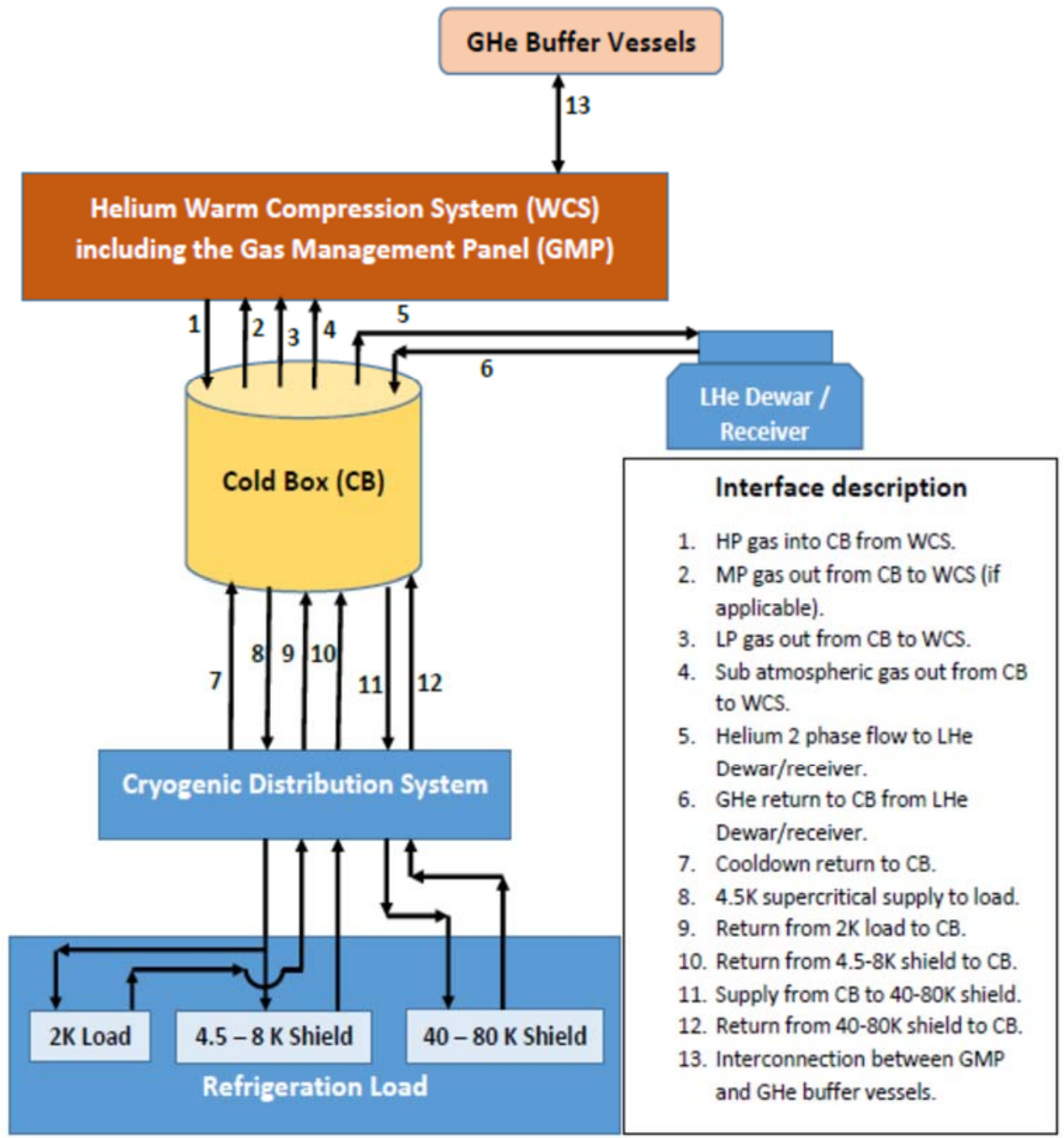

Figure 3.96: Simplified Schematic showing the SHCP, the CDS and the distribution of cryogenic refrigeration to load (cryomodules).

Table 3.36 presents the helium volumes for each cryomodule and the total volume of the liquid helium in all cryomodules. 
Table 3.36: Helium inventory requirements of PIP-II cryomodules and cryo-distribution system

\begin{tabular}{l|c|c}
\hline \hline & $\begin{array}{c}\text { Number of } \\
\text { cryomodules }\end{array}$ & $\begin{array}{c}\text { Required Helium } \\
\text { Inventory }\left[\mathrm{sm}^{3}\right]^{*}\end{array}$ \\
\hline HWR & 1 & 390 \\
\hline SSR1 & 2 & 408 \\
\hline SSR2 & 7 & 1998 \\
\hline LB650 & 11 & 2333 \\
\hline HB650 & 4 & 1696 \\
\hline CDS & - & 972 \\
\hline \multicolumn{1}{c}{ Total } & & $\mathbf{7 7 9 7}$ \\
\hline \hline
\end{tabular}

* $\mathrm{sm}^{3}$ - standard cubic meter

\subsubsection{Steady State Operation of the SHCP}

The different steady state operation modes of the proposed SHCP are listed in Table 3.37. The major features of the steady state operation are:

- Ability to cope with the cooling loads for each of the steady state operation modes listed in Table 3.37.

Table 3.37: Steady State Modes of operation of the SHCP

\begin{tabular}{l|c|c|c|c}
\hline \hline Circuit & $\begin{array}{c}\text { LHe } \\
{[\mathrm{g} / \mathrm{s}]}\end{array}$ & $\begin{array}{c}4.5 \mathrm{~K} \text { Standby } \\
{[\mathrm{W}]}\end{array}$ & $\begin{array}{c}\text { CW operation } \\
{[\mathrm{W}]^{*}}\end{array}$ & $\begin{array}{c}\text { Maximum } \\
\text { Capacity [W] }\end{array}$ \\
\hline $2 \mathrm{~K}$ & N.A. & N.A. & $\geq 1,750$ & $\geq 1,900$ \\
\hline $4.5 \mathrm{~K}$ & TBD* $^{*}$ & - & - & - \\
\hline LTTS & - & $\geq 1,150$ & $\geq 1,150$ & $\geq 1,500$ \\
\hline HTTS & - & $\geq 7,000$ & $\geq 7,000$ & $\geq 9,100$ \\
\hline \hline
\end{tabular}

${ }^{*}$ To be determined, proposed and demonstrated by the vendor.

- In the " $4.5 \mathrm{~K}$ stand by" mode, the only heat load applicable is the static heat load (heat in leak) of cryogenic distribution system and testing components.

- In the "Maximum capacity" mode, along with the static heat load, the dynamic heat load due to magnet current and RF power being turned on, also comes into the picture. This mode represents the maximum SHCP refrigeration capacity at $2 \mathrm{~K}$ to deal with the maximum heat load considering all the safety factors.

- The "Pulsed operation" and "CW operation" modes represent the design condition of the SHCP with all the dynamic and static heat loads but without considering the safety factors and loss in the cryogenic distribution system.

- Apart from these refrigeration modes, there exists a provision of a helium liquefaction mode at $4.5 \mathrm{~K}$.

- Complete exclusion of liquid nitrogen pre-cooling in the SHCP design. 
- Capability of transition from one operation mode to another with minimal manual intervention.

- Automatic reaction to changes in heat load by reducing or increasing SHCP performance without active participation of an operator.

\subsubsection{Transient Operation of the SHCP}

The transient operation modes of the SHCP involves the cool down, warm up and responses to fault conditions and normal shut down. The major features of the cool down mode are:

- Capability of cooling down the SHCP CB either alone or together with the Linac cryomodules (CM).

- $\mathrm{CB}$ to operate in a mode of liquefaction at rising level once the Linac CM cold masses reach a temperature below $5 \mathrm{~K}$.

- Capability of supporting cool down of LTTS and/or HTTS circuits prior to, or simultaneously with, the cooling of the $2 \mathrm{~K}$ circuit.

- Pump down to achieve $2 \mathrm{~K}$ by switching on the $\mathrm{CC}$ train to start only when the $4.5 \mathrm{~K}$ circuit is in normal operation, the Linac CMs are filled with $4.5 \mathrm{~K}$ liquid helium (LHe) and when the cool down of the sub-atmospheric circuits including the header is completed.

- Capability to cope with different pump down volumes right from one CM to the entire Linac CM set.

- Capability to re-start pump down after a scheduled or un-scheduled stoppage of the CC train.

- Capability of pumping down a single CM in case of its replacement/maintenance.

- Capability of pumping down both with or without the sub-atmospheric header.

The major features of the warm up mode are:

- Capability of warming up the $\mathrm{CB}$ either alone or together with the Linac cryomodules (CM).

The major features of the SHCP responses to fault conditions are:

- Capability of withstanding heat load variation, without a trip or significant excursion of operating parameters.

- Provision of protection of plant components in the event of beam line vacuum loss and insulation vacuum loss in CMs.

- Support (without damage to plant components) of abnormal shutdown modes such as those due to component trip, failure of compressor system, utilities interruption and other emergency trips.

- Capability to cope with unexpected controls or communication interruption or failure without damage to plant components.

- Capability of plant restart without warm-up or purge of its circuits after incidents such as power glitch, cooling water outage, etc.

- Capability of TEX and CC restart without warm-up or purge of cold circuits.

- Capability of plant restart without warm-up or purge of its circuits after reset of an interlock that caused a component or element false trip.

The major features of the SHCP normal shut down mode are:

- Capability of plant shutdown via either local or remote command without damage to components.

- Normal shutdown mode sequence to bring about pressurizing of all circuits (including the normally sub-atmospheric circuits) to above atmospheric pressure to protect these from 
possible contamination.

\subsubsection{Interfaces of the SHCP}

The major interfaces of the SHCP includes that between the CB and WCS, CDS and LHe Dewar. The CB will be connected to the WCS by at maximum four main helium lines (HP delivery, LP return, sub-atmospheric return and MP return) without counting auxiliary lines for bearing gas, purging requirements, etc. The physical interface between the SHCP CB and the SRF Linac CDS will be one single transfer line port incorporating six lines (Figure 3.96, Table 3.38). The port would contain a vacuum barrier to separate the insulation vacuum of the transfer line from that of the CB.

The LHe Dewar, apart from providing a buffer supply of liquid helium during regular operation, will be used during the acceptance of the SHCP. The physical interface between the CB and the transfer lines leading up to the LHe Dewar will be bayonet couplings. The requirements to process parameters at the interface between the SHCP and the CDS as well as the LHe Dewar for each of the steady state operation modes are presented in Table 3.39.

Table 3.38: CDS transfer line sizes

\begin{tabular}{l|l}
\hline \hline Line description & Line size (NPS) \\
\hline 4.5K Supercritical helium (supply) & 2" Schedule 10 \\
\hline Sub - atmospheric helium (2K return) & 10" Schedule 5 \\
\hline LTTS return & 2" Schedule 10 \\
\hline HTTS supply & 2" Schedule 10 \\
\hline HTTS return & 2" Schedule 10 \\
\hline Cool down (return) & 3" Schedule 10 \\
\hline \hline
\end{tabular}

Table 3.39: SHCP interface with the CDS/LHe dewar

\begin{tabular}{l|c|c|c|c}
\hline \multirow{2}{*}{ Circuit } & \multicolumn{2}{|c|}{ Supply } & \multicolumn{2}{c}{ Return } \\
\cline { 2 - 5 } & $\mathrm{P}[\mathrm{MPa}]$ & $\mathrm{T}[\mathrm{K}]$ & $\mathrm{P}[\mathrm{MPa}]$ & $\mathrm{T}[\mathrm{K}]$ \\
\hline $2 \mathrm{~K}$ & - & - & $2.7 \mathrm{e}-3$ & $\leq 3.8$ \\
\hline $4.5 \mathrm{~K}$ supercritical & $0.22 \leq \mathrm{P} \leq 0.4$ & $\leq 4.5$ & - & - \\
\hline LHe & 0.12 & $\begin{array}{c}\text { Sat. } \\
\text { Temperature }\end{array}$ & $\begin{array}{c}\text { LHe Dewar } \\
\text { pressure }-\Delta \mathrm{P}_{1}{ }^{*}\end{array}$ & $\begin{array}{c}\text { Sat. } \\
\text { Temperature }\end{array}$ \\
\hline LTTS & - & - & $0.22 \leq \mathrm{P}^{2} \leq 0.4$ & $\leq 9$ \\
\hline HTTS & $0.3 \leq \mathrm{P} \leq 1.8$ & $35-40$ & $\mathrm{P}-\Delta \mathrm{P}_{2}{ }^{\#}$ & $\leq 80$ \\
\hline \hline
\end{tabular}

${ }^{*} \Delta \mathrm{P}_{1}$ is the pressure drop in LP return of the transfer line (about $5 \mathrm{kPa}$ )

${ }^{\#} \Delta \mathrm{P}_{2}$ is the pressure drop in the HTTS line as provided in Table 3.34. 


\subsubsection{Infrastructure and Utilities Requirements}

The major PIP-II cryogenic infrastructure consists of the Warm Compression System (WCS) building, the Cold Box (CB) building and the interconnection between the CB building and the PIPII tunnel, housing the Cryogenic Distribution System (CDS). The design of the cryogenic infrastructure will include adequate provisions to minimize transmission of vibration to the PIP-II tunnel in order to prevent excitation of microphonics in the SC cavities.

The major requirements to the WCS building are:

- High bay area: 60’ x 200' (Floor) with a 25 metric ton EOT crane.

- Electrical power: 3 phase, $4.16 \mathrm{kVAC}, 60 \mathrm{~Hz}$ with minimum power requirement of about 3.5 MW; 3 phase, $480 \mathrm{VAC}, 60 \mathrm{~Hz}$ with minimum power requirement of about $200 \mathrm{~kW}$ for auxiliary equipment; 1 phase, $120 \mathrm{VAC}, 60 \mathrm{~Hz}$ incidental requirements $(20 \mathrm{~kW})$. Class 2 (AC power from UPS) for operating the control panels.

- Cooling water: About 1500 gpm with a supply temperature of about $85-95^{\circ} \mathrm{F}$ and allowable rise in temperature of about $18^{\circ} \mathrm{F}$.

- Ventilation blower: $300 \mathrm{~kW}$ approx.

- Instrument air: 500 gpm approx.

The major requirements to the $\mathrm{CB}$ building are:

- High bay area: 50' x 125' (Floor) with a 15 metric ton EOT crane and a side bay area.

- Electrical power: 3 phase, $480 \mathrm{VAC}, 60 \mathrm{~Hz}$ with minimum power requirement of about 100 $\mathrm{kW}$ for auxiliary equipment; 1 phase, $120 \mathrm{VAC}, 60 \mathrm{~Hz}$ incidental requirements $(30 \mathrm{~kW})$. Class 2 (AC power from UPS) for operating the control panels.

- Cooling water: About 200 gpm with a supply temperature of about $50-60{ }^{\circ} \mathrm{F}$ and allowable rise in temperature of about $9{ }^{\circ} \mathrm{F}$.

- Instrument air: 500 gpm approx. 


\subsection{Instrumentation}

Various beam instrumentation and diagnostics systems are necessary to characterize and monitor the beam parameters and the performance in all PIP-II sub-accelerators. For startup and initial beam commissioning we need to provide, at a minimum, beam instruments in order to observe:

- Beam intensity,

- Beam position / orbit,

- Transverse beam profiles,

- Beam phase / timing.

Beside these core beam instrumentation systems, additional beam diagnostics will be needed. These instruments will be used to characterize such beam parameters as the beam emittance, longitudinal bunch profile and tails, transverse beam halo, bunch-by-bunch chopping efficiency and advanced beam emittance measures.

When possible the beam instrumentation and diagnostics systems must be able to operate over a wide bandwidth from few microsecond long pulses for machine commissioning and tuning to long operational pulses. In addition, these systems must be compatible with $\mathrm{CW}$ beam operation. Table 3.40 presents an estimate of quantities of different beam instrumentation systems by location for the linac and the $800 \mathrm{MeV}$ beam transport line. In the table the SC linac also includes the linac extension capable to include 4 additional HB650 cryomodules.

Table 3.40: Estimate of beam instrumentation in the Linac and $800 \mathrm{MeV}$ transfer line

\begin{tabular}{l|c|c|c|c|c|c|c}
\hline \hline Location & $\begin{array}{c}\text { Beam } \\
\text { current }\end{array}$ & $\begin{array}{c}\text { Transverse } \\
\text { position } \\
\text { /phase }\end{array}$ & $\begin{array}{c}\text { Transverse } \\
\text { profiles }\end{array}$ & $\begin{array}{c}\text { Longitudinal } \\
\text { profiles }\end{array}$ & $\begin{array}{c}\text { Beam } \\
\text { loss }\end{array}$ & $\begin{array}{c}\text { Transverse } \\
\text { emittance }\end{array}$ & $\begin{array}{c}\text { Bunch } \\
\text { extinction }\end{array}$ \\
\hline LEBT & 2 & N/A & - & - & - & 1 & - \\
\hline MEBT & 2 & 12 & $4+2^{*}$ & $2^{*}$ & TBD & 1 & 1 \\
\hline SC linac & 5 & 57 & $10^{*}$ & $10^{*}$ & $\begin{array}{c}2 \text { per } \\
\text { CM }\end{array}$ & - & - \\
\hline $\begin{array}{l}\text { Transport } \\
\text { line }\end{array}$ & 2 & 57 & $4^{*}$ & TBD & $\begin{array}{c}1 \text { per } \\
\text { FE }\end{array}$ & 1 & - \\
\hline \hline
\end{tabular}

$\downarrow$ one per focusing element (solenoid, quad, doublet, triplet), $\mathrm{CM}=$ cryo-module,

* = laser wire

The high beam intensity / power and the presence of superconducting technologies require a reliable, fail safe machine protection system (MPS) to prevent quenches in cryogenic elements or damage due to an uncontrolled loss of the high power beam. This system will primarily be based on beam loss monitors (BLM) and beam intensity monitors such as toroids and dedicated ring pickups (see details in Section 3.8).

\subsubsection{Beam Position and Phase Measurement System}

Beam orbit monitoring is the most fundamental measurement and powerful diagnostics tool in an accelerator. PIP-II requires a large number of new warm and cold beam position monitors (BPM) for the linac and $800 \mathrm{MeV}$ transfer beam line. Both the warm and cold BPMs must be capable of measuring all 3 coordinates: horizontal and vertical position and beam phase relative to the RF, as well as a relative measure of the beam intensity. The beam line design includes a single four-button 
BPM located near each beam focusing element (solenoid, quad, doublet, triplet). The geometry of all BPMs will be similar with their aperture slowly increasing with acceleration. The stiles of BPMs are presented in Table 3.41. Transport line BPMs are required to measure only one coordinate: $x$ if located near $\mathrm{F}$ quad, and $y$ if located near D quad.

Table 3.41: Styles of PIP-II BPMs

\begin{tabular}{|c|c|c|c|c|}
\hline Style & Quantity & $\begin{array}{l}\text { Aperture } \\
{[\mathrm{mm}]}\end{array}$ & $\begin{array}{c}\text { Operating } \\
\text { temperature }\end{array}$ & Location (quantity) \\
\hline MEBT & 12 & 28.5 & warm & MEBT \\
\hline HWR & 8 & 36 & cold & HWR \\
\hline SSR1 & 8 & 33 & cold & SSR1 \\
\hline SSR2 & 21 & 43 & cold & SSR2 \\
\hline SC linac & 20 & 45 & warm & $\begin{array}{lll}\text { LB650 (11), } & \text { HB650(4), } & \text { linac } \\
\text { extension }(5)\end{array}$ \\
\hline Transport line & 56 & 45 & warm & Arc 1(19), Arc 2(33), beam dump (4) \\
\hline Beam split & 1 & 160 & warm & In front of transfer line Lambertson \\
\hline
\end{tabular}

The signal duration is approximately proportional to the BPM aperture and shortens with beam acceleration as shown in Figure 3.97 for the HWR style BPM [127].
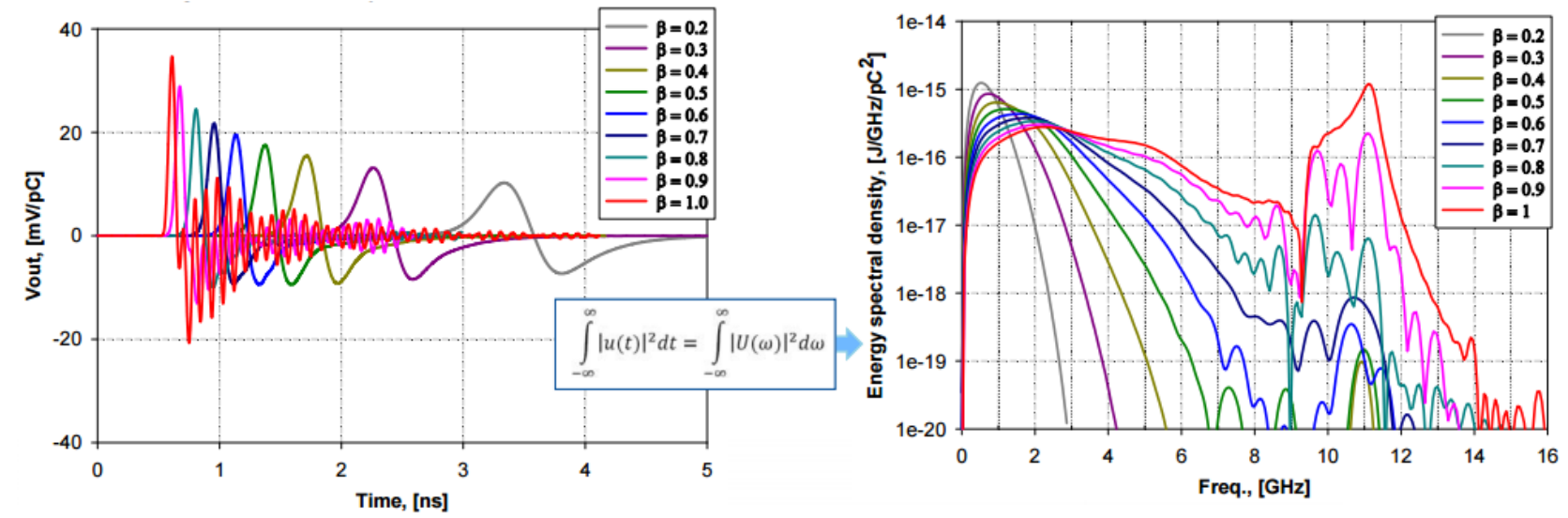

Figure 3.97: Numerical simulations of BPM signals produced by a bunch moving with different velosities: left - time domain signals, right - corresponding spectra; rms length is $4 \mathrm{~mm}$.

\section{BPM Measurement Modes}

There are two modes that need to be supported by the BPM measurements for PIP-II commissioning and operation. The first mode is assigned for the pulsed beam at a single trajectory. It is applicable to all BPMs upstream of the MEBT chopper and to all other BPMs when the MEBT chopper is off. The second mode is assigned for pulsed beam split into two trajectories. This occurs only for the four BPMs located in the MEBT chopper region and only when the chopper is operational. In this case the chopper kicker splits the beam into two streams propagating downstream and oscillating relative to each other at the betatron frequency. Table 3.42 presents the resolution requirements for the BPM system for these two beam modes for beam pulses with duration at least $10 \mu \mathrm{s}$. 
Table 3.42: BPM measurement requirements for one- and two-trajectory modes

\begin{tabular}{l|c|c}
\hline \hline & One-trajectory Mode & Two-trajectory Mode \\
\hline Transverse position, $\mu \mathrm{m}$ & 10 & 100 \\
\hline Phase, degrees of $162.5 \mathrm{MHz}$ & 0.05 & 0.5 \\
\hline Relative intensity, $\%$ & 1 & 2 \\
\hline \hline
\end{tabular}

\section{Warm MEBT BPM Pickups}

The MEBT includes 12 warm BPMs, one for each focusing element. Each BPM is mounted to a quadrupole of a MEBT quad doublet or triplet (focusing element). For a low energy beam, buttontype pickups provide a good compromise between amplitude and time responses. Prototype fourbutton BPMs have been designed and installed for testing in the MEBT of PIP-II Injector Test beamline. The left pane of Figure 3.98 shows the BPM view, and the right pane shows the BPM mounted to the iron of a MEBT focusing quadrupole magnet. The total BPM length is 3". Its maximum diameter is 2.7" and a beam aperture 1.12". The BPM is composed of non-magnetic stainless steel and utilizes $20 \mathrm{~mm}$ diameter button pickups. The design is similar to the FRIB BPM design [128].
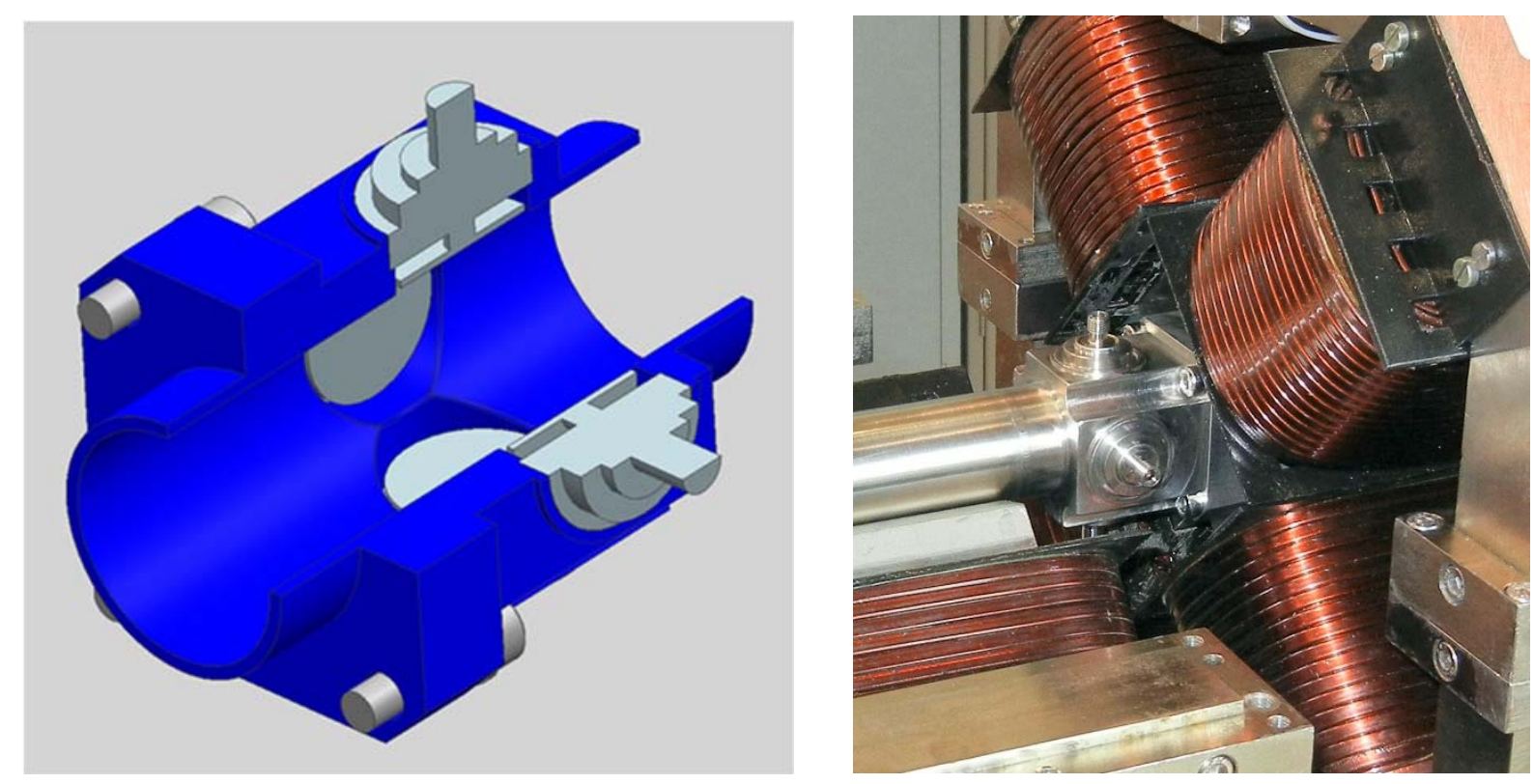

Figure 3.98: Section view of a prototype MEBT BPM (left) and prototype BPM mounted to a MEBT quadrupole (right).

\section{Warm Linac and Transfer Line Pickups}

After HWR, SSR1 and SSR2, focusing elements for the rest of the linac will reside in warm sections between the eleven LB 650 and four the HB 650 cryomodules. Each focusing element will have a four-button BPM of the SC linac style. The total number of BPMs for the 650 section of the linac is 20. These BPMs will be mounted to the iron of one of the focusing element quadrupoles, in a design similar to the MEBT BPMs. This quadrupole magnet design will also be utilized in the 800 $\mathrm{MeV}$ transfer line, which will also include a BPM for each focusing element. A total of 57 BPMs will be installed in the $800 \mathrm{MeV}$ transfer line. 


\section{Cold BPM Pickups}

The linac HWR, SSR1 and SSR2 cryo-modules will contain 37 cold BPM pickups which will be located inside the HWR, SSR1 and SSR2 cryo-modules and attached to each solenoid (focusing element). Their design must meet UHV, cryogenic and clean room requirements. Left pane of Figure 3.99 shows a view of BPM pickup design and the right pane shows an assembled prototype BPM to be used in the SSR1 cryomodule.

Beam instrumentation within a cryogenic environment needs extra care to meet ultra-high vacuum, cryogenic and clean room requirements simultaneously. Therefore, it is limited to beam orbit monitoring with button BPM pickups due to its simple mechanical design and good reliability.

The signal performance of the button-style BPM for $\mathrm{H}^{-}$beams has been simulated [127]. For a low-energy beam the button-type pickups yield a good compromise between amplitude and time responses. Figure 3.100 shows the time and frequency domain responses of a button BPM for a lowenergy beam with $\beta=0.2$ which corresponds to the end of the first SSR 1 cryomodule. The calculations were performed with CST Studio particle wake-field solver. For $5 \mathrm{~mA}$ RFQ current the bunch charge is $30.8 \mathrm{pC}$, which, in accordance with Figure 3.100, corresponds to a peak voltage on each BPM electrode of about $0.25 \mathrm{~V}$. As shown in Figure 3.97, the voltage grows approximately linearly with an increase of beam velocity $\beta$; and the pulse duration decreases approximately inversely proportional to $\beta$. Here we take into account that the bunch length is shorter than the duration of a single particle signal in the course of entire acceleration.
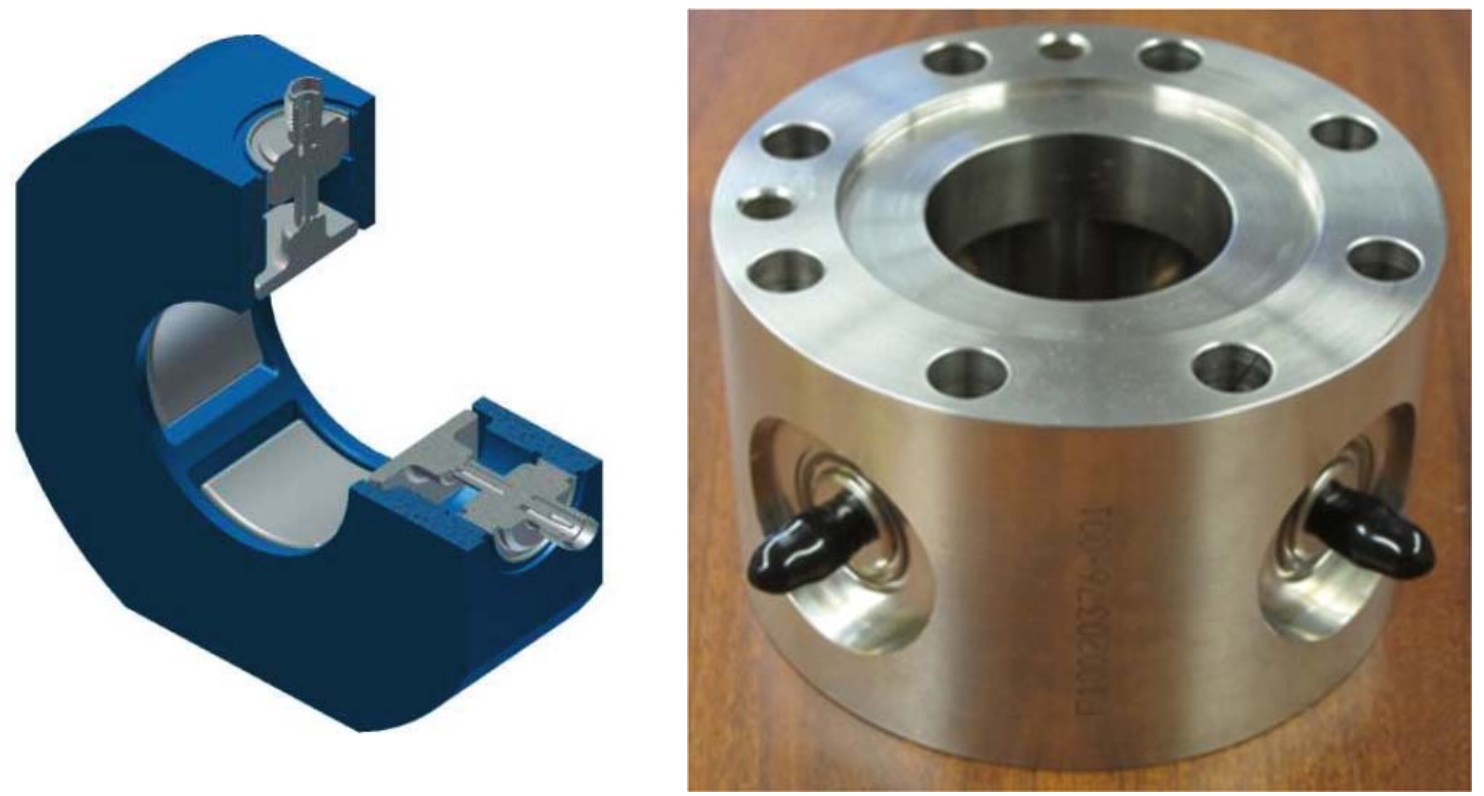

Figure 3.99: Section view of prototype cold BPM (left) and a prototype SSR1 cold BPM assembly (right).

\section{BPM Data Acquisition}

The standard BPM readout electronics for PIP-II will measure position, intensity, and phase using direct digital down-conversion by measuring the amplitude of the $1^{\text {st }}$ beam harmonic $(162.5 \mathrm{MHz})$ for each electrode. In addition, the BPM readout will also take measurements at the $3^{\text {rd }}$ harmonic in an attempt to make a relative bunch length measurement [129]. This $1^{\text {st }}$ and $3^{\text {rd }}$ harmonic acquisition system will be tested at the PIP-II Injector Test accelerator. A simplified block diagram of the readout electronics is shown in Figure 3.101. 

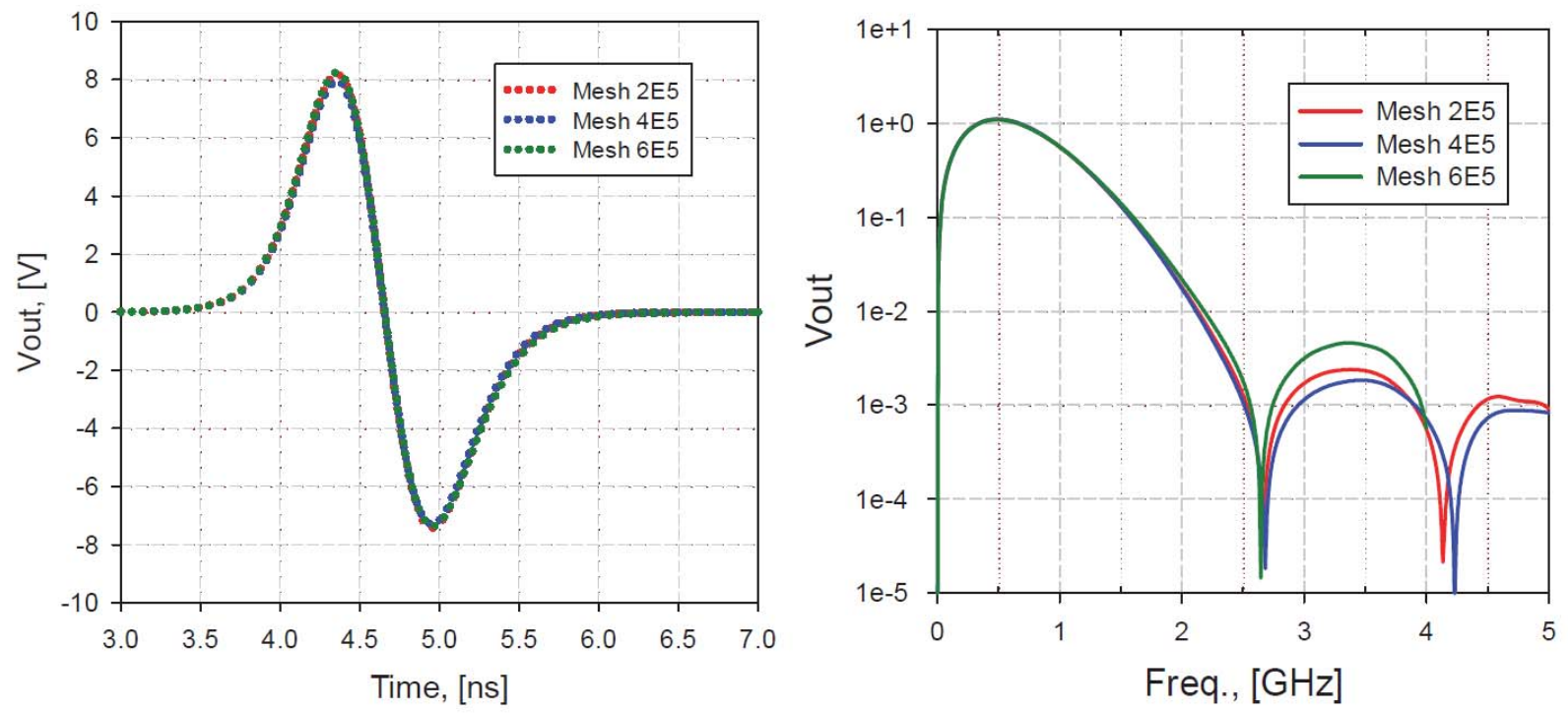

Figure 3.100: BPM output voltage in time (left) and frequency (right) domains versus mesh size (CST Studio, $4 \mathrm{~mm}$ rms, $\beta=0.15,1 \mathrm{nC}$ bunch).

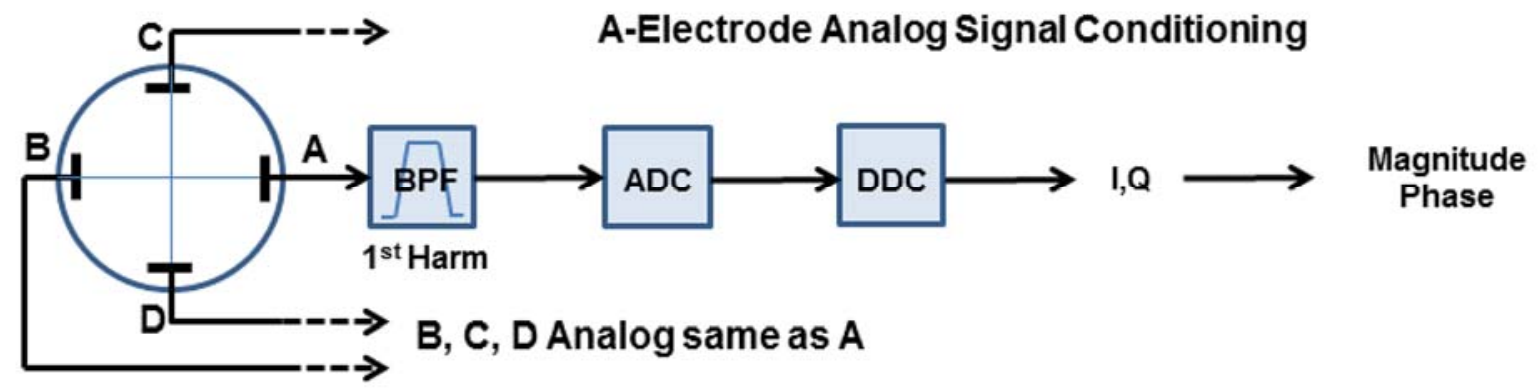

Figure 3.101: Block diagram for BPM electronics. The third harmonics part is simular and is not shown.

Figure 3.102 presents benchtop stretched wire measurements of a prototype MEBT BPM and a prototype cryo-module BPM. The stretched-wire measurements show non-linear distortions in the position measurements due to finite button pickup size. A 2D polynomial fit to the difference over sum in each plane will be used to correct nonlinearities in position and intensity responses of the button pickups. In addition, the calculated position correction will include a compensation for beam velocity which also affects the measurement.

As seen in Figures 3.97 and 3.98, the $1^{\text {st }}$ harmonic gives adequate signal for a low-energy beam. However, if the signal strength will be found inadequate at higher beam energies, we will then change the readout electronics to operate at the 2 nd harmonic.

In the MEBT chopper region, the BPM system needs to measure simultaneously two different beam paths. This will require the BPM system to have sufficiently high bandwidth to make bunchby-bunch transverse position measurements. The proposed BPM electronics will not allow highbandwidth bunch-by-bunch measurements because of the $1^{\text {st }}$ harmonic bandpass filter. To acquire bunch-by-bunch information, high bandwidth oscilloscopes will be used to directly sample the button which will provide reasonable signal integration and transverse position resolution. Although oscilloscopes have high-bandwidth signal acquisition, they are relatively slow in signal processing. 
Therefore, the MEBT chopper bunch-by-bunch measurements will not acquire measurements for every PIP-II beam pulse.
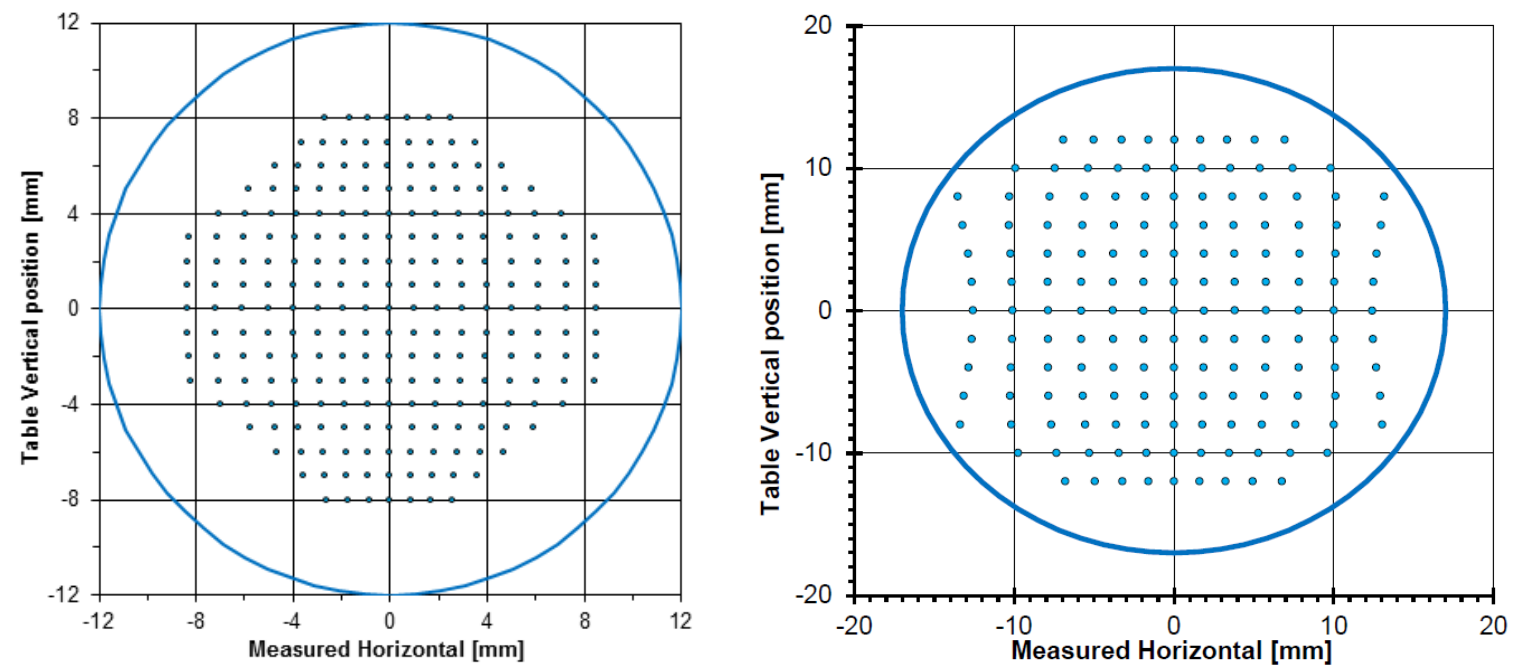

Figure 3.102: Benchtop stretched wire mapping of a prototype MEBT BPM (left) and a prototype cryomodule BPM (right).

\subsubsection{Beam Loss Measurement System}

Beam loss monitoring is an important tool for beam tuning as well as a critical part of machine protection. The PIP-II beam loss monitoring system will also help to prevent excessive activation of materials.

PIP-II will utilize three types of Beam Loss Monitor (BLM) devices through the linac and the linac-to-Booster transport line. The primary BLM devices will be ionization chambers. Ionization chambers have a relatively good response time ( $\sim$ few $\mu$ s), are a simple in design and are resistant to radiation damage. The other two BLM devices will be fast Photomultiplier Tube (PMT) based detectors and neutron detectors. Both the ionization chamber detectors and PMT detectors are sensitive to charged particles, however the scintillator-based PMT detectors have a faster response time ( $\sim 10 \mathrm{~ns})$, adjustable gain and are sensitive to lower energy gamma rays. The BLM detectors will be evenly distributed along the linac and $800 \mathrm{MeV}$ transport line with ionization detectors located near focusing elements between the cryo-modules and neutron detectors located near the middle of cryo-modules. The PMT-based detectors will mostly be distributed at the low-energy end of the linac $(\sim<100 \mathrm{MeV})$ and at key locations that require fast beam loss measurements. The beam loss monitoring system will also include a number of movable BLM detectors to be used to study specific beam loss issues as they occur during commissioning and beam tuning. In addition, there will be used the fourth type of beam loss monitor, the total loss monitor, described in Section 3.7. Such monitors better characterize the total beam loss but without detailed information on its location.

\section{Beam Loss Monitor Signal Processing}

Since several types of BLMs will be utilized in PIP-II, the signal processing will vary depending on the particular application of the given monitor. Fast PMT based signals for machine protection will be processed to provide both a Fast Shutdown Signal (FSD) to the MPS as well as integrated to provide a tuning signal. The time scales for these signals are a few microseconds with cable delays included. FPGA processing time for FSD is only a few nanoseconds. Ionization type loss monitor 
signals will be digitized at a sampling rate of $125 / 250 \mathrm{MHz}$ and various thresholds for losses will be established according to the damage potential and diagnostic protection requirements. Figure 3.103 shows a typical block diagram of a VME based signal processing scheme for a PMT-based beam loss monitor.

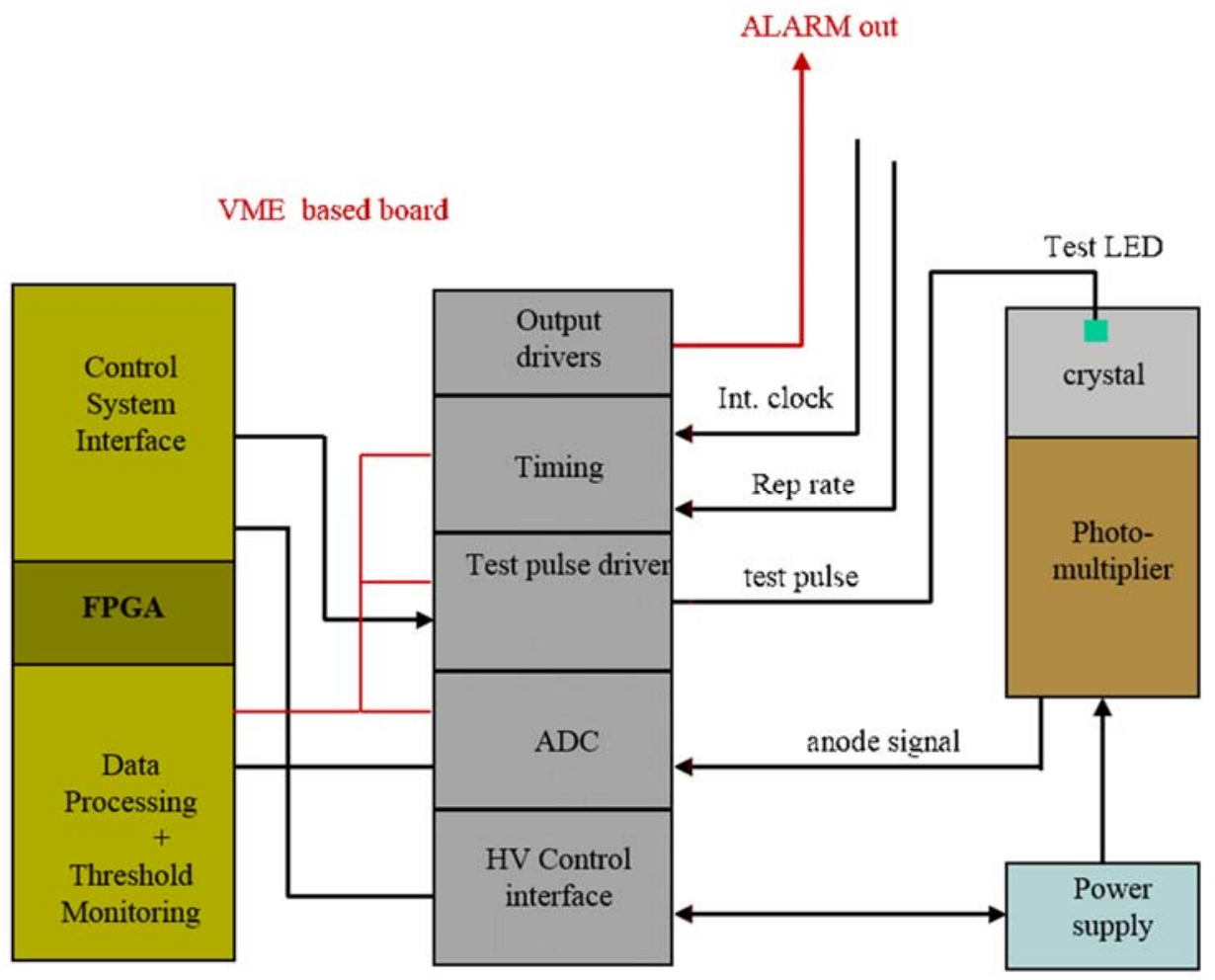

Figure 3.103: Example of PMT-based beam loss monitor electronics.

\subsubsection{Beam Intensity Measurement System}

Protection of the PIP-II superconducting linac will require high precision measurements of pulsed and, in the future, CW beam currents as well as an accurate measure of beam current loss computed from the difference of beam currents at different locations. Such measurements are an important supplement to the BLM system. The beam current will be measured by a combination of different beam current instruments. The primary instruments will include DCCTs and beam toroids. In addition, a relative measure of beam current will be made by the BPM system as well as dedicated ring pickups.

The primary measure of absolute beam current will be via DCCTs and beam toroids. Standard beam toroids have the advantage of being less expensive than DCCTs and having a wide bandwidth to measure pulsed beam but are $\mathrm{AC}$ coupled and so cannot measure $\mathrm{CW}$ beam. DCCTs can measure $\mathrm{CW}$ beam but cannot measure short beam pulses and are generally more expensive. PIP-II will employ both types of instruments to adequately cover the proposed range of PIP-II beam timing structures. PIP-II will also utilize the BPM system to give a relative measure of the beam intensity.

In addition, several dedicated ring pickups will be employed in the PIP-II linac to give an independent measure of the relative beam current. The ring pickup is similar to a single button BPM and will utilize rectifying electronics to generate a measure of the PIP-II beam pulse. Figure 3.104 shows a view of the ring pickup to be tested in the PIP-II Injector Test accelerator and the response of the rectifying electronics to a $20 \mu$ s test beam signal. 
Both the BPM intensity and the ring pickup intensity, as well as absolute current from the beam toroids, can be utilized by the differential beam current monitor system to detect fast beam loss for the machine protection system. A similar differential beam current monitor system has been employed by the SNS accelerator [130].
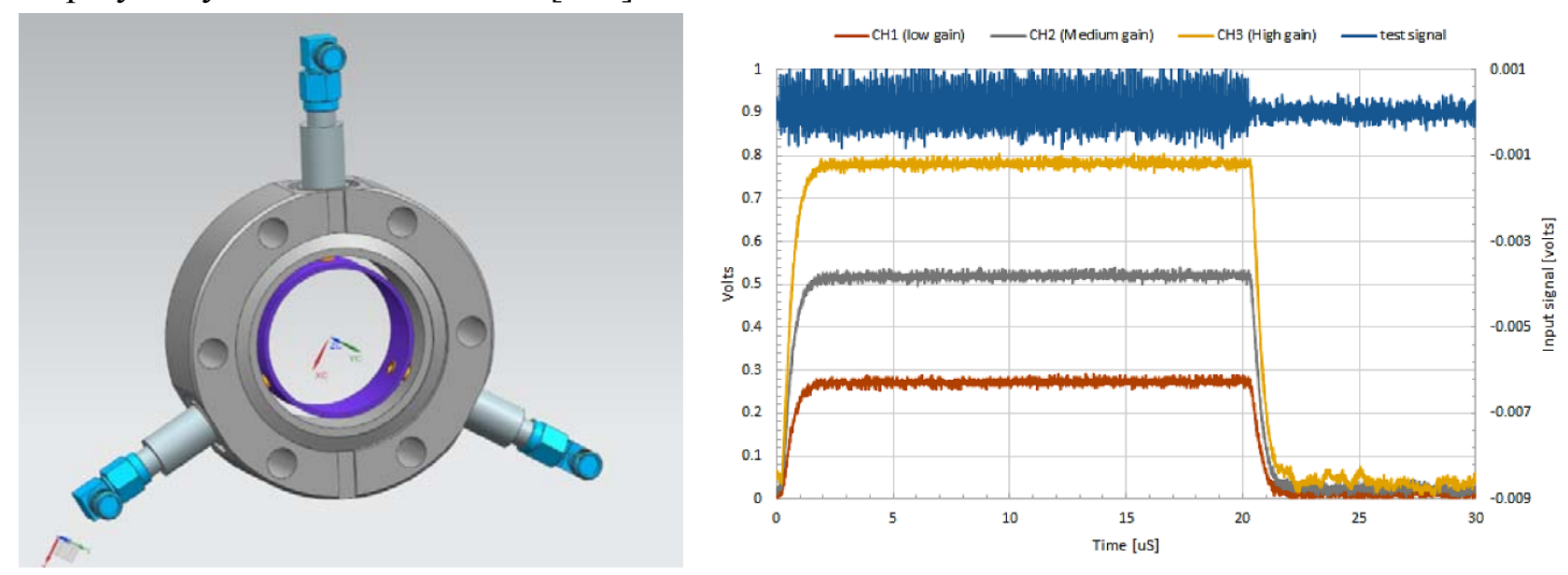

Figure 3.104: Drawing of a prototype ring pickup (left) and response of the ring pickup rectifying electronics to simulated $20 \mu \mathrm{s} 162.5 \mathrm{MHz}$ test signal (right)

\subsubsection{Beam Transverse Profile Measurement System}

We are considering two types of transverse wire profiling methods for PIP-II. The first method would use photo-disassociation of $\mathrm{H}^{-}$by laser radiation, and the second method would be a traditional wire scanner. Because of the minimal interaction with the beam, the laser-based profile monitor is our primary choice. In the vicinity of superconducting cryomodules it normally should be the only method to use. However, traditional wire scanners are a proven technology in accelerators and they will be considered as a backup choice for certain areas of the linac and $800 \mathrm{MeV}$ transfer line. Table 3.43 shows the preliminary requirements for beam transverse profile measurements. In addition to these two primary choices for transverse profiles we will utilize transverse scrapers in the LEBT and MEBT to produce beam profile measurements.

Table 3.43: Requirements for beam transverse profile measurements.

\begin{tabular}{|l|l|}
\hline Parameter & Value \\
\hline Spatial resolution & $<0.1 \mathrm{~mm}$ \\
\hline Signal dynamic range & $>10^{3}$ \\
\hline Spatial range & $\pm 15 \mathrm{~mm}$ \\
\hline
\end{tabular}

\section{Laser-based Transverse Profile Measurements}

PIP-II will utilize transverse laser profile monitors throughout the linac and $800 \mathrm{MeV}$ transport line. Laser-based transverse profile measurements of $\mathrm{H}^{-}$beam has been demonstrated by different groups [131,132]. SNS at Oak Ridge National Lab has implemented laser-based profile measurements as part of its operation [133]. Traditionally, the technique of photo-disassociation $\left(\mathrm{H}^{-}\right.$ $\left.+\gamma \rightarrow \mathrm{H}^{\mathrm{o}}+\mathrm{e}^{-}\right)$is accomplished with high peak power lasers and signal detection through the collection of electrons. However, SNS has demonstrated a longitudinal profile measurement in their MEBT utilizing a low peak power laser with electron collection. A low peak power laser produces 
far fewer photo-disassociations and, hence, a smaller signal.

For PIP-II we will construct a transverse $\mathrm{H}^{-}$beam profiling instrument utilizing a low peak power, high rep-rate fiber laser with optical fiber transport to the accelerator and synchronous signal detection. The system will detect this small signal through narrow-band synchronous detection of a modulated laser pulse train. Figure 3.105 shows a diagram of the proposed laser profile monitor. This system utilizes a high rep-rate, picosecond, mode-locked laser (MLL) that is locked to the accelerator LLRF. The laser pulse train amplitude is modulated by a variable RF signal that is asynchronous to the accelerator RF. The laser pulse train is then transported to the beam line via optical fiber and scanned across the beam. Modulated intensity of $\mathrm{H}^{-}$beam is observed at downstream BPM(s) with the synchronous lock-in detection. Some systems, where space is available, will additionally include beam profile measurements with electron detection for profile verification. Where bunch lengths are not too short, longitudinal profile scans are measured by changing MLL phase relative to the LLRF. A prototype version of the laser profile monitor will be tested at the PIP-II Injector Test accelerator.

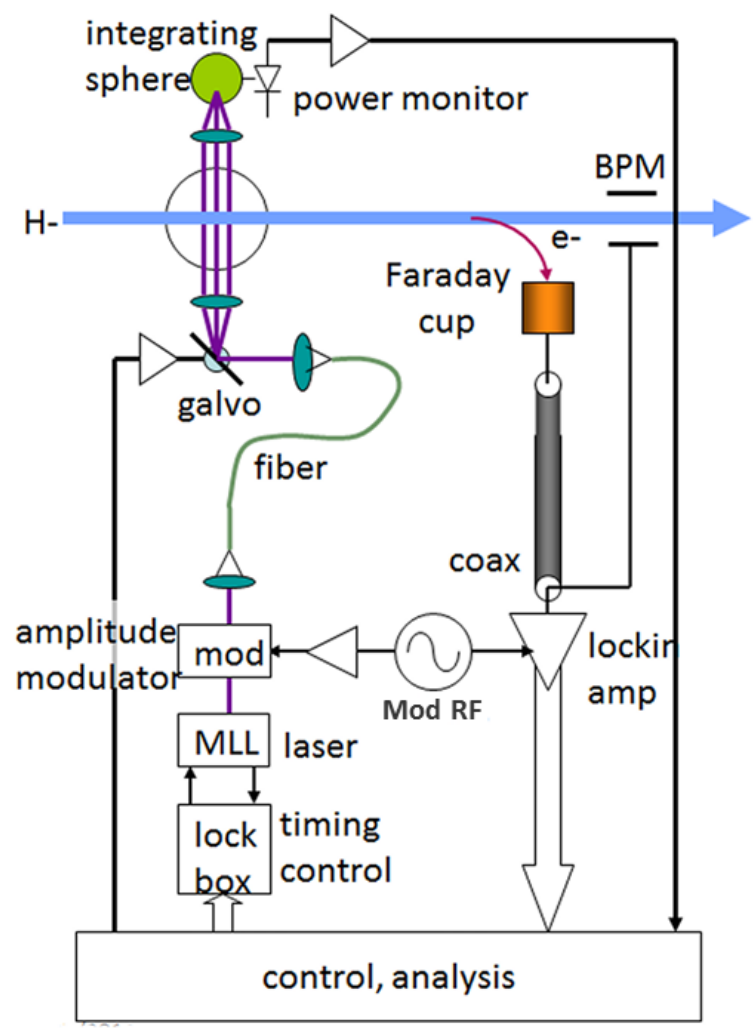

Figure 3.105: Proposed transverse and longitudinal beam profile system. Drawing courtesy of $R$. Wilcox, LBNL [134].

\section{Wire Scanner Transverse Profile Measurements}

Wire scanner systems have been a traditional method used for beam transverse profile measurements. They have the advantage of being a straightforward and well-understood technique. They have the disadvantage of being invasive, causing beam loss, and also for being fairly slow. We will utilize wire scanners as a secondary profile measurement method in the MEBT and the $800 \mathrm{MeV}$ transport line. It is unadvisable to use such systems in close vicinity to SC cryomodules due to spattering of wire material by the beam and possible micro-particle formation due to blistering. A prototype wire scanner will be tested in the PIP-II Injector Test accelerator. 


\subsubsection{Other Beam Measurement Systems}

\subsubsection{Beam Transverse Emittance Measurements}

Transverse emittance measurements are a highly useful and versatile tool for the tuning and operation of an accelerator. There are various techniques that can be used to determine the transverse emittance. PIP-II will employ two types of emittance measurements for transverse phase-space: (1) Allison-type emittance scanners and (2) laser-based emittance scanners.

In addition to these phase-space measurement techniques, PIP-II will also make rms emittance measurements utilizing quadrupole scans and transverse profile monitors. These rms measurements will occur at locations that can provide a necessary combination of focusing elements and profile monitors.

\section{Allison-Type Emittance Scanners}

An emittance scanner for intense low-energy ion beams, based on sweeping electric fields, was proposed in 1983 [135]. This type of emittance scanner has been typically classified as an Allisontype transverse emittance scanner. Typically, such scanners have been utilized for ion source and LEBT beam emittance measurements [136]. A functional representation of an Allison scanner illustrating the two slit design with electric field deflecting plates is shown in Figure 3.106. The angular scan is performed with voltage change on the deflecting plates, and the coordinate scan is performed with mechanical displacement of the monitor with a step motor. PIP-II will utilize two Allison scanners: one for the LEBT and one for the MEBT.

A water-cooled Allison scanner has been built and tested at the LEBT of PIP2IT for measuring ion source emittance [137]. The scanner has been operated extensively for both pulsed and DC 30 $\mathrm{keV} \mathrm{H} \mathrm{H}^{-}$beam up to beam current of $10 \mathrm{~mA}$. Figure 3.107 shows a set of phase space measurements taken in the PIP-II Injector Test LEBT with this Allison scanner. This figure illustrates the capability of the Allison emittance scanner.

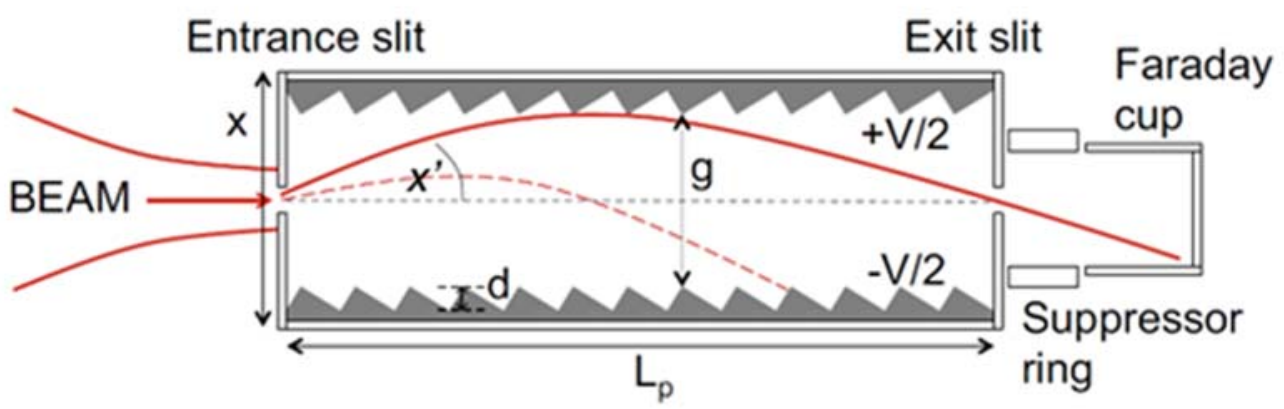

Figure 3.106. Pictorial representation of an Allison-type scanner with an illustration of the bending properties of the electric deflecting plates.

\section{Laser-Based Emittance Scanners}

Measurements of transverse phase space and emittance at the end of the PIP-II linac will be performed with a laser-based emittance scanner. A functional diagram of a laser-based emittance scanner is shown in Figure 3.108. A laser-based scanner has the advantage of minimal beam interaction enabling it to make measurements for both pulsed and $\mathrm{CW}$ beam. A similar, but not identical, technique has been implemented at both the SNS and LINAC4 accelerators [138, 139]. 

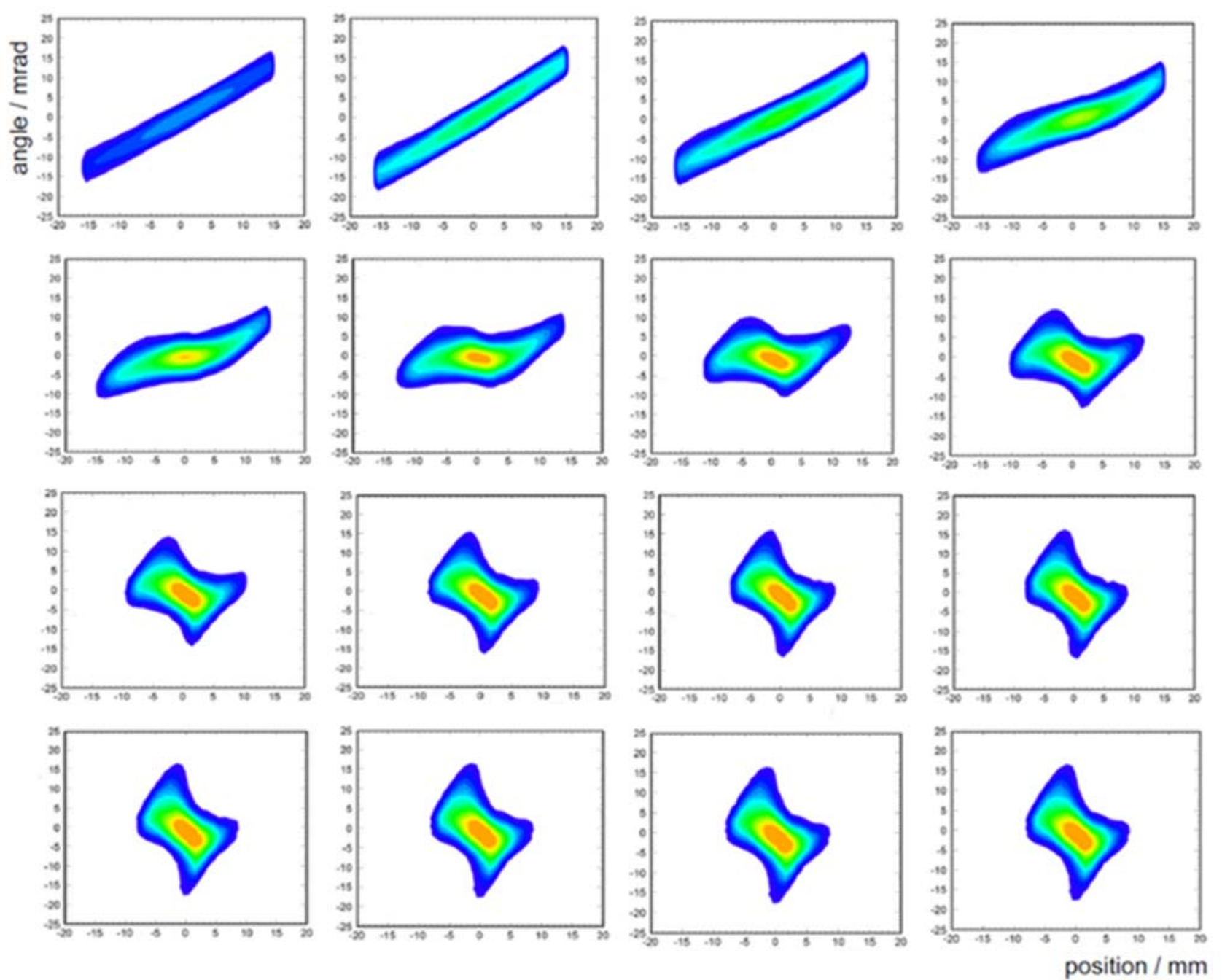

Figure 3.107: An example of evolution of the transverse phase space on time after pulse beginning taken in the LEBT of the PIP-Injector Test accelerator for a 2 ms pulsed, $5 \mathrm{~mA} \mathrm{H}^{-}$beam with $0.125 \mathrm{~ms}$ time steps. The evolution is happening due to beam space charge nuetralization.

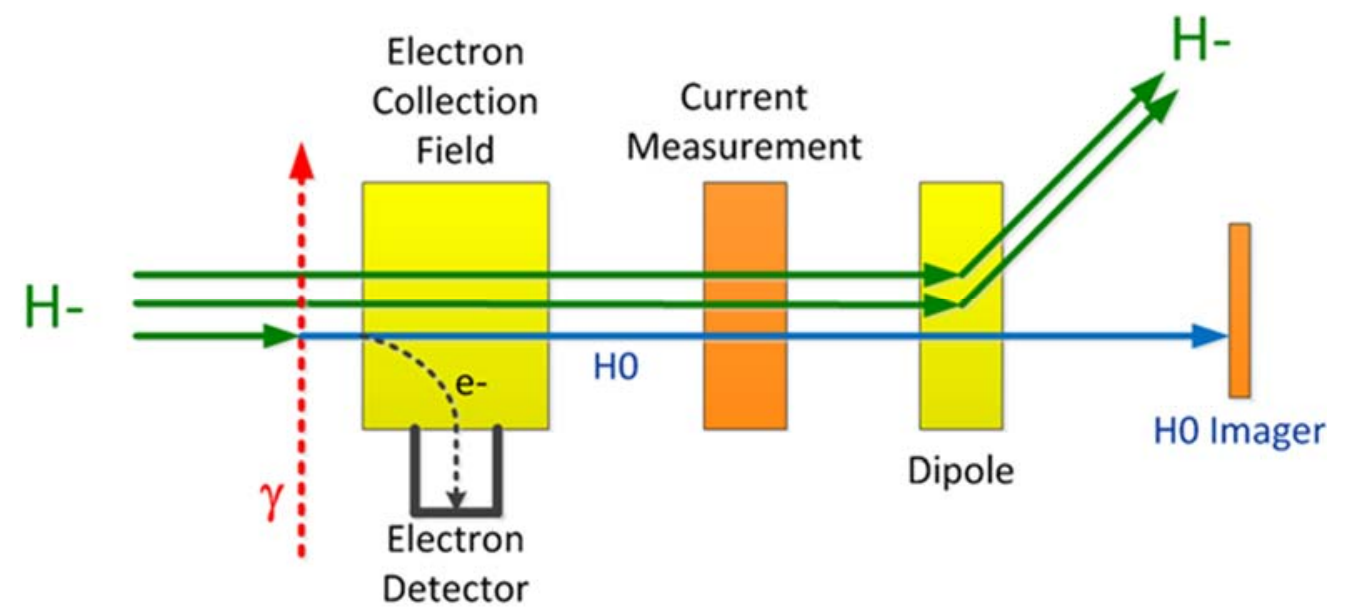

Figure 3.108: Functional diagram of a laser-based transverse emittance monitor. 


\subsubsection{Beam Longitudinal Profile Measurements}

The PIP-II longitudinal rms bunch length changes from several hundred picoseconds in the MEBT to as short as 4 picoseconds at the end of the linac. Measurement of the longitudinal profile for these short bunches can be difficult. As discussed earlier, PIP-II will utilize laser-based profiling for both transverse and longitudinal profile measurements for bunches of adequate length. SNS has demonstrated that laser-based longitudinal profile measurements are possible for MEBT beam [140].

However, laser-based measurements of longitudinal profiles become difficult when the time of the transverse bunch crossing by light becomes close to the bunch duration. For PIP-II this happens when beam velocity becomes comparable to the speed of light ${ }^{11}$. Additionally, the bunch duration is decreasing with acceleration requiring a shorter length of the laser pulse. Measurement of longitudinal profiles at the linac end requires a laser pulse length of $\sim 200$ femtoseconds or less. While it is possible to generate these short laser pulses with sufficient power, it is difficult to transport the laser pulse without its lengthening due to dispersion in a fiber. If laser-based measurements are found to be impractical for very short bunch length measurements, PIP-II will explore other measurement options, such as optical transition radiation techniques.

\subsubsection{MEBT Chopper Extinction Measurements}

The PIP-II MEBT chopper will be able to arbitrarily remove individual bunches from the beam. The chopping efficiency of bunch removal needs to be verified to a level of better than one part in $10^{-4}$. PIP-II will utilize a high-bandwidth resistive wall current monitor (RWCM) to measure the integrated field produced by individual beam bunches. Integration of the RWCM signal will be accomplished using a fast oscilloscope. This technique is similar to the Fermilab sampled bunch display systems used to measure longitudinal particle distribution of individual bunches in the Tevatron and Main Injector accelerators [141]. Figure 109 shows a block diagram of the proposed MEBT bunch extinction system.

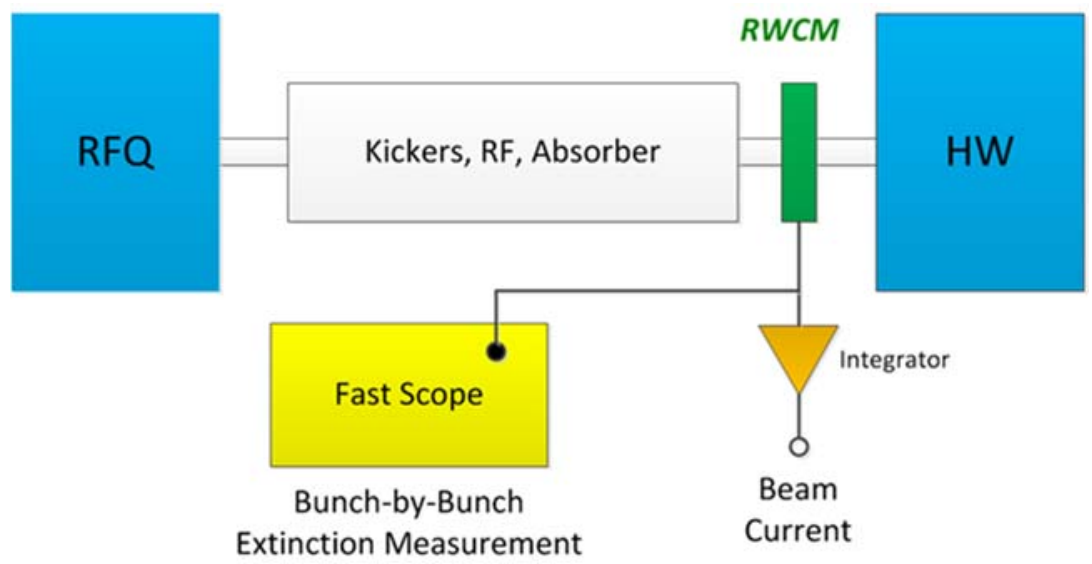

Figure 3.109: Block diagram of the PIP-II MEBT extinction measurement system.

\subsubsection{Beam Halo Measurements}

For any superconducting accelerator, measurement and control of the longitudinal and transverse beam halo is important. Typically, beam halo measurements are defined to be at the intensity level of $10^{-4}$ to $10^{-6}$ of the core beam [142]. This places a large dynamic range requirement for any halo measurement system.

\footnotetext{
${ }^{11}$ The transverse and longitudinal bunch sizes are quite close for entire acceleration in the SC linac.
} 
For transverse beam halo measurements and control in the MEBT, PIP-II will primarily utilize electrically isolated scrapers. Such scrapers, with current readout, have been tested in the PIP-II Injector Test accelerator. Wire scanners will also be used to measure transverse beam halo but in order to protect the superconducting linac, wire scanners will be limited to operation in the MEBT and the $800 \mathrm{MeV}$ transport line. Also, the LEBT and MEBT emittance scanners will be utilized to make transverse tails and halo measurement.

In addition, the laser-based profile monitors also can measure transverse tails of bunch charge distribution. However, their sensitivity to tails is probably limited to $\sim 10^{-3}$. Measurement of longitudinal bunch tails and halo is more difficult. PIP-II will utilize laser-based profiling to measure bunch tails but, in comparison to the transverse measurements, their dynamic range is additionally affected by tails in the laser pulse. 


\subsection{Controls}

The control system is responsible for control and monitoring of accelerator equipment, machine configuration, timing and synchronization, diagnostics, data archiving, and alarms. PIP-II will use an evolution of the Fermilab control system ACNET [143]. This is the system that is used in the main accelerator complex and also at the FAST and PIP2IT test facilities [144]. As shown in Figure 3.110, ACNET is fundamentally a three tiered system with frontend, central service, and user console layers. Frontend computers directly communicate with hardware over a wide variety of field buses. User console computers provide the human interface to the system. Central service computers provide general services such as a database, alarms, application management, and frontend support. Communication between various computers is carried out using a connectionless protocol over UDP. Subsystems developed by collaborators based on the EPICS control system can be integrated into the main system.

The scale of the control system is expected to be similar to that of the Fermilab complex when the Tevatron was operating. The system should support up to $1 \mathrm{M}$ device properties. Time stamping must be provided so that all data from the PIP-II linac can be properly correlated with that from the existing complex. Services for alarms, data archiving, and machine configuration save and restore should be common for the entire complex. The control system should contribute less than $1 \%$ to operational unavailability. The high beam power implies the need for a sophisticated machine protection system to avoid damage to the accelerator due to errant beam pulses.

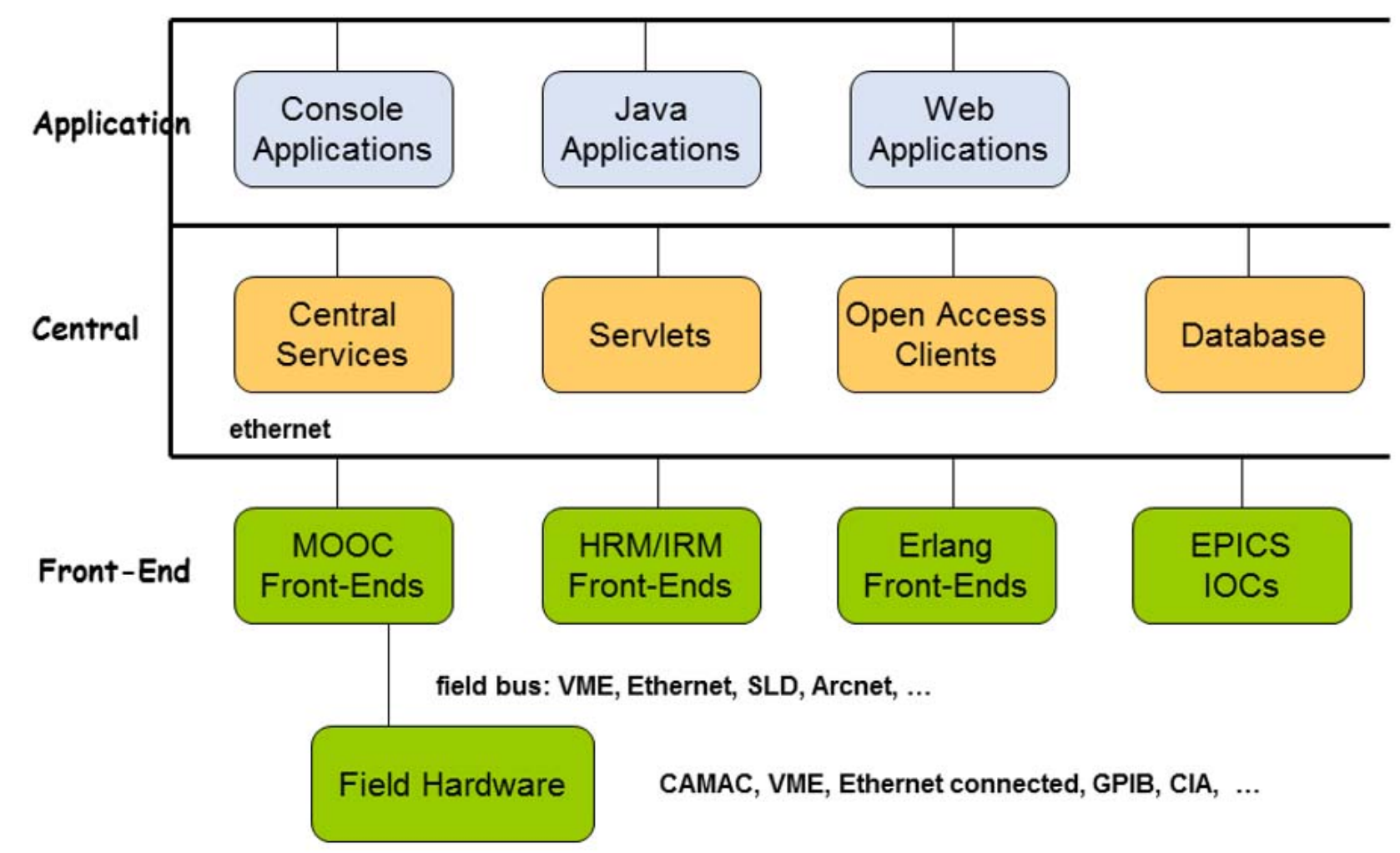

Figure 3.110: The ACNET control system.

The current control system makes use of a 2 tiered timing system for the accelerators. The present top level is a $10 \mathrm{MHz}$ based clock system (TCLK) that provides basic system co-ordination and data acquisition timing. The second level clock systems are specific to each accelerator and synchronous to beam in that machine (MIBS, RRBS, PCLK, NML-CLK \& HINS_CLK). The beam sync clocks 
have base frequencies that are derived from the machine's RF to provide bunch level timing for devices such as intensity monitors, BPMs and kickers. Relevant clock events are reflected from one level of clock system to the other as needed to support operations and data acquisition. There is an additional system called MDAT that provides various machine data to the complex. Among MDAT's data are frames for individual machine states (MI, Recycler, BSSB and Switchyard), MI bus related data (programmed \& measured currents and momenta) along with various intensity monitor readings.

A new upper level timing system (ACLK) will be developed that is expected to provide a major enhancement over the TCLK and MDAT links presently used in the main complex. A simple prototype has been developed based on a $1 \mathrm{Gbps}$ data link that adds a data payload and cycle stamp to each clock event transmission. The latter will allow reliable correlation of data across different frontends. A critical requirement of the new system will be its ability to be synchronous with the existing TCLK as legacy systems around the complex that can only listen to TCLK (and MDAT) will need to be supported for the foreseeable future.

It is expected that the ACLK design will serve as the basis for a new Linac beam synchronous clock based on requirements for the SC linac with reflected ACLK events, cycle stamps and data payloads as needed. This clock in relation to ACLK will function in an analogous fashion to the existing PCLK at P2IT to TCLK today.

It is highly desirable to have a single control system operating the entire complex rather than separate systems for the new linac and for older parts of the system. There should only be a single copy of core services such as alarms and data archiving. Software applications should be able to access any device in the system. This model simplifies development and operation, and reduces long term maintenance costs of the complex. The current control system covering the Booster, Main Injector and Recycler represents a large investment in both software and hardware and it is not practical to replace it completely by the start of PIP-II operations. The strategy will be to update gradually parts of the system before and during PIP-II construction, and in support of the PIP2IT test facility. This upgrade will include modernizing the application software environment as well as replacing obsolete hardware. Upgraded timing and machine protection systems will be developed for the PIP-II linac that will accommodate the legacy hardware in the existing parts of the complex. These systems as well as linac control software will be prototyped at P2IT.

It is recognized that some equipment will be developed outside of Fermilab by institutions with expertise in the EPICS control system. Also it may be appropriate in some cases to use commercial hardware that comes with embedded EPICS software. It is planned to support integration of EPICS frontends and some core applications in the Fermilab control system. This has been demonstrated in several different ways at different levels of the control system at previous test facilities. The current ACNET system provides a gateway to EPICS IOCs at the frontend level. The control system will specify standard interfaces between its internal components as well as with technical equipment. This will make integration, testing, and software development easier and more reliable and reduce the long term maintenance load. Also standard interfaces allow parts of the system to be more easily upgraded if required for either improved performance or to replace obsolete technologies. Only portions of the system need be changed while the core architecture of the control system remains the same.

\section{Operation at $20 \mathrm{~Hz}$}

Increasing the Booster repetition rate from 15 to $20 \mathrm{~Hz}$ will require a significant change in the control system. The current timing system is based on a $15 \mathrm{~Hz}$ signal derived from the $60 \mathrm{~Hz}$ line voltage along with a $15 \mathrm{~Hz}$ signal generated by the Booster GMPS. These are transmitted out to the 
rest of the complex as TCLK events generated via the Timeline Generator (TLG). These events will have to be changed to $20 \mathrm{~Hz}$ events. The shorter time between events and beam pulses will have to be accounted for by software changes to the TLG which generates the main timing signals for the various accelerators. A variety of systems perform software tasks on each $15 \mathrm{~Hz}$ pulse and each will have to be examined to ensure there is sufficient time to complete their task when the timing moves to $20 \mathrm{~Hz}$. Though this is a major change that impacts many parts of the control system, it is currently believed that both PIP-II and the remaining parts of the existing complex will be able to adapt. 


\subsection{Radiation Safety and Radiation Shielding Design}

Design requirements and radiation limits for accelerators and beam transport lines are provided by the Fermilab Radiological Controls Manual (FRCM). The manual requires well-engineered designs that maintain occupational and environmental radiation exposures as low as reasonably achievable (ALARA) and that are compliant with applicable regulations and DOE Orders. At minimum, the accelerator shielding design is to have passive shielding elements designed to enable areas external to shielding to be classified as minimal occupancy. Minimal occupancy is defined to mean any area which is not normally occupied by people more than 1 hour in 8 consecutive hours. Dose rates for potential exposure to radiological workers in areas without continuous occupancy are to be ALARA and such that individuals do not receive more than $20 \%$ of the applicable limits. The design goal for dose rates in areas of continuous occupancy is to be less than an average of 0.05 $\mathrm{mrem} / \mathrm{hr}$ and, if possible, as low as is reasonably achievable. Reliance on active systems such as radiation safety interlocks and/or beam line instrumentation to achieve radiation safety goals should be chosen only if passive elements cannot, in view of planned accelerator operations, reasonably achieve the level of protection required by the FRCM. Discharges of radioactive liquid to the environment should be kept ALARA. Materials and components should be selected to minimize the radiological concerns, both occupational and environmental. Where removable contamination might be associated with accelerator operations, provisions should be made in facility designs for the containment of such material. Internal radiation exposure is to be minimized in accordance with ALARA principles by the inclusion of engineered controls such as ventilation, containment, filtration systems, where practicable and with appropriate administrative procedures. Efficiency of maintenance, decontamination, operations, and decommissioning should be maximized.

The FRCM specifies the manner in which radiological posting requirements are to be determined. The maximum dose is that which can be delivered under the worst credible accident in that area, taking into consideration circumstances and controls, which serve to limit the intensity of the maximum beam loss and/or its duration. Some examples of accident scenarios are (1) beam intensity significantly greater than the nominal beam intensity; (2) unanticipated beam losses; and (3) single pulse full machine loss on an element. The maximum dose is to be determined through the Safety Analysis, which shall document calculations and measurements of possible radiation exposures, radiation shielding, beam optics and other relevant information. The Safety Analysis must be reviewed and approved by the SRSO prior to construction and/or operation of the beam.

The FRCM specifies requirements for entry controls. Accelerator/beam line areas are to be posted and controlled for the normal operating conditions when the Safety Analysis proves that delivering the maximum dose to an individual is unlikely. Accelerator/beam line areas are to be posted and controlled for accident conditions when the Safety Analysis describes a scenario in which it is likely that the maximum dose may be delivered to an individual.

A Safety Analysis for PIP-II beam operation and the application of the FRCM design requirements to PIP-II are described in the remainder of this section.

\subsubsection{Radiation Limits}

\section{Safety Analysis}

A PIP-II upgrade will change the operation of SC Linac from the pulsed to $\mathrm{CW}$ regime. That will enable linac operation with megawatt scale beam power. To determine the range of normal and accident beam loss conditions, this Safety Analysis considers both the machine operation in the pulsed and CW regimes as described in Section 1. 
Accelerator components such as cryomodules and beam pipes can be damaged or destroyed very quickly by beam power even at the levels envisioned for PIP-II operation in the pulsed regime. Consequently, the control of beam loss through a machine protection system is a primary design consideration for PIP-II. The principal design features required for the control of beam loss in the SC Linac and Linac-to-Booster Beam Transport include precision alignment of all accelerator components, precise control of beam focusing, elimination of RF jitter, and precision control of beam orbit. Operation of PIP-II accelerators and beam lines without precision controls could easily result in beam losses exceeding $100 \mathrm{~W} / \mathrm{m}$. The machine protection system (MPS) is required to ensure that all beam control features are functional and operating as intended. The loss of any control feature will cause the machine protection system to inhibit beam acceleration at the ion source and LEBT. The MPS should be capable to stop machine operation within about $10 \mu$ s of sensing an abnormal condition.

PIP-II accelerators and beam lines accelerate and transport $\mathrm{H}^{-}$beams. The principal beam loss mechanisms are related to stripping electrons from $\mathrm{H}^{-}$ions; the causes of stripping include $\mathrm{H}^{-}$ion collisions with residual gas, blackbody photon interactions, Lorentz force (magnetic stripping), and intrabeam stripping. The major contribution to beam loss comes from intrabeam stripping and this has been determined to be below $0.1 \mathrm{~W} / \mathrm{m}$ (see Section 2.1.4). Losses from the remaining mechanisms are significantly smaller (see Section 2.2.1).

The beam power delivered by the Fermilab Booster has risen significantly over the lifetime of this accelerator, while the radiation shielding available over the Booster beam enclosure has remained fixed. Improvements resulting from PIP and other machine upgrades have led to increasing beam power while controlling and reducing beam losses. An interlocked radiation detector array has been a necessary protective measure at the Booster accelerator to compensate for the fixed shielding inventory. The most recent PIP upgrades have raised the Booster beam power to $80 \mathrm{~kW}$ while keeping the beam losses at fixed or reduced levels. Since it was not practicable to increase Booster radiation shielding, it was necessary to continue with reliance upon an interlocked radiation detector system to limit the severity and duration of beam loss conditions. Reliance upon an interlocked radiation detector system makes it implicit that radiation levels are controlled at defined, normal loss conditions. That is, the normal loss condition is the de facto maximum beam loss condition. The maximum normal loss condition, is limited by the FRCM, while the nominal upper limit is set by the laboratory's well established shielding review process.

The MI-8 line and the Main Injector accelerator are heavily shielded with 24.5 feet of earth equivalent shielding. An addition of radiation shielding to the MI-8 line and the Main Injector accelerator would be both costly and of limited utility. For example, at a beam energy of $8 \mathrm{GeV}$, a $22.5 \mathrm{~kW}$ beam loss is required to exceed the $1 \mathrm{mrem} / \mathrm{hr}$ on the surface of the shielding berm. Various machine protection features inhibit the continuous loss of beam power at this level. As is the case for the Booster accelerator, the shielding assessment process is employed to ensure the limits of the FRCM are observed for the MI8 line and the Main Injector. If found to be necessary, a comprehensive, interlocked radiation detection system (TLM) described below could be employed to ensure compliance with all requirements of the FRCM.

The shielding prescription required by this Safety Analysis for the SC Linac and the Linac-toBooster Transfer Line is discussed below.

\section{Facility Design Beam Loss Level}

The conclusion of the above Safety Analysis is that the average beam loss for the SC Linac and the Linac-to-Booster Beam Transport Line under normal conditions will be of the order of $0.1 \mathrm{~W} / \mathrm{m}$. 
Machine protection systems will monitor the performance of beam focusing, beam orbit, RF stability, and machine alignment. Machine protection systems will reduce accelerator beam power or inhibit accelerator operation in the event the precision control of the accelerator is lost. The reaction time of the machine protection systems under consideration is lower than $10 \mu \mathrm{s}$. Therefore, only operation under normal conditions should be possible. For the purposes of the facility shielding design, it is assumed that the peak average beam loss will be $10 \mathrm{~W} / \mathrm{m}$, a factor of 100 higher than what is expected during nominal beam operating conditions.

\section{Facility Radiological Design Goals}

The design goals for the PIP-II SC Linac and the Linac-to-Booster Beam Line meet or exceed the minimum requirements of the FRCM stated above. The design goals are:

1. Permit unlimited occupancy for all service buildings, shielding berms, parking lots, control rooms, and associated areas. By design, radiation levels are to be kept below $0.05 \mathrm{mrem} / \mathrm{hr}$ in all accessible locations outside of the beam enclosures for normal operating conditions, based upon an assumed continuous beam loss of $10 \mathrm{~W} / \mathrm{m}$. The actual nominal beam loss condition described in the Safety Analysis is expected to be about $0.1 \mathrm{~W} / \mathrm{m}$.

2. Permit inspection and maintenance activities within tunnel enclosures while maintaining personnel radiation exposure due to residual activation of accelerator components and beam enclosures at levels as low as reasonably achievable. At $0.1 \mathrm{~W} / \mathrm{m}$, the residual dose rates should not exceed about $15 \mathrm{mrem} / \mathrm{hr}$.

3. Limit radiation exposure due to air activation both within the beam enclosure during inspection and maintenance activities and at the site boundary.

4. Limit ground water and surface water activation to levels well below regulatory standards.

5. Prevent the activation of accelerator component surfaces to avoid the generation of removable radioactivity.

6. Minimize the activation of accelerator components which can impact their useful service life.

\subsubsection{Radiological Design Requirements}

Nominal beam loss throughout the PIP-II accelerator and beam lines is expected to be about 0.1 $\mathrm{W} / \mathrm{m}$. Machine Protection Systems will limit or inhibit beam operations within $10 \mu$ s sensing a machine fault. The design requirements for radiation shielding discussed below are based upon an assumed continuous beam loss of $10 \mathrm{~W} / \mathrm{m}$. The consequences of the activation of accelerator components, enclosure structures, air, water, and removable contamination are discussed in terms of the expected nominal beam loss of $0.1 \mathrm{~W} / \mathrm{m}$ as defined in the Safety Analysis.

\section{Radiation Shielding}

A typical cross section conceptual design of the PIP-II accelerator enclosure is shown in Figure 3.111 with the enclosure height of 13.6 feet indicated. The passive shielding of 13.5 feet will vary along the length of the enclosure from 13.5 feet at the north end to 18.5 feet at the south end. A cross-section of Linac-to-Booster transfer line near the Booster is shown in Figure 3.112. This transport line will be crossing the beam line delivering the $120 \mathrm{GeV}$ MI beam to the experimental areas (former Tevatron/Main Ring tunnel). The tunnel height is 8 feet and the passive shielding is 18.5 feet. The line will be crossing the Tevatron enclosure near its ceiling (see details in Section 2.2.2). 


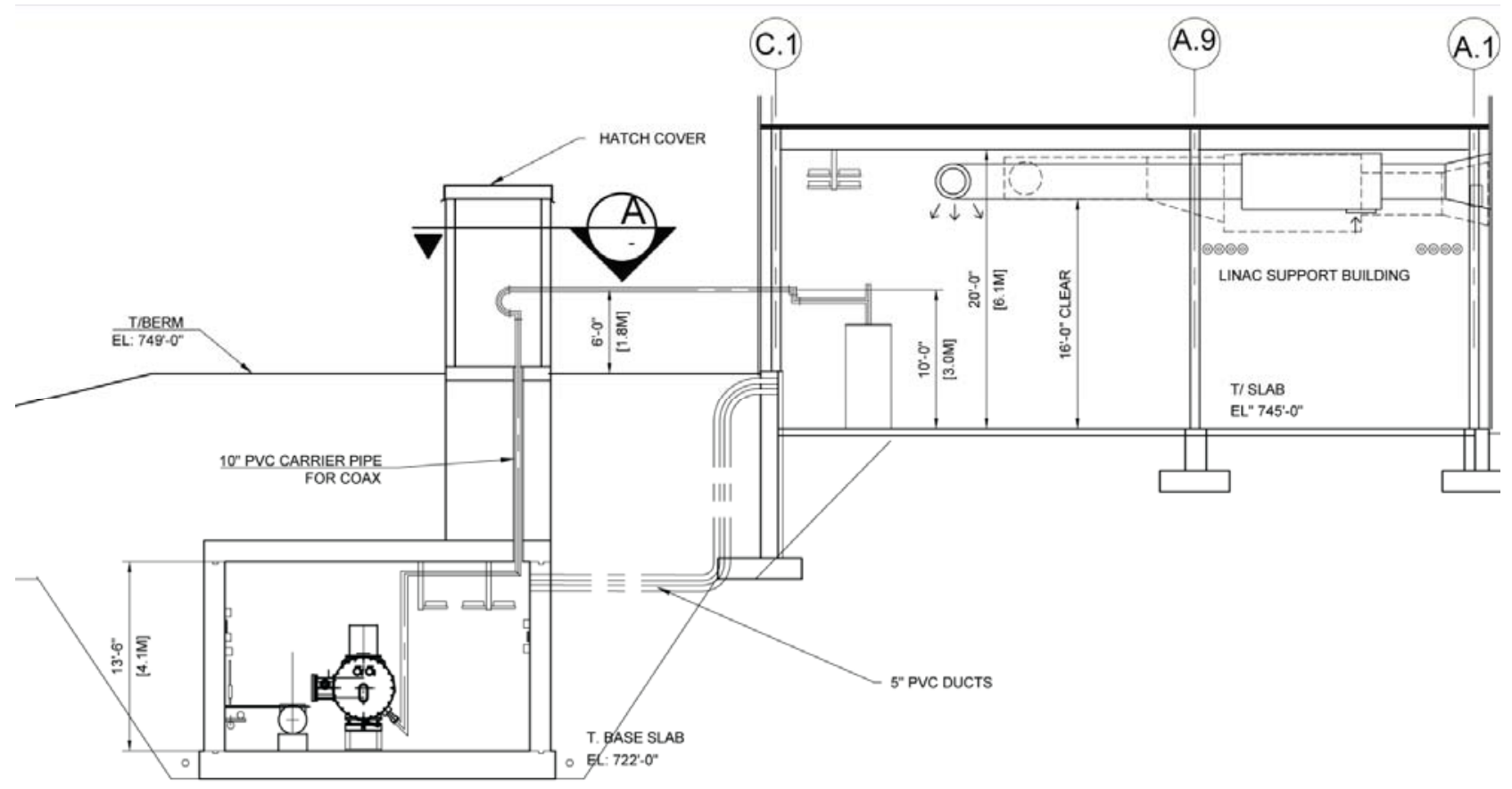

Figure 3.111: An early concept for the cross section of PIP-II linear accelerator enclosure

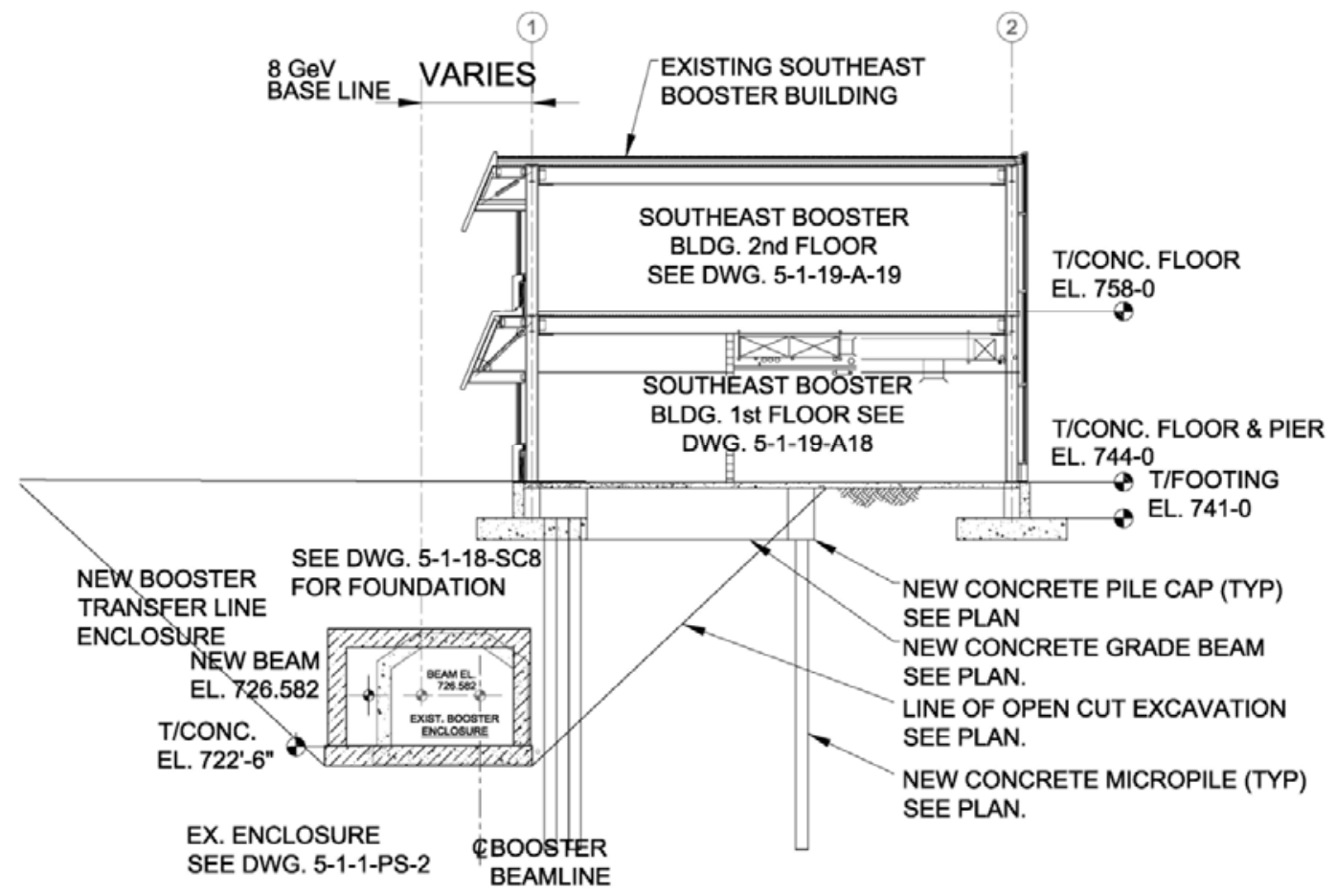

Figure 3.112: Cross-section of $800 \mathrm{MeV}$ transport line near the Booster accelerator.

An established parameterization [145] is used to determine the radiation dose equivalent rate as a function of energy $(\mathrm{GeV})$, distance (feet), and angle with respect to incident beam direction (degrees) from a low energy proton beam $(<1 \mathrm{GeV})$ incident upon a target. The parameterization determines the dose per incident proton (mrem) at one foot as: 


$$
\left(E, r, \theta_{s}\right)=2 \times 10^{-5}\left(1+\mathrm{E}^{0.6}\right)\left(\frac{1-e^{-3.6 E^{1.6}}}{\left(0.3048 r\left(\theta_{s}+\frac{40}{\sqrt{E}}\right)\right)^{2}}\right)
$$

The neutrons produce a major fraction of the radiation behind the shield. For neutron energies below $1 \mathrm{GeV}$, the attenuation length in concrete strongly depends on the energy of neutrons. The mean free path of low energy neutrons relative to the high energy asymptote has been parameterized as [145]:

$$
\frac{\lambda_{L E}}{\lambda_{H E}}=1-0.8 e^{-5^{*} E}
$$

The reduction in radiation dose rate as a function of energy $(\mathrm{GeV})$ and concrete shield thickness (feet) is:

$$
A\left(E, T_{\text {conc }}\right)=10^{\frac{-T_{\text {conc }}}{3}\left(1-0.8 e^{-5 E}\right)}
$$

In the below dose rate estimates, the peak neutron energy, $E(\mathrm{GeV})$, is taken to be equal to the beam energy. This simplification is conservative in that the actual neutron energies are necessarily lower and hence lead to better attenuation provided by the concrete shielding than indicated by Eq. (3.9). In addition, the dose equivalent per neutron conversion factor is taken as a constant value of $40 \mathrm{fSv} / \mathrm{n}$ over the full range of the neutron spectrum. Thus the resulting shielding estimates are implicitly conservative.

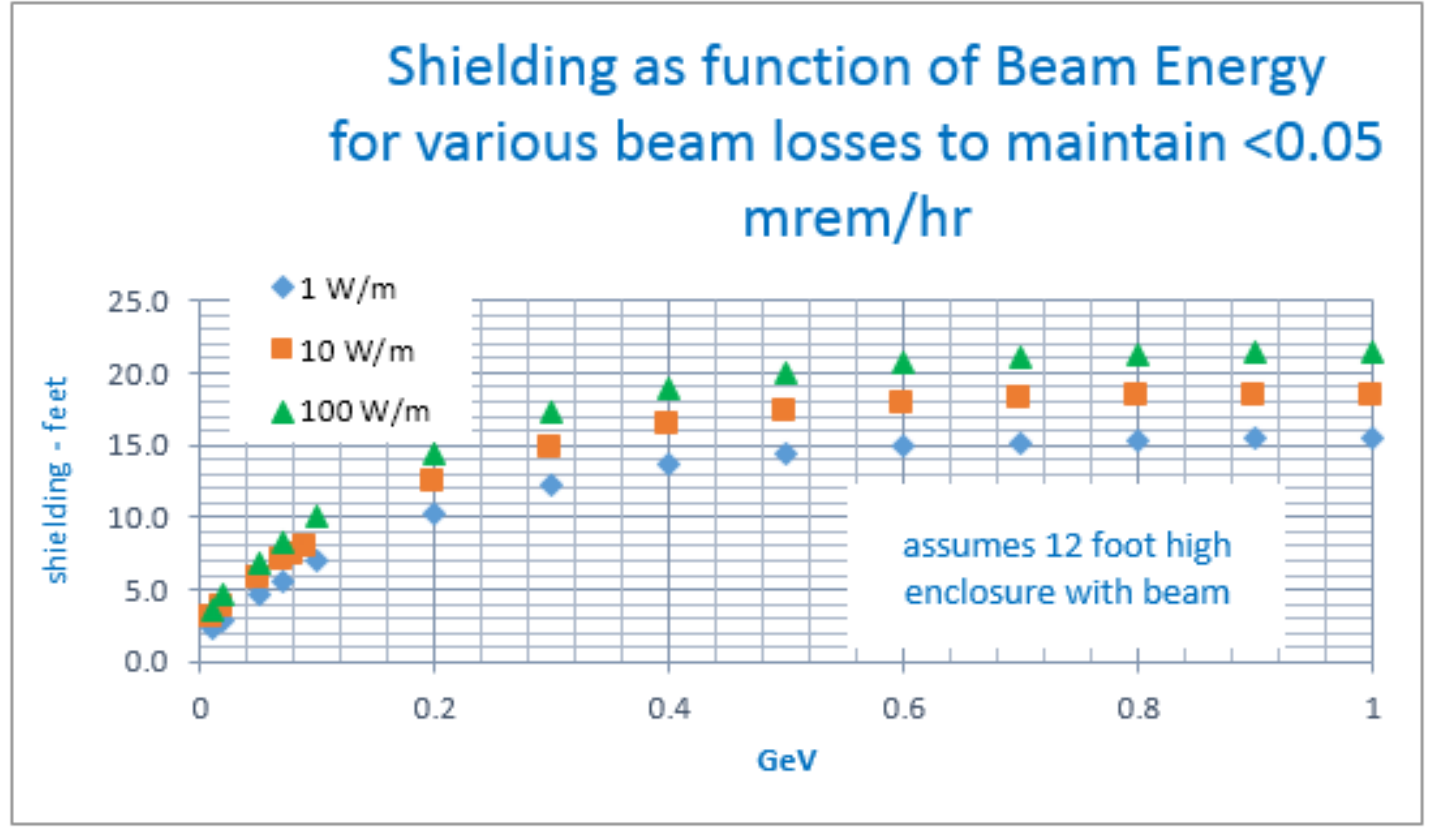

Figure 3.113: Radiation shielding requirements as a function of beam energy and beam power loss for a PIP-II beam enclosure

The radiation shielding required to limit radiation dose rates to $0.05 \mathrm{mrem} / \mathrm{hr}$ for beam energy in the range $[0,1] \mathrm{GeV}$ and various levels of beam loss is shown in Figure 3.113. The shielding requirement varies with beam energy and assumed maximum beam power loss, and it is different for normal and accident conditions. The choice of shielding thickness will take into account a number 
of factors including the confidence level given to the Safety Analysis including consideration of the projected loss mechanisms and the machine protection system.

The Safety Analysis should also determine a layout and a cross-section of the wave guides delivering power for $650 \mathrm{MHz}$ cryomodules.

An active protection system based on the Total Loss Monitor (TLM) has been developed and implemented. It can be used to guarantee the limitation of any given beam power loss. The use of a TLM system will help to fix the level of beam power loss, and, as a consequence, fix the amount of radiation shielding required.

The TLM is an argon/ $\mathrm{CO}_{2}(80 \% / 20 \%)$ gas filled ion chamber of variable length with an applied bias voltage. Beam loss in the vicinity of the ion chamber produces a charge whose magnitude is proportional to the amount of beam loss. The TLM response to an $8 \mathrm{GeV}$ proton beam loss made under controlled conditions measured over a wide range of bias voltage and over two decades of beam intensity has been determined as shown in Figure 3.114. The response has been shown to be independent on the TLM length. At the nominal bias of 800 volts, the TLM response to $8 \mathrm{GeV}$ proton beam loss is about $3 \mathrm{nC} / \mathrm{E} 10$ protons. Preliminary scaling laws, to be verified in further TLM development work, can be used to predict TLM response at other energies.

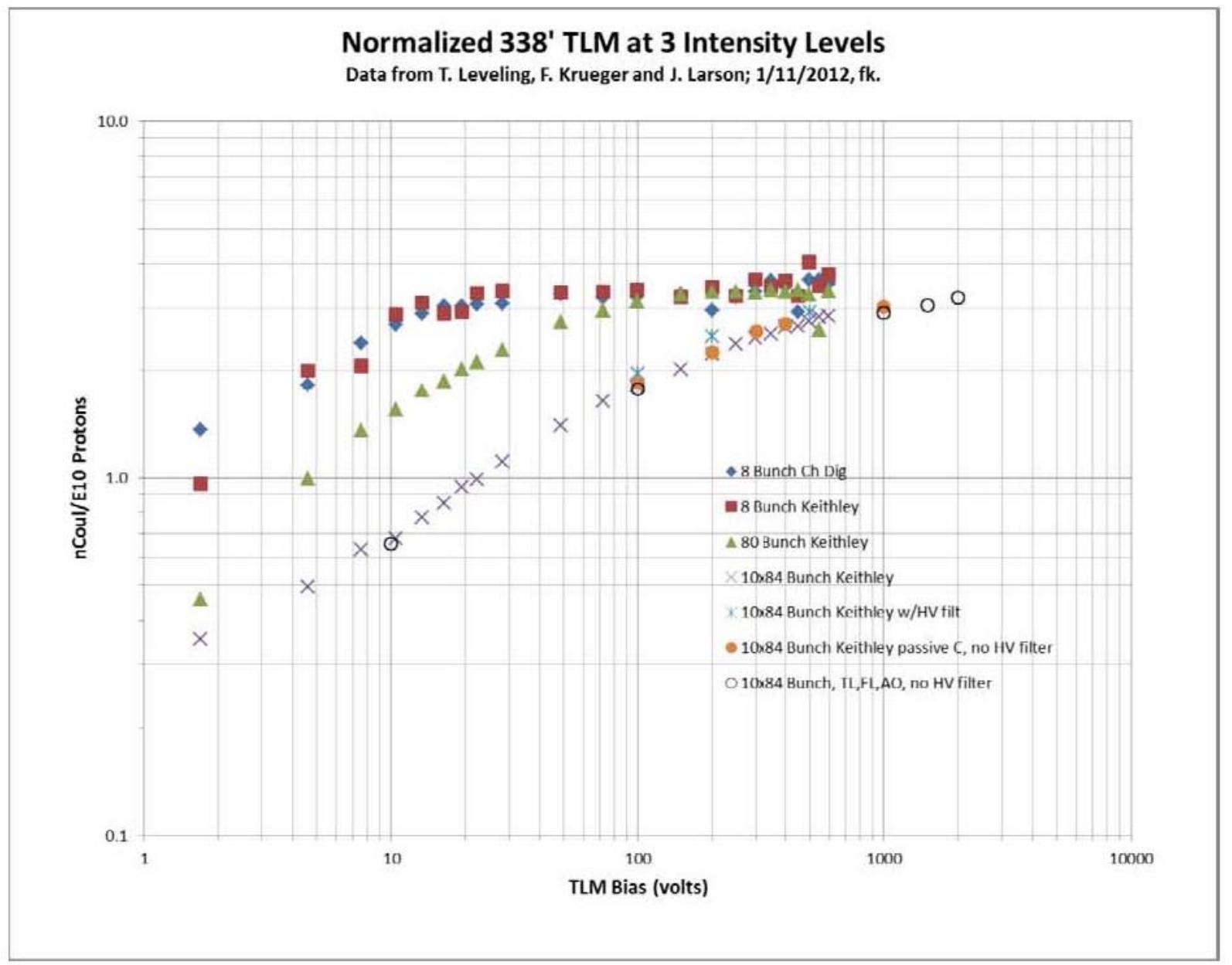

Figure 3.114: Response of 338 foot (103 meter) TLM as a function of applied bias voltage over 2 decades of beam intensity

The response can be scaled to beam energy down to $1 \mathrm{GeV}$ by the relationship: 


$$
\frac{3 \mathrm{nC}}{10^{10} \text { protons }} \times\left(\frac{E}{8 \mathrm{GeV}}\right)^{0.8}
$$

The response to beam energy below $1 \mathrm{GeV}$ remains to be determined.

A feature of the TLM system is that an interlock trip level can be established to limit beam loss to $1 \mathrm{~W} / \mathrm{m}$ or virtually any other beam power loss. A TLM, as presently conceived, does not distinguish between distributed losses and single point beam losses. The process to set TLM trip levels consists of two steps: (1) establish the total charge to be collected for a distributed loss, e.g., $1 \mathrm{~W} / \mathrm{m}$, (2) evaluate the shielding considering that the total charge is deposited at any single location. If the shielding is sufficient for the maximum charge collection rate at any location, then the TLM can effectively limit both the distributed beam loss and worst case single point beam loss.

Based upon significant TLM response characterization studies completed for the 2017 Booster Shielding Assessment, it should be possible to limit PIP-II beam loss with a TLM system which begins at the HWR cryomodule and continues through the entire accelerator and the beam transport line.

\section{Residual Activation of Accelerator Components and Structures}

Residual radiation levels in beam transport lines and accelerators due to operational beam losses must be controlled in order to conduct maintenance activities while keeping personnel radiation exposure as low as reasonably achievable (ALARA). For $1.6 \mathrm{MW}$ beam power, even $0.1 \%$ loss would result in very high residual radiation levels which would render a beam enclosure access difficult and maintenance near loss points extraordinarily difficult. A sensitive machine protection system, which inhibits the beam operation when significant losses are present, is required to allow access and maintenance activities as historically enjoyed at Fermilab.

For design purposes, a loss rate of 3 to $10 \mathrm{~W} / \mathrm{m}$ results in a dose rate of about $100 \mathrm{mR} / \mathrm{hr}$ at one foot from beam line components within magnets following a 30 day irradiation period and 1 day of cool down. A loss of $0.25 \mathrm{~W} / \mathrm{m}$ results in a dose rate of about $100 \mathrm{mR} / \mathrm{hr}$ at one foot from low mass components such as beam pipes for the same irradiation/cooling period. Radiation levels considered acceptable are typically at least a factor of 5 less than these levels. For example, for a typical magnet beam loss location at $2 \mathrm{~W} / \mathrm{m}$, the fractional beam power loss is $1 \mathrm{ppm}$. A sensitive machine protection system will be required to quickly identify and suspend operation in the event such losses occur.

In the Safety Analysis, projected normal losses due to intrabeam scattering and other loss mechanisms are below $0.1 \mathrm{~W} / \mathrm{m}$. The machine protection system as presently conceived should limit beam loss to $<0.25 \mathrm{~W} / \mathrm{m}$. Consequently, residual activation of the accelerator, beam line components, and tunnel structures should be comparable to or less than levels tolerated in existing and previous Fermilab accelerators. While the machine protection system would serve to protect the accelerator and beam line components, the TLM system would serve in a parallel role as a personnel safety system to limit residual activation of accelerator components.

\section{Air Activation}

Air activation must also be characterized for the projected PIP-II operations. Based upon the anticipated losses described in the safety analysis, the combination of anticipated normal beam loss and the machine protection system should serve to limit the total beam loss levels at or below those produced at existing facilities. Based upon projected losses from the Safety Analysis, no significant air activation is anticipated. While the machine protection system would serve to protect the accelerator and beam line components, the TLM system would serve in a parallel role as a personnel 
safety system to limit air activation within accelerator enclosures.

\section{Water Activation}

The site chosen for the new PIP-II accelerator and beam line enclosures is inside the former Tevatron ring. In order to evaluate surface and ground water activation, a geological survey (core borings) will be required to understand ground water migration rates at this site since no data presently exists. An estimate of surface and ground water activation is necessary in order to ensure compliance with regulatory requirements for surface and ground water. However, based upon losses projected by the Safety Analysis, no significant surface water or ground water activation is anticipated. The machine protection system would serve to limit the total beam loss that would also determine the level of surface water and ground water activation. The TLM system would serve a parallel, redundant role to also limit surface and ground water activation.

\section{Radioactive Surface Contamination}

Radioactive surface contamination happens together with the activation of accelerator and beam line components. Maintenance activities are rendered more complicated when radioactive surface contamination is present due to prescriptions for the use of personnel protective equipment including coveralls, gloves, shoe covers, and other protective measures. It is possible in megawatt-scale beam power machines to produce very significant levels radioactive surface contamination at beam loss locations. However, as indicated in the Safety Analysis, the nominal beam power losses are expected to be approximately $0.1 \mathrm{~W} / \mathrm{m}$, about a factor of 100 below the beam power loss required to produce the onset of measurable radioactive surface contamination. Consequently, radioactive surface contamination on the accelerator and beam line components, and on the tunnel structures should be comparable to or less than levels tolerated in the existing and previous machines.

\section{Lifetime of Machine Components}

Based upon the level of beam loss projected by the Safety Analysis and also upon experience with existing accelerator and beam line facilities, machine component lifetimes should, in general, be on the order of many decades. 


\subsection{Machine Protection System}

The PIP-II linac is being designed to accelerate up to $2 \mathrm{~mA}$ average beam current. Initially the machine will provide $800 \mathrm{MeV}$ high intensity beams for the Fermilab neutrino program and should be capable of working in a pulsed $(0.54 \mathrm{~ms}, 20 \mathrm{~Hz})$ mode for injection into the Booster. That corresponds to $1.1 \%$ duty factor and an average beam current of $22 \mu \mathrm{A}$. The future mode of PIP-II operation is to deliver a quasi-CW beam simultaneously to multiple users (e.g. muon experiments). A quasi-CW beam implies that the beam current is constant when averaged over one period with duration of few microseconds, where the bunch sequence inside one period is set by requirements coming from experiments and, from the accelerator points of view, the bunch sequence is arbitrary (i.e. cannot be determined at the design stage, and can be changed at any time in the course of machine operation). This planned upgrade to $\mathrm{CW}$ operation implies that the total beam current and damage potential will be greater than in any present HEP hadron linac.

A robust Machine Protection System (MPS) shall protect the linac and its components from direct beam induced damage and excessive radiation damage. This Machine Protection System (MPS) will be a part of the entire Fermilab complex MPS responsible for protection of equipment in PIP-II and associated downstream machines from beam induced damage.

The main goal of the MPS is to protect the machines from beam induced damage; thereby inhibiting the beam in case of excessive beam loss, equipment failures, or operator request. In achieving that objective, the system will also provide the following features:

- Manage beam intensity and permit limits of MPS designated devices while providing post mortem data to the control system,

- Provide a comprehensive overview of the machine state and readiness status to subsystems and the broader complex,

- Provide a global synchronization trigger for beam related system fault analysis,

- Provide linac beam status to the accelerator complex control system,

- Provide high availability and fail safe operation where possible,

- Manage and display MPS alarms.

The MPS is not a personnel safety system, however, it is required to operate in a fail-safe manner. The design will take into account the redundant implementation of critical MPS components where possible to reduce probability of costly damage and corresponding downtime.

\subsubsection{MPS Design}

The PIP-II MPS will comprise of a logic system that takes in signals from various sub-systems and drives permits to beam enabling devices. These devices interacting with the MPS will be divided into primary and secondary categories based on how critical they are to mitigating beam damage. Primary devices are main actuators for beam and should guarantee that, when they function properly, no dramatic damage can be caused by the beam even if protection through secondary devices fail. Both categories include sensing and beam-inhibiting devices.

The primary beam-inhibiting devices are located at the Ion Source and in the Low Energy Beam transport (LEBT) section. They will include the LEBT chopper, the LEBT dipole, the Ion Source modulator and the Ion Source bias power supply. These Primary devices shall not be masked for normal operations. However, there will be situations, such as MPS commissioning, when masking some of these devices will likely be required. In such cases, one can only mask these devices after receiving permission from the machine leader, MPS coordinator, and Operations Department, as a 
form of administrative configuration control. Special instrumentation and diagnostics specifically developed for MPS use are included as primary devices. These will include a beam transmission loss system which compares beam current at various locations along the linac as well as permit signals from users indicating their readiness to receive beam from the PIP-II linac.

The secondary beam inhibiting devices are those devices whose malfunctioning will not create dramatic damage; either because the effects can be detected and mitigated by the primary devices, or because the inclusion of the devices into the MPS is for the protection of the device itself (e.g. insertion devices). These secondary devices further decrease the probability of damage and possible irradiation of components. The list of secondary sensing devices includes: the system providing the beam request sequence from the accelerator complex, status signals from the Linac subsystems, e.g. RF amplifiers, magnet power supplies, quench detection system, cryogenic system, LCW, the control system etc. In addition, these also include malfunctioning subsystems which can affect the beam delivery (e.g. RF amplifier) thereby dropping the Linac beam permit.

Devices and systems that are beam losses indicators (like radiation monitors, current signals induced on scrapers by the beam), vacuum gauges, valves and positions of insertion devices will also be capable to inhibit the beam.

Examples of beam-inhibiting secondary devices are the MEBT chopper, switching magnets, separators, and beam stops that can prevent the beam entering an alarmed area.

General protocols of interaction between the secondary devices and MPS should be described in PIP-II MPS Technical Requirement Specifications (TRS), while details of specific levels and timings may be described separately.

\subsubsection{Protection of the Warm Frontend}

Detection of beam losses in the PIP-II warm frontend is performed mainly by secondary devices, in particular, by the scraping system. Complicated trajectories, which vary from bunch to bunch, and the changing beam structure along the beam line, make it impractical to designate a small set of devices to be primarily responsible for the beam loss detection. On the other hand, lower beam power density, negligible residual radiation, lower sensitivity of the warm elements to beam losses and their lower cost to replace, decreases the potential for critical beam-induced damage. The robustness of the warm frontend should be achieved by the redundancy in secondary devices which protect the warm frontend.

\subsubsection{MPS Configuration}

The MPS will be the collection of all subsystems involved in the monitoring and safe delivery of beam to the dumps or designated user and not limited to any particular subsystem or diagnostic device. It has connections to several external devices and sub-systems. Figure 3.115 shows a conceptual overview diagram of the MPS. The top layer (1) comprises signal providers such as beam loss monitors, beam position monitors, magnet power supplies etc. Systems at this level send alarms or status information to the MPS logic subsystems (permit system) which issues a permit based on the comprehensive overview of all inputs and requests. Only simple digital signals (e.g. on-off, OKalarm) are transmitted. All devices or subsystems that are determined to be pertinent to protecting the machine or necessary for machine configuration are included. The permit system layer (2) of the MPS will be FPGA based and is thus fully programmable to handle complex logic tasks. The logic here will be designed to ensure safe operating conditions by monitoring operational input, chopper performance, the status of critical devices and by imposing limits on the beam power. The final layer of the system shows the main actuators (3). This will comprise all points where the MPS logic may 
act on the operation of the machine to prevent beam from being produced or transported.

Three functional layers

to the system:

1. Signal interface

2. Main Logic layer (permit system) includes slot ' 0 ' interface

3. Primary and Secondary Actuators for beam inhibit

MPS comprises a logic system that takes in low level signals from various beam instruments and interlock systems and drives permits to low energy beam enable devices.

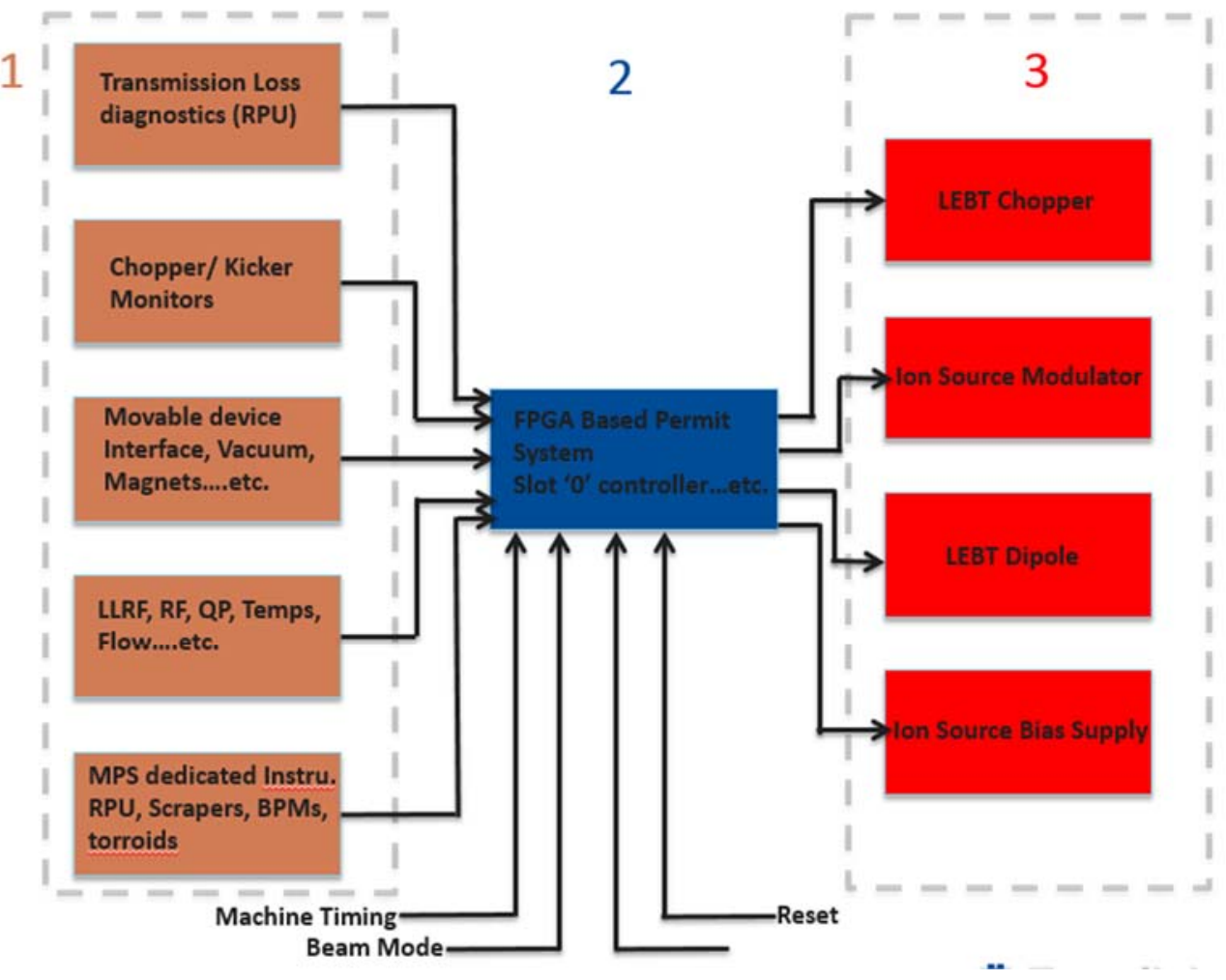

Figure 3.115: MPS Conceptual Layout

The comprehensive overview of the entire machine will be obtained by monitoring of all relevant inputs from machine diagnostics and critical systems affecting safe operation.

The protection system model is based on experience gained from commissioning and operating the SNS accelerator. Its peak current specifications are about 20 times higher than the PIP-II peak beam current specification, and its copper to SC cavity transition occurs at $187 \mathrm{MeV}$. Above 200 $\mathrm{MeV}$ the PIP-II MPS hardware design and placement can be modeled after the SNS system. The PIP-II MPS system will not need response times as stringent as the SNS because of lower peak currents. The challenge for the PIP-II MPS comes from the protection of low energy cryomodules $(2.1 \mathrm{MeV}-150 \mathrm{MeV})$ where the radiation created by beam losses has difficulty penetrating the cryomodule and beam pipe.

The Linac supplies the beam if an MPS permit is issued. The distribution of the beam between users is defined by the time sequence in the MEBT chopper and by bending magnet(s) according to users' requests summed in the Beam Switch Sum Box (BSSB).

Removing a request by a user inhibits the beam to this user but generally allows running the Linac beam to other users.

In a case of observing of an un-requested beam or a failure requiring a fast and reliable termination of the beam, the user drops the permit, interrupting the entire Linac beam.

The entire protection system interfaces with the accelerator control system and machine timing system for configuration management, timing and post mortem analysis as shown in Figure 3.116. The operational modes, operational logic, reaction time and complexity of inputs will differ based on the machine configuration and damage potential at various stages of the accelerator complex. 


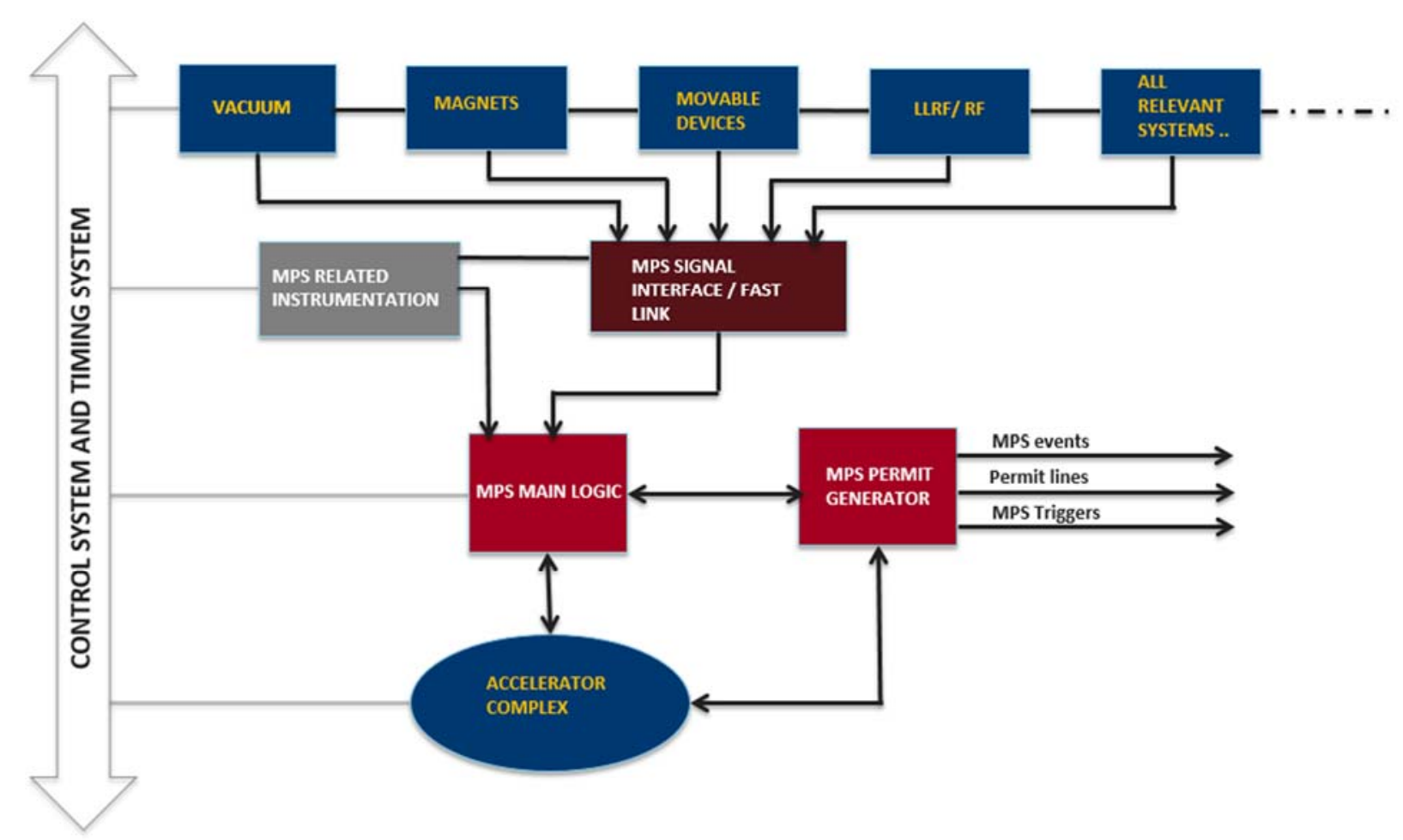

Figure 3.116: Conceptual MPS layout integrated with control system.

\subsubsection{Specifications for MPS Primary Systems}

A drop of the beam permit will inhibit the Linac beam at the entrance of the RFQ within $10 \mu \mathrm{s}$ after the PIP-II MPS receives the signal. Specific times scales relevant to interfacing to the global accelerator complex will be based on the mitigating damage potentials at downstream machine location, specific hardware limitation and signal time-of-flight issues. A separate interface document shall describe interaction with the MPS of Fermilab Accelerator Complex, including these time requirements and protocols.

In the PIP-II linac the primary systems for comparing beam current losses (Transmission Loss System) will identify the beam losses expressed as a difference between readings at specified locations and shall drop the beam permit if it detects a beam loss $>500 \mu \mathrm{A}$ averaged over $1 \mu \mathrm{s}$ sliding time window, and $>5 \mu \mathrm{A}$ averaged over one power line period (1/60 s) for operation in the CW regime. The primary system will also drop the permit if it detects a large deviation of the measured beam pattern from the expected one. This deviation is deemed large when the average current measured in a $30 \mu$ s sliding time window exceeds the expected value by more than $20 \%$ of the beam current or $20 \mu \mathrm{A}$, whichever is larger. The MPS will inhibit beam in a single step or with a two-step process designed to limit downtime. Beam inhibits by the LEBT chopper will occur within $10 \mu \mathrm{s}$ after a system drops its beam permit. This time is measured as the difference between the permit being removed at the failure location and the disappearance of the beam at the entrance of the RFQ. Alternatively, if the average beam current measured by designated primary system devices doesn't drop below measureable level within $15 \mu$ s of the fall of the beam permit, the Ion Source modulator, LEBT dipole and the Ion Source bias power supply will be turned off in a fail safe manner. MPS will be capable of initiating both steps at once in cases considered as potentially severe such as bad vacuum or failed subsystems. Scenarios with only one step is envisioned primarily to inhibit the beam in response to RF sparking, where recovery is typically fast and the beam can be restored 
automatically.

The MPS latching scheme will be capable of differentiating between the fault inputs. It will either latch the status of all failed system inputs on a fall of the permit and do not restart beam until an operator command to reset the permit and resume beam operations, or, for some pre-set fault inputs, resume operation automatically after a given time.

\subsubsection{Protection System R\&D}

Protecting the superconducting cavities from low energy protons losses where the particle energies are too low to produce significant detectable radiation will be a major part of the developmental work needed to effectively inject beam without quenches. To achieve this, we will need to research sensitive means for measuring these losses and develop an effective feedback for machine protection. In addition, we plan to achieve the following goals as a result of designing, constructing and operating the PIP2IT MPS:

- Understand and verify acceptable loss rates in the room temperature sections,

- Develop a strategy to monitor chopped beam from the MEBT,

- Estimate the particle shielding effects of superconducting cavities and cryomodules,

- Develop effective algorithms for the FPGA based logic system,

- Demonstrate effective integration with controls/instrumentation and all subsystems,

- Understand dark current effects as it relates to protection issues.

In order to protect the accelerator from damage as the beam transitions from the room temperature sections of the machine to the superconducting sections, some specialized instrumentation may be developed at PIP2IT. Developing an effective algorithm to monitor the beam position as a feedback to machine protection will be of interest for both PIP2IT and PIP-II.

\subsubsection{MPS Dedicated Instrumentation}

Several diagnostic instruments will be dedicated to provide MPS related functions and will be integral to MPS ability to determine errant beam events. These devices will be outside of the scope of normal diagnostic operation related to machine tuning and calibration. In particular, we mention here ring pickups (see Section 3.5), MEBT kicker protection electrodes and scrapers. Several ring pickups will be dedicated to monitor the LEBT chopper performance, monitor beam intensity and provide transmission loss information to the MPS. In addition, electronics designed to monitor both MEBT kicker electrodes and machine scraper currents will provide fast comparative analysis of beam-induced current signals from these devices. It is planned to provide both digital and analog integration methods for monitoring excessive beam loss of these devices. Figure 3.117 is a schematic diagram of the integration process that will be implemented. The integration criterion will be based on the characteristic time constant required to prevent damage to a given device. Ideally the total interruption time interval will limit the beam power to which the given device can be exposed below its damage potential limits. 


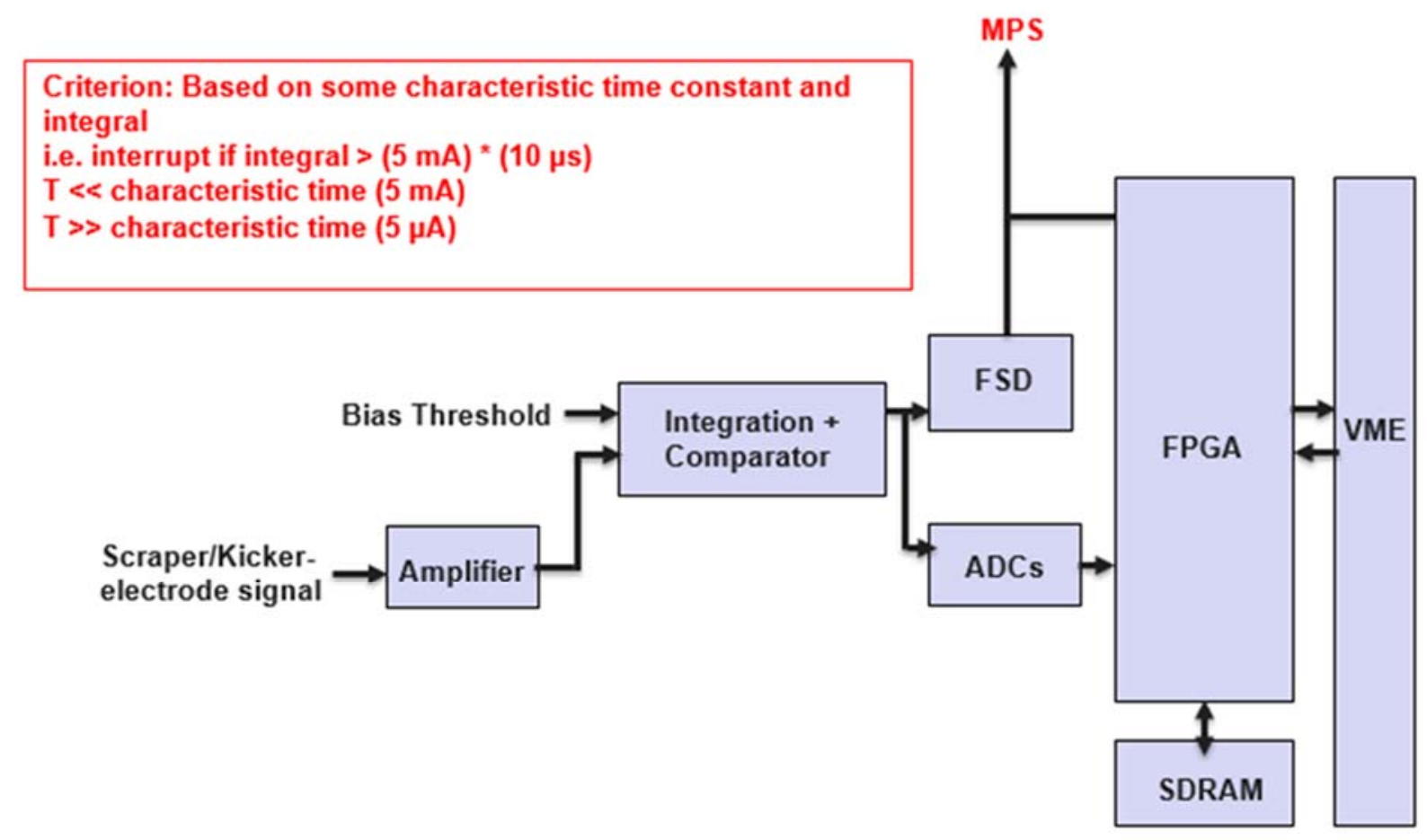

Figure 3.117: Schematic diagram of signal processing. 


\section{Siting and Conventional Facilities}

\subsection{Introduction}

The PIP-II conventional facilities will house the accelerator components and support equipment required to install and operate the PIP-II linac and transfer line. The Conventional Facilities portion of the project includes the management, planning, design and construction of new structures, buildings and utilities as well as modifications to existing structures required to install and operate the PIP-II accelerator.

The PIP-II conventional facilities scope includes the elements of work normally included in conventional construction such as earthwork, utilities, structural concrete, structural steel, architectural cladding, finishes, roofing, plumbing, process piping, heating ventilation and air conditioning (HVAC), fire protection, fire detection, lighting and electrical. This also includes the work required to extend the utilities to the project site, excavation associated with the below grade cast-in-place concrete enclosures, creation of a shielding berm and site restoration.

The PIP-II conventional facilities will consist of the following five (5) functional areas:

1. Site Work

The Site Work consists of the extension of existing utilities to the PIP-II site, wetland mitigation, roadwork and parking area, hardstands, storage tank foundations and related work to provide the supporting infrastructure.

2. Linac

The Linac functional area consists of the below grade Frontend and Linac Enclosure and the associated above grade Linac Support Building. The Linac Enclosure will house the PIP-II accelerator components as well as provide space for support functions. The Linac Support building will run parallel to the below grade Linac Enclosure and house the components required to operate the PIP-II accelerator. The Linac Support Building includes a high bay service building with a loading dock and related services to accommodate the installation and servicing of beamline components. The Linac Support building will be designed to accommodate visitor tours as part of the Fermilab outreach program.

3. Transport Line

The Transport Line functional area includes the below grade enclosures to house the beamline components required to transport the proton beam from the new Linac Enclosure to the existing Booster Enclosure and includes the conventional construction work required to cross the existing Main Ring tunnel as well as the work required to transport the beam into the existing Booster accelerator enclosure. This functional area also includes the space and equipment to house the Beam Dump.

4. Cryogenics Plant

This functional area consists of the conventional construction required to install, house and operate the cryogenic plant to support PIP-II accelerator operations.

5. Mechanical Plant

This functional area consists of the conventional construction required to install, house and operate the cooling systems to support PIP-II accelerator operations.

The scope of the conventional facilities portion of the PIP-II project will be realized through several design and construction packages. This is intended to provide a logical and constructible sequence to reduce the construction period to a minimum. Further design iterations will be required to optimize the construction packaging based on programmatic and funding limitations. The design 
methodology and construction means and methods for the conventional facilities work are expected to be similar to that which has been employed on the Fermilab site for decades.

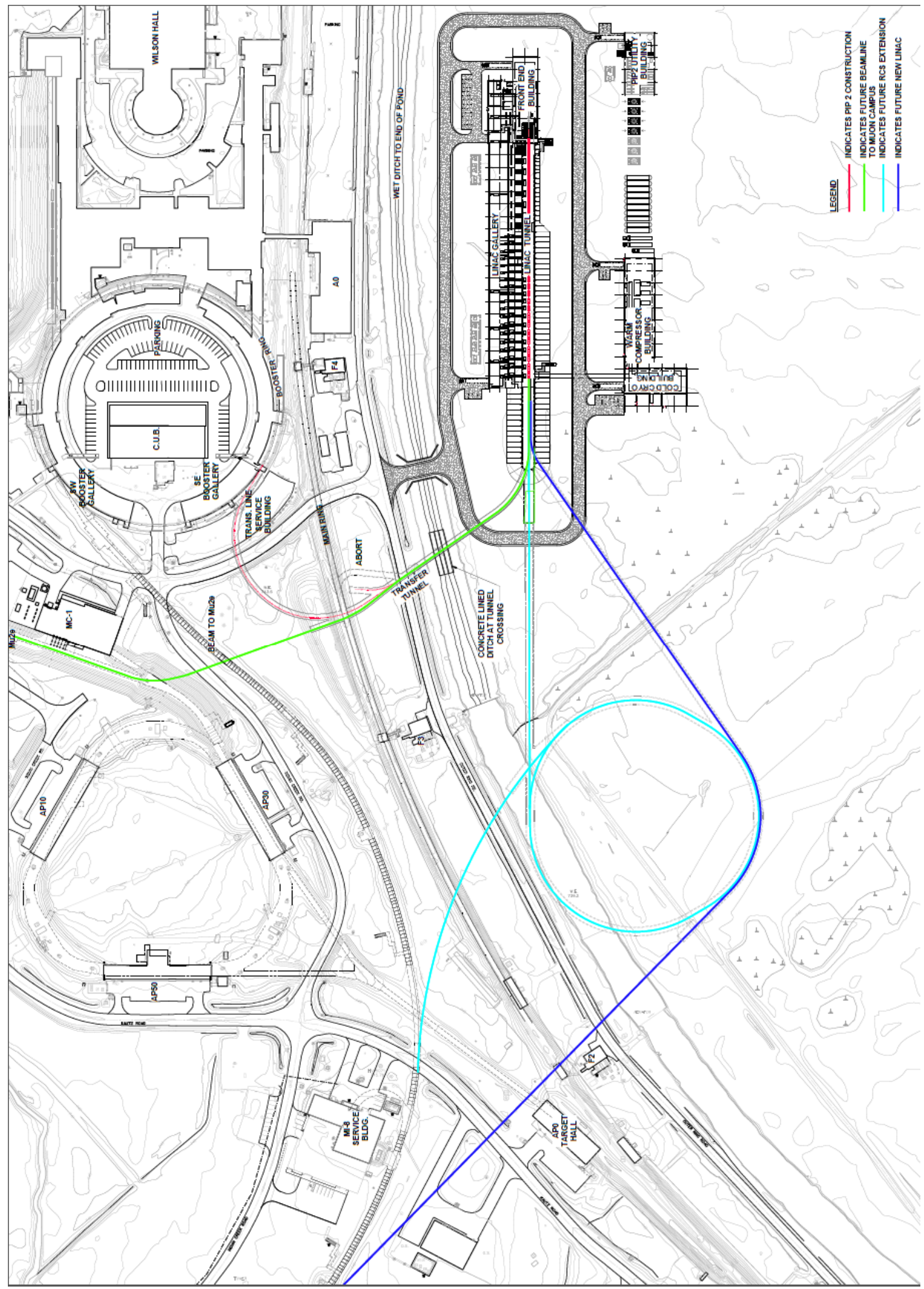

Figure 4.1: Plan view of PIP-II facilities and location of possible future expansion opportunities. 


\subsection{Siting}

The location of the PIP-II facility is driven primarily by the physics requirement for close proximity to the existing Booster accelerator (see Figures 1.1 and 4.1) and access to existing infrastructure. The location in the Main Ring infield, adjacent to the Footprint area of the Fermilab campus, allows direct access to existing electrical, water, and cryogenic infrastructure currently located in the vicinity. In addition, the Main Ring infield location is well suited to extensions of chilled water service from the existing Central Utility Building (CUB). At the same time, the Main Ring infield location provides space for future expansion opportunities.

The siting of PIP-II facility was chosen to minimize the impact to existing known wetlands within the Main Ring (Tevatron) infield as well as conform to the 2015 Fermilab Campus Master Plan [146] which has designated the area east of Wilson Hall as the Superconducting Linac Complex.

Surface construction for the PIP-II facility includes new buildings, site improvements, roadwork and parking to allow access from the Fermilab Central Campus.

Underground construction includes the Linac Enclosure, the Transfer Line enclosure, and Beam Dump enclosure. The Linac enclosure is sited at the same elevation as the Booster and the Main Ring tunnel. The Transfer Line enclosure will cross the Main Ring tunnel which holds the existing 120 $\mathrm{GeV}$ transfer line used for beam delivery to the Fixed Target Area Switchyard, which is assumed to continue its operation in the PIP-II era. The floors of two tunnels will be on the same level, but the PIP-II Transfer Line will rise up and come down in vicinity of the existing $120 \mathrm{GeV}$ line; and it will cross the Main Ring tunnel near its ceiling.

\subsection{Requirements}

The requirements for the PIP-II conventional facilities scope of work were developed from stakeholder input, organization processes and enterprise assets. The main sources of stakeholder inputs are the other subproject managers gathered from regularly scheduled meetings. The requirements employed in the design and construction of the PIP-II conventional facilities documented in the PIP-II document database [146].

\subsubsection{Organizational Processes}

Organizational Processes provide institutional requirements for the design, construction and operations of all projects built and operated at Fermilab. For the PIP-II conventional facilities these requirements are derived from the Policies and Procedures of the Fermilab Directorate, Accelerator Division (AD), and the PIP-II project. All applicable DOE orders and standards are included in these requirements. A selection of applicable standards is listed below:

- DOE Order 151.1C - Comprehensive Emergency Management System

- DOE Order 413.3B - Program and Project Management for the Acquisition of Capital Assets, Change 1 issued 11/29/10

- DOE Order 414.1C - Quality Assurance

- DOE Order 420.1B - Facility Safety

- DOE Order 430.1B - Real Property Asset Management (2/8/08)

- DOE Order 430.2B - Departmental Energy, Renewable Energy and Transportation Management

- DOE Order 450.1A - Environmental Protection Program (6/4/08)

- DOE STD-1066-99 - Fire Protection Design Criteria 
- DOE STD-1073-2003 - Configuration Management

- DOE Guide 420.1-2 - Guide for the Mitigation of Natural Phenomena Hazards for DOE Nuclear Facilities and Non-Nuclear Facilities

- 10 CFR 835 - Radiological Protection Program

- 10 CFR 851 - Worker Safety and Health Program

- $\quad 10$ CRF 851.23 - Safety and Health Standards

- Internal Fermilab permits and work notifications as described in the Fermilab ES\&H Manual (FESHM)

- Fermilab Director's Policy Manual (http://www.fnal.gov/directorate/Policy_Manual.html)

- Fermilab Engineering Manual (http://www.fnal.gov/directorate/documents/FNAL_Engineering_Manual.pdf)

\subsubsection{Enterprise Standards}

Enterprise standards from regulatory agencies, code bodies and trade organizations also provide requirements for the design and construction of the PIP-II conventional facilities. The Fermilab Engineering Standards Manual provides a comprehensive listing of applicable and adopted building codes and design standards. The applicable standards are listed below:

- Codes, Standards, and Guidelines

- International Building Code (IBC) - 2009 Edition

- International Energy Conservation Code - 2009 Edition

- International Fire Code - 2009 Edition

- International Mechanical Code - 2009 Edition

- Minimum Design Loads for Buildings and Other Structures - ASCE 7-05

- Building Code Requirements for Structural Concrete - ACI 318-05

- Specification for Structural Steel Buildings - AISC 360-05

- Building Code Requirements for Structural Concrete and Commentary - ACI 318-08

- Building Code Requirements for Masonry - ACI 530-05

- Illinois Plumbing Code - 2004

- Illinois Department of Public Health Codes

- Illinois IEPA

- NFPA 101 Life Safety Code - 2009 Edition

- NFPA 13 - Standard for the Installation of Sprinkler Systems - 2010 Edition

- NFPA 24 - Standard for the Installation of Private Fire Service Mains and Their Appurtenances - 2010 Edition

- NFPA 30 - Flammable and Combustible Liquids Code - 2008 Edition

- NFPA 55 - Compressed Gases and Cryogenic Fluids Code - 2010 Edition

- NFPA 70 - National Electrical Code - 2008 Edition

- NFPA 70E - Standard for Electrical Safety in the Workplace - 2009 Edition

- NFPA 72 - National Fire Alarm Code - 2010 Edition

- NFPA 80 - Fire Doors and Fire Windows - 2010 Edition

- NFPA 90A - Standard for the Installation of Air-Conditioning and Ventilating Systems 2009 Edition

- NFPA 90B - Standard for the Installation of Warm Air Heating and Air Conditioning Systems - 2009 Edition 
- NFPA 92A - Standard for Smoke-Control Systems utilizing Barriers and Pressure Differences - 2009 Edition

- NFPA 92B - Standard for Smoke Management Systems in Malls, Atria, and Large Spaces - 2009 Edition

- NFPA 110 - Emergency and Standby Power Systems - 2010 Edition

- NFPA 115 - Standard for Laser Fire Protection - 2008 Edition

- NFPA 780 - Standard for the Installation of Lightning Protection Systems (and UL 96A) -2008 Edition

- ASHRAE Standard 90.1-2004 Energy Standard for Buildings Except Low-Rise Residential Buildings

- ANSI/HFES 100-2007 - Human Factors Engineering of Computer Workstations

- ANSI 17.1 Safety Code for Elevators and Escalators

- ANSI/ASHRAE Standard 62.1-2004 Ventilation for Acceptable Indoor Air Quality

- ANSI/AIHA Z9.5-2003 Standards for Laboratory Ventilation

- ANSI/ASME B31.3 - Process Piping (2002)

- ANSI 31.9 - Building Services Piping (1996)

- Occupational Safety and Health Administration (OSHA)

- Underwriters Laboratory

- ICC/ANSI A117.1 - 2003 Standard for Accessible and Usable Buildings and Facilities Illinois Accessibility Code

- ADA Accessibility Guidelines for Buildings and Facilities (ADAAG) - 2004 will be used for those areas of facility not exempted by Fermilab policy

- Illinois Accessibility Code

\subsubsection{Performance Requirements}

The performance requirements listed below describe the project specific requirements that exceed or are not addressed in the applicable building codes and standards requirements contained in Organizational Process or Enterprise Standards listed above.

\subsubsection{Architectural Considerations}

The design of the above grade buildings will be developed based on the 2015 Fermilab Campus Master Plan [147] including the desire that "New buildings and structures should be designed to be fresh, inviting, innovative, dynamic and forward-looking." To this end, the buildings will incorporate the appropriate portions of the design guidelines including:

- Entrances and ground floors that are welcoming;

- Entrances that are evident in the daytime and at night;

- The ground floor will emphasize transparency;

- Service and utilities areas will be located so as to not negatively affect pedestrian paths or building entrances;

The architectural finishes inside the buildings will generally be exposed construction suitable for equipment installation and operation.

Floor surfaces in the Linac Enclosure and lower level of the Linac Support Building will receive an epoxy finish. 


\subsubsection{Underground Enclosures}

Open cut excavation techniques are anticipated for the construction of the below grade enclosures. After the concrete enclosure is constructed and damp proofed the enclosures will be backfilled using stone around the enclosure, followed by suitable clays and silts, covered with topsoil and seeded. Shielding berms will be constructed using maintainable slopes with a 1' vertical rise to 3' of horizontal run.

\subsubsection{Structural Systems}

The structural systems for the PIP-II conventional facilities are expected to be constructed utilizing conventional methods similar to systems utilized at Fermilab over the past 40 years.

Below grade enclosures will be constructed of a cast-in-place concrete, including base, wall and roof slabs. An alternate construction method utilizing precast concrete sections will be investigated during subsequent design phases. These enclosures will be designed to support the shielding loads.

The above grade buildings will be a braced steel frame with pre-finished metal siding with structural steel and where applicable designed to accommodate overhead bridge cranes.

The structural systems for the Warm Compressor Station of the Cryogenics Plant will require vibration isolation in order to avoid impacting the operation of the linac SC cavities. An Engineering Note from the LCLS-II project (LCLSII-4.8-EN-0326-R0) titled "Vibration Measurements at the JLAB Cryoplant and Linac [148] noted that compressors at Jefferson Laboratory generated ground motion that resulted in cavity detuning and adversely affected superconducting linac operations. It was also noted that the level of motion decreased rapidly with distance from the cryo compressors with a finding that compressors located $30 \mathrm{~m}$ (98.5 feet from the Linac is possible without impacting operations. In addition, the engineering note included a recommendation to mount the compressors on an isolated foundation. For PIP-II, the cryogenic compressors will be located approximately $61 \mathrm{~m}$ (200 feet) away from the Linac and be mounted on isolated foundations.

The flatness and levelness of the new floor slabs built as part of the conventional facilities will be designed for normal construction tolerances and a ASTM E1155 floor flatness value of F(F) 25 and a floor levelness $\mathrm{F}(\mathrm{L})$ of 20 .

\subsubsection{Mechanical Systems}

The HVAC systems for the PIP-II surface building will conform to ASHRAE 90.1, ASHRAE 62, applicable NFPA requirements and applicable sections of the Fermilab Engineering Standards Manual.

Mechanical systems and building automation systems controls will be designed based on Fermilab standards and in accordance with ASHRAE 90.1.

All plumbing work to be designed in accordance with Illinois Plumbing Code and Standard Specifications for Water \& Sewer Main Construction in Illinois.

Heating, Ventilation and Air Conditioning Design Parameters are as following:

Linac Support Building - High Bay:

- Temperature: 74 degrees F (+/- $2.5 \mathrm{~F})$

- Humidity: $\quad 55 \%$ maximum relative humidity, no minimum

Linac Support Building - Gallery Space:

- Temperature: 74 degrees F (+/- 2.5 F)

- Humidity: $\quad 55 \%$ maximum relative humidity, no minimum 
Linac Enclosure:

- Temperature: 74 degrees F (+/- $2.5 \mathrm{~F})$

- Humidity: $\quad 55 \%$ maximum relative humidity, no minimum

Transport Line Enclosure:

- Temperature: winter - 74 degrees F (+/- $2.5 \mathrm{~F})$ / summer - 80 degrees F (+/- $5 \mathrm{~F})$

- Humidity: $\quad 55 \%$ maximum relative humidity, no minimum

Cryogenics Plant - Warm Compressor Station:

- Temperature: winter - 68 degrees F (+/- $5 \mathrm{~F})$ / summer - 90 degrees F (+/- 5 F)

- Humidity: $\quad 55 \%$ maximum relative humidity, no minimum

Cryogenics Plant - Cold Box Station:

- Temperature: winter - 68 degrees F (+/- 5 F) / summer - 90 degrees F (+/- 5 F)

- Humidity: $\quad 55 \%$ maximum relative humidity, no minimum

Cryogenics Plant - Control Room:

- Temperature: winter - 68 degrees F (+/- 5 F) / summer - 78 degrees F (+/- 5 F)

- Humidity: $\quad 55 \%$ maximum relative humidity, no minimum

The mechanical systems in the Linac Support Building are designed to accommodate two different operating conditions: Pulsed Mode (PM) and Continuous Wave (CW). The design for each operating condition is driven primarily by the heat load rejected to air by the RF equipment housed in the gallery of the Linac Support Building. Most of heat will be intercepted by LCW. Still, considerable heat load goes to air.

Under pulsed mode linac operations the heat load will be handled by a HVAC system utilizing ducted air handling units located throughout the gallery space. Chilled Water (CHW) will be used to provide a cooling medium for this mechanical equipment. The source of the $\mathrm{CHW}$ for this system will be the existing chillers in Central Utility Building (CUB). The CHW supply and return piping will be extended to the PIP-II project site. Currently, the CHW system has the capacity to accommodate the anticipated PIP-II loads.

The capacity of the existing CUB CHW system is approximately 250 tons which is sufficient for cooling the RF equipment heat load under PM operation. The increased heat load under $\mathrm{CW}$ operation and the limited capacity of the CHW plant at CUB require supplemental cooling.

In order to provide cooling under $\mathrm{CW}$ linac operations, a supplemental chilled water system will be used to provide the $\mathrm{CHW}$ used under $\mathrm{CW}$ linac operations. This chiller system will be located in the PIP-II Utility Building and supply and return piping will be routed underground to the Linac Service Building. The mechanical system inside the gallery space will be based on ducted air handling units.

The overall HVAC system is designed to be modular and operated at the lower heat loads in PM operations using CHW from CUB and supplemented using the new chiller located in the PIP-II Utility Building to operate at the higher heat loads anticipated during $\mathrm{CW}$ operations.

Industrial Cooling Water (ICW) will be used to provide a cooling medium for the cryogenic compressors housed in the Warm Compressor Station of the Cryogenics Plant. The existing site wide ICW will be extended to the PIP-II project site where it will be strained/filtered to achieve the PIP-II water quality requirements. In order to provide a cooling medium that meets the cryogenic compressor requirements, the ICW supply will be routed through a heat exchanger located in the PIP-II Utility Building. This will allow the compressor side of the heat exchanger to be a closed 
loop system with water treated to meet the cryogenic compressors requirements. The ICW will be discharged into a new return ditch and routed to existing return routes to Casey's Pond.

A series of evaporative fluid coolers will be used to provide a cooling medium for the Low Conductivity Water (LCW) system without the use of chillers. This modular design approach will provide the direct cooling of the LCW system without the need for heat exchangers, or cooling ponds. ICW was considered as a cooling medium, however the site-wide ICW system lacks the capacity to provide this cooling.

The Linac Enclosure will be designated as an Oxygen Deficiency Hazard (ODH) location and will require a protection system. The Conventional Facilities design will provide the mechanical equipment including fans, louvers and ductwork for the ODH mitigation system. The sensors, controls and programming of the systems will be the responsibility of the controls department.

\subsubsection{Electrical Systems}

The electrical power for PIP-II facility will be provided by extending the existing site wide medium voltage feeder system to the project site in new concrete encased power duct bank.

The conventional facilities portion of the work will include the medium voltage distribution including ductbank, feeders, switches, transformers and incoming service feeds. The conventional facilities portion of the experiment power will end at the incoming service panel.

The house power including general power, lighting and power for mechanical equipment will be the responsibility of conventional facilities.

Emergency lighting and exit signage will be installed as part of the conventional facilities portion of work in accordance with Fermilab guidelines and requirements.

A UFER style ground will be installed that includes connection of the concrete reinforcing to the building ground system and structural elements of the above grade surface buildings.

Basis networking will be installed as part of the conventional facilities portion of the work. This includes extension of the site wide data/communication network to the PIP-II project site. The surface buildings will be provided with basic networking infrastructure including VOIP phone lines, connections to building automation systems and wireless access points. Extension and improvement of the data/communication system for experimental equipment will be done as part of the equipment installation.

The surface buildings will include electronic access control based on the existing site wide security system. This will include access to main building entrances.

\subsubsection{6. $\quad$ Fire Protection Systems}

Fire Alarm/Fire Suppression systems will be designed in accordance with the applicable sections of the Fermilab Engineering Standards Manual.

Automatic sprinkler systems will be designed to a minimum of an Ordinary Hazard Group 2 classification, in accordance with National Fire Protection Association (NFPA) latest edition. The most commonly used NFPA standards relative to automatic sprinkler systems are: 13, 20, 25, 318 and 750. Automatic sprinklers will be installed in buildings. Automatic sprinklers are not required in the enclosures or vertical exit passageways, based on the Main Injector Life Safety Fire Protection Analysis. Automatic sprinklers were installed in the Main Injector at enclosure stairs connecting to surface buildings and for 50' on either side of the stair alcove. These assumptions will be validated during subsequent design phases by life safety consultants.

The below grade enclosures will be designed to allow for safe passage of personnel through the 
enclosure during operations and installation. Egress shall be spaced so that travel distances are no greater than 300 feet where there are two paths of travel to an exit and no more than 50 feet when sprinklered (25 feet without sprinklers) where there is a single path of travel to a vertical exit. Doors will be located at the enclosure levels at each exit but since the vertical distance to the exit discharge is less than thirty feet double doors are not required. All exits from below grade enclosures will lead to an exit discharge without requiring travel through a building.

Fire alarm systems will be designed with a minimum standby power (battery) capacity. These batteries will be capable of maintaining the entire system in a non-alarm condition for 24 hours, in addition to 15 minutes in full load alarm condition. The most commonly used NFPA standards relative to fire alarm systems are: 70,72,90A, and 318. Manual pull stations and alarm notifications will be provided in enclosures and buildings. In addition, fire extinguishers will be provided in accordance with FESHM Chapter series 6000.

The facility will be equipped with a hard-wired, zoned, general evacuation fire alarm system consisting of:

- Manual fire alarm stations at the building exits

- Sprinkler system water flow and valve supervisory devices

- Combination fire alarm horn/strobe located throughout the building

- A 24 volt addressable fire alarm control panel

- Connection to the site wide FIRUS monitoring system

- Smoke detection as required.

\subsubsection{High Performance Sustainable Buildings}

Fermilab is committed to designing, locating, constructing, maintaining and operating its facilities in an energy efficient and sustainable manner that strives to achieve a balance that will realize maximum attainable reuse and recycling of depletable resources, in an economically viable manner and consistent with Fermilab's mission and goals. To accomplish that, Fermilab complies with Guiding Principles for the Federal Leadership in High Performance and Sustainable Buildings (Guiding Principles). This direction is taken from the Fermilab Director's Policy 3. The project goal is to comply with the DOE's Guiding Principles for High Performance and Sustainable Buildings.

\subsubsection{Radiation Safety}

The conventional facilities will be designed to incorporate the applicable radiation safety requirements as contained in FESHM Section 1100 to reduce exposure to as low as reasonable achievable (ALARA) levels, including shielding labyrinths, exit passageways and passive shielding. The conceptual design is based on a preliminary review of the expected beam intensities and historic data from similar equipment. A complete radiation assessment will be conducted in subsequent project phases. The results of these assessments will be incorporated into the conventional facilities design.

The conventional facilities portion of the project will incorporate physical space for radiation safety interlock system, but the installation of the equipment, extension of monitoring devices and programming of the system will be done as part of the equipment installation. These systems will be designed in accordance with the FRCM Chapter 10 Radiation Safety Interlock Systems.

In accordance with current FRCM requirements, all sump discharges from the Linac Enclosure and the Transport Line Enclosure will be discharged to cooling ponds or ditches. 


\subsection{Conventional Facilities Scope}

The description of the scope of work for the PIP-II conventional facilities are listed below by functional area.

\subsubsection{Site Work Scope}

The site work includes wetland mitigation, roadwork and parking area, hardstands, storage tank foundations and related work to provide the supporting infrastructure for PIP-II.

The Main Ring infield has known wetlands which have influenced the siting of the PIP-II facilities. The wetlands in the vicinity of the proposed PIP-II project site were initially delineated and characterized in 2010. In the spring of 2016, these wetlands were revisited to further refine the location and impact of PIP-II. The 2016 wetland assessment report [149] was used as a basis for siting decisions and efforts were made to avoid the impact on existing wetlands and minimize unavoidable impacts during both the construction period as well as during operation of the new facility.

Wetland delineation of the project area and project plans will be submitted to the U.S. Army Corps of Engineers to determine if a permit would be required under Section 404 of the Clean Water Act. The mitigation strategy, if needed, is to purchase suitable wetland credits for those areas of unavoidable impact.

The existing South Booster Road will be reconfigured in order to allow for the installation of the Transport Line and associated shielding.

New access roads will provide vehicular access to the PIP-II facility from existing Fermilab roads. These roads will be constructed in a similar manner to existing Fermilab roads and will be suitable for all weather access. The access road will intersect the existing Main Ring Road at two locations to prevent dead-ends. Paved parking will be provided for vehicles at major entries to the buildings along with a gravel hardstand that will provide a staging area during installation. A paved approach to the at-grade loading dock with suitable truck maneuvering space will be provided.

The existing A0 cooling pond is currently used for cooling existing beamline components in the Main Ring tunnel as well as a return path for Industrial Cooling Water (ICW) discharge from cryogenic loads in the A0 service building. The existing pond will be reconfigured and upgraded to serve as a return ditch for the ICW system. This will include a modernization of the pond banks.

Some existing Fermilab utilities will be extended for use by the PIP-II. These utilities include Industrial Cooling Water (ICW), Domestic Water Service (DWS), Natural Gas (Gas), Chilled Water (CHW), Sanitary Sewer (SS), data/communication and electrical power. Figure 4.2, below indicates the location of existing utilities in the vicinity of the PIP-II site.

The ICW service will be extended from the existing lines located near the intersection of Booster Tower Road and Main Ring Road. The existing site wide ICW service is fed from Casey's Pond at the north end of the Fermilab site where it is filtered and treated as it is pumped into the piping network. This service is anticipated to supply treated ICW for fire protection in the sprinkler system and hydrants in addition to 1,400 gallons per minute of process cooling to serve as a cooling medium for the cryogenic compressors. The ICW discharge will be piped west of the Main Ring tunnel so that will flow to Casey's Pond via existing cooling ponds and return ditches.

The existing DWS service will be extended from the intersection of Booster Tower Road near the Central Utility Building (CUB) to provide potable water uses and make up water for process systems. As part of the subsequent design phases, a looped system connecting to other segments of the site wide DWS system will be investigated and incorporated as required. 


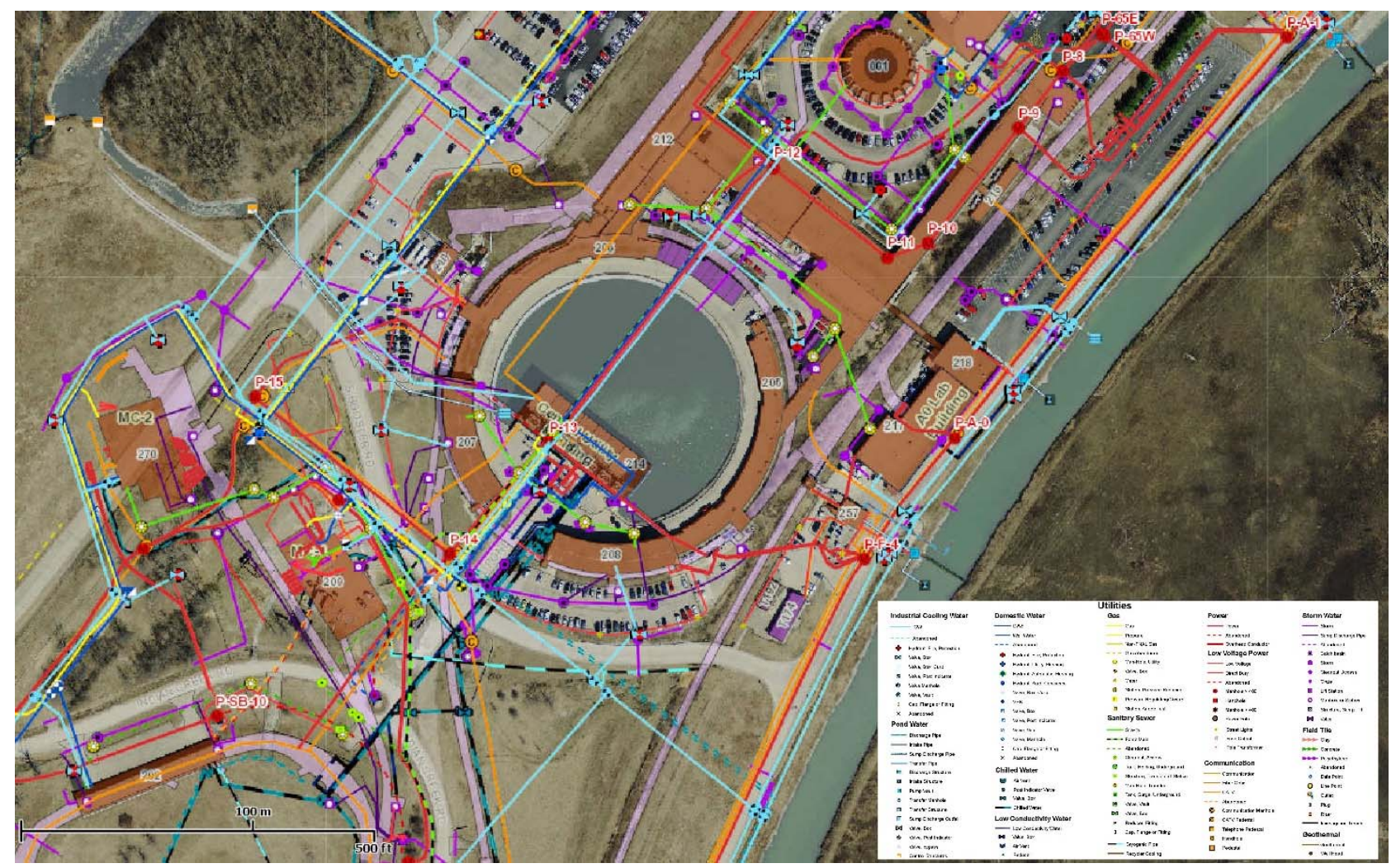

Figure 4.2: GIS Map in PIP-II visinity.

PIP-II will connect to the existing SS system at Booster Tower Road near CUB. The PIP-II SS system will accommodate discharges from the toilet facilities as well as backwash from process loads. A new lift station at the PIP-II facility will collect the sanitary discharge locally and pump it via a force main to a new manhole installed in the existing SS system.

The existing natural gas (NG) service will be extended from the existing site wide network at Booster Tower Road near CUB. The NG will provide a fuel source for HVAC heating.

The existing CUB chillers have the capacity to supply 300 tons of CHW to PIP-II for cooling. Connection to the CHW Supply and CHW Return lines will be made near Booster Tower Road near CUB and be routed to PIP-II.

Radioactive Water (RAW) systems will be utilized for the programmatic equipment in the Beam Dump. The RAW system, based on existing Fermilab system designs, will be double isolated from surface water and will reject the heat to the LCW system. The design, procurement and installation of the RAW systems are included in the accelerator portion of the project.

The existing 13.8 KV site wide electrical feeder system will be extended to PIP-II to provide a looped feed served primarily from the Master Substation (MSS). A backup up feeder, capable of powering critical portions of PIP-II will be served from the existing Kautz Road Substation (KRS). Connection to the MSS network will be accomplished at manhole P71 in the Main Ring and installed in a new concrete encased ductbank to the PIP-II site. A new substation, consisting of transformers, air switches and related electrical gear will be installed adjacent to the PIP-II Linac Service Building and the Cryogenics Plant.

The existing data and communication system will be extended in new duct banks to PIP-II from existing below grade ductbanks along the Main Ring Road. The connection location is assumed to be the existing communication manhole located adjacent to the Booster Tower. 


\subsubsection{Linac}

The Linac work includes the below grade, cast-in-place concrete Linac Enclosure and the above grade Linac Support Building.

The above grade and below grade portions of the Linac will be designed to include egress, construction type, emergency lighting, exit signage and smoke control ventilation in accordance with the IBC (International Building Code) and NFPA. Automatic sprinkler systems for the Linac Support Building will comply with the standards for an Ordinary Hazard Group 1 classification, in accordance with latest edition of the National Fire Protection Association's (NFPA) Codes and Standards.

The below grade Linac Enclosure is sized to accommodate the length of a $0.8 \mathrm{GeV}$ linac which includes space to accommodate a possible future upgrade of the Linac energy through the installation of four (4) HB650 cryomodules.

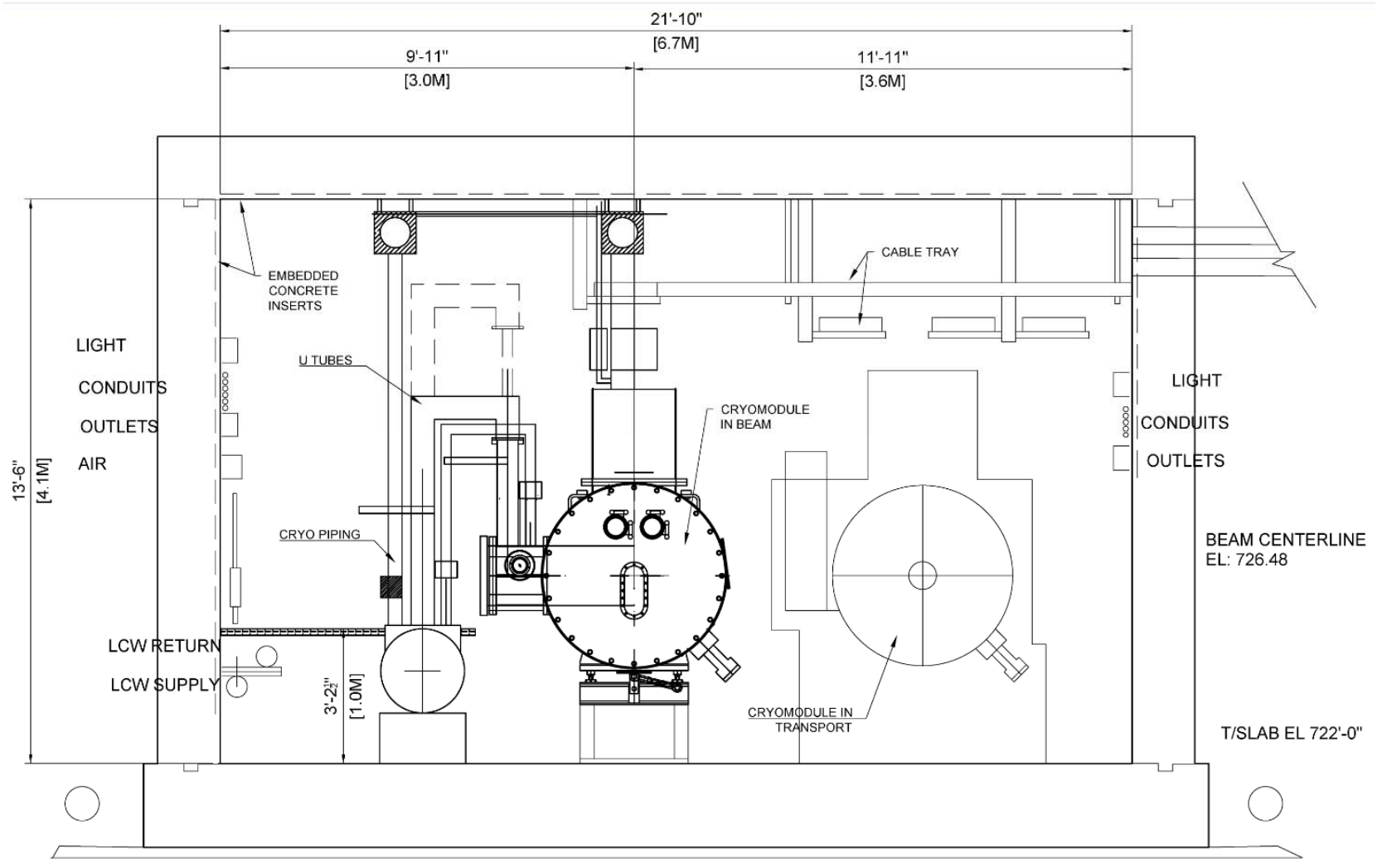

Figure 4.3: Cross section of linac enclosure (downstream view).

The Linac Enclosure will provide space for the linac hardware, penetrations for utilities (power, water, cryogens) and cabling, as well as for equipment installation and maintenance. The Linac Enclosure will also accommodate the logistics of installation, repair and removal of beamline components and related support equipment. Figure 4.3 depicts a typical cross section through the Linac Enclosure showing the space required to accommodate the beamline components as well as the space required for installation. Figure 4.4 shows a plan view of the enclosure.

The depth below grade of the Linac Enclosure is based on the beamline components matching the elevation of the existing Booster component elevation. This places the base slab of the Linac Enclosure at elevation $722.215 \mathrm{ft}$. $(220.131 \mathrm{~m})$ or approximately 25 feet below existing grade.

The below grade enclosure will have code compliance exit stairways provided to conform to the 
required maximum distance to an exit. These exit stairs will be configured to maintain the radiation safety shielding requirements. Fire detection will be via air sampling and line type sensors.

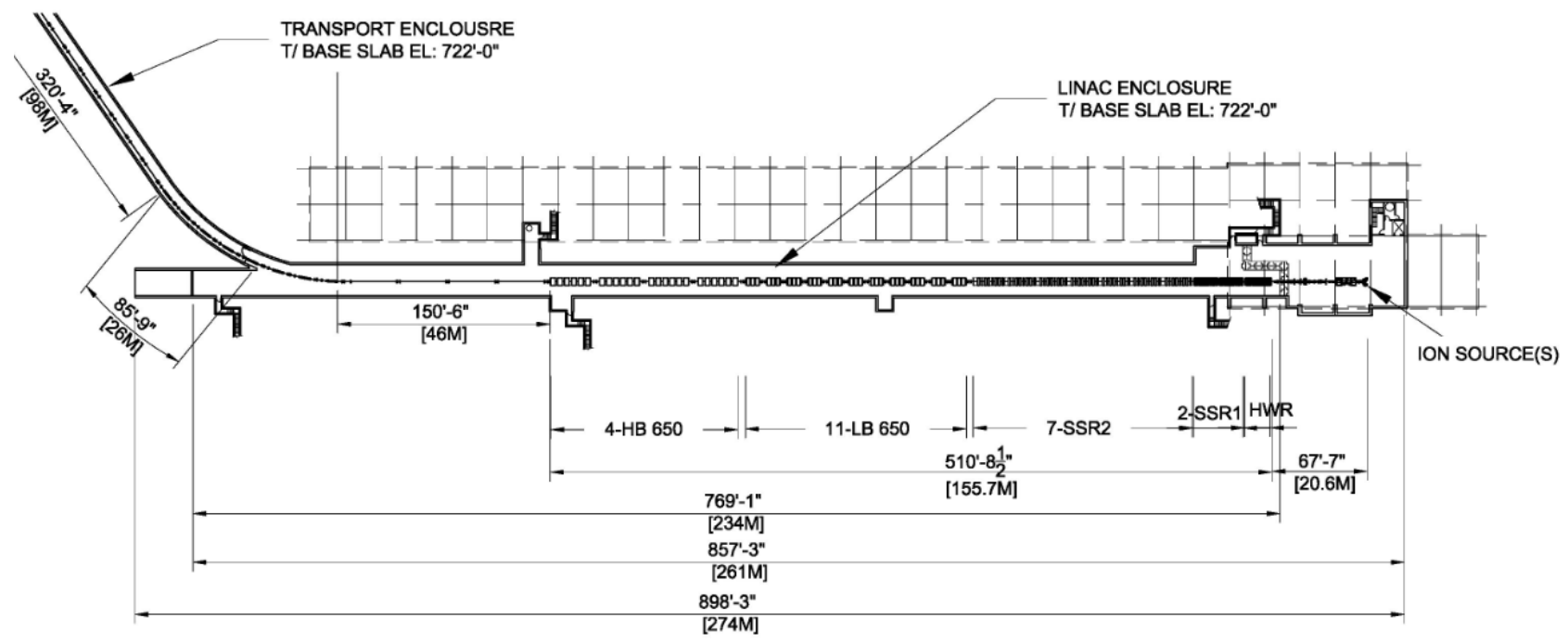

Figure 4.4: Enclosure level plan (top view).

The interior walls and ceiling of the Linac Enclosure will be painted and the exterior will be moisture proofed to provide a safe and dry semi-conditioned space for personnel and equipment. The below grade structures will be flanked with underdrain piping that will negate the hydraulic pressure on the walls and roof of the enclosure. The underdrains will be routed to duplex sumps that will discharge water to existing surface water features and away from the structure.

The walls and ceiling of the Linac Enclosure will be fitted with channel inserts to allow for the support of cable trays, cooling water, electrical conduits and fire detection equipment. Convenience outlets, 120/208VAC, will be provided at least every sixty feet along the walls. 480V/60 amp welding outlets will be provided spaced at 400 feet along the length of the Linac Enclosure.

In addition to required emergency and exit lighting, light fixtures will be provided to supply a minimum of 20 foot-candles. A percentage of these lights will be on uninterruptible power supply (UPS) circuits to provide emergency lighting during power failures.

The Linac Enclosure will be ventilated with neutral, dehumidified air as required by code. The underground air flow will include the provision for Oxygen Deficiency Hazard (ODH) ventilation. The conventional facilities will provide the mechanical equipment and ductwork for ODH ventilation while the controls, sensors, programming and commissioning will be accomplished as part of the equipment installation.

The south (downstream) end of the Linac Enclosure will include an elevator to accommodate the movement of the test carts, diagnostic equipment and related items that are needed for Linac operations and maintenance. Note that a passage from the downstream to the upstream end of the linac (high bay) will be obstructed by the Linac-to-Booster transfer line located at the same height as the linac or $1.3 \mathrm{~m}$ above the tunnel floor.

The construction of the below grade structure will utilize traditional "open cut and cover" method. This method has been used successfully at Fermilab for the construction of the majority of shielded enclosures on-site of a similar depth and use.

Based on a preliminary shielding assessment, the design of the Linac Enclosure will accommodate 5.6 meters (18.5 feet) of earth equivalent passive shielding in order to achieve an unlimited occupancy of the Linac Service Building and surrounding spaces. In general, this will be 
accomplished with an earthen berm with maintainable side slopes. For road crossings and areas where berms are not feasible, the design will utilize steel plates to achieve the required equivalent shielding. The 5.6 meters (18.5 feet) of shielding is based on a preliminary assessment (see Section 3.7) which will be finalized during the final design. Shielding documentation will be prepared and initial approval for construction obtained prior to the start of construction.

The Linac Support Building will provide space for the support services required to install, operate and maintain the PIP-II beamline components. The Linac Support Building contains three (3) spaces as described below:

High Bay: The high bay portion of the Linac Support Building will provide space for unloading, staging and assembling beamline components. This includes an at-grade loading dock with a 30 ton overhead bridge crane for moving equipment from grade to the below grade portion of the high bay.

The below grade portion of the high bay will be sized to accommodate the low energy portion of the PIP-II beamline components that do not require radiation shielding as well as space for staging and preparing beamline components for installation. The below grade portion of the high bay will also contain the support equipment and infrastructure required to operate the adjacent beamline components.

Gallery: The gallery space of the Linac Support Building will house the equipment needed to operate the beamline components in the adjacent, below grade Linac Enclosure. The 210 meter (690 foot) gallery will be constructed parallel to the below-grade Linac Enclosure and will provide penetrations for utilities, controls, cooling water, cryogens and related operational services. The south (downstream) end of the gallery will include access to the elevator to Linac Enclosure to accommodate the movement of the test carts, diagnostic equipment and related items that are needed for Linac operations and maintenance.

Support: The support space of the Linac Support Building will house the equipment and services required to support the operation of the building. This includes the following functions:

Process Water Equipment Room to house heat exchangers, pumps with vibration isolation, controls and related equipment for the Low Conductivity Water (LCW) system used to cool the beamline components and power amplifiers. Since this space is driven by water containing components, if necessary, this space could be housed in a separate building rather than contained with the Linac Support Building.

Mechanical that supports the operation of the facility.

Electrical equipment that supports the Linac Support Building including the incoming electrical service switchgear, panel boards, and related power supplies. The switchgear will serve conventional facilities equipment, the programmatic equipment and the HVAC systems. This room will house the electrical panels serving the lights, outlets and general building power.

Control Room to house monitoring/control equipment, conference room space for 8-10 people and adjacent workspace;

Visitor's Area suitable for use by outreach programs of up to 15 people;

Building Services including a building manager's office, toilet rooms and janitor's closet.

\subsubsection{Transport Line}

The Transport Line will house the beamline components required to bring the proton beam from the downstream end of the Linac Enclosure the existing Booster accelerator. The 225 meter (737 foot) long Transport Line enclosure will generally have a 3 meter (10 foot) wide by 2.45 meters ( 8 
foot) high cross section.

In order to accommodate the crossing of the new PIP-II transport line and the existing Main Ring beamline, the PIP-II beamline will rise up and over the existing Main Ring beamline components. A portion of the existing precast Main Ring tunnel will be removed and replaced with a cast-in-place concrete structure capable of accommodating both beamlines. This portion of the Transport Line enclosure will have a $3.4 \mathrm{~m}$ (11 foot) high enclosure.

A 9 meter (30 foot) long extraction enclosure stub will be constructed to accommodate possible future beamlines to the Muon Campus region.

The construction of the Transport Line enclosure will utilize traditional "open cut and cover" methods in which earth material is excavated, the concrete beamline enclosure is constructed and the completed enclosure is covered with the excavated material with granular material of the sides of the enclosure to facilitate drainage. This method has been used successfully at Fermilab for the construction of the majority of shielded enclosures on-site.

The interior walls and ceiling of the Transport Line enclosure will be painted and the exterior concrete surface will be moisture proofed to provide a safe, dry semi-conditioned space for personnel and equipment. The enclosure will be flanked with underdrain piping that will negate the hydraulic pressure on the walls and roof of the enclosure. The underdrains will be routed to a duplex sump that will discharge water onto grade and away from the enclosure. The walls and ceiling of the enclosure will be fitted with channel inserts to allow for the support of cable trays, cooling water piping, electrical conduits and fire detection equipment.

Convenience outlets, 120/208VAC, will be provided every sixty (60) feet along the wall on the side of the beamline. Welding outlets, $60 \mathrm{amp} / 480 \mathrm{~V}$, will be provided at two (2) locations along the length of the enclosure. In addition to the required emergency and exit lighting, light fixtures will be provided to supply a minimum of 20 foot-candles. A percentage of these lights will provide emergency lighting during power failures. Lighting will be controlled by the lighting control panel in the Linac Support Building.

The Transport Line enclosure will be separated from the Linac Enclosure by an air barrier to contain the evaporated cryogens within the Linac Enclosure. Fire detection will be via air sampling and line type sensors. The fire detection devices will report to the fire panel in MC-1.

A Beam Dump enclosure will connect to the Transport Line enclosure to house the $50 \mathrm{~kW}$ beam Dump, radioactive cooling water (RAW) system and related equipment. The beam dump enclosure will be on the right side (looking downstream) of the transport line tunnel. That determines that the passage along the tunnel will be at its left side.

The floor of the Transport Line enclosure will match the elevation of the Linac Enclosure to facilitate the installation of beamline components.

The Transport Line enclosure will be installed beneath the existing Booster Tower East parking lot and building to allow for the PIP-II beam to intercept the existing Booster beamline at the existing Long 11 straight section, which results in minimal displacements of the existing Booster tunnel equipment and reduces the interference with existing support services.

The installation of the Transport Line enclosure will require a partial demolition and temporary support of the existing Booster Tower East building to excavate and install the cast-in-place concrete connection to the existing Booster enclosure.

The Booster Tower Southeast parking lot will be replaced with a shielding berm similar in style and construction to that constructed when the Main Injector $8 \mathrm{GeV}$ line was installed at Booster Tower West. Vehicular access to existing electrical equipment in the northeast corner of the parking 
lot will be provided for maintenance of the electrical equipment.

The Transport Line will be designed to support up to 5.6 meters (18.5 feet) of earth and concrete shielding in order to provide for "unlimited occupancy" of all above ground areas accessible to the general public. The Transport Line enclosure include code required egress paths. The 5.6 meters (18.5 feet) of shielding is based on a preliminary assessment which will be finalized during the final design. Shielding documentation will be prepared and initial approval for construction obtained prior to the start of construction.

Space for beamline power supplies, control equipment and related equipment will be provided in the one bay of the Linac Support Building.

\subsubsection{Cryogenics Plant}

The PIP-II Cryogenics Plant will provide space for the cryogenics equipment for the PIP-II accelerator components. The Cryogenics Plant will be located at the downstream end of the Linac Enclosure in order to be positioned to allow for future expansion. The Cryogenics Plant contains three (3) primary spaces as described below:

Cold Box Station: The Cold Box Station will contain the equipment to install, operate and maintain the cold box. This includes the following criteria:

- Building Size: 15 m x 40 m (50 feet x 131 feet);

- Overhead crane with a capacity of 15 tons;

- Overhead door $5 \mathrm{~m}$ x $5 \mathrm{~m}$ (17 feet x 17 feet);

- Maximum floor loading: 20,000 kg/square meter (4,096 pounds/square foot);

- Space for five (5) 10,000 liter (2,641 gallons) dewars;

- 565 liters/minute (150 gallons per minute) of chilled water;

- Coordination Center to house 4-8 people;

- Control Room to accommodate equipment, monitors and related control equipment;

- Control Room and Coordination Center should have an isolated HVAC system that is capable of pressuring the room to reduce the oxygen deficiency hazard of the space;

Warm Compressor Station: The Warm Compressor Station will contain the equipment to install, operate and maintain the compressor and related equipment to support the Cold Box Station. This includes the following criteria:

- Building Size: 20 m x 30 m (66 feet x 100 feet)

- Overhead crane with a capacity of 25 tons;

- Overhead door $5 \mathrm{~m}$ x $5 \mathrm{~m}$ (17 feet x 17 feet);

- Maximum floor loading: 20,000 kg/square meter (4,096 pounds/square foot);

- Space for five (5) 10,000 liter (2,641 gallons) dewars;

- The cooling medium for the cryogenics compressors can be industrial cooling water (ICW) if it meets PIP-II quality requirements. A review of the previous tests of the ICW indicate that the water is generally acceptable, but will require additional filtration to meet the solids requirements.

Exterior Space: The exterior space for the Cryogenics Plant will provide for access to the Cold Box Station and Warm Compressor Station. This includes the following criteria:

- Space for ten (10) 113,000 liter (30,000 gallon) storage tanks and related piping;

- Space for one (1) 34,000 liter (9,000 gallon) liquid nitrogen dewar and related piping;

- Space for one (1) truck mounted mobile purifier; 
- Space for tanker truck for servicing the storage tanks/dewar;

- Parking for 8-10 vehicles;

- Loading dock access to both the Warm Compressor Station and the Cold Box Station;

- Underground utility tunnel that connects the Cryogenics Plant to the Linac Enclosure.

\subsubsection{Utility Building}

The PIP-II Utility Building will house the mechanical infrastructure to cool the Cryogenics Plant, LCW systems and HVAC systems. The Utility Building will be located at the upstream end of the Linac Enclosure and will include the following:

- Heat exchangers, pumps, electrical equipment and controls for the LCW and CHW systems;

- Water treatment and filtration systems;

- Electrical equipment;

- Control Room;

- Exterior space for cooling towers.

The Utility Building is sized to accommodate the anticipated equipment for both pulsed mode and continuous wave mode operations. 


\subsection{Site Power Requirements}

An estimate of site install power for the SC linac and the beam transfer line to the Booster is given in Table 4.1.

The following items were included into the power estimate:

- $\quad$ RFQ and MEBT RF: includes RF sources powering RFQ and MEBT bunching cavities operating in $\mathrm{CW}$ regime.

- $\quad$ SC Linac RF: includes RF sources powering SC cavities to accelerate $2 \mathrm{~mA}$ of beam to 0.8 $\mathrm{GeV}$ with $\mathrm{RF}$ in $\mathrm{CW}$ regime. Magnets: Includes all power supplies for quads, solenoids, dipoles and trim magnets.

- Support Electronics: includes power to low voltage racks used to support cavity operations including LLRF, interlocks, machine protection, instrumentation, timing, and control communication.

- Cryogenic Systems: Based on the CHL upgrade described in Section 3.4.

- $\quad$ LCW (Low Conductivity Water): The primary load is cooling of the RF sources and power supplies for dipoles.

- $\quad$ HVAC (heating, ventilation and air-conditioning): The primary loads are RF power not removed by the LCW system and the removal of heat from equipment galleries.

- Conventional Systems: Power required for other linac/beamline components (vacuum pumps), and for occupied spaces.

A typical operating power will be lower than the installed power and will be determined by the regime of operation.

Table 4.1: Site power estimates

\begin{tabular}{l|c}
\hline \hline System & Wall-Plug Power \\
\hline RFQ and MEBT RF & 320 \\
\hline SC linac RF & 8000 \\
\hline Magnets (quads, solenoids, dipoles) & 300 \\
\hline Support Electronics & 150 \\
\hline Cryogenic Systems & 3250 \\
\hline LCW & 200 \\
\hline Chilled water & 500 \\
\hline HVAC & 200 \\
\hline Conventional Systems & 300 \\
\hline Total & $\mathbf{1 3 2 2 0}$ \\
\hline \hline
\end{tabular}




\subsection{Fluids Systems}

Process Fluid Systems (LCW, RAW and compressed air) are critical for the successful operation of the PIP-II accelerator facility. These fluid systems provide both process temperature control and process manipulation for the various equipment that powers and operates the PIP-II machine. The scope of the PIP-II process fluid systems include the frontend enclosure and gallery, the SC LINAC enclosure and gallery, the transport line enclosure to Booster and associated service building, and the beam dump (see Figure 4.5).

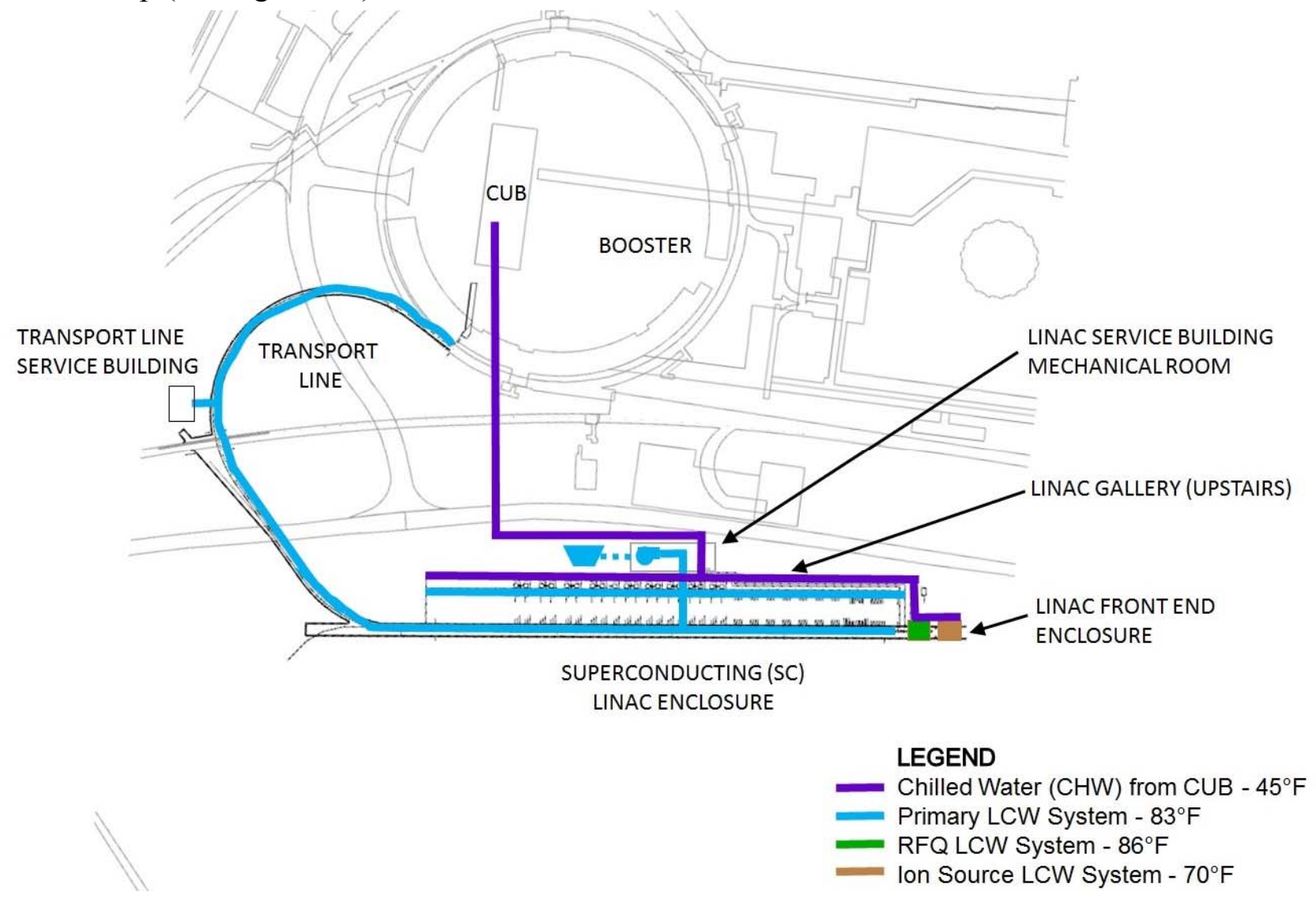

Figure 4.5: The scope layout of the different process water systems.

\subsubsection{Process Temperature Control - Water/HVAC}

Heat loads in the PIP-II enclosure can be budgeted in two places - heat load reacted to water and heat load reacted to ambient air and the associated HVAC system.

\section{Heat Load to Water}

The Heat Load to Water portion of the PIP-II Process control is composed of 4 different types of process water systems that work together to remove a total of $6.5 \mathrm{MW}$ of heat load from PIP-II equipment. These systems include a primary (main) low conductivity water (LCW) system, an RFQ standalone system, a standalone system for the Ion source, and a RadioActive Water (RAW) system for the beam dump.

\section{Primary (Main) Low Conductivity Water (LCW) System}

The primary low conductivity water system, also known as the PIP-II LCW System, will provide 
the bulk of the process temperature control to water cooled equipment throughout the footprint of the PIP-II accelerator. This footprint includes the frontend enclosure and gallery, the SC LINAC enclosure and gallery, the Linac-to-Booster transport line enclosure and associated transport line service building, and the SC Linac beam dump. Primary users of the PIP-II LCW System include radio-frequency power amplifiers (RFPA), circulators, and RF loads located in the RF gallery portion of the frontend and SC section of the PIP-II accelerator. Each of the accelerator beamline RF cavity sections are powered by an RF network that depend on a precise and reliable water system for process temperature control. The RF sections that power the LB650 and HB650 cavity sections of the accelerator are the largest users of the Primary LCW System at approximately $80 \%$ of the total water flow and power dissipation. The pumps for the PIP-II LCW system will be located in the SC-Linac Gallery. Heat load rejection from the LCW system will involve the use of a specially designed fluid cooler tower system. This heat rejection technology will yield a reliable process equipment temperature control within the range of $83 \mathrm{~F}$ to $88 \mathrm{~F}$. The fluid cooler tower system will be located adjacent to the PIP-II Utility Building. The supply pressure for the PIP-II LCW System will be approximately 100 PSIG. Figure 4.6 shows a typical LCW pump room.

Table 4.2: Power Rejection and Flow Rate Summary for PIP-II Primary LCW System and Heat rejection via fluid cooler tower system

\begin{tabular}{|c|c|c|}
\hline Equipment* & $\begin{array}{l}\text { Total Heat } \\
\text { Dissipation } \\
(\mathrm{KW})\end{array}$ & $\begin{array}{l}\text { Flow } \\
\text { Rate } \\
\text { (GPM) } \\
\text { Total } \\
\end{array}$ \\
\hline \multicolumn{3}{|l|}{ LEBT } \\
\hline Subtotal & 20.0 & 11.4 \\
\hline \multicolumn{3}{|l|}{ RFQ" (162.5 MHz) } \\
\hline Subtotal & 300.0 & 168.0 \\
\hline \multicolumn{3}{|l|}{ MEBT } \\
\hline Subtotal & 56.4 & 32.1 \\
\hline \multicolumn{3}{|l|}{ HWR (162.5 MHz) } \\
\hline Subtotal & 82.8 & 31.1 \\
\hline \multicolumn{3}{|l|}{ SSR1 (325 MHz) } \\
\hline Subtotal & 164.1 & 61.7 \\
\hline \multicolumn{3}{|l|}{ SSR2 (325 MHz) } \\
\hline Subtotal & 1063.1 & 399.8 \\
\hline \multicolumn{3}{|l|}{ LB 650 (650 MHz) } \\
\hline Subtotal & 2086.2 & 784.6 \\
\hline \multicolumn{3}{|l|}{ HB $650(650 \mathrm{MHz})$} \\
\hline Subtotal & 2577.1 & 969.2 \\
\hline \multicolumn{3}{|l|}{ Transport Line } \\
\hline Subtotal & 200.0 & 75.2 \\
\hline Total & 6549.8 & 2533.0 \\
\hline
\end{tabular}

* The RFQ information shown in the table above include RF equipment located in the gallery only. The RFQ and Ion Source device each have separate cooling systems and are not included in this table. 


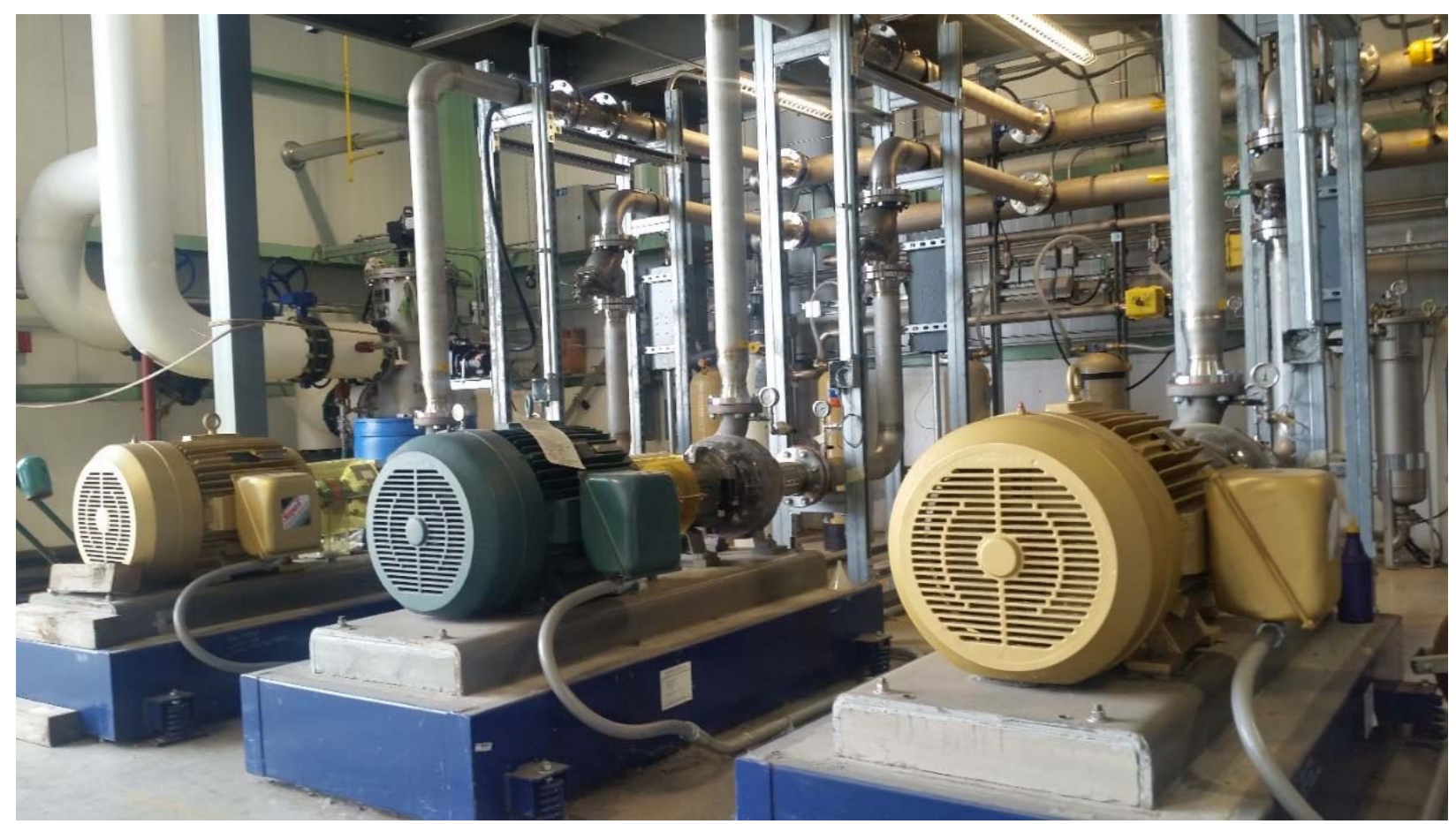

Figure 4.6: Typical LCW pump room.

\section{RFQ LCW System}

Dynamic tuning and resonance control of the RFQ is accomplished with cooling water; there are no mechanical tuners in the RFQ. Water Temperature control for the RFQ will involve a complex network of LCW systems. The RFQ RF power amplifiers, circulators, and RF loads located in the frontend gallery will utilize the PIP-II Primary LCW System for general temperature control. However, the RFQ itself located in the enclosure will require an independent customized cooling system arrangement specially designed for precise temperature control of the separate vane and wall cooling circuits. In order to control the resonant frequency of the RFQ, separate (independent) circulating LCW cooling systems will be designed for each section, indirectly connected to one distribution cooling skid. LCW circuit supply temperature for the separate vane and wall sections of the RFQ may vary for tuning, but in stable operation has to be kept within required $\pm 0.1^{\circ} \mathrm{C}$ of a preset temperature of $86^{\circ} \mathrm{F}$. Cooling water at $70^{\circ} \mathrm{F}$, supplied by a distribution cooling skid, is injected into wall and vane water loops. Water injection rate is controlled by the flow control valves. Water is mixed in helical mixers before it is delivered to wall and vane sections of the RFQ. Maximum LCW flow rate is 87 GPM for vane circuit and 172 GPM for wall circuit. Heat rejection will take place in the distribution cooling skid via heat exchanger with the $45^{\circ} \mathrm{F}$ building chill water (CHW). A system has been developed and built for the PIP-II Injector Test, as shown in Figures 4.7 and 4.8.

\section{Ion Source LCW Skid}

The Ion Source may require an independent cooling system located in the frontend enclosure in close proximity to the ion source. In the PIP-II injector test, this is a closed-loop chiller. Studies will be performed to determine if the chiller is required for PIP-II, or if a warmer supply temperature of the Primary LCW system can be tolerated. In the event that a separate system is required, heat rejection will take place in the distribution cooling skid via heat exchanger with the $45^{\circ} \mathrm{F}$ building CHW. The independent cooling system will need be designed to remove $40 \mathrm{KW}$ heat rejection via delivery of $24 \mathrm{GPM}$ of LCW at $90 \mathrm{PSIG}$ and supply temperature of $70^{\circ} \mathrm{F} \pm 1^{\circ} \mathrm{F}$. 


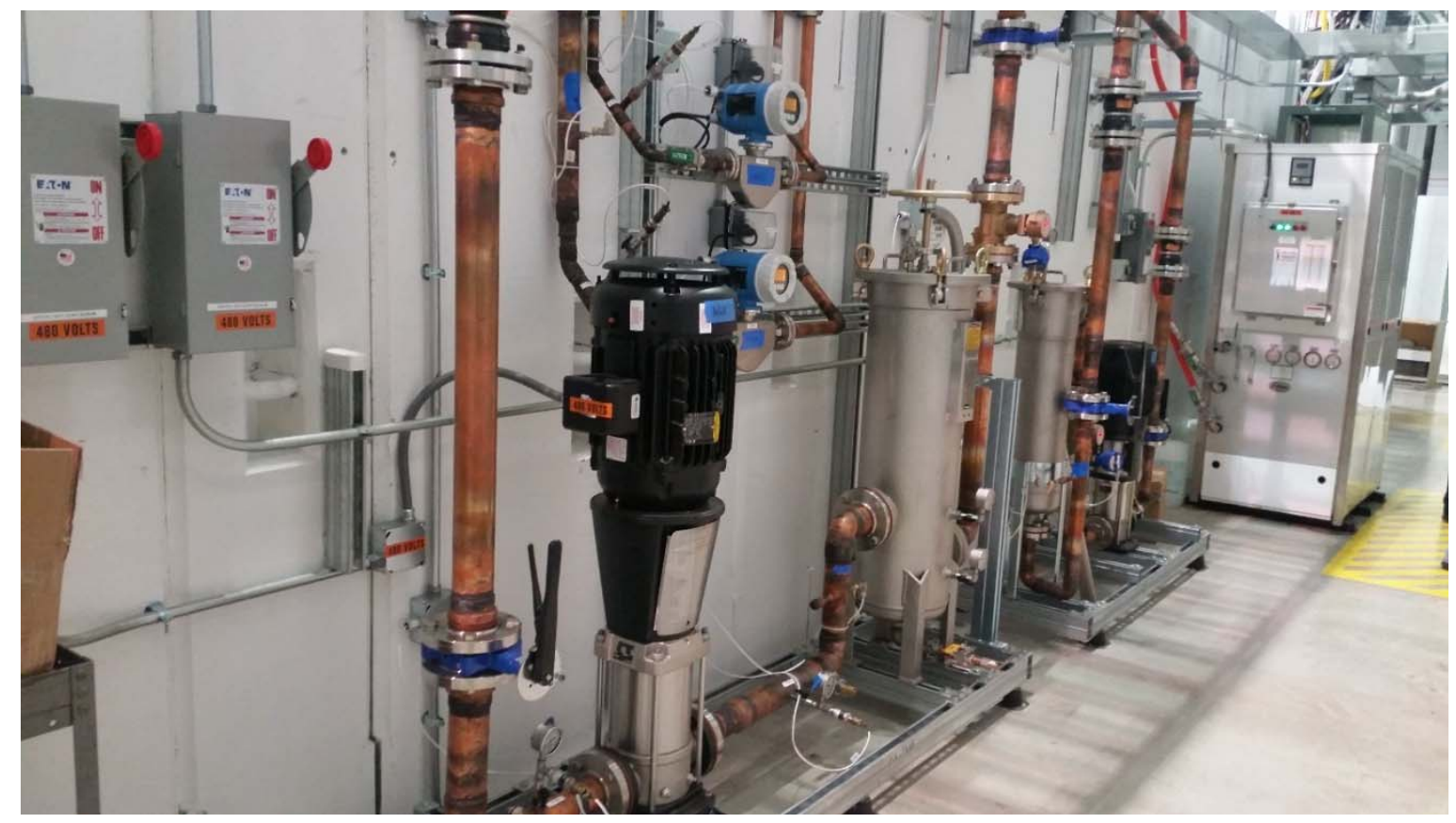

Figure 4.7: RFQ LCW showing separate Vane and Wall LCW skids. These skids incorporate pumps, filtration, and precision flow measurement.

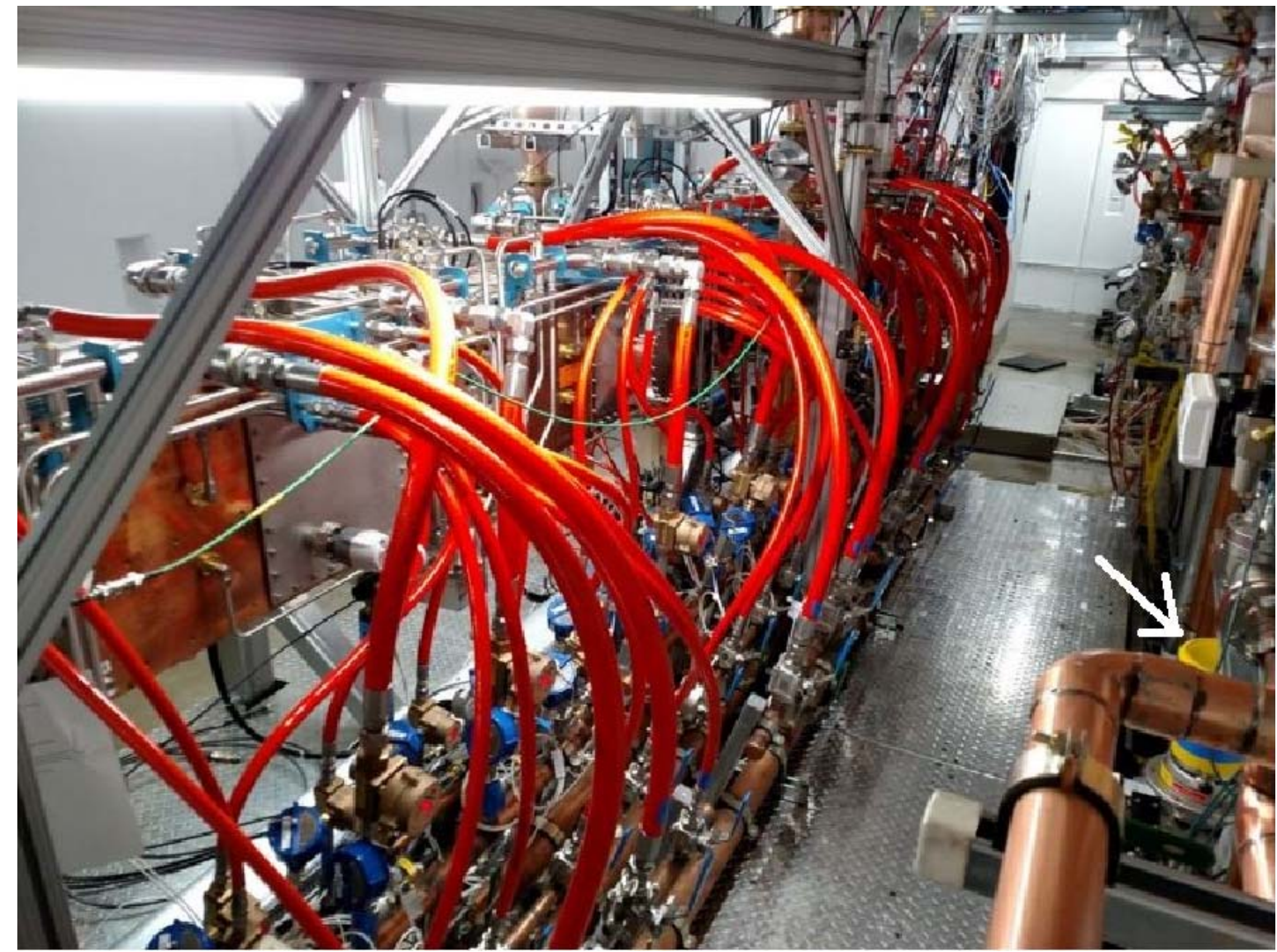

Figure 4.8: RFQ Cooling system. Dynamic mixing valve introducing cold water from the cooling skid is marked by white arrow. Cold water mixes with the main recirculating flow at a helical mixer below the false floor, and then flows to manifolding distributing balanced flow to each RFQ module and circuit. 


\section{Beam Dump}

There will be a beam dump for $800 \mathrm{MeV}$ beam coming out of the linac. It is expected that RAW system will be required for cooling of the beam abort. Heat dissipation is up to $50 \mathrm{KW}$. The RAW system would reject heat to the PIP-II Primary LCW system.

\section{Heat Load to Air (HVAC)}

The heat load to air portion of the PIP-II Process control is focused primarily in the RF gallery and frontend sections of the PIP-II LINAC Service building and is based on an assumed heat load dissipated in air equal to $15 \%$ of the total of $6.5 \mathrm{MW}$ of heat load removed from the PIP-II equipment. Heat rejection will take place via multiple ducted air handling units cooled with chilled water for the loads operating in the pulsed regime of operation. For the heat load anticipated in the continuous wave $(\mathrm{CW})$ regime of operation, additional chilled water based air handling equipment will be installed. Very little heat rejection to air is expected in the SC LINAC or transport line enclosures.

\subsubsection{Process Manipulation - Compressed Air}

The PIP-II accelerator facility will require process manipulation for the operation of the accelerator, including assembly, commissioning, and operation of vacuum compressors, cryogenic equipment, and various pneumatically operated equipment and instrumentation.

To supply compressed air to the necessary equipment within the PIP-II area, the air will be pressurized (compressed), processed (filters, dryers) and transmitted (piping) to various points of use throughout the PIP-II accelerator enclosure and gallery footprint:

Pressurization - The ambient air will be pressurized by screw air compressors with vibration isolation to a range between 90 and 120 PSIG. In order to protect various equipment there will be pressure regulators installed throughout the delivery system where needed.

Processing - To remove solid particulates, moisture and oil the compressed air is directed through two filters with automatic drains and refrigerated or desiccant air dryers. To ensure that proper air pressure and moisture content is delivered to points of use, monitoring pressure and dew point meters are piped in the system.

Transmission - Compressed air is to be distributed in copper piping and delivered to points of use throughout the PIP-II accelerator enclosure and gallery footprint.

PIP-II will have two independent compressed air systems:

1) Application I - Cryogenic Compressor Room - Compressed air system that supplies air to equipment located in the cryogenic compressor room, nitrogen dewar tank valves and cryo-plant equipment. It will be located in the Cryogenic Compressor Room.

This system is going to require two different air dryers.

a) Refrigerated air dryer that will keep dew point at or below $(+40 \mathrm{~F})$.

Air will be distributed to vacuum pumps, compressors, purifiers, inventory control, distribution box, etc. It will also be used as house air for air powered tools.

b) Desiccant dryer, that will keep dew point around $(-40 \mathrm{~F})$

Air will be distributed to cryogenic equipment that requires low dew point compressed air.

2) Application II - General Accelerator - Compressed air system that supplies air to equipment in the PIP-II accelerator enclosure, gallery, and general equipment that is located outside the cryogenic compressor room footprint. This compressed air system will be located in the PIP-II Utility Building and routed to the Linac Service Building via underground piping.

This system is going to require two different air dryers also. 
a) Refrigerated air dryer that will keep dew point at or below (+40 F).

This compressed air will be distributed to RFQ couplers, vacuum valves and will be used as house air for air powered tools also.

b) Desiccant dryer, that will keep dew point around (-40 F). This air will be distributed via distribution tubing to HWR, SSR1, and SSR2 couplers, where it will be used to prevent excessive cooling or condensation at the coupler ceramic windows.

Figure 4.9 shows typical compressed air arrangement.

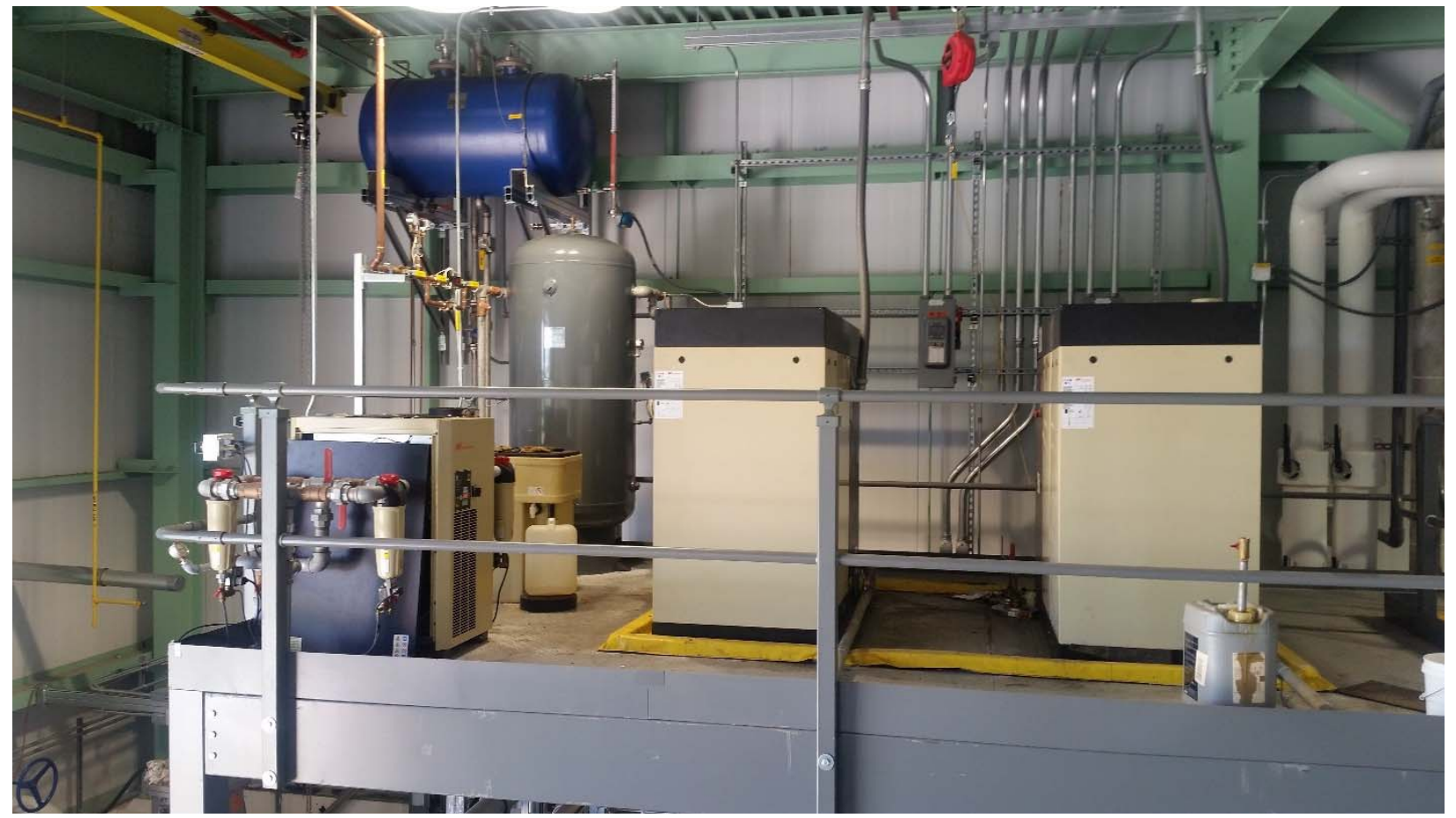

Figure 4.9: Typical compressed air arrangement. Screw compressors, dryers, filters, and distribution piping shown 


\section{References}

[1] "Building for Discovery: Strategic Plan for U.S. Particle Physics in the Global Context", Report of the Particle Physics Project Prioritization Panel, May 2014, http://science.energy.gov/ /media/hep/hepap/pdf/May-2014/FINAL_P5_Report 053014.pdf

[2] LBNE Conceptual Design Report Volume 2: The Beamline at the Near Site, October 2012, http://lbne2-docdb.fnal.gov/cgi-bin/RetrieveFile?docid=4317; filename=CDR-beam-volume101812-reduced.pdf

[3] Proton Improvement Plan website, http://www-ad.fnal.gov/proton/PIP/PIP_index.html

[4] The PIP-II Reference Design Report, V. Lebedev editor, June 2015, http://pip2docdb.fnal.gov/cgi-bin/RetrieveFile?docid=1\&filename=PIP-II RDR v0.0.pdf\&version=1

[5] G. E. Krafczyk, " Booster $20 \mathrm{~Hz}$ tests", Beams-doc-4777, http://beamdocs.fnal.gov/AD-public/DocDB/ShowDocument?docid=4777

[6] M. Plum, et.al., "STATUS OF THE SNS RING POWER RAMP UP", Proceedings of EPAC08, p. 3560-3562.

[7] I. Kourbanis, et.al., "Progress Toward Doubling the Beam Power at Fermilab's Accelerator Complex", Proceedings of IPAC 2014 (FERMILAB-CONF-14-187-AD).

[8] "Project X LEBT: Functional Requirement Specifications", TeamCenter Document \#ED0001289; uncontrolled copy in Project X Document 912: http://projectXdocdb.fnal.gov/cgi-bin/ShowDocument?docid $=912$

[9] S. Nagaitsev, et. al. "The Project-X Injector Experiment: A Novel High Performance Frontend for a Future High Power Proton Facility at Fermilab", Proceedings of PAC-2013, p. 374.

[10] Ji, Q and Staples, J, Project X Document 897: "Project X H' Ion Source Acceptance Test and Future Plan", http://projectx-docdb.fnal.gov/cgi-bin/ShowDocument?docid=897

[11] G. Romanov, et. al. "Project-X RFQ EM-design", IPAC2012, New Orleans, May 2012; FERMILAB-CONF-128-TD

[12] K.R. Crandall et al. "RFQ Design Codes", Los Alamos Report LA-UR-96-1836 (1996, Revised, Dec. 7 2005)

[13] “PXIE MEBT Functional Requirements Specification”, TeamCenter Document \#ED0001303; uncontrolled copy in Project X Document 938: http://projectxdocdb.fnal.gov/cgi-bin/ShowDocument?docid $=938$

[14] Yakovlev, V, "SRF Linac Technology Development", Proceedings of the XXVI Linac Conference (LINAC12), Tel-Aviv, 2012.

[15] Ciovati, G, "Review of High Field Q Slope, Cavity Measurements", in Proc. SRF2009: 90 (2009).

[16] Project X Document 590: “Assumption about Q values in CW linac”, http://projectxdocdb.fnal.gov/cgi-bin/ShowDocument?docid $=590$

[17] Reschke, D, "Analysis of the RF results of recent 9-cell cavities at DESY", TTC-Report 2009-01, 2009

[18] Brunner, O, et al, "Assessment of the basic parameters of the CERN Superconducting Proton Linac", PRSTAB 12, 070402, 2009

[19] Technical Specifications for High RRR Grade Niobium Sheet for Use in Superconducting Radio Frequency Cavities, Fermilab Specification: 5500.000-ES-371037 Rev B, Fermilab, Technical Division. 
[20] Chase, B., Peterson, Th. and Yakovlev, V.,"A Strategy for Cavity Resonance Control in the Project X CW Linac,” 02.20.2011, Project X Document 827-v1, ProjectX Database, http://projectx-docdb.fnal.gov/cgi-bin/ShowDocument?docid=827.

[21] Optics files supporting optics for the SC linac and transfer line. PIP-II Document 119; http://pip2-docdb.fnal.gov:8080/cgi-bin/ShowDocument?docid=119

[22] V. Lebedev, et.al. "Intrabeam stripping in $\mathrm{H}^{-}$linacs", Linac-2010, Tsukuba, Japan, Sep. $12-$ 17, 2010.

[23] A. Shishlo, J., et.al. "First Observation of Intrabeam Stripping of Negative Hydrogen in a Superconducting Linear Accelerator" Plum, Phys. Rev. Lett. 108, 114801 (2012).

[24] Krivosheev, O. and Mokhov, N. "Tolerable beam loss at High-Intensity Proton Machines", Fermilab-Conf-00/185, Aug. 2000.

[25] J.-P. Carneiro, "H- Stripping Equations and Application to the High Intensity Neutrino Source", Beams-doc-2740, http://beamdocs.fnal.gov/AD-public/DocDB/DocumentDatabase

[26] G.H. Gillespie, Phys. Rev. A 15, 563 (1977) and 16, 943 (1977), Nucl. Intr. \& Meth. B 2, 231 (1984) and B 10/11, 23 (1985).

[27] A. Drozhdin, "H- beam collimation in the transfer line from $8 \mathrm{GeV}$ linac to the Main Injector”, Beams-doc-2740, June 2008, https://beamdocs.fnal.gov/AD-private/DocDB/ShowDocument?docid=2201

[28] J. Lackey, et. al., "Operation and Performance of the New Fermilab Booster $\mathrm{H}^{-}$Injection System”, FERMILAB-CONF-07-248-AD.

[29] M. Popovic, et. al., "A Proposed $\mathrm{H}^{-}$Injection System for the Fermilab Booster”, Beams-doc 1784-v1, April 2005.

[30] M.S. Gulley et al., Phys. Rev. A 53, 3201 (1996).

[31] W. Chou, M. Kostin and Z. Tanga, "Stripping Efficiency and Lifetime of Carbon Foils," http://arxiv.org/abs/physics/0611157

[32] V. Lebedev and V. Shiltsev (Eds.), “Accelerator Physics at the Tevatron Collider”, Springer, 2014.

[33] Yu. Tokpanov, V. Lebedev, W. Pellico, "MEASUREMENTS OF MAGNETIC PERMEABILITY OF SOFT STEEL AT HIGH FREQUENCIES”, Proceedings of IPAC2012, New Orleans, Louisiana, USA.

[34] James L. Crisp and Brian J. Fellenz, "Measured Longitudinal Beam Impedance of Booster Gradient Magnets", Fermilab-TM-2145, March 22, 2001.

[35] V. Lebedev and C. Bhat, "Booster Transition Crossing," http://pxie.fnal.gov/PIPIImeetings/TransitionCrossing.pdf

[36] V. A. Lebedev, J.-F. Ostiguy and C.M. Bhat, "BEAM ACCELERATION AND TRANSITION CROSSING IN THE FERMILAB BOOSTER”, HB-2016.

[37] J.-F. Ostiguy, V. Lebedev and C.M. Bhat, "MODELING LONGITUDINAL DYNAMICS IN THE FERMILAB BOOSTER SYNCHROTRON”, IPAC-16.

[38] W. Pellico, "From PIP to PIP-II," https://beamdocs.fnal.gov/AD/DocDB/0047/004700/002/Booster-PIPII-v1.pdf

[39] King-Yuen Ng, "Longitudinal Coupled Bunch Instability in the Fermilab Booster", FERMILAB-FN-0464; KEK-87-17. 
[40] A. Burov and V. Lebedev, FERMILAB-TM-2492-AD (2011)

[41] A. Macridin et al., Phys. Rev. ST-AB, 14, 061003 (2011)

[42] A. Burov, Phys. Rev. ST-AB, 12, 044202 (2009)

[43] A. Burov, Phys. Rev. ST-AB, 17, 021007 (2014)

[44] A. Chao, "Physics of Collective Beam Instabilities in High Energy Accelerators", John Wiley \& Sons, Inc., Eq. (6.58), (1993)

[45] A. Macridin et al., FERMILAB-CONF-13-431-CD (2013).

[46] Synergia home - https://web.fnal.gov/sites/Synergia/SitePages/Synergia\%20Home.aspx

[47] A. Burov, Phys. Rev. Accel. Beams, 19, 084402 (2016)

[48] J. Wei, "Transition Crossing", in "Handbook of Accelerator Physics and Engineering" (edited by A.W. Chao and M. Tigner), World Scientific, $2^{\text {nd }}$ Printing (2002).

[49] A. Burov, FERMILAB-PUB-11-174-AD (2011)

[50] See Eq. (6.240), p. 360. in Ref. [51]

[51] A. Burov, FERMILAB-PUB-16-279-AD (2016).

[52] "Project X and PXIE Ion Source Functional Requirement Specifications," TeamCenter Document \#ED0001288; uncontrolled copy in Project X Document 968:, http://projectXdocdb.fnal.gov/cgi-bin/ShowDocument?docid=968

[53] http://www.d-pace.com/

[54] R. D’Arcy et al., Nucl. Instrum. And Methods in Phys. Res. A 815 (2016) 7-17.

[55] A. Shemyakin and L. Prost, "Scheme for Low Energy Beam Transport with a nonneutralized section”, FERMILAB-TM-2599-AD (2015); arXiv:1504.06302 [physics.acc-ph]

[56] "PXIE LEBT Beam Transverse Emittance Station FRS”, TeamCenter Document \#ED0001294; uncontrolled copy in Project X Document 1077: http://projectxdocdb.fnal.gov/cgi-bin/ShowDocument?docid=1077

[57] "Project X RFQ functional physics requirements", TeamCenter Document \#ED0001300; uncontrolled copy in Project X Document 894: http://projectx-docdb.fnal.gov/cgi$\underline{\text { bin/ShowDocument?docid }=894}$

[58] Virostek, S, et al, "Design and analysis of the PXIE CW Radio-Frequency Quadrupole (RFQ)", in Proc. of IPAC2012, New Orleans, Louisiana, USA, 2012, THPPC034.

[59] Ratti, A, et al, "The Design of a High Current, High Duty Factor RFQ for the SNS", in Proc. of EPAC '00, Vienna, Austria, 2000, pp. 495-497.

[60] S. Kazakov, et al, "DESIGN OF 162.5 MHZ CW MAIN COUPLER FOR RFQ", LINAC 2014, Geneva, Switzerland, THPP049, p. 960.

[61] Sang-ho Kim, et.al. "Stabilized operation of the Spallation Neutron Source radio-frequency quadrupole”, Phys. Rev. ST Accel. Beams 13, 070101 (2010)

[62] "PXIE MEBT quadrupoles specifications", TeamCenter Document \#ED0001312; uncontrolled copy in Project X Document 933: http://projectx-docdb.fnal.gov/cgibin/ShowDocument?docid $=933$

[63] "FRS for Bunching cavity for PXIE MEBT", TeamCenter Document \#ED0001307; uncontrolled copy in Project X Document 1071: http://projectX-docdb.fnal.gov/cgibin/ShowDocument?docid=1071

[64] Romanov, G, et al, "CW room temperature re-buncher for the Project X frontend" in Proc. 
of IPAC'12, New Orleans, USA, 2012, THPPP063

[65] "PXIE MEBT kicker specifications", TeamCenter Document \#ED0001305; uncontrolled cone in Project X Document 977: http://projectx-docdb.fnal.gov/cgibin/ShowDocument?docid=977

[66] Diebele, C and Aleksandrov A., "Experimental study of the SNS MEBT chopper performance”, in Proc. of IPAC'10, Kyoto, Japan, 2010, MOPD063.

[67] Lebedev, V, et al, "Progress with PXIE MEBT chopper", in Proc. of IPAC'12, New Orleans, USA, 2012, WEPPD078.

[68] "Functional specifications for PXIE MEBT absorber", TeamCenter Document \#ED0001304; uncontrolled copy in Project X Document 964: http://projectx-docdb.fnal.gov/cgibin/ShowDocument?docid $=964$

[69] A. Shemyakin and C. Baffes, High power density test of PXIE MEBT absorber prototype, Proc. of LINAC'14, August 31 - September 5, 2014, Geneva, Switzerland, THPP055.

[70] "Functional specifications for PXIE MEBT scrapers", TeamCenter Document \#ED0001306; incontrolled copy in Project X Document 1067: http://projectx-docdb.fnal.gov/cgibin/ShowDocument?docid=1067

[71] R.Webber et al, "Overview of the High Intensity Neutrino Source Linac R\&D Program at Fermilab”, LINAC'08, Victoria, BC, Canada, 2008.

[72] Iouri Terechkine, et al, "Cw Room Temperature Re-Buncher for the PIP-II Linac", IPAC2014, Dresden, Germany, 2014.

[73] Mustapha, B., et al., "A Ring-Shaped Center Conductor Geometry for a Half-Wave Resonator," in Proc. of IPAC'12, New Orleans, Louisiana, USA, 2012, Pg. 2289.

[74] Fuerst, J., et al., "Niobium to Stainless Steel Braze transition Development," in Proc. of SRF'03, Travemunde, Lubeck, Germany, 2003, Pg. 305.

[75] American Society of Mechanical Engineers Boiler and Pressure Vessel Code, Section VII 2010.

[76] Fuerst, J., “The ATLAS Energy Upgrade Cryomodule,” in Proc. of SRF'09, Berlin, Germany, 2009, Pg. 52.

[77] Conway, Z., et al., "Achieving High Peak Fields and Low residual Resistance in Half-Wave Cavities," in Proc. of SRF'15, Whistler, British Columbia, Canada, 2015, WEBA05.

[78] Fuerst, J., et al., "Assembly Installation and Commissioning of the ATLAS Upgrade Cryomodule," in Advances in Cryogenic Engineering 55A, edited by J.G. Weisend et al., American Institute of Physics, New York, New York, 2010, Pg. 815.

[79] Conway, Z., et al., "Assembly and Commissioning of a new SRF Cryomoudle for the ATLAS Intensity Upgrade," in Advances in Cryogenic Engineering 59, edited by J.G. Weisend et al., Anchorage, Alaska, USA, 2013, Pg. 1829.

[80] Process Piping: American Society of Mechanical Engineers Code for Pressure Piping B31.32012.

[81] SSR1 Cryomodule Design for PXIE, T. Nicol et al., Proceedings of PAC2013, Pasadena, CA, USA, THPMA09

[82] Apollinari, G., et al., "Development of 325 MHz Single Spoke Resonators at Fermilab", FERMILAB-PUB-08-303-TD.

[83] L. Ristori et al., "Design, Fabrication and Testing of Single Spoke Resonators at Fermilab", 
in Proc. of SRF2009, Berlin, Germany, THPPO011

[84] SSR1 cavity Functional Requirement Specifications: Teamcenter ED0001317

[85] Guidelines for the Design, Fabrication, Testing and Installation of SRF Cavities, FESHM Chapter 5031.6, TD-09-005, Fermi National Accelerator Laboratory

[86] Passarelli, D., et. al., "Methodology for the Structural Design of Single Spoke Accelerating Cavities at Fermilab", in Nuclear Instruments and Methods in Physics Research Section A, Volume 834C, 2016, Pages 1-9

[87] Sukhanov, A., et al., "Result of Cold Tests of The Fermilab SSR1 Cavities", in Proc. Of LINAC2014, Geneva, Switzerland, THPP057.

[88] Hocker, A., et. al., "RF Tests of Dressed $325 \mathrm{MHz}$ Single Spoke Resonators at 2 K", in Proc. of LINAC2014, Geneva, Switzerland, MOPP055.

[89] Passarelli, D. et. al., "High-Vacuum Simulations and Measurements on the SSR1 Cryomodule Beam-line”, in Proc. of SRF2015, Whistler, Canada, TUPB074.

[90] Kneisel, P., "Effect of Cavity Vacuum on Performance of Superconducting Niobium Cavities", in Proc. of the 1995 Workshop on RF Superconductivity, Gif-sur-Yvette, France.

[91] Knobloch, J., Padamsee, H., "Reduction of the surface resistance in superconducting cavities due to gas discharge", in Proc. of 8th Workshop on RF Superconductivity, Padova, Italy.

[92] Focusing Lens for SSR1 Cryomodule Project X Injector Experiment (PXIE) Functional Requirements Specification: Teamcenter ED0001315.

[93] Lunin, A., et al., "Development of a Low-Beta Button BPM for PXIE Project", in Proc. of IBIC2013, Oxford, UK.

[94] $325 \mathrm{MHz}$ Coupler Functional Requirement Specification: Teamcenter ED0001777.

[95] Pronitchev, O., et al., "Mechanical Design of a High Power Coupler for the PIP-II $325 \mathrm{MHz}$ SSR1 RF Cavity", in Proc. of SRF2015, Whistler, Canada, THPB091.

[96] Kazakov, S., et al., "Testing of $325 \mathrm{MHz}$ Couplers at Test Stand in Resonance Mode", in Proc. of SRF2015, Whistler, Canada, THPB098.

[97] Nicol, T.H., et al., "Design and Analysis of the SSC Dipole Magnet Suspension System", in Supercollider 1, p. 637 (1989).

[98] Paparella, R. "Overview of Recent Tuner Development on Elliptical and Low-Beta Cavities", in Proc. of SRF2015, Whistler, BC, Canada, FRAA01.

[99] Pischalnikov, Y., et al., “Tests of a Tuner for a 325MHz SRF Spoke Resonator", in Proc. of 2011 Particle Accelerator Conference, New York, NY, USA, TUP080.

[100] Passarelli, D., et al., "SSR1 Tuner Mechanism: Passive and Active Device", in Proc. of LINAC2014, Geneva, Switzerland, TUPP052.

[101] Passarelli, D., et. al., "Performance of the Tuner mechanism for SSR1 Resonators During Fully Integrated Tests at Fermilab", in Proc. of SRF2015, Whistler, Canada, THPB061.

[102] Schappert, W., et al., "Resonance Control for Narrow-Bandwidth, Superconducting RF Applications", in Proc. of SRF2015, TUPB095, Whistler, BC, Canada

[103] 325 MHz SSR2 Superconducting RF Cavities Functional Requirements Specification: Teamcenter ED0001854.

[104] Focusing Lens for SSR2 Cryomodule Project X Injector Experiment (PXIE) Functional Requirements Specification: Teamcenter ED0003642. 
[105] Kumud Singh, "PIP-II Solenoid Focusing lens for SSR2 Cryomodule”, PIP-II document 55, http://pip2-docdb.fnal.gov/cgi-bin/DocumentDatabase/

[106] Schappert,W, et al, "Microphonics Control for Project X," PAC2011, New York, March 28, 2011, TUP086.

[107] V.Yakovlev, et al, "HOMs in the Project-X CW Linac," Int. Workshop on Higher-Order Mode Damping in Superconducting RF Cavities, Cornell University, October 11-13, 2010, http://www.Ins.cornell.edu/Events/HOM10/rsrc/LEPP/Events/HOM10/Agenda/MA3 Yakov lev.pdf

[108] Functional Requirement Specification 650 MHz, Beta .61 CRYOMODULE CAVITIES: Teamcenter ED0001830.

[109] Functional Requirement Specification $650 \mathrm{MHz}$, Beta .61 DRESSED CAVITY, Teamcenter ED0001834.

[110] Functional Requirement Specification 650 MHz, Beta 92 CRYOMODULE CAVITIES, Teamcenter ED0001322.

[111] Functional Requirement Specification $650 \mathrm{MHz}$, Beta .92 DRESSED CAVITY: Teamcenter ED0001321.

[112] S.-H. Kim, "HOM Experiences at the SNS SCL," SPL HOM workshop, CERN, June 25-26, 2009. http://indico.cern.ch/event/57247/session/1/contribution/9/material/slides/1.pdf

[113] Project X: Accelerator Reference Design, S. D. Holmes (editor), http://arxiv.org/abs/1306.5022

[114] Functional Requirement Specification: 325 MHz Coupler, Teamcenter ED0001777.

[115] Functional Requirement Specification: $650 \mathrm{MHz}$ Coupler, Teamcenter ED0003645.

[116] A. Grassellino, A. Romanenko, O. Melnychuk, Y. Trenikhina, A. Crawford, A. Rowe, M. Wong, D. Sergatskov, T. Khabiboulline, F. Barkov, 2013 Supercond. Sci. Technol. 26 102001 (Rapid Communication).

[117] A. Romanenko, A. Grassellino, A. C. Crawford, D. A. Sergatskov, and O. Melnychuk, Appl. Phys. Lett. 105, 234103 (2014).

[118] Functional Requirement Specification: Half Wave Resonator Cryomodule, Teamcenter ED0001313.

[119] Functional Requirement Specification: 325 MHz SSR1 cryomodule, Teamcenter ED0001316.

[120] Functional Requirement Specification: 325 MHz SSR2 cryomodule, Teamcenter ED0001829.

[121] Functional Requirement Specification: PIP-II linac alignment, Teamcenter ED0005936.

[122] N.V. Mokhov, C. James, The MARS Code System User's Guide, http://www-ap.fnal.gov/MARS/

[123] N. V. Mokhov, S.I. Striganov, "MARS15 Overview", in Proc. of Hadronic Shower Simulation Workshop, Fermilab, AIP Conf. Proc. 896 (2007) pp. 50-60.

[124] N. Mokhov, P. Aarnio, Y. Eidelman, K. Gudima, A. Konobeev, V. Pronskikh, I. Rakhno, S. Striganov, I. Tropin, "MARS15 code developments driven by the intensity frontier needs," Progress in Nuclear Science and Technology 4, 496 (2014).

[125]Foster, GW, Chou W, E. Malamud E (ed), "Proton Driver Study II", Chapter 16, FermilabTM-2169, (May 2002). 
[126]P.Treitea, et al., Design, project execution, and commissioning of the $1.8 \mathrm{~K}$ superfluid helium refrigeration system for SRF cryomodule testing, ICEC/ICMC 2014

https://indico.cern.ch/event/244641/session/25/contribution/41.pdf

[127]A. Lunin, et al., "Development of a Low-Beta Button BPM for the PXIE Project," TUPC14, IBIC2013, Oxford, UK

[128]J. Crisp, et al.. "Position and Phase Measurements of Microampere Beams at the Michigan State University REA3 Facility," THPAC20, PAC2013, Pasadena, CA

[129]C. Drennan, Privite communications.

[130]W. Blokland, C. Peters, “A New Differential and Errant Beam Current Monitor for the SNS Accelerator," THAL2, IBIC2013, Oxford, UK

[131]R. Connolly, et al., "Beam-Energy and Laser Beam-Profile Monitor at the BNL Linac," TUPSM011, BIW2010, Santa Fe, NM

[132]Y. Liu, et al., "Laser Based Diagnostics for Measuring H- Beam Parameters," WEOCN1, PAC2011, New York, NY

[133]Y. Liu, et al., "Laser Applications: $\mathrm{H}^{-}$Beam Photo-Detachment and Push Button Diagnostics," TUCP02, BIW2012, Newport News, VA

[134]R. B. Wilcox, et al., “A Low-Power Laser Wire with Fiber Optic Distribution,” TUPD53, DIPAC2011, Hamburg Germany

[135]P. W. Allison, et al., “An Emittance Scanner for Intense Low-Energy Ion Beams,” IEEE Transactions on Nuclear Science, Vol. NS-30 (1983)

[136]M. P. Stockli, et al., "Emittance Studies with an Allison Scanner,” Rev. Sci. Instrum. 77, 03B706 (2006)

[137]R. D'Arcy, et al., “Characterization of the PXIE Allison-type Emittance Scanner,” Nucl. Inst. A 815 (2016)

[138] Y. Liu, et al., "Laser Wire Based Transverse Emittance Measurements of $\mathrm{H}^{-}$Beam at Spallation Neutron Source," MOPHA041, IPAC2015, Richmond, VA.

[139] T. Hofmann et al, "Design of a Laser-Based Profile Monitor for LINAC4 Commissioning at $50 \mathrm{MeV}$ and $100 \mathrm{MeV}$ " Proceedings of IBIC 2015, Melbourne, Australia.

[140]A. Zhukov, et al., "Longitudinal Laser Wire at SNS," MOCYB3, IBIC2014, Monterey, CA

[141]R. Thurman-Keup, et al., "Longitudinal Bunch Monitoring at the Fermilab Tevatron and Min Injector Synchrotrons," Jinst 6 T10004 (2011)

[142]A. Aleksandrov, "Beam Halo Characterization and Mitigation," FRXBA01, IPAC2016, Busan, Korea

[143]Cahill, K, et al, “The Fermilab Accelerator Control System”, ICFA Beam Dyn. Newslett. 47:106-124, 2008

[144]Patrick, J and Lackey, S, "Control Systems for Linac Test Facilities at Fermilab", Proc. Linac08, Victoria, BC, 2008

[145] Sullivan, AH, "A Guide to Radiation and Radioactivity Levels Near High Energy Particle Accelerators", Nuclear Technology Publishing, (1992).

[146]PIP-II Document Database (http://pip2-docdb.fnal.gov/)

[147]Fermilab Campus Master Plan (http://fess.fnal.gov/master_plan/index.html)

[148] Vibration Measurements at the JLAB Cryoplant and Linac, dated 18NOV14, (http://pip2- 
docdb.fnal.gov:8080/cgi-bin/ShowDocument?docid=122)

[147] Wetland Assessment Report, dated 29JUL16, (http://pip2-docdb.fnal.gov:8080/cgibin/ShowDocument?docid=159) 


\section{Appendix A: Beam Transport to the Upgrade of Mu2e Experiment}

The Mu2e experiment is presently under construction at Fermilab. The experiment will search for the charged-lepton-flavor-violating process of coherent muon-to-electron conversion in the presence of a nucleus with a sensitivity of four orders of magnitude beyond current limits [1]. Mu2e will be taking the $8 \mathrm{GeV}$ proton beam from the Booster which time structure will be, then, reformatted in the Recycler and the Delivery Ring. A slow extraction from the Delivery ring will create pulses of $3 \cdot 10^{7}$ protons per pulse with the total width of about $250 \mathrm{~ns}$ and following at intervals of $1.7 \mu \mathrm{s}$. The beam is transported through the M4 beamline to the production target inside a high-field superconducting solenoid. The proton beam power on the target will be around $8 \mathrm{~kW}$.

Studies have been carried out to explore the possibility for an upgrade path of the Mu2e experiment exploiting the PIP-II beam [2-3]. The bunch-by-bunch chopping system located in the MEBT can create beam pulses of a required width $(\sim 100 \mathrm{~ns})$ and repetition rate $(\sim 1 \mu \mathrm{s})$. Operations of the SC Linac in CW mode with $5 \mathrm{~mA} \mathrm{RFQ} \mathrm{current} \mathrm{and} \mathrm{the} \mathrm{required} \mathrm{bunch} \mathrm{structure} \mathrm{will} \mathrm{generate}$ $100 \mathrm{~kW}, 800 \mathrm{MeV}$ beam at the Mu2e upgrade target. Efficiencies of slow pion production (per unit power) required for the Mu2e are quite close for 0.8 and $8 \mathrm{GeV}$. Therefore, it is expected that with moderate changes to the Mu2e facility a usage of PIP-II beam should increase the experiment sensitivity by a factor 10 . The lowering of beam energy also excludes antiproton production representing a major background source.

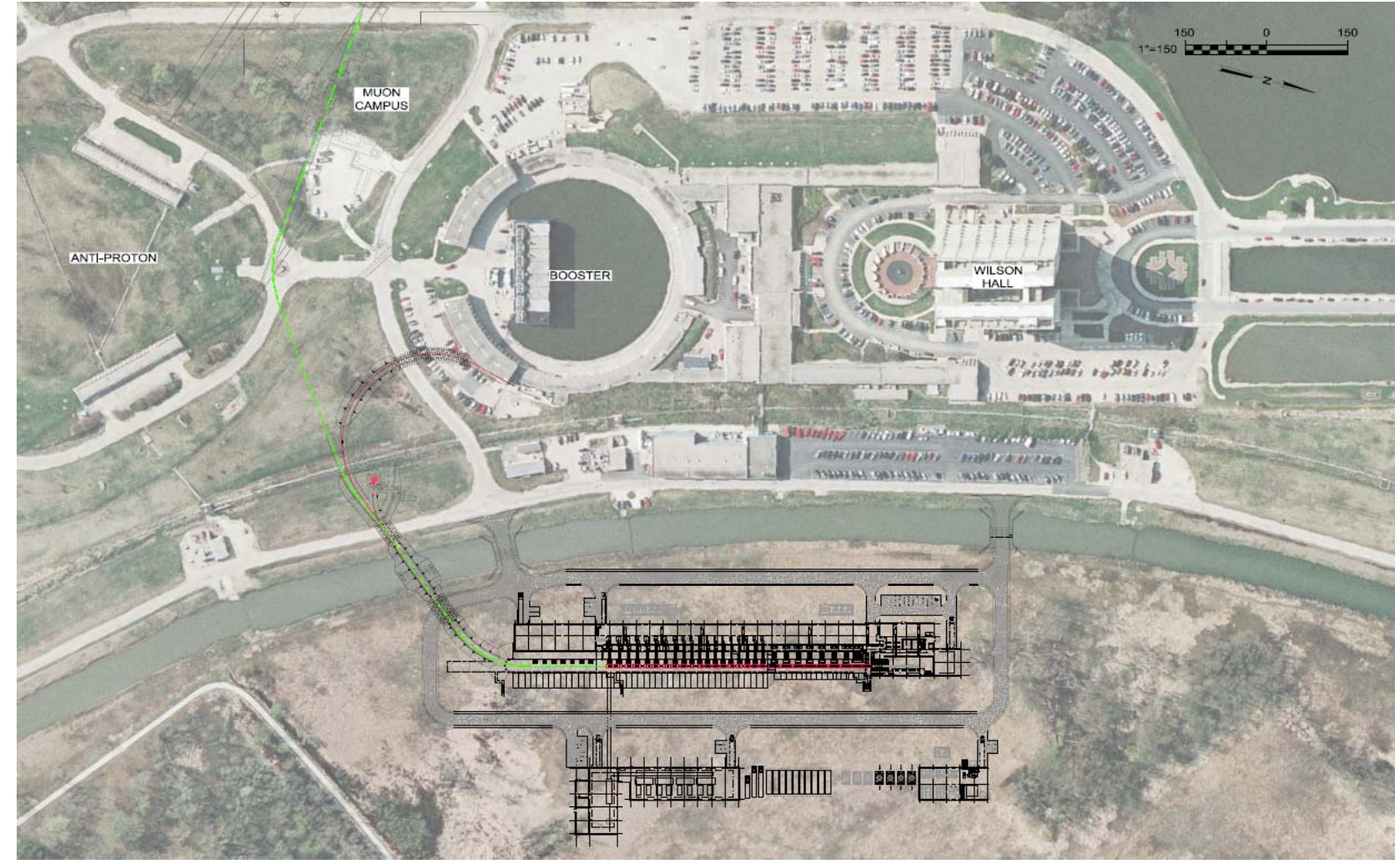

Figure A1.1: CAD drawing of the PIP-II Linac with enclosure, Transfer line to Booster (red), Beam Dump (red) and Mu2e upgrade (green) on the Fermilab site.

The beam transport from the PIP-II SC Linac to the production target is forked from the Linac-toBooster transfer line with the switch system described in Section 2.2.2. Then it is merged with the existing M4 line. Figure A1.1 presents a layout of the Linac-to-Booster Transfer Line, the Dump 
Line and the line to the Mu2e upgrade. The fast corrector of the beam switch directs the beam to the corresponding aperture of the septum magnet. The trajectory of extracted beam is symmetric to the one leading to the dump. Similar to the transport line, the Mu2e line has FODO focusing. But because of the geometrical constraints the cell length and quadrupole strengths are different from those of the Booster transport. The focusing (defocusing) quadrupoles of this line are powered in series from a different power supply than the ones in the Booster transfer line. The cell length is $11.1 \mathrm{~m}$, and the phase advances per cell are 105 and $80 \mathrm{deg}$. for horizontal and vertical planes, respectively. The corresponding strengths of the quadrupoles are about $0.27 \mathrm{~m}^{-1}$ and $-0.24 \mathrm{~m}^{-1}$ for focusing and defocusing quadrupoles, respectively $\left(\mathrm{L}=20 \mathrm{~cm}, \mathrm{G}=0.66\right.$ and $-0.59 \mathrm{kG} / \mathrm{cm}$ at $\left.E_{\text {kin }}=800 \mathrm{MeV}\right)$.

After the Lambertson magnet the transport to the Mu2e upgrade continues with a (horizontally) defocusing quadrupole and a second half of a regular FODO cell followed by another cell, with no bending dipoles. At this point there is enough horizontal separation from the Booster transfer line. The following 2 cells cross the Tevatron tunnel in the same way used for the transport to the Booster, using 4 rolled dipole magnets to raise the beam elevation near the ceiling of the tunnel. After crossing the Tevatron Tunnel the line does not come back to the elevation of the SC Linac/Booster since to reach the Mu2e upgrade area it needs to cross also MI- 8 line that transports the $8 \mathrm{GeV}$ proton beam from Booster to Recycler. The elevation of MI-8 line is around $3.23 \mathrm{~m}$ below the SC Linac, while the elevation of the beam at the crossing with the Tevatron tunnel is around $1.27 \mathrm{~m}$ above the SC Linac. The elevation of line M4 at the joining point with the Mu2e upgrade transport line is around $1.80 \mathrm{~m}$ above the SC Linac. With these geometrical constraints the choice in the design is to keep constant the elevation of the Mu2e upgrade transport after the crossing with the Tevatron tunnel until it arrives in proximity of line M4. The vertical separation with line MI-8 at the crossing is then around $4.5 \mathrm{~m}$ that should be enough to keep their enclosures separated. An engineering study needs to be performed to check the feasibility and cost of this choice. A third arc with rolled dipoles connects the Mu2e upgrade transport to M4 line.

The 2 FODO cells that cross the Tevatron tunnel have dipoles of the same family used in the Booster transport. The following 9 cells form a straight transport with no dipoles and cross line MI8 , as explained. The next 4 cells form a third arc that connects the Mu2e upgrade transport to M4 line. This arc includes 7 rolled dipoles of the same type used for the other transport lines but with a magnetic field of $2.38 \mathrm{kG}$. At the end of this arc the horizontal and vertical dispersions are both negligible. The strategy adopted for the upgrade of M4 line is to keep it as close as possible to the design of the Mu2e experiment with same element types and configurations. PIP-II He beam has smaller geometrical emittance compared to the $8 \mathrm{GeV}$ proton beam from the Booster so that keeping the same optics of the original M4 line will not require larger apertures of elements and vacuum chamber. To this end the magnetic field needs to be scaled down proportionally to the momentum of the PIP-II beam at $800 \mathrm{MeV}$ from the momentum of the Booster beam at $8 \mathrm{GeV}$.

In order to match the beam parameters to the required values along the transport the first 4 quadrupoles after the Lambertson magnet and the last 4 before the junction with line M4 have different strengths and will be each powered by a single power supply.

Figure A1.2 shows the optics functions of the entire beam transport starting from the end of the SC Linac and coming to the end of M4 line. Initially the line coincides with the SC Linac-to-Booster line but then, in the first straight, the line forks to the Mu2e upgrade line itself. 


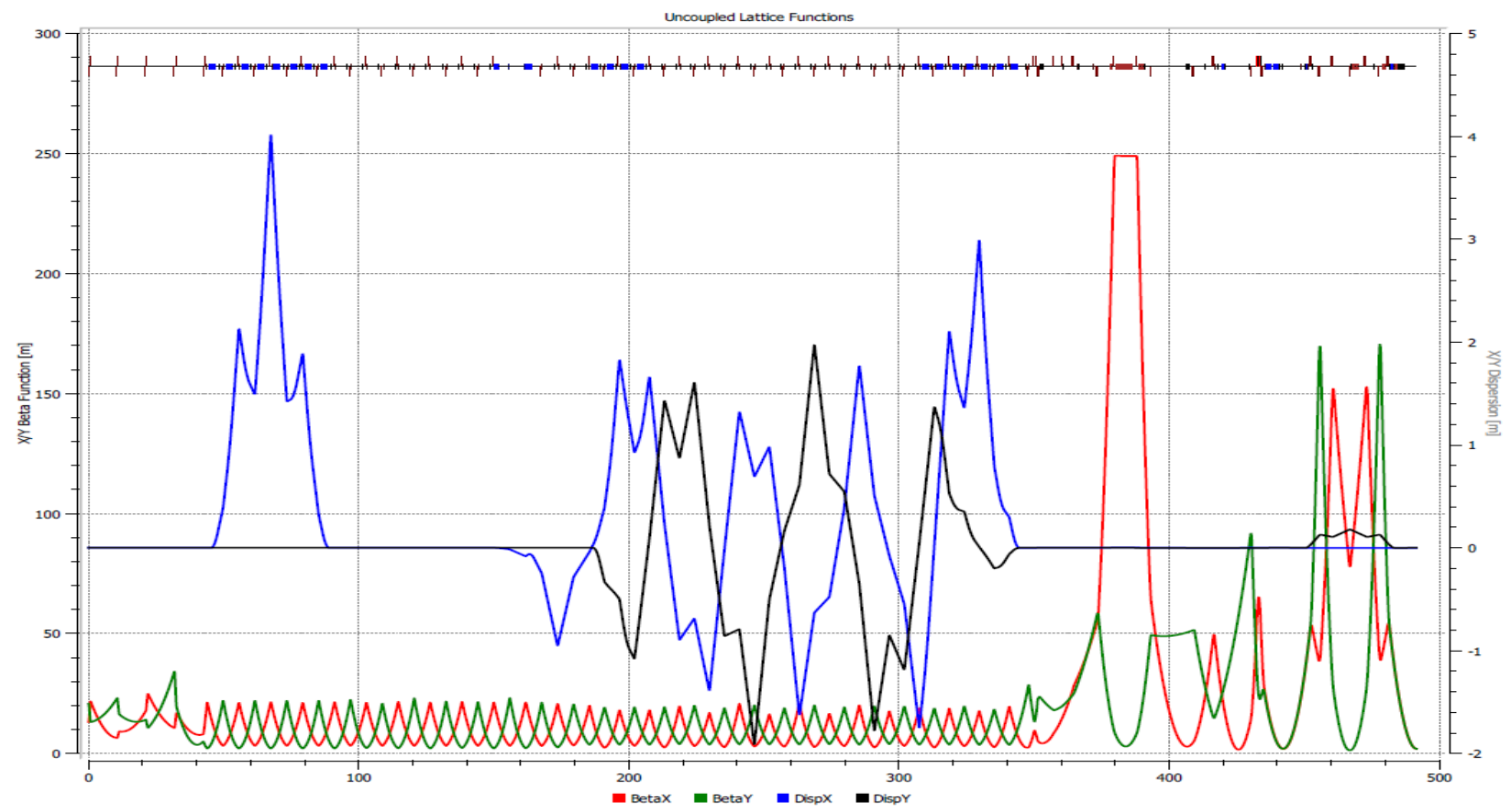

Figure A1.2: Optical functions of the beam from the end of the SC Linac to the end of line M4 when the fast corrector of the switch is set to direct the beam to the Mu2e upgrade.

\section{References to Appendix A}

[1] L. Bartoszek et al., "Mu2e Technical Design Report", arXiv:1501.05241v2 [physics.ins-det].

[2] K. Knoepfel et al., "White paper for the APS Division of Particles and Field Community Summer Study", arXiv:1307.1168v2 [physics.ins-det].

[3] V. Cirigliano et al., Phys. Rev. D80, 013002 (2009). 